\title{
FLORA CRITICA
}

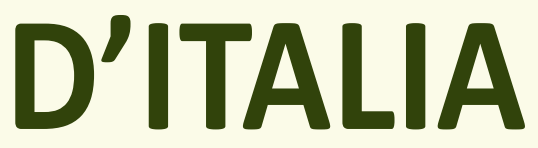

0
0
$\frac{8}{8}$
0
0
0
0

1
8
8
8
8
8
0
0

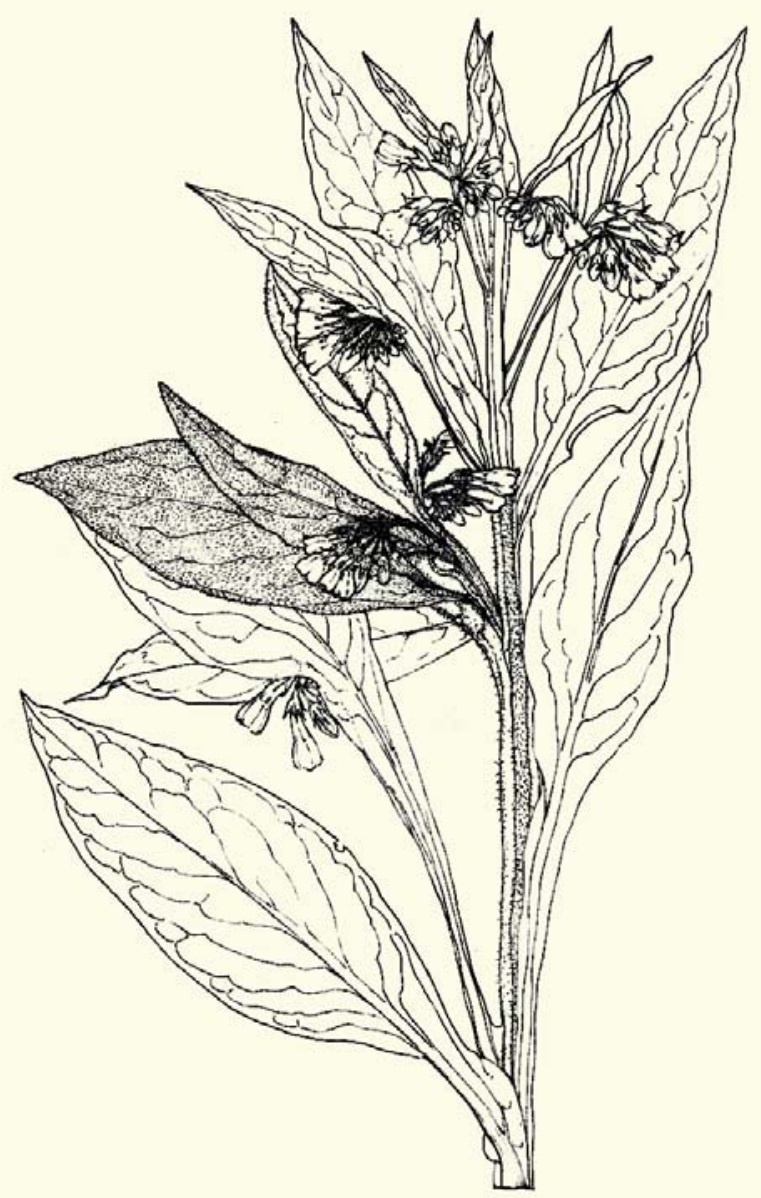

Boraginaceae - Boragineae Lorenzo Cecchi \& Federico Selvi 


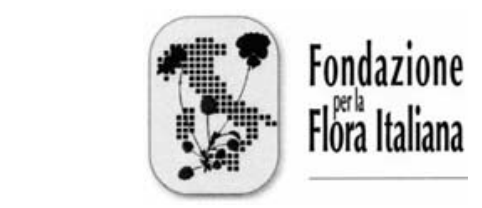

\section{FLORA CRITICA D'ITALIA}

Edito dalla Fondazione per la Flora Italiana con il supporto della Società Botanica Italiana e il contributo della Fondazione Internazionale pro Herbario Mediterraneo

\section{Comitato Editoriale}

Lorenzo Peruzzi (Pisa) (Coordinatore), Gianniantonio Domina (Palermo) (Segretario), Lorenzo Cecchi (Firenze), Giovanni Cristofolini (Bologna), Werner Greuter (Palermo), Enio Nardi (Firenze), Francesco M. Raimondo (Palermo), Federico Selvi (Firenze), Angelo Troìa (Palermo)

\section{Boraginaceae - Boragineae}

Versione 1.0, pubblicata online il 03.08.2017

Lorenzo Cecchi ${ }^{1} \&$ Federico Selvi ${ }^{2}$

${ }^{1}$ Università degli Studi di Firenze, Museo di Storia Naturale, Sezione Botanica “Filippo Parlatore", via G. La Pira 4, 50121 Firenze, Italia.

${ }^{2}$ Università degli Studi di Firenze, Dipartimento di Scienze delle Produzioni Agroalimentari e dell'Ambiente (DISPAA), Laboratorio di Botanica, piazzale delle Cascine 28, 50144 Firenze, Italia 



\section{Fondazione per la Flora Italiana}

Atto costitutivo: Palermo, Orto Botanico, 28/09/2007

Primo Consiglio di Amministrazione (2009 - 2012): Carlo Blasi, Donato Chiatante, Bruno Corrias, Giovanni Cristofolini e Francesco Maria Raimondo

Attuale Consiglio di Amministrazione: Carlo Blasi, Donato Chiatante, Giovanni Cristofolini, Enio Nardi, Lorenzo Peruzzi, Francesco Maria Raimondo, Maria Consolata Siniscalco.

(C) 2017 Fondazione per la Flora Italiana, tutti i diritti riservati

Composizione Grafica

Gianniantonio Domina, Lorenzo Cecchi \& Angelo Troia

Immagine di copertina

Symphytum officinale, disegno di L. Cecchi

Pubblicato online il 03.08.2017

su http://www.floraditalia.it

DOI: 10.17773/FI_Ita_Boragineae1.0 



\section{Boraginaceae Juss.} BORAGINACEE

Boraginaceae Juss., Gen. PI.: 128. 1789. nom. cons., non Adans., Fam. PI. 2: 173. 1763, ('Borragines') nom. illeg.

Tipo: Borago L.

(=) Anchusaceae Vest, Anleit. Stud. Bot.: 274, 302. 1818 ('Anchusoideae'). Tipo: Anchusa L.

(=) Buglossaceae Hoffmanns. \& Link, Fl. Portug. 1: 63. 1809 ('Buglossinae'). Tipo: Buglossum Mill.

(=) Cerinthaceae Martynov, Tekhno-Bot. Slovar: 120. 1820 ('Cerinthoides'); Bercht. \& J.Presl, Přir. Rostlin: 244. 1820.

Tipo: Cerinthe L.

(=) Onosmaceae Martinov in Tekhno-Bot. Slovar: 437.1820 ('Onosmoides'). Tipo: Onosma L.

(=) Echiaceae Raf., FI. Tellur. 2: 61.1837 ('Echidia'). Tipo: Echium L.

(=) Cynoglossaceae Döll, Rhein. Fl.: 406. 1843. Tipo: Cynoglossum L.

Bibliografia LÅngStRÖM \& CHASE 2002, HILGER \& al. 2004 [Boragineae], 2005, THOMAS \& al. 2008 [Lithospermeae], CECCHI \& SELVI 2009 [Lithospermeae], WEIGEND \& al. 2009, 2010a [Lithospermeae], NAZAIRE \& HUfFORD 2012, WeIGEND \& al. 2013, 2014, CHACón \& al. 2016, Weigend \& al. in KUBITZKI \& al. 2016.

DESCRIZIONE Erbe annue, bienni o perenni, più raramente arbusti o piccoli alberi (nelle regioni subtropicali), spesso diffusamente ricoperte da tubercoli o tricomi, non aromatiche. Foglie spiralate, raramente opposte (Asperugo), senza stipole. Infiorescenze terminali cimose, a maturazione progressiva, tipicamente scorpioidi, di rado corimboso-panicolate. Fiori ermafroditi, tetraciclici e generalmente pentameri, talora (Anchusella) con riduzione del numero degli stami. Calice generalmente gamosepalo attinomorfo, dialisepalo solo in Cerinthe, con sepali ineguali inseriti a livelli diversi lungo l'asse fiorale. Corolla gamopetala, spesso attinomorfa, più raramente zigomorfa (nella flora italiana nei generi Anchusella, Echium L., Lycopsis e, in parte, Cerinthe L.); invaginazioni della corolla (pliche o "squame") e peli sono spesso presenti alla fauce, con probabile funzione di organi per la presentazione secondaria del polline. Stami inseriti sul tubo corollino. Stilo lineare-subulato, ginobasico, cioè inserito alla base del gineceo, sulla stessa superficie contigua al ricettacolo (ginobase) sulla quale sono ancorate le distinte porzioni dell'ovario; stigma clavato, capitato, bilobo o tetralobo (non in Italia: Arnebia Forssk.). Ovario supero, bicarpellare, generalmente tetraloculare e tetraspermo, nettamente di- o tetralobato; frutto eremocarpico, cioè suddiviso in 2-4 nucule di- o monosperme (mericarpi) separate dalla ginobase e recanti una chiara cicatrice in corrispondenza della superficie di attacco. Germinazione epigea.

NUMERI Nonostante il numero cromosomico sia noto ad oggi per non più del $35 \%$ dei CROMOSOMICl taxa descritti per la famiglia, e per specie e sottospecie in larga maggioranza 
europee e nordamericane, la gamma di variazioni del complemento cromosomico risulta assai ampia (Weigend \& al. in KUBITZKI \& al. 2016). I numeri cromosomici di base più comuni sono $x=6,7$ e 8 ; più raramente si riscontrano valori di $x=4,9$ o 10, o altri ritenuti di origine secondaria: $x=11,12,13,14,15$, 17 e 19. Frequente è la formazione di complessi poliploidi, sia all'interno di generi che, più raramente, all'interno della stessa specie, che in tal modo si trova a includere diversi citotipi; altrettanto ben documentata è la tendenza alla disploidia discendente o la presenza di B-cromosomi. Tra le specie italiane, il numero cromosomico più basso, $2 n=12$, è condiviso da alcune specie diploidi di Onosma L., mentre quello più elevato, $2 n=144$, è presente in alcuni citotipi di Symphytum tuberosum, interpretabile sia come dodecaploide in base 12 (GRAU 1968b, 1971) che come 18-ploide in base 8 (MURín \& MÁJOVSKI 1982).

BIologia Piante quasi esclusivamente entomofile, di rado ornitofile (alcune specie di RIPRODUTTIVA Lithospermum L. americane) o cleistogame (alcune popolazioni di Neatostema apulum (L.) I.M.Johnst., ma carattere non fissato geneticamente; oss. pers.); prevale l'allogamia, spesso associata a polimorfismo stilare (ercogamia); sono altresi molto diffusi anche i meccanismi di autofecondazione. Dispersione dei semi principalmente per esozoocoria, grazie alla presenza di strofioli lipidici alla base dei mericarpi ("elaiosomi") attrattivi per le formiche (mirmecocoria: la maggior parte delle Boragineae), o di appendici uncinate (glochidie) atte ad aderire al vello dei mammiferi o al piumaggio degli uccelli e variamente distribuite sulla superficie dei mericarpi o su quella esterna del calice caduco (nei generi di Boragineae sudamericane Moritzia DC. ex Meisn. e Thaumatocaryon Baill. e nella maggior parte delle Cynoglosseae); più raramente si ha dispersione per anemo- o idrocoria.

DISTRIBUZIONE Famiglia che annovera circa 85 generi e 1600-1700 specie, a distribuzione pressochè cosmopolita, ma con ampie lacune distributive nelle regioni a clima equatoriale e tropicale umido; i suoi principali centri di diversità specifica si trovano nelle aree a clima sub-tropicale o temperato arido, cioè nelle regioni meridionali del regno olartico (sottoregno tetidico, regioni mediterranea e irano-turanica) e nel regno capense, ma importanti aree di più recente differenziazione si hanno anche in Nord- e Sudamerica, Australia e Africa tropicale. Si contano in Italia 31 generi, 26 dei quali con almeno una specie autoctona.

\section{NOTE}

SISTEMATICA In contrasto con APG $(2009,2016)$, ma in linea con l'aggiornamento già acquisito sull'APG website (STEVENS 2016) e con le più recenti sinossi edite dal Boraginales Working Group (boraginales.myspecies.info/; CHACón \& al. 2016, LUEBERT \& al. 2016), la famiglia è qui trattata in senso stretto, separatamente da altri gruppi di pari rango nell'ambito dell'ordine Boraginales.

Si tratta di una scelta diversa da quella cautelativamente adottata nella prima sinossi tassonomica per questa Flora (CECCHI \& SELVI 2014), indotta dalla sempre più approfondita conoscenza della filogenesi dell'ordine Boraginales Juss. ex Bercht. \& J. Presl (WEIGEND \& al. 2014). Secondo la maggior parte degli specialisti del gruppo, il sistema di più famiglie è infatti l'interpretazione più appropriata e coerente col grado di effettiva separazione evolutiva tra i diversi gruppi (Fig. 1). Le Boraginaceae vengono a loro volta suddivise in tre linee monofiletiche fondamentali, corrispondenti alle sottofamiglie Echiochiloideae Weigend, Boraginoideae e Cynoglossoideae Weigend (con 8 tribù che da sole comprendono oltre l'80\% delle specie note; CHACón \& al. 2016; Fig. 2); nella flora italiana si trovano rappresentanti di 7 differenti tribù, riferibili alle sole sottofamiglie 
Boraginoideae e Cynoglossoideae.

PROPRIETÀ E USI Le parti vegetative della maggior parte delle Boraginaceae contengono elevate quantità di polisaccaridi complessi (mucillagini) che trattengono l'acqua e conferiscono loro notevoli capacità reidratanti, emollienti e depurative; diverse specie sono note nella medicina tradizionale per la cura di febbri e processi infiammatori, se non per quella di specifiche patologie, infezioni o forme di avvelenamento. L'olio ricavato dai semi di alcune specie è un eccellente integratore ricco di acidi grassi poliinsaturi. Dai succhi rossi delle radici di alcune Boraginaceae si ottengono tinture naturali per tessuti e prodotti cosmetici. In cucina, i fiori di alcune specie sono impiegati per arricchire le insalate o per confezionare canditi e confetture, mentre le foglie si possono consumare cotte, in purezza o mescolate ad altre verdure, per conferire a contorni, salse o ripieni una migliore consistenza e un sapore delicato ma caratteristico, simile a quello del cetriolo. La presenza di alcaloidi pirrolizidinici epatotossici, neurotossici e potenzialmente cancerogeni, tuttavia, ne suggerisce un uso saltuario e moderato, ed ha comportato la rimozione dal commercio di una parte consistente dei prodotti farmaceutici tradizionalmente ottenuti da queste piante. Molte specie di questa famiglia, infine, per il colore vivace dei fiori sono coltivate nei giardini e talora riprodotte e selezionate in cultivar ornamentali, ovvero impiegate dagli apicoltori come piante mellifere.

ChIAVE Delle 1. Mericarpi più o meno appiattiti dorso-ventralmente, spesso glochidiati o SOTTOFAMIGLIE alati, raramente privi di tali ornamentazioni ma in tal caso in piante con foglie apparentemente opposte e calice accrecente in due segmenti bratteiformi (Asperugo) o con infiorescenze lungamente o completamente ebratteate (Amsinckia, Myosotis) sottofamiglia Cynoglossoideae

- Mericarpi ovoidi o trigoni, non appiattiti dorso-ventralmente, mai glochidiati o alati, con infiorescenze spesso regolarmente bratteate sottofamiglia Boraginoideae 


\section{Boraginoideae Arn.} BORAGINOIDEE

Boraginoideae Arn., Encycl. Brit. 122. 1832 ('Borageae').

Tipo: Borago L.

(=) Echioideae Burmeist., Handb. Naturgesch.: 272. 1837 ('Echieae').

Tipo: Echium L.

DESCRIZIONE Fiori generalmente grandi e con pliche faucali prominenti. Ginobase piatta. Nucule da ovoidali a triangolari-ovoidi (trigone), lisce o tubercolate, mai glochidiate né alate.

DISTRIBUZIONE Gruppo a gravitazione tetidica, con radiazioni secondarie in Asia orientale e nelle Americhe. Entrambe le tribù (Boragineae e Lithospermeae Dumort.) sono ampiamente rappresentate in Italia.

\section{NOTE}

SISTEMATICA Nonostante i modesti caratteri diagnostici e sinapomorfici, la relazione filogenetica tra Boragineae e Lithospermeae è stata ripetutamente confermata dalle analisi molecolari (CHACON \& al. 2016; Fig. 2).

ChIAVE delle Tribù 1. Mericarpi provvisti alla base di un cercine più o meno ispessito e in genere di un elaiosoma sporgente tribù Boragineae

- Mericarpi senza cercine basale né elaiosoma tribù Lithospermeae 
Boragineae Rchb. BORAGINEE

Boragineae Rchb., Fl. Germ. Excurs. 1(3): 340. 1831.

Tipo: Borago L.

= Symphyteae D.Don, Edinburgh New Philos. J. 13: 239. 1832.

Tipo: Symphytum L.

= Anchuseae W.D.J.Koch, Syn. Fl. Germ. Helv.: 497. 1837.

Tipo: Anchusa L.

DESCRIZIONE Erbe annue o perenni, ispide o sericee. Foglie alterne. Cime generalmente bratteate. Pliche faucali in genere presenti e ben sviluppate, raramente poco rilevate (Borago subgen. Buglossites) ovvero sostituite da bande di tricomi (Pulmonaria). Stigma troncato o capitato, raramente bilobo o debolmente bifido. Nucule ovoidi, erette o ricurve, più o meno compresse lateralmente e carenate ventralmente, con base stipitata (Moritzia, Pentaglottis, Thaumatocaryon) o più comunemente allargata in un cercine ispessito; cicatrice basale più o meno circolare, dotata di uno strofiolo centrale (elaiosoma) ricco di sostanze lipidiche; pericarpo non calcificato.

DistRIBUZIONE Attenendosi al trattamento tradizionale di Anchusa s.l. (si veda però la nota nella scheda relativa al genere) le Boragineae comprendono almeno 17 generi (Fig. 3), i due più precocemente divergenti (Moritzia e Thaumatocaryon) endemici del regno neotropico, i restanti 15 del Vecchio Mondo, con la maggior parte delle specie distribuite tra le regioni del sottoregno tetidico e un piccolo contingente di rappresentanti nelle regioni africane sudano-zambesiana (orientale) e capense. In Italia sono rappresentati con certezza 12 dei 13 generi presenti in Europa (tutti coll'eccezione di Gastrocotyle Bunge), con un totale di 36 specie, delle quali 29 autoctone e 7 esotiche. Delle 36 totali, tuttavia, solo 29 (26 autoctone e tre esotiche) si trovano stabilmente sul nostro territorio, mentre sette ( 3 probabilmente a margine dell'areale naturale e 4 esotiche) vi compaiono o vi sono comparse solo occasionalmente senza costituire popolamenti stabili per più di 25 anni. Il rappresentante di un tredicesimo genere (Trachystemon orientalis) e una quinta specie di Nonea ( $N$. rosea) vanno al momento esclusi dalla flora italiana, in quanto le sole testimonianze accertate della loro presenza allo stato spontaneo in Italia si riferiscono a effimeri popolamenti contigui a piante mantenute in coltivazione, senza che vi siano prove effettive di una loro reale emancipazione dalla coltura.

ChIAVE DEI GenerI 1. Fiori lungamente peduncolati (fino a $3 \mathrm{~cm}$ ); antere mucronate e filamenti all'apice con appendice acuta o subrotonda

3. Borago

- Fiori brevemente peduncolati (massimo $1 \mathrm{~cm}$ ) o sessili; antere non mucronate, filamenti senza appendice ..... 2

2. Corolla tubuloso-campanulata con lobi molto brevi; stilo sporgente; squame lanceolate e acute 4. Symphytum

- Corolla ipocrateriforme, infundibulare o rotata, con lembo più o meno allargato e lobi lunghi almeno $3-4 \mathrm{~mm}$; stilo incluso; squame brevi e più o meno arrotondate ... 3

3. Mericarpi con cercine basale poco sviluppato, inseriti sul ricettacolo attraverso un breve stipite eccentrico 
- Mericarpi con cercine ben sviluppato, senza stipite 4

4. Corolla rotata con tubo breve (ca. $2 \mathrm{~mm}$ ) e lembo piatto o quasi; squame faucali papillose 5

- Corolla ipocrateriforme o infundibulare con tubo lungo ( $\geq 5 \mathrm{~mm}$ ); squame faucali con tricomi allungati

5. Specie rizomatose; infiorescenze di cime ebratteate 2. Brunnera

- Specie non rizomatose; infiorescenze di cime bratteate 9. Cynoglottis

6. Corolla zigomorfa con lembo inclinato 7

- Corolla attinomorfa con lembo piano 8

7. Infiorescenze di cime ramificate, mai arrossate; tubo corollino piegato a S; stami fertili 5; stigma bilobo 10. Lycopsis

- Infiorescenze di cime semplici, arrossate; tubo corollino diritto, lembo incurvato; stami fertili 2; stigma bifido

11. Anchusella

8. Infiorescenze con fiori sessili, capitato-aggregate, contratte anche nel frutto e con lunghe brattee patenti; mericarpi a forma di elmetto con fessura laterale e cercine basale plicato-dentato 8. Hormuzakia

- Infiorescenze con fiori peduncolati, allungate e più o meno lasse in frutto; mericarpi senza fessura laterale e con cercine arrotondato, rugoso, non dentato

9. Squame almeno tanto lunghe che larghe, occludenti e sporgenti dalla fauce 12. Anchusa

- Squame brevi, più o meno pelose ma non occludenti nè sporgenti dalla fauce 10

10. Squame prolungate longitudinalmente all'interno del tubo in 5 linee di peli; stami inseriti alla base del tubo corollino; stilo ca. $1 \mathrm{~mm}$ 5. Melanortocarya

- Squame non prolungate in linee longitudinali di peli dentro il tubo; stami inseriti a metà o più in alto nel tubo corollino; stilo lungo almeno $4 \mathrm{~mm}$ 11

11. Mericarpi rugoso-reticolati, trasversalmente ovoidi con becco laterale (oblungo-eretti in N. lutea); fauce con 5 distinte squame più o meno pelose; specie annue o perenni

- Mericarpi lisci, ovoido-eretti; fauce con anello continuo di peli; specie sempre perenni 


\section{Pentaglottis Tausch 0 PENTAGLOTTIDE}

Pentaglottis Tausch, Flora 12(2): 643. 1829.

(三) Caryolopha Fisch. \& Trautv., Ind. Sem. Hort. Petrop. 3: 31. 1837.

Tipo (cfr. TAUSCH 1829: 643; Fischer \& Trautvetter in FISCHER \& al. 1837: 32): $P$. sempervirens (L.) Tausch.

Biblografia SelVI \& BigazZI 1998.

EtIMologia Dal greco $\pi \varepsilon ́ v t \varepsilon$ (pente), "cinque", e $y \lambda \tilde{\omega} \tau \tau i \varsigma$ (glottis), "ligulato" (a sua volta da $\gamma \lambda \tilde{\omega} \sigma \sigma \alpha$, glossa, "lingua"), "a cinque lingue", per le vistose pliche faucali sporgenti al centro del lembo corollino.

DESCRIZIONE Erbe perenni, ispide. Foglie basali ovate e picciolate, le cauline progressivamente ridotte e presto sessili. Cime brevi e contratte, bratteate, singole o appaiate. Calice profondamente diviso, accrescente alla fruttificazione. Corolla a lembo rotato, con pliche molto prominenti, ma fauce pervia. Stami brevi, inseriti in alto nel tubo corollino, ma inclusi. Mericarpi ovoidi a orientamento riflesso, cioè curvati rispetto ad uno stipite che decorre lungo tutta la faccia adassiale, separandosene brevemente in prossimità della superficie basale di attacco.

BIologIA Piante allogame entomofile. La dispersione dei semi avviene per mirmecocoria. RIPRODUTTIVA

DISTRIBUZIONE Genere monotipico, endemico europeo, la cui sola specie è autoctona nelle provincie iberica e atlantica meridionale, frequente altrove in coltivazione e talora naturalizzata.

\section{NOTE}

SISTEMATICA Genere monotipico separatosi precocemente dal clado che include le altre Boragineae del Vecchio Mondo (HILGER \& al. 2004, WEIGEND \& al. 2010a), superficialmente simile ad Anchusa, cui era un tempo assimilato, ma distinto da esso sia da evidenti caratteri macromorfologici (in particolare i mericarpi stipitati eccentricamente alla base, carattere ancestrale condiviso con gli americani Moritzia e Thaumatocaryon, e la corolla con tubo corollino assai breve), palinologici e micromorfologici, che dal singolare corredo cromosomico. Simile a Brunnera per le foglie e la corolla con tubo breve e lembo rotato.

\subsection{Pentaglottis sempervirens (L.) Tausch O PENTAGLOTTIDE}

Pentaglottis sempervirens (L.) Tausch in Flora 12(2): 643. 1829.

(三) Anchusa sempervirens L., Sp. PI. 1: 134. 1753 三 Caryolopha sempervirens (L.) Fisch. \& Trautv., Ind. Sem. Hort. Petrop. 3: 32. 1837 三 Buglossum sempervirens (L.) All., Fl. Pedem. 1: 48. 1785.

Locus classicus: "in Anglia, Hispania".

Lectotipo (SELVI \& BIGAZZI 1998: 129): [pianta coltivata in Germania o in Francia] "Lipsiae in horto Deurlingi, Lutetiae in horto Medico", s.d., s.coll., Herb. Burser 
Vol. XIV(2): 21 (UPS-BURSER!).

Altro materiale originale (CECCHI \& SELVI 2015b: 632): [pianta coltivata nei Paesi Bassi], s.d., s.coll., Herb. Clifford 47.1 (BM 557915!).

EtIMOLOGIA Dal latino sempervirens, sempreverde, per l'habitus perenne che la distingue da altre specie incluse da LINNEO (1753) in Anchusa.

DESCRIZIONE Fusti eretti di 3-10 dm; indumento di dense setole patenti, lunghe fino a 1,5 $\mathrm{mm}$, miste a peli più brevi. Foglie strettamente ovate, acute, con margini interi o leggermente crenato-ondulati, le basali con lamina ampia fino a $10 \times 35 \mathrm{~cm}$, decorrente su un picciolo lungo fino a $15 \mathrm{~cm}$, le cauline a base cuneata. Cime pauciflore (5-15 fiori), inserite all'ascella di foglie cauline lungo la parte superiore del fusto, le terminali, appaiate all'ascella di foglie opposte; brattee nettamente minori delle foglie cauline, lunghe più o meno quanto il calice. Calice 3-5 mm all'antesi, fino a $8 \mathrm{~mm}$ nel frutto, diviso fin quasi alla base in lobi lineari-lanceolati. Corolla con tubo bianco, allargato verso l'alto, lungo circa 4 $\mathrm{mm}$, e lembo blu intenso, raramente più chiaro, con toni violacei solo prima della maturazione, con lobi arrotondati e diametro di $8-10 \mathrm{~mm}$; pliche faucali papillose. Stami con filamento subnullo e antere raggiungenti le pliche faucali. Mericarpi di 1,5-2 $\mathrm{mm}$, con cercine incospicuo e superficie nerastra, minutamente papillosa. (Fig. 4)

FENOLOGIA II solo saggio italiano recante una data di raccolta è quello recente del Comasco, di un esemplare in piena fioritura raccolto all'inizio di maggio; le osservazioni su esemplari sia naturali che coltivati indicano che la specie nei nostri climi fiorisce per gran parte della primavera, tra aprile e giugno, e matura i frutti a partire da maggio, fino ad estate inoltrata.

NUMero $2 n=22$, riscontrato su popolazioni spagnole (ElenA-Rosselló \& al. 1987, LUQUe CROMOSOMICO 1989).

ECologia Boschi, argini, siepi, luoghi freschi e ombrosi a margine dei coltivi o delle strade, su substrato generalmente acido; presente nelle aree di origine tra il livello del mare e la media montagna (fino a $1600 \mathrm{~m}$ ); in Italia è stata trovata solo a quote collinari, tra 250 e 500 m circa.

DISTRIBUZIONE Specie europea occidentale, originaria della provincia iberica e della parte continentale di quella atlantica, diffusa anche più a nord e a ovest, fino alle isole britanniche e all'Europa centrale, ma probabilmente in seguito a naturalizzazione da piante coltivate. In Italia (Fig. 5) la sua presenza è documentata solo da rare raccolte in Lombardia e Veneto, a stento compatibili con una naturale rarefazione al margine orientale dell'areale, più probabilmente interpretabili come popolamenti alloctoni ed effimeri.

\section{NOTE}

ALTRI NOMI VOLGARI Buglossa falsa borrana, buglossa ovata.

Distribuzione Le antiche segnalazioni per il Piemonte (ALLIONI 1785: 48), sono frutto di confusione con Anchusa azurea (Colla 1835, oss. pers.). 


\section{Brunnera Steven 0 BRUNNERA}

Brunnera Steven in Bull. Soc. Imp. Naturalistes Moscou 24(1): 582. 1851.

Tipo (CECCHI \& SELVI 2015b: 632): B. macrophylla (Adams) I.M.Johnst.

Etimologia Genere dedicato allo svizzero Samuel Brunner (1790-1844), botanico ed esploratore, che nella prima metà dell' 800 raccolse piante, tra l'altro, sul Caucaso e in Siberia.

DESCRIZIONE Erbe perenni rizomatose, pubescenti o ispide per indumento dimorfo di setole rade miste a peli brevi più densi. Foglie ovate o cuoriformi, a margine intero o debolmente ondulato, le basali grandi e picciolate, le cauline più o meno gradualmente ridotte, sessili. Cime ebratteate, pauciflore, riunite in infiorescenze composte all'apice del fusto o singolarmente inserite all'ascella di foglie cauline; fiori lungamente peduncolati. Corolla con tubo quasi bianco, breve, incluso nel calice, e lembo rotato azzurro o blu, diviso in lobi arrotondati; pliche faucali prominenti ma non occludenti. Antere subsessili. Stilo breve, incluso. Mericarpi in numero ridotto per aborto, ovoidi o globosi, eretti, con superficie rugoso-costoluta, cercine marcato e strofiolo sporgente.

Numero $2 n=12$, comune a tutte le specie note (BIGAZZI \& SelVI 2001, StePANOV 1994); per CROMOSOMICO $B$. sibirica sono inoltre riportati diversi corredi derivati, sia poliploidi che aneuploidi $(2 n=14,24,36,72$; MALAKHOVA 1990, Stepanov 1994, Stepanov \& MURATOVA 1995).

Bıologia Piante allogame, entomofile. Dispersione dei semi per mirmecocoria; RIPRODUTTIVA propagazione vegetativa per rizoma.

DISTRIBUZIONE Tre specie, una nella regione irano-turanica occidentale e due nella circumboreale asiatica; una di queste ultime, coltivata anche nei giardini italiani a scopi ornamentali, si è recentemente naturalizzata in Toscana.

\subsection{Brunnera macrophylla (Adams) I.M.Johnst. O BRUNNERA DEL CAUCASO}

Brunnera macrophylla (Adams) I.M.Johnst. in Contr. Gray Herb. 73: 541924.

(三) Myosotis macrophylla Adams in Beitr. Naturk. (Weber \& Mohr) 1: 46. $1805 \equiv$ Anchusa myosotidiflora Lehm., PI. Asperif. Nucif. 234. 1818 三 B. myosotidiflora (Lehm.) Steven in Bull. Soc. Imp. Naturalistes Moscou 1: 582. 1851, nom illeg. Locus classicus: [Georgia] "in Iberiae sylvaticis, umbrosis". Lectotipo (CECCHI \& SELVI 2015b: 632): [Georgia] "ex Iberia", s.d., s.coll. (MW!). Altro materiale originale (CECCHI \& SELVI 2015b: 632): [Georgia] "in valle Ananuriae Iberiae", s.d., Adams (B-W 3274!).

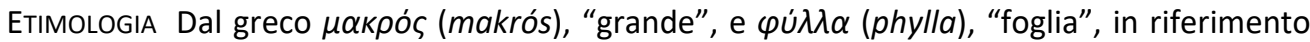
alle foglie basali molto ampie, rispetto a quelle di ogni altro membro del genere Myosotis, entro il quale la specie fu originariamente descritta per l'aspetto dei fiori. 
DESCRIZIONE Erba perenne rizomatosa, con fusti eretti di $20-80 \mathrm{~cm}$, brevemente pubescente e scabra per peli corti appressati, con brevi setole più lunghe sui fusti e i margini fogliari. Foglie basali lungamente picciolate, a lamina ampia e cuoriforme, $15 \times$ $20 \mathrm{~cm}$, le cauline nettamente minori, progressivamente ridotte verso l'alto, ovato-lanceolate, sessili. Cime con fiori su peduncoli sottili di 2-4 mm, alla fruttificazione allungati fino a $1 \mathrm{~cm}$ o più, patenti o deflessi. Calice piccolo, 1,5 $\mathrm{mm}$, diviso più o meno fino a metà in lacinie triangolari-lanceolate. Corolla di 3$5 \mathrm{~mm}$ di diametro, con lembo blu; pliche faucali molto rilevate, bianche e pubescenti. Mericarpi subglobosi di circa $3 \mathrm{~mm}$, con superficie bruno-scura, percorsa da creste longitudinali marcate e strofiolo molto sporgente di 1-1,5 mm. (Fig. 6)

FENOLOGIA I pochi saggi disponibili dall'unica stazione italiana sono di esemplari raccolti in giugno e recanti sia fiori che frutti maturi; le osservazioni su esemplari in coltivazione e i dati diffusi tra i floricoltori suggeriscono trattarsi di specie a ciclo riproduttivo relativamente breve, nei nostri climi a fioritura tardo primaverile, con rilascio di nucule mature dalla fine di maggio e prolungato non oltre i primi di luglio.

Numero $2 n=12$, su popolazioni autoctone in Georgia (DAVLIANIDZE 1985) e Turchia CROMOSOMICO (BIgAZZI \& SELVI 2001) e su piante coltivate (SMITH 1932); i conteggi 2n = 8, 48 riportati per piante coltivate (STREY 1931) o dell'estremo oriente russo (Probatova \& al. 2001) sono incerti, il secondo quasi certamente attribuibile all'affine $B$. sibirica Steven, che mostra maggiore variabilità cariotipica.

ECologIA Margini e radure in boschi umidi montani, attorno ai $900 \mathrm{~m}$.

DISTRIBUZIONE Pianta di origine pontico-caucasica, coltivata per ornamento nelle regioni a clima temperato e localmente naturalizzata in Europa, Nordamerica e Nuova Zelanda. In Italia (Fig. 7) si è solo recentemente spontaneizzata sull'Appennino Tosco-Romagnolo nei pressi di Badia Prataglia, ov'è ancora rara e localizzata ma in costante espansione (FrIGNANI \& al. 2006) e nel parco di Villa d'Este a Tivoli (IAMONICO \& al. 2014).

NOTE

NomenclatURA Benchè nel protologo di Anchusa myosotidiflora sia esplicito il rimando alla specie già descritta da Adams come Myosotis macrophylla, la creazione di un secondo nome è giustificata per evitare il conflitto con $A$. macrophylla Desf.; è invece illegittima la sua ricombinazione in Brunnera, visto che in tale ambito la priorità dell'epiteto creato da Adams torna ad essere l'unico criterio valido. 


\section{Borago L.} BORRAGINE

Borago L., Sp. PI. 1: 137. 1753 [Gen. PI., ed. 5: 67. 1754.]

Tipo (BRITTON \& BROWN 1913: 92-93, confermato da Hitchcock in HITCHCOCK \& GREEN 1929: 128): B. officinalis L.

(=) Buglossites Moris, Index Seminum (Torino): 32. 1845.

Tipo (cfr. MoRIs 1845: 32): Buglossites laxiflorus (Lam. ex DC.) Moris, ('laxiflora') [Borago pygmaea (Lam. ex DC.) Chater \& Greuter].

BibliografiA SelVI \& al. 2006a.

Etimologia Nome di antica origine, già in uso nel Latino medievale, secondo alcuni derivante da una forma dialettale dell'arabo قرع أبــ (abū 'araq), letteralmente "fonte di sudore", in virtù dell'uso di $B$. officinalis come diaforetico; secondo altri dal latino borra, veste o tessuto ruvido, per l'aspetto e la consistenza delle foglie; secondo altri ancora addirittura dal celtico barrach, uomo coraggioso, per I'uso del vino di borragine come bevanda propiziatoria alla battaglia da parte dei guerrieri di quel popolo.

DESCRIZIONE Erbe annue o perenni, ispide per setole robuste e tubercolate. Foglie sopra verde-scuro, con lamina scabra e rugoso-bollosa, a margini eroso-ondulati, sotto più chiare, con nervature prominenti, le basali con lamina ovata o oblunga e picciolo più o meno distinto, le cauline e bratteali minori e progressivamente ridotte, finalmente sessili, a base cordata o brevemente decorrente sul fusto. Infiorescenza con cime molto lasse, bratteate, molto distese alla fruttificazione; fiori su lunghi peduncoli incurvati nella porzione distale, i fruttiferi decisamente deflessi; brattee progressivamente ridotte, le inferiori spesso raggiungenti o superanti il calice, le superiori più brevi del peduncolo. Calice diviso fin quasi alla base in lacinie triangolari-acuminate, accrescente nel frutto. Corolla con tubo brevissimo o subnullo e lembo campanulato (subgen. Buglossites) oppure rotato-riflesso (sottogenere Borago), diviso in lobi ovati; fauce con o senza pliche faucali (rispettivamente, subgen. Borago e subgen. Buglossites). Stami inseriti presso la base del tubo, con filamenti dilatati in una base sacciforme dalla quale emerge una lunga appendice abassiale; antere conniventi e appressate allo stilo, mucronate. Stimma capitato. Nucule ovoidi, erette, a superficie scura, rugoso-reticolata, e cercine ispessito.

NUMERI Nonostante si tratti di un piccolo gruppo, dall'analisi cariologica di quattro delle CROMOSOMICI cinque specie note emerge un'ampia variabilità nei corredi cromosomici: $2 \mathrm{n}=$ $12,16,18,30,32,48$, con numeri aploidi $n=6,8,9,15$. Si ritiene più plausibile (Selvi \& al. 2006a) che dal numero cromosomico di base ancestrale $p=8$, condiviso con molti membri della stessa tribù e ancora riscontrabile in entrambi i sottogeneri in assetto sia diploide che tetra- ed esaploide $(2 n=16,32,48)$, siano derivati per disploidia discendente e ascendente tutti i restanti numeri. Meno probabile l'origine da $p=6$, condiviso con pochi altri gruppi di antica origine, tra i quali Brunnera, e oggi noto solo per una specie (B. trabutii Maire) con chiari caratteri derivati.

BIologiA Piante entomofile, allogame ma generalmente autocompatibili.

RIPRODUTTIVA Distribuzione dei semi per mirmecocoria, nelle specie sciafile del subgen. Buglossites, che crescono spesso in luoghi stillicidiosi o soggetti al periodico 
scorrimento di acque meteoriche, integrata da idrocoria.

DISTRIBUZIONE Genere subendemico della regione mediterranea, a gravitazione centrooccidentale, con quattro specie relativamente rare e localizzate, endemiche di aree relativamente ristrette nelle provincie marocchina meridionale, mediterranea meridionale e liguro-tirrenica, e una quinta ( $B$. officinalis) a più ampia distribuzione, spesso sinantropica, diffusa in coltivazione o come avventizia anche ben oltre i limiti del suo areale originario, nella regione circumboreale europea, in Nordamerica e altrove.

\section{NOTE}

SISTEMATICA Genere probabilmente di antica origine, le cui relazioni non sembrano ancora del tutto accertate; appare filogeneticamente vicino Symphytum (HILGER \& al. 2004) ma con probabili, strette relazioni anche con altri generi precocemmente divergenti nella tribù, quali Brunnera, Trachystemon D.Don e Pentaglottis (WEIGEND \& al. 2010a), con ciascuno dei quali mostra sinapomorfie più o meno evidenti. Si presenta diviso in due linee evolutive distinte di antica separazione, corrispondenti a due sottogeneri che per le notevoli differenze non sarebbe inopportuno trattare alla stregua di generi distinti: Buglossites e Borago. II primo, verosimilmente ancestrale, comprende le specie perenni a corolla campanulata, endemiche del sistema sardo-corso, ove sono legate ad ambienti umidi e ombrosi. II secondo, con caratteri tipicamente derivati, include invece le specie annuali a corolla con lembo rotato o riflesso, presenti in Nordafrica e nel resto dell'areale, generalmente eliofile e mediamente più tolleranti l'aridità.

ChIAVE Delle SPECIE 1. Erba annua con robusti fusti eretti; corolla ipocrateriforme con pliche faucali prominenti, trapezoidali, lembo rotato e lobi di $6 \times 13 \mathrm{~mm}$; mericarpi di 6-10 mm (subgen. Borago)

2. B. officinalis

- Erba perenne con esili fusti prostrati o ascendenti; corolla da campanulata a quasi cilindrica, senza pliche faucali prominenti, con lobi di 1-3 mm; mericarpi di 2-4 mm (subgen. Buglossites)

2

2. Corolla campanulata, azzurra, lunga il doppio del calice $(9-11 \mathrm{~mm})$; appendice del filamento staminale tridentata all'apice; mericarpi 2-3,5 mm

3. B. pygmaea

- Corolla strettamente campanulata o quasi cilindrica, bianca o con lievissime sfumature azzurre, subeguale al calice (3-4 $\mathrm{mm}$ ); appendice staminale con punta semplice; mericarpi 1,7-2 $\mathrm{mm}$ 1. B. morisiana

\subsection{Borago morisiana Bigazzi \& Ricceri BORRAGINE DI MORIS}

Borago morisiana Bigazzi \& Ricceri in Webbia 46(2): 192. 1992.

Locus classicus: [Italia, Sardegna] "Isola di San Pietro (Sardegna sudoccidentale); Calavinagra. Fosso umido con fondo sabbioso a circa 50-100 m dal mare [...] Flumini major [...] in irriguis montium"

Olotipo (cfr. BIGAZZI \& RICCERI 1992: 194): [Italia, Sardegna] "Isola di San Pietro (Sardegna sud-occidentale); Calavinagra. Fosso umido con fondo sabbioso a circa 50-100 m dal mare", 28/08/1988, Bigazzi (FI 2373!; iso- B 10 0365357!, 10 0360070!, FI 2374!, 2375!, MA 525451!).

Paratipi (BIGAZZI \& RICCERI 1992: 194-196): [Italia, Sardegna] "Flumini major", s.d., s.coll., Herb. Moris (TO); "in irriguis montium”, s.d., s.coll. (FI 7411!). 
(=) Buglossites laxiflorus var. parviflorus Moris, Fl. Sardoa 3: 137. 1859 ('parviflora'). Locus classicus: [Italia, Sardegna] "In humentibus irriguisve montanis".

Lectotipo (BIGAZZI \& RICCERI 1992: 192): [Italia, Sardegna] "Flumini major", s.d., s.coll., Herb. Moris (TO!).

(=) B. laxiflora var. micranthos Guşul. in Bul. Fac. St. Cernauti 2: 440. 1928.

Locus classicus: [Italia, Sardegna] non specificato.

Lectotipo (BIGAZZI \& RICCERI 1992: 192): [Italia, Sardegna] “Sardinia” 1836, Moris (K).

EtIMologia Specie dedicata a Giuseppe Giacinto Moris (1796-1869), botanico torinese, autore della Flora Sardoa (1837-1859) e primo a riconoscere i caratteri distintivi di questa entità.

DESCRIZIONE Erba perenne, ramificata alla base, con fusti esili, verdi, prostrato-ascendenti di 1-4 dm, ispida per setole patenti lunghe $0,7 \mathrm{~mm}$. Foglie basali picciolate, oblungo-lanceolate, arrotondate all'apice, 4-5 × 6-16 cm. Cime semplici o poco ramificate, con fiori su peduncoli di $6-20 \mathrm{~mm}$; brattee ovato-lanceolate. Calice 22,5 mm all'antesi, fino a 6-7 mm nel frutto, con lacinie largamente triangolari. Corolla strettamente campanulata o quasi tubulosa, bianca o con tenui sfumature azzurre sul lembo, lunga circa $3 \mathrm{~mm}$ e appena sporgente dal calice, con lembo diviso in lobi ovati ad apice arrotondato. Stami inclusi, con appendice del filamento superante le antere, oblunga e ristretta in punta semplice. Mericarpi 1,5-2 mm. (Fig. 8)

FENOLOGIA Specie a fioritura prolungata, da aprile fino alla tarda estate, con rilascio progressivo dei frutti dalla metà di maggio ad agosto-settembre.

NUMERO 2n = 18 (SELVI \& al. 2006a), sia su materiale proveniente dal locus classicus (isola CROMOSOMIco di S. Pietro) che dall'unica altra stazione nota oggi in Sardegna (Laconi). II conteggio $2 n=16$ riportato da BIGAZZI \& RICCERI (1992) sembra frutto di errore.

Ecologia Rocce e sabbie umide lungo corsi d'acqua temporanei o canaletti di scolo delle acque meteoriche, 0-650 m.

DistRIBUZIONE Pianta estremamente rara, endemica di Sardegna (Fig. 9), dov'è nota attualmente per due sole aree circoscritte, nella parte centro-meridionale dell'isola, presso Laconi, e nella sola Cala Vinagra sull'isola di S. Pietro (locus classicus); nella località di Fluminimaggiore indicata da Moris non è stata più ritrovata ed è forse scomparsa, mentre è probabilmente errata la segnalazione per la Gallura riportata da ARRIGONI (2013: 223).

\section{NOTE}

SISTEMATICA Endemismo relittuale, di grande interesse filogenetico in quanto diploide, a differenza dell'affine $B$. pygmaea (poliploide con $\mathrm{x}=8$ ), a sua volta già considerata un tipo arcaico all'origine del genere. Sono entrambe legate ad ambienti umidi, ma $B$. morisiana è più termofila e confinata in stazioni primarie fortemente conservative, mentre $B$. pygmaea è capace di colonizzare anche stazioni secondarie e antropizzate.

CONSERVAZIONe L'estrema localizzazione ed esiguità delle popolazioni di B. morisiana rendono incerte le sue prospettive di sopravvivenza, in modo particolare nel suo locus classicus, ove lo sfruttamento turistico espone il sito a profonde e rapide alterazioni ambientali. La specie è attualmente considerata in pericolo (EN) secondo i criteri IUCN (RossI \& al. 2013). 


\subsection{Borago officinalis L. BORRAGINE COMUNE}

Borago officinalis L., Sp. PI. 1: 137. 1753.

Locus classicus: "hodiae in Normannia ad Colbeck et alibi in Europa; venit olim ex Aleppo [Siria]".

Lectotipo (Edmondson in JARVIS \& al. 1993: 25): s.loc, s.d., s.coll., Herb. Linneo 188.1 (LINN!).

Altro materiale originale (CECCHI \& SELVI 2015b: 635): [pianta coltivata nei Paesi Bassi] "Borago floribus coeruleis", s.d., Herb. Clifford 44.1.1 (BM 557895!); "Borago floribus albis", s.d., Herb. Clifford 44.1.2 (BM 557896!).

Etimologia Dal latino officinalis, "officinale", in riferimento all'impiego delle foglie e dei fiori di questa specie in erboristeria.

DESCRIZIONE Erba annua a fusti eretti, robusti, ramificati, di 2-6 dm, ispido-pungente per setole rigide e patenti, lunghe fino a $3,5 \mathrm{~mm}$. Foglie basali lamina largamente ovata di 5-15 × 10-30 cm, ad apice acuto, nettamente distinta dal robusto picciolo alato. Infiorescenza ampia con cime spesso arrossate; fiori su peduncoli lunghi fino a mm, all'ascella di brattee assai più brevi. Calice diviso fino alla base in lacinie lineari di $8-10 \mathrm{~mm}$, accrescente nel frutto fino a $20 \mathrm{~mm}$. Corolla blubrillante, di rado più chiara, con tubo di $2-3 \mathrm{~cm}$ e lembo rotato con lobi patenti, acuti, 8-12 mm; pliche faucali molto prominenti, a profilo trapezoidale, leggermente bilobe, internamente puberule. Stami fortemente sporgenti, con antere scure e appendice del filamento molto vistosa, violetto-porporina, lineare, raggiungente circa la metà di queste. Mericarpi $4-5 \mathrm{~mm}$. (Fig. 10)

FENOLOGIA A causa di fioriture tardive o del realizzarsi di più cicli riproduttivi nell'arco dello stesso anno, il periodo di fioritura di questa specie può coprire anche in singole stazioni un ampio intervallo di tempo, compreso tra l'inizio dell'inverno (gennaio) e l'autunno inoltrato (ottobre), pur culminando nei mesi primaverili; nucule mature possono trovarsi già dalla fine di marzo, ma l'apice della fruttificazione si ha tra quella di maggio e luglio.

Numero $2 n=16$, da piante laziali (D'AMATO \& MARCH 1983), umbre (D'AMATO \& MARCHI CROMOSOMICo 1983) e toscane (SELVI \& al. 2006a, COPPI \& al. 2007); lo stesso numero è stato riscontrato su materiale proveniente da Polonia (Turåla-Szybowska in PogAN \& al. 1989), Slovacchia (HINDÁKOVÁ \& al. 2005) e Spagna (LUQUE 1989).

ECologia Campi, incolti, ruderi, margini stradali e ferroviari, arginature, spesso sinantropica, generalmente tra 0 e $800 \mathrm{~m}$, raramente nel piano montano fino a $1400 \mathrm{~m}$.

DISTRIBUZIONE Specie comune allo stato spontaneo nella regione mediterranea centrooccidentale, che probabilmente ne rappresenta l'areale ancestrale, ma ormai largamente diffusa anche in gran parte della circumboreale europea, nonché coltivata e naturalizzata in molte altre zone a clima temperato del globo, come il Nordamerica, I'Asia, la Nuova Zelanda. In Italia è presente e molto comune in tutte le regioni, isole comprese. (Fig. 11)

NOTE

ALTRI NOMI VOLGARI Borrana, borrana officinale, buglossa vera. 
PROPRIETÀ E USI Pianta nota in erboristeria per le proprietà emollienti, idratanti e diuretiche di foglie e cime, legate all'elevato contenuto di mucillagini, per le quali ne vengono preparati decotti o infusi per il trattamento delle infiammazioni dell'apparato respiratorio o del cavo orale, dell'artrite, dei calcoli alla cistifellea. In cucina le foglie e i germogli giovani, dal sapore vagamente simile a quello del cetriolo, possono essere cotti per essere consumati, come tali o in preparazioni più elaborate, quali frittate, condimenti per la pasta, ripieni di prodotti da forno etc., sia in purezza che mescolati con altre erbe di campo; i fiori vengono talora impiegati nelle insalate per conferir loro sapore e colore, e si aggiungevano anticamente al vino per confezionare una bevanda ritenuta capace di infondere coraggio e buon umore. I semi, infine, sono impiegati per l'estrazione di un olio utile in farmacia, per la cura aspecifica di una vasta gamma di patologie (malattie cardiovascolari, neoplasie etc.) grazie alla presenza in alta concentrazione di acidi grassi poliinsaturi.

\subsection{Borago pygmaea (Lam. ex DC.) Chater \& Greuter BORRAGINE SARDA}

Borago pygmaea (Lam. ex DC.) Chater \& Greuter in Bot. J. Linn. Soc. 65: 261. 1972.

(三) Campanula pygmaea Lam. ex DC., Fl. Franç. ed. 3, 3: 705. 1805. Locus classicus: [Francia] "originaire de l'isle de Corse".

Olotipo (cfr. Chater \& Greuter in HEYwOoD 1972: 261): [Francia] "isle de Corse", s.d., s.coll., Herb. Lamarck 53.95 (P-LA 356218!).

(=) Anchusa laxiflora DC. in DC. \& Lam., FI. Franç., ed. 3, 3: 631. $1805 \equiv$ B. laxiflora (DC.) Fisch., Cat. Jard. PI. Gorenki, ed. 2: 27. 1812, nom. illeg., non. Poir. in Lam, Encycl. Suppl. 1: 693. 1811 三 Buglossites laxiflorus (DC.) Moris, Index Seminum [Turin]: 32.1845 ('laxiflora').

Locus classicus: [Francia] "isle de Corse".

Lectotipo (CECCHI \& SELVI 2015b: 636): [Francia] "Corse", s.d., Labillardiére, ex Herb. Desfontaines (FI-W 129105 !).

ETIMologia Dal latino pygmaeus, "pigmeo"; pur adattandosi bene anche al confronto con la più diffusa e robusta $B$. officinalis, la specie allora meglio conosciuta, l'epiteto fu inizialmente creato coll'idea che dovesse riferirsi ad una specie di Campanula, e basato su un singolo, modesto frammento di non immediata identificazione.

DESCRIZIONE Erba perenne, ramificata alla base, con fusti esili, spesso arrossati, prostratoascendenti di 2-7 dm, ispida per setole patenti-retrorse lunghe fino a $1 \mathrm{~mm}$. Foglie basali picciolate, oblungo-lanceolate, arrotondate all'apice, 4-5 ×6-16 cm. Cime semplici o poco ramificate, con fiori su peduncoli lunghi fino a $3 \mathrm{~cm}$; brattee ovato-lanceolate. Calice di 4-5 $\mathrm{mm}$ all'antesi, fino a $8 \mathrm{~mm}$ nel frutto, con lacinie strettamente triangolari o lineari-lanceolate. Corolla largamente campanulata, azzurra, lunga 6-7 mm e sporgente dal calice, con lembo diviso in lobi ovati ad apice acuto o subacuto. Stami inclusi, con appendice del filamento superante le antere, oblunga e tricuspidata all'apice. Mericarpi 3-4 mm. (Fig. 12)

FENOLOGIA Specie a fioritura piuttosto prolungata, perlopiù tardo-primaverile e estiva, con frutti maturi rilasciati a partire dalla fine di maggio e fino ad agosto- settembre.

NUMERO $2 n=30$ nella maggior parte delle popolazioni sarde fin qui studiate (SELVI \& al. CROMOSOMICo 2006a); $2 n=48$ in piante dell'isola di Capraia e del centro-nord della Corsica 
(SELVI \& al. 2006a); in almeno una località della Sardegna centro-meridionale (DIANA-CORRIAS 1980) e nella parte meridionale della Corsica (STREY 1931, CONTANDRIOPOULOS 1962, SELVI \& al. 2006a, COPPI \& al. 2007) sono stati invece riscontrati citotipi di passaggio con $2 n=32$. Si ritiene plausibile che il numero crosomico di base $p=8$, condiviso con $B$. officinalis e con ipotetiche popolazioni diploidi estinte di $B$. pygmaea stessa, rappresenti lo stato ancestrale del genere, dal quale sarebbero derivati da un lato i citotipi tetraploide $(2 n=32)$, esaploide $(2 n=48)$ e ipopoliploide $(2 n=30)$ di $B$. pygmaea, dall'altro, come anticipato nella scheda del genere, i numeri base secondari riscontrabili in $B$. trabutii Maire $(2 n=12)$ e $B$. morisiana $(2 n=18)$, rispettivamente per disploidia discendente e ascendente (SELVI \& al. 2006a).

ECOLOGIA Boscaglie umide, argini di ruscelli, sorgenti e stillicidi, in luoghi sia ombreggiati che esposti al sole, generalmente tra 200 e $800 \mathrm{~m}$, di rado nel piano montano fino a $1400 \mathrm{~m}$.

DISTRIBUZIONE Endemismo insulare, circoscritto alla provincia liguro-tirrenica occidentale, in particolare al distretto sardo-corso, diffuso, sia pure in modo sporadico, in tutta la Sardegna, la Corsica e il versante occidentale dell'isola di Capraia. (Fig. 13)

\section{NOTE}

ALTRI NOMI VOLGARI Borrana campanellina, borrandella.

DISTRIBUZIONE Un campione di questa specie conservato a Parigi (P00529044 ${ }^{1}$ ) risulta raccolto a Cadice, sulla costa atlantica dell'Andalusia, in Spagna, ma si tratta certamente di pianta coltivata o occasionalmente sfuggita alla coltivazione.

${ }^{1}$ http://mediaphoto.mnhn.fr/media/1441366440519PwemevJxFocUkJmm

PROPRIETÀ E USI Le foglie vengono talora raccolte, soprattutto in Corsica, per scopi alimentari; possono impiegarsi, con egual parsimonia, come quelle della più comune $B$. officinalis, come verdura cotta o nelle misticanze di erbe per la preparazione di pietanze più elaborate e prodotti da forno. 


\section{Symphytum L. CONSOLIDA}

Symphytum L., Sp. PI. 1: 136. 1753; Gen. PI., ed. 5: 66. 1754.

Tipo (BRITTON \& BRown 1913: 92, confermato da Hitchcock in HITCHCOCK \& GREEN 1929: 128): S. officinale L.

Bibliografia BuCKNALL 1913, BotTega \& GaRBARI 2003.

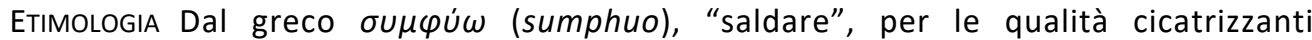
attribuite alla specie tipo (S. officinale); il nome volgare "consolida", che ben si adatterebbe anche alla capacità di trattenere il terreno del robusto apparato radicale della stessa pianta, va probabilmente riferito alla stessa origine.

DESCRIZIONE Erbe perenni con fusti eretti e apparato radicale robusto, fittonante o rizomatoso-tuberoso; indumento ispido-irsuto più o meno denso, costituito in proporzioni variabili da peli di diversa morfologia e dimensione (ghiandole stipitate, < 0,1 $\mathrm{mm}$; aculeoli uncinati, 0,1-0,7 mm; peli brevi diritti, 0,3-0,5 $\mathrm{mm}$; setole, $0,5-1,5(2) \mathrm{mm}$ ). Foglie con lamina acuminata, le basali e cauline inferiori picciolate, le cauline superiori e progressivamente ridotte, quelle dell'ultima coppia ascellanti l'infiorescenza, invariabilmente sessili, opposte o subopposte. Infiorescenza ebratteata, costituita da cime scorpiodi appaiate, spesso molto allungate alla fruttificazione; fiori nutanti, peduncolati. Calice cilindrico-campanulato, accrescente alla fruttificazione. Corolla generalmente clavato-infundiboliforme, con tubo cilindrico allungato e lembo dilatato, obconico o urceolato, brevemente inciso (ipocrateriforme, con tubo breve e lobi profondi patenti o riflessi, solo in alcune specie del Mediterraneo orientale, attribuite in passato al genere Procopiania Gușul.); superficie esterna papillosa o ghiandolosa; squame faucali molto prominenti, da lineare-subulate a strettamente triangolari, papillose ai margini. Stami con antere conniventi. Stilo filiforme, sporgente; stimma piccolo e intero. Mericarpi ovoidi, carenati, eretti o obliqui, da lisci a rugosi, con cercine basale rilevato e coste più o meno rilevate.

NUMERI Forse il genere più variabile dell'intera famiglia dal punto di vista CROMOSOMICl cariologico, Symphytum mostra un'amplissima gamma di variazioni del corredo cromosomico, sia infra- che interspecifiche. I numeri cromosomici delle popolazioni studiate $(2 \mathrm{n}=18,20,22,24,28,30,32,34,36,40,41$, $42,43,44,45,46,47,48,56,60,64,72,76,82,84,96,100,104,120,128$, 144) corrispondono ad assetti sia diploidi che auto- o allopoliploidi, ma rivelano anche gli effetti di ibridazione, fenomeni introgressivi e processi di aneuploidia discendente. II valore di $2 n=144$, dodecaploide, registrato per una popolazione di $S$. tuberosum, è il maggiore noto per l'intera famiglia. Sono sei i numeri cromosomici di base fin qui riscontrati: $x=7,8$, $10,11,12$ e 15.

BIOLOGIA Piante generalmente entomofile e allogame. Dispersione dei mericarpi RIPRODUTTIVA prevalentemente per mirmecocoria.

DISTRIBUZIONE Genere ampio di circa 35 specie, alcune delle quali forse di origine ibrida, cui 
andrebbero aggiunti i numerosi, ma spesso solo presunti, ibridi descritti nelle rispettive zone di sovrapposizione, naturali o artificiali che siano; sono distribuite nella regione mediterranea, nelle province centro-occidentali di quella circumboreale e in alcune aree limitrofe della regione irano-turanica. In Italia ne sono presenti sei, di cui due esotiche e una terza rappresentata da tre sottospecie, una delle quali endemica in Sicilia.

\section{NOTE}

Sistematica Le poche specie attribuite in passato al genere Procopiania Gușul., endemiche della provincia mediterranea orientale (isole egee), si distinguono nettamente per il lembo corollino profondamente inciso in lacinie strette e circinnato-riflesse; il gruppo, monofiletico, appare però chiaramente annidato all'interno dell'albero evolutivo di Symphytum (SANDBRINK \& al. 1990, HACIOĞLU \& ERIK 2011), e sembra più appropriata la sua distinzione come categoria infragenerica.

ChIAVE Delle SPECIE 1. Piante alte più di $50 \mathrm{~cm}$, con rizoma obliquo o verticale, fusiforme; corolla bianca, porporino-violetta o roseo-celeste 2

- Piante alte non più di $35 \mathrm{~cm}$, con rizoma orizzontale uniformemente ingrossato, oppure sottile con ingrossamenti alternati; corolla giallo-pallida

2. Foglie a lamina largamente ovata; corolla infundibuliforme ... 5. S. orientale

- Foglie a lamina ellittico-lanceolata; lembo corollino campanulato o urceolato-campanulato 3

3. Fusto almeno in parte alato, in alto irsuto-strigoso, con setole diritte ed esili, talora miste ad altre più forti e falcate; calice lungo da 6 a $10 \mathrm{~mm}$; mericarpi lisci 4

- Fusto senza ali, in alto nettamente strigoso per la presenza di sole setole robuste e falcate; calice minore di 4-5 $\mathrm{mm}$; mericarpi rugosi 1. S. asperum

4. Fusto alato su tutta la lunghezza, con ali larghe $2-8 \mathrm{~mm}$; corolla a lembo campanulato 4. S. officinale

- Fusto alato solo in parte, con ali più strette di $2 \mathrm{~mm}$; corolla a lembo urceolato-campanulato

6. S. tanaicense

5. Corolla lunga 10-15 $\mathrm{mm}$; pliche faucali nettamente sporgenti 2. S. bulbosum

- Corolla lunga 15-20 $\mathrm{mm}$; pliche faucali incluse 6

6. Rizoma sottile, con ingrossamenti bulbiformi ben distanziati; foglie contratte o attenuate alla base, mai decorrenti su ali del fusto; filamento staminale lungo circa quanto l'antera 3. S. gussonei

- Rizoma uniformemente ingrossato; foglie decorrenti sul picciolo, talora prolungate in strette ali sul fusto; filamento staminale lungo non più della metà dell'antera

7. S. tuberosum

\subsection{Symphytum asperum Lepech. $O$ CONSOLIDA RUGOSA}

Symphytum asperum Lepech. in Nova Acta Acad. Sci. Imp. Petrop. Hist. Acad. 14: 442, t. 7. 1798. 
Locus classicus: [pianta coltivata in Russia] "in horto Academico [S. Pietroburgo]", da semi raccolti "in jugo montium Caucasi Rossici [Russia]". Lectotipo (CECCHI \& SELVI 2015b: 638): disegno in LEPECHIN (1798: t. 7!).

Etimologia Dal latino asper, "ruvido", "rugoso", riferito all'indumento ispido- strigoso.

DESCRIZIONE Radice fittonante e fusto ampiamente ramificato, (3)5-12(15) dm, angoloso, con ali nulle o incospicue; indumento ruvido-scabroso per robuste setole falcate che, specie verso la parte superiore del fusto e le venature inferiori delle foglie, si sostituiscono progressivamente ad altre più lunghe ed esili. Foglie basali con lamina ovato-ellittica, ad apice acuminato e base tronca o cordata, fino a $7-12 \times 15-20 \mathrm{~cm}$; foglie cauline non o solo brevemente decorrenti sul fusto. Cime con (5)10-20 fiori su peduncoli di 5-10 mm. Calice di (2)4-5 mm, diviso per metà o più in lacinie strettamente ellittiche, arrotondate all'apice. Corolla obconica, (11)12-15(20) mm, l'immatura roseo-lillacina, poi celeste, distintamente villosa sulla superficie esterna, con tubo nettamente sporgente dal calice e lembo gradualmente allargato. Mericarpi di circa 2,5 × 4,5 mm, ristretti alla base, con superficie nerastra, percorsa da creste reticolate e rugoso-granulata. (Fig. 14)

FENOLOGIA Fiorisce tra maggio e agosto, con la maturazione dei frutti che si protrae fino all'inizio dell'autunno.

NUMERO $2 \mathrm{n}=32$, verificato sia su popolazioni avventizie in Danimarca (GADELLA \& CROMOSOMICO KLIPHUIS 1975) e Regno Unito (GAdelLA \& KLIPHUIS 1974, 1978, PerRING 1975) che su popolazioni naturali del Caucaso georgiano (GAGNIDZE \& al. 1985) e russo (GRAU 1968b, GADELLA \& KLIPHUIS 1972). II conteggio di $2 n=40$ riportato da BRITTON (1951) per piante nordamericane va riferito, secondo GADELLA \& KLIPHUIS (1978), all'ibrido con S. officinale, S. × uplandicum (vedi scheda relativa). Ugualmente, piante coltivate, e dichiaratamente sterili, con $2 n=$ 45 (ShIRATO \& al. 1985) interpretate come pentaploidi, potrebbero rappresentare una delle forme introgressive intermedie tra $S$. officinale e $S$. $\times$ uplandicum, che in ogni caso rientrano sotto quest'ultimo binomio.

Ecologia Specie montana, che allo stato spontaneo cresce tra 500 e $2600 \mathrm{~m}$ di quota ma è diffusa soprattutto nei prati rocciosi d'altitudine, oltre i $1600 \mathrm{~m}$ (KURTTO 1982). In Italia viene talora coltivata come foraggio per maiali o conigli e si è sporadicamente naturalizzata in contesti sinantropici, presso i coltivi e in ambienti freschi e umidi, dai fondovalle alle vallate alpine, tra 500 e $1100 \mathrm{~m}$.

DISTRIBUZIONE Specie alloctona, originaria della provincia caucasica e della parte orientale di quella eusina, in un'area compresa tra l'Anatolia nord-orientale, il Caucaso russo e l'Azerbaijan, coltivata da oltre due secoli in molti paesi a clima temperato, per ornamento o come foraggera, e talora naturalizzata nell'Europa centro-settentrionale e forse in Nordamerica (KURTTO 1982, GADELLA 1984). In Italia è stata segnalata sporadicamente come spontanea in diverse località del centro-nord, sempre in contesti sinantropici. (Fig. 15) 
NOTE

SISTEMATICA Symphytum asperum appartiene ad una delle stirpi più diversificate e complesse del genere, originaria dell'area caucasica; tra le specie presenti sul nostro territorio, quella filogeneticamente più affine sembra essergli S. orientale (HACIOĞLU \& ERIK 2011), che se ne distingue però nettamente per l'indumento più morbido, le foglie più larghe e la corolla clavata, bianca. Oltre che per l'indumento, S. asperum si distingue inoltre da S. officinale e $S$. tanaicense, dei quali in parte condivide l'ecologia, per le foglie mediamente più larghe, a lamina troncato-cordata, per il calice più breve, per la corolla a lembo obconico, azzurra a maturità.

Nonostante la distanza sul piano sia morfologico che corologico appaia in alcuni casi netta, le barriere riproduttive tra molte specie di Symphytum finiscono spesso col risultare effimere allorchè queste si trovino a crescere, promiscuamente, nello stesso luogo. Oltre a $S$. × uplandicum, un popolamento del quale è stato recentemente osservato nel Piacentino (cfr. sezione dedicata agl'ibridi), ci pare pertanto opportuno segnalare in questa nota altri due nototaxa derivati da $S$. asperum che, sia pur ancora mai segnalati in Italia, potrebbero comparirvi nelle zone di contatto interspecifico: 1) S. $\times$ norvicense Leaney \& C.L.O'Reilly, descritto come ibrido tra S. asperum e S. orientale (per quanto ne sappiamo, non esistono attualmente località italiane in cui le due parentali, entrambe avventizie, vengano in contatto, ma non si può escludere che questo possa accadere di qui a breve in alcune località dell'Italia centro-settentrionale); 2) S. $\times$ perringianum P.H.Oswald \& P.D.Sell, descritto come ibrido tra $S$. $\times$ uplandicum e $S$. orientale L.

PROPRIETÀ E USI Introdotto inizialmente in Europa centrale come foraggio per maiali, conigli e capre, poi diffusosi anche per uso ornamentale, $S$. asperum viene talora preferito nei giardini all'autoctono $S$. officinale per la minore invasività e la mancata emissione di sostanze allelopatiche nocive alla coesistenza con altre piante decorative ${ }^{1}$.

${ }^{1}$ http://www.luontoportti.com/suomi/fi/kukkakasvit/tarharaunioyrtti

\subsection{Symphytum bulbosum K. F. Schimp. CONSOLIDA BULBOSA}

Symphytum bulbosum K. F. Schimp. in Flora 8(1): 17. 1825.

(E) S. tuberosum subsp. bulbosum (K. F. Schimp.) P. Fourn., Quatre FI. France: 747. 1937 ES. tuberosum var. bulbosum (K. F. Schimp.) Nicotra, Syll. FI. Sic.: 39. 1893. Locus classicus: [Germania] "in ipsis vineis Heidelbergae et forsan aliis Germaniae et Galliae [Francia] locis".

Lectotipo (BOTTEGA \& GARBARI 2003: 254): [Germania] "Heidelberg", s.d., Schimper (REG 79480; iso- M 188158!, 188159!).

Altro materiale originale (CECCHI \& SELVI 2015b: 638): [Germania] "in vineis Heidelbergae", 04/05/1824, Schimper (M 188163!).

(=) S. clusii C. C. Gmel., Fl. Bad. 4: 144. 1826.

Locus classicus: [Germania] "Circa Heidelberg in vineiis".

Lectotipo (CECCHI \& SELVI 2015b: 639): [Germania] "prope Heidelberg in vineis passim", 1823, Gmelin (KR!).

Altro materiale originale (CECCHI \& SELVI 2015b: 639): [Germania] "ex vineis prope Heidelberg", s.d., Gmelin (KR!).

(=) S. punctatum Gaud., FI. Helv. 2: 41.1828.

Locus classicus: [Svizzera] "in Helvetia transalpina".

Tipo non designato. 
(=) S. zeyheri K. F. Schimp. in Flora 12(2): 418. 1829 三 S. bulbosum subsp. zeyheri (K. F. Schimp.) Nyman, Consp. Fl. Eur.: 510. 1881 E S. bulbosum var. zeyheri (K. F. Schimp.) Nicotra, Syll. Fl. Sic.: 39. 1893.

Locus classicus: [Italia, Sicilia] non specificato.

Lectotipo (CECCHI \& SELVI 2015b: 639): [Italia, Sicilia] “Palermo", s.d., Zeyher (FR 31985 !).

Etimologia Dal latino bulbosus, "dotato di bulbo(i)", in riferimento ai tuberi globosi che si formano ai nodi del rizoma orizzontale, altrimenti sottile.

DESCRIZIONE Rizoma orizzontale sottile, recante tuberi subglobosi distanziati; fusto semplice o poco ramificato, non alato, 2-3 dm; indumento scabro di setole miste a peli brevi sugli organi epigei, con aculeoli uncinati presenti anche su quelli ipogei; peli ghiandolari radi o assenti. Foglie a lamina ovato-acuminata, nelle basali e cauline inferiori a base cordata, lunga fino a $13 \mathrm{~cm}$, le superiori a base cuneatoattenuata. Cime dell'infiorescenza ciascuna con 5-10 fiori; peduncoli fiorali di 2$3 \mathrm{~mm}$. Calice di 6-7 $\mathrm{mm}$, profondamente diviso in lacinie strettamente triangolari. Corolla giallastra di $1-1,5 \mathrm{~cm}$, papilloso-ghiandolosa all'esterno, divisa in parti uguali tra il tubo cilindrico-obconico e il lembo campanulato, diviso per circa la metà in lacinie triangolari erette; pliche faucali strettamente triangolari-lanceolate di 6-8 mm, sporgenti di 1-3 mm oltre la corolla. Stami di 4$5 \mathrm{~mm}$ con filamento lungo quanto l'antera. Stilo sporgente dalla corolla per 1-4 $\mathrm{mm}$. Nucule di 3-4 $\mathrm{mm}$ a superficie rugosa, bruno-verdastra, percorsi da costolature pronunciate, con cercine dentato e strofiolo molto sporgente (3 $\mathrm{mm}$ ). (Fig. 16)

FENOLOGIA La fioritura si verifica prevalentemente tra marzo e maggio, ma può essere anticipata fin dalla fine di febbraio e protrarsi raramente fino ai primi di luglio; $i$ frutti maturano a partire dalla seconda metà di aprile e vengono rilasciati fino all'inizio dell'estate.

Numero $2 n=84$ su piante calabresi (PeruzZI 2003), $2 n=90$ su piante siciliane (CAMPo \& CROMOSOMICO ROMANO 1995), $2 n=104$ su piante toscane e coltivate (BOTTEGA \& al. 2001), 2n = 120 su piante venete (GADELLA \& KLIPHUIS 1978). In Europa, il numero $2 n=84$ è stato verificato da GRAU (1971) anche su esemplari originari del locus classicus, mentre quelli di $2 n=72$ (STREY 1931) e 78 (TARNAVSCHI 1948) sono stati considerati poco attendibili dallo stesso GrAU (1971) e da GADELLA \& KLIPHUIS (1978). In letteratura si riportano ancora $2 n=48$ per piante greche e $2 n=96$ per piante coltivate di origine ignota (STEARN 1986, JOHNSON \& BRANDHAM 1997).

EcologIA Boschi, siepi, incolti freschi e umidi. spesso lungo i corsi d'acqua, su suoli sciolti e pingui, tra 0 e $500 \mathrm{~m}$.

DISTRIBUZIONE Specie prevalentemente mediterranea, a gravitazione centro-orientale, diffusa e comune nelle province liguro-tirrenica, adriatica, balcanica e mediterraneoorientale, tra la Francia meridionale e la Corsica a ovest e la Turchia nordoccidentale a est, più rarerafatta verso nord, ove penetra spontaneamente nella parte meridionale delle contigue provincie circumboreali-europee, o si trova come alloctona naturalizzata (a nord fino all'Inghilterra). In Italia è comune nella parte insulare e peninsulare, verso nord fino all'area apuana, e alla bassa pianura padana, facendosi più rara a partire dalla sinistra idrografica del Po (pianura veneta) fin sul versante meridionale delle Alpi centro-orientali. (Fig. 17) 
NOTE

ALTRI NOMI VOLGARI Consolida minore.

SISTEMATICA Anticamente sinonimizzata con $S$. tuberosum, questa specie se ne distingue nettamente per la forma e struttura degli organi ipogei e ancor più per la corolla tubulosocampanulata raccorciata, che lascia sporgere visibilmente le pliche faucali (BÉGUINOT 1900). Quanto a S. zeyheri, segnalato spesso dai floristi dell' 800 come vicariante di $S$. bulbosum nell'Italia meridionale e insulare, si distinguerebbe dalla forma tipica per l'indumento più ispido di setole riflesse, le foglie grigio-verdi nel fresco e annerenti nel secco, le pliche corolline decisamente più sporgenti $(2,5-3,5(4) \mathrm{mm})$; la sua separazione fu tuttavia messa in dubbio già da LITARDIÈre (1955: 94), e ribadita dal monografo Pawlowski in TUTIN \& al. (1972: 103-105), nè allo stato delle nostre conoscenze sembrano esservi ragionevoli motivi per rimetterla in discussione.

\subsection{Symphytum gussonei F. W. Schultz CONSOLIDA DI GUSSONE}

Symphytum gussonei F. W. Schultz in Arch. Fl.: 27. 1874, in Flora 58: 218. 1875.

(झ) S. tuberosum var. gussonei (F. W. Schultz) Nicotra, Syll. FI. Sic.: 39. 1893. Locus classicus: [Italia, Sicilia] non specificato.

Lectotipo (BOTTEGA \& GARBARI 2003: 251): [Italia, Sicilia] "In nemoribus", s.d., Gussone, Herb. Gussone ("Symphytum mediterraneum Koch.”, NAP!).

(=) S. tuberosum var. australe Strobl in Flora 67: 624. 1884 ('australis'). Locus classicus: [Italia, Sicilia] "S. Guglielmo ob Castelbuono und am monte S. Angelo ob Cefalù, im Valle del Sapone [...] im Waldern unterhalb Gibilmanna und in den Nebroden [...] auch noch an einigen anderen punkten Siziliens".

Lectotipo (CECCHI \& SELVI 2015b: 640): [Italia, Sicilia] in nemoribus - Mirto", s.d., Todaro Fl. Sic. 1393 (ADMONT!; iso- FI 7406!, FI 7407!, P 529278!, 529279!, 529280!, 529281!, TO!).

Etimologia Specie dedicata al botanico campano Giovanni Gussone (1787-1866), direttore dell'Orto Botanico a Palermo e poi a Napoli, allievo di Michele Tenore e autore di importanti opere sulla flora della Sicilia e del Meridione, che per primo raccolse questa specie attribuendola erroneamente a $S$. mediterraneum [S. tuberosum subsp. angustifolium].

DESCRIZIONE Rizoma sottile con tuberi subglobosi ben distanziati; fusto semplice o poco ramificato, $15-20 \mathrm{~cm}$, privo di ali; indumento scabro di setole miste a peli brevi sugli organi epigei, con aculeoli uncinati presenti anche su quelli ipogei; peli ghiandolari radi o assenti. Foglie (escluse quelle ascellanti l'infiorescenza) 5-8, a lamina largamente ovata o ovato-elittica, 3-6 × 6-11 cm, con base cuneata e picciolo ben distinto, spesso molto allungato in quelle basali e inferiori, più o meno largamente alato. Infiorescenza spesso su un internodo nettamente più lungo della foglia prossimale, con cime di 5-10 fiori ciascuna; peduncoli fiorali di 2-4 mm, con cime di 5-10 fiori. Calice di 6-8 mm, profondamente diviso in lacinie strettamente triangolari. Corolla di $1,5-2 \mathrm{~cm}$, papilloso-ghiandolosa all'esterno, infundiboliforme, oscuramente divisa in parti uguali tra il tubo bianco e il lembo bianco-giallastro, talora soffuso di rosso, inciso per non più di $3 \mathrm{~mm}$ in lacinie largamente triangolari; pliche faucali strettamente triangolarilanceolate, 1-2 × 5-6 mm, incluse. Stami equamente divisi tra filamento e 
antera. Nucule subsferiche di $3 \mathrm{~mm}$ di diametro, a superficie rugosa, brunoverdastra, percorse da costolature pronunciate e con strofiolo sporgente di circa $1 \mathrm{~mm}$. (Fig. 18)

FENOLOGIA Pianta a fioritura primaverile-estiva, tra marzo e luglio, che matura i frutti tra maggio e agosto.

Numero $2 n=96$, ottenuto da due diverse stazioni del Palermitano (BotTega $\&$ al. 2001). CROMOSOMICO

ECologia Boschi di latifoglie su suoli acidi e pingui, nella fascia collinare e montana inferiore, tra 200 e $1100 \mathrm{~m}$.

DISTRIBUZIONE Endemismo piuttosto raro, vicariante di S. tuberosum in Sicilia, dov'è presente soprattutto sul versante nord di Madonie, Nebrodi e Peloritani, ovvero delle catene montuose della parte centrosettentrionale e nordoccidentale dell'isola, tra il Messinese e il Palermitano. (Fig. 19)

\section{NOTE}

\section{ALTRI NOMI VOLGARI Consolida siciliana.}

SISTEMATICA Recenti analisi di filogenesi molecolare (HACIOĞLU \& ERIK 2011) confermano il rapporto di stretta affinità, già evidenziato da PAWLOWSKI (1971), tra S. gussonei e S. tuberosum, sua vicariante sul continente. La pianta siciliana se ne distingue piuttosto agevolmente per il rizoma sottile con ingrossamenti distanziati, le foglie più nettamente picciolate, l'internodo particolarmente allungato che precede l'infiorescenza, i filamenti staminali allungati e i mericarpi subsferici. La struttura del rizoma e la dimensione relativa di filamento e antere sono caratteri condivisi con S. bulbosum, specie simpatrica che tuttavia si riconosce con facilità per la corolla abbreviata e le pliche faucali sporgenti.

\subsection{Symphytum officinale L. CONSOLIDA MAGGIORE}

Symphytum officinale L., Sp. PI. 1: 136. 1753.

Locus classicus: "in Europae umbrosis subhumidis".

Lectotipo (GADELLA 1984: 1063): s.loc, s.d., s.coll., Herb. Linneo 185.1 (LINN!).

(=) S. patens Sibth., Fl. Oxon.: 70. 1794 I S. officinale var. purpureum Pers., Syn. PI. 1: 161.1805 三 S. officinale var. patens (Sibth.) Nyman, Consp. Fl. Eur.: 509. 1881.

Locus classicus: [Regno Unito] "Banks of the Thames by Caversham". Lectotipo (CECCHI \& SELVI 2015b: 640): [Regno Unito] s.d., s.coll., Herb. Sherard 321a (OXF!).

(=) S. bohemicum F. W. Schmidt, FI. Boëm. 3: 13. 1794 E S. officinale var. bohemicum (F. W. Schmidt) Nyman, Consp. Fl. Eur.: 509. $1881 \equiv$ S. officinale subsp. bohemicum (F. W. Schmidt.) Čelak. in Sitzungsber. Königl. Böhm. Ges. Wiss., Math.-Naturwiss. Cl. 1891(1): 29. 1891 三 S. officinale var. typicum f. bohemicum (F. W. Schmidt) Fiori in Fiori \& Bég., FI. Italia 2(3): 377. 1902.

Locus classicus: [Repubblica Ceca] "in pratis paludosis ad Albim fluvium [fiume Labe] non procul Melnik in der Auen; etiam bei der Stephansüberfuhr [Štěpánský Přívoz]". 
Lectotipo (KIRSCHNER \& al. 2007: 351): [Repubblica Ceca] "De pratis udis Bohemiae ad Melnik", s.d., Schmidt (PRC).

(=) S. officinale var. ochroleucum A. DC. in DC., Prodr. 10: 37. 1846.

Locus classicus: non indicato.

Lectotipo (CECCHI \& SELVI 2015b: 641): disegno in MüLLER (1777: t. 664!).

Etımologia Dal latino officinalis, "officinale", in riferimento all'impiego delle foglie e dei rizomi di questa specie nella farmacopea popolare e in erboristeria.

DESCRIZIONE Robusta radice fittonante verticale, ramificata; fusto eretto e ramificato, 5-12 $\mathrm{dm}$, percorso da ali di 2-8 $\mathrm{mm}$ decorrenti dalla lamina delle foglie cauline e superanti l'internodo inferiore; indumento scabro, persistente nel secco, riflesso sul fusto, costituito da setole miste a peli brevi e radi peli ghiandolari, con aculeoli uncinati presenti solo sugli organi fotosintetici. Foglie acuminate, a nervature fortemente sporgenti sulla pagina inferiore e margine da piano a visibilmente ondulato, le basali molto grandi, $10-15 \times 20-50 \mathrm{~cm}$, con lamina da lanceolata a ovato-lanceolata, le cauline lanceolate, con lamina largamente decorrente sul fusto. Cime dense con 20-30 fiori su peduncoli di 3-4 mm. Calice di 5-8 mm, diviso per $1 / 2-2 / 3$ in lacinie ovato-lanceolate appressate alla corolla, pubescente su tutta la superficie. Corolla da bianca a porporino-violetta, tubuloso-campanulata o tubuloso-urceolata (non sempre ristretta nella porzione distale), di 1,3-1,5 cm, esternamente papilloso-ghiandolosa, con brevi lacinie triangolari (circa $1 \mathrm{~mm}$ ) riflesse; pliche faucali strettamente triangolari, $0,7 \mathrm{~mm}$, incluse. Stami di 5-6 mm, con filamento circa la metà dell'antera, e connettivo sporgente tra le logge. Stilo brevemente sporgente dalla corolla. Mericarpi ovoidi, di circa 4,5 $7 \mathrm{~mm}$, con superficie liscia, nera, e denti generalmente eretti sul cercine basale; strofiolo sporgente $1 \mathrm{~mm}$. (Fig. 20)

FENOLOGIA Fiorisce più o meno continuativamente tra marzo e i primi di ottobre, con una prima fase di fioritura culminante nella tarda primavera e una seconda nella prima parte dell'autunno; i frutti maturano tra maggio e la fine di ottobre.

NUMERO I conteggi effettuati per questa specie polimorfa su numerosissime popolazioni CROMOSOMICO naturali indicano la presenza di due citotipi principali, il diploide $2 n=24$ (cui possono aggiungersi fino a 4 cromosomi $B$ sovrannumerari) e il tetraploide $2 n=$ 48. II diploide, meno frequente, è sempre associato a piante con fiori bianchi, comuni solo nella parte meridionale dell'areale: tali sono due popolazioni di Val d'Aosta (Gadella \& Kliphuis 1970, 1972) e Toscana (PeruzZI \& al. 2000, 2001), e con esse altre in Francia (GADELLA \& al. 1983, MEKKI \& al. 1987), Germania (GAdella \& KliphUIS 1972), Paesi Bassi (GAdella \& KliphUIS 1967, 1972, MeKKI \& al. 1987), Polonia (GADELLA \& KLIPHUIS 1972, WCISŁO 1972), Regno Unito (GADELLA \& KLIPHUIS 1972, 1974, PeRRING 1975), Slovacchia (GADELLA \& KLIPHUIS 1972, Murín \& MÁJOVSKI 1982) e Ungheria (GADELLA \& KLIPHUIS 1972, MEKKI \& al. 1987). II tetraploide, al contrario, corrisponde generalmente a piante con fiori purpureovioletti, fenotipo prevalente nell'Europa centrale e settentrionale; tali sono risultate piante provenienti da Austria (GADELLA \& KLIPHUIS 1972), Belgio (GADELLA \& KLIPHUIS 1972, GADELLA \& al. 1983), Bielorussia (DMItrieVA \& PARFENOV 1985, PARFENOV \& DimitrieVA 1988), Bulgaria (GAdelLA \& KLIPHUIS 1983), Francia (GADELLA \& KLIPHUIS 1972), Germania (GADELLA \& KLIPHUIS 1972, BOTTEGA \& al. 2001), Paesi Bassi (GAdella \& KLIPHUIS 1963, 1967b, 1972, Gadella 1983), Polonia (GAdella \& KLIPHUIS 1972, WCISŁO 1972), Regno Unito (GADELLA \& KLIPHUIS 1972, 1974, PERRING 1975), Repubblica Ceca (GAdelLA \& KLIPHUIS 1972, KrahulcovÁ 1988), Repubblica di Macedonia (SEKOVSKI \& JovANOVSKA 1983), Romania (TARNAVSCHI 1948, GADELLA 
\& KLIPHUIS 1972), Serbia (GAdelLA \& KLIPHUIS 1972), Slovacchia (GAdella \& KLIPHUIS 1972, MuRín \& MÁJOVSKI 1982), Slovenia (GADELLA \& KLIPHUIS 1972, Van Loon \& Kieft in LöVE 1980b), Svizzera (GADELLA \& al. 1983) e Ungheria (GADELLA \& KLIPHUIS 1972). A questi si aggiunge poi tutta una serie di dati relativi a corredi aneuploidi che viene brevemente discussa nelle note in fondo a questa scheda.

ECologIA Prati umidi, boschi ripari, spesso sugli argini e lungo i fossi, tra 0 e $1700 \mathrm{~m}$.

DISTRIBUZIONE Specie mesofila a larghissima diffusione, prevalentemente circumborealepaleartica (dalla provincia europeo-atlantica a ovest a quelle siberiane a est), molto meno frequente, e forse in qualche caso solo avventizio, più a sud, nella regione mediterranea centro-orientale, con poche stazioni isolate tra I'Italia meridionale e la Turchia. In Italia è comune in tutte le regioni settentrionali, raggiungendo in penisola il suo primo limite meridionale nel Valdarno e sul monte Vettore nelle Marche, per poi comparire con isolate stazioni sul versante tirrenico dell'Italia meridionale, tra Lazio e Sicilia; riportata anche per la Sardegna (ARRIGONI 2013: 256-257), dov'è tuttavia dubbia la sua presenza allo stato spontaneo. (Fig. 21)

\section{NOTE}

ALTRI NOMI VOLGARI Alo, erba confermo, erba di San Lorenzo, erba rustica, naro, orecchia d'asino, sinfito.

SISTEMATICA Specie piuttosto polimorfa, soprattutto per la larghezza e lunghezza delle ali che decorrono dalla base delle foglie lungo il fusto, per il colore della corolla e per la strigosità dell'indumento, che in effetti varia, aumentando, anche in una stessa pianta, tra la primavera e l'estate. Le tipiche popolazioni di S. bohemicum, circoscritte a stazioni subalofile nel nord della Repubblica Ceca, si distinguerebbero secondo KIRSCHNER \& al. (2007) per il corredo diploide $2 n=24$, ma per quanto discusso anche in questa scheda non si tratta evidentemente nè di un carattere esclusivo di queste, nè di un'area geografica chiaramente circoscritta, tale da giustificare alcuna separazione a rango infraspecifico. Per l'ibrido potenziale con S. asperum, S. × uplandicum, si veda la nota in calce all'altro parentale.

NUMERI CROMOSOMICI Secondo GAdella \& KLIPHUIS (1973) le popolazioni di "S. officinale" con corredo $2 n=40$, riscontrate con una certa frequenza nell'Europa nord-occidentale, a ovest fino al Regno Unito (GADELLA \& KLIPHUIS 1974), all'Irlanda (GADELLA \& al. 1983) e all'Islanda (Löve \& LÖVE 1956), e note in particolare dai Paesi Bassi e dagli stati limitrofi, principale area oggetto delle loro ricerche (GADELLA \& KLIPHUIS 1963, 1967b, 1971, 1972, HUIZIG \& al. 1982, GADELLA $\&$ al. 1983), vanno in realtà riferite a due gruppi distinti: da un lato l'ibrido tra S. asperum $(2 n=32)$ e popolazioni tetraploidi di $S$. officinale, ovvero $S . \times$ uplandicum, dall'altro una stirpe diversa, non meglio identificata. Quest'ultima fu trattata in seguito dagli stessi autori come insieme di citotipi di S. officinale s.str., privi di valore tassonomico (GADELLA \& KLIPHUIS 1984, 1985), ma era già stata assimilata dagli stessi autori (GADELLA \& KLIPHUIS 1967) e da GraU (1971) a S. officinale subsp. uliginosum, ovvero a S. tanaicense. Seguendo questo punto di vista, tutta la serie di conteggi compresi tra $2 n=41$ e 47, riscontrati per popolazioni del Belgio e dei Paesi Bassi (GADELLA \& KLIPHUIS 1963, 1967b, 1971, Gadella 1972), del Regno Unito (GADELLA \& KLIPHUIS 1974), e su piante sterili coltivate in Giappone (SHIRATO \& al. 1985) e forse altrove (DATTA 1933, secondo GADELLA \& KLIPHUIS 1963, non verificato), andrebbe interpretata come sciame degli ibridi interspecifici e delle forme introgressive tra $S$. officinale e una delle due suddette entità. Analogamente, accanto a occasionali fenomeni di poliploidizzazione e disploidia a livello infraspecifico, che non possono in assoluto escludersi, appare plausibile che a forme ibride possano riferirsi anche gli altri corredi "anomali" fin qui riportati per piante del "gruppo" di S. officinale: $2 n=26$ (Paesi Bassi; Gadella \& Kliphuis 1963), $2 n=36$ (piante coltivate; StREY 1931, SUzUKA 1950), 2n = 56 (Slovacchia; GAdella \& KLIPHUIS 1978). 
PROPRIETÀE USI Le mucillagini rilasciate nell'infuso acquoso o alcolico della radice hanno effetto emolliente, decongestionante, espettorante e analgesico. Per la presenza di allantoina, il cataplasma ottenuto con la poltiglia della radice mista a olio d'oliva è impiegato come cicatrizzante nella cura di piccole ferite, bruciature o escoriazioni, e si ritiene capace di stimolare la formazione del callo osseo dopo le fratture. Foglie e radici trovano un moderato uso in campo alimentare, come verdura cotta o in infusi, ma come per molte altre specie della famiglia ne viene sconsigliato l'uso interno per la presenza di alcaloidi pirrolizidinici tossici. Per la sua rusticità è usato in agricoltura organica per il miglioramento dei terreni e nelle pratiche di sovescio, o coltivato (ma con parsimonia) come foraggera, analogamente a quanto avviene anche per altre specie affini alloctone in Italia. Le foglie macerate in acqua per la produzione di concimi ricchi in potassio, e alcuni organi, opportunamente trattati, possono trovare un qualche uso in cosmetica. La specie è infine talora coltivata nei giardini per il suo valore ornamentale.

\subsection{Symphytum orientale L. CONSOLIDA ORIENTALE}

Symphytum orientale L., Sp. PI. 1: 136. 1753.

Locus classicus: [Turchia] "juxta Costantinopoli rivulos".

Lectotipo (KURTTO 1985: 330): "Symphytum Constantinopolitanum, Borraginis folio \& facie, flore albo", disegno in BuXBAUM (1740: 36, t. 68!).

EtıMologiA Dal latino orientalis, "orientale", per la distribuzione geografica.

DESCRIZIONE Robusta radice fittonante verticale, ramificata; fusto eretto e ramificato, 5-7 $\mathrm{dm}$, raramente alato; indumento denso e vellutato, costituito da setole particolarmente allungate $(1-1,5 \mathrm{~mm})$, miste a peli brevi e aculeoli, entrambi uncinati, sugli organi fotosintetici, e a radi peli ghiandolari perlopiù sulle nervature di foglie e calice. Foglie basali molto grandi, lungamente picciolate, con lamina largamente ovata, lunga fino a $30 \mathrm{~cm}$ o più, ad apice arrotondato, base cordata e nervature fortemente sporgenti sulla pagina inferiore; le cauline largamente ovate, con lamina a base arrotondata. Cime dense con oltre 20 fiori ciascuna, su peduncoli di 2-3 mm. Calice di 5-9 mm, diviso per 1/4-1/2 in lacinie ovato e attuse appressate alla corolla. Corolla bianca, infundiboliforme, di 1,1$1,7 \mathrm{~cm}$, esternamente papillosa, con tubo sporgente dal calice e brevi lacinie largamente triangolari (circa $1 \mathrm{~mm}$ ) riflesse; pliche faucali strettamente triangolari, 0,6 $\mathrm{mm}$, incluse. Stami di $4-5 \mathrm{~mm}$, con filamento lungo più o meno quanto l'antera, e connettivo sporgente tra le logge. Stilo sporgente dalla corolla per 2-4 mm. Mericarpi ovoidi, talora leggermente ricurvi, di circa $1 \times 1,5$ $\mathrm{mm}$, con superficie rugosa, bruna, e strofiolo appena sporgente $(0,5 \mathrm{~mm})$. (Fig. 22)

FENOLOGIA Specie a fioritura primaverile, prolungata talora dalla metà di marzo ai primi di luglio, che disperde frutti maturi a partire dalla fine di aprile, fino ad estate inoltrata.

NUMero $2 n=32$ in esemplari naturalizzati in Toscana (BOTTEGA \& GARBARI 2003). II dato CROMOSOMICO conferma quello già ottenuto tanto da esemplari in coltivazione (GRAU 1971) quanto da popolazioni naturali in Bulgaria (Markova \& Goranova in KAMARI \& al. 1995) e Turchia (GADELLA \& KLIPHUIS 1978), mentre conteggi precedenti riportavano numeri maggiori $(2 n=62,63$; TARNAVSCHI 1948). 
ECologIA Specie forestale, in natura tipica di boschi di Pinus nigra e valli fluviali ombrose, tra 0 e $1500 \mathrm{~m}$; da noi perlopiù in parchi e ambienti antropizzati, talora anche in radure e incolti assolati.

DISTRIBUZIONE Specie alloctona di origine pontico-caucasica, endemica del Caucaso e della Turchia centro-settentrionale, coltivata per ornamento e naturalizzata in varie zone a clima mediterraneo o submediterraneo. In Italia si trova allo stato subspontaneo nelle aiuole abbandonate e negli incolti all'interno o nei pressi di alcuni giardini e orti botanici, dai quali si è diffuso e sporadicamente naturalizzato già a partire dalla fine del XIX secolo in alcune regioni centrali; localmente infestante i coltivi (osservazioni personali nella piana fiorentina). (Fig. 23)

\section{NOTE}

SISTEMATICA Per l'ibrido potenziale con $S$. asperum, $S . \times$ norvicense, si veda la nota in calce all'altro parentale.

\subsection{Symphytum tanaicense Steven CONSOLIDA RUSSA}

Symphytum tanaicense Steven, in Bull. Soc. Imp. Nat. Moscou 24: 1851.

(三) S. officinale subsp. tanaicense (Steven) Soó in Bot. Közlem. 28: 127. 1931. Locus classicus: [Russia] "ad Tanain inferiorem".

Olotipo (cfr. BotTega \& GaRBarI 2003: 258): [Russia] "ad Tanain inferiorem", 06/1817, Steven (H 1535841!).

(=) S. uliginosum A. Kern. in Oesterr. Bot. Z. 13: 227. 1863 三 S. officinale subsp. uliginosum (A. Kern.) Nyman, Consp. Fl. Eur.: 509. 1881 三 S. officinale var. typicum f. uliginosum (A. Kern.) Fiori in Fiori \& Bég., Fl. Italia 2(3): 377. 1902. Locus classicus: [Ungheria] "In pratis uliginosis prope Pest".

Lectotipo (CECCHI \& SELVI 2015b: 641): [Ungheria] "Sumpfwiesen am Rakos bei Pest", s.d., Kerner (WU 69901!; iso- WU 69902!, 69904!; "Sumpfige Wiesen längs d. Rakosbrache bei Pest", WU 69903!).

EtıMologia Dal latino tanaicensis, "del fiume Tánaïs" (in greco Távaïs), antico nome del Don, legato alla città di Tana, che sorge alla sua foce sul mare d'Azov.

DESCRIZIONE Robusta radice fittonante verticale, ramificata; fusto eretto e ramificato, 7-9 $\mathrm{dm}$, percorso da ali incospicue, di $1-3 \mathrm{~mm}$, che normalmente non raggiungono dalla base di ogni foglia l'internodo inferiore; indumento scabro, rado, perlopiù caduco nel secco, costituito in prevalenza di peli brevi e peli ghiandolari, con aculeoli uncinati presenti solo sugli organi fotosintetici e setole lunghe rare, concentrate sulle nervature della pagina inferiore delle foglie. Foglie basali molto grandi, 5-9 $\times 15-30 \mathrm{~cm}$, con lamina ovato-lanceolata, acuminata, a nervature fortemente sporgenti sulla pagina inferiore, assenti all'antesi; le cauline con lamina lanceolata, strettamente decorrente sul fusto. Cime dense con 20-30 fiori su peduncoli di 3-4 mm. Calice di circa $10 \mathrm{~mm}$, diviso per 4/5 in lacinie ovato-lanceolate divergenti e glabrescenti, con peli solo sui margini e le nervature. Corolla porporino-violetta, tubuloso-urceolata (ristretta nella porzione distale), di circa $1,5 \mathrm{~cm}$, esternamente papilloso-ghiandolosa, con brevi 
lacinie triangolari (circa $1 \mathrm{~mm}$ ) riflesse; pliche faucali strettamente triangolari, 0,7 mm, incluse. Stami di 5-6 mm, con filamento circa la metà dell'antera, e connettivo sporgente tra le logge. Stilo brevemente sporgente dalla corolla. Mericarpi ovoidi, di circa 4,5 × $7 \mathrm{~mm}$, con superficie liscia, marrone-scuro, e denti riflessi sul cercine basale; strofiolo sporgente $1 \mathrm{~mm}$. (Fig. 24)

FENOLOGIA Fiorisce tra aprile e giugno e matura i frutti fino alla fine dell'estate.

NUMERo Corredo disploide, $2 n=40$, verificato sia su piante toscane (PERUzZI $\&$ al. 2001) CROMOSOMICO che slovacche (MÁJOVSKÝ \& UHRíKovÁ 1985, MURín \& MÁsOVSKI 1987). A questa entità vanno inoltre riferiti i conteggi $2 n=40$ attribuiti a $S$. officinale s.l., per i quali si rimanda alla nota in calce alla scheda di quella specie.

ECOLOGIA Prati umidi torbosi, sponde lacustri, canneti, attorno a livello del mare (tra (10)0 e $10 \mathrm{~m}$ ).

DISTRIBUZIONE Specie quasi esclusivamente circumboreale-europea, presente più o meno diffusamente tra la provincie europee atlantica centro-orientale (Paesi Bassi) e quelle centrali e orientali (fino alla Russia meridionale), raggiungendo nella parte settentrionale della provincia liguro-tirrenica (regione mediterranea) le latitudini più meridionali, forse quale relitto post-glaciale. La sua presenza in Italia è estremamente rarefatta ed è stata fino ad oggi accertata solo per poche stazioni del Valdarno inferiore in Toscana; stazioni storiche nella pianura friulana presso Pordenone, nell'Agro Pontino (basso Lazio) e, forse, nel Bellunese (Veneto) restano da confermare. (Fig. 25)

\section{NOTE}

NomenclatURA Se trattata come sottospecie di S. officinale (cfr. nota successiva), la priorità nomenclaturale, limitata per l'Art. 11.2 dell'ICN entro ciascun rango (MCNEILL \& al. 2012), va alla combinazione di Nyman dell'epiteto "uliginosum" anzichè a quella di Soó per "tanaicense".

SISTEMATICA Specie debolmente distinta da S. officinale, benché i caratteri diagnostici si mantengano anche nelle stazioni ove crescono in sostanziale simpatria. L'affinità tra le due è evidente non solo sul piano morfologico, ma anche su quello chemiotassonomico (JAARSMA \& al. 1989) e cariologico. Vi è una chiara interfertilità degli ibridi tra $S$. tanaicense e il citotipo tetraploide di S. officinale, che in condizioni sperimentali possono dar luogo a sciami di individui e popolazioni aneuploidi capaci di riprodursi sessualmente senza apparenti, forti barriere riproduttive (GADELLA \& KLIPHUIS 1971). BUCKNALL (1913), pur trattandolo erroneamente in sinonimia di S. patens (il cui tipo ha fusto largamente alato), riteneva $S$. tanaicense endemico della Russia europea, altrove solo introdotto; in tal caso, se cioè alle deboli barriere riproduttive corrispondesse una separazione geografica tra le due entità, potrebbe darsi come rango tassonomico più appropriato quello di sottospecie. GADELLA \& KLIPHUIS $(1973,1984,1985)$, al contrario, non gli attribuivano alcuna valenza tassonomica, limitandosi a segnalarne informalmente la corrispondenza con le popolazioni aneuploidi $(2 n=40)$ di S. officinale.

CONSERVAZIONE Entità rara per l'Italia, ove sembra circoscritta a pochissime stazioni relitte, ma non così nel suo areale compessivo. Pertanto il suo status di conservazione secondo i criteri della IUCN non è stato valutato su scala globale, ma risulta a pericolo critico (CR) su quella nazionale (Rossı \& al. 2013) per le gravi minacce che interessano le modeste aree umide ove sono confinate le nostre isolate popolazioni. 


\subsection{Symphytum tuberosum L. CONSOLIDA FEMMINA}

Symphytum tuberosum L., Sp. PI. 1: 136. 1753.

Locus classicus: [Germania] "in Germania australi".

Lectotipo (STEARN 1985: 177): s.loc, s.d., s.coll., Herb. Linneo 185.3 (LINN!).

Etimologia Dal latino tuberosus, "tuberoso", per il rizoma ingrossato e nodoso.

DESCRIZIONE Rizoma robusto, nodoso, con ingrossamenti più o meno distanziati; fusto semplice o poco ramificato, $15-25(50) \mathrm{cm}$, parzialmente alato per decorrenza delle lamine fogliari; indumento scabro di setole miste a peli brevi sugli organi epigei, con aculeoli uncinati presenti anche su quelli ipogei; peli ghiandolari radi o assenti. Foglie solo cauline, a lamina da ellittico-lanceolata a largamente ovata, acuminate all'apice, con base cuneato-attenuata, decorrente, e picciolo breve o nullo. Infiorescenza su un internodo raramente più lungo della foglia prossimale; peduncoli fiorali di 2-4 $\mathrm{mm}$. Calice di 7-10 $\mathrm{mm}$, profondamente diviso in lacinie strettamente triangolari. Corolla di $1,5-2,3 \mathrm{~cm}$, papillosoghiandolosa all'esterno, infundiboliforme, oscuramente divisa in parti uguali tra il tubo bianco e il lembo bianco-giallastro, raramente soffuso di rosso, inciso per non più di $3 \mathrm{~mm}$ in lacinie largamente triangolari, riflesse; pliche faucali strettamente triangolari, incluse. Stami di 4-5 mm con filamento $<1 / 2$ dell'antera. Stilo sporgente dalla corolla per 1-3 mm. Nucule largamente ovoidi, a superficie rugosa, bruno-verdastra, percorse da costolature pronunciate e con strofiolo sporgente di circa $1 \mathrm{~mm}$.

NUMERO È nota una grande varietà sia di citotipi che di possibili numeri base $(2 n=18,32$, CROMOSOMICo 48?, 64, 72, 76, 80?, 82, 84, 96, 100, 112?, 120, 128, 144), per la quale non era stata messa in evidenza fin qui alcuna chiara corrispondenza né con le variazioni morfologiche né, se non localmente, con l'area geografica di provenienza del materiale analizzato. Recenti studi (MURín \& MÁJOVSKI 1982, KoBRLovÁ \& al. 2016) suggeriscono al contrario che le due sottospecie corrispondano invece a due distinti citotipi (si veda per questo la nota in calce alla scheda della subsp. angustifolium).

DISTRIBUZIONE Specie polimorfa, ampiamente distribuita in vasta parte dell'area euromediterranea e presente in tutte le province meridionali della regione circumboreale europea e in quelle settentrionali della mediterranea; l'areale complessivo spazia da nord a sud tra la Gran Bretagna a la Calabria, e da ovest a est tra la penisola iberica e quella anatolica. Tradizionalmente si accetta la distinzione in due sottospecie debolmente separate sul piano morfologico, quella tipica a gravitazione occidentale, la subsp. angustifolium vicariante più orientale, unica in Italia (BOTTEGA \& GARBARI 2003) e diffusa a partire dalla provincia centro-europea verso est.

ECologia Boschi mesofili di latifoglie, su suolo fresco e pingue, perlopiù nella fascia planiziale e collinare, più raramente in quella montana $(0-1850 \mathrm{~m})$.

\section{NOTE}

ALtRI NOMI VOLGARI Consolida paternostrale, consolida tuberosa.

SISTEMATICA In linea con un concetto di specie più ampio, già adottato per altri generi in questa 
trattazione, e con la definizione delle sottospecie quali entità morfologicamente poco distinte e geograficamente vicarianti, si è tentati di ricondurre sotto un più ampio concetto di "S. tuberosum s.l." anche S. gussonei, sulla base di evidenze sperimentali che le vedono vicine non solo dal punto di vista morfologico ma anche da quello filogenetico (HACIOĞLU \& ERIK 2011); ce ne asteniamo per l'ancora incompleta conoscenza del grado di isolamento tra le due entità, che potrebbe ancora essere incompleto sul piano biologico, ma è invece assoluto su quello corologico. Per l'aspetto della corolla e l'evidente sovrapposizione degli areali, entrambe le entità sono invece nettamente distinte dall'affine S. bulbosum, come già efficacemente osservato da BÉGUINOT (1900).

\subsubsection{Symphytum tuberosum}

subsp. angustifolium (A. Kern.) Nyman CONSOLIDA A FOGLIE STRETTE

Symphytum tuberosum subsp. angustifolium (A. Kern.) Nyman, Consp. Fl. Eur.: 510. 1881.

(E) S. angustifolium A. Kern. in Öst. Bot. Z. 13: 227. 1863.

Locus classicus: [Ungheria] "in silvis frondosis Hungariae. In circulo pilisiensi ad pedem montis Slanitzka prope Csabam".

Lectotipo (BOTTEGA \& GARBARI 2003: 247, 249): [Ungheria] "Slaniztka bei Csaba", s.d., Kerner (WU 69897!; iso- WU 69896!, 69898!, 69899!).

(=) S. mediterraneum W. D. J. Koch, Syn. Fl. Germ. Helv. 1: 500.1837 三. tuberosum var. mediterraneum (W. D. J. Koch) Fiori in Fiori \& Bég., Fl. Italia 2(3): 378. 1902.

Locus classicus: [Francia] "prope Telonem".

Neotipo (CECCHI \& SELVI 2015b: 641): [Francia] “Var - Toulon", 28/03/1846, Puiseux (P 529286!; iso- P 529265!).

(=) S. nodosum Schur, Enum. Pl. Transsilvaniae: 468. $1866 \equiv$ S. tuberosum subsp. nodosum (Schur) Soó in Acta Geobot. Hung. 4: 192. 1941.

Locus classicus: [Romania] "In Felsenspalten der Glimmerschiefer-Gebirge: Surul, Götzenberg".

Tipo non designato.

(=) S. tuberosum f. miniatum Fiori in Fiori \& Bég., FI. Italia 2(3): 378. 1902.

Locus classicus: [Italia, Lombardia] "a Cava Carbonara nel Pavese", [Veneto] "al Cansiglio".

Neotipo (CECCHI \& SELVI 2015b: 642): [Italia, Lombardia] "locis arenosis aridis subumbrosis prope Carbonara (PV) non longe a Ticino flumine, solo siliceo", 04/1905, Traverso, FI. It. Exs. 945 ( "Symphytum tuberosum L.", FI7408!; isoFI7409!, GE!, MOD!).

Etimologia Dal latino angustus, "stretto", e folium, "foglia", per le foglie in genere da ovatoa strettamente lanceolate.

DESCRIZIONE Rizoma spesso, ma ingrossamenti (tuberi) irregolari più o meno distanziati. Foglie cauline intermedie (escluse quelle ascellanti l'infiorescenza) 3-8, a lamina da ellittico-lanceolata a ovato-ellittica, 2-4 × 6-13 cm. Infiorescenza pauciflora, con 3-8(20) fiori. Pliche faucali di 2-2,5 × 4,5-6 mm. Stami con filamento non più lungo di $1 / 3$ dell'antera, spesso subnullo. Nucule $3 \times 4 \mathrm{~mm}$. 
FENOLOGIA Pianta a fioritura primaverile, raramente anche estiva, nella quale fiori maturi, a seconda della quota e delle condizioni ambientali, possono trovarsi già da metà marzo e in talora fino alla fine di luglio; i frutti maturano di conseguenza tra la seconda metà di aprile e il termine dell'estate.

NUMERo Per le popolazioni italiane di S. tuberosum subsp. angustifolium si riportano tre CROMOSOMICo livelli di ploidia: $2 n=96$ in piante venete (JAARSMA \& al. 1990), lo stesso riscontrato anche in popolazioni provenienti da Austria (KOBRLOVÁ \& al. 2016), Germania (GraU 1968b, JAARSMA \& al. 1990, LIPPERT 2006, KoBRLOVÁ \& al. 2016), Polonia (WCISto 1972, JAARSMA \& al. 1990, KobRLOVÁ \& al. 2016), Repubblica Ceca (GAdella \& KLIPHUIS 1978, KobrLovÁ \& al. 2016), Russia (JAARSMA \& al. 1990), Slovacchia (Kobrlová \& al. 2016), Slovenia (GRAU 1968b, GAdelLA \& KLIPHUIS 1978), Spagna (LUQUE 1989) e Ungheria (KoBRLOVÁ \& al. 2016); $2 n=120$ in piante toscane (BOTTEGA \& al. 2001), corrispondente a quello di almeno una popolazione francese (JAARSMA \& al. 1990); $2 n=144$ ancora da materiale toscano (GRAU 1968b). In altri paesi europei è tuttavia documentata per questa entità una serie molto più ampia e diversificata di numeri cromosomici: $2 n=18$ in Romania (TARNAVSCHI 1948), $2 \mathrm{n}=32$ in Slovacchia, Ungheria, Ucraina, Romania (MURín \& MÁJOVSKI 1982, KobRLOVÁ \& al. 2016) e Repubblica Ceca (KoBRLOVÁ \& al. 2016), 2n = 64 in Grecia (van Loon \& Oudemans in LöVE 1982a) e Turchia europea (GADELLA \& KLIPHUIS 1978, JAARSMA \& al. 1990; vedi anche SANDBRINK \& al. 1990), $2 n=72$ in Germania (STREY 1931, MARKova \& IVANOVA 1970) e Bulgaria (MARKOVA \& IVANOVA 1970), 2n = 76 in Germania (STREY 1931), $2 n=82$ in Romania (TARNAVSCHI 1948), $2 n=84$ nel Regno Unito (GADELLA \& KLIPHUIS 1978), 100 in Germania (GRAU 1968b). A questi, infine, si aggiungono altri 3 livelli stimati, non basati su conteggio diretto ma su misure al citofluorimetro a flusso (KoBRLovÁ \& al. 2016): DNA-esaploide $(2 n=48$ ?) in Repubblica Ceca, decaploide $(2 n=80$ ?) in Austria e Repubblica Ceca, tetradecaploide $(2 n=112$ ?) in Germania e Repubblica Ceca.

DISTRIBUZIONE Secondo la circoscrizione accettata da BOTTEGA \& GARBARI (2003) si tratterebbe della sottospecie a più ampia distribuzione, esclusa solo dalla parte occidentale dell'areale della specie e unica in Italia. È presente e relativamente frequente in quasi tutte le regioni italiane, con l'eccezione di Sicilia, Sardegna e Valle d'Aosta. (Fig. 27)

\section{NOTE}

SISTEMATICA Anche se alcuni recenti trattamenti floristici relativi a territori dell'Europa centrale (MIREK \& al. 2002, FISCHER \& al. 2008, JÄGER 2009, KIRÁLY \& al. 2011, DANIHELKA \& al. 2012) considerano $S$. tuberosum come una singola specie altamente polimorfa, studi cariologici suggeriscono l'esistenza di almeno due taxa infraspecifici (Murín \& MÁsovski 1982, KOBRLOVÁ \& al. 2016). Le indagini mostrano una corrispondenza tra i due livelli di ploidia prevalenti con i due morfotipi e la loro diversa ecologia. II dodecaploide $(2 n=96)$ sarebbe proprio delle popolazioni di faggeta montana o sub-montana e corrisponde morfologicamente al tipo della specie; in boschi termofili di querce caducifoglie e carpino bianco è invece diffuso il morfotipo angustifolium, a corredo tetraploide $(2 n=32)$; tutti i restanti citotipi sono solo sporadici e rappresenterebbero forme aneuploidi di transizione. La conoscenza della variabilità cariotipica delle popolazioni del resto dell'areale è ancora troppo frammentaria ma, se la suddetta corrispondenza fosse confermata, la presenza della sottospecie tipica andrebbe certamente rivalutata anche per l'Italia. 


\section{SOTTOSPECIE DA CONFERMARE}

\section{Symphytum tuberosum}

subsp. tuberosum

\section{CONSOLIDA FEMMINA TIPICA}

DESCRIZIONE Rizoma spesso con ingrossamenti (tuberi) molto ravvicinati. Foglie cauline intermedie (escluse quelle ascellanti l'infiorescenza) 6-12, a lamina ovatoellittica, generalmente con rapporto tra lunghezza e larghezza $<3$. Infiorescenza con 8-16(40) fiori. Pliche faucali 1-2 × 6-7 mm. Stami con filamento lungo da $1 / 3$ a $1 / 2$ dell'antera.

DISTRIBUZIONE Secondo l'interpretazione tradizionale, la sottospecie tipica sarebbe vicariante della subsp. angustifolium nella parte occidentale dell'areale, diffusa in particolare nelle province atlantica e iberica, e in misura minore in quella europea centrale, fino alla Germania meridionale (locus classicus) e all'arco alpino occidentale, e a sud in quella balearica. Secondo BOTTEGA \& GARBARI (2003) tutte le segnalazioni italiane per questa sottospecie (da Piemonte, Lombardia e Veneto) sarebbero frutto di confusione con la subsp. angustifolium. Tuttavia la sua presenza in Italia potrebbe essere confermata per l'esistenza di popolazioni con corredo cromosomico dodecaploide $(2 n=96)$; indagini cariologiche più approfondite appaiono necessarie in tal senso (KoBRLOVÁ \& al. 2016).

\section{IBRIDI}

\section{Symphytum $\times$ bicknellii Buckn. CONSOLIDA DI BICKNELL}

Symphytum $\times$ bicknellii Buckn. in J. Linn. Soc., Bot. 41: 552. 1913.

Locus classicus: [Italia, Liguria] "Val Borghetto, Bordighera [...] Vallecrosia, Bordighera".

Lectotipo (CECCHI \& SELVI 2015b: 643): [Italia, Liguria] "Piani di Vallecrosia, sotto gli ulivi", 17/04/1895, Bicknell (Herb. Bicknell, Istituto Internazionale di Studi Liguri, Bordighera!).

Altro materiale originale (CECCHI \& SELVI 2015b: 643): [Italia, Liguria] "Val Borghetto, presso Bordighera", 12/03/1889, Bicknell (Herb. Bicknell, Istituto Internazionale di Studi Liguri, Bordighera!).

(=) S. bulbosum F.W.Schimp. $\times$ S. tuberosum L.

Etimologia Ibrido dedicato al raccoglitore dei campioni tipo, il britannico Clarence Bicknell (1842-1918), italiano d'adozione, pastore anglicano, esperantista ma anche eclettico uomo di scienza, fondatore dell'omonimo museo di Storia Naturale a Bordighera.

DESCRIZIONE Rizoma con ingrossamenti oblunghi; fusto semplice o poco ramificato, parzialmente alato per decorrenza delle lamine fogliari. Foglie basali con lamina 
a base arrotondata e picciolo nettamente distinto, le cauline inferiori a base attenuata e decorrente, con picciolo breve o nullo. Corolla di 11-13 mm, due volte maggiore del calice; pliche faucali brevemente sporgenti.

DISTRIBUZIONE Noto con certezza per le sole raccolte originali, nell'estremo ponente ligure.

\section{Symphytum $\times$ ferrariense C. Massal. CONSOLIDA FERRARESE}

Symphytum $\times$ ferrariense C. Massal. in Boll. Soc. Bot. Ital. 1913(6): 78. 1913. Locus classicus: [Italia, Emilia Romagna] "Orto botanico dell'Università di Ferrara".

Olotipo (cfr. KURTTO 1981: 19): [Italia, Emilia Romagna] “Cultum Hort. Bot. Ferrarae", s.d., Massalongo (FER!).

Paratipo (CECCHI \& SELVI 2015b: 644): [Italia, Emilia Romagna], "cresciuto nell’Orto Botanico di Ferrara", 01/05/1913, Massalongo (FI 7136!).

(=) S. $\times$ floribundum Shuttlew. ex Buckn. in J. Linn. Soc., Bot. 41: 531. 1913. Locus classicus: [Francia] "Var [...] Hyères [...] Dans le propriété de Mme. la Comtesse de Beauregard à Hyères (Var, France) [...] Lieux herbeaux, bord des fossés, Hyères [...] Hyères. Le Long d'un conduit d'eau dans le jardin même de Chateau Beauregard à Hyères [...] Aups, Var. Lieux humides le long des prairies". Lectotipo (KURTTO 1981: 19): [Francia] "Le Long d'un conduit d'eau dans le jardin même de Chateau Beauregard à Hyères”, 8-30/05/1871, Shuttleworth (K).

(=) S. officinale L. $\times$ S. orientale L.

Etimologia Nome riferito all'origine orticola del primo esemplare descritto, raccolto dall'autore nelle aiuole dell'orto botanico di Ferrara.

DESCRIZIONE Radice robusta e fittonante; fusto alto e ramificato; denso indumento composto prevalentemente di setole lunghe e dense, sul fusto miste anche a peli uncinati più brevi e robusti. Foglie cauline strettamente ovato-lanceolate, con lamina contratta in un breve picciolo, più o meno decorrente su questo e sul fusto. Calice diviso in lacinie triangolari-lanceolate, da acute a subacute. Corolla da bianco-sporca a violetto-pallida, con lobi da deflessi a eretti e pliche faucali da subulate a strettamente lanceolate, a margini sia interi che debolmente ondulati. Frutti generalmente assenti.

DISTRIBUZIONE Raramente osservato tra la fine del XIX secolo e l'inizio del XX nelle zone di contatto tra le due parentali tra le province centro-europea e ligure-tirrenica, ovvero in poche località del dipartimento di Var, nel sud della Francia, e nell'orto botanico di Ferrara (locus classicus).

\section{NOTE}

NOMENCLATURA La sinonimia con $S$. $\times$ floribundum è stata qui indicata, nonostante tale nome non sia mai stato usato per piante italiane, in considerazione del fatto che viene talora ascritto (http://www.ipni.org/) a "Shuttlew. ex Nyman, Consp. Fl. Eur. 509. 1881", il che gli darebbe priorità rispetto a $S$. ferrariense (1913). Tale ascrizione è in realtà erronea (ICN Art. 36.1; McNeILl \& al. 2012) perché prima del 1913 il binomio di Shuttleworth fu sempre e solo citato come sinonimo di S. mediterraneum W.D.J.Koch (Kurrto 1981), senza alcuna valida pubblicazione. 
SISTEMATICA E Si tratta di un ibrido perlopiù sterile tra S. officinale e S. orientale, osservato in Italia, per DISTRIBUZIONE la prima e unica volta, nell'orto botanico di Ferrara, dov'era cresciuto spontaneamente a fianco delle specie parentali. Le foglie sono mediamente di forma intermedia a quella delle due specie, così come la larghezza delle pliche faucali; l'indumento di setole perlopiù lunghe e lanose si avvicina maggiormente a quello di $S$. orientale, benché più rado e con qualche pelo uncinato, più robusto, sul fusto. Secondo KURTTo (1981) allo stesso andrebbero riferite altre due entità con caratteri più o meno intermedi tra le suddette parentali, ovvero $S$. floribundum e $S . \times$ hyerense Pawł. (= S. officinale $\times S$. floribundum) che, in quest'ordine, segnano più o meno un gradiente continuo di transizione, forse una sequenza introgressiva, verso $S$. officinale. Entrambe furono descritte sulla base di alcuni reperti sterili raccolti tra il 1871 e il 1892 in una piccola area della Francia meridionale a est di Tolone, e a lungo confuse con S. mediterraneum, descritta per la stessa area ma riferibile invece a $S$. tuberosum. Le piante crescevano sempre in luoghi fortemente antropizzati, dov'era plausibile che esemplari delle due parentali entrassero in contatto, pur non essendovi testimonianza diretta della presenza di S. orientale in coltivazione. II fatto che non siano mai più state osservate da oltre un secolo conferma la loro incapacità di costituire popolazioni stabili in natura.

\section{Symphytum $\times$ uplandicum Nyman CONSOLIDA DELL'UPLAND}

Symphytum $\times$ uplandicum Nyman, Syll. Fl. Eur.: 80. 1855 .

Locus classicus: [Svezia] "Upland".

Lectotipo (CECCHI \& SELVI 2015b: 645): [Svezia] “Upsalia”, s.d., Ångström (come "Symphytum asperrimum", S 14-32982!).

(=) S. asperum Lepech. $\times$ S. officinale L.

EtimologiA Da "Uppland", provincia svedese della regione dello Svealand, immediatamente a nord di Stoccolma, area di provenienza dei campioni originali.

DISTRIBUZIONE Pianta piuttosto comune, segnalata diffusamente in Europa e Nordamerica anche al di fuori delle aree di sovrapposizione tra gli areali delle due specie parentali, e spesso più abbondante di queste come esotica. La sua presenza in Italia ci è nota attualmente solo per un campione piemontese e per una popolazione recentemente rinvenuta nel Piacentino, ma non è escluso che ve ne siano altre nelle zone ove è stato coltivato $S$. asperum.

SISTEMATICA E Ibrido tra S. asperum e S. officinale, include sia i prodotti d'incrocio di prima generazione, DISTRIBUZIONE da tempo diffusi in coltivazione come vigorose piante ornamentali, sia lo sciame di forme intermedie che possono prodursi per introgressione o reincrocio, recanti tutta la gamma di modeste variazioni che segnano il passaggio verso la forma tipica del parentale dominante (perlopiù S. officinale; GADELLA \& KLIPHUIS 1969, 1971, 1975, 1984). Secondo BuCKNALL (1913) la pianta di Nyman corrisponderebbe ad un'altra già descritta in precedenza dal Caucaso, S. peregrinum Ledeb., il cui epiteto di conseguenza avrebbe priorità nomenclaturale, $\mathrm{ma}$ in assenza di verifiche sperimentali preferiamo attenerci al punto di vista degli autori più recenti, che li considerano quali entità distinte (GADELLA \& KLIPHUIS 1978, 1983). Generalmente $S$. x uplandicum ha indumento di aspetto intermedio tra le due parentali; la lamina è cordata nelle foglie basali e cuneata nelle cauline, con base non decorrente sul fusto, o decorrente per non più di $1 \mathrm{~cm}$; il calice eguaglia o supera di poco la metà del tubo corollino; la corolla è campanulato-obconica, mai veramente urceolata, e il suo colore a maturità è azzurro-porporino (nè bianco, nè perfettamente viola o azzurro); la superficie dei mericarpi, quando questi arrivino a maturità, può essere da leggermente lucida a rugoso-granulata. La barriera riproduttiva tra le parentali è debole (GADELLA \& KLIPHUIS 1969) e l'ibrido può propagarsi tanto per via 
vegetativa quanto per seme; $S . \times$ uplandicum può quindi trovarsi in natura sia nelle zone di sovrapposizione tra i popolamenti delle due parentali che in stazioni indipendenti, e in alcuni paesi europei è addirittura più comune di quelle. 


\section{Melanortocarya Selvi, Bigazzi, Hilger \& Papini o MELANORTOCARIA}

Melanortocarya Selvi, Bigazzi, Hilger \& Papini in Taxon 55: 915. 2006.

Tipo (cfr. SelVI \& al. 2006b: 915): M. obtusifolia (Willd.) Selvi, Bigazzi, Hilger \& Papini.

Bibliografia SelVI \& al. 2006b.

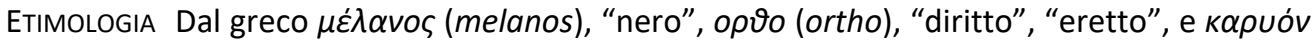
(káryon), "noce", cioè "a nucula eretta e nera", in riferimento al caratteristico mericarpo di tipo "pulmonarioide" che si distingue nettamente da quelli tipici di Nonea, genere da cui questo è stato segregato.

DESCRIZIONE Erbe annue con indumento ispido. Foglie basali largamente spatolate, le cauline e bratteali progressivamente minori, ovato-ellittiche, sessili. Infiorescenze bratteate, semplici o poco ramificate, con fiori su peduncoli brevi o subnulli, deflessi alla fruttificazione. Calice diviso per circa metà in lacinie triangolareovate erette, cilindrico-campanulato all'antesi, alla fruttificazione notevolmente accrescente, rigonfio e sub-sferico (ventricoso). Corolla ipocrateriforme, con tubo lungo e lobi rotati; pliche faucali sostituite da bande longitudinali di peli intercalate ai petali, che dalla fauce si estondono internamente al tubo per oltre metà della sua lunghezza. Stami a filamento subnullo, inseriti alla base del tubo corollino, con antere che non raggiungono la parte inferiore delle bande pubescenti. Stilo molto breve, incluso, con stigma bilobo. Mericarpi ovoidi, eretti, subacuti, a superficie nera lucida, minutamente puberuli, con cercine biancastro molto rilevato.

NUMERI Invariabilmente $2 \mathrm{n}=20$ nell'unica specie nota, talora con uno o due cromosomi CROMOSOMICI B sovrannumerari.

BIologia Piante entomofile, ma certamente autogame, come dimostra la posizione degli RIPRODUTTIVA organi riproduttivi (stami e pistillo) molto arretrata rispetto alla fauce, carattere condiviso solo con Neatostema e, fuori dalla nostra flora, con le specie di Anchusa del sottogenere Buglossoides (Tausch) Gușul. La dispersione dei mericarpi, come nella maggior parte delle Boragineae, è mirmecocora.

DISTRIBUZIONE Genere monotipico distribuito nella provincia mediterranea orientale, donde sconfina marginalmente in quella pontica a nord e in quella mesopotamica ad est; altrove solo alloctono.

\section{NOTE}

ETIMologia II vocabolo melanortocarya è stato inteso dagli autori come modifica del termine orthocaryum, a nucula eretta, già utilizzato da Alphonse de Candolle in CANDolle (1846: 28) per definire una sezione che includeva le specie di Nonea con mericarpi eretti; le altre ad esservi incluse (tra le quali $N$. lutea), tuttavia, condividono soltanto questo carattere con $M$. obtusifolia, ma presentano nucule di tipo "noneoide" (rugose e variamente sculturate) e risultano dei veri membri di Nonea anche alla luce dei dati molecolari (Selvi \& al. 2006b).

SISTEMATICA Genere monotipico presumibilmente antico, Melanortocarya è stato recentemente separato da Nonea sulla base di analisi molecolari secondo cui occupa una posizione precocemente divergente rispetto alla linea evolutiva che avrebbe poi condotto all'evoluzione di Nonea (incl. Elizaldia Willk.) e Pulmonaria (incl. Paraskevia W. Sauer \& 
G. Sauer); se ne distingue facilmente per le bande pubescenti che dalla fauce corollina si protendono in profondità nel tubo, per gli stami inseriti alla base del tubo stesso, lo stilo brevissimo (con stimma bilobo minutamente papilloso) e i mericarpi neri, lucidi ed eretti, di tipo "pulmonarioide". Importanti caratteri distintivi si trovano anche a livello palinologico, visto che i granuli pollinici di Melanortocarya, oltre che minori, sono visibilmente contratti sul piano equatoriale e presentano solo tre aperture, mentre quelli di Nonea sono ovoidi e con un numero di aperture maggiore (carattere chiaramente derivato).

5.1. Melanortocarya obtusifolia (Willd.) Selvi, Bigazzi, Hilger \& Papini 0 NONNEA AZZURRA

Melanortocarya obtusifolia (Willd.) Selvi, Bigazzi, Hilger \& Papini in Taxon 55: 915. 2006.

(三) Lycopsis obtusifolia Willd., Sp. PI. 1: 780.1798 $\equiv$ Nonea obtusifolia (Willd.) DC. in DC. \& Lam., Fl. Franç., ed. 3, 3: 626. 1805.

Locus classicus: [Grecia] "in Chio [...] in Lesbo".

Lectotipo (SELVI \& al. 2006b: 915): [Grecia] "in Chio", s.d., Tournefort 1701 (B-W 3389!).

Etimologia Dal latino obtusus, "ottuso", e folium, "foglia", per il profilo più frequente dell'apice fogliare.

DESCRIZIONE Erba annua, ramificata alla base in numerosi fusti prostrato-ascendenti, 10-25 $\mathrm{cm}$, ispido-pubescente per se tole patenti a base tubercolata, annerente alla disseccazione. Foglie verde scuro, visibilmente maculate di bianco per i grossi tubercoli calcificati alla base delle setole maggiori, con margine intero o leggermente ondulato; le basali oblungo-spatolate, secche all'antesi, le cauline ovato-ellittiche, a base tronca e apice da acuto a ottuso, 0,8-1,5 $\times 2-4 \mathrm{~cm}$. Infiorescenze brevi, semplici o poco ramificate, con brattee maggiori dei fiori. Calice di 4-5 $\mathrm{mm}$ all'antesi, fino a $8-10 \mathrm{~mm}$ alla fruttificazione, diviso fino ametà in denti triangolari. Corolla con tubo biancastro di 3-4 $\mathrm{mm}$ e lembo azzurro di 4$5 \mathrm{~mm}$ di diametro, con lobi ovato-orbicolari; pliche faucali densamente pubescenti e prolungate in basso. Antere di 0,7 mm. Stilo di circa $1 \mathrm{~mm}$. Mericarpi 2,5-3 mm, nero lucidi, con cercine biancastro. (Fig. 28)

FENOLOGIA Specie a fioritura precoce, primaverile o tardo-invernale (marzo-maggio), in cui la maturazione dei frutti si conclude entro la tarda primavera al termine dell'effimero ciclo vitale della pianta.

Numero $2 n=20$, verificato sia su piante greche (GraU 1971, Constantinidis in KAMARI \& al. CROMOSOMICO 1996), che turche (SELVI \& BIGAZZI 2002); sono stati osservati fino a due cromosomi B sovrannumerari (Constantinidis in KAMARI \& al. 1996).

ECOLOGIA Terofita di ambienti aridi, propria delle garighe mediterranee e della vegetazione steppica o predesertica, ove può trovarsi dal livello del mare fino ad almeno $600 \mathrm{~m}$; da noi è stat trovata solo come esotica sinantropica in stazioni ruderali in area urbana o periurbana, a quote inferiori a $100 \mathrm{~m}$.

DISTRIBUZIONE Specie subendemica della provincia mediterranea orientale, a partire dalla Grecia orientale fino alle isole egee, alla Turchia e ai paesi del Medio Oriente; da 
qui si spinge a nord, con poche stazioni sulle coste bulgare meridionali del Mar Nero, nella provincia pontica, e probabilmente penetra indefinitamente ad est nella provincia mesopotamica. In Italia è esotica casuale; fu raccolta e segnalata diffusamente in diverse località dell'area urbana di Roma tra il il 1876 e il 1897, poi, sembra, ancora nel 1983 nell'Agro Pontino (Bassani, Herb. Bassani, fide Anzalone \& al. 2010: 271), da allora mai più ritrovata e forse scomparsa. (Fig. 29)

\section{NOTE}

Nomenclatura Prima che ne fosse svelata la vera identità, i primi esemplari italiani della specie, raccolti nel 1876 da un giardiniere dell'Orto Botanico di Roma nei dintorni della città, furono provvisoriamente etichettati da Giuseppe De Notaris (1805-1877) come "Nonnea romana", senza che alcuna pubblicazione vi facesse seguito. Uno di questi si trova a Verona (VER), corredato di una lunga lettera d'accompagnamento del De Notaris indirizzata al collega Caro Massalongo (1852-1928) che ne spiega le circostanze del ritrovamento, ma ve ne sono probabilmente anche Roma (RO-HR) e a Berlino (B), dove Romualdo Pirotta (1853-1936) ne spedì almeno uno a Paul Wilhelm Magnus (1844-1914) per aver conferma della sua identità. PIROTTA (1886: 119) riferì poi del ritrovamento di numerosi altri esemplari nelle stesse zone, citando il binomio di De Notaris e corredandolo di una lunga descrizione, ma lo fece al solo scopo di confermarne l'identità con N. obtusifolia. Per questo "Nonea romana De Not. ex Pirotta" è un nome invalido che non richiede alcuna tipificazione. 


\section{Pulmonaria L.} POLMONARIA

Pulmonaria L., Sp. PI. 1: 135. 1753; Gen. PI., ed. 5: 65. 1754.

Tipo (JARVIS \& al. 1993: 80): P. officinalis L.

\section{Bibliografia Kerner 1878, SAUer 1975, Bolliger 1982.}

Etimologia Dall'aggettivo latino sostantivizzato pulmonarius, "buono per i polmoni", in riferimento all'uso delle foglie nella medicina tradizionale, come antinfiammatori ed espettoranti.

DESCRIZIONE Erbe perenni brevemente rizomatose (una sola specie in Grecia con radice fittonante), con fusti eretti o eretto-ascendenti alti fino a $15-50 \mathrm{~cm}$; denso indumento eteromorfo, talora ghiandoloso, sulla pagina superiore delle foglie mature costituito in parti variabili di diversi tipi di peli semplici (aculeoli brevissimi $\leq 0,2 \mathrm{~mm}$, conici, lunghi da 1 a 2,5 volte la larghezza; peli brevi, esili, di 0,3-0,6 mm; setole robuste di 0,6-3 mm), misti talora a ghiandole stipitate (peli ghiandolari) o sessili (microghiandole). Foglie acuminate, di aspetto e consistenza feltrosi, spesso cosparse di macchie più chiare del colore di fondo; le basali in pieno sviluppo (foglie estivali e autunnali) grandi, più o meno nettamente picciolate; le cauline e bratteali assai più piccole, sessili. Infiorescenze semplici o ramificate in poche cime oscuramente scorpioidi, irregolarmente bratteate; fiori peduncolati. Calice diviso per circa un terzo della lunghezza totale (raramente fino alla metà) in lacinie triangolari-ovate, cilindrico-campanulato all'antesi, più o meno accrescente (campanulato o ventricoso) alla fruttificazione. Corolla infundiboliforme, con tubo bianco, gradualmente pigmentato in alto, e lembo con lobi arrotondati, eretto-patenti (mai del tutto rotati), di colore purpureo-violaceo, più raramente rosso o azzurro; pliche faucali sostituite da areole di tricomi costituenti un anello più o meno continuo. Piante eterostile, con filamenti staminali inseriti a metà del tubo corollino o alla base di questo, ma antere sempre incluse. Stilo, rispettivamente, incluso o sporgente dalla fauce; stimma capitato o debolmente bilobo. Nucule ovoidi nere o nerastre, erette, lisce, lucide o pubescenti, con cercine basale marcato e strofiolo allargato, cupuliforme.

NUMERI Grande variabilità cariologica $(2 n=14,16,18,20,22,24,26,28,30)$, con corredi CROMOSOMICl diploidi, tetraploidi o disploidi. L'origine più probabile di questi ultimi è l'ibridazione seguita da una rapida "normalizzazione" del corredo cromosomico, ovvero da fenomeni di disploidia discendente e, più raramente, ascendente. Che i corredi a numero dispari $(2 n=21,25,27)$ siano osservati raramente, in piante isolate, è indice che i prodotti dell'ibridazione tra diversi citotipi sono perlopiù transitori. II più probabile numero cromosomico di base ancestrale è $p$ $=7$ (Bolliger 1982), e i corredi più diffusi sono il diploide con $2 n=14$ e il tetraploide con $2 n=28$. Sono stati talora osservati cromosomi $B$ sovrannumerari.

BIOLOGIA Piante entomofile, dicogame proterandriche, autoincompatibili, con presenza di RIPRODUTTIVA forme eterostile all'interno della maggior parte delle popolazioni studiate e abbondante produzione di nettare. La dispersione dei frutti, grazie allo strofiolo che funge da attrazione e da organo di presa, è perlopiù mirmecocora, come nella stragrande maggioranza dei membri delle Boragineae. 
DISTRIBUZIONE Al genere Pulmonaria vengono ascritte attualmente circa 20 specie della regione circumboreale euro-asiatica e delle aree montane e collinari di quella mediterranea settentrionale, in larga maggioranza circoscritte al subcontinenete europeo (una sola, $P$. dacica Simonk., con estensione dell'areale alla regione asiatica orientale). In molti casi, tuttavia, si tratta di microspecie differenziate per il cariotipo, e solo debolmente sul piano morfologico ("citospecie"), e non possiamo escludere che studi più approfonditi inducano ad una riduzione del rango di alcune di esse; in attesa che questi studi siano sviluppati in modo organico ed esteso, nella presente trattazione dobbiamo riconoscere per l'Italia cinque specie, con una sottospecie endemica.

\section{NOTE}

SISTEMATICA La sistematica di Pulmonaria è da sempre materia di difficile trattazione: per quanto oggi più chiara nelle sue dinamiche, crediamo sia ancora ben lungi dall'essere definitivamente risolta. Se un modello come quello presentato nelle due edizioni della Flora Analitica $d^{\prime}$ Italia (Fiori \& Paoletti 1902, Fiori 1926), con numerose varietà e forme riunite in una singola macrospecie ( $P$. officinalis) può apparire troppo riduttivo, nondimeno l'approccio moderno sembra portare ad un'eccessiva "atomizzazione" del gruppo. I motivi di questo contrasto sono due facce della stessa medaglia: da un lato, la difficoltà di conciliare deboli diversità morfologiche con grandi diversità cariologiche; dall'altro, la possibilità che queste ultime derivino da una complessa evoluzione reticolare che coinvolge più o meno direttamente tutte o quasi tutte le stirpi conosciute, in molteplici direzioni e combinazioni, che di fatto configura un singameone per il quale qualunque sistema tassonomico gerarchicamente strutturato appare inadeguato.

Sul piano strettamente morfologico, il primo grande monografo del genere fu KERNER (1878), che vi incluse 12 specie articolate in tre sezioni (sect. Asperae A.Kern., sect. Strigosae A.Kern. e sect. Molles A.Kern.), individuando per primo, nel tipo di indumento delle foglie basali, un criterio diagnostico fondamentale; qualche decennio dopo PAWLOWSKI (1962), trattando delle specie ungheresi, vi avrebbe aggiunto quello della struttura del rizoma (lunghezza e spessore delle innovazioni), ma con minor successo. Una soluzione molto più parsimoniosa di quella di Kerner fu proposta da Gams in HEGI (1927: 2209-2220), per il quale esisterebbero solo quattro grandi macrospecie di origine terziaria, mentre tutte le altre stirpi meno differenziate sarebbero da ricondurre al rango di sottospecie geografiche.

$\mathrm{Ma}$, come abbiamo anticipato, questo punto di vista, all'apparenza aderente alla modesta diversità fenotipica delle varie entità, sembra scontrarsi con evidenze biologiche di tutt'altro assetto. II quadro sistematico-tassonomico più rigoroso e completo a nostra disposizione è infatti quello proposto da SAUER (1975) e Bolliger (1982), principalmente sulla base di informazioni di natura cariologica. Attraverso un'imponente indagine condotta sui numeri cromosomici di centinaia di popolazioni europee, essi hanno evidenziato una correlazione tra morfologia (caratteri deboli ma costanti), livello di ploidia e distribuzione geografica, riconoscendo un numero di entità tassonomiche assai superiore al passato. In tempi recenti, uno studio di filogenesi molecolare (KIRCHNER 2004) ha conseguito un'efficace caratterizzazione genetica di quattro specie indagate con marcatori popolazionali ad alta risoluzione (RAPD), ma ha ottenuto solo scarse informazioni applicando solo i classici marcatori di sequenza, nucleari e plastidiali, a tutte le altre; se da un lato si è trattato di un lavoro ampio, meritevole e promettente, esso è quindi ancora incapace di costituire un riferimento unitario che aggiorni o sostituisca le precedenti sinossi.

In attesa di ulteriori novità, pertanto, il nostro trattamento non può che seguire l'approccio generale offerto da queste ultime. Sulla base dei caratteri delle foglie mature, il genere si presenta in Italia con tre "stirpi" principali di facile distinzione, che potrebbero corrispondere ad altrettante "macrospecie" filogeneticamente distinte. La prima comprende le popolazioni riferibili al complesso (?) di P. angustifolia-australis, caratterizzate da foglie lanceolate e indumento di setole relativamente dense, più 0 
meno uniformi. La seconda quelle del complesso (?) di $P$. hirta-vallarsae-stiriaca, con foglie ovali-acuminate, a base spesso cuneata, e indumento dimorfo, con setole rade miste a tricomi nettamente più brevi e numerosi. La terza e ultima, rappresentata dalla sola $P$. officinalis, si distingue per le foglie mature con lamina in genere cordatocuoriforme e indumento apparentemente monomorfo, in realtà costituito spesso da setole rade miste ad aculeoli brevissimi, conici, visibili solo al microscopio. Più difficile è la distinzione delle relative "microspecie" o "citospecie". Le entità europee del complesso (?) di P. hirta-stiriaca-vallarsae, per esempio, appaiono tra loro molto simili e per quelle italiane è stato dimostrato un discreto grado di interfertilità (PuPPI \& CRISTOFOLINI 1996), sicchè il loro isolamento riproduttivo pare incompleto dal punto di vista biologico (VOSA \& PISTOLESI 2004). Una situazione simile sembra interessare anche il complesso (?) di $P$. angustifolia-australis, lasciando solo $P$. officinalis nella condizione di specie relativamente ben differenziata e di facile distinzione.

PROPRIETÀ E USI Come molte piante della famiglia definite "officinali", le proprietà curative attribuite in passato alle specie di Pulmonaria si basava sull'infondata convinzione che le malattie dovessero curarsi con elementi naturali di aspetto che ricordasse i sintomi o l'organo colpito. Nella fattispecie, quello delle foglie ampie, feltrose e spesso macchiettate di bianco, richiama sia la struttura spugnosa dei polmoni che l'escreato, suggerendone I'utilità nel combattere le patologie del sistema respiratorio, pur senza alcun riscontro scientifico. Foglie e fiori si possono invece impiegare in cucina come verdure cotte o aggiunte decorative nelle insalate, ma vale anche in questo caso il monito di farne un uso parsimonioso, per la presenza di alcaloidi pirrolizidinici potenzialmente epatotossici.

CHIAVE DELLE SPECIE II criterio più importante e sicuro per la determinazione delle specie consiste nell'esame della forma e dell'indumento delle foglie basali adulte, estivali e autunnali, cioè di quelle sviluppatesi pienamente dopo il termine della fioritura; la qualità dell'indumento riportata in chiave e nelle singole descrizioni dev'essere verificata sulla loro pagina superiore, a circa $1 \mathrm{~cm}$ dalla nervatura mediana.

1. Foglie basali adulte con lamina lanceolata, almeno quattro volte più lunga che larga, la base decorrente sulle ali di un picciolo indistinto; pagina superiore con setole di lunghezza variabile, ma mai nettamente dimorfe ... 2

- Foglie basali adulte con lamina ovato-acuminata, meno di tre volte più lunga che larga, distintamente picciolate; pagina superiore con setole sparse miste a peli o aculeoli più brevi

2. Rizoma esile, 2-6 $\mathrm{mm}$ di diametro, con innovazioni ben distanziate; lamina delle foglie basali cosparsa di macchie piccole, regolari e distinte, con numerose ghiandole sessili o stipitate 5. P. stiriaca

- Rizoma robusto, 5-10 $\mathrm{mm}$ di diametro, con innovazioni ravvicinate; lamina delle foglie basali generalmente immacolata, priva di ghiandole o con sparsi peli ghiandolari brevemente stipitati .... ... 3

3. Foglie basali adulte strettamente lanceolate, 6-9 volte più lunghe che larghe, le cauline da lineari a largamente lanceolate, a base cuneata; indumento di setole omogenee e brevi (ca. 0,6 mm), senza ghiandole; corolla azzurra, glabra sotto la fauce 1. P. angustifolia

- Foglie basali adulte largamente lanceolate, 4-6(7) volte più lunghe che larghe, le cauline ovato lanceolate, a base troncato-cordata; indumento di setole molto eterogenee $(0,5-1,5 \mathrm{~mm})$, miste a rade ghiandole brevemente stipitate; corolla violetto-porporina, glabrescente o sparsamente pubescente sotto la fauce ................................................... 2. P. australis

4. Foglie basali adulte con lamina a base cordata, sopra con sparsa copertura di setole lunghe e brevi peli ghiandolari, sul resto della superficie con densi aculeoli conici ( $\leq 0,2 \mathrm{~mm}$; subsp. officinalis), o glabra (subsp. marzolae); lato 
interno del tubo corollino glabro sotto l'anello di peli

4. P. officinalis

- Foglie basali adulte con lamina a base da troncata a cuneato-attenuata, senza aculeoli, le setole miste a peli brevi di almeno $0,3 \mathrm{~mm}$; lato interno del tubo corollino più o meno pubescente sotto l'anello di peli . 5

5. Foglie basali adulte a margine ondulato, con macchie assenti o verdastre e poco evidenti e setole sparse tra densi peli brevi (25-70 per $\mathrm{mm}^{2}$ )

6. P. vallarsae

- Foglie basali adulte a margine piano, con macchie biancastre, ampie e spesso confluenti, e setole miste a radi peli brevi (meno di $25-70$ per $\mathrm{mm}^{2}$ ) 3. P. hirta

\subsection{Pulmonaria angustifolia $\mathrm{L}$. POLMONARIA A FOGLIE STRETTE}

Pulmonaria angustifolia L., Sp. PI. 1: 135. 1753.

(三) Lithospermum graminifolium Viv., Elench. Pl.: 23. 1802, non Viv. in Ann. Bot. 1(2): 163. 1804 三 P. officinalis var. angustifolia (L.) Fiori, Nuov. Fl. Ital. 2(2): 278. 1926.

Locus classicus: "in Pannonia, Helvetia, Suecia".

Lectotipo (Selvi in CAFFERTY \& JARVIS 2004: 803): [Svezia] "Gotland, Skara”, s.d., s.coll., Herb. Linneo 184.1 (LINN!).

(=) P. azurea Besser, Prim. Fl. Galiciae Austriac. 1: 150. $1809 \equiv$ P. officinalis var. azurea (Besser) Fiori in Fiori \& Bég., FI. Italia 2(3): 372. 1902.

Locus classicus: [Polonia] "in scopolis vallis Oycow".

Tipo non designato.

Etimologia Attributo composto dal latino angustus, stretto, e folia, foglia, a foglie strette.

DESCRIZIONE Rizoma breve e robusto, 5-10 mm di diametro, con innovazioni brevi $(<5 \mathrm{~cm})$. Foglie prive di macchie, le basali adulte lunghe almeno fino a $17 \mathrm{~cm}$, con lamina strettamente lanceolata, 6-9 volte più lunga che larga, con base attenuatodecorrente e picciolo indistinto; indumento della pagina superiore costituito da setole piuttosto brevi e omogenee (ca. 0,6 mm), senza peli ghiandolari; foglie cauline e bratteali da lineari a largamente lanceolate, a base cuneata. Calice fiorale lungo $8-10 \mathrm{~mm}$. Corolla di $12-14 \mathrm{~mm}$, con tubo glabro sotto l'anello di peli faucali e lembo di 8-10 mm di diametro, azzurro-bluastro, con toni violaceoporporini solo nei fiori immaturi. (Fig. 30)

FENOLOGIA Specie a fioritura tardo-primaverile ed estiva, tra la seconda metà di aprile e la fine di luglio, che matura i frutti tra la fine di giugno e agosto.

NUMERO Corredo diploide con $2 \mathrm{n}=14$, verificato sia su piante piemontesi e lombarde CROMOSOMICO (SAUER 1975) che su popolazioni provenienti da Austria (SAUER 1975, Dobeš in Dobeš \& ViteK 2000), Bielorussia (SEMERENKo 1990), Francia (SAUER 1975, Bolliger 1982), Germania (SAUer 1975, SAUer \& GRUber 1979, LIPPERT 2006), Polonia (PogAn \& al. 1989), Slovacchia (MÁJovskÝ \& Murin 1980), Svezia (SAUER 1975, LÖVKVIST \& HULTGÅRD 1999). Secondo SAUER (1975), conteggi diversi riportati in letteratura $(2 \mathrm{n}=20,24,28$; TARNAVSCHI 1935, Bolliger 1982) sono dovuti ad errori di identificazione. 
ECOLOGIA Boschi di latifoglie, boscaglie e prati aridi, su terreni acidi, nella maggior parte dell'areale europeo a quote collinari, a partire da $100 \mathrm{~m}$ di quota, da noi segnalata invece solo dalla fascia collinare a quella subalpina, tra 550 e $1600 \mathrm{~m}$ di quota.

DISTRIBUZIONE Specie delle province europee centrale e orientale, distribuita da nord a sud tra la parte meridionale della Penisola scandinava e le Alpi centro-occidentali (delle quali occupa quasi esclusivamente il versante nord) e da ovest a est tra la Germania e la Russia europea. In Italia sconfina solo sporadicamente tra le Alpi Marittime e il massiccio del Brenta, ma la maggior parte delle segnalazioni storiche per questa specie vanno riferite alla vicariante $P$. australis. (Fig. 32)

\section{NOTE}

ALTRI NOMI VOLGARI Borrana celestina.

Nomenclatura Come giustamente osservato da Peruzzi \& Soldano in Nepl \& al. (2010: 385) il nome "Lithospermum graminifolium" pubblicato da VIVIANI (1802), pur coll'evidente proposito di riferirsi alla "Pulmonaria suffruticosa" di Linneo (oggi Moltkia suffruticosa (L.) Brand.), per via del riferimento diretto a $P$. angustifolia $\mathrm{L}$. dev'essere trattato quale un sinonimo omotipico di questa (non però superfluo e invalido, né illegittimo, perché formalmente nome di rimpiazzo in Lithospermum, stante già l'esistenza di L. angustifolium Forssk. del 1785; ICN Artt. 6.11, 7.4; MCNEILL \& al. 2012). L'epiteto "graminifolium" di Viviani non pare sia mai stato ricombinato in seguito, nè come Lithospermum nè sotto altri generi, con riferimento al lavoro del 1802, ma sempre e soltanto alla seconda pubblicazione dell'autore (quella del 1804), nella quale ci pare fosse suo chiaro intento di sottolineare la distinzione di questa specie ed evitare di ripetere l'errore, visto che manca ogni riferimento alla precedente o ad alcun nome linneano. Secondo questa interpretazione anche quello del 1804 è un nome nuovo validamente pubblicato, illegittimo perché omonimo del precedente, ma valido perchè eterotipico rispetto ad esso, e come tale riferibile ai sinonimi di $M$. suffruticosa.

SISTEMATICA II complesso di P. angustifolia s.I. attende ancora una valida sistemazione. Secondo SAUER (1975), accanto alla specie linneana, tipica delle fasce planiziale e collinare dell'Europa centrale e orientale, diploide con $2 n=14$, ne esistono almeno altre due strettamente vicarianti: una, $P$. kerneri Wettst., anch'essa diploide, endemica del settore alpinoorientale, l'altra, $P$. visianii, tetraploide o ipotetraploide $(2 n=26,28)$, di quello adriaticoorientale, tra l'Istria e la Dalmazia. Più incerta è la relazione con $P$. australis, il cui corredo ipotriploide $(2 \mathrm{n}=20)$ di probabile origine ibrida (SAUER 1987), potrebbe teoricamente derivare sia da parentali di questo stesso gruppo che di gruppi diversi. A sostegno di una stretta relazione con $P$. angustifolia s.str. è la piccola distanza genetica ottenuta da KIRCHNER (2004) con marcatori popolazionali, comparabile con quella che gli stessi marcatori (RAPD) hanno rilevato tra altre entità conspecifiche. In presenza di esemplari incompleti o immaturi la distinzione tra le due specie resta comunque assai difficile. Pulmonaria angustifolia s.str. si distingue da $P$. australis per la forma e l'indumento delle foglie (più strette, con setole mediamente più brevi e omogenee e prive di peli ghiandolari), per i fiori complessivamente minori e il tubo corollino internamente glabro sotto l'anello di peli faucali.

IBRIDI Una popolazione ibrida tra la vera P. angustifolia e "P. saccharata" (da intendersi come $P$. vallarsae subsp. apennina), con caratteri intermedi e corredo cromosomico $2 n=18$, è stata rinvenuta in un'area di simpatria tra le parentali da SAUER (1975), sul versante settentrionale delle Alpi Marittime francesi, a poca distanza dal confine italiano, e non è escluso che l'incrocio possa aver luogo anche sul nostro versante. Per il presunto ibrido tra " $P$. angustifolia" (più probabilmente $P$. australis) e $P$. officinalis, descritto per I'Italia come $P$. $\times$ hybrida (si veda la scheda a termine della trattazione del genere), non si hanno ad oggi riscontri cariologici. 


\subsection{Pulmonaria australis (Murr) W. Sauer POLMONARIA SUDALPINA}

Pulmonaria australis (Murr) W. Sauer in Biblioth. Bot. 131: 56. 1975.

(三) P. angustifolia var. australis Murr in Dörfl., Herb. Norm., Sched. Cent. XLV: 138, no. 4480. 1903.

Locus classicus: [Italia, Trentino Alto Adige] "Tirolia australis. In collibus et montibus circa "Trient." 450 m. s. m.".

Lectotipo (SAUER 1975: 56): [Italia, Trentino Alto Adige] "Tirol, Trient, Chegul", 19/05/1869, Lièvre (IBF).

Altro materiale originale (CECCHI \& SELVI 2015b: 647): [Italia, Trentino Alto Adige] "Tirolia australis. In collibus et montibus circa "Trient." 450 m. s. m.", 04/1901, Murr (B 10 0365437!; iso - KFTA 1858!, 1859!, P 505742, 505743!, 505744!, 505745 !).

(-) P. visianii auct. (H. Merxmüller. \& W. Sauer in HeYwood 1972: 100, p. p.) non Degen \& Lengyel, Fl. Veleb. 2: 569. 1937.

EtIMologia Dal latino australis, "meridionale", in riferimento alla distribuzione circoscritta al versante meridionale delle Alpi.

DESCRIZIONE Rizoma breve e robusto, di 5-10 mm di diametro, con innovazioni brevi $(<5 \mathrm{~cm})$. Foglie generalmente prive di macchie, le basali adulte lunghe fino a $40 \mathrm{~cm}$, con lamina largamente ellittico-lanceolata, 4-6 volte più lunga che larga, con base attenuato-decorrente e picciolo indistinto; indumento della pagina superiore costituito da setole di lunghezza diversa, comprese tra 0,5 e 1,5 mm, miste a rade ghiandole brevemente stipitate; foglie cauline e bratteali ovato-lanceolate, a base da arrotondata a cordato-amplessicaule. Calice fiorale lungo 9-12 mm. Corolla di 13-17 mm, con tubo sparsamente pubescente o glabrescente sotto l'anello di peli faucali e lembo di $10-15 \mathrm{~mm}$ di diametro, violetto-porporino. (Fig. 31)

FENOLOGIA Specie a fioritura perlopiù primaverile precoce, ma ritardata a quote più elevate, dove può protrarsi fino ad estate avanzata; i mericarpi maturano e vengono rilasciati a partire dalla fine di aprile fino a settembre.

NUMERO $2 n=20$, da interpretarsi come ipotriploide, verificato sia su piante lombarde e CROMOSOMICo del Trentino Alto Adige che su popolazioni di stazioni poco distanti dal nostro confine in Austria (SAUER 1975), Francia (SAUER 1975, BOlliger 1982) e Slovenia (SAUER 1975); un solo conteggio aneuploide, $2 \mathrm{n}=18+1 \mathrm{~B}$, per una pianta dell'Alto Adige (SAUER 1975).

ECologiA Boschi mesofili di latifoglie o conifere, su terreni acidi, tra 100 e 2200 m di quota.

DISTRIBUZIONE Endemismo della provincia centro-europea meridionale, vicariante meridionale di $P$. angustifolia su quasi tutto il versante alpino meridionale; più frequente sulle Alpi centro-orientali, si spinge a ovest con stazioni più isolate fino alle Alpi Cozie francesi, ma sconfina dal nostro territorio soprattutto a nord, nell'Austria meridionale, e a est, nell'Istria slovena e croata. In Italia è diffusa nella maggior parte delle regioni settentrionali. (Fig. 32) 


\section{NOTE}

TIPO Nella scheda $n^{\circ} 4480$ dell'Herbarium Normale di Dörfler, del 1903, il basionimo fu validamente pubblicato sulla base di due raccolte originali: la prima, quella di Lièvre del 1869, citata dall'autore nella diagnosi in calce alla scheda e successivamente indicata come "olotipo" da SAUER (1975: 56), corrisponde ad un campione che l'autore aveva già citato quattro anni prima (MURR 1899: 152) sotto altro nome ("Pulmonaria tuberosa Freyn apud Murr"); la seconda è rappresentata dalla serie di duplicati raccolti dallo stesso Murr nel 1901 e distribuiti a numerosi erbari con un'etichetta che ricalcava fedelmente il testo della stessa scheda. Per questo motivo il campione conservato in IBF non solo deve considerarsi un lectotipo anziché un olotipo, ma, se dovesse risultare ormai distrutto ("Almost the entire collection was flooded in 1985. All specimens were covered with mud. 2015 restoration of vascular plants from the main herbarium was finishe $^{\prime \prime 1}$ ), ciascuno dei duplicati distribuiti da Dörfler con l'etichetta n. 4480 risulterebbe egualmente valido per la designazione di un nuovo lectotipo.

${ }^{1}$ http://sweetgum.nybg.org/science/ih/herbarium details.php?irn=126158

SISTEMATICA Pulmonaria australis è spesso confusa con $P$. angustifolia, specie assai simile ma apparentemente molto più rara sul nostro territorio; in presenza di esemplari incompleti o immaturi la distinzione tra le due specie è pressochè impossibile. Tuttavia, accanto ad evidenze sperimentali (KIRCHNER 2004) che testimoniano un loro probabile, diretto legame filogenetico (si veda la nota per $P$. angustifolia), sia il corredo cromosomico che alcuni caratteri diagnostici negli esemplari pienamente sviluppati consentono di distinguere le due forme tipiche: $P$. australis è caratterizzata dalla lamina fogliare mediamente più larga (di rado debolmente maculata), dall'indumento delle foglie basali adulte eteromorfo e debolmente ghiandoloso e dai fiori maggiori, con tubo corollino mai del tutto glabro sotto l'anello di peli faucali.

\subsection{Pulmonaria hirta L.} POLMONARIA CHIAZZATA

Pulmonaria hirta L., Sp. PI., ed. 2, 2: 1667. 1763.

Locus classicus: [Italia, Toscana] "in Etruriae montibus".

Lectotipo (Selvi \& Cristofolini in CAFFERTY \& JARVIS 2004: 803): "Pulmonaria Fragariae odore", disegno in BOCCONE (1697: t. 105!).

Epitipi (Selvi in CAFFERTY \& JARVIS 2004: 803): [Italia, Toscana] "Prov. Arezzo: montane Abies-Fagus woods close around the walls of Eremo di Camaldoli, 1100 m", 26/03/2002, Selvi (FI 2356!; iso- BM!); ibidem, 31/05/2002, Cristofolini (FI 2366 !; iso- BM!).

(=) P. picta Rouy, FI. Fr. 10: 297. 1908.

Locus classicus: [Italia] "Italie surtout centrale".

Neotipo (PUPPI \& CRISTOFOLINI 1996: 15): [Italia, Toscana] “Cerbaie, nel bosco di Valle Lupitana" 06/05/1951, Contardo (FI 7410!).

(-) P. saccharata auct. (H. Merxmüller. \& W. Sauer in HeYwood 1972: 101, PIGNATTI 1982: 410) non Mill., Gard. Dict., ed. 8. n. 3. $1768 \equiv$ P. officinalis var. saccharata (Mill. non sensu orig.) Fiori in Fiori \& Bég., Fl. Italia 2(3): 371. 1902.

Etimologia Dal latino hirtus, "irto", "ispido", in riferimento all'indumento delle foglie e dei fusti caratterizzato da setole maggiori e più rade in questa che nelle altre specie di questa stirpe. 
DESCRIZIONE Rizoma breve e robusto, $4-10 \mathrm{~mm}$ di diametro, ma innovazioni lunghe fino a 20 $\mathrm{cm}$. Foglie spesso cosparse di evidenti macchie biancastre ampie, irregolari e spesso confluenti, le basali adulte con lamina lunga fino a $25 \mathrm{~cm}$, ovata, 2-3 volte più lunga che larga, a base cuneata e margine piano, e picciolo ben distinto; indumento della pagina superiore rado $\left(<25\right.$ tricomi per $\left.\mathrm{mm}^{2}\right)$, costituito prevalentemente da setole di lunghezza compresa tra 1,2 e 1,5 mm, miste a peli più brevi e ghiandole stipitate o sessili molto rade; foglie cauline e bratteali ovato-lanceolate, a base da arrotondata a cordato-amplessicaule. Calice fiorale lungo $10-13(18) \mathrm{mm}$. Corolla di $15-20 \mathrm{~mm}$, con tubo pubescente sotto l'anello di peli faucali e lembo fino a $15 \mathrm{~mm}$ di diametro, violettoporporino. (Fig. 33)

NUMero Popolazioni tetraploidi con $2 \mathrm{n}=28$ sono presenti in Toscana (SAUER 1975, CROMOSOMICO CAPINERI 1986, VOSA \& PISTOLESI 2004) e Umbria (VOSA \& PISTOLESI 2004); i corredi aneuploidi con $2 n=25,26,26+b$ o 27 occasionalmente riportati per piante provenienti da Emilia Romagna (PUPPI \& CRISTOFOLINI 1996, SAUER 1975), Lazio (PUPPI \& CRISTOFOLINI 1996), Liguria (SAUER 1975), Toscana (VOSA \& PISTOLESI 2004) e persino Abruzzo (BLAISE \& CARTIER 1982), dove la specie non sembra presente, possono essere interpretati come ibridi, variamente emancipati dalle parentali, con $P$. vallarsae subsp. apennina, che ha $2 n=22$. Se accettiamo la separazione dei citotipi italiani in altrettante entità distinte, i conteggi con $2 n=22$ dalle Alpi occidentali, in territorio sia francese che italiano (Liguria e Piemonte; SAUER 1975, VOSA \& PISTOLESI 2004), e dall'Appennino centro-settentrionale in Liguria (Bolliger 1982), Emilia Romagna (PUPPI \& CRISTOFOLINI 1996, VosA \& PISTOLESI 2004), Toscana (MerXmüller \& GraU 1969) e Lazio (PupPI \& CRISTofolinI 1996) vanno riferiti invece esclusivamente a quest'ultima.

ECOLOGIA Su suolo pingue, generalmente in boschi mesofili di latifoglie, tra la fascia collinare e il limite superiore delle faggete (tra 300 e $1500 \mathrm{~m}$ ), ma con stazioni di bassa quota che possono spingersi al di sotto dei $100 \mathrm{~m}$ ed altre più elevate in boschi di abete, peccio e larice, almeno fino a 1600 m sulle Alpi Marittime.

DISTRIBUZIONE Specie della provincia liguro-tirrenica settentrionale, donde sconfina in una modesta porzione di quella centro-europea meridionale. Distribuita tra le Alpi Marittime e l'Appennino centro-meridionale, in Italia prevale decisamente sul versante tirrenico (poche e da verificare le stazioni segnalate su quello orientale degli Apennini, in provincia adriatica, tra le Marche e l'Abruzzo; PupPI \& CRISTOFOLINI 1996) e non sembra spingersi più a sud dei colli laziali. (Fig. 34)

\section{NOTE}

Nomenclatura A lungo il nome di riferimento utilizzato per questa specie e per l'aggregato di microspecie ad essa collegate, in Italia e non solo, è stato quello di $P$. saccharata, derivato dal latino saccharum, zucchero, quindi dall'aggettivo saccharatus, zuccherato, in riferimento alla maculatura chiara, abbondante e confluente, della lamina fogliare, che nelle forme tipiche ricorda incrostazioni zuccherine. L'epitipificazione del binomio $P$. hirta con materiale toscano ha inequivocabilmente chiarito l'identità della nostra pianta, indipendentemente dalla scelta di considerare quella di Miller quale specie distinta (PuPPI \& CRISTOFOLINI 1996) o sottospecie di questa.

SISTEMATICA Pulmonaria hirta appartiene ad un complesso di specie allopatriche con caratteri omogenei, che potrebbero essere eguamente trattate come entità infraspecifiche. L'analisi condotta da PUPPI \& CRISTOFOLINI (1996) suggerisce uno stretto legame di P. hirta s.str. con $P$. affinis Jord., diffusa dalla Francia centro-settentrionale fino alla regione pirenaica, e con $P$. saccharata, pianta che, a dispetto del protologo, sarebbe circoscritta 
alla parte centro-orientale della provincia europeo-atlantica, presente con certezza solo in Belgio e Olanda (vedasi anche LACAITA 1927). Se l'insieme di queste entità costituisca effettivamente una singola "macrospecie" è questione che meriterebbe ulteriore approfondimenti. VOSA \& PISTOLESI (2004) avanzano ragionevoli dubbi sulla solidità di un rigido concetto di "citospecie", visto che la plasticità cariologica ridurrebbe le barriere tra citotipi formalmente incompatibili, e suggeriscono addirittura che tutto il complesso $P$. hirta-vallarsae (non è chiaro fino a che punto inclusivo) possa essere trattato come tale. L'ipotesi ci sembra plausibile, ma non può essere confermata che con più approfonditi studi, possibilmente corroborati da analisi molecolari; nell'attesa, riteniamo più prudente mantenere un assetto tassonomico più tradizionale, "fino a prova contraria". Dobbiamo comunque precisare che, per l'estesa sovrapposizione dell'areale appenninico dei due principali citotipi italiani, $P$. hirta s.str. $(2 n=28)$ e $P$. vallarsae s.l. $(2 n=22)$, essi non possono trattarsi a rango di sottospecie l'uno dell'altro, come invece proposto da PerUzzI (2010: 531).

IBRIDI Come anticipato nella sezione dedicata ai numeri cromosomici, piante di " $P$. saccharata s.l." con corredi aneuploidi dell'Appennino centro-settentrionale, quasi sempre rinvenute in condizione di simpatria con $P$. hirta e $P$. vallarsae subsp. apennina, possono considerarsi prodotti diretti o indiretti del loro incrocio, la cui insorgenza è peraltro ben documentata da test di laboratorio (PUPPI \& CRISTOFOLINI 1996). Va comunque notato che si tratta di ritrovamenti occasionali, che non sembrano dar luogo a popolamenti stabili, nè tantomeno indipendenti.

\subsection{Pulmonaria officinalis $\mathrm{L}$. POLMONARIA MAGGIORE}

Pulmonaria officinalis L., Sp. PI. 1: 135. 1753.

(三) P. vulgaris Mérat, Nouv. Fl. Env. Paris: 70. 1812, nom. illeg.

Locus classicus: "in Europae nemoribus".

Lectotipo (Selvi in CAFFERTY \& JARVIS 2004: 804): "Florentiae, Monspelii in horto Regio", Herb. Burser XIV(2): 51, individuo sul lato sinistro del foglio (UPSBURSER!).

Etimologia Dal latino officinalis, "officinale", in riferimento all'impiego delle foglie di questa specie in medicina, per la cura delle malattie polmonari.

DesCrizIONE Rizoma allungato e sottile, 3-6(10) $\mathrm{mm}$ di diametro, con innovazioni di 4-7 cm. Foglie più o meno chiaramente maculate, le basali adulte con lamina largamente ovata, 1,5-2 volte più lunga che larga, a base cordata o cuoriforme, e picciolo largamente alato; indumento della pagina superiore con setole sparse, di lunghezza variabile tra 0,5 e $1,5 \mathrm{~mm}$, miste a radi peli ghiandolari e in genere (subsp. officinalis) a densi aculeoli conici $(<0,2 \mathrm{~mm})$; foglie cauline e bratteali ovate, a base cordato-amplessicaule. Calice fiorale lungo 9-11 mm. Corolla di 13-20 mm, con tubo glabro sotto l'anello di peli faucali, e lembo di 10$16 \mathrm{~mm}$ di diametro, roseo-vinoso. (Fig. 35)

FENOLOGIA Fioritura culminante tra marzo e maggio, a seconda della quota anticipata anche alla fine dell'inverno, tra gennaio e febbraio, o ritardata fino ad agosto; mericarpi maturi sono rilasciati da metà primavera ad estate inoltrata.

Numero $2 n=16$; si rimanda al trattamento delle sottospecie per i dettagli sulle fonti CROMOSOMICO bibliografiche. 
DISTRIBUZIONE Specie delle province europee atlantica e centrale. In Italia è presente con due sottospecie, una delle quali endemica. (Fig. 36)

NOTE

NomencLAtURA II binomio P. vulgaris fu validamente pubblicato (ICN Art. 36.1; MCNeILL \& al. 2012) perché creato con l'idea che l'originale linneano fosse ambiguo e che la "Pulmonaria officinalis" di Linneo potesse differire dalle piante in seguito citate da altri autori con lo stesso nome, cioè senza una formale assimilazione della pianta di Mérat col tipo di Linneo conservato a Uppsala. Tuttavia l'autore non specificò a quale delle diverse interpretazioni di "P. officinalis" dovesse riferirsi la pianta da lui descritta, lasciando di fatto irrisolta l'identità della sua specie in assenza di materiale originale. Per questa ragione, trattandosi di nome ampiamente negletto nella letteratura recente, la questione è stata risolta selezionando quale suo lectotipo lo stesso già designato per $P$. officinalis (CECCHI \& SELVI 2015b: 649).

IBRIDI Dell'ibrido tra $P$. officinalis e "P. angustifolia" (più probabilmente $P$. australis) non si hanno altre segnalazioni che non quella di Kerner, che basandosi su piante del Tirolo italiano vi descrisse la notospecie $P$. $\times$ hybrida. Tale circostanza, pur plausibile, in assenza di saggi mirati o di esemplari spontanei freschi su cui condurre un'analisi cariologica, non può ovviamente essere accertata (si veda la scheda del presunto ibrido a termine della trattazione del genere).

ChIAVE delle 1. Lamina delle foglie basali vistosamente maculata, con setole miste a sparsi SOTTOSPECIE peli ghiandolari e a densi aculeoli ................................ 1. subsp. officinalis

- Lamina delle foglie basali oscuramente maculata, priva di aculeoli 2. subsp. marzolae

\subsubsection{Pulmonaria officinalis subsp. officinalis POLMONARIA MAGGIORE TIPICA}

(=) P. maculata F. Dietr., Vollst. Lex. Gärtn. 7: 657. 1807 三 P. officinalis L. f. maculata (F. Dietr.) Fiori in Fiori \& Bég., Fl. Italia 2(3): 371. 1902.

Locus classicus: [pianta coltivata in Germania] "im hiesigen Garten [nei giardini locali]".

Tipo non designato.

DESCRIZIONE Foglie basali adulte a base tipicamente cuoriforme, con macchie bianche numerose, spesso confluenti, e una densa copertura di aculeoli conici misti alle setole e ai radi peli ghiandolari della superficie superiore. (Fig. $35 \mathrm{~A}-\mathrm{C}$ )

Numero $2 n=16$, verificato sia su piante alpine della Lombardia e del Trentino Alto Adige CROMOSOMICO (SAUER 1975, ASTUti \& al. 2014) che su popolazioni venete dei Colli berici ed Euganei (SAUER 1975) e di varie località dell'Appennino ligure e tosco-emiliano (CAPINERI 1986, VOSA \& PISTOLESI 2004). Questi dati confermano quelli di numerosi conteggi effettuati su piante raccolte in altri paesi europei: Austria (SAUER 1975, Dobeš in DOBEŠ \& al. 1997; DOBEŠ \& VITEK 2000), Bosnia-Erzegovina (SAUER 1975), Bulgaria (SAUER 1975), Cechia (Javůrková in LöVE 1981: 857), Croazia (SAUER 1975), Francia (SAUER 1975), Germania (SAUER 1975, SAUER \& Gruber 1979), Regno Unito (SAUER 1975), Romania (SAUER 1975), Slovacchia (Vachova in MÁJOVSKÝ 1974, Murin IN MÁJOVSKÝ 1978, MÁJOVSKÝ \& MURIN 1980), Slovenia (SAUER 1975, Druskovic \& Lovka in STACE 1995), Svezia (LöVKVIST \& HULTGÅRD 1999), Svizzera (SAUER 1975, BOlLIGER 1978). I conteggi di $2 n=24$ riportati per piante svizzere 
(Bolliger 1978) e di 2n = 14 per piante bielorusse (DMITRIEVA 1986), macedoni (SEKOVSKI \& JOVANOVSKA 1983) e polacche (MIZIANTI \& al. 1981) si riferiscono certamente ad entità diverse.

ECOLOGIA Boschi mesofili di latifoglie, su suolo pingue, in genere a quote collinari, ma nota da stazioni di pianura, quasi a livello del mare, fino ad almeno $2000 \mathrm{~m}$.

DISTRIBUZIONE Entità delle provincie europee atlantica e centrale. In Italia è perlopiù circoscritta alla fascia collinare e montana dei rilievi alpini e prealpini, scendendo a quote inferiori solo nella pianura padana settentrionale e in quella veneto-friulana; assai più rara nell'Appennino centro-settentrionale. (Fig. 36)

\section{NOTE}

Nomenclatura A differenza di quanto erroneamente riportato da CECCHI \& Selvi (2015b: 650), il binomio "Pulmonaria maculosa" (anziché "maculata"), non è mai stato validamente pubblicato perché riportato con rango ambiguo da LIEBLEIN (1784: 56), poi solo elencato tra i sinonimi di $P$. officinalis (e comunque frutto di un chiaro errore di trascrizione del nome di Dietrich) da REICHENBACH (1831: 338).

\subsubsection{Pulmonaria officinalis subsp. marzolae POLMONARIA DEL MONTE MARZOLA}

Pulmonaria officinalis subsp. marzolae G. Astuti, Peruzzi, Cristof. \& P. Pupillo in Phytotaxa 186(3): 155. 2014.

Locus classicus: [Italia, Trentino Alto Adige] "Marzola (Trento), lungo il sentiero Bivio II - Sella Marzola, 46 $01^{\prime} 32^{\prime \prime} \mathrm{N}, 11^{\circ} 10^{\prime} 50^{\prime \prime} \mathrm{E}, 1640 \mathrm{~m}$ [...] Marzola (Trento), lungo il sentiero Bivio II - rifugio Bailoni, $46^{\circ} 01^{\prime} 11^{\prime \prime} \mathrm{N}, 11^{\circ} 10^{\prime} 56^{\prime \prime} \mathrm{E}, 1640 \mathrm{~m}[. .$. Marzola (Trento), lungo il sentiero Bivio II - rifugio Bailoni, $1620 \mathrm{~m}, 46^{\circ} 01^{\prime} 23^{\prime \prime} \mathrm{N}$, $11^{\circ} 10^{\prime} 56^{\prime \prime} \mathrm{E}$ [...] Marzola (Trento), sopra Bivio I, $1600 \mathrm{~m}, 46^{\circ} 02^{\prime} 07^{\prime \prime} \mathrm{N}, 11^{\circ} 11^{\prime} 14^{\prime \prime} \mathrm{E}$ [...] Marzola (Trento), sotto Bivio I, a sx del sentiero, $46^{\circ} 02^{\prime} 11^{\prime \prime} \mathrm{N}, 11^{\circ} 11^{\prime} 14^{\prime \prime} \mathrm{E}$, $1570 \mathrm{~m}^{\prime \prime}$.

Olotipo (cfr. Astutı \& al. 2014: 155): [Italia, Trentino Alto Adige] “Marzola (TN), sentiero bivio II, sella Marzola, 1640 m”, 19/06/2013, Pupillo (FI 7164!).

Paratipi (Astuti \& al. 2014: 156): [Italia, Trentino Alto Adige]

"Marzola (Trento), lungo il sentiero Bivio II - rifugio Bailoni, 46 $01^{\prime} 11^{\prime} \mathrm{N}$,

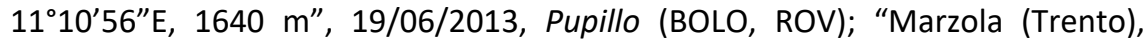
lungo il sentiero Bivio II - rifugio Bailoni, $1620 \mathrm{~m}, 46^{\circ} 01^{\prime} 23^{\prime \prime} \mathrm{N}, 11^{\circ} 10^{\prime} 56^{\prime \prime} \mathrm{E}$ ", 08/06/2013, Pupillo (BOLO); "Marzola (Trento), sopra Bivio I, $1600 \mathrm{~m}, 46^{\circ}$

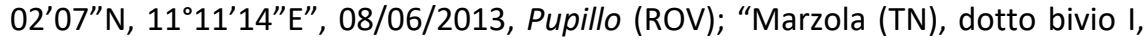
sinistra del sentiero, $1570 \mathrm{~m}$ ", 08/06/2013, Pupillo (FI 7165!).

(=?) P. tridentina Evers in Verh. Zool.-Bot. Ges. Wien 46: 74. 1896.

Locus classicus: [Italia, Trentino Alto Adige] "Trient, Schlucht des Salébaches, Goccia d'Oro, Mori, Monte Brugino; Val di Ledro, Malga Dromaë in dumetis".

Lectotipo (CECCHI \& SelVI 2015b: 650): [Italia, Trentino Alto Adige] "Tirolia australis: Mori, Monte Brugino, in dumetis; Tridentum in faucibus torrentis Salé; Goccia d'Oro", [25.04, 25.05, 15.12] 1893, Evers 8648 (GZU 272989!).

Altro materiale originale (CECCHI \& SELVI 2015b: 650): [Italia, Trentino Alto Adige] "Tirolia australis: Mori, Monte Brugino, in dumetis; Tridentum in faucibus torrentis Salé; Goccia d'Oro", [25.04, 25.05, 15.12] 1893, Evers 8648 (GZU 
272986!, 272987!, 272988!); “In angustiis torrentis Salé; Goccia d'Oro - Val di Ledro, Biacesa, in dumetis Malgae Dromaë", [03.1891, 05.1895], Evers (GZU 272990 !, 272991!).

Etimologia Dal nome del monte Marzola, posto tra la valle dell'Adige e la Valsugana a oriente della città di Trento, locus classicus per questa entità di recentissima descrizione.

DESCRIZIONE Foglie basali adulte a base più spesso tronca, di rado cuoriforme, con macchie verdastre poco evidenti e prive di aculeoli sulla pagina superiore. (Fig. $35 \mathrm{D}-\mathrm{E}$ )

Numero $2 n=16$, da materiale del locus classicus (Astutı \& al. 2014). CROMOSOMICO

ECOLOGIA Boschi mesofili di latifoglie, su suolo pingue di matrice calcareo-dolomitica, tra 1200 e 1700 m di quota.

DISTRIBUZIONE Endemismo molto localizzato, noto per poche località del Trentino sud-orientale (Fig. 36).

\subsection{Pulmonaria stiriaca A. Kern. POLMONARIA DELLA STIRIA}

Pulmonaria stiriaca A. Kern., Monogr. Pulmon.: 36. 1878.

Locus classicus: [Austria] "inter Kapfenberg et Aflenz in Stiria superiore".

Lectotipo (SAUER 1973: 6): [Austria] "Im Thörlgraben bei Aflenz", 1849, Kerner (WU 69918).

Altro materiale originale (CECCHI \& SELVI 2015b: 650): [Italia, Friuli Venezia Giulia] "waldige Stellen auf Sanstein boden westlich von Görz", 1869, Krašan, Herb. Kerner (WU 77331!; iso- WU 77332!).

Etimologia Dal latino stiriacus, "della Stiria", regione dell'Austria sud-orientale da cui proviene il materiale tipico.

DESCRIZIONE Rizoma esile, 2-6 mm di diametro, con innovazioni lunghe fino a $15 \mathrm{~cm}$ o più. Foglie generalmente cosparse di evidenti macchie biancastre, di rado confluenti, le basali adulte lunghe fino a a $35 \mathrm{~cm}$, con lamina ovata, 2-4 volte più lunga che larga, a margine piano e base attenuata, decorrente su un picciolo alato lungo in genere da $1 / 3$ a $1 / 2$ di essa; indumento della pagina superiore costituito da dense setole di lunghezza molto variabile, miste a microghiandole e ad abbondanti peli ghiandolari; foglie cauline a base cordato semiamplessicaule, le inferiori ovate, le superiori progressivamente ristrette. Calice fiorale lungo (7)10-13(16) $\mathrm{mm}$. Corolla di $15-20 \mathrm{~mm}$, il tubo con pubescenza discontinua sotto l'anello di peli faucali, costituita da piccole bande alternate ai petali, il lembo fino a $16 \mathrm{~mm}$ di diametro, densamente pubescente sopra la fauce, azzurrolillacino. (Fig. 37)

FENOLOGIA Fiorisce precocemente in primavera, tra marzo e maggio, completando la maturazione dei frutti entro l'inizio dell'estate.

NUMERO II citotipo prevalente è $2 n=18$, verificato sulla maggior parte delle popolazioni CROMOSOMICO austriache esaminate (SAUER 1973, 1975), ma se ne conosce anche uno con $2 n=$ 24, presente sia in Austria che in Slovenia (SAUER 1973); sempre dalla Slovenia 
proveniva anche I'unico esemplare di Pulmonaria per il quale sia stato conteggiato $2 n=30$ (SAUER 1975), interpretato da SAUER (1987) come ibrido tra i due (ammettendo la partecipazione di un gamete non ridotto del primo).

ECologia Boschi di latifoglie, su ogni tipo di substrato (suoli di matrice sia silicea che calcarea o serpentinosa; SAUER 1973), nella fascia collinare inferiore, tra 200 e $500 \mathrm{~m}$.

DistribuzIONE Endemismo delle Alpi orientali (alla confluenza delle province centro-europea, adriatica e balcanica). Già segnalato nel protologo di Kerner (1878) per la valle dell'Isonzo nei pressi di Gorizia, la sua presenza marginale in Italia è stata recentemente confermata da una raccolta nel Friuli orientale, nella valle del Natisone (Fig. 38).

\section{NOTE}

SISTEMATICA Specie apparentemente collegata al complesso di $P$. hirta-vallarsae; si distingue in particolare da quest'ultima, che le è geograficamente più prossima, per il rizoma più sottile, per la lamina delle foglie basali nettamente più stretta, gradualmente assottigliata nel picciolo e quasi sempre vistosamente maculata, e per la corolla generalmente più chiara e con toni più azzurri che porporini. Attraverso un complesso modello di evoluzione dei cariotipi, SAUER (1987) ha ipotizzato un'origine ibrida dei due principali citotipi di $P$. stiriaca da altre specie parapatriche, col contributo sia di quelle orientali a cariotipo diploide ancestrale $2 n=14$ ( $P$. carnica W.Sauer, P. rubra Schott.), che di quelle occidentali a cariotipo disploide derivato ( $P$. australis, $P$. vallarsae). Secondo un'ipotesi alternativa, già avanzata da SIMONKAI (1904) e sostenuta da alcune evidenze molecolari (KIRCHNER 2004), le linee parentali coinvolte sarebbero addirittura quelle di $P$. mollis e $P$. officinalis. La questione è quindi tutt'altro che risolta.

\subsection{Pulmonaria vallarsae A.Kern.} POLMONARIA ITALICA

Pulmonaria vallarsae A. Kern., Monogr. Pulmon.: 33. 1878.

(E) P. officinalis var. vallarsae (A. Kern.) Fiori in Fiori \& Bég., Fl. Italia 2(3): 371. 1902.

Locus classicus: [Italia, Trentino Alto Adige] "in Tirolia australi [...] in ripa fluvii Leno in superiore parte Vallarsae".

Lectotipo (PUPPI \& CRISTOFOLINI 1996: 16): [Italia, Trentino Alto Adige] "Tirolia australis, Vallarsa", ca. 1870, Kerner (WU 69919!; iso- WU 71728!, 71729!, 71730!, 71731!, 71732!, 71733!, 71734!, 71735!, 71736!, 71737!, 71738!, 71739!, 71740!, 71741!, 71742!, 71743!, 71744!, 71745!, 71746!, 71747!, 71748!, 71749!, 71750!, 71751!, 71752!, 71753!, 71754!, 71755!)

Altro materiale originale (CECCHI \& SELVI 2015b: 652): [Italia, Trentino Alto Adige] "Unter Al'ometo in Vallarsa, jenseits der kleinen über den Leno führenden Brücke", 1868, Kerner (WU 69920!; iso-, "Im Gebüsche nächst der kleinen Brücke, welche über den Leno führt am Wege von Rauschi nach Al'ometo", WU 69921!, 69922!).

Etimologia Da Vallarsa, località dell'alta val di Leno, prossima al confine tra Trentino e Veneto, dalla quale proviene il campione tipo.

DESCRIZIONE Rizoma robusto di 4-10 mm di diametro, con innovazioni di $3-5 \mathrm{~cm}$. Foglie 
unicolori o con macchie verdastre poco marcate e mai confluenti, le basali adulte con lamina lunga fino a $30 \mathrm{~cm}$, ovata, 3-5 volte più lunga che larga, a base cuneata o troncata e margine ondulato, e picciolo ben distinto; indumento della pagina superiore denso (25-70 tricomi per $\mathrm{mm}^{2}$ ), costituito per oltre i $4 / 5$ da peli brevi, con setole rade e ghiandole stipitate o sessili più o meno abbondanti; foglie cauline e bratteali ellittico-lanceolate, ovato-lanceolate, a base da arrotondata a cordato-amplessicaule. Calice fiorale lungo $9-11 \mathrm{~mm}$. Corolla di 13-22 $\mathrm{mm}$, con tubo da densamente pubescente a glabrescente sotto l'anello di peli faucali e lembo di 8-18 $\mathrm{mm}$ di diametro, da porporino chiaro a violaceo (Fig. 39).

NUMERI $2 n=22$, già interpretato sia come ipertriploide $(2 n=3 x+1)$ originato CROMOSOMICI dall'incrocio tra corredi diploide ( $P$. carnica?) e tetraploide (?) con lo stesso numero base $x=7$ (SAUER 1975), ma più probabilmente ibrido stabilizzato di un diploide con $2 n=16$ ( $P$. officinalis) e di un tetraploide con $2 n=28$ ( $P$. hirta; cfr. VOSA \& PISTOLESI 2004). Tale corredo è costante e condiviso da tutte le popolazioni studiate di entrambe le sottospecie.

DISTRIBUZIONE Specie endemica o sub-endemica italiana; distribuita a cavallo delle province centro-europea, liguro-tirrenica e adriatica, è presente con due sottospecie ad areale disgiunto sui due lati della pianura padana, l'una a nord-est sulle Alpi e Prealpi orientali, tra Veneto e Trentino Alto Adige, l'altra a sud, tra le Alpi Marittime a nord-ovest, ove probabilmente sconfina in Francia, e il Gargano e I'Appennino calabro-lucano a sud-est, prevalentemente sul versante adriatico. (Fig. 40)

\section{NOTE}

SISTEMATICA Simile a $P$. hirta, se ne distingue in genere per gli scapi fiorali più elevati e le infiorescenze più lasse, ma sopratutto per le foglie a margini ondulati, con macchie meno marcate o assenti e l'indumento più ricco di peli brevi e ghiandole. SAUER (1987) suggerisce che questa specie sia nata come ipertriploide $(2 n=21+1)$ a partire dall'ibrido tra popolazioni diploidi del gruppo di $P$. rubra-carnica $(2 \mathrm{n}=14)$ e altre tetraploidi di " $P$. saccharata" (cioè $P$. hirta, $2 n=28$ ). Al contrario, VOSA \& PISTOLESI (2004), ipotizzano una sua origine ibrida tra i ceppi tetraploidi di $P$. hirta $(2 n=28)$ e quelli diploidi di $P$. officinalis $(2 n=16)$. A favore di una stretta relazione tra $P$. hirta e $P$. vallarsae, le due principali specie italiane, depongono anche i risultati dei test di incrocio interspecifico (PUPPI \& CRISTOFOLINI 1996), secondo i quali il grado di interfertilità sarebbe addirittura maggiore tra la prima e ciascuna delle due sottospecie della seconda che non tra queste ultime. Tuttavia, sia la presunta origine ibrida che la parziale sovrapposizione di areale tra $P$. vallarsae subsp. apennina e $P$. hirta nelle Alpi Marittime e nell'Appennino settentrionale, nonostante la quale si mantiene una distinzione piuttosto netta tra i due popolamenti e i due citotipi, non consente di trattarle alla stregua di sottospecie, come proposto da PeruzzI (2010). Ciò, beninteso, a meno di trattare come terza sottospecie di $P$. hirta anche la tipica $P$. vallarse, che dall'apennina risulta difficilmente distinguibile.

ChIAVE delle 1. Pagina superiore delle foglie basali adulte con peli ghiandolari pluricellulari SOTTOSPECIE (di lunghezza paragonabile a quella delle setole) 2-3 volte più abbondanti di quelli unicellulari (brevemente stipitati o sessili); picciolo verde, spesso largamente alato (ala di circa $2 \mathrm{~mm}$ ) 1. subsp. vallarsae

- Pagina superiore delle foglie basali adulte con peli ghiandolari pluricellulari (di lunghezza paragonabile a quella delle setole) 2-3 volte meno abbondanti di quelli unicellulari (brevemente stipitati o sessili); picciolo verdastrovioletto, strettamente alato (ala $<1 \mathrm{~mm}$ ) 


\subsubsection{Pulmonaria vallarsae subsp. vallarsae POLMONARIA DELLA VALLARSA}

DESCRIZIONE Indumento sulla lamina superiore delle foglie basali adulte con con rapporto tra setole e peli brevi circa 1:9 e peli ghiandolari pluricellulari (lunghi più o meno quanto le setole) 2-3 volte più abbondanti di quelli unicellulari (brevemente stipitati); picciolo verde, largamente alato (ala di circa $2 \mathrm{~mm}$ ) . (Fig. 39 A, B, E)

FENOLOGIA Fiorisce tra marzo e maggio, con maturazione dei frutti, a seconda della stazione, tra la tarda primavera e l'inizio dell'estate.

Numero $2 \mathrm{n}=22$, su materiale proveniente dal locus classicus o da stazioni vicine in CRomosomico Trentino Alto Adige (MerXmüller \& Grau 1969, SAUer 1975, PupPI \& CristofolinI 1996) e Veneto (SAUER 1975).

ECOLOGIA Faggete o arboreti di conifere, su suolo pingue, tra la fascia collinare e il limite superiore dei boschi montani (300 e $1600 \mathrm{~m}$ ).

DISTRIBUZIONE Endemismo italiano ad areale piuttosto limitato, circoscrito ai settori prealpino e alpino-orientale, tra la valle orientale dell'Adige a sud-ovest e le Dolomiti bellunesi, in val Cordevole, a nord-est. (Fig. 40)

6.6.2. Pulmonaria vallarsae subsp. apennina (Cristof. \& Puppi) L. Cecchi \& Selvi POLMONARIA APPENNINICA

Pulmonaria vallarsae subsp. apennina (Cristof. \& Puppi) L. Cecchi \& Selvi, PI. Biosyst. 149(4): [653-]654. 2015.

(三) P. apennina Cristof. \& Puppi in Webbia 51(1): 17. 1996 三 P. hirta subsp. apennina (Cristof. \& Puppi) Peruzzi in Inform. Bot. Ital 42(2): 531. 2010.

Locus classicus: [Italia, Emilia Romagna] "Parco Talon, Casalecchio di Reno [...]". Olotipo (cfr. PUPPI \& CRISTOFOLINI 1996: 18): [Italia, Emilia Romagna] “Parco Talon, Casalecchio di Reno", 26/04/1996, Puppi (FI 7200!; iso- BOLO).

Paratipi (cfr. PUPPI \& CRISTOFOLINI 1996: 18-20): [Italia, Liguria] "San Lazzaro Reale (IM), dal frantoio di Ca di Abbi presso il fiume Impero", 08/08/1937, Corradi (FI 7211!); "lungo la strada Viozene - Ponte di Nava", 08/09/1948, Corradi \& al. (FI 7210!); "Pignone, tra Monterosso al Mare e la SS Aurelia, su calcare, $200 \mathrm{~m}$ ", 03/04/1982, Marchetti (SIENA); "val di Vara su argille a palombini, 125 m”, s.d., Marchetti (SIENA); [Piemonte] "Cuneo, Certosa", 04/03/1946, Berluti (FI 7403!); "Montezemolo (CN), 300 m", 22/05/1986, Cristofolini (BOLO); "Priero (CN), 500 m", 22/05/1986, Cristofolini (BOLO); "val Bormida (CN), 300 m", 22/05/1986, Cristofolini (BOLO); Roccavione (CN), s.d., Biadego (VER!); [Emilia Romagna] "colli dell'Osservanzia presso Bologna", 05/04/1890, Fiori (FI 7207!); "Parma, base umida delle sponde del Baganza", 06/04/1920, Minio (FI 7208!); "Appennino tosco-emiliano, passo del Lupo, 1500 m", 25/05/1955, Chiarugi (FI 7209!); “Appennino modenese, Sestola, 1020 m”, 08/1962, s.coll. (RO-HG!); "valle Tresinaro (RE), 300 m", 1984, Ubaldi (BOLO); "m.te Adone (BO), 400 m", 04/1985, Tomaselli (BOLO); "parco Talon di Casalecchio di Reno (BO), 90 m", 
09/09/1985, Puppi (BOLO); "Vergato, Rio Croara (BO), 300 m, 05/1987, Tomaselli (BOLO); "Corniolo (FC), 600 m", 11/1986, Agostini (BOLO); "S. Benedetto in Alpe (FC), 414 m, 03/1987, Agostini (BOLO); "Strigara (FC), 500 m", 03/1987, Agostini (BOLO); "Modigliana (FC)", 1988, s.coll. (BOLO); "S. Lazzaro di Savena (BO), 60 m", 1990, Bugamelli (BOLO); [Toscana] "Montopoli", 07/04/1863, Beccari (FI 7204!); "Florentia in umbrosis", 02/05/1886, Levier (ROHG); "dalla Calla a Poggio Scali per la Giogana", 17/06/1912, Baccarini (FI 7205!); "Versilia, tra Stazzema e la foce di Petrosciana", 30/03/1923, Chiarugi (FI 7201!); "Cafaggiolo (FI), fonte della Docciola, 07/07/1940, Beni (FI 7202!); "Firenze, bordi di un ruscello per andare al viale dei Colli", 05/1945, Contardo \& Corradi (FI 7203!); "m.te Falterona, valle di San Godenzo, nel castagneto fra Castagno e Ripalta, 700-900 m", 08/04/1952, Forasassi (FI 7404!); "m.te Falterona, valle di San Godenzo, Capanna Lama, a SO di castagno, 850 m ca.", 02/05/1952, Forasassi (FI 7266!); "m.te Falterona, valle di San Godenzo, La Macia sul crinale fra m.te Falterona e m.te Massicaia, 1260 m", 02/05/1952, Forasassi (FI 7405!); "m.te Falterona, La Burraia, foresta di Campigna, 14501068", 23/05/1952, Corradi \& al. (FI 7206!); "passo di Viamaggio - Pieve Santo Stefano (AR)", 19/04/1976, Ubaldi (BOLO); "Pieve Santo Stefano - La Verna (AR)", 19/04/1976, Ubaldi (BOLO); "Alpi Apuane, presso Redicesi di Massa”, 08/04/1978, Marchetti (SIENA); "Alpi Apuane, nei pressi di Bibola sotto Aulla (MS)", 16/04/1980, Marchetti (SIENA); "Le Pizzorne (LU), 900 m”, 24/03/1985, Tomaselli (BOLO); "Alpi Apuane (MS), 125 m”, 20/04/1985, Tomaselli (BOLO); [Marche] "Monastero - valle del Mulino (PU)", 1972, Ubaldi (BOLO); "Sasso Simone (PU)", 01/05/1976, Ubaldi (BOLO); "m.te Carpegna (PU), 900 m", 31/05/1983, Ubaldi (BOLO); "Camerino, 350 m”, 26/10/1990, Tibiletti (BOLO); "S. Angelo presso Matelica, 550 m", 26/10/1990, Tibiletti (BOLO); "S. Michele, tra Matelica e Fabriano, 300 m", 26/10/1990, Tibiletti (BOLO); "Amandola (AP), 590 m”, 28/10/1990, Tibiletti (BOLO); "Visso (MC), 520 m”, 28/10/1990, Tibiletti (BOLO); [Umbria] “Gubbio, Padule, 510 m", 27/10/1990, Tibiletti (BOLO); "Scheggia, presso Gubbio, 680 m", 27/10/1990, Tibiletti (BOLO 508133); [Lazio] "m.ti Albani (RM)", 13/04/1881, Pedicino (VER); "in montibus prope Albano", 21/02/1920, Lacaita (RO-HR); "Riano (RM)", 1980, Caprolati (RO-HR); "m.te Terminillo", 06/1983, Anzalone (RO-HR); "m.ti Simbruini, zona Trevi - Valle Pietra", 24/05/1985, Anzalone (RO-HR); "Colli Albani, nel castagneto presso Valle Oscura", s.d., Lusina (RO-HR); "Colli Albani tra Cappuccini e Albano", s.d., Biadego (RO-HR); "S. Cesareo, nel bosco", s.d., Cacciato (RO-HR); [Abruzzo] "Monte di Mezzo prope Castel di Sangro", 23/05/1837, Gussone, Herb. Gussone (NAP); "Parco Nazionale d'Abruzzo, verso Bisegna", 02/06/1956, Anzalone (ROHR); "Tagliacozzo (AQ)", 02/04/1961, s.coll. (RO-HR); "Senarica (TE), 700 m", 09/1986, Zanotti (BOLO); "Majella, Palena, 1000 m”, 07/1987, Cristofolini \& al. (BOLO); "Castel di Sangro", s.d., Cesati (RO-HC; iso- WU-Kerner); "m.te Sirente”, s.d., Pellegrini (RO-HR); [Molise] "tra S. Massimo e Campitello, 1200 m", 26/05/1971, Bianchini 8331 (VER!); "faggeta alle pendici del m.te Miletto, 1450 m", 01/07/1968, Bianchini 8330 (VER); [Campania] "selve di * sopra Maddaloni e bosco di Querciacupa presso Valle", 10/04/1838, Gussone, Herb. Gussone (NAP); "in montibus supra Amalfi, 800 m", 21/02/1920, Lacaita (RO-HR); "m.te Cervati (SA), 1000 m", 07/1987, Ubaldi (BOLO); "m.te Gelbison (SA), 1600 m", 07/1987, Ubaldi (BOLO); [Basilicata] "Potenza, bois de Quercus cerris, sol argileux, 1000 m", 24/04/1933, Gavioli (RO-HR); "Potenza, in silva Aria Silvana, 900-1200 m", 26/04/1936, Gavioli (RO-HR); "La Maddalena, bosco di cerrofaggio, 1300 m", 08/1977, Ubaldi (BOLO); "Villa d'Agri", 06/1991, Puppi (BOLO); 
[Puglia] "Gargano, Foresta Umbra, 800 m", 04/05/1982, Bianchini (VER); [Calabria] "in montibus di Pecoraro", 1877, Arcangeli (RO-HR); "m.ti da Giffone a Serra S. Bruno", 1907, Pasquale \& Lacaita (NAP); s.loc., s.d., Longo (RO-HR).

(-) P. mollis auct. (TenORE 1831: 84) non Hornem., Hort. Bot. Hafn. 1: 179. 1813.

Etimologia Dal latino apenninus, "degli Apennini”, in riferimento all'areale del taxon.

DESCRIZIONE Indumento sulla lamina superiore delle foglie basali adulte con con rapporto tra setole e peli brevi circa 1:4 e peli ghiandolari pluricellulari (lunghi più o meno quanto le setole) 2-3 volte meno abbondanti di quelli unicellulari (brevemente stipitati); picciolo verdastro-violetto, strettamente alato (ala $<1 \mathrm{~mm}$ ). (Fig. $39 \mathrm{C}$, D)

FENOLOGIA Pianta a fioritura primaverile precoce, talora anticipata ai primi di marzo, assai raramente protratta oltre la prima metà di giugno; i frutti maturi vengono rilasciati in genere a partire dalla seconda metà di aprile e fino all'inizio dell'estate.

NUMERO $2 n=22$, verificato sia su piante determinate direttamente come $P$. apennina che CROMOSOMICO su popolazioni aneuploidi attribuite in precedenza a " $P$. saccharata" $O$ " $P$. vallarsae" ma presumibilmente riferibili a questo taxon, provenienti dalle Alpi Marittime Francesi (SAUER 1975) e da varie regioni dell'Italia peninsulare: Abruzzo (PupPI \& CRISTOFOLINI 1996, MeRXMÜlleR \& GRAU 1969), Basilicata (SAUER 1975, CAPINeri 1986), Calabria (MerXmüller \& Grau 1969, SAUer 1975, Peruzzi \& CesCa 2003), Emilia Romagna (SAUER 1975, PUPPI \& CRISTOFOlINI 1996, Vosa \& Pistolesi 2004), Lazio (CAPInerI 1986), Puglia (MerXmüller \& Grau 1969, SAUer 1975) e Toscana (CAPINERI 1986, VosA \& PISTOLESI 2004). In una popolazione apuana sono stati rilevati anche due cromosomi $B$ sovrannumerari (CAPINERI 1986); per altri corredi aneuploidi $(2 n=25,26,26+B$ o 27$)$ si veda quanto annotato in questo campo per $P$. hirta.

ECOLOGIA Boschi di latifoglie, su suolo pingue, a quote perlopiù collinari $(0-800 \mathrm{~m})$ nella parte settentrionale dell'areale, progressivamente più in alto, fino alla fascia di faggeta $(1000 \mathrm{~m})$ verso sud.

DISTRIBUZIONE Sottospecie prevalentemente appenninica, diffusa tra le Alpi Marittime (dove forse sconfina in Francia) e l'Appennino calabro, lungo il contatto tra le province liguro-tirrenica, europea-centrale e adriatica, ma assai più diffusa sul versante adriatico che su quello tirrenico (Fig. 40).

\section{NOTE}

SISTEMATICA La distinzione di questa sottospecie da quella tipica, alpina, apparentemente supportata dall'evidente disgiunzione degli areali (che tuttavia potrebbe essersi prodotta in epoca relativamente recente con la deforestazione medioevale della pianura padana), si basa su deboli differenze quantitative nell'indumento delle foglie adulte e sulle ali del picciolo meno sviluppate, ma trova conferma anche in elementi chemiotassonomici e nei risultati di esperimenti d'incrocio, dai quali si sono ottenuti meno nucule mature dagli incroci tra le due sottospecie che da quelli di entrambe con P. hirta (PUPPI \& CRISTOFOLINI 1996). Appare invece piuttosto arbitrario, sia dal punto di vista morfologico che corologico (vista la loro condizione di parziale simpatria), la sua ricombinazione come sottospecie di P. hirta (PERUZZI 2010: 531), a meno che tutta una serie di altre entità egualmente collegate non le vengano ricondotte in egual modo, con un'operazione potrebbe essere plausibile, ma ad oggi lungi dall'avere sufficiente supporto sperimentale. 
ECologia Si tratta di entità mediamente più mesofila dell'affine $P$. hirta, che le è parzalmente simpatrica.

\section{SPECIE DA ESCLUDERE}

\section{Pulmonaria mollis Hornem. POLMONARIA MOLLE}

Pulmonaria mollis Hornem., Hort. Bot. Hafn. 1: 179. $1813 \equiv$ P. officinalis var. mollis (Hornem.) Fiori in Fiori \& Bég., Fl. Italia 2(3): 372. 1902.

Locus classicus: "in Austria?".

Olotipo (cfr. SAUER 1974: 245): [Germania] "Bavaria”, s.d., Vahl (C 10008768!).

(=) P. tuberosa Schrank. in Nova Acta Phys.-Med. Acad. Caes. Leop.-Carol. Nat. Cur. 9: 97. 1818, nom. illeg., non Schmidt ex Mart. in Denkschr. Königl. Akad. Wiss. München 5: 175. $1817 \equiv$. officinalis var. tuberosa (Schrank) Fiori in Fiori \& Bég., FI. Italia 2(3): 372. 1902.

Locus classicus: [Austria] "in Carinthia"; [Germania] "in Bavaria prope Rosenheim"; [pianta coltivata in Germania] "in horto [Monacense]".

Lectotipo CECCHI \& SELVI 2015b: 675): [pianta coltivata in Germania] "H[orto]. Mon[acense]." (M 158762!).

(=?) P. angustifolia var. oblongata Schrad. ex Roem. \& Schult., Syst. Veg., ed. 15 bis 4: 744.1819 I P. vulgaris var. oblongata (Schrad.) Nyman, Consp. Fl. Europ.: 512. $1881 \equiv P$. officinalis var. tuberosa f. oblongata (Schrad. ex Roem. \& Schult.) Fiori in Fiori \& Bég., Fl. Italia 2(3): 372. 1902.

Locus classicus: non specificato.

Tipo non designato.

(-) P. tuberosa Schmidt ex Mart. in Denkschr. Königl. Akad. Wiss. München 5: 175. 1817 (P. mollis subsp. alpigena W. Sauer).

Locus classicus: [Germania] "In Bavariae subalpinis prope Rosenheim". Lectotipo (CECCHI \& SELVI 2015b: 675): [Germania] "albo in sylv.", 1814, [Schmidt?] (BR 8484291!).

DESCRIZIONE Rizoma robusto con innovazioni brevi. Foglie prive di macchie, d'estate più o meno distintamente grigiastre, le basali adulte con lamina lunga fino a $30 \mathrm{~cm}$ o più, ellittica, 3-4 volte più lunga che larga, a base cuneata e margine talora ondulato, e picciolo breve, largamente alato ma piuttosto distinto; indumento della pagina superiore denso e vellutato, composto di peli brevi di 0,2-0,5 mm misti a setole rade di 1-3 $\mathrm{mm}$ e a ghiandole lungamente stipitate, i tre tipi di tricomi in rapporto di circa 100:10:1; foglie cauline ellittico-lanceolate, a base troncata o cordato-amplessicaule. Calice fiorale lungo 11-12 mm. Corolla con tubo densamente pubescente sotto l'anello di peli faucali e lembo anch'esso pubescente, da azzurro-lillacino a viola intenso, raramente rosso.

DISTRIBUZIONE Specie endemica della provincia europea centrale, ov'è largamente diffusa, con due sottospecie vicarianti, sull'arco alpino (subsp. alpigena W. Sauer) e sulle più vaste distese planiziali e collinari dell'Europa media (subsp. mollis). Le popolazioni più meridionali si trovano sul versante settentrionale delle Alpi, senza raggiungere il nostro paese. 


\section{NOTE}

Nomenclatura Pulmonaria mollis, nome accettato per la specie che Kerner (1878) descrisse come $P$. mollissima A. Kern., è spesso attribuito per errore a "Wulfen ex Hornem.", nonostante alcuni riscontri bibliografici (SAUER 1974) facciano ritenere che si tratti di "Wolff" anziché di "Wulfen". Tuttavia entrambe le attribuzioni sono scorrette (ICN Art. 46.5; MCNEILL \& al. 2012), perché Hornemann si limitò a citare una pianta inviatagli da Martin Vahl "sub nomine P. mollis Wulfen" senza disporre di alcuna diagnosi scritta da tale "Wulfen" o "Wolff".

Di un'altra entità tenuta per buona da Kerner e citata per la flora italiana, "Pulmonaria tuberosa Shrank", SAUER (1972) sostiene che sia un "nomen confusum", la cui tipificazione è impedita per la parziale mescolanza del materiale originale, e perché le informazioni fornite dal protologo potrebbero adattarsi tanto a $P$. collina W. Sauer quanto a $P$. mollis subsp. alpigena (SAUER 1974). In realtà tra i campioni conservati nell'erbario di Monaco ce n'è uno autenticato da Schrank e determinato dallo stesso Sauer come sottospecie tipica di $P$. mollis, la cui designazione come lectotipo ha risolto definitivamente la questione. Il binomio di Schrank è comunque illegittimo perchè sinonimo eterotipico posteriore della pianta descritta l'anno prima da Martius su materiale di Schmidt, il cui neotipo corrisponde invece a $P$. mollis subsp. alpigena.

Quanto al binomio $P$. oblongata, che resta formalmente da tipificare, lo status di ibrido che Kerner (1878) le attribuiva tra $P$. tuberosa Schrank e $P$. mollissima ci induce a porla tentativamente in quest'ambito. Anche se non ci risultano campioni italiani determinati, secondo la prima trattazione di Fiori in FIORI \& PAOLETTI (1902), come P. officinalis "var. tuberosa" o come sua "f. oblongata", è molto probabile che l'uso di entrambe i nomi in luogo della tipica "var. azurea" (sinonimo di $P$. angustifolia) servisse a distinguere diversi morfotipi di $P$. australis.

SISTEMATICA II complesso di $P$. mollis s.l. è quello a più ampia distribuzione di tutto il genere, essendo presente in molte delle province eurasiatiche della regione circumboreale, da ovest a est tra la Baviera e la Siberia, spingendosi a nord fino alla Germania centrale, a sud in tutti i Balcani, sconfinando nella provincia mediterranea orientale fino ai monti del Peloponneso. Entro questi limiti, le popolazioni dell'Europa sud-orientale e dell'Asia vanno riferite secondo SAUER (1987) ad una specie morfologicamente e cariologicamente distinta ( $P$. dacica Simonk.), mentre l'areale di $P$. mollis s.str. sarebbe limitato all'Europa centrale. Le occasionali segnalazioni di questa specie lungo il bordo meridionale delle Alpi si devono a probabile confusione con $P$. australis, dalla quale si distingue per le foglie basali più larghe e per l'indumento più denso, con ghiandole lungamente stipitate, oltre che per il corredo cromosomico $2 n=18$. II binomio " $P$. mollis" erroneamente attribuito a Reichenbach e riferito da TENORE (1831) a piante del sud Italia, d'altra parte, va certamente inteso per $P$. vallarsae subsp. apennina (PUPPI \& CRISTOFOLINI 1996).

\section{IBRIDI}

\section{Pulmonaria $\times$ hybrida A. Kern. POLMONARIA IBRIDA}

Pulmonaria $\times$ hybrida A. Kern., Monogr. Pulmon.: 31. 1878.

Locus classicus: [Italia, Trentino Alto Adige] "prope Brixinam in Tirolia". Lectotipo (CECCHI \& SELVI 2015b: 654): [Italia, Trentino Alto Adige] “Brixen”, 1863, Kerner (BM 752603!).

(=?) P. australis $\times P$. officinalis.

DISTRIBUZIONE Segnalata un'unica volta nei pressi di Bressanone, in Trentino Alto Adige, ove convivono le due specie parentali. 


\section{NOTE}

SISTEMATICA Pulmonaria hybrida è stata descritta da KeRneR (1878) come ibrido tra "P. angustifolia" (forse $P$. australis) e $P$. officinalis. Come ricordato più volte, il corredo aneuploide di molte "citospecie" descritte per il genere Pulmonaria è stato spesso spiegato come frutto della stabilizzazione di stirpi ibridogene, magari a seguito ad "assestamenti" legati a fenomeni di disploidia discendente o ascendente. Sebbene forse poco frequente, l'ibridazione in Pulmonaria è quindi un fenomeno conosciuto e diffuso tra i rappresentanti di molte delle stirpi che entrino in qualche modo in contatto tra loro. All'interno di un gruppo tanto omogeneo, però, tanto l'identità di presunti nototaxa quanto quella di nuovi ibridi occasionali è di fatto impossibile da accertare col solo esame di campioni d'erbario, senza un'analisi cariologica. Questo è il caso di $P$. hybrida, entità nota per il solo campione tipo. La sua natura ibrida sembra comunque plausibile, sia perchè il materiale originale è stato rinvenuto in presenza di entrambe le presunte parentali (KERNER 1878), sia perché presenta obiettivamente caratteri macro- e micromorfologici intermedi tra esse. Differisce infatti da $P$. angustifolia s.l. (compresa $P$. australis) per le foglie più brevi (a base attenuata ma lamina largamente lanceolata), maculate e coperte di densi aculeoli, e da $P$. officinalis per il profilo stesso delle foglie, l'indumento costituito da setole più dense miste ad aculeoli più lunghi (2-3 volte più lunghi che larghi), la corolla azzurro-lillacina. 


\section{Nonea Medik.} NONNEA

Nonea Medik., Philos. Bot. 1: 31. 1789.

Tipo (JANCHEN 1953: 210): Lycopsis pulla L. (N. pulla (L.) DC.

(=) Onochilis Mart. in Denkschr. Königl. Akad. Wiss. München 5e: 177. 1817. Tipo (cfr. MARTIUS 1817: 177): Onochilis pulla Mart. [N. vesicaria (L.) Rchb.].

Bibliografia SelVI \& al. 2002, 2006b.

Etimologia Genere dedicato, con errata trascrizione, al botanico tedesco Johann Philipp Nonne (1729-1772), professore a Erfurt e autore nel 1763 di un Flora su quel territorio (Flora in Territorio Erfordensi Indigena).

DESCRIZIONE Erbe annue o perenni, con fusti da prostrati a eretti e indumento di peli molli o rigidi più o meno densi, spesso anche ghiandolari. Foglie acuminate, le basali oblanceolate, con lamina a base cuneata e decorrente e picciolo più o meno indistinto, le cauline e bratteali assai più piccole, da lineari-lanceolate a oblanceolate, sessili. Infiorescenze bratteate, semplici o ramificate in poche cime oscuramente scorpioidi, pauciflore; fiori peduncolati, i peduncoli deflessi alla fruttificazione. Calice diviso per non più della metà in lacinie triangolareovate erette, cilindrico-campanulato all'antesi, alla fruttificazione notevolmente accrescente, rigonfio e sub-sferico (ventricoso). Corolla infundibuliforme, da attinomorfa a leggermente zigomorfa, con tubo lungo e lobi del lembo da eretti a eretto-patenti (mai del tutto rotati), di colore da bianco a viola scuro o quasi nero; pliche faucali talora presenti e ben rilevate, talaltra sostituite da ciuffi distinti o da un anello continuo di tricomi, solo di rado la corolla internamente glabra. Stami a filamento spesso brevissimo o subnullo, inseriti nella metà superiore del tubo corollino, inclusi o parzialmente sporgenti. Stilo incluso; stimma capitato o debolmente bilobo. Nucule da largamente ovoidi a reniformi, orientate perpendicolarmente rispetto all'asse fiorale, a superficie più o meno reticolato-rugosa, con cercine basale assai marcato, raramente dentato, e strofiolo altrettanto evidente.

NUMERI Come nell'affine genere Pulmonaria, è documentata in Nonea una notevole CROMOSOMICl variabilità di citotipi, con corredi da diploidi a decaploidi $(2 n=14,16,18$, (19), $20,22,28,30,32,36,40,44,54,59,60,100)$, talora con cromosomi B sovrannumerari. L'ipotesi di SELVI \& BIGAZZI (2002) è che lo stato ancestrale sia quello più frequente tra i citotipi perenni anatolici, montani, con $x=10$, e che $i$ corredi con diverso numero cromosomico di base, in particolare con $x=9,8,7$, progressivamente più diffusi tra le specie annuali, rappresentino un adattamento a cicli vitali più brevi attraverso la disploidia discendente.

BIOLOGIA Specie entomofile, perlopiù allogame, talora autogame. Dispersione delle RIPRODUTTIVA nucule mediata da formiche (mirmecocoria).

DISTRIBUZIONE Genere altamente polimorfo e in attiva speciazione, comprende circa 35 specie nel sottoregno tetidico e della parte sud-orientale della regione circumboreale europea, dalla penisola iberica all'Asia centro-occidentale passando per il Nordafrica, il Mediterraneo orientale, le coste del Mar Nero e il Caucaso, con massima diversità e probabile area di origine compresa tra la provincia pontica e quelle della regione irano-turanica nord-occidentale. In Italia sono 3 le specie 
autoctone, la quarta ( $N$. lutea) essendosi naturalizzata in epoca storica e restando vincolata a poche stazioni in ambito sinantropico.

NOTE

Sistematica Con l'inclusione delle 3 specie nordafricane già riferite ad Elizaldia Willk. (= N. sect. Phaneranthera A.DC) e la separazione di Melanortocarya, Nonea costituisce un gruppo chiaramente monofiletico, evolutosi da un progenitore comune con Pulmonaria, dal quale si distingue per il calice maggiormente accrescente alla fruttificazione, l'assenza di eterostilia, i mericarpi opachi e sculturati; presenta un'elevata variabilità morfologica, palinologica e cromosomica, frutto probabilmente di una recente radiazione evolutiva (SELVI \& al. 2006b), ma nessuna delle linee evolutive rilevate dall'analisi filogenetica trova corrispondenza con le sezioni descritte da CANDOLle (1846: 27-34).

ChIAVE deLle SPECIE 1. Pianta perenne; corolla con lembo inclinato, violaceo-nerastro .... 3. N. pulla

- Pianta annua; corolla con lembo piano, bianca, gialla o porporina .............. 2

2. Pianta non glutinosa, senza peli ghiandolari; corolla bianca; mericarpi reniformi

1. N. echioides

- Piante ispido-glutinose con peli ghiandolari; corolla gialla o porporina; mericarpi ovoidi, non reniformi 3

3. Corolla porporina, con lembo di 3-5 $\mathrm{mm}$ di diametro; mericapi obliquamente ovoidi, più larghi che lunghi 4. N. vesicaria

- Corolla gialla, con lembo di 8-12 mm di diametro; mericarpi erettooblunghi, più lunghi che larghi 2. N. lutea

\subsection{Nonea echioides (L.) Roem. \& Schult. NONNEA BIANCA}

Nonea echioides (L.) Roem. \& Schult., Syst. Veg., ed. 15 bis , 4: 71. 1819.

(E) Lycopsis echioides L., Sp. PI., ed. 4, 1: 781. $1762 \equiv$ Anchusa ventricosa Sm. in Sibth. \& Sm., Fl. Graec. 2: 581836 三 N. ventricosa (Sm.) Griseb., Spic. Fl. Rumel. 2(4): 98. 1844 三 Aipyanthus echioides (L.) Steven in Bull. Soc. Imp. Naturalistes Moscou 1: 600. 1851 三 N. alba subsp. ventricosa (Sm.) Nyman, Consp. Fl. Europ.: 512. 1881.

Locus classicus: [Armenia] "in America [lege Armenia; EDMONDSON 1977]". Lectotipo (EDMONDSON 1977: 29): [Spagna] 14/05/1753, Löfling, 71/7, ex Herb. Alströmer (S-LINN!).

Altro materiale originale (CECCHI \& SeLVI 2015b: 654): [Spagna] "Ex Hispania”, s.d, Löfling 146, 71/9, ex Herb. Casström (S 9-35911!); s.loc, s.d., s.coll., Herb. Linneo 190.6 (LINN!).

(=) N. alba DC. in DC. \& Lam., Fl. Franc. (DC. \& Lamarck), ed. 3., 6: 420. $1815 \equiv N$. ventricosa (Sm.) Griseb. f. alba (DC.) Fiori in Fiori \& Bég., Fl. Italia 2(3): 373. 1902.

Locus classicus: [Francia] "dans les blés sur les deux rives du Rhône audessous d'Avignon, à Tarascon et à Aramon".

Lectotipo (CECCHI \& SELVI 2015b: 655): [Francia] "Tarascon et Aramon, 2 rives du Rhone", 1810, Requien, Herb. Candolle 4542 (G-DC 137568!). 
Etimologia Dal nome del genere di boraginacee Echium e dal suffisso latino -oides, che indica affinità, probabilmente per la superficiale somiglianza dell'indumento ispido di questa specie con molte di quelle del genere Echium.

DESCRIZIONE Erba annua con fusti ascendenti numerosi dalla base, (5)15-35(50) cm, ispidopubescente, per rade setole a base tubercolata miste a brevi peli più densi e a peli pluricellulari, ghiandolari e non. Foglie oblungo-spatolate, acute, a margine generalmente ondulato o sinuato-dentato, le basali secche all'antesi, le cauline 0,5-1,5 × 2-6 cm, oblungo-lanceolate, a base talora decorrente, ottusomucronate. Infiorescenze semplici o poco ramose, con cime allungate fino a $10(20) \mathrm{cm}$ alla fruttificazione; brattee ovato-lanceolate lunghe alpiù $3,5 \mathrm{~cm}, \mathrm{a}$ base asimmetrica, decorrenti su un solo lato; peduncoli di $1 \mathrm{~mm}$ alla fioritura, poi allungati fino a $4 \mathrm{~mm}$ e spesso deflessi. Calice diviso fino a $1 / 4-1 / 3$ della lunghezza in lobi triangolari, lungo 4-5 $\mathrm{mm}$ all'antesi ma rigonfio e accresciuto fino a 8-10(12) $\mathrm{mm}$ alla fruttificazione. Corolla bianco-sporca di $6-8 \mathrm{~mm}$, infundibulare e leggermente zigomorfa, con tubo incluso nel calice, appena ricurvo, e lembo di (2,5)4-5 mm di diametro, a lobi arrotondati e spesso lievemente increspati; pliche faucali bilobe, pubescenti. Stami inclusi, inseriti a metà del tubo, con antere di 0,6-1 mm. Mericarpi reniformi, 1,5-2 × 2,5-3,5 mm, privi di becco laterale, con cercine basale incospicuo e superficie grigiastra, papillosa e debolmente reticolata (Fig. 41).

FENOLOGIA II solo saggio italiano a noi noto fu raccolto a fine maggio e reca sia fiori che frutti. Il clima nelle stazioni pugliesi è comunque compatibile col resto dell'areale, dove la specie fiorisce e fruttifica tipicamente nell'arco dei mesi primaverili e conclude il suo ciclo maturando le ultime nucule entro la prima metà di giugno.

NUMERO $2 n=16$, verificato sia su piante spagnole (LUQUE 1995) che turche (SELVI \& BIGAZZI CROMOSOMICO 2002).

ECOLOGIA Incolti aridi, rupestri e sassosi, più raramente in luoghi erbosi e su terreni più pingui a margine dei coltivi, generalmente su substrato calcareo, dal livello del mare fin oltre i $1700 \mathrm{~m}$, in Italia però solo a quote planiziali.

DISTRIBUZIONE Specie mediterranea a larga diffusione, presente più o meno diffusamente in tutte le province settentrionali nelle province. In Italia però è stata raccolta solo anticamente in alcune località pugliesi; da allora non è stata mai più ritrovata ed è forse scomparsa (Fig. 42).

\section{NOTE}

NomenclatuRa Edmonson (1977) spiega con dovizia di dettagli perché la contraddizione tra il locus classicus indicato nel protologo e l'effettiva origine del tipo, oltre che di grafia, sia anche di sostanza. L'equivoco sarebbe derivato dalla confusione generata dai molti omonimi eterotipici del genere Echioides, che avrebbe indotto Linneo a riferire il campione in suo possesso, raccolto da Löfling in Spagna, alla pianta ben diversa che Tournefort aveva descritto dall'Armenia come Echioides orientale (oggi Huynhia pulchra (Willd. ex Roemer \& Schultes) Greuter \& Burdet). 


\subsection{Nonea lutea (Desr. ex Lam.) DC. 0 NONNEA GIALLA}

Nonea lutea (Desr. ex Lam.) DC., in DC. \& Lam., Fl. Franç., ed. 3, 3: 626. 1805.

(三) Lycopsis lutea Desr. ex Lam., Encycl. 3: 657. 1792.

Locus classicus: [pianta coltivata in Francia] "cultivée au Jardin du Roy". Lectotipo (CECCHI \& SELVI 2015b: 650): s.loc., s.d., s.coll., Herb. Lamarck 59.179 (PLA 357408!).

(=) Anchusa dubia Nocca, Ticin. Hort. PI. 1: 5, t. 3. 1800.

Locus classicus: [pianta coltivata in Italia, Lombardia] [Hortus ticinensis].

Lectotipo (CECCHI \& SELVI 2015b: 656): disegno in NOCCA (1800: t. 3!).

(=) Lycopsis setosa Lehm., PI. Asperif. Nucif.: 269. $1818 \equiv$ N. setosa (Lehm.) Roem. \& Schult., Syst. Veg., ed. 15 bis , 4: 754. 1819.

Locus classicus: [Armenia] "In Iberia".

Olotipo (cfr. CECCHI \& SELVI 2015b: 655): "ad Caucasum", s.d., Adams (MEL 1010645!).

EtIMOlogia Dal latino luteus, "giallo", in riferimento al colore della corolla.

DESCRIZIONE Erba annua, semplice o ramificata alla base in pochi fusti eretto-ascendenti, 20$60 \mathrm{~cm}$, pubescente-ghiandolosa, con setole sparse di varia lunghezza. Foglie verde brillante, con superficie punteggiata di radi tubercoli biancastri e margine da piano a leggermente ondulato, le basali largamente oblanceolato-spatolate, ad apice ottuso o mucronulato, le cauline e bratteali da ellittico-lanceolate a ovate, a base cordato-amplessicaule, 0,5-2 × 2-7 cm. Infiorescenza eretta, semplice o lassamente ramificata, con brattee maggiori del calice. Calice diviso fino a metà in denti triangolare-acuti, lungo 6-8 mm all'antesi, fino a $15-17 \mathrm{~mm}$ alla fruttificazione. Corolla infundibulare giallo limone, più pallida sul lembo che alla fauce, con tubo di 6-7 mm e lembo di 8-12 $\mathrm{mm}$ di diametro, con lobi largamente arrotondati e poco incisi; fauce con areole brevemente pubescenti. Stami con antere di 1,6 mm, inclusi. Mericarpi eretto-oblunghi, ottusi, 5-6,5 $\mathrm{mm}$, con superficie grigiastra rugoso-reticolata e cercine basale incospicuo (Fig. 43).

FENOLOGIA Fiorisce tra marzo e giugno, talora fino ai primi di luglio, maturando i frutti durante tutta la primavera e l'inizio dell'estate.

NUMERo $2 n=14$, verificato su piante della popolazione esotica dell'orto botanico di CROMOSOMICO Firenze (oss. pers.); lo stesso era già stato riscontrato su materiale proveniente dall'Iran (Aryavand in LöVE 1971) e dalla Turchia (SELVI \& BIGAZZI 2002).

ECOLOGIA Specie in origine di ambienti rocciosi e prativi della fascia collinare. In Italia si trova in stazioni ruderali e negli incolti di aree urbane o antropizzate, in pianura e collina, tra 0 e $950 \mathrm{~m}$.

DISTRIBUZIONE Specie a gravitazione prevalente nell'area del Caucaso, presente dalla Russia meridionale, all'Anatolia nord-orientale, all'Iran nord-occidentale, sporadica ed esotica altrove. In Italia è circoscritta ad ambienti strettamente sinantropici, talora naturalizzata; fu raccolta a partire dalla seconda metà del XIX secolo in diverse città del centro-nord, e in alcune di queste, sia pure senza apparenti tendenze ad espandersi, si trova tuttora. (Fig. 44) 


\subsection{Nonea pulla (L.) DC. NONNEA PERENNE}

Nonea pulla (L.) DC. in DC. \& Lam., Fl. Franç., ed. 3, 3: 626. 1805.

(三) Lycopsis pulla L. in Loefl., Iter Hispan.: 66-67, 81, 302. 1758, nom. cons. prop. $\equiv$ Lycopsis pulla L., Syst. Nat., ed. 10, 2: 916. 1759, nom. illeg. ミ N. erecta Bernh., Syst. Verz.: 127. 1800.

Locus classicus: [Spagna] "i Madrid och nog i St. Fernando".

Lectotipo (KAZMI 1971: 676): s.loc, s.d., s.coll., Herb. Linneo 190.2 (LINN!), typ. cons. prop. (CECCHI \& al. 2014: 1132).

Etimologia Dal latino pullus, "scuro", riferito al colore della corolla. Rimasto di ambigua attribuzione fino a tempi recenti (CECCHI \& al. 2014), l'epiteto linneano era stato rimpiazzato da Bernhardi con l'aggettivo erectus, "eretto", opportunamente scelto per distinguere la specie dall'altra a fiori scuri già nota a quei tempi , l'annuale - e prostrata - N. vesicaria.

DESCRIZIONE Erba perenne ramificata alla base in fusti eretti, $20-50 \mathrm{~cm}$, pubescente-ispida per setole sparse e più o meno ghiandolosa in alto. Foglie grigio-verdi con tinte bluastre, le basali da lanceolate a oblanceolate, 0,7-3 × 3-10 cm, acute, a margine piano e intero, le cauline e bratteali strettamente ellittico-lanceolate, a base cordato-amplessicaule. Cime ramificate, con fiori spesso unilaterali, subsessili all'antesi, e brattee maggiori del calice. Calice diviso fino a $1 / 3$ in denti triangolari acuti, 6-8 $\mathrm{mm}$ all'antesi e fino a 12 alla fruttificazione. Corolla violaceo-scura, quasi nerastra, infundibulare e leggermente zigomorfa, con tubo arcuato di 5-7 $\mathrm{mm}$ e lembo inclinato di 5-8 $\mathrm{mm}$ di diametro; squame faucali poco rilevate, pubescenti. Stami con antere di 1,6 mm, inclusi. Mericarpi obliquo-ovoidi con becco laterale e cercine basale ispessito, 2-3 $\times 4-5 \mathrm{~mm}$, con superficie rugoso-papillosa scura, spesso puberula, percorsa da alcune coste ben rilevate, in corrispondenza delle quali il cercine basale risulta debolmente dentato. (Fig. 45)

FENOLOGIA Fioritura tardo-primaverile ed estiva, tra maggio e agosto, con rilascio delle nucule mature a partire da giugno e fino alla fine dell'estate.

NUMERo $2 n=14$ in piante della Slovacchia (Uhrikova in MÁJOVSKÝ 1978), 2n = 20 in piante CROMOSOMICO della Repubblica Ceca (Javurková-Jarolímová in MĚsíčEK \& JAVURKOVÁ-JAROLíMOVÁ 1992). Piante dell'Iran con $2 n=22$ (GHAfFARI $1996^{1}$ ) e dell'India con $2 n=28$ (SIDHU $1979^{1}$ ), potrebbero appartenere a specie simili, in passato ricondotte a sottospecie di questa, visto che in nessuno dei due paesi ci risultano popolazioni nè naturali nè avventizie della tipica $N$. pulla.

${ }^{1}$ Secondo http://www.tropicos.org/Name/50159390?projectid=9

ECologia Pianta prevalentemente di ambienti steppici continentali, da noi trovata solo in campi e incolti aridi montani, tra 400 e $1650 \mathrm{~m}$.

DISTRIBUZIONE Specie a gravitazione eurasiatica, ad ampia distribuzione, nota per le province europee centrale e orientale, verso sud fino all'area del Caucaso e alla provincia armeno-iraniana (SELVI \& BIGAZZI 2001). In Italia è da sempre rara e localizzata, forse perchè solo esotica; la sua presenza attuale è confermata per una sola stazione recentemente scoperta in Val d'Aosta, ma fu raccolta anticamente anche in Val di Susa e forse (PIGNATTI 1982) sul Carso Triestino. (Fig. 46) 


\section{NOTE}

NomenclatURA II nome "Lycopsis pulla" di Linneo fu pubblicato una prima volta nell'Iter Hispanicum (1758), opera postuma di Pehr Löfling (1729-1756), poi nella decima edizione del Systema Naturae (1759). Sfortunatamente, è evidente come Linneo intendesse riferirsi nei due casi a piante diverse. L'aggettivo latino "pulla" ("scura") è mutuato dal polinimio "Echioides flore pullo" di RIVINUS (1690), già elencato da LINNEO (1753) tra i sinonimi di Lycopsis vesicaria, che non a caso ha corolle viola scuro. Nell'indice dell'Iter Hispanicum di Löfling, lo stesso epiteto fu attribuito impropriamente (forse dallo stesso Linneo) ad una pianta annuale simpatrica di $N$. vesicaria, oggi nota come $N$. echioides, nonostante lo stesso LöFLING (1758: 66-67) avesse osservato che: "den jag ej kan tro ár Lycopsis Hort. Ups. 35.2 eller Echioides flore pullo Riv. emedan denne altid har hvita blommor. Velez menar, at det kunde vara Pulmonaria chia echii folio verrucosa, calyce vesicario, flore albo. Tournef. cor. 6" ["non posso crederla la Lycopsis Hort. Ups. 35.2 o la Echioides flore pullo Riv. perché ha fiori bianchi. Direi che possa essere la Pulmonaria chia echii folio verrucosa, calyce vesicario, flore albo. Tournef. cor. 6"]. L'anno successivo LINNEO (1759) confuse definitivamente le cose anche tra le due specie europee a fiore scuro di "Lycopsis", distinguendole solo sulla base del portamento, la prostrata - com'era anche la pianta di Löfling - come L. vesicaria, l'eretta come L. pulla, nome che per contrasto con la prima "L. pulla" diventava un sinonimo eterotipico posteriore, e dunque illegittimo! La prova più evidente di questa mutata intenzione è il campione designato come tipo per questa seconda "Lycopsis pulla", che non corrisponde affatto alla pianta spagnola bensì alla specie perenne centro- ed est-europea, che da CANDOLLE \& LAMARCK (1805) in poi è nota come Nonea pulla. Trattandosi di nome illegittimo, perchè illegittimo ne era il basionimo, BERNHARDI (1800) propose in soluzione di questo complesso problema nomenclaturale il suo $\mathrm{N}$. erecta, lungamente negletto e recuperato solo in alcune opere recenti. Per risolvere la questione definitivamente, tuttavia, il nome di $N$. pulla è stato recentemente confermato da Cecchi \& al. (2014) proponendo come typus conservandum del nome di Löfling lo stesso già designato per quello di Linneo e anticipando così il basionimo formale per la specie dal 1759 al 1758.

\subsection{Nonea vesicaria (L.) Rchb. NONNEA PURPUREA}

Nonea vesicaria (L.) Rchb., Fl. Germ. Excurs. 1: 338. 1831.

(三) Lycopsis vesicaria L., Sp. PI. 1: 138. $1753 \equiv N$. decumbens Moench, Methodus: 422. 1794, nom. illeg.

Locus classicus: "in Europae australi".

Lectotipo (Qaiser in JAFRI \& EL-GADI 1979: 26): [pianta coltivata in Svezia] "H[ortus].U[ppsaliensis].", s.d., s.coll., Herb. Linneo 190.1 (LINN!).

Altro materiale originale (CECCHI \& SELVI 2015b: 657): [pianta coltivata nei Paesi Bassi] s.d., s.coll., Herb. Clifford 47.1 (BM 557889!).

(=) Lycopsis nigricans Desr. in Lam., Encycl. 3: 655. 1792.

Locus classicus: [pianta coltivata in Francia] "au Jardin du Roi".

Lectotipo (CECCHI \& SELVI 2015b: 657): s.loc., s.d., s.coll., Herb. Lamarck 59.178 (PLA 357407!).

(=) Echioides nigricans Desf., Fl. Atlant. 1: 163. $1798 \equiv$ N. nigricans (Desf.) DC. in DC. \& Lam., Fl. Franç., ed. 3, 3: 626. 1805 三 Onochilis pulla Mart. in Denkschr. Königl. Akad. Wiss. München 5e: 177. 1817.

Locus classicus: [Marocco] "in arenis prope Tozzer". 
Lectotipo (CECCHI \& SELVI 2015b: 658): disegno in ZANONI (1742: t. 38!).

Etimologia Da vesicaria, nome latino per la fabacea Colutea arborescens L., arbusto i cui legumi sono rigonfi egualmente ai calici fruttiferi di tutte le specie di Nonea; per Linneo l'epiteto era discriminante come specie del genere Lycopsis, che non ne comprendeva altre dal calice tanto accrescente alla fruttificazione.

DESCRIZIONE Erba annua, molto ramificata alla base in fusti eretto-ascendenti, $20-60 \mathrm{~cm}$, ispido-setosa per lunghe setole e peli pluricellulari misti a densi peli brevi, in alto ghiandolosa e spesso arrossata. Foglie a margine piano e intero o leggermente sinuato-ondulato, le inferiori spatolate, lunghe al massimo $3 \mathrm{~cm}$, secche all'antesi, le cauline e bratteali strettamente ellittico-lanceolate, 0,7-2 $\times$ 3-8 cm. Infiorescenza eretta, poco ramificata, con brattee superiori più o meno nettamente falcate, molto maggiori dei fiori. Calice diviso fino a metà in denti triangolare-acuti, lungo 5-7 mm all'antesi, fino a 8-12 $\mathrm{mm}$ alla fruttificazione. Corolla strettamente tubulare-infundiboliforme, bicolore, con tubo bianco di 8$10 \mathrm{~mm}$ e lembo porporino molto scuro, di appena 3-4 $\mathrm{mm}$ di diametro, con lobi suberetti; fauce con areole brevemente pubescenti. Stami con antere di 1,2 $\mathrm{mm}$, inclusi ma raggiungenti la fauce corollina. Mericarpi obliquo-ovoidi, con cercine basale ispessito e superficie grigiastra, papillosa e reticolata, percorsa da coste marcate (Fig. 47).

FENOLOGIA Pianta a fioritura precoce, primaverile e forse anche tardo invernale, della quale possono rinvenirsi frutti maturi, o addirittura individui già completamente in fruttificazione, a partire da marzo, ma non oltre l'inizio dell'estate.

Numero $2 n=30$, verificato su una popolazione siciliana (Coppi $\&$ al. 2006 ) e conforme a CROMOSOMICO quanto già conteggiato su piante provenienti da Portogallo (FERNANDES \& QUEIRÓS 1971, GRAU 1971, Fernándes \& LeITÃo 1972) e Spagna (VALdÉs \& al. 1978, LUQUe 1995).

ECOLOGIA Specie psammofila di ambienti retrodunali o collinari interni, con vegetazione erbacea terofitica, raramente ruderale. Diffusa tra il livello del mare e il piano montano (fino a $1800 \mathrm{~m}$ ); da noi si trova solo in luoghi perlopiù prossimi al litorale, tra 0 e $100 \mathrm{~m}$.

DISTRIBUZIONE Specie mediterraneo-occidentale, diffusa perlopiù nelle province iberica, sudoccidentale e balearica, raggiunge il suo limite a est nella parte meridionale della provincia liguro-tirrenica. In Italia è rara, essendone l'areale limitato a poche stazioni della Sicilia occidentale e meridionale (Fig. 48).

\section{NOTE}

ALTRI NOMI VOLGARI Lingua bovina negra, nonnea porporina.

Sistematica Nonea vesicaria condivide il numero cromosomico piuttosto insolito $2 n=30$ (forse originatosi come ibrido aberrante dall'unione di un gamete semplice ed uno non ridotto con $n=x=10$ ) con due delle tre entità nordafricane in precedenza ascritte al genere Elizaldia; tale affinità, che si accompagna ad altri caratteri cariologici comuni, è supportata dai risultati di recenti analisi filogenetiche basate su un ampio campionamento specifico e geografico, che dimostrano l'appartenenza delle tre entità ad un piccolo clado ben definito all'interno del genere. 


\section{SPECIE DA ESCLUDERE}

\section{Nonea rosea (M.Bieb.) Link O NONNEA ROSA}

Nonea rosea (M.Bieb.) Link, Enum. Hort. Berol. Alt. 1: 1671821.

(三) Anchusa rosea M.Bieb., Fl. Taur.-Caucas. 1: 125. 1808.

Locus classicus: [Russia, Daghestan] "in hortis et vineis ad fluvium Terek, circa Kisljar [Kizljar]".

Lectotipo (A. Baytop in DAvIS 1979: 408): [Russia, Daghestan] "in hortis et vineis ad fluvium Terek, circa Kisljar", s.d., Bieberstein (LE).

DESCRIZIONE Erba annua, $20-30 \mathrm{~cm}$, ispido-setosa per lunghe setole e peli pluricellulari misti a densi peli brevi. Foglie lineari-lancelate, fino a 0,5-1 $\times 11 \mathrm{~cm}$. Calice diviso fino a metà in denti subulato-acuminati, lungo 4-6 mm all'antesi, fino a $12 \mathrm{~mm}$ alla fruttificazione. Corolla di $15-18 \mathrm{~mm}$, campanulata, da roseo-porporina a brunastra, con lembo di 4-8 $\mathrm{mm}$ di diametro e pliche faucali semilunari di 0,4 $\times$ 0,9 $\mathrm{mm}$. Mericarpi eretti, oblungo-ovoidi, 3-4 $\mathrm{mm}$, con cercine basale liscio e superficie grigia, puberula, da liscia a rugosa. (da A. Baytop in DAVIS 1979: 407408).

DISTRIBUZIONE Province dell'area caucasica, da noi solo esotica casuale in ambito sinantropico in Toscana, in epoca storica.

\section{NOTE}

DistRIBUZIONE L'unico saggio della specie a noi noto per l'Italia è quello di una pianta "spontanea" derivata da individui coltivati e raccolta nel 1902 in Toscana, in Casentino (Vallombrosa, nata spontanea nell'orto botanico, 15/10/1902, Fiori, FIAF!); ma si trattò certamente di una spontaneizzazione mancata ed effimera, data l'assenza di ulteriori, successive segnalazioni. 


\section{Hormuzakia Guşul. ORMUZACHIA}

Hormuzakia Guşul. in Publ. Soc. Nat. Roman. 6: 8. 1923.

(三) Anchusa subgen. Hormuzakia (Guşul.) Chamb., Notes Roy. Bot. Gard. Edinb. 35: 298. 1977.

Tipo (cfr. GuşulEAC 1923: 8): H. aggregata (Lehm.) Guşul.

Biblografia BIgAZZI \& al. 1999.

Etimologia Genere dedicato al naturalista austro-rumeno Constantin von Hormuzaki (18621937), entomologo e biogeografo.

DESCRIzIONE Piante annue o perenni, ispide. Foglie basali strette, con picciolo indistintamente separato dalla lamina, le cauline e bratteali minori e sessili. Cime bratteate, con fiori inizialmente sessili, all'ascella di brattee nettamente più lunghe. Calice profondamente diviso in lacinie strette. Corolla ipocrateriforme con tubo lungo e stretto, internamente pubescente alla base e occluso in alto da pliche faucali prominenti e irsute; lembo rotato, relativamente piccolo. Stami inseriti in alto sul tubo corollino. Stimma capitatogloboso. Mericarpi ovoido-compressi, il corpo principale ricurvo e orientato perpendicolarmente all'asse fiorale, con cercine basale assai spesso e inciso; elaiosoma cospicuo.

NUMeRI $2 n=16$, verificato sulla sola specie tipo del genere.

CROMOSOMICI

BIOLOGIA Piante entomofile, parzialmente autocompatibili ma in popolazioni naturali RIPRODUTTIVA prevalentemente allogame. La dispersione dei semi è mirmecocora; la tipica dentatura del cercine basale sembra un adattamento all'ancoraggio al substrato sabbioso incoerente.

DISTRIBUZIONE Due specie nella regione mediterranea sud-orientale e in quella saharo-arabica nord-orientale, una largamente distribuita tra la Sicilia, l'Africa settentrionale e il Vicino Oriente, l'altra strettamente endemica di una modesta area nel deserto del Negev, in Israele.

\section{NOTE}

SISTEMATICA Filogeneticamente vicina ad Anchusa subgen. Buglossum Guşul. (HILGer \& al. 2004), con cui tra l'altro condivide le squame faucali lungamente irsute ed occludenti, Hormuzakia merita la distinzione in un genere a sè stante per diverse autoapomorfie micro- e macromorfologiche, la più vistosa delle quali è indubbiamente rappresentata dalla morfologia dei mericarpi "a forma di elmetto", col corpo piegato e il cercine inciso, che accomuna chiaramente le due specie ad oggi note.

\subsection{Hormuzakia aggregata (Lehm.) Guşul.} BUGLOSSA AGGREGATA

Hormuzakia aggregata (Lehm.) Guşul. in Publ. Soc. Nat. Roman. 6: 8. 1923. 
(三) Anchusa aggregata Lehm., Pl. Asperif. Nucif. 1: 219. 1818.

Locus classicus: [Grecia] "prope Athenas".

Lectotipo (SELVI \& BIGAZZI 1998: 128): “Anchusa parviflora” disegno in SIBTHORP \& SMITH (1813: t. 167!).

(-) Anchusa parviflora auct. (Smith in SIBTHORP \& SMITH 1806: 117) non Willd., Sp. PI., ed. 4, 1(2): 759. 1797.

(-) Echium humile auct. (Gussone 1842: 219) non Desf., Fl. Atlant. 1: 165. $1798 \equiv$ Anchusa humilis (Desf.) I. M. Johnst. in Contr. Gray Herb. 73: 55. 1924.

EtimologiA Dal latino aggregatus, "aggregato", "denso", in riferimento alle cime fiorali nelle quali i fiori restano appressati anche con la fruttificazione.

DESCRIZIONE Pianta annua, ramificata alla base, con fusti spessi, carnosi, eretti o prostratoascendenti, 8-50 cm, e indumento ispido-pungente di setole bianche, molto fitte e robuste, lunghe fino a $2 \mathrm{~mm}$. Foglie lineari o oblanceolate, a margine leggermente eroso-dentato o ondulato, le basali da sublineari o strettamente oblanceolate, $0,5-2 \times 6-12 \mathrm{~cm}$, secche all'antesi. Cime per lo più semplici, dense e molto contratte anche alla fruttificazione, con brattee lineari lunghe fino a 2,5 $\mathrm{cm}$, spesso regolarmente alternate e patenti nell'infruttescenza, e fiori sessili. Calice $5 \mathrm{~mm}$ all'antesi, diviso fino alla base in lacinie acuminate, il fruttifero accrescente, globoso, lungo fino a $10 \mathrm{~mm}$ e quasi bianco per le setole ancor più fitte che sugli altri organi vegetativi. Corolla con tubo quasi bianco, sporgente dal calice, di 6-7 mm, e lembo da azzurro pallido a azzurro-porporino o blu cobalto intenso, di $5 \mathrm{~mm}$ di diametro, con lobi strettamente obovato-oblunghi. Stili inseriti alla fauce, con antere di 1,6 $\mathrm{mm}$. Mericarpi generalmente 2 per aborto, $2,5 \times 3,5 \mathrm{~mm}$, incurvati, fessurati lateralmente in corrispondenza del becco acuto, con superficie bruno-chiara, minutamente papillosa e tubercolata, senza coste, e cercine basale molto ampio e sporgente, plicato-dentato (Fig. 49).

FENOLOGIA Fiorisce durante tutti i mesi primaverili, talora già nel tardo inverno, maturando i frutti tra aprile e l'estate inoltrata.

NUMERo $2 n=16$, su piante siciliane (COPPI \& al. 2006), in accordo con quanto rilevato su CROMOSOMICO materiale proveniente da Israele (BIGAZZI \& al. 1999).

Ecologia Psammofita dei litorali sabbiosi o dei deserti interni, da noi circoscritta alle zone costiere, perlopiù nei retroduna consolidati, non esposti direttamente ai venti, ma pochi metri sul livello del mare.

DISTRIBUZIONE Specie a gravitazione mediterraneo-orientale, distribuita tra le province mediterranee liguro-tirrenica, orientale e meridionale e le zone interne delle adiacenti regioni saharo-arabica e irano-turanica, tra l'Algeria a ovest e la Siria a est. In Italia è molto rara, essendo il suo areale limitato a poche stazioni lungo il litorale meridionale della Sicilia (Fig. 50).

\section{NOTE}

NomenclatURA II lectotipo scelto per questa specie, una tavola esplicitamente citata nel protologo, coincide perfettamente con quella che l'autore ebbe a pubblicare appena tre anni dopo (LehmanN 1821: 27); nonostante la tavola della Flora Graeca di Sibthorp e Smith intendesse ritrarre l'Anchusa parviflora Willd. È invece arduo stabilire l'esatta identità di quest'ultima, perchè il tipo è un frammento incompleto di difficile attribuzione (un'etichetta con nota autografa di Pierre Edmond Boissier suggerisce che possa persino trattarsi di un Echium). 
ALTRI NOMI VOLGARI Buglossa aggregata, buglossa viperina.

CONSERVAZIONE Specie localmente vulnerabile, la cui distribuzione italiana è assai ridotta e localizzata, in ambienti fortemente soggetti a disturbo antropico per le attività connesse allo sfruttamento turistico del litorale. 


\section{Cynoglottis (Guşul.) Vural \& Kit Tan CINOGLOTTIDE}

Cynoglottis (Guşul.) Vural \& Kit Tan in Notes Roy. Bot. Gard. Edinburgh 41: 71. 1983.

(E) Anchusa subgen. Cynoglottis Guşul. in Publ. Soc. Nat. Roman. 6: 11. 1923.

Tipo (cfr. GuŞULEAC 1923: 11): Anchusa barrelieri (All.) Vitm. (C. barrelieri (All.) Vural \& Kit Tan).

Bibliografia BigazZI \& SelVI 2001.

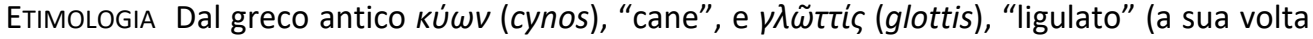
da $\gamma \lambda \hat{\omega} \sigma \sigma \alpha$, glossa, "lingua"), "a lingua di cane", probabilmente in riferimento alla consistenza feltrosa delle foglie e alla somiglianza degli organi vegetativi con quelli delle specie del genere Cynoglossum (tribù Cynoglosseae).

DESCRIZIONE Erbe perenni, a fusi eretti, semplici o ramificati, densamente pubescenti o ispide. Foglie da oblanceolato-spatolate a sublineari, le basali a lamina decorrente, con picciolo alato indistinto, le cauline progressivamente ridotte, cuneate o cordate, sessili. Infiorescenze ramificate in cime numerose, bratteate. Calice profondamente inciso. Corolla ipocrateriforme, con tubo più breve del calice, lembo rotato e pliche faucali piccole. Nucule strettamente ovoidi, erette, debolmente reticolate, prive di becco e con cercine basale incospicuo.

NUMERO I citotipi prevalenti sono il diploide e tetraploide in base 9, ovvero $2 n=18$ e $2 n=$ CROMOSOMICO 36. Tuttavia in seno ad alcune delle stesse popolazioni euploidi studiate, sia nella specie tipica che della sua vicariante orientale $(C$. chetikiana Vural \& Kit Tan) sono stati riscontrati individui a corredo aneuploide con $2 n=12,16,17,24$, suggerendo che $p=6$ sia il numero cromosomico di base ancestrale, metre $x=$ 8, 9 siano derivati secondariamente a seguito di ibridazione e disploidia ascendente.

BIOLOGIA Specie allogame entomofile, perlopiù autoincompatibili, prive di polimorfismo RIPRODUTTIVA stilare; marcata specializzazione per l'impollinazione mediata da imenotteri del genere Colletes Latreille, 1802 (MüLLER \& KUHLMANN 2003). Distribuzione mirmecocora delle nucule.

DISTRIBUZIONE Due specie vicarianti in Europa e Asia occidentale, distribuite tra la regione mediterranea centro-orientale, la circumboreale europea e l'irano-turanica occidentale, a ovest fino alla parte continentale della provincia ligure-tirrenica, ad est fino a quella settentrionale della provincia mesopotamica e a quella meridionale della provincia est-europea. In Italia è presente la sola specie tipica.

\section{NOTE}

SISTEMATICA Simile ad Anchusa, ma con calice più profondamente inciso, tubo corollino abbreviato e mericarpi oblungo-eretti. Ulteriori carattere distintivi a supporto della sua definitiva separazione a rango di genere indipendente (VURAL \& TAN 1983) sono forniti dalla morfologia pollinica e dal corredo cromosomico con $x=9$. Le più strette relazioni filogenetiche appaiono quelle con Anchusa subgen. Buglossellum, circostanza per la quale il genere Anchusa presenta un assetto evolutivo decisamente parafiletico (BIGAzZI \& SeLVI 2001, SelVI \& al. 2004). 


\subsection{Cynoglottis barrelieri (All.) Vural \& Kit Tan BUGLOSSA DI BARRELIER}

Cynoglottis barrelieri (All.) Vural \& Kit Tan in Notes Roy. Bot. Gard. Edinburgh 41(1): 71. 1983.

(三) Buglossum barrelieri All., Fl. Pedem. 1: 48. 1785 E Anchusa barrelieri (All.) Vitm., Summa PI. 1: 388. 1790.

Locus classicus: [Italia, Piemonte] "inter Rocavion, \& Robilant, \& in valle Sturae prope Demont".

Lectotipo (SELVI \& BIGAZZI 1998: 127): [Italia, Piemonte] "a Rocavion ad Robilant secus viam, et in valle Sturae secus viam demontis", s.d., Bellardi, Herb. Bellardi (TO!).

(=) Anchusa longifolia Lam., Encycl. 1: 504. 1785.

Locus classicus: [Italia?] "Nous présumons que cette plante [...] croît naturellement en Italie".

Lectotipo (CECCHI \& SELVI 2015b: 660): [Italia] “Buglossum Alpinum, perenne Italicum, caeruleum minus foliis angustioribus, bullis minutissimis exasperatis", s.d., s.coll. (P 680159!; iso-, "Buglossum cynoglossi vulgaris folio, flore amoene coeruleo. D.Sherard “, P 680160!).

EtIMOLogia Specie dedicata a Jacques Barrelier (1606-1673), frate domenicano e botanico francese, autore della famosa opera Plantae per Galliam, Hispaniam et Italiam observatae, iconibus aeneis exhibitae, pubblicata postuma nel 1714.

DESCRIZIONE Erba bienne o perenne, con robusta radice fittonante e fusti striati eretti, ramosi, alti 3-6 dm; denso indumento monomorfico di peli appressati, morbidi, di circa $0,6 \mathrm{~mm}$. Foglie acute, con margini piani ed interi o oscuramente e irregolarmente ondulato-incisi, le basali oblanceolato-spatolate, fino a $3 \times 12$ $\mathrm{cm}$, le cauline più brevi e sessili, a base cordato-amplessicaule. Infiorescenza ricca, molto ramificata, con cime allungate e fiori su peduncoli di circa $1 \mathrm{~mm}$; brattee da lineari a lanceolato triangolari, 2-5(7) $\mathrm{mm}$. Calice diviso per oltre i 2/3 in lacinie lineari erette e ottuse, all'antesi lungo $3 \mathrm{~mm}$, alla fruttificazione fino al doppio o oltre. Corolla con tubo bianco di 1,5-2 mm, lievemente allargato nella metà superiore, e lembo azzurro intenso, rotato, con lobi arrotondati, $6-8 \mathrm{~mm}$ in diametro; pliche faucali bianche, papillose, non occludenti la fauce. Stami con filamento subnullo e antere di ca. $1 \mathrm{~mm}$, parzialmente sovrapposte alle pliche. Mericarpi 3,5 mm, oblungo-ovoidi, eretti, con cercine basale alto, ma poco rilevato, e superficie brunastra o nerastra, densamente papillosa, solcata da un reticolo di coste longitudinali.

NUMERo Piante del citotipo diploide, $2 n=18$, o tetraploide, $2 n=36$, sono state CROMOSOMICO riscontrate in entrambe le sottospecie note. Occasionali numeri aneuploidi, fino ad oggi noti solo per quella tipica, sono stati interpretati come prodotto di disploidia discendente (BIGAZZI \& SELVI 2001).

DISTRIBUZIONE Specie endemica europea, presente con due sottospecie poco differenziate, vicarianti in un ampio areale compreso tra la provincia liguro-tirrenica e quella balcanica, ed estesa ad aree limitrofe delle provincie europee centrale e orientale e mediterranea orientale. La sottospecie tipica vive principalmente in Italia, donde sconfina appena verso nord, sul versante alpino francese, e a est, con alcune popolazioni disgiunte in Grecia, ov'è perlopiù sostituita dalla subsp. serpentinicola (Rech. f.) Vural \& Kit Tan. 
NOTE

ALTRI NOMI VOLGARI Buglossa pannocchiuta.

\subsubsection{Cynoglottis barrelieri subsp. barrelieri BUGLOSSA DI BARRELIER TIPICA}

DESCRIZIONE Robusta pianta bienne o perenne, alta fino più di $80 \mathrm{~cm}$, poco ramificata alla base. Foglie basali oblanceolate larghe almeno $15 \mathrm{~mm}$ e lunghe fino a $25 \mathrm{~cm}$ o più, a margine oscuramente ondulato-crespo. Corolla fino a $15 \mathrm{~mm}$ di diametro (Fig. 51).

NUMERO Tutte le popolazioni italiane fin qui esaminate, provenienti da Calabria (BıgAzZI \& Cromosomico Selvi 2001), Lazio (D’Amato \& Trojani 1985) e Toscana (BigazZI \& SelVi 2001), hanno corredo diploide $2 \mathrm{n}=18$, in accordo con quanto osservato sia su piante coltivate (LEVITSKY 1940) che su popolazioni greche (BIGAZZI \& SELVI 2001). La presenza di piante con corredi aneuploidi $(2 n=16,17)$ in almeno una popolazione della Grecia orientale è interpretata come il risultato di un fenomeno di disploidia discendente (BIGAZZI \& SELVI 2001), in linea con dati storici ottenuti da materiale coltivato di origine sconosciuta (STREY 1931, SMITH 1932). Il citotipo tetraploide, $2 n=36$, è stato ad oggi riscontrato solo nei Balcani, in Bulgaria (Markova in LöVE 1983; Markova \& Goranova in KAMARI \& al. 1995) e Grecia (CoPPI \& al. 2006).

FENOLOGIA Pianta a fioritura tardo primaverile ed estiva, prevalentemente tra maggio e luglio, di rado già alla fine di aprile; la fruttificazione avviene tra giugno e agosto, prolungandosi talora fino ai primi di settembre.

ECologia Pendii rocciosi, pascoli aridi, radure, incolti e margini di strada, tra il piano collinare e quello montano, 200-1800 m.

DISTRIBUZIONE Distribuita più o meno continuamente tra le Alpi sud-occidentali e il massiccio del Pollino, relativamente comune solo in Piemonte e nell'Appennino centrale; assente dalle isole (Fig. 52).

\section{NOTE}

SISTEMATICA La subsp. serpentinicola è endemica dell'area balcanica, ove occupa generalmente fasce altitudinali più elevate, o affioramenti ofiolitici; si distingue da quella tipica per essere sempre perenne, legnosa alla base, e con più abbondanti ramificazioni basali, che le conferiscono un aspetto cespuglioso. 


\section{Lycopsis L.} LICOPSIDE

Lycopsis L., Sp. PI. 1: 138. 1753; Gen. PI., ed. 5: 68. 1754.

(三) Anchusa subgen. Lycopsis (L.) Guşul., Bul. Fac. Sti. Cernauti 1: 77. 1927. Tipo (BRITTON \& BROWN 1913: 93, confermato da Hitchcock in HITCHCOCK \& GREEN 1929: 128): L. arvensis L.

Biblografia SelVI \& BigazZI 1998.

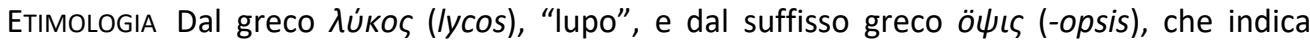
somiglianza, in riferimento forse all'indumento ispido, o forse alla corolla obliqua che richiama l'aspetto delle fauci aperte di un lupo (si veda anche Valdés in TALAVERA \& al. 2012: 363).

DESCRIZIONE Erbe annue con indumento ispido-strigoso. Foglie più o meno crespatoondulate, le basali gradualmente attenuate nel picciuolo, con lamina decorrente, le cauline e bratteali progressivamente ridotte, sessili, spesso da cordato-auricolate a semiamplessicauli. Infiorescenza ramificata, bratteata, fortemente allungata al termine dell'antesi. Corolla zigomorfa, col tubo bianco nettamente piegato a $\mathrm{S}$, incluso nel calice, e lembo azzurro, obliquo, a lobi ineguali; pliche faucali presenti, bianche, lanoso-papillose, occludenti o quasi la fauce. Stami inseriti a metà del tubo corollino, inclusi. Stimma capitato-ovoide, bilobo, incluso. Nucule largamente ovali, orientate perpendicolarmente all'asse fiorale, con becco breve e cercine basale prominente, percorse da coste ottuse.

NUMERo Numero base $x=8$; corredo diploide $(2 n=16)$ o ottaploide $(2 n=48)$. CROMOSOMICO

BIOLOGIA Specie chiaramente allogame, entomofile, la corolla zigomorfa specializzata per RIPRODUTTIVA coevoluzione con gli impollinatori. Dispersione principalmente mirmecocora dei semi.

DISTRIBUZIONE Due sole specie nelle regioni europea, mediterranea e irano-turanica occidentale, una delle quali, la più diffusa, autoctona nell'Italia settentrionale, I'altra, a gravitazione orientale, osservata in Italia solo come avventizia in rare occasioni.

\section{NOTE}

ALTRI NOMI VOLGaRI Buglossa, lingua di bue.

Sistematica II genere appartiene al grande complesso di Anchusa s.l., all'interno del quale i dati molecolari (HILGER \& al. 2004) supportano importanti sinapomorfie morfologiche nella necessità di riconoscere questo ed altri gruppi a rango di generi indipendenti. La più evidente differenza tra Lycopsis e i tipici rappresentanti di Anchusa è costituita dalla corolla zigomorfa, con gli stami inseriti nel tubo a livelli diversi, in aggiunta ad alcuni caratteri peculiari nella micromorfologia dei granuli pollinici (BIGAZZI \& SELVI 1998).

ChIAVE Delle SPECIE 2. Cime contratte con fiori sessili; brattee subeguali al calice; tubo corollino 5$7 \mathrm{~mm}$, incurvato verso la metà; calice fruttifero rigonfio .....

- Cime lasse con fiori distanziati e peduncolati (1,5-2 mm); brattee nettamente superanti i fiori; corolla 4-5 mm, con tubo incurvato nella metà inferiore; calice fruttifero aperto a stella 2. L. orientalis 


\subsection{Lycopsis arvensis $\mathrm{L}$. BUGLOSSA MINORE}

Lycopsis arvensis L., Sp. PI. 1: 139. 1753.

(三) Anchusa arvensis (L.) M. Bieb., Fl. Taur.-Cauc. 1: 123. 1808.

Locus classicus: "in Europae agris".

Lectotipo (SElVI \& al. 1996: 306): “In Misnia, Helvetia, Dania”, Herb. Burser XIV(2): 26 (UPS-BURSER!).

Etimologia Dal latino arvensis, "campestre", per l'ambiente in cui vive la specie.

DESCRIZIONE Erba con fusti eretti o ascendenti, ramosi, 2-5 dm, ispida per dense setole patenti, rigide e tuberculate, di ca. $2 \mathrm{~mm}$, miste a peli più brevi. Foglie con margini ondulati o eroso-dentati, acute, le basali spatolate, $1-3 \times 4-12 \mathrm{~cm}$, le cauline e bratteali da lineari a oblungo-lanceolate. Infiorescenza con brattee subeguali ai fiori e peduncoli fiorali subnulli, i fruttiferi allungati fino a $5 \mathrm{~mm}$. Calice diviso fin quasi alla base in denti acuminati, all'antesi $4-5 \mathrm{~mm}$, nel frutto fino a $8 \mathrm{~mm}$, eretti e più o meno richiusi sui mericarpi. Corolla con tubo di 5-6 $\mathrm{mm}$ e lembo azzurro chiaro, raramente biancastro, di 4-6 $\mathrm{mm}$ di diametro, a lobi arrotondati; pliche faucali bianco-lanose, occludenti la fauce. Mericarpi $2 \times 3,5$ $\mathrm{mm}$, a superficie debolmente rugosa (Fig. 53).

FENOLOGIA Specie a fioritura prolungata, a seconda della stazione estesa dall'inizio della primavera, talora già dalla fine dell'inverno, fino ad estate inoltrata, con maturazione delle nucule tra aprile e settembre.

NUMERO Corredo esaploide, $2 n=48$, riscontrato sia su materiale italiano, proveniente da CRomosomico Piemonte (BIGAzZI \& al. 1997) e Val d'Aosta (GAdelLA \& KLIPHUIS 1970) che su piante coltivate (VASUDEVAN 1975) o popolazioni naturali o naturalizzate provenienti da Bielorussia (DMITRIEVA \& PARFEnov 1983, DMITRIEVA 1986), Canada (Gervals 1981), Islanda (Löve \& LöVe 1956), Polonia (Målecka in PogAN \& al. 1989), Slovacchia (Uhrikova in MÁJovskÝ 1976), Spagna (LUQUE 1983), Svezia (LÖVKVIST \& HULTGÅRD 1999) e Svizzera (D'AMATo 1989). Poco probabili gli antichi conteggi di $2 n=27$ ottenuti per la Germania da SVENSSON (1925) e TISCHLER (1935).

ECologIA Incolti, campi, bordi di strade e ambienti ruderali, dal livello del mare al piano montano, fino a $1600 \mathrm{~m}$ di quota.

DISTRIBUZIONE Specie a larga distribuzione eurasiatica, autoctona tra la provincia iberica e buona parte di quella europea orientale, ma più comune in quelle della regione circumboreale e meno nell'area mediterranea; avventizia in Nord America e in varie zone di transizione tra la regione circumboreale asiatica, l'irano-turanica e l'asiatica orientale, nella Siberia Meridionale e in Cina. In Italia è assai rara e sporadica, probabilmente solo avventizia, nel centro-sud, ma era presente e diffusa in tutte le regioni settentrionali fino a pochi decenni fa; in epoca recente è divenuta invece poco frequente anche nel settentrione, regredendo spesso a poche stazioni relitte (Fig. 54).

\section{NOTE}

SISTEMATICA ED II corredo esaploide di questa specie è probabilmente derivato da quello diploide di $L$. ECOLOGIA orientalis, e sembra conferirgli maggiore competitività anche in ambienti meno 
conservati, sinantropici; non a caso L. arvensis, in assenza di pratiche di agricoltura intensiva, si comporta da specie sinantropica nelle zone agricole e pastorali, sia all'interno del suo areale che come alloctona. La quasi totalità delle segnalazioni per Italia peninsulare e insulare va riferita ad Anchusella cretica, confusa con questa per la corolla zigomorfa, che tuttavia si distingue per avere tubo corollino diritto e solo il lembo obliquo.

\subsection{Lycopsis orientalis $L . O$ BUGLOSSA ORIENTALE}

Lycopsis orientalis L., Sp. PI.: 139. 1753.

(三) Anchusa ovata Lehm., PI. Asperif. Nucif. 1: 222. 1818 三 Anchusa orientalis (L.) Rchb., Icon. Fl. Germ. Helv. (Rchb.) 18: 63 1858, nom. illeg., non L., Sp. PI. 1: 133. $1753 \equiv$ L. arvensis subsp. orientalis (L.) Kuntze in Trudy Imp. S.-Peterburgsk. Bot. Sada 10: 216. 1887 E Anchusa arvensis subsp. orientalis Nordh. in Norsk Fl. 526. 1940.

Locus classicus: "in Oriente".

Neotipo (Güner \& Duman in CAFFERTY \& JARVIS 2004: 803): [Turchia] "B5 Nevsehir: Ürgüp, 1.080m, lake shores, wet places", 22/06/1989, Vural 5360 \& al. (GAZI; iso- ISTF, AIBU).

Etimologia Dal latino orientalis, "orientale", per l'areale orientale rispetto alla vicariante $L$. arvensis.

DESCRIZIONE Erba con fusti eretti o ascendenti, ramosi, 1-4 dm, ispida per dense setole patenti, rigide e tuberculate, di ca. $2 \mathrm{~mm}$, miste a peli più brevi. Foglie con margini ondulati o eroso-dentati, acute, le basali spatolate, le cauline e bratteali ovato-lanceolate. Infiorescenza con brattee nettamente superanti i fiori e peduncoli evidenti già all'antesi, nel frutto lunghi 9-11(17) $\mathrm{mm}$. Calice diviso fin quasi alla base in denti acuminati, all'antesi 4-6 mm, nel frutto fino a $10 \mathrm{~mm}$, patenti a stella. Corolla con tubo di 4-5 mm, curvato nella metà inferiore, e lembo azzurro con lobi ineguali di 2-3 mm; pliche faucali bianco-lanose, non del tutto occludenti la fauce. Mericarpi $1,5 \times 3 \mathrm{~mm}$, a superficie fortemente rugosa (Fig. 55).

FENOLOGIA Specie a fioritura precoce, che inizia già alla fine dell'inverno e porta alla maturazione dei primi mericarpi già in aprile; i due saggi piemontesi di questa specie, effettuati in pieno autunno, recano tuttavia sia fiori che frutti maturi, e insieme a osservazioni su esemplari in coltivazione e a dati riferiti a stazioni climaticamente simili nell'areale d'origine indicano la possibilità di una seconda fase di fioritura, tardo-estiva e autunnale.

NUMERO $2 n=16$, verificato sia su piante coltivate di origine sconosciuta (STREY 1931) che CROMOSOMICO su popolazioni autoctone in India (VASUDEVAN 1975), Iran (Aryavand in LöVE 1977), Repubblica Ceca (KIRSCHNER \& al. in LöVE 1982b) e Turchia (Bigazzi \& al. in KAMARI \& al. 2000) e avventizie in Portogallo (LUQUE 1980).

ECologia Diffusa nelle aree di origine in aree rocciose o campestri della fascia altocollinare e montana, tra 850 e $2300 \mathrm{~m}$; in Italia solo avventizia in aree ruderali o sinantropiche, a quote collinari (200-500 m). 
DISTRIBUZIONE Specie a gravitazione mediterraneo-orientale, con areale esteso ad est fino all'Asia centrale e a sud fino al Corno d'Africa (verosimilmente avventizia nella parte nord-occidentale della penisola iberica). In Italia fu raccolta in due sole località del Piemonte nel 1972, ove cresceva come avventizia occasionale, senza esservi mai più riscontrata da allora, ma è stata forse recentemente ritrovata in due altre stazioni isolate, in Veneto ed Emilia Romagna (Fig. 56). 


\section{Anchusella Bigazzi, E.Nardi \& Selvi ANCUSELLA}

Anchusella Bigazzi, E.Nardi \& Selvi in PI. Syst. Evol. 205: 253. 1997.

(三) Anchusa subgen. Rivinia Greuter in Candollea 20: 202. 1965.

Tipo (GREUTER 1965: 202, BIGAZZI \& al. 1997: 253): Anchusella variegata (L.) Bigazzi, E.Nardi \& Selvi.

BIBLIOGRAFIA BIGAZZI \& al. 1997.

Etimologia Diminutivo dell'affine genere Anchusa (inteso in senso stretto), col diminutivo ellus, per il minore sviluppo che le specie di questo genere mostrano - in habitus e organi riproduttivi - rispetto alla maggior parte di quelle cui erano originariamente assimilate.

DESCRIZIONE Erbe annue, ispido-strigose, da prostrato-ascendenti ad ascendenti, spesso ramificate alla base e arrossate in alto. Foglie con superficie bollosa e margini repando-dentati, muniti di rigide setole su ciascun dente, le basali e cauline inferiori da lineare-lanceolate a spatolate, decorrenti su un picciuolo indistinto, le cauline ridotte, triangolare-ovate, sessili. Infiorescenza semplice, bratteata. Calice diviso fin quasi alla base in lobi lanceolati, eretti alla fruttificazione. Corolla zigomorfa, con tubo diritto o lievemente curvo per una leggera strozzatura nella metà inferiore, e lembo obliquo; androceo ridotto per aborto, con due soli stami nella parte ventrale (adassaiale) del tubo, con filamenti brevi e appiattiti e antere relativamente grandi, accompagnati da tre staminodi rudimentali atrofici; stigma bifido con lobi troncati obliquamente. Nucule obliquamente ovoidi, con cercine basale incospicuo.

Numero $2 n=16$, corredo diploide con $x=8$, in entrambe le specie note per questo CROMOSOMICO genere.

BIOLOGIA Piante entomogame, parzialmente autocompatibili ma prevalentemente RIPRODUTTIVA allogame in natura, prive di polimorfismo stilare. Frutto a dispersione prevalentemente mirmecocora.

DISTRIBUZIONE Due specie nella regione mediterranea centro-orientale, delle quali una sola è presente in Italia.

\section{NOTE}

NomencLATURA II lectotipo del genere è un disegno (RIvinus 1690: t. 9) chiaramente corrispondente alla specie greca $A$. variegata (L.) Bigazzi, E.Nardi \& Selvi, nonostante vi si legga il nome "Buglossoides cretica". Secondo l'Art. 53.3 dell'ICN (MCNEILL \& al. 2012), il nome sottogenerico Rivinia non è utilizzabile al rango di genere per l'alta possibilità di essere confuso col sinonimo Rivina L. (1753) delle Phytolaccaceae.

SISTEMATICA Le analisi molecolari condotte sull'intero complesso supergenerico di Anchusa s.l. collocano Anchusella in più stretta affinità con Anchusa subgen. Anchusa di quanto non lo siano gli altri sottogeneri (HILGER \& al. 2004). La distinzione del genere Anchusella all'interno del gruppo, e in particolare da Lycopsis, col quale condivide la corolla zigomorfa, è comunque supportata da differenze sia macro- e micromorfologiche, quali l'infiorescenza sempre semplice, la forma non attinomorfa né sigmoide della corolla, la riduzione dell'androceo e i granuli pollinici tetracolpati, che cariologiche (GREUTER 1965, SELVI \& al. 1997). 


\subsection{Anchusella cretica (Mill.) Bigazzi, E.Nardi \& Selvi BUGLOSSA CRETESE}

Anchusella cretica (Mill.) Bigazzi, E. Nardi \& Selvi in PI. Syst. Evol. 205: 257. 1997.

(三) Anchusa cretica Mill., Gard. Dict., ed.8: $\mathrm{n}^{\circ} 7.1768 \equiv$ Lycopsis cretica (Mill.) Holub in Preslia 49: 359.

Locus classicus: [pianta coltivata in Francia] "H[orto]. R[egio]. Par[isiense].". Lectotipo (BIGAZZI \& al. 1997: 257): "1 Herbar. Miller", s.d., s.coll., Herb. Miller (BM!).

(=) Lycopsis bullata Cirillo, PI. Rar. Neap. 1: 34. 1788.

Locus classicus: [Italia, Campania] "in ruderatis ubique circa urbem".

Lectotipo (SELVI \& BIGAZZI 1998: 124): disegno in CIRILLO (1788: t.11, f. 3!).

(=) Lycopsis arvensis f. albiflora Ten. ex Fiori in Fiori \& Bég., Fl. Italia 2(3): 373. 1902. Locus classicus: [Italia] non specificato.

Tipo non designato.

(=) Lycopsis variegata f. albiflora Trotter in Boll. Soc. Bot. Ital. 1905: 37. $1905 \equiv$ Lycopsis variegata var. albiflora (Trotter) Trotter in Boll. Soc. Bot. Ital. 1917: 73. 1917.

Locus classicus: [Italia, Campania] "nei dintorni di Avellino".

Tipo non designato.

(-) Lycopsis variegata auct. (BERTOLONI 1835-1836: 338; ARCANGELI 1882: 490; PARLATORE \& CARUEL 1886: 902; FIORI 1926: 280) non L., Sp. Pl. 1: 138. 1753.

Etimologia Dal latino creticus, "di Creta", nome erroneamente attribuito a questa pianta da Miller, sulla base di esemplari coltivati che egli riteneva originari di quell'isola, nonostante la pianta non vi cresca.

DESCRIZIONE Erba alta da 5 a $50 \mathrm{~cm}$, ispida per rigide setole patenti di 1-1,5 $\mathrm{mm}$, a base tubercolata. Foglie basali e cauline inferiori 5-15 × 20-70 mm, acute. Cime spesso arrossate, dense, con fiori subsessili unilaterali e brattee linearilanceolate, minori del calice alla fruttificazione. Lobi calicini di 5,5 $\mathrm{mm}$. Corolla con tubo di 6-7 mm, e lembo di 7-8 $\mathrm{mm}$ di diametro, con lobi arrotondati, azzurro-intenso con venature biancastre; fauce nettamente obliqua, con pliche faucali bianco-lanose occludenti. Antere di $1,7 \mathrm{~mm}$ raggiungenti le squame. Stigma bifido con lobi obliquo-troncati. Nucule acute con superficie nerastra, papilloso-rugosa (Fig. 57).

FENOLOGIA Specie a fioritura primaverile o tardo invernale, può iniziare il rilascio delle nucule già attorno alla fine di marzo, per terminarla non oltre la fine di giugno o i primi di luglio, con la chiusura del ciclo vitale.

Numero $2 n=16$, su piante pugliesi (Grau 1968b), laziali (Altamura \& al. 1984, D'Amato CROMOSOMICO 1989) e toscane (BIGAZZI \& al. 1997), a conferma di quanto osservato anche su materiale greco (Strid \& Franzen in LÖVE 1981: 838).

ECOLOGIA Prevalentemente xerofitica, in pascoli e incolti aridi, arbusteti radi e garighe soggetti a forte aridità estiva, di rado in luoghi più umidi, soprattutto su substrato calcareo, 0-1500 m. 
DISTRIBUZIONE Provincie liguro-tirrenica, adriatica e mediterraneo-orientale, tra la Sicilia, la penisola italiana centro-meridionale e il Peloponneso, passando per i Balcani occidentali tra l'Istria e l'Epiro. In Italia è specie largamente distribuita in Sicilia e nelle regioni meridionali fino alla Campania e alla Basilicata; assai più rara in Puglia (sul Gargano) e, risalendo verso nord sul versante tirrenico, in tutto il Lazio e in poche località della bassa Maremma Toscana (Fig. 58).

\section{NOTE}

ALTRI NOMI VOLGARI Lingua bovina verrucosa.

SISTEMATICA Spesso confusa con A. variegata, tipo del genere, che tuttavia è endemica della Grecia meridionale e delle isole egee, facilmente distinguibile per l'habitus prostratoascendente, la corolla bianca con chiazze porporine sui lobi e alla fauce, i mericarpi percorsi alla base da coste marcate, ortogonali alla superficie di adesione, e alcuni caratteri micromorfologici. 


\section{Anchusa L.} BUGLOSSA

Anchusa L., Sp. PI. 1: 133. 1753; Gen. PI., ed. 5: 64. 1754.

Tipo (BRITTON \& BROWN 1913: 93, confermato da Hitchcock in HITCHKOCK \& GREENE 1929: 127): A. officinalis L.

(=) Buglossum Mill., Gard. Dict., ed. 4. 1754.

Tipo non designato.

Bibliografia Selvi \& BigazZI 1998.

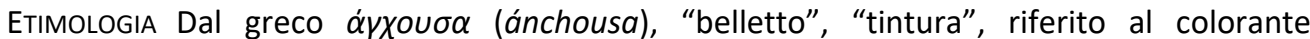
ottenuto dalle radici, proprio di alcune specie del genere ma in particolare di $A$. tinctoria (L.) L., oggi ascritta al genere Alkanna.

DESCRIZIONE Erbe annuali, bienni o perenni, con indumento ispido-strigoso, mono- o dimorfico. Foglie intere o crespato-ondulate, le basali più o meno gradualmente attenuate nel picciuolo, con lamina decorrente, le cauline e bratteali progressivamente ridotte, sessili, spesso da cordato-auricolate a semiamplessicauli. Fiori peduncolati in infiorescenze terminali, spesso bratteate e ramificate, considerevolmente allungate alla fruttificazione, di rado con fiori isolati all'ascella di brattee uguali alle altre foglie cauline ( $A$. litorea). Calice parzialmente o completamente diviso, spesso notevolmente accrescente nel frutto. Corolla da blu scuro a violetto, raramente rosa pallido o quasi bianca, rotata o campanulata, con tubo allungato e pliche faucali evidenti, di vario aspetto. Stami inclusi, con filamento più breve dell'antera. Stimma capitatoovoide, bilobo. Nucule quattro o meno per aborto, obliquamente ovoidi o erette, rugoso-reticolate, con cicatrice basale circondata da un cercine più o meno ispessito, crenulato o leggermente denticolato.

NUMERI Generalmente $n=x=8$, con la sola eccezione della greca $A$. thessala Boiss. \& CROMOSOMICl Sprun. (sottogenere Buglossellum), che ha numero aploide $\mathrm{n}=6$. II corredo diploide $2 \mathrm{n}=16$ è di gran lunga prevalente, ma esistono anche specie tetraploidi con $2 n=32$.

BıologıA Piante allogame, talora con polimorfismo stilare (ercogamia) più o meno RIPRODUTTIVA marcato, ma spesso (con l'eccezione di $A$. officinalis e $A$. undulata) capaci anche di autofecondazione. Dispersione dei mericarpi principalmente affidata alle formiche (mirmecocoria), secondariamente anche per idrocoria (oss. pers.).

DISTRIBUZIONE II maggior genere della tribù Boragineae, con circa 30 specie a gravitazione prevalentemente mediterranea, assai meno diversificato nelle adiacenti province delle regioni circumboreale europea e irano-turanica; due sole specie endemiche africane estendono l'areale verso sud, in aree nettamente disgiunte della regione sudano-zambesiana ( $A$. affinis $\mathrm{R}$. Br. dell'Eritrea) e addirittura capense ( $A$. capensis Thunb. del Sudafrica). In Italia Anchusa è rappresentato da dieci specie, sei delle quali costituiscono un complesso di schizoendemismi circoscritto al sistema sardo-corso.

\section{NOTE}

ETIMOLogiA La parola latina buglossum, all'origine del termine che in molte lingue indica le specie di Anchusa come alcune di generi più o meno affini, deriva dal greco Boüৎ (bous), "bue", e 
$\gamma \lambda \omega \dot{\sigma \sigma \alpha}$ (glossa), "lingua", quindi "lingua di bue", come il nome volgare dei rappresentanti di questo e di vari generi affini, in riferimento alla forma e alla consistenza feltrosa delle foglie.

ALTRI NOMI VOLGARI Coda di volpe, lingua bovina, lingua di bue.

Sistematica Con la separazione degli affini Anchusella, Gastrocotyle Bunge, Hormuzakia, Lycopsis e Phyllocara Gușul., il genere Anchusa assume un assetto parafiletico, costituito da quattro sottogruppi ben differenziati da caratteri del fiore e del frutto, che qui consideriamo ancora come sottogeneri, ma che potrebbero egualmente essere trattati a rango di generi indipendenti (HILGER \& al. 2004): A. subg. Anchusa, subg. Buglossellum Gușul., subg. Buglossoides (Tausch) Gușul., subg. Buglossum (Mill.) Gușul. Con l'eccezione della sola $A$. azurea, che appartiene a quest'ultimo e si distingue facilmente per le infiorescenze prive o quasi di brattee, le squame faucali lungamente irsute e i mericarpi grossi ed eretti, tutte le specie presenti in Italia appartengono al sottogenere tipico, caratterizzato dalle cime regolarmente bratteate, la pubescenza delle pliche faucali più breve e i mericarpi ovoidi orientati obliquamente rispetto all'asse fiorale.

Nell'ambito di quest'ultimo, i taxa di Anchusa endemici del sistema sardo-corso ( $A$. capellii, A. crispa, A. formosa, A. litorea, A. montelinasana e A. sardoa) costituiscono a loro volta un sottoinsieme morfologicamente omogeneo e filogeneticamente distinto, contraddistinto dal portamento prostrato- o eretto-ascendente, dal tubo corollino abbreviato, dal lembo rotato, dalle nucule piccole e, dal punto di vista ecologico, dalla stretta limitazione a substrati di natura silicea. All'interno di questo clado, il divario tra le entità costiere e quelle montane, ristrette a stazioni remote e puntiformi, rende plausibile l'ipotesi di una derivazione delle prime dalle seconde, ovvero della differenziazione di taxa a ciclo breve, in ambienti secondari, a partire da ceppi orofitici, lungo una o più linee derivate. Meno chiara appare invece l'origine del clado stesso. I dati molecolari a nostra disposizione (COPPI \& al. 2008, MANSION \& al. 2009, A. Coppi com. pers.) lo pongono sorprendentemente in relazione diretta con le sole due specie endemiche africane ( $A$. affinis e $A$. capensis), la cui separazione risalirebbe appena al tardo Pliocene, meno di 3 milioni di anni fa. Si esclude pertanto che la radiazione evolutiva sulle due isole sia iniziata con il distacco della placca sardo-corsa dal continente europeo, ca. 30 milioni di anni fa, accettando piuttosto l'idea che abbia avuto luogo in tempi assai più brevi dopo l'insediamento di progenitori africani per dispersione via terra (durante l'abbassamento dei livelli del mare nei periodi glaciali) o via mare (sulla resistenza dei mericarpi alla salinità, si veda QUILICHINI \& DEBUSSCHE 2000).

PROPRIETÀ E USI L'impiego delle foglie e delle cime immature di Anchusa in erboristeria e in cucina, così come quello della tintura estraibile dalla radice in cosmesi, è noto soprattutto per la specie tipo, $A$. officinalis, e in misura minore per $A$. azurea, con proprietà e controindicazioni pressappoco uguali a quelle già discusse per Borago o Pulmonaria. Le stesse applicazioni si confanno certamente anche a molte, se non tutte, delle altre specie del genere, benchè nessuna sia mai esplicitamente citata nei testi di erboristeria.

ChIAVE DELlE SPECIE 1. Infiorescenza ebratteata o con poche brattee isolate, non ascellanti i peduncoli fiorali; calice diviso fin quasi alla base in lacinie strettamente lineari, non accrescente alla fruttificazione; tubo corollino in alto completamente occluso dai ciuffi di peli lungamente prominenti dalle pliche faucali; mericarpi oblungo-ovoidi, lunghi 6-9 mm, eretti, grigio-brunastri (subgen. Buglossum) 1. A. azurea

- Infiorescenza regolarmente bratteata, con poche brattee isolate sterili; calice diviso per non più di $2 / 3$ della lunghezza in lacinie da lineari a largamente triangolari, accrescente alla fruttificazione; tubo corollino pervio o occluso da pliche faucali brevemente pubescenti; mericarpi ovoidi, lunghi al massimo $4,5 \mathrm{~mm}$, orientati obliquamente rispetto all'asse fiorale, da bruno-scuri a neri (subgen. Anchusa) 
2. Pianta eretta con cime dense e vistosamente scorpioidi, le brattee bruscamente ridotte rispetto alle foglie cauline superiori, quelle superiori assai minori del calice; corolla con tubo $>6 \mathrm{~mm}$, sporgente dal calice, e lembo campanulato, diviso in lobi deltoidi; antere $>2 \mathrm{~mm}$ 3

- Pianta da prostrata a eretto-ascendente con cime spesso incospicue e solo oscuramente scorpioidi, le brattee gradualmente ridotte rispetto alle foglie cauline e tutte $\geq$ calice; corolla con tubo $<5,5 \mathrm{~mm}$, incluso nel calice, e lembo rotato, diviso in lobi arrotondati; antere $<2 \mathrm{~mm}$ (gruppo degli endemismi sardo-corsi)

3. Pianta perenne con indumento monomorfico di peli morbidi, perlopiù non tubercolati; foglie in genere con margine piano; corolla con lembo largo circa quanto la lunghezza del tubo; stigma capitato-depresso ... 4

- Pianta bienne con indumento dimorfico composto da peli morbidi brevi misti a lunghe setole a base tubercolata; foglie in genere a margine più o meno regolarmente ondulato; corolla con lembo largo meno della lunghezza del tubo; stigma oblungo-ellissoidale, spesso con apice acuto sterile

10. A. undulata

4. Foglie larghe meno di $1 \mathrm{~cm}$; calice $4-6 \mathrm{~mm}$, con lobi ottusi; corolla giallopallida 7. A. ochroleuca

- Foglie più larghe di $1 \mathrm{~cm}$; calice $5-7 \mathrm{~mm}$, con lobi acuti; corolla violettoporporina, raramente bianca o rosata 8. A. officinalis

5. Foglie basali largamente lanceolate o oblanceolate, $15-30 \mathrm{~mm}$ di larghezza, le cauline da largamente lineari a lanceolate, con margine piano o debolmente ondulato; calice fruttifero $>10 \mathrm{~mm}$ (orofite) .....

- Tutte le foglie da lineari a strettamente oblanceolato-spatolate, di larghezza $<15 \mathrm{~mm}$, con margine spesso increspato-ondulato; calice fruttifero < 10 $\mathrm{mm}$ (psammofite)

6. Calice diviso per più della metà della sua lunghezza; corolla con tubo di 6,5 $\mathrm{mm}$ e lembo di 10-12 $\mathrm{mm}$ di diametro

- Calice diviso per meno della metà della sua lunghezza; corolla con tubo di 5 $\mathrm{mm}$ e lembo di 7-8 $\mathrm{mm}$ di diametro

7. Indumento pungente per setole rade di $3 \mathrm{~mm}$; foglie fino a $3 \times 14 \mathrm{~cm}$; infiorescenza ramificata distalmente in 2-3 cime relativamente brevi e dense anche alla fruttificazione; peduncoli fruttiferi in genere $<5 \mathrm{~mm}$, patenti; calice fruttifero ventricoso-urceolato, con denti riflessi e nervi costati prominenti

4. A. formosa

- Indumento ispido ma non pungente, con fitte setole $<2,5 \mathrm{~mm}$; foglie fino a $2,5 \times 8 \mathrm{~cm}$; infiorescenza ramificata fin dalla metà, con cime lasse alla fruttificazione; peduncoli fruttiferi fino a $7 \mathrm{~mm}$, deflessi; calice fruttifero tubuloso-campanulato, senza coste prominenti

6. A. montelinasana

8. Pianta annua a radice esile; fiori poco numerosi, isolati e distanziati all'ascella di brattee uguali alle foglie cauline, spesso emergenti fin dalla base dei brevi fusti; corolla con lembo $<5 \mathrm{~mm}$; antere non sovrapposte alle squame faucali

5. A. litorea

- Piante bienni o perenni, raramente annue, ma con fittone robusto; cime cospicue, in infiorescenze terminali, la parte inferiore sterile del fusto evidente, foglie bratteali gradualmente ma nettamente ridotte rispetto alle foglie cauline; corolla con lembo $>5 \mathrm{~mm}$; antere sovrapposte alle squame faucali

9. Fusti eretto-ascendenti; foglie basali di larghezza $>1 \mathrm{~cm}$; brattee lungamente superanti i fiori; calice internamente pubescente, alla 
fruttificazione tubuloso, 9-10 $\mathrm{mm}$

9. A. sardoa

- Fusti prostrato-diffusi; foglie basali di larghezza $<10 \mathrm{~mm}$; brattee subeguali ai fiori, o minori di questi; calice internamente glabro, alla fruttificazione subgloboso-urceolato, 6-8 $\mathrm{mm}$

3. A. crispa

\subsection{Anchusa azurea Mill. BUGLOSSA ITALICA}

Anchusa azurea Mill., Gard. Dict., ed. 8: $\mathrm{n}^{\circ} 9.1768$.

Locus classicus: [pianta coltivata nel Regno Unito] non specificato.

Neotipo (SelVI \& BIGAZZI 1998: 130): [Italia, Puglia] "margini di campi lungo I'autostada Napoli-Bari presso il Lago di Capacciotti all'Ofanto", 04/05/1995, Bigazzi \& Selvi (FI 2358!).

(=) A. italica Retz., Observ. Bot. 1: 12. 1779.

Locus classicus: non indicato.

Lectotipo (SELVI \& BIGAZZI 1998: 130): “Anchusa besleri N” (LD!).

(=) A. italica var. angustifolia Guss., Fl. Sic. Syn. 1: 220. $1843 \equiv$ A. italica f. angustifolia (Guss.) Fiori in Fiori \& Bég., FI. Italia 2(3): 375. 1902 三 A. azurea var. angustifolia (Guss.) Fiori, Nuov. Fl. Ital. 2(2): 281.1926.

Locus classicus: [Italia, Sicilia] "in Sicilia et in Alicuri, Filicuri, Saline, Lipari".

Lectotipo (CECCHI \& SELvi 2015b: 665): [Italia, Sicilia] "in arvis, Palermo", s.d., Gasparrini, Herb. Gussone (NAP!).

(=) A. italica var. humilis Ten., Syll. PI. Fl. Neapol.: 82. 1831 = A. italica Retz. f. humilis (Ten.) Fiori in Fiori \& Bég., Fl. Italia 2(3): 375. 1902.

Locus classicus: [Italia, Campania] "in campis argillosis cultis insularum Inarimes et Caprearum".

Lectotipo (CECCHI \& SelVI 2015b: 665): disegno di "Anchusa paniculata" in SIBTHORP \& SMITH (1813: t. 63!).

Epitipo (CeCCHI \& Selvi 2015b: 665): [Italy, Campania] “Principato Ultra”, s.d., Gussone, Herb. Tenore (NAP!).

Etimologia Dall'aggettivo latinizzato azureus, "azzurro", "color del cielo", a sua volta derivato dall'arabo لازورد (lāzaward) e poi dal latino medievale lazulum o lazur, "cielo", per la tinta blu delle corolle mature.

DESCRIZIONE Erba perenne vigorosa, a base lignificata e fusti eretto-diffusi, più o meno ramificati, elevati fino a $1,5 \mathrm{~m}$; indumento denso, ispido-strigoso, composto di setole ialine patenti e rigide, a base tubercolata, lunghe fino a 2,5 $\mathrm{mm}$. Foglie a margine intero e apice acuto, le basali lanceolate, $1,5-4 \times 6-15 \mathrm{~cm} 0$ più. Infiorescenza panicolata, con cime numerose, molto allungate alla fruttificazione; brattee spesso assenti o presenti in numero minore dei fiori, più brevi di questi, triangolari-lanceolate; peduncoli eretto-patenti di $1-2 \mathrm{~cm}$. Calice diviso fino alla base in denti lineari-lanceolati di 7-11 $\mathrm{mm}$, il fruttifero fino a 20 $\mathrm{mm}$. Corolla ipocrateriforme, con tubo da biancastro a roseo-lillacino di 7-12 $\mathrm{mm}$ e lembo rotato di $10-15 \mathrm{~mm}$ di diametro, con lobi arrotondati di colore blu intenso, con toni porporini solo negli stadi immaturi; pliche faucali completamente occludenti, coperte di densi tricomi eretti, bianchi. Stilo generalmente incluso, talora appena sporgente dalle pliche faucali; stimma 
bilobo, i due lobi globosi e divergenti. Stami inseriti poco sotto la fauce, con antere raggiungenti le squame, lunghe $2,5 \mathrm{~mm}$. Mericarpi di 6-9 $\times 2-3 \mathrm{~mm}$, oblunghi ed eretti, ottusi, con superficie grigio-marrone percorsa da costolature molto rilevate (Fig. 59).

FENOLOGIA Fioritura principalmente tra aprile e giugno, talora anticipata fino ai primi di marzo nelle stazioni più calde e meridionali, o posticipata fino alla tarda estate in quelle più fresche; i frutti maturano a partire da metà maggio.

NUMERO Tetraploide con $2 n=32$, numero verificato su piante sarde (VALSECCHI 1976), CROMOSOMICO piemontesi e valdostane (Löve \& Löve in LöVE 1982b), toscane, laziali e lucane (D'AMATO \& TROJANI 1985) e in accordo con quanto già riportato da LEVITSKY (1940) e poi riscontrato su materiale proveniente da Bulgaria (MARKOVA \& IVANOVA 1971, Markova \& Goranova in KAMARI \& al. 1995), Iran (GHAFFARI 1996), Portogallo (FERnÁNDES \& LeITÃo 1972) e Spagna (LÖVE \& KJELQUIST 1974, VALDÉS \& al. 1978, LUQUE 1983).

ECOLOGIA Prati incolti, margini di vie e coltivi, ambienti ruderali, occasionalmente in garighe e pendii aridi, più frequente dal livello del mare al piano collinare, ma presente anche a quote maggiori, fino a $1500 \mathrm{~m}$.

DISTRIBUZIONE Diffusa in tutta la regione mediterranea e in quella irano turanica fino al Pakistan, anche se nella parte sud-orientale del bacino del Mediterraneo è spesso sostituita dalla vicariante $A$. strigosa Banks \& Sol.; da queste si estende, forse solo come avventizia, verso nord fino ad ampia parte delle province europee atlantica e centrale, verso sud almeno in parte di quella saharo-arabica e sudano-zambesiana; è certamente alloctona in altre aree del globo a clima simile, come il Nordamerica e il Sudafrica. In Italia è presente e relativamente comune in gran parte del territorio, in particolare nelle zone a clima più mite o caldo delle regioni insulari e peninsulari, rarefacendosi significativamente nelle regioni settentrionali (Fig. 60).

\section{NOTE}

NomenCLATURA L'assenza di materiale originale di Miller nell'erbario del British Museum di Londra, unitamente ad alcune ambiguità nel protologo, ha indotto in passato molti autori a considerare il binomio A. azurea come nomen confusum, preferendole il sinonimo posteriore $A$. italica, col quale la pianta è spesso citata anche, e a maggior ragione, nelle opere inerenti la nostra flora. La neotipificazione proposta da SELVI \& BIGAzZI (1998) ha invece confermato una sinonimia già da tempo largamente riconosciuta, dirimendo definitivamente la questione nomenclaturale. Quanto alla possibile sinonimia con $A$. biceps, si veda la nota per $A$. officinalis.

ALTRI NOMI VOLGARI Buglossa azzurra, buglossa volgare, lingua di bue comune.

SISTEMATICA Anchusa azurea è l'unico rappresentante in Italia del sottogenere Buglossum, di cui fa parte insieme all'asiatica $A$. strigosa Banks \& Sol.; come discusso nelle note introduttive al genere, si tratta di una linea parafiletica rispetto al sottogenere tipico, più vicina ai generi Hormuzakia e Phyllocara, che secondo i principi della tassonomia filogenetica sarebbe meritevole d'essere distinta come genere indipendente.

PROPRIETÀ E USI I fiori e le parti giovani della pianta, prima che diventino troppo ispide, possono trovare in erboristeria e in cucina gli stessi impieghi (e le stesse controindicazioni) che per $A$. officinalis, Borago officinalis o Pulmonaria, con proprietà emollienti, diuretiche e antinfiammatorie. Lo stesso dicasi per la tintura rossa estraibile dal fittone, che trovava, soprattutto in passato, un certo uso in cosmetica. 


\subsection{Anchusa capellii Moris BUGLOSSA DI CAPELLI}

Anchusa capellii Moris, Stirp. Sard. El. 2: 6. 1827.

(E) A. hybrida subsp. capellii (Moris) Nyman, Consp. Fl. Europ.: $511.1881 \equiv$ A. crispa var. capellii (Moris) Illario, Arch. Bot. 11: 261. $1935 \equiv$ A. undulata subsp. capellii (Moris) Vals., Webbia 30: 57. 1976.

Locus classicus: [Italia, Sardegna] non specificato.

Lectotipo (SELVI 1998: 267): [Italia, Sardegna] "in Sardiniae montibus", 1825, Moris, Herb. Moris (TO).

Altro materiale originale (CECCHI \& SeLVI 2015b: 666): [Italia, Sardegna] "in pascuis summi montis S. Vittoria Esterzili", s.d., Moris, Herb. Moris (TO).

Etimologia Specie dedicata a Carlo Matteo Capelli (1765-1831), uomo politico protagonista del primo Risorgimento italiano e studioso variegato. Fu successore di Giovanbattista Balbis e predecessore di Moris alla direzione dell'orto botanico e alla cattedra di Medicina di Torino, nonchè referente in patria del Moris stesso durante le spedizioni in Sardegna che gli consentirono, tra l'altro, la scoperta di questa nuova entità.

DESCRIZIONE Erba bienne o perenne a vita breve, con fusti prostrato-ascendenti lunghi fino a $40 \mathrm{~cm}$, ramificati dalla base; indumento dimorfo, ispido per rigide setole ialine tuberculate, lunghe fino a $3 \mathrm{~mm}$, miste a densi peli brevi. Foglie verde-scuro, lievemente bollose e con margine da intero e piano a leggermente dentatoondulato, le basali oblanceolato-spatolate, 1-3,5 × 4-14 cm. Infiorescenze ramificate in cime molto allungate alla fruttificazione, con brattee lunghe più 0 meno quanto il calice e peduncoli fruttiferi sensibilmente allungati, fino a 6-7 $\mathrm{mm}$. Calice di 5-6 mm, diviso fino a circa 2/3 della lunghezza in denti subacuti, nel frutto largamente campanulato e fino a oltre $1 \mathrm{~cm}$. Corolla ipocrateriforme con tubo bianco di 5-6 mm, incluso nel calice, e lembo rotato fino a $12 \mathrm{~mm}$ di diametro, blu intenso, talora con toni porporini e macchie o bande radiali più chiare; pliche faucali bianco-lanose, molto prominenti e occludenti la fauce. Antere di $2 \mathrm{~mm}$, parzialmente sovrapposte alle pliche faucali. Polimorfismo stilare, con individui brevistili e longistili in rapporto di circa 3:1. Mericarpi di $2 \times$ $3 \mathrm{~mm}$ scarsi, obliquo-ovoidi, con becco laterale e superficie tuberculato-rugosa, bruno-scura o nerastra, percorsa da coste reticolate e con cercine basale prominente (Fig. 61).

FENOLOGIA Fiorisce nella tarda primavera, maturando i frutti generalmente tra la fine di maggio e i primi di agosto.

NUMERO $2 n=16$, su piante provenienti dal m.te Santa Vittoria di Esterzili, locus classicus CROMOSOMICO (VALSECCHI 1976; Bigazzi \& al. in KAMARI \& al. 2000).

Ecologia Anfratti rocciosi nei canali franosi e negli ambienti pascolati di quota, su substrato siliceo, tra 730 e $1200 \mathrm{~m}$.

DISTRIBUZIONE Endemismo puntiforme, noto attualmente per una singola stazione sul crinale del monte Santa Vittoria di Esterzili, nella Sardegna sud-orientale. Anticamente segnalata una sola volta anche per il vicino rilievo del Taccu de Sadali, ma qui non più ritrovata (Fig. 62). 
NOTE

ALTRI NOMI VOLGARI Buglossa del monte Santa Vittoria, buglossa sdraiata.

ConserVAzIONE Specie assai rara, a rischio di scomparsa per l'estrema limitatezza dell'areale, la tendenza a fluttuazioni demografiche, e la suscettibilità al pascolo. Classificata come in pericolo critico (CR) nella lista rossa regionale (CONTI \& al. 1997) e come vulnerabile (VU) dalla IUCN.

\subsection{Anchusa crispa Viv. BUGLOSSA CRESPA}

Anchusa crispa Viv., App. Fl. Cors. Prodr. 1: 1. 1825.

(三) Lycopsis crispa (Viv.) Bertol., Fl. It. 2: 337. 1835 三 A. hybrida var. crispa (Viv.) Nyman, Consp. Fl. Eur.: 510. 1881 三 Anchusa undulata var. crispa (Viv.) Fiori, Nuova Fl. It. 2: 282. 1926 三 A. crispa var. vivianii Illario, Arch. Bot. 11: 261. 1935, nom. illeg.

Locus classicus: [Francia] "in Corsicae collibus".

Lectotipo (SelVI \& BIGAZZI 1998: 134): [Francia] s.d., [Viviani] (“m. Viviani 1826”, Herb. De Candolle, G 144099!).

Etimologia Dal latino crispus, "crespo", in riferimento al margine fortemente crespoondulato delle foglie.

DESCRIZIONE Erba generalmente bienne o perenne a vita breve, di rado annua, ramificata alla base, prostrato-diffusa o -ascendente, con fusti lunghi fino a $35 \mathrm{~cm}$, fortemente ispida per robuste setole patenti lunghe fino a $2,5 \mathrm{~mm}$, tubercolate alla base, miste a peli più brevi e densi. Foglie da lineari a strettamente lanceolate, ottuse, con margine fortemente crespo-ondulato, le basali in rosette lasse, 3-9 $\mathrm{mm} \times 6$ $10 \mathrm{~cm}$, talora marcescenti all'antesi. Infiorescenze ramificate in cime lasse ma poco allungate dopo l'antesi, regolarmente bratteate, con fiori su peduncoli brevi o subnulli, ma allungati dopo l'antesi fino a 2(6) $\mathrm{mm}$. Calice campanulato di 3-4 mm, diviso da per $1 / 3-1 / 2$ in denti ottusi, glabri all'interno, dopo l'antesi subgloboso-urceolato e fino a $5 \times 7,5 \mathrm{~mm}$. Corolla da bianca a blu-violetta, con tubo di 4-5 $\mathrm{mm}$ e lembo rotato di 5-7 $\mathrm{mm}$ in diametro, diviso in lobi arrotondati. Antere lunghe circa $1,5 \mathrm{~mm}$, lievemente sovrapposte alle pliche faucali. Stigma capitato-ovoide, bilobo. Mericarpi $1,3 \times 2,1 \mathrm{~mm}$, obliquo-ovoidi con becco laterale, bruno-grigiastri, con superficie rugoso-reticolata e cercine basale incospicuo (Fig. 63).

FENOLOGIA Fiorisce durante tutta la primavera, da marzo a giugno, rilasciando mericarpi maturi a partire dalla seconda metà di maggio, fino alla prima metà di luglio.

NUMero $2 \mathrm{n}=16$, su piante da popolazioni sarde di entrambe le sottospecie (VALSECCHI CROMOSOMico 1976, D’Amato 1989, Selvi \& BigazzI 1998, Selvi \& Bigazzi in KamaRI \& al. 2000), peraltro in accordo con precedenti conteggi su popolazioni corse della sottospecie tipica (CONTANDRIOPOULOS 1962).

ECologIA Sabbie marittime e dune stabilizzate a poca distanza dal mare, talora nei retroduna con bassa e rada vegetazione arbustiva pioniera, a meno di $10 \mathrm{~m}$ di quota.

DISTRIBUZIONE Raro endemismo sardo-corso, circoscritto a poche località costiere nel centro- 
sud della Corsica e nella Sardegna settentrionale, con due sottospecie debolmente differenziate (Fig. 64).

\section{NOTE}

ALTRI NOMI VOLGARI Lingua bovina crespa.

SISTEMATICA Variazioni nel colore dei fiori e nei rapporti dimensionali tra calice e brattee rendono in certi casi debole la distinzione tra le due sottospecie, le cui popolazioni appaiono peraltro strettamente parapatriche, senza un'evidente disgiunzione; i dati genetici ne confermano tuttavia la separazione (COPPI \& al. 2008).

CONSERVAZIONE A dispetto dell'errata indicazione geografica nel protologo che, dovuta certamente ad un errore di etichettatura, l'indicherebbe come specie collinare (così data erroneamente anche da ARRIGONI 2013: 262), I'areale della specie è limitato a pochi tratti di costa fortemente disturbati dalle attività connesse agli insediamenti balneari, ed è potenzialmente minacciata dalla progressiva espansione dell'aliena invasiva Carpobrotus acinaciformis (L.) L. Bolus (Aizoaceae). La specie è attualmente classificata come in pericolo (EN) dalla IUCN, ed entrambe le sottospecie lo sono nella lista rossa della flora italiana (RossI \& al. 2013); recentemente (BACCHETTA \& al. 2008) era stata avanzata la proposta di trasferirle tra le entità a pericolo critico (CR, subsp. crispa) e vulnerabili (VU, subsp. maritima).

ChIAVE delle 1. Foglie basali strettamente oblanceolate, larghe più di $5 \mathrm{~mm}$; brattea nel SOTTOSPECIE fiore superante il calice; calice diviso in lobi ovato-lanceolati per $1 / 2-1 / 3$ 1. subsp. crispa

- Foglie basali lineari, larghe 3-4 mm; brattea nel fiore più breve del calice; calice diviso in lobi ovato-arrotondati per circa $1 / 3$ 2. subsp. maritima

12.3.1. Anchusa crispa subsp. crispa BUGLOSSA CRESPA TIPICA

DESCRIZIONE Foglie basali strettamente oblanceolate, larghe $5-9 \mathrm{~mm}$. Fiori all'ascella di brattee più lunghe del calice. Calice diviso da $1 / 3$ a 1/2 in lobi ovato-lanceolati (Fig. $63 \mathrm{~A}-\mathrm{E}$ ).

DISTRIBUZIONE Assai rara, esclusiva della Corsica centro-meridionale, ove si trova sia su spiagge del versante tirrenico che di quello occidentale, e della Sardegna nordoccidentale, tra Porticciolo e Porto Torres, e ancora ad est presso Isola Rossa (Fig. 64).

\subsubsection{Anchusa crispa}

subsp. maritima (Vals.) Selvi \& Bigazzi BUGLOSSA MARITTIMA

Anchusa crispa subsp. maritima (Vals.) Selvi \& Bigazzi in Pl. Biosystems 132(2): 136. 1998.

(三) A. maritima Vals., Boll. Soc. Sarda Sci. Nat. 26: 311. 1988. 
Locus classicus: [Italia, Sardegna] "Dune della spiaggia di Badesi".

Olotipo (cfr. VALSECCHI 1988: 311): [Italia, Sardegna] "Dune della spiaggia di Badesi", 20/04/1966, Valsecchi (SS).

Paratipi (VALSECCHI 1988: 314): [Italia, Sardegna] "San Pietro a mare presso Codaruina", 05/06/1964, Valsecchi (SS); "Spiaggia Paradiso (Codaruina)", 20/04/1966, Valsecchi (SS); ibidem, 04/06/1967, Valsecchi (SS); ibidem, 29/05/1970, Valsecchi (SS); "Li Junchi", 12/05/1965, Valsecchi (SS); "Badesi", 16/05/1969 (SS, FI 7137!); "Codaruina, in loc. Li Junchi presso la foce del fiume Coghinas", 16/05/1969, Valsecchi (SS, FI 2357!); "Spiagga Badesi" 06/05/1969, Valsecchi (SS); "Torre di Vignola", 29/05/1970, Valsecchi (SS); "Spiaggia dell'Isola Rossa", Maiore, 04/05/1970 (SS); "La Ciaccia, 02/05/1970, Valsecchi (SS); "La Marinedda", 1974, Dolcher (SS).

Etimologia Dal latino maritimus, "marittimo", in riferimento all'ecologia di psammofita litoranea di questa pianta.

DESCRIZIONE Foglie basali lineari, larghe 3-4 mm. Fiori all'ascella di brattee più brevi del calice. Calice diviso fino a $1 / 3$ in lobi ovato-arrotondati (Fig. 63F).

DISTRIBUZIONE Rarissimo endemismo sardo, localmente abbastanza abbondante ma circoscritto a poche spiagge del litorale settentrionale, tra Castelsardo e Torre Vignola (Fig. 63F).

\section{NOTE}

SISTEMATICA Inquadrata dapprima invalidamente come varietà di $A$. undulata (VALSECCHI 1976), poi descritta a rango specifico, quest'entità è invece chiaramente conspecifica della tipica $A$. crispa, dalla quale si distingue per pochi caratteri diagnostici; le piante della popolazione più orientale, a Torre Vignola, mostrano tra l'altro caratteri intermedi tra le due, rendendone ancor più tenue la distinzione.

\subsection{Anchusa formosa Selvi, Bigazzi \& Bacchetta BUGLOSSA FORMOSA}

Anchusa formosa Selvi, Bigazzi \& Bacchetta in PI. Biosyst. 131(2): 104. 1997.

Locus classicus: [Italia, Sardegna] "Sulcis Nord-Orientale (Cagliari), versante NE del Monte Lattias, fra le rocce granitiche nell' alveo secco del Rio Su Fundu, su suolo siliceo sabbioso-detritico, c. 850 m".

Olotipo (cfr. SElVI \& al. 1997: 104): [Italia, Sardegna] "Sulcis Nord-Orientale (Cagliari), versante NE del Monte Lattias, fra le rocce granitiche nell' alveo secco del Rio Su Fundu, su suolo siliceo sabbioso-detritico, c. 850 m", 18/05/1997, Selvi \& Bigazzi (FI 2369; iso- CAG, FI 2370, K, SS).

Paratipi (SELVI \& al. 1997: 105): [Italia, Sardegna] "Mt Lattias nell' alveo del Rio Su Fundu, detrito granitico, c. 780 m”, 18/05/1997, Selvi \& Bigazzi (FI); “Mt Lattias (Agro di Uta, CA), nel Canale del Longufresu su suolo siliceo scheletrico, esp. E-SE, c. 720 m”, 13/07/1997, Bacchetta \& Mossa (CAG); "Mt Lattias, alla base della cresta nel canale di Sennamanna, detrito granitico, c. 830 m", 13/07/1997, Bacchetta \& Mossa (CAG).

Etimologia Dal latino formosus, "formoso", "florido", in relazione all'aspetto particolarmente vigoroso e ai colori vivaci delle foglie e dei fiori. 
DESCRIZIONE Erba bienne o perenne a vita breve, con fusti prostrato-ascendenti lunghi fino a $40 \mathrm{~cm}$, ramificati dalla base; indumento dimorfo, ispido per rigide setole ialine lunghe fino a $3,5 \mathrm{~mm}$, miste a densi peli brevi. Foglie verde brillante, intere o solo oscuramente crespo-ondulate, con setole piuttosto rade, le basali oblanceolato-spatolate, $1,5-3 \times 8-18 \mathrm{~cm}$. Infiorescenze ramificate in cime piuttosto brevi anche alla fruttificazione, e perlopiù distali, con brattee minori o subeguali al calice, e peduncoli brevi, da subnulli a $<5 \mathrm{~mm}$. Calice fiorifero di 5-6 $\mathrm{mm}$, tubuloso, diviso per meno della metà in denti strettamente triangolari; il fruttifero ventricoso-urceolato, costato per i nervi prominenti, lungo fino a 1 $\mathrm{cm}$. Corolla ipocrateriforme con tubo bianco o roseo-lillacino di 5-6 mm, talora un po' sporgente dal calice, e lembo rotato di 7-9 $\mathrm{mm}$ di diametro, blu-violetto con toni porporini; pliche faucali bianco-lanose, molto prominenti e occludenti la fauce. Antere di 1,7 mm, parzialmente sovrapposte alle pliche faucali. Stilo di ca. $5 \mathrm{~mm}$, incluso. Mericarpi di ca. $2 \mathrm{~mm}$, obliquo-ovoidi, con becco laterale e superficie tuberculato-rugosa, nerastra, percorsa da coste reticolate e con cercine basale incospicuo (Fig. 65).

FENOLOGIA Fiorisce tra aprile e giugno, raramente fino a luglio, iniziando a disperdere i mericarpi da maggio, fino ad estate inoltrata.

Numero $2 n=16$, su piante sarde del locus classicus (monte Lattias; Selvi \& al. 1997). CROMOSOMICO

ECOLOGIA Brecciai e detriti granitici in canaloni rupestri, su versanti umidi e perlopiù ombrosi esposti ad est, tra 650 e $850 \mathrm{~m}$.

DISTRIBUZIONE Rarissimo endemismo sardo del Sulcis orientale, circoscritto a due modeste popolazioni sul versante orientale dei monti Lattias e Arcosu (Fig. 66).

NOTE

CONSERVAZIONE Specie soggetta a notevoli fluttuazioni demografiche in relazione agli eventi meterorici annuali ed al conseguente regime idrico dei ripidi canaloni in cui cresce. Nonostante la su estrema rarità, per la quale ne è stato proposto l'inserimento nella categoria delle vulnerabili (VU) della IUCN (BACCHETTA \& al. 2008), non appare tuttavia minacciata direttamente da fattori antropici, essendo tali stazioni assai repote e interamente comprese in aree naturali protette (Parco Regionale del Sulcis, Oasi WWF e SIC di Monte Arcosu).

\subsection{Anchusa litorea Moris BUGLOSSA LITORANEA}

Anchusa litorea Moris in Atti Congr. Sci. Ital. Genova, 8: 566. 1846.

(E) A. crispa var. litorea (Moris) Illario, Arch. Bot. 11: 261. 1935.

Locus classicus: [Italia, Sardegna] "in arena mobilis maris Oristano" (MoRIS 1827: 32, 1829: 9-10).

Lectotipo (VALSECCHI 1988: 323): [Italia, Sardegna] "in arenosis maritimis circa Oristano", 1826, Moris, Herb. Moris ("Anchusa crispa Moris"; TO).

Altro materiale originale (CECCHI \& SELVI 2015b: 669-670): [Italia, Sardegna] "in arenosis maritimis circa Oristano", 1826, Moris, Herb. Moris (“Anchusa crispa Moris"; TO). Paralectotype (here designated): [Italy, Sardinia] "in arena mobilis 
insula St. Pietro", s.d., s.coll., Herb. Moris ("Anchusa crispa / Lycopsis crispa Bertol." / Anchusa verrucosa"; TO); "circa * Oristano", s.d., s.coll., Herb. Moris ("Anchusa maritima Nob."; TO); "in arenis mobilis maritim. inter Terralba et Oristano, 04/1827, s.coll., Herb. Moris (TO); in arenosis maritimis circa Oristano, s.d., Moris, Herb. Moris ("Anchusa crispa Nob."; TO); s.loc., s.d., s.coll., Herb. Moris ("Anchusa crispa non Viv. ! nec DC!"; TO); s.loc., s.d., Moris ("Anchusa crispa Viv."; FI 7139!).

EtimologiA Dal latino litoreus, "litoraneo", per la distribuzione strettamente costiera della specie.

DESCRIZIONE Erba annua di 3-10(20) cm, ascendente o eretta, semplice o poco ramificata alla base, ispida per rade setole patenti, tubercolate, lunghe fino a $2 \mathrm{~mm}$, miste a peli brevi più numerosi. Foglie lineari, $0,4-0,6(0,8) \times 2-6(8) \mathrm{cm}$, acute, con margini eroso-increspati e oscuramente dentati, le basali spesso essiccate all'antesi, le cauline o bratteali poco ridotte rispetto a queste. Infiorescenza povera, lassa, con fiori distanziati, emergenti all'ascella delle foglie cauline o bratteali, spesso fin quasi dal livello del suolo, su peduncoli fino a $3 \mathrm{~mm}$. Calice diviso fino a $2 / 3$ in lacinie lineari, il fiorifero tubuloso, lungo ca. $3,5 \mathrm{~mm}$, il fruttifero maggiore, campanulato. Corolla ipocrateriforme, da bianca ad azzurro pallido, con tubo di 3-4 mm e lembo subrotato di 3,5-4 $\mathrm{mm}$ di diametro. Antere di $1,2 \mathrm{~mm}$, non raggiungenti le squame. Mericarpi di $2 \mathrm{~mm}$ scarsi, con becco laterale e superficie grigiastra densamente tuberculato-papillosa, percorsa da coste reticolate e con cercine basale sottile (Fig. 67).

FENOLOGIA Fiorisce tra marzo e la prima metà di giugno, cominciando a rilasciare le nucule già all'inizio di aprile e completandone la maturazione con la chiusura del ciclo vitale, entro l'inizio dell'estate.

Numero 2n=16, su piante sarde del locus classicus (S'Ena Arrubia; VALSECCH 1976). CROMOSOMICO

ECologIA Psammofita, limitata alle sabbie marittime e ai sistemi dunali e retrodunali litoranei, sia in luoghi esposti che in microstazioni più riparate nelle formazioni arbutive a Juniperus, tra 0 e $20 \mathrm{~m}$.

DISTRIBUZIONE Specie rarissima, endemica dei litorali sabbiosi della Sardegna sud-occidentale, oggi presente con certezza solo nel complesso dunale di Piscinas presso Is Arenas di Arbus (Fig. 68).

\section{NOTE}

NomenclatURA II binomio fu pubblicato negli atti dell'ottava riunione degli scienziati italiani di Genova, a rettifica dell'errata attribuzione dei campioni fatta da Moris nel primo e nel terzo fascicolo delllo Stirpium sardoarum elenchus (Moris 1927, 1929), rispettivamente come A. arvensis e poi come $A$. crispa. Solo nella prima citazione si cita il luogo della raccolta, motivo per cui il locus classicus risulta pubblicato 19 anni prima del protologo.

SISTEMATICA Specie ben distinta nell'ambito del complesso degli endemismi sardo-corsi del genere Anchusa, tra i quali si distingue facilmente soprattutto per il ciclo vitale annuale, che solo raramente si è riscontrato in coltivazione anche in $A$. crispa, e l'infiorescenza rada, coi fiori apparentemente isolati all'ascella di foglie cauline indistinte. Per questi caratteri fu inclusa in una serie distinta (Litorea) nelle due edizioni della Flora Analitica d'Italia (FIORI \& BÉGUINOT 1902, FIORI 1926), e per la profonda incisione del calice, che le accomuna, fu raggruppata con A. capelli nella sezione Litorea da GuşULEAC $(1927,1929)$.

CONSERVAZIONE Una delle specie più rare e a rischio della nostra flora, classificata come in pericolo critico 
(CR) sia dalla IUCN che nella lista rossa nazionale (RossI \& al. 2013), la cui vulnerabilità appare ulteriormente aumentata dalla ridottissima variabilità genetica, conseguente all'elevato tasso di autofecondazione. Apparentemente scomparsa in gran parte dell'areale storicamente documentato, che spaziava dalla costa sassarese occidentale a nord e le isole di S. Pietro e S. Antioco a sud, non fu più osservata dal 1980 fino al 2005, anno in cui il suo ritrovamento presso Marina di Arbus fugò serie ipotesi sulla sua definitiva estinzione. Benchè la dispersione delle nucule col vento e il breve ciclo vitale la rendano certamente soggetta a notevoli fluttuazioni demografiche, con rapida scomparsa e ricomparsa di popolamenti effimeri anche a discrete distanze, la sua presenza è attualmente accertata solo sui complessi dunali di Is Arenas ad Arbus. Legata ad ambienti solitamente soggetti ad intenso disturbo per le attività balneari, $A$. litorea deve probabilmente la propria sopravvivenza anche all'interdizione all'accesso che su gran parte di quel litorale vige per la presenza di un'estesa Colonia Penale.

\subsection{Anchusa montelinasana Angius, Pontec. \& Selvi BUGLOSSA DEL MONTE LINAS}

Anchusa montelinasana Angius, Pontec. \& Selvi in Syst. Biodivers. 6: 166. 2008. Locus classicus: [Italia, Sardegna] "Gonnosfanadiga (Cagliari), Monte Linas sotto punta Sa Cabixettas, $1070 \mathrm{~m}, 39^{\circ} 26.559 \mathrm{~N}-8^{\circ} 37.598 \mathrm{E}$ ".

Olotipo (cfr. Angius \& al. in BACCHETTA \& al. 2008: 166): [Italia, Sardegna] "Gonnosfanadiga (Cagliari), Monte Linas sotto punta Sa Cabixettas, $1070 \mathrm{~m}$, $39^{\circ} 26.559 \mathrm{~N}-8^{\circ} 37.598 \mathrm{E}$ ", 20/05/2005, Angius \& al. (CAG; iso- FI 2603, BM).

EtıMologiA Dall'aggettivo latinizzato montelinasanus, "del monte Linas", luogo di origine del tipo e unica stazione ad oggi conosciuta.

DESCRIZIONE Erba bienne o perenne a vita breve, con fusti prostrato-ascendenti lunghi fino a $40 \mathrm{~cm}$, ramificati dalla base; indumento dimorfo, ispido per rigide setole ialine a base tubercolata lunghe fino a $2 \mathrm{~mm}$, miste a densi peli brevi. Foglie verde brillante, con margine da intero e piano a leggermente dentato-ondulato, con setole piuttosto rade, le basali oblanceolato-spatolate, 1-2 $\times 5-10 \mathrm{~cm}$. Infiorescenze ramificate con cime molto allungate alla fruttificazione e brattee subeguali al calice; peduncoli fioriferi di $1-2 \mathrm{~mm}$, i fruttiferi fino a $8 \mathrm{~mm}$, riflessi. Calice fiorifero di 5-6 mm, tubuloso, diviso per ca. $1 / 3$ in denti triangolari-ovati, il fruttifero ventricoso-urceolato, costato per i nervi prominenti, lungo fino a 1 $\mathrm{cm}$. Corolla ipocrateriforme con tubo bianco o roseo-lillacino di $5 \mathrm{~mm}$ e lembo subrotato di 7-9 $\mathrm{mm}$ di diametro, blu-violetto, spesso con toni porporini e bande radiali più chiare; pliche faucali bianco-lanose, molto prominenti e occludenti la fauce. Antere di $1,8 \mathrm{~mm}$, parzialmente sovrapposte alle pliche faucali. Stilo di ca. $5 \mathrm{~mm}$, incluso. Mericarpi di ca. $2 \mathrm{~mm}$, obliquo-ovoidi, con becco laterale e superficie tuberculato-rugosa, nerastra, percorsa da coste reticolate e con cercine basale incospicuo (Fig. 69).

FENOLOGIA Fiorisce nella tarda primavera, maturando i frutti nell'arco di un mese, tra la fine di maggio e i primi di agosto.

Numero $2 n=16$ su piante sarde del locus classicus (monte Linas; BACCHETtA $\&$ al. 2008). CROMOSOMICO

ECOLOGIA Brecciai e detriti granitici in canaloni rupestri, in stazioni spesso riparate da alte erbe o arbusti ma su versanti relativamente esposti, orientati ad est, tra 1000 e $1200 \mathrm{~m}$. 
DISTRIBUZIONE Endemismo puntiforme, noto solo da tre canaloni franosi in una stretta fascia altitudinale prossima alla vetat del monte Linas, nella Sardegna sud-occidentale (Fig. 70).

NOTE

SISTEMATICA Anchusa montelinasana appartiene chiaramente al complesso di entità schizoendemiche isolate sui principali massicci silicei paleozoici della Sardegna meridionale, geneticamente distinto e probabilmente precursore di quello delle psammofite costiere del sistema Sardo-Corso. I caratteri che la distinguono rispetto alle altre due specie appaiono più o meno intermedi. Con $A$. formosa, geneticamente e geograficamente più prossima, condivide il calice poco inciso e costato alla fruttificazione, le tinte porporine della corolla e i mericarpi con cercine incospicuo; ad $A$. capellii, di cui appare complessivamente più gracile, si avvicina invece per l'indumento delle foglie più denso, le cime molto distese e i peduncoli fruttiferi molto allungati e deflessi.

CONSERVAZIONE Non ancora inserita nella lista rossa nazionale (Rossi \& al. 2013), ne è stato recentemente proposto l'inserimento tra le specie in pericolo (EN) della IUCN (BACCHETTA \& al. 2008).

\subsection{Anchusa ochroleuca M.Bieb. $O$ BUGLOSSA OCROLEUCA}

Anchusa ochroleuca M.Bieb., Fl. Taur.-Cauc. 1: 125, 421. 1808.

Locus classicus: [Russia] "in campis apricis herbidis caspio-caucasicis, wolgicis circa Zarizyn et ad Tyram prope Bender".

Lectotipo (CECCHI \& Selvi 2015b: 670): [Russia] "[ad Sarepta] ad Wolgam et Tanain", s.d., Bieberstein (B-W 03319-02!).

Altro materiale originale (CECCHI \& SELVI 2015b: 671): [Russia] "[ad Sarepta]", s.d., Bieberstein (B-W 03319-01!).

(=) A. ochroleuca var. rosea Marches., Fl. Trieste: 382. $1897 \equiv$ A. ochroleuca f. rosea (Marches.) Fiori in Fiori \& Bég., Fl. Italia 2(3): 375. 1902.

Locus classicus: [Italia, Friuli Venezia Giulia] "presso alla stazione di Draga". Tipo non designato.

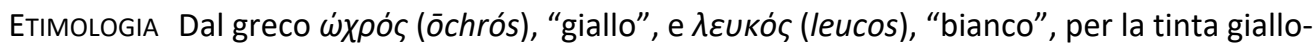
pallida della corolla.

DESCRIZIONE Robusta erba perenne con fusti primari eretti, alti fino a $7 \mathrm{dm}$, e molte ramificazioni secondarie; indumento perlopiù monomorfico di peli semplici, riflessi sul fusto, crespi e retrorsi sulla pagina inferiore delle foglie, che diventa dimorfico su quella superiore per la presenza di più rade setole, di circa $1 \mathrm{~mm}$, con base tubercolata. Foglie strettamente lanceolate, piane, a margine integerrimo o oscuramente ondulato, le basali indistintamente picciuolate, lunghe fino a $18 \mathrm{~cm}$, le cauline minori $(8-12 \mathrm{~cm})$, sessili, a base da cuneata a tronca. Infiorescenza ricca e ramificata, con 4-16 cime 5-30-flore appaiate, non allungate alla fruttificazione; peduncoli di $0-2 \mathrm{~mm}(3 \mathrm{~mm}$ quello alla biforcazione delle cime), non allungati dopo l'antesi; brattee triangolarilanceolate di 5(15) mm, generalmente assai minori del calice. Calice di $3 \times 5-8$ $\mathrm{mm}$, diviso da un terzo alla metà in lacinie lineari con apice da arrotondato a debolmente retuso e margine scarioso e cigliolato; tubo accrescente alla 
fruttificazione fino a 5/6 del totale. Corolla ipocrateriforme, $10-17 \mathrm{~mm}$, giallo pallido, con tubo bianco eguagliante il calice e lembo largamente campanulato con lobi ottusi lunghi da un quarto a un terzo del totale; pliche faucali $2 \mathrm{~mm}$, densamente pubescenti. Antere $2 \mathrm{~mm}$, raggiungenti appena la base delle pliche. Mericarpi di 2,5 × 4,5 mm, obliquo-ovidi, con becco laterale e superficie rugosotuberculata, grigiastra, percorsa da coste reticolate e con cercine basale ispessito (Fig. 71).

FENOLOGIA I pochi saggi disponibili testimoniano di esemplari in fiore e in frutto nelle due stazioni italiane sia in giugno che in settembre, suggerendo per il nostro territorio un ciclo biologico del tutto analogo a quello di $A$. officinalis, con fioritura primaverile ed estiva e maturazione dei frutti protratta fino all'autunno.

Numero $2 n=16$, verificato su piante bulgare (Markova in Löve 1983, Markova \& CROMOSOMICO Goranova in KAMARI \& al. 1995).

ECOLOGIA Specie di ambienti planiziali continentali, da noi trovata solo su ruderi, massicciate ferroviarie e alvei torrentizi, tra il livello del mare e circa $400 \mathrm{~m}$ di quota.

DISTRIBUZIONE Specie tipica delle vaste aree planiziali del sud della provincia europea orientale, si estende a est in quella aralo-caspica e a ovest in quella centro-europea, in Ungheria e Romania, e in quella balcanica, in Bulgaria; coltivata per ornamento, è stata segnalata come avventizia in buona parte dell'Europa centrooccidentale, verso nord-ovest fino alle isole britanniche. In Italia fu osservata per oltre trent'anni, tra la fine del XIX secolo e i primi decenni del XX, nei pressi della stazione ferroviaria (oggi abbandonata) di Draga - Sant'Elia sul Carso triestino, a poche decine di metri dall'attuale confine italo-sloveno, e nella bassa valle del Frigido in Versilia, ma nessuno dei due popolamenti sembra sopravvissuto oltre quel periodo (Fig. 72).

\section{NOTE}

SISTEMATICA Assai simile ad A. officinalis, se ne distingue per le foglie più strette, sempre intere, con indumento dimorfico sulla pagina superiore, per il calice mediamente più breve e il lembo della corolla giallo-pallido; da non confondere con A. officinalis var. ochroleuca Boiss., variante a fiori gialli della prima, descritta dalla Macedonia.

IBRIDI POTENZIALI A. × baumgartenii (Nyman) Gușul. è l'ibrido con A. officinalis, che ha caratteri intermedi nell'indumento, nel profilo dei lobi calicini e nel colore della corolla, e talora è fertile (STACE 1995: 649).

\subsection{Anchusa officinalis $\mathrm{L}$.} BUGLOSSA COMUNE

Anchusa officinalis L., Sp. PI. 1: 133. 1753.

Locus classicus: "ad Europae ruderata, vias, agros".

Lectotipo (SELVI \& al. 1996: 306): [pianta coltivata nei Paesi Bassi] Herb. Clifford: 46.1A (BM 557910). 
(=) A. angustifolia L., Sp. Pl. 1: 133. $1753 \equiv$ A. officinalis subsp. angustifolia (L.) Nyman, Consp. Fl. Eur.: 510. $1881 \equiv$ A. officinalis f. angustifolia (L.) Fiori in Fiori \& Bég., Fl. Italia 2(3): 375. 1902.

Locus classicus: "in Italia, Germania".

Lectotipo (Selvi in CAFFERTY \& JARVIS 2004: 800): s.loc, s.d., s.coll. (LINN-HL 182.2).

(=?) A. biceps Vest in Flora 4(10): 148. 1821.

Locus classicus: [Italia, Veneto] "in pago Suove [Soave] non procul a Mantua". Tipo non designato.

(=) A. arvalis Rchb., Iconogr. Bot. Pl. Crit. 3: 83, t. 297 (470). $1825 \equiv$ A. officinalis var. arvalis (Rchb.) Nyman, Consp. Fl. Eur.: 510. 1881.

Locus classicus: [Germania] "in arvis sterilibus viisque [...] sicut e Flora Dresdensis".

Lectotipo (CECCHI \& Selvi 2015b: 672): [Germania] “Anchusa arvalis Rchb. Tab. 297", s.d., s.coll., Herb. Reichenbach (W 1889-0286076).

Etimologia Dal latino officinalis, "officinale", in riferimento all'impiego delle foglie e dei fiori di questa specie in erboristeria.

DESCRIZIONE Erba generalmente perenne, di rado bienne, con fusti eretti, 2-8 dm, più o meno ramosi, e indumento monomorfico di setole patenti, dense ma relativamente morbide, lunghe fino a $1,5 \mathrm{~mm}$. Foglie di solito con margine intero e piano, talora irregolarmente ondulate o crespe, le basali oblanceolate, 1,5-3,5 × 5$13(30) \mathrm{cm}$. Infiorescenza in genere ricca e ramificata, con numerose cime scorpioidi molto allungate alla fruttificazione; peduncoli brevissimi o subnulli all'antesi, 1-3 mm con la maturazione del frutto; brattee subeguali al calice. Calice di 5-7 mm, diviso per metà o meno in denti strettamente triangolari, alla fruttificazione sacciforme ed ingrossato fino a $12 \mathrm{~mm}$. Corolla ipocrateriforme, con tubo bianco o roseo di $8 \mathrm{~mm}$ e lembo largamente campanulato, violettopurpureo, più raramente rosa o bianco, di (6)8-10(12) $\mathrm{mm}$ di diametro e con lobi ottusi; pliche faucali bianco-lanose, prominenti. Antere $2,2 \mathrm{~mm}$, raggiungenti appena la base delle pliche. Mericarpi di 2,5 $\times 4,5 \mathrm{~mm}$, obliquoovidi, con becco laterale e superficie rugoso-tuberculata, grigiastra, percorsa da coste reticolate e con cercine basale ispessito.

FENOLOGIA A seconda della stazione la fioritura può aver luogo già all'inizio della primavera, ovvero prolungarsi fino alla fine di ottobre, e il rilascio delle nucule, la cui maturazione richiede circa un mese, iniziare ad aprile o terminare in autunno inoltrato.

NUMERO Diploide con $2 n=16$, verificato su materiale del Trentino Alto Adige (D'AMATO \& CROMOSOMICO TROJANI 1985); stesso valore è stato ottenuto da diverse altre popolazioni europee della specie, provenienti da Austria (Kiehn \& al. in DOBEŠ \& VITEK 2000), Bulgaria (Loon \& Setten in LöVE 1982b, Markova \& Goranova in KAMARI \& al. 1995; si veda anche LOON 1987), Grecia (Strid in LÖVE 1980c), Polonia (SKALINSKA 1974), Serbia (Loon \& Kieft in LÖVE 1980b), Slovacchia (Hindakova in MÁJOVSKÝ 1976) e Svezia (LÖVKVIST \& HULTGÅRD 1999).

ECOLOGIA Margini dei campi e incolti, bordi stradali, prati e ambienti ruderali, dal livello del mare fino al $2000 \mathrm{~m}$.

DISTRIBUZIONE Specie ad ampia distribuzione in Europa e in minor misura nell'Asia occidentale, con almeno tre varianti geografiche riconosciute a rango sottospecifico o varietale (SElvi \& BIgAZZI 2003). Diffusa e comune nelle provincie europee 
atlantica, centrale e orientale e in quella eusina (pontica), $A$. officinalis si spinge ulteriormente a nord nella provincia nord-europea, occupando gran parte della Fennoscandia centro-meridionale, più raramente e meno diffusamente a sud, in ridotte aree limitrofe della regione mediterranea; è coltivata e spesso avventizia altrove, comprese ampie parti del Nordamerica. In Italia si trova più o meno sporadicamente, con la sola sottospecie tipica, in tutte le regioni settentrionali, soprattutto in pianura padana e sui bassi rilievi di tutto l'arco alpino, benchè resti da confermare la sua presenza in Liguria (SELVI \& BIGAZZI 1998); nel centrosud è sostituita dalla vicariante $A$. undulata (subsp. hybrida).

\section{NOTE}

ALTRI NOMI VOLGARI Coda di volpe, sugamiele.

SISTEMATICA Specie assai variabile nella forma e nelle dimensioni di foglie, brattee e parti fiorali (in particolare dei denti calicini). Anchusa angustifolia e A. arvalis corrispondono a morfotipi con foglie lineari o sublineari, spesso con fiori ridotti e indumento lievemente più sviluppato della norma; individui con questo aspetto sono stati segnalati sporadicamente nell'Italia nord-orientale e talora erroneamente determinati anche come A. leptophylla, che invece è specie ben distinta, a diffusione mediterraneo-orientale e anatolica. Le numerose segnalazioni di $A$. officinalis per la penisola, in area mediterranea, sono invece da riferire a $A$. undulata subsp. hybrida.

Alcuni autori (GuşuLEAC 1927, 1929, Chater in TUTIN \& al. 1972) hanno ritenuto che Anchusa biceps Vest, descritta dal Mantovano (VEST 1821: 148), non fosse altro che una forma con più marcata lobatura dello stigma di $A$. azurea, alla quale sembrerebbe riferirsi il protologo parlando di peli delle pliche faucali proiettati verso il tubo corollino ("squamae faucis in tubum demittunt pilos") e di 2-3 nucule abortive per fiore ("semina... plerumque 2-3 abortiva"). Tuttavia, in assenza di materiale valido per la tipificazione sia nell'erbario Vest a GJO che in altre collezioni, resta altrettanto sensata l'ipotesi avanzata da Caruel in Parlatore \& Caruel (1886), che potesse trattarsi di $A$. officinalis, specie più diffusa dell'altra nel Mantovano e forse più compatibile con l'indumento morbido e il lembo non completamente rotato della pianta descritta da Vest ("Folia... molliter hirsuta... Corolla infundiboliformis"). A meno di una neotipificazione, la questione resta ad oggi irrisolta per la irreperibilità del materiale originario.

Proprietàe usı Le foglie e le cime di $A$. officinalis trovano gli stessi impieghi di quelle di Borago officinalis, in erboristeria per la preparazione di decotti con proprietà emollienti, diuretiche e antinfiammatorie, in cucina come semplice verdure cotte o come ingrediente di stufati o ripieni; anche in questo caso se ne raccomanda un uso parsimonioso per evitare di assumere un eccesso di alcaloidi epatotossici.

\subsubsection{Anchusa officinalis subsp. officinalis BUGLOSSA COMUNE TIPICA}

DESCRIZIONE Foglie basali e cauline inferiori largamente lanceolate, subottuse, di solito subamplessicauli e senza getti sterili ascellari. Cime molto allungate e ramificate. Calice diviso per non più di metà della lunghezza. Lembo corollino da 8 a $12 \mathrm{~mm}$ di diametro (Fig. 73).

DISTRIBUZIONE La sottospecie più comune e diffusa in quasi tutto l'areale specifico, sostituita con certezza solo in alcune località greche dalla subsp. intacta (Griseb.) Selvi \& Bigazzi. Tutte le popolazioni italiane corrispondono decisamente al tipo linneano (Fig. 74). 


\subsection{Anchusa sardoa (Illario) Selvi \& Bigazzi BUGLOSSA DI PORTO CONTE}

Anchusa sardoa (Illario) Selvi \& Bigazzi in PI. Biosystems 132(2): 136. 1998.

(E) A. crispa var. sardoa Illario, Arch. Bot. Forlì 11: 261. 1935.

Locus classicus: [Italia, Sardegna] "In arenis maritimis Porto Conte".

Lectotipo (SELVI \& BIGAZZI 1998: 136): [Italia, Sardegna] "Porto Conte", s.d., s.coll., Herb. Moris (TO).

Etimologia Dal latino sardous, "sardo", in riferimento alla distribuzione strettamente endemica di questa specie in Sardegna.

DESCRIZIONE Erba bienne o perenne a vita breve, con fusti eretto-ascendenti lunghi fino a 35 $\mathrm{cm}$, fortemente ispida per robuste setole patenti tubercolate alla base, lunghe fino a $3 \mathrm{~mm}$, miste a peli più brevi e densi. Foglie da lineari a strettamente lanceolate, ottuse, con margine fortemente crespo-ondulato, le basali 9-12 mm $\times 10-15 \mathrm{~cm}$, spesso marcescenti all'antesi. Infiorescenze ramificate in cime relativamente pauciflore (3-10), lasse, molto allungate dopo l'antesi, con brattee 1,5-3 volte più lunghe dei fiori e peduncoli brevi o subnulli, allungati dopo l'antesi fino a 2(5) $\mathrm{mm}$. Calice campanulato di 3-4 $\mathrm{mm}$, diviso per ca. la metà o poco più in denti strettamente lanceolati, ottusi, ispidi anche internamente, dopo l'antesi subgloboso-urceolato e allungato fino a ca. $1 \mathrm{~cm}$. Corolla azzurro-lillacino pallida, con tubo di 4-5 $\mathrm{mm}$ e lembo rotato di 5-7 $\mathrm{mm}$ in diametro, diviso in lobi a profilo quadrato. Antere lunghe circa 1,5-2 mm, lievemente sovrapposte alle pliche faucali. Stigma capitato-ovoide. Mericarpi $1,5 \times 2,5 \mathrm{~mm}$, obliquo-ovoidi con becco laterale, bruno-grigiastri, con superficie rugoso-reticolata e cercine basale incospicuo (Fig. 75).

FENOLOGIA Fiorisce tra aprile e giugno, iniziando la disseminazione a partire da maggio, fino ad estate inoltrata.

Numero $2 \mathrm{n}=16$, su piante del locus classicus e unica popolazione conosciuta (Porto CROMOSOMIco Conte; VAlSeCCHI 1976, SelVI \& BIGAZZI 1998).

ECologIa Sistemi dunali litoranei, su sabbie consolidate, talora negli erbosi all'interno di pinete artificiali in prossimità della costa, 0-10 m.

DISTRIBUZIONE Endemismo puntiforme, limitato alla baia di Porto Conte, nella Sardegna nordoccidentale (Fig. 76).

\section{NOTE}

Nomenclatura Per il portamento eretto la specie fu erroneamente citata da Moris come $A$. undulata nel terzo volume della Flora sardoa (MoRIS 1858-1859: 141), nè mai pubblicata da quell'autore con altri nomi, benchè egli notasse alcune differenze della pianta sarda sia rispetto al tipo linneano di $A$. undulata che all'iconografia della Flora Graeca di Sibthorp (raffigurante la subsp. hybrida). La stessa interpretazione fu accettata da VALSECCHI (1976) e seguita ancora nelle sinossi floristiche più recenti (PIGNATTI 1982, GREUTER \& al. 1984), nonostante questa specie presenti tutti i caratteri propri del complesso degli endemismi sardo-corsi (si veda a tal proposito la nota sistematica nella scheda del genere), come già evidenziato da ILLARIO (1935).

ALTRI NOMI VOLGARI Buglossa sarda. 
CONSERVAZIONE Specie altamente minacciata per l'estrema localizzazione in un'area soggetta ad erosione costiera e soprattutto a disturbo antropico, sia per le attività connesse al turismo estivo (calpestio, parcheggio) che per la piantumazione di pinete artificiali. Sia a livello nazionale che internazionale, $A$. sardoa compare nella lista delle entità in pericolo critico (CR) della IUCN (RossI \& al. 2013).

\subsection{Anchusa undulata L. BUGLOSSA ONDULATA}

Anchusa undulata L., Sp. PI. 1: 133. 1753.

Locus classicus: "in Hispaniae, Lusitaniae pratis". Lectotipo (VALDÉS 1981b: 109): “in Hispaniae, Lusitaniae pratis", s.d., Löfling 144a, (LINN-HL 182.3).

Etimologia Dal latino undulatus, "ondulato", in riferimento al margine sinuoso delle foglie, soprattutto le basali.

DESCRIZIONE Erba generalmente bienne, di rado perenne, con fusti generalmente eretti (prostrati o ascendenti solo nella subsp. sartorii (Guşul.) Selvi \& Bigazzi, mediterranea orientale), 2-6 dm, più o meno ramosi, e indumento dimorfico di setole rigide patenti, tubercolate, lunghe fino a $2 \mathrm{~mm}$, miste a peli più brevi e densi. Foglie da lineari a largamente oblanceolato-spatolate, con margine più o meno sinuoso-ondulato, le basali 1-2,5 $\times 4-15 \mathrm{~cm}$, spesso marcescenti all'antesi. Infiorescenza ricca e ramificata, con numerose cime scorpioidi molto allungate alla fruttificazione; brattee ovato-lanceolate, cordate, assai più brevi del calice; peduncoli minori di $2 \mathrm{~mm}$ all'antesi, fino a $8 \mathrm{~mm}$ con la maturazione del frutto. Calice di 5-8 mm, diviso in genere per meno della metà (fino a $2 / 3$ nella subsp. sartorii $\mathrm{e}$ in alcune popolazioni libiche di incerta collocazione) in denti strettamente triangolari, il fruttifero sacciforme e assai ingrossato, lungo fino a $15 \mathrm{~mm}$. Corolla ipocrateriforme, con tubo bianco di 7-8 $\mathrm{mm}$ e lembo largamente campanulato, violetto-purpureo, variabile, anche in seno alla stessa popolazione, tra 5 e $15 \mathrm{~mm}$ di diametro, con lobi deltoidi, subacuti; pliche faucali bianco-lanose, prominenti e occludenti la fauce. Antere $2,3 \mathrm{~mm}$. Stilo variabile (ercogamia); stigma ellissoidale, spesso con breve punta apicale sterile. Mericarpi di $2 \times 4 \mathrm{~mm}$, obliquo-ovidi, con becco laterale e superficie rugosotuberculata, nerastra, percorsa da coste reticolate e con cercine basale ispessito (Fig. 77).

NUMERO $2 n=16$, verificato sia su popolazioni occidentali della sottospecie tipica (LUQUE CROMOSOMICo 1980, 1983, Devesa \& al. in LöVE 1984), che su piante riferibili alla sottospecie sartorii (SELVI \& BIGAZZI 2003) e alla subsp. hybrida (per la quale si rimanda alla scheda che segue).

DISTRIBUZIONE Presente in tutte le province della regione mediterranea, ov'è rappresentata da almeno tre sottospecie distinte per portamento e caratteri fiorali secondari. La tipica è diffusa nella parte occidentale dell'areale, tra le province iberica e mediterranea sud-occidentale; a partire dalla parte sud-orientale di quest'ultima, fino a gran parte di quella orientale, è sostituita dalla subsp. hybrida, unica presente in Italia, e localmente nell'area egea, dalla subsp. sartorii (Fig. 78). 
NOTE

SISTEMATICA Pianta assai variabile per aspetto e dimensioni relative sia degli organi vegetativi che delle parti fiorali, in relazione alle condizioni ambientali. Secondo SeLVI (1998) e SeLVI \& BIGAZZI (2003) se ne possono riconoscere tre sottospecie, cui forse potrebbe aggiungersene una quarta circoscritta alle popolazioni della Cirenaica, caratterizzate dal calice maggiormente inciso (SELVI 1998). Le due sottospecie a gravitazione mediterranea centro-orientale, collegate da alcune popolazioni con caratteri intermedi, sono accomunate dalla posizione abbassata delle antere; quella tipica, mediterraneooccidentale, al contrario, si distingue per gli stami inseriti alla stessa altezza delle pliche faucali, con le antere del tutto sovrapposte ad esse, nonchè per il polline con tre aperture anzichè quattro, come solitamente si riscontra nei rappresentanti del sottogenere tipico.

\subsubsection{Anchusa undulata}

subsp. hybrida (Ten.) Bég. BUGLOSSA ONDULATA ORIENTALE

Anchusa undulata subsp. hybrida (Ten.) Bég. in Nuovo G. Bot. Ital. 17(4): 634. 1910.

(E) A. hybrida Ten., Prodr. Fl. Nap.: XIV. $1811 \equiv$ A. undulata var. hybrida (Ten.) Fiori, Nuov. Fl. Ital. 2(2): 267. 1926

Locus classicus: [Italia] non specificato.

Lectotipo (SELVI 1998: 267): [Italia, Campania] "inter Capua et S. Agata”, 1811, s.coll., Herb. Tenore (NAP!).

Altro materiale originale (SELVI 1998: 266): [Italia, Campania] "in arvis circa Neapolim", s.d., Tenore 118 (FI 2371!); s.loc., s.d., Tenore (FI 2372!).

(=) A. crispata C.Presl, Fl. Sicul.: xxxiii. $1826 \equiv$ A. hybrida subsp. capellii var. crispata (C. Presl) Nyman, Consp. Fl. Europ.: 511. 1881 三 A. hybrida var. crispata (C. Presl) Nicotra, Syll. FI. Sic.: 39. 1893.

Locus classicus: [Italia, Sicilia] non specificato.

Tipo non designato.

(=) A. hybrida f. subintegrifolia Fiori in Fiori \& Bég., Fl. Italia 2(3): 376. 1902.

Locus classicus: [Italia] non specificato.

Lectotipo (SELVI \& BIGAZZI 1998: 134): [Italia] "in arvis", Tenore, Herb. Tenore (NAP!).

(=) A. undulata f. angustata Fiori in Fiori \& Bég., Fl. Italia 2(3): 376. 1902.

Locus classicus: [Italia] non specificato.

Lectotipo (CECCHI \& SELVI 2015b: 674): [Italia, Calabria] "S. Maria presso Catanzaro", 07/05/1883, Andrea Fiori, Herb. Fiori (FI 7153!).

EtIMologiA Da latino hybridus, "ibrido", in riferimento alla presenza di caratteri ritenuti intermedi tra la tipica $A$. undulata e $A$. officinalis, come fu precisato dallo stesso autore, a un anno dalla pubblicazione del Prodromo, nella Flora Napolitana (TENORE 1812: 46).

DESCRIZIONE Stami inseriti a circa $2 / 3$ della lunghezza del tubo corollino, con antere non o appena raggiungenti le pliche faucali. Stigma spesso con un'appendice sterile tra i due lobi (Fig. 77 A-C, E, F). 
FENOLOGIA II periodo di fioritura è molto ampio, di solito esteso dall'inizio della primavera a quello dell'estate, ma, a seconda della stazione, può iniziare già alla fine dell'inverno o terminare in pieno autunno; i frutti maturano in circa un mese, prevalentemente tra aprile e luglio.

Numero $2 n=16$ in piante provenienti dalla Toscana (SELVI \& BIGAZZI 1998), dal Lazio CROMOSOMICO (CAPINERI \& al. 1978, D'AMATO 1989) e dalla Sardegna (VALSECCHI 1976), in accordo coi conteggi effettuati su materiale bulgaro (Markova \& Goranova in KAMARI \& al. 1995) e turco (Bigazzi \& al. in KAMARI \& al. 2000).

ECOLOGIA Pascoli e ambienti rocciosi, garighe e pendii aridi, margini delle strade, ambienti ruderali, luoghi incolti e coltivi, su ogni genere di substrato, comune soprattutto da livello del mare alla fascia collinare, ma rinvenuta anche in quello montano inferiore fino a $1300 \mathrm{~m}$.

DISTRIBUZIONE Entità a gravitazione centro-orientale, presente a partire dal sud-est della Francia e dalla fascia litorale e sub-litorale algerina e assai comune da qui fino a tutta la provincia mediterranea orientale, tra la Turchia anatolica e l'Egitto, passando per le aree occidentali dei paesi del Vicino Oriente. In Italia è vicariante di $A$. officinalis in tutte le aree a clima mediterraneo (province ligurotirrenica e adriatica), soprattutto in penisola a partire dall'arco ligure e dalla Romagna meridionale verso sud, ma ancora piuttosto rara e sporadica fino alla Toscana centrale e sulle isole maggiori (Fig. 78).

NOTE

ALTRI NOMI VOLGARI Buglossa ibrida.

\section{SPECIE DA ESCLUDERE}

\section{Anchusa aegyptiaca (L.) DC. BUGLOSSA EGIZIANA}

Anchusa aegyptiaca (L.) A.DC. in DC., Prodr. 10: 48. 1846.

(三) Lycopsis aegyptiaca L., Sp. PI.: 138. 1753.

Locus classicus: [Egitto] "in Aegypto".

Neotipo (Mill in CAFFERTY \& JARVIS 2004: 802): [Giordania] "Petra", 15/03/1974, Boulos \& Al-Eisawi 6290 (E 284765; iso- IABH).

DESCRIZIONE Erba annua, eretto ascendente, 5-35 cm, ispido-strigosa per indumento dimorfo di robuste setole tubercolate miste a peli brevi. Foglie spesso tubercolatobollose, a margine eroso-ondulato, ottuse, le basali largamente oblanceolate, 10-15 × 25-40 mm. Infiorescenze bratteato-fogliose, i fiori non necessariamente inseriti all'ascella delle foglie bratteali, e assai minori di queste; peduncoli fiorali 2-3 $\mathrm{mm}$, nel frutto allungati fino a oltre $2 \mathrm{~cm}$ e più o meno patenti o ricurvi. Calice di circa $5 \mathrm{~mm}$, diviso fin quasi alla base in lobi lineari-lanceolati. Corolla ipocrateriforme con tubo bianco, sporgente dal calice, e lembo rotato, giallo pallido, di 4-6 mm di diametro, la fauce occlusa da pliche fortemente sporgenti e arcuate verso l'esterno. Stami inseriti circa alla metà del tubo, due 
legegrmente più in alto degli altri. Stilo incluso. Nucule 2-3 $\times 4-5 \mathrm{~mm}$, orientate quasi parallelamente all'asse fiorale, con becco pronunciato. superficie rugosocostata, grigiastra, e spesso cercine basale.

DISTRIBUZIONE Specie a gravitazione orientale, occupa buona parte della provincia mediterranea orientale, in particolare molte delle isole egee, e da questa si spinge ad est e a sud in zone più aride interne delle regioni irano-turaniana $\mathrm{e}$ saharo-arabica, appartenenti alle province mesopotamica ed egitto-arabica.

NOTE

DISTRIBUZIONE Segnalata dubitativamente per la Sicilia in alcune Flore recenti (ZANGHERI 1976, PIGNATTI 1982, GREUTER \& al. 1984), la sua presenza in Italia non è confermata da alcun reperto, nè appare probabile alla luce del suo quadro distributivo; qualora fosse mai realmente comparsa nel nostro territorio, la sua presenza andrebbe comunque considerata accidentale ed effimera.

\section{GENERE DA ESCLUDERE}

\section{Trachystemon D. Don o TRACHISTEMONE}

Trachystemon D. Don in Edinburgh N. Philos. J. 13: 239. 1832.

Tipo (cfr. DoN 1832: 239): Trachystemon orientalis (L.) G.Don.

DESCRIZIONE Robusta erba rizomatosa, ispida. Infiorescenza panicolata con cime scorpioidi bratteate e fiori lungamente peduncolati. Calice accrescente alla fruttificazione. Corolla divisa in lobi lineari fortemente ricurvi a elica; pliche faucali distribuite in due serie di 5 , le inferiori villose, le superiori papillose. Stami fortemente sporgenti, conniventi, con filamento pubescente alla base e assai più lungo delle antere. Nucule ovoidi, rugoso-reticolate, con margine basale fortemente ispessito. (modificata da J. R. Edmondson in DAVIS 1979: 386-387).

DISTRIBUZIONE Genere mototipico originario ed endemico della provincia eusina.

\section{Trachystemon orientalis (L.) D. Don o BORRAGINE ORIENTALE}

(三) Borago orientalis L., Sp. PI. 1: 138. 1753 三 Psilostemon orientalis (L.) DC. ex A. DC., Prodr. 10: 36. 1846.

Locus classicus: [Turchia] "circum Costantinopolin".

Lectotipo (Edmondson in CAFFERTY \& JARVIS 2004: 800): s.loc., s.d., s.coll., Herb. Linneo 188.5 (LINN!).

DESCRIZIONE Pianta di (20)30-40(60) cm, poco ramificata, con rizoma tuberoso, nerastro, e indumento di peli retrorsi. Foglie basali ampie, largamente ovato-cordate, lungamente picciolate e con lamina lunga fino a $20 \mathrm{~cm}$; foglie cauline nettamente minori, più strettamente ovate, sessili e amplessicauli. Brattee acute, da lanceolate a oblanceolate. Calice di 3-4 mm, fino a $7 \mathrm{~mm}$ alla 
fruttificazione, con lobi triangolari-ottusi. Corolla blu-violacea, con tubo di 4-6 $\mathrm{mm}$, lobi di 6-8 $\mathrm{mm}$ e pliche faucali superiori bianche e smarginate. Stilo sporgente dal cono staminale. Nucule brunastre, carenate. (modificata da J. R. Edmondson in DAVIS 1979: 387).

DISTRIBUZIONE Specie eusina, distribuita tra la Bulgaria orientale e il Caucaso occidentale per tutta la fascia settentrionale della penisola anatolica, sul versante nord delle Alpi Pontiche. In Italia, come altrove in Europa, è stato coltivato negli orti e giardini botanici e si è occasionalmente spontaneizzato negli stessi luoghi, senza apparentemente mai affrancarsi dallo stretto ambito sinantropico.

\section{NOTE}

DISTRIBUZIONE Specie segnalata per l'Italia soltanto come esotica casuale nelle aiuole dell'orto botanico di Firenze (Chiarugi in PASSERINI \& al. 1928), nelle quali fu osservata almeno fino al 1938 (spontanea nei prati del Giardino ex Botanico di Firenze, 13/04/1938, Corradi, FI!). Nel "Giardino dei Semplici" si trova ancora, effettivamente, un nucleo stabile di questa specie, ma si tratta della progenie di un singolo, nuovo individuo, proveniente della Turchia nord-orientale e messo a dimora nel 2002. La specie tende ad espandersi e ad occupare autonomamente nuovi spazi, ma la sua sola presenza in un giardino non è sufficiente a trattarla come entità davvero stabilizzata, essendo oltretutto scomparso il popolamento originario citato da Chiarugi. 


\section{FONTI VERIFICATE UTILIZZATE PER LA DISTRIBUZIONE ITALIANA DELLE SPECIE}

Anchusa azurea LIGURIA: torrente Sturla, presso Genova, 08/1856, Ardissone, FI. PIEMONTE: Torino, lungo il Sangone, 05/09/1919, Vatova, FI - Domodossola, s.d., Rossi, FI - envir. d'Alba, *, s.d., Bourgeau, FI. LOMBARDIA: Oltrepò, Canneto, 24/07/1858, Rampoldi, FI - in graminosis prope Belforte *, 05/1883, Groves, FI - Mantova, s.d., Barbieri, FI. TRENTINO ALTO ADIGE: Ala, s.d., Kerner, FI. VENETO: Bassano, 900 m, 07/1899, Parsi, FI - S. Maria in Stelle, loc. Bianchini (VR), 100 m, 22/06/1982, Bianchini \& Di Carlo, Bianchini 13557, 13558, VER - Pagnaghe presso Tregnago (VR), 300 m, 31/08/1995, Ziviani, Bianchini 21681, VER - Sassonegro, Euganei, 290 m, 31/05/2004, Lasen, Herb. Lasen - colli Berici, Mossano (VI), presso il cimitero, $70 \mathrm{~m}$, prato, 29/04/2007, Scortegagna, MNAV. FRIULI VENEZIA GIULIA: Muggia, 06/1882, Marchesetti, FI - cortile Istituto tecnico Zomon (UD), 06/06/1941, Fornaciari, MFU-Forojuliense 10785. EMILIA ROMAGNA: Castrocaro, pago ab urbe Forlì Emilia paucum dissitum, ad agrorum margines, ad vias etc., 20/06/1877, Sommier, FI - S. Vittore presso Bologna, 14/06/1891, Fiori, FI - campi a Casinalbo (MO), 28/05/1902, Fiori, FI - Piandelagotti, Radici, 01/07/1910, Lunardi, FI - Parma, Vigheffio, principio del greto (anche orlo di un campo), 01/05/1920, Minio, FI - Sassatella, siepe, 22/08/1924, Lunardi, FI - dintorni di Mondaino, campi in riposo e luoghi erbosi incolti nell'alta valle del Rio Salso tra Ca' Scaramuccia e S. Andrea, 125-275 m, suolo argilloso o argilloso-sabbioso, 01/05/1970, Brilli-Cattarini \& al., PESA. TOSCANA: Monteroni d'Arbia, nelle crete senesi, nei coltivati, 10/06/1894, Mari, FI - insula Gorgona, sopra la Colonia, 21/05/1899, Sommier, FI - insula Capraria, haud procul portu, in arvis, 24/05/1910, Sommier, FI - Firenze, Greve in Chianti, 28/04/1927, Corradi, FI - San Vincenzo, Donoratico, pineta ovest, strada maestra, 14/05/1933, Gabrielith \& al., FI - Comune di Pontassieve, S. Brigida, tra le Lucole e il santuario della Madonna del Sasso, 24/04/1954, Gaito, FI - alta val Tiberina, Montauto, 23/05/1955, Chiarugi \& al., FI - Montepulciano, colline argillose della val d'Orcia, 300-600 m, 15/05/1960, Antoni, FI - Poggio a Caiano (FI), bosco di Buonistallo, zona alta, lungo il muro di cinta fino alla zona dei coltivi, 16/05/1964, Maugini, FI - Appennino tosco-emiliano, Sasso di Castro, pascoli del versante O, 30/07/1965, Margheri, FI - Massa (MS), a lato del Frigido appena al livello dell'autostrada, 25/04/1974, Soldano, Herb. Soldano 471 - Arcipelago toscano, isola d'Elba, Bagnaia, nella valle, 16/04/1976, Fossi Innamorati, FI - Talamone, coltivi lungo la strada sopra il convento, 07/05/1982, Arrigoni \& al., FI - al monastero di S. Maria della Sambuca (LI), 20/06/1985, Ansaldo, PI - m.te Labbro (GR), margini di coltivi, ca. $800 \mathrm{~m}$, 03/06/1992, Baldini \& Luccioli, FI - Piombino (LI), valle di Riotorto, 02/07/1993, Cenerini, $\mathrm{PI}$ - m.te Argentario (GR), tra le Tre Fonti e P.ta Telegrafo, $400 \mathrm{~m}$ ca., margini di gariga, calcare, 07/1994, Baldini, FI - S. Martino ad Ulmiano (PI), fosso di scolo asciutto affluente del Fosso Martraverso, 22/05/2000, Pedullà, PI - Sesto Fiorentino (FI), lungo via Pratese in zona Osmannoro, 03/05/2005, Cecchi 371, Herb. Cecchi - Monteverdi Marittimo (PI), Poggio Badia, 04/06/2008, Maggini, PIAGR. MARCHE: m.te Conero, luoghi erbosi incolti (ex-coltivi) nel versante NO al Pian Grande, 400-425 m, suolo calcareo o più o meno decalcificato, 21/05/1966, Brilli-Cattarini, PESA - dintorni di Castelleone di Suasa, campi coltivati e luoghi erbosi incolti sui colli tra la Croce del Termine ed il Fosso della Volpara, 225-250 m, suolo argilloso, 02/05/1968, Brilli-Cattarini \& al., PESA - dintorni di Jesi, campi coltivati e luoghi erbosi incolti lungo il Fosso Maccarata presso il ponte di Mazzangrugno, ca. 55 m, suolo argilloso, 04/05/1968, Brilli-Cattarini \& al., PESA - dintorni di Massignano, campi in riposo sui colli lungo la strada tra Massignano e Montefiore all'Aso, 275-300 m, suolo sabbioso, argilloso-sabbioso o ghiaioso, 14/05/1971, BrilliCattarini \& Sialm, PESA - dintorni di Matelica, campi in riposo e luoghi erbosi incolti lungo la strada tra Matelica ed Esanatoglia presso Villa Vincenza, ca. $380 \mathrm{~m}$, suolo argilloso- 
calcareo, humus nullo, 19/05/1971, Brilli-Cattarini \& Sialm, PESA - gruppo del m.te Murano - Valmontagnana, campi coltivati e in riposo nel Pian delle Mosche a ONO del m.te Sassone, ca. $650 \mathrm{~m}$, suolo calcareo, humus nullo, 05/06/1971, Brilli-Cattarini \& Sialm, PESA - gruppo del Montigno, luogjhi erbosi asciutti nel basso versante $\mathrm{S}$ del $\mathrm{m}$.te Maggio sotto la Fonte della Rocca di Sotto, ca. $750 \mathrm{~m}$, suolo calcareo, humus scarso o nullo, 02/06/1972, Brilli-Cattarini \& al., PESA - dintorni di Lunano, luoghi erbosi incolti asciutti sulla dorsale tra Lupaiolo e Ca' Scheggiola, ca. $485 \mathrm{~m}$, suolo prevalentemente argilloso, humus nullo, 30/05/1973, Brilli-Cattarini \& al., PESA - dintorni di Rotella, luoghi erbosi incolti asciutti in loc. Piaggiole presso il ponte sul Fosso dell'Inferno, ca. $325 \mathrm{~m}$, suolo prevalentemente arenaceo, humus nullo, 11/06/1973, Brilli-Cattarini \& al., PESA dintorni di Senigallia, campi coltivati e luoghi erbosi incolti alla Bruciata nella valle del Cesano, 100-125 m, suolo argilloso, humus nullo, 08/05/1976, Brilli-Cattarini, PESA dintorni di Castelfidardo, luoghi erbosi incolti presso il margine occidentale della Selva di Castelfidardo, 50-75 m, suolo prevalentemente argilloso-sabbioso, humus nullo, 28/05/1976, Brilli-Cattarini, PESA - Serre d'Acquapartita, luoghi erbosi incolti a Col di Battaglia, ca. $600 \mathrm{~m}$, suolo arenaceo-argilloso, humus nullo, 31/05/1977, Brilli-Cattarini, PESA - dintorni di Montefortino, campi incolti in Contrada Chiurchiello, 600-650 m, suolo arenaceo, humus nullo, 12/06/1991, Brilli-Cattarini \& al., PESA. UMBRIA: argine del fosso delle Macerine, Montalera, 28/07/1881, Tanfani, FI - Gubbio, m.te che sovrasata alla città, 20/08/1885, Tanfani, FI - m.te Subasio, 28/06/1886, Pirola, CAT-Frizzi, 1415 Circoscrizione di Terni, territorio di Stroncone, luoghi coltivati, 09/08/1893, Mari, FI Norcia, Forca Canapine, scendendo lungo la strada poco prima del bivio di Castelluccio, 07/07/1977, Arrigoni, FI - calanchi di Fabro (TN), 10/05/2001, Abruzzese \& Gigante, PERU. LAZIO: in apricis aridis Campaniae, Itri alla Civita, 06/1871, Terracciano, FI - Palo, 14/05/1882, Tanfani, FI - Ponza d'Arcinazzo, 15/08/1882, Tanfani, FI - prope Terracina, lungo la via di Gaeta, 23/05/1893, Sommier, FI - Tivoli, Contrada degli Arci, 16/05/1903, Missoni, FI - Roma, Ostia Antica, agli scavi, 18/07/1958, Lanza, FI - arcipelago ponziano, isola di Ventotene, 29/05/1967, Anzalone, FI - Roma, periferia NE, incolti a NE dell'Ateneo Salesiano, 70 m, 10/09/1976, Lucato 40, VER. ABRUZZO: m.te Velino, ai piedi del versante di Rosciolo, 800-1500 m, 08/09/1902, Vaccari, FI - fra Civitellaroveto e Meta passando per Peschiera, 500-1000 m, 02/07/1904, Vaccari, FI - Majella, stazione ferroviaria di Scansano, 29/06/1906, Vaccari, FI - presso Trasacco nella Marsica (AQ), deposito di legna, $670 \mathrm{~m}, 12 / 08 / 2007$, Di Carlo 3430, VER - bordo strada dopo il fontanile a Vigliano, frazione di Scoppito (AQ), 930 m, 26/07/2010, Di Carlo 4081, VER - Torino di Sangro $(\mathrm{CH})$, a margine tra i campi e aree ruderali presso vecchie cave abbandonate, 27/05/2015, Biaggini \& al., Boraginales Working Group 2015.005, B, BLAT, BONN, BR, BRNM, FI, K, M, MA, P, SBBG. MOLISE: Termoli, 04/05/1893, Martelli, FI - Capracotta, margini strade, $1400 \mathrm{~m}, 04 / 05 / 1916$, Biadego, VER - Venafro (IS), lungo la strada ed il fosso al bivio per Pozzilli, 11/06/1971, Steinberg \& Bavazzano, FI - Cantalupo nel Sannio (IS), nel greto del fiume il Rio, 12/06/1971, Steinberg \& Bavazzano, FI - Guardiaregia, 700 m, 20/06/1978, Bianchini 8339, VER - lago di Occhito (CB), 450 m, 23/06/1979, Bianchini 8324, VER. CAMPANIA: inter sata montana, Taburno, 06/1874, Terracciano, FI - Amalfi, Majori, strada * a margini presso al chiesa di S. Francesco, 23/04/1918, Biadego, VER m.te Alburno, parete rocciosa presso Polla, 10/06/1948, De Philippis, FI - Guardia (BN), falde rocciose del m.te Ciesco, 14/06/1971, Steinberg \& Bavazzano, FI - m.ti Picentini, V. Sabato, incolti, siepi, 600-900 m, 28/07/1973, Moraldo, FI - passo Perrone, $1100 \mathrm{~m}$, 20/06/1978, Bianchini 8340, VER - presso Grotta Minarda (AV), s.d., Bruni, FI. BASILICATA: Potenza, m.te Montocchio, in pascuis saxosis, 900 m, 24/06/1937, Gavioli, FI - Pignola, in herbosis, loco Pantani vocato, 800 m, 24/05/1938, Gavioli, FI - Potenza, in herbosis, loco Macchia Romana vocato, 800 m, 07/1940, Gavioli, FI. PUGLIA: in incultis prope Hydruntum, 06/1875, D* Cesare, FI - isole Tremiti, S. Domino, 26/05/1906, 
Cecconi, FI - Gargano, gariga a Sideritis sicula in Valle Carbonara (m.te S. Angelo), 600650 m, 26/05/1968, Moggi, FI - margini della strada tra Deliceto e Bovino, ca. 650 m, 09/06/1968, Nardi \& Bavazzano, FI - m.ti della Daunia, Motta Montecorvino, Crocella di Motta, lungo i campi e incolti produttivi, 13/06/1971, Steinberg \& Bavazzano, Fl. CALABRIA: in aridis collium prope Palizzi, 05/06/1877, Arcangeli, FI - Catanzaro, margini erbosi delle strade nei colli, sotto Bellavista, 05/1894, Micheletti, FI - Cosenza, dintorni del castello, 18/05/1950, Corra, FI - La Sila, ai piedi di Valle Grande (Longobucco), 700 m, 16/06/1950, Sarfatti \& Corradi, FI - massiccio del Pollino, lungo la strda presso Civita, 22/05/2007, Cecchi \& Coppi, foto. SICILIA: Militello val di Noto, 04/1820, s.coll., PAL 64052 - in aridis supra li Ficarazzi, 29/03/1828, s.coll., PAL 60050 - tra Vittoria e Terranova, lungo le vie, 18/05/1873, Aiuti, FI - in campis et collibus reg. inferioribus, Palermo, 05/1900, Ross H. Sic. 265, GE - insula Lampedusa, 06/1906, Martorana, FI Messina, Capo Sant'Alessio, 13/04/1957, Gramuglio, FI - m.te Cammarata (AG), 04/05/1993, Di Martino \& Piazza, PAL 70707 - Calatafimi (TP), Contrada Calemici, exp. O, $420 \mathrm{~m}$, dans les terrains incultes, 24/07/1996, Certa, PAL 64021 - tra Cerami e Nicosia (EN), 950 m, negli incolti, 21/05/2003, Certa \& Carratello, PAL 70545 - Chiusa Sclafani, c. da Lissandro (PA), N 3741.738', E 13²0.037', incolti, 27/04/2010, Certa, PAL 87610 Terrapilata, s.d., s.coll., PAL 64055 - Pantelleria, s.d., Calcara, FI - Avola, in campis arenosis aridis, vel humidis, s.d., Bianca, FI - Ficuzza, in arvis, s.d., Lojacono, FI - Siracusa, s.d., Cassia, FI - Castelbuono, 5.s.d., Minà 213, FI. SARDEGNA: spiaggia di Cagliari, 06/05/1879, Biondi, FI - Fertilia dopo il bivio per Olmedo, 08/05/1971, Arrigoni \& Nardi, FI - Oliena, pendici N della Scala di Sovana, 18/05/1979, Arrigoni \& al., FI - Villanovatulo, scendendo verso il Flumendosa, substr. siliceo, 09/06/1989, Arrigoni \& al., Fl.

Anchusa capellii SARDEGNA: in pascuis summi montis S. Vittoria Esterzili, s.d., Moris, Herb. Moris, TO [materiale originale] - Taccu de' Sadali, 24/07/1898, Martelli It. Sard. 99, FI.

Anchusa crispa SARDEGNA: Alghero a Capo Caccia, 17/05/1895, Martelli It. Sard. 386, FI - Sassari, Porto subsp. crispa Palmas presso I'Argentiera, 06/05/1971, Arrigoni \& Nardi, FI - Porto Torres, dune alla destra dello Stagno di Pilo, 18/06/1972, Valsecchi, FI - Gallura, retroduna della spiaggia La Marinedda all'Isola Rossa, 04/07/1998, Selvi, FI-HB 98.01 - isola dell'Asinara, Fornelli, Cala Spalmadore, retroduna con vegetazione psammofila a dominanza di Matthiola tricuspidata, sul lato orientale, $40^{\circ} 59^{\prime} 20.17^{\prime \prime} \mathrm{N}, 8^{\circ} 13^{\prime} 20.31 " \mathrm{E}, 2 \mathrm{~m}, 15 / 04 / 2015$, Biaggini \& al., $\mathrm{Fl}$, Herb. Cecchi.

Anchusa crispa SARDEGNA: Dune della spiaggia di Badesi, 20/04/1966, Valsecchi, SS [olotipo] - Gallura, subsp. maritima Valledoria alla foce del Coghinas, campi incolti sabbiosi, 16/05/1997, Bigazzi \& Selvi, FIHB 97.05 .

Anchusa formosa SARDEGNA: Sulcis Nord-Orientale (Cagliari), versante NE del Monte Lattias, fra le rocce granitiche nell' alveo secco del Rio Su Fundu, su suolo siliceo sabbioso-detritico, c. 850 m, 18/05/1997, Selvi \& Bigazzi, FI 2369 [olotipo], CAG, FI 2370, K, SS [isotipi] - Su Scavoni, Monte Arcosu, Siliqua (CA), su graniti in disfacimento a $850 \mathrm{~m}$, esp. NNE $15^{\circ}$, 27/10/2001, Bacchetta, FI-HB 01.39.

Anchusa litorea SARDEGNA: in arenosis maritimis circa Oristano, 1826, Moris, Herb. Moris, TO [lectotipo] - isola S. Pietro, in litore arenoso Spalmadore grande, 03/05/1894, Martelli, FI - S. Antioco (CA), Le Saline, 05/05/1975, Milia \& Mossa, CAG-Sardegna - spiaggia di Arborea, 04/04/1981, Mossa, CAG-Sardegna - Arbus (CA), colonia penale di Is Arenas, sistemi dunali, 15 m, 39³1'089" N, 8²5'965" E, 12/05/2005, Angius \& al., CAG-Sardegna.

Anchusa SARDEGNA: Gonnosfanadiga (Cagliari), Monte Linas sotto punta Sa Cabixettas, $1070 \mathrm{~m}$, montelinasana $39^{\circ} 26.559 \mathrm{~N}-8^{\circ} 37.598 \mathrm{E}$ ", 20/05/2005, Angius \& al., CAG [olotipo], Fl 2603, BM [isotipi] 
Anchusa ochroleuca FRIULI VENEZIA GIULIA: Trieste, stazione di Draga, 06/1920, Marchesetti, FI. TOSCANA: sponda sinistra del Frigido (MS) a valle del ponte sulla via Aurelia, 07/1928, Pellegrini, FI.

Anchusa officinalis PIEMONTE: Trino Vercellese, sponde del Po, 15/09/1918, Gresino, FI - Stupinigi (TO), lungo il Sangone, 04/06/1920, Ferrari \& Fontana 3771, FI - Cossato (BI), sponda sinistra del Cervo a SO della Spolina, 01/05/1992, Soldano, Herb. Soldano 8437 - prov. Cuneo, alta valle Gesso, Terme di Valdieri, incolto lungo strada per il Valasco, $1390 \mathrm{~m}$, 24/07/2006, Selvi, FI-HB 06.31. VALLE D'AOSTA: da La Thuille a Près S. Didier lungo un viottolo, 09/08/1909, Camperio, FI - Valtournenche, lungo la statale, tra Ussin e Buisson, $1150 \mathrm{~m}$ ca., 09/06/1985, Bovio 394, Herb. Bovio - St. Nicolas, scarpate di una strada sterrata sopra Evian, 1010 m, 13/10/1992, Bovio \& Rosset, AO-N.SFV 940. LOMBARDIA: lungo I'Oglio a Grumone, 18/08/1866, Parlatore, FI - lungo la circonvallazione di Lodi, sulle rive della fossa di separazione, 30/06/1878, Mazzucchelli, FI - Malgrate, m.te Barro, 08/04/1890, Lordelli, FI - Valtellina, SS dello Stelvio, sotto Bormio su uno scarico in loc. Le Prese, 17/07/1974, Ricceri \& Steinberg, FI - Mantova città, area ruderale erbosa presso il consorzio di bonifica, impianto idrovoro Valsecchi, 24/06/1995, Bigazzi \& Selvi, FI, FI-HB 95.06 - Val Camonica, Monno (BS), dintorni del campo sportivo, 1000 m, 10/08/1999, Martini, MFU-Martini 4571. TRENTINO ALTO ADIGE: Alpi trentine, Cavalese, suolo prfirico, 1000-1250 m, 26/06/1922, Fiori, FI - Vipiteno, prati fra Vipiteno e Novale, detriti misti, 950-1000 m, 31/05/1952, Zenari, FI - val di Sole, bivio per Ortisé, 20/06/1962, Ricceri, $\mathrm{FI}$ - val Senales, rocce e prati nel lariceto rado lungo la strada presso il lago di Vernago, 1690 m, 14/07/1974, Ricceri \& Steinberg, FI - strada per Malga Lozen, 1450 m, 22/06/1994, Lasen, Herb. Lasen - Agumes, Prato allo Stelvio, 1020 m, 03/06/2003, Lasen, Herb. Lasen. VENETO: sopra i muri dell'Orto Botanico di Padova, s.d., Vaccari, FI Legnago val di Cimbro, aridi, 28/08/1896, Bolzon, FI - litorale veneto, delta del Po, Ariano Polesine (RO), Duna Gozzi, incolto in duna fossile, 22/05/2005, Scortegagna, MNAV m.te Venda, colli Euganei (PD), 525 m, ruderale, 25/04/2007, Argenti \& Argenti, Herb. Argenti - Verona, lungo il fiume Adige a Palazzina, $60 \mathrm{~m}, 09 / 06 / 2010$, Di Carlo 4035, VER Saviner di Rocca Pietore (BL), ruderale, 1020 m, 29/05/2011, Argenti \& Argenti, Fl, Herb. Argenti. FRIULI VENEZIA GIULIA: alla foce del Timavo, 07/1878, Marchesetti, FI Firmano, 08/1890, Della Torre, MFU-Forojuliense 10788. EMILIA ROMAGNA: bosco della Mesola (FE), margine strada, 26/05/2002, Argenti \& Argenti, Herb. Argenti.

Anchusa sardoa SARDEGNA: Porto Conte, s.d., s.coll., Herb. Moris, TO [lectotipo].

Anchusa undulata LIGURIA: Borzoli, villa Doria, 22/05/1883, Gestro, FI - nei seminati a Voltri, 06/1891, Fiori, subsp. hybrida FI. TOSCANA: Maremma toscana, San Vincenzo, 30/04/1870, Sommier, FI - Viareggio, 06/1872, Bargagli, FI - insula Gorgona, verso il camposanto, 01/04/1899, Sommier, FI colli fra Lucca e Quiesa, ad agrorum margines, 21/11/1899, Sommier, FI - Firenze, alla stazione di Campomarte, 17/05/1908, Fiori, FI - Arezzo, presso il cavalcavia dell'Olmo, nei campi, 17/04/1912, Savelli, FI - Vallombrosa, al Masso del Saltino, substr. siliceo, 1020 m, 11/08/1912, Fiori, FI - Camucia (AR), luoghi incolti lungo le strade, 265 m, 04/1930, Tani, FI - m.te Cetona, 29/04/1943, Negri \& Corradi, FI - monti dell'Uccellina, lungo l'itinerario di Castelmarino e fra le rocce nei dintorni della torre, 14/05/1982, Arrigoni \& al., FI - m.te Argentario (GR), Olmo, vicino alla fattoria abbandonata, 14/04/1987, Baldini, FI Suvereto (LI), Montioni, 06/06/1993, Cenerini, PI - isola di Pianosa (LI), Bagni d'Agrippa Belvedere - Sembolello, 09/05/2000, Baldini \& Vivona, FI - nel bosco vicino agli argini di un affluente del Lente, Sorano, $380 \mathrm{~m}$, tufo, 11/07/2002, Viciani, FI - Capalbio (GR), margine stradale tra la strada litoranea e la ferrovia, proseguendo per Ansedonia, 0-5 m ca., ruderale, 13/03/2004, Sforzi \& Senesi, FI. MARCHE: Pesaro, luoghi erbosi incolti presso il cimitero, circa $10 \mathrm{~m}$, suolo argilloso-sabbioso, humus nullo, 20/05/1974, BrilliCattarini, PESA - dintorni di Offagna, campi incolti e luoghi erbosi incolti sul m.te della 
Crescia, 325-350 m, suolo prevalentemente sabbioso, humus nullo, 19/04/1978, BrilliCattarini, PESA - dintorni di Ascoli Piceno, ruderati e luoghi erbosi lungo la SS Salaria presso S. Emidio alle Grotte, $175 \mathrm{~m}$, suolo arenaceo, humus nullo, 19/04/1985, BrilliCattarini \& Gubellini, PESA - Maceratese, s.d., s.coll., FI. UMBRIA: presso Chiesa Trevi, 11/09/1886, Batelli, Herb. Batelli, PERU - Trasimeno al Bracci, sponda meridionale, 06/1887, Cicioni, Herb. Cicioni, PERU 1174711 - lungo il F. Nestore alla Morcella, 07/07/1887, Cicioni, Herb. Cicioni, PERU 1175411 - circondario di Terni, dintorni di Stroncone, sui margini della strada, 31/08/1890, Mari, FI. LAZIO: in herbosis apricis, Itri alla Civita, 06/1871, Terracciano, FI - Palo, lungo la ferrovia, 14/05/1882, Tanfani, FI Palombara Sabina alla chiesa di S. Giovanni in Argentella, 05/04/1904, Vaccari, FI - mare di Corneto alle saline, 15/05/1910, Vaccari, FI - pineta di Castel Fusano (RM), 15/07/1958, Lanza, FI - Anzio, Lido dei Pini, dune consolidate, 04/06/1976, Ricceri \& Steinberg, FI - Castelli Romani, Gallicano nel Lazio (RO), via Maremmana inferiore, Colle, on the SSW slope of the hill, $170 \mathrm{~m}$, among grass and shrub vegetation recovering after fire [...], 06/04/1993, Iberite \& al., SEEM 16361, FI - Roma, Sant'Agnese fuori le mura, 14/09/1996, Vaccari, FI - Appennino laziale, Teverina, Bagnoregio (VT), muro in tufo, 06/04/2007, Scortegagna, MNAV - Viterbo, sorg. Bullicame, 340 m, incolto, 07/04/2007, Scortegagna, MNAV. ABRUZZO: lungo il canale del Fucino all'Emissario, 18/08/1875, Cherici, FI - arene marittime a Castellammare Adriatico, 17/07/1886, Fiori, FI - dintorni del Campo Federale, L'Aquila, 730 m, incolti, 25/04/1974, Tammaro, SFI 34, FI. MOLISE: Termoli, Nuova Cliternia, ca. 10 m, 21/04/1960, Di Vincenzo 196, Bianchini 409, VER. CAMPANIA: inter Capua et S. Agata, 1811, s.coll., Herb. Tenore, NAP [lectotipo] - in pascuis demissis, Demanio di Calvi, 05/1870, Terracciano, $\mathrm{FI}$ - in vineis et incultis arenosis, ad vias supra Casamicciola a Coccofreddo, 11/09/1875, Levier, FI - m.ti Tisati presso Caserta, 26/02/1899, Frizzi, CAT-Frizzi, 1422 - nei vigneti del Vesuvio (Comune di S. Sebastiano), 12/04/1907, Pellanda, FI - Portici (NA), loco dicto Bosco Gussone, 50 m, solo siliceo, 05/04/1910, Pellanda, FIE 2144, FI - fra Rocchetta e Formicola, bordo stradale, 30/04/1994, Bigazzi, FI-HB 94.05. BASILICATA: in arenosis, Melfi presso I'Ofanto, 04/1860, Terracciano, FI - Potenza, 07/1880, Biondi, FI - Bernalda negli oliveti, contrada S. Angelo, 05/1918, Gavioli, FI - in silva Montecchio ad pendices m.tis Vulture, loco le Grotticelle dicto, solo siliceo-vulcanico, ca. 400 m, 09/04/1922, Gavioli, FI - Tolve, in arenosis et glareosis secundum flumen Fiumara di Tolve dictum, 250-300 m, 02/06/1924, Gavioli, FI - Matera, piazzale chiesa della Palomba, 15/05/1950, Negri \& Messeri, FI. PUGLIA: in agris circa S. Severo, solo calcareo, 10-100', 06/06/1875, Porta \& Rigo It. Ital. I/ 381, FI, VER - in arvis S. Pietro, Manduria, 04/1876, Profeta, $\mathrm{FI}$ - in pascuis maritimis prope Gallipoli, 06/1883, Groves, $\mathrm{FI}$ - dintorni di Taranto, 06/1890, A.Vaccari, FI - nella sabbia alla riva dell'Ofanto a Canne, 17/05/1897, Palanza, FI - presso Spinazzola, 08/06/1898, Fiori, FI - stazione di Rocchetta S. Venere, 02/06/1899, Fiori, FI - presso Otranto (LE), substr. calcare, $30 \mathrm{~m}$ ca., 31/05/1913, Fiori, FI - Bari a S. Girolamo, arene marittime, 05/1931, Zodda, FI - rocce calcaree della Gravina di Puglia, 300-330 m ca., 12/06/1968, Nardi \& Bavazzano, FI - Castellaneta, macchia in loc. La Gaudella, 30/04/1975, Steinberg \& Ricceri, FI - Lizzano, lungo la spiaggia fra Marina di Torricella e Trullo di mare, 02/05/1975, Steinberg \& Ricceri, FI - spiaggia di Brindisi, 07/06/1982, Brullo \& Signorello, CAT-generale 36896, 36897 [sub Nonea ventricosa] - Lesina, Torre Fortore, $10 \mathrm{~m}$, 19/04/1983, Bianchini \& Di Carlo, Bianchini 6728, VER - Martina Franca, bosco delle Pianelle, 07/05/1984, Brullo \& al., CAT-generale 36899 [sub Nonea ventricosa]. CALABRIA: in herbidis secus viam prope Bianco nuovo, 12/06/1877, Arcangeli, FI - S. Maria presso Catanzaro, 07/05/1883, Andrea Fiori, Herb. Fiori, FI 7153 [lectotipo di $A$. undulata f. angustata] - Cosenza, lungo il fiume Busento, 18/05/1950, Corradi, FI - Sila, bivio Garga, 31/05/1955, Sarfatti, FI - Marina di Catanzaro, sulla spiaggia, 03/04/1960, Padula, FI - Cassano allo lonio presso le Grotte di Sant'Angelo, 
detrito calcareo, 13/05/1994, Bigazzi \& Montori, FI, FI-HB 94.04 - spiaggia lungo la SS 106 Jonica, in loc. Bivio di Camini, Monteserace Marina (RC), 2 m, 10/04/2003, Martini, MFUMartini 5334. SICILIA: Palermo, nella spiaggia della Renella, 03/1838, Parlatore, FI - Etna a m.te S. Leo, 12/05/1905, Cavara, FI - Nicolosi sotto m.te Pizzuto, 01/05/1829, s.coll., PAL 64009. SARDEGNA: Foresta Burgos, 10/06/1974, Valsecchi, FI - Arbus (CA), rio S. Anna, esp. $175 \mathrm{~S}$, inclinazione $3^{\circ}, 39^{\circ} 29^{\prime} 535^{\prime \prime} \mathrm{N}, 8^{\circ} 27^{\prime} 792^{\prime \prime}, 03 / 06 / 2005$, Angius \& al., CAG-Sardegna, FI-HB 05.19, Herb. Cecchi 409.

Anchusella cretica TOSCANA: Ansedonia, prope il Tombolo di Feniglia, 16/03/1883, Forsyth Major, FI Maremma orbetellana, fra Nunziatella e Capalbiaccio, in arvis incultis, 27/03/1894, Sommier, FI - Castell'Azzara (GR), vetta del m.te Penna, nei pascoli rocciosi e cigli rupestri, calcare, 950-1050 m, 24/05/1995, Selvi, SFI 815, FI. LAZIO: Velletri, castagneto sopra la strada dei laghi a colle Petrona, 24/05/1938, Senni 316, FI - foresta demaniale del Circeo, bosco misto a farnia, sughera, ontano etc., in loc. Palazzo e Pantano, 23/04/1965, Bavazzano \& Ricceri, FI - Viterbo, poggio della Pallanzana, 21/06/1985, Macchiati, FI. CAMPANIA: Ischia, cratère brisè du Mt. Rotaro, 16/06/1864, Bolle, FI Salerno, Pesto, 13/04/1903, Passerini, FI - Pompei, nei campi, suolo vulcanico, 16/02/1906, Fiori, FI - Neapolis, in herbosis et ad margines silvarum, secundum viae quae e loco Pigna ad Camaldoli ducit, 200 m, solo siliceo, 15/03/1913, Guadagno, FIE 2141, FI S. Agata, penisola sorrentina, grotta di Berta, 02/04/1920, Biadego, VER - m.te Alburno, lecceta da Ottati a Costa la Croce, 550-900 m, 29/05/1951, De Philippis \& Moggi, FI penisola Sorrentina, Agerola, incolti erbosi, ca. 600 m, 10/05/1995, Bigazzi \& Selvi, FI-HB 95.14. BASILICATA: Vulture a Monticchio, regione submontana, 10/06/1898, Fiori, FI Muro Lucano, in pascuis, loco Difesa Grande vocato, 900 m, 08/06/1929, Gavioli, FI Pignola, bosco di Acquafredda, 29/05/1933, Gavioli, FI - Abriola, m.te Arioso, in herbosis, loco Le Murge vocato, 1300 m, 19/06/1933, Gavioli, FI - Rionero in Vulture (PZ), m.te Vulture, tra Rionero e laghi di Monticchio, $820 \mathrm{~m}$, margine strada, $\mathrm{N} \mathrm{40} 55.186^{\prime}, \mathrm{E}$ $15^{\circ} 37.360 ', 06 / 06 / 2003$, Conti \& Tinti, APP 5382 - Pietrapertosa (PZ), Dolomiti lucane, pascoli aridi, 03/05/2004, Conti \& Tinti, APP 20268. PUGLIA: Gargano, m.te Croce, lungo la via rotabile, suolo calcareo, $800 \mathrm{~m}, 12 / 07 / 1915$, Fiori, FI - Accadia (FG), versante $\mathrm{S}$ del m.te Tre Titoli, pascolo, 27/05/2011, Carruggio, BI 36030, 36354 - Biccari (FG), m.te Cornacchia, Toppo Pescara, brometo, 28/05/2011, Carruggio, BI 36029 - circa Lesina, s.d., $T^{*}$, VER. CALABRIA: lungo le strade nei colli di S. Leonardo (CZ), 01/05/1896, Micheletti, FI - Reggio Calabria, m.te Basilicò, loc. Tre Aie, faggeta, ca. 1100 m, 02/06/1955, Chiarugi \& al., FI - m.te Pollino, pascoli intorno al fortino a N di Campo Tenese, 1050-1100 m ca., calcari e scisti, 20/05/1972, Arrigoni \& al., FI - presso la diga di Tarsia verso Corigliano, ai margini della strada, 20/04/1989, Del Prete \& al., PI - Monte Cocuzzo (CS), prati rocciosi verso la vetta, ca. 1300 m, 13/05/1994, Bigazzi, FI-HB 94.11 - Sila Piccola, Villaggio Mancuso (CS), cumulo di terra smossa nella pineta a Pinus nigra nei pressi del centro visite in località Monaco, ca. 1300 m s. I. m., 23/05/2007, Cecchi \& al. 1093, Fl-HB 08.41, Herb. Cecchi - Papasidero (CS), Timpone Sirio, 860 m, 12/05/2009, Di Marco, PI - sotto Maida, s.d., Ricca, FI - Simeri Crichi (CZ), mosaico di pascoli, ex coltivi, gariga e sughereta degradata su un poggio presso l'abitato, 11/05/2015, Biaggini \& al., Boraginales Working Group 2015.002, B, BLAT, BONN, BR, BRNM, FI, K, M, MA, P, SBBG - Aspromonte, alla base di costoni calcarei nei colli sopra San Luca, $800 \mathrm{~m}, 13 / 05 / 2015$, Biaggini \& al., foto!. SICILIA: Giardini, non longe a Taurominio, ad viam, 02/04/1873, Sommier, FI - m.te Antennamare presso Messina, 05/1892, A.Vaccari, $\mathrm{FI}$ - pendici dell'Etna (CT), versante di Adrano, $1300 \mathrm{~m}, 12 / 05 / 1986$, Bianchini \& Di Carlo, Bianchini 11284, VER - m.te Soro, $37^{\circ} 56^{\prime} \mathrm{N}, 14^{\circ} 41^{\prime} \mathrm{E}, 1700-1800 \mathrm{~m}$, brown woody soil on quartzarenite, 09/06/1990, Raimondo \& al., OPTIMA It. Med. III 1804, PAL 68230 - Cesarò, San Fratello (ME), presso torrente Torti I, N 3751'951', E 1440.376', incolti, 09/06/2004, Certa \& al., PAL 87315 - 
Mandanici, s.d., Borzì, FI - Mirto, ad sylvarum margines, 5.s.d., Todaro, FI. - In Fl è inoltre conservato un campione della Lombardia (Mantova, s.d., s.coll., Fl), ma è probabile si tratti o di uno scambio di cartellino, o di pianta coltivata o affrancatasi solo in modo effimero dalla coltivazione. Segnalata anche per le isole Eolie (Pasta \& Lo Cascio 2002).

Borago morisiana SARDEGNA: Flumini major, s.d., s.coll., Herb. Moris, TO [paratipo] - S. Pietro, cala del Becco, 01/05/1952, Martinoli, FI - Isola di San Pietro (Sardegna sud-occidentale); Calavinagra. Fosso umido con fondo sabbioso a circa 50-100 m dal mare, 28/08/1988, Bigazzi, FI 2373 [olotipo], B 10 0365357, 10 0360070, FI 2374, 2375, MA 525451 [isotipi] Laconi (NU), rocce bagnate di travertino presso una cascatella nel bosco del Rio Bau Oni presso la cantoniera di Funtanamela, 670 m, 06/06/2003, Bacchetta \& al., FI-HB 03.02.

Borago officinalis LIGURIA: San Remo, 1888, Bazzanti, FI - Monterosso al Mare, loco Fegina dicto, 18/09/1925, Montale, Gavioli 9049, FI - Caprina (SP), Campazzi, Montemarcello, 06/05/1994, Maccini, PI - Diano *, 4.s.d., Ricca 220, FI. PIEMONTE: Baceno, 750 m, 01/06/1918, Biadego, VER - Baldissero, 18/05/1919, Vatova, FI - * di Argentera, orti, 29/07/1919, Biadego, VER - Graglia, prato, 596 m, 05/10/1996, Belmondo, UTV 1659. VAL D'AOSTA: Aosta, 20/04/1899, Vaccari, FI - Courmayeur, 1300 m, 19/07/1959, Bianchini 475, VER - Saint Cristophe, a Veynes, presso i giardini, $775 \mathrm{~m}, 10 / 1993$, Bovio \& Giunta, AO-N.SFV 1064 - Torgnon (AO), margini di sentiero montano, 1800 m, 07/1994, D'Amato, UTV 1668. LOMBARDIA: lungo i fossetti presso Cassano d'Adda, 04/1881, Mazzucchelli, FI - Cisano, ad oras Benaci in cultis, 04/1889, Goiran, VER. TRENTINO ALTO ADIGE: Mölten ob Vilpian, Unterlage porphyr., 1000 m, 01/06/1902, Red*, Pfaff 934, PAD-Triveneto. VENETO: Veronae, in collibus veronensibus, in cultis, 04/1891, Goiran, VER - Monselice (Rocca), 07/1894, Paoletti, GE - lago di Garda, campi e luoghi coltivati a Malaspina, 07/04/1895, Goiran, VER - in un campo a Valdobbiadene, $280 \mathrm{~m}, 28 / 10 / 1896$, Pampanini, FI - Euganei sul m.te Lonzina, 05/1898, Fiori, FI - spontanea negli orti di Padova e Bassano, 06/1925, Vaccari, FI - Grumolo Pedemonte di Zugliano (VI), fossi, 08/1971, Fenogli, MNAV - Lamon, 600 m, 20/07/1984, Lasen, Herb. Lasen - Rio Celarda, 230 m, 04/07/1992, Lasen, Herb. Lasen - Schio, C. Ramone, 700 m, *, 25/02/1994, Scortegagna, MNAV - Sappada (BL), 1200 m, incolto, nitrofila, 31/08/2007, Argenti \& Argenti, Herb. Argenti. FRIULI VENEZIA GIULIA: fossa di porta Ronchi, Aquileia (UD), 15/04/1904, Corgnali, MFU-Forojuliense 10792 - Trieste (TS), piccola scarpata dietro piazzale Cagni, 30 m, 31/03/1998, Sannini, MFU-Martini 4574 - S. Pietro al Natisone, 3.9.s.d., Minio, FI - Cividalese, in un campo ad Albana, s.d., s.coll., MFU-Forojuliense 10794. EMILIA ROMAGNA: Fontana - Saliceta S. Giuliano, 02/06/1883, Fiori, FI Vallisnera presso Culagna, 06/07/1882, Fiori, FI - Scandiano, 14/04/1884, Fiori, FI Polenta, 15/05/1887, Chelussi, FI - dintorni di Forli, 05/1888, Chelussi, FI - al sole presso Casalecchio (BO), 07/05/1904, Fiori, FI - Piandelagotti, in un prato, 15/07/1955, Lunardi, FI - parco fluviale di Casola Valsenio (RA), pendii assolati del torrente, 01/05/2000, Buoso, BOLO 506827 - Parco di Monteveglio (BO), margine di un bosco di latifoglie, zona ombrosa, $130 \mathrm{~m}$, 05/05/2002, Nucciotti, BOLO 40635 - Ferrara, prati presso le mura estensi occidentali, 15/04/2010, Piccoli, FER. TOSCANA: Orciatico pr. Volterra, in agris, 05/10/1897, Stefanini, FI - insula Gorgona, 21/05/1899, Sommier, FI - presso Sinalunga, nel giardino del Castelletto e nei campi sottostanti, 31/07/1913, Savelli, FI - Bagni di Lucca, luoghi erbosi, campi, 120 m, 06/1930, Tani, FI - Sansepolcro, Golini, 17/07/1932, Pichi Sermolli 205, FI - Buggiano, 08/04/1933, Pichi Sermolli 204, FI - Garfagnana, Ceserana, 23/04/1934, Losacco, Pichi Sermolli 8964, FI - Val d'Elsa, Castelfiorentino, fattoria di Coiano, 09/04/1951, Marsili, FI - Cerbaie, lungo il Rio Nero, 29/04/1951, Contardo, FI - alta Lunigiana, alta valle del Taverone, 06/07/1951, Ferrarini, FI - macchia di Monteverro presso Capalbio (GR), 100 m ca., esp. S, 18/04/1968, Nardi \& Ricceri, FI isola di Capraia (LI), 18/04/1973, Peccenini, Herb. Peccenini - m.te Pisano, M. S. Giuliano, 
19/05/1982, Simonetti, PI - palude Diaccia, 25/05/1982, Bicchi, PI - isola d'Elba, Barbarossa - porto Azzurro, 26/03/1983, Del Prete, PI - Suvereto (LI), Poggio Martelloni, 18/04/1993, Cenerini, PI - isola di Giannutri (GR), campo sportivo, 20/02/1999, Baldini, FI - fossetto umido di scolo nei campi nella piana antistante Asciano (PI), 20/04/1999, Pedullà, PI - isola del Giglio, Campese, 42 $22^{\prime} 00^{\prime \prime} \mathrm{N}, 10^{\circ} 53^{\prime} 20^{\prime \prime} \mathrm{E}$, ruderalbeeinflußte Wiese, 15/05/1999, Dressler \& Will, FR 573, FI - Sesto Fiorentino (FI), lungo via Pratese in zona Osmannoro, 23/04/2005, Cecchi 365, Herb. Cecchi. MARCHE: dintorni di Pesaro, campi coltivati e luoghi erbosi incolti lungo il Fosso di Selva Grossa presso Case Bruciate, ca. $30 \mathrm{~m}$, suolo prevalentemente argilloso, 14/05/1967, Brilli-Cattarini \& al., PESA dintorni di Ancona, luoghi erbosi incolti tra il colle del Trave e il Montirozzo, ca. $200 \mathrm{~m}$, suolo prevalentemente argilloso, humus subnullo, 09/04/1984, Brilli-Cattarini \& Gubellini, PESA - dintorni di Porto d'Ascoli, luoghi erbosi incolti sul litorale della Sgariglia, ca. $3 \mathrm{~m}$, suolo prevalentemente argilloso-sabbioso, humus nullo, 09/04/1984, BrilliCattarini \& Gubellini, PESA - dintorni di S. Lorenzo in Campo, luoghi erbosi in colti sui colli tra Mirabello e Roncaglia, ca. $150 \mathrm{~m}$, suolo argilloso-calcareo, humus nullo, 03.05.* , BrilliCattarini \& al., PESA - dintorni di Polverigi, campi coltivati e luoghi erbosi incolti lungo la strada in loc. Ponte di *, ca. 50 m, suolo argilloso, 05.05.*, Brilli-Cattarini, PESA - dintorni di Civitanova Marche, luoghi erbosi incolti lungo la strada da Porto Civitanova a Civitanova Alta, ca. $30 \mathrm{~m}$, suolo prevalentemente argilloso, 10.05.*, Brilli-Cattarini \& al., PESA - valle del Cesano, luoghi erbosi incolti assolati lungo la strada provinciale Cesanese tra Ponte del * Castelvecchio, ca. $60 \mathrm{~m}$, suolo prevalentemente argilloso, 13.05.*, BrilliCattarini, PESA - dintorni di Porto S. Elpidio, luoghi erbosi * nell'alveo del Tenna presso la foce, 1-2 m, suolo prevalentemente argilloso-calcareo, 25.05.*, Brilli-Cattarini, PESA. UMBRIA: m.te Subasio, orto delle Carceri, 24/07/1886, Frizzi, CAT 1416 - Perugia, prativi presso la clinica veterinaria dell'Università, 17/06/2015, Cecchi, fotografia inedita. LAZIO: dintorni di Roma, littorale romano presso Pratica, 1875, Siemoni, FI - margine superiore del lago di Albano sopra Marino, prati, 25/04/1936, Senni 106, FI - Monterotondo (RM), 04/1951, Steinberg, FI - m.te Circeo, versante NNE, ai margini della strada basale e negli incolti, $18 \mathrm{~m}, 10 / 04 / 1964$, Padula, FI - terreno di scarico lungo la strada Sperlonga - Itri, presso Sperlonga, $100 \mathrm{~m}$ ca., 21/04/1968, Nardi \& Ricceri, FI - argine del Garigliano presso l'estuario, 22/04/1968, Nardi \& Ricceri, FI - Torre in Pietra (RM), margine di bosco di latifoglie, ca. 50 m, 26/03/1994, Anelli, UTV 1665 - Poggio Olivastro, Canino (VT), incolto presso sorgente di acqua calda, 05/03/2000, Luciani, UTV 16678 - Caio - Bassano in Teverina (VT), vicino a terreno concimato, 05/03/2001, Purchiaroni \& al., UTV 16657 Parco Regionale dei Monti Simbruini (RM), lungo sentiero, 800 m, 21/05/2004, Baliva, UTV 23842 - Roviano (RM), Leveta, prato incolto prospiciente l'abitato, $500 \mathrm{~m}$, 22/05/2005, Riccioluti, UTV 23803 - Barbarano Romano (VT), Pian de Nasse, incolto molto sfruttato dal pascolo, 04/06/2007, Scoppola, UTV 27852 - m.te Civita, Spigno Saturnia (LT), valletta pianeggiante arida con rosacee e pero selvatico, ca. $600 \mathrm{~m}$, 18/04/2008, Santilli \& Palmieri, UTV 28296. ABRUZzo: Teramo, in herbidis, 05/1948, Zodda, BI 24996 - Caramanico Terme (PE), valle dell'Orfento presso il bivio per Decontra, ca. $650 \mathrm{~m}, 08 / 04 / 2000$, Conti, APP 12413 - Palena - Lettopalena (CH), vallone di Izzo, 618 $\mathrm{m}$, incolto, N 42 $00.031^{\prime}$, E $14^{\circ} 08.808^{\prime}, 17 / 04 / 2004$, Di Renzo, APP 14119 - Torino di Sangro (CH), versante sul mare, 23/04/2009, Conti \& al., APP 40997 - Liscia (CH), nei pressi di loc. Duodo, $484 \mathrm{~m}$, coord. 463037, 4643979, 33T, 29/04/2009, locchi \& Bartolucci, APP 37215 - Anversa degli Abruzzi (AQ), Gole del Sagittario, tra Cavuto e la frana, riva sinistra del fiume, incolti e pascoli, 08/07/2009, Conti, APP 41595. MOLISE: Venafro (IS), lungo la strada ed il fosso al bivio per Pozzilli, 11/06/1971, Steinberg \& Bavazzano, FI - valle del Trigno (IS), Contrada Penna, 298 m, coord. 463059, 4628177, 33T, 30/04/2009, locchi \& Bartolucci, APP 37398. CAMPANIA: Ischia al Bagno, 18/05/1849, Gussone, Herb. Gussone, NAP - Pesto (SA), 13/04/1903, Passerini, FI - 
coltivati ai colli di Sorrento, 10/11/1919, Biadego, VER - margini erbosi sulla strada Atrani Minori, 13/11/1919, Biadego, VER. BASILICATA: Potenza, in glareosis rivi Rio Freddo dicti, 800 m, 05/1903, Gavioli 3785, FI. PUGLIA: Gargano, lungo la strada da S. Severo a S. Marco di Lamis, 09/05/1893, Martelli, FI - Laterza, letto e sponde della Gravina di Laterza, sotto il paese, 26/04/1975, Steinberg \& Ricceri, FI - Gagliano del Capo, vallone profondo a mare, fra S. Maria di Leuca e Marina di Novaglie, 03/05/1975, Steinberg \& Ricceri, FI - Gargano, Manfredonia e dintorni, $10 \mathrm{~m}, 30 / 04 / 1982$, Bianchini \& Di Carlo, Bianchini 5712, VER- in incultis prope Hydruntum, 05/1878, Groves, FI - campus universitario di Bari, 18/03/1988, D'Amico \& Sburlino, BI 30300 - Monopoli, Lama Belvedere, 12/11/2004, Angiulli, BI 35298. CALABRIA: marina di Catanzaro, vicino al mare, sulla spiaggia, 03/04/1960, Padula, FI Papasidero (CS), Castiglione, 343 m, 03/04/2010, Di Marco, PI - Palagani Mendicino, 500 m, 28/04/1976, Morrone \& Cassano, CLU 1339 - da Trebisacce a Rocca Imperiale, lungo il litorale, 23/03/1978, Provenzano, CLU 1342 - Cassano Ionico (CS), Casino Drago, 500 m, 16/04/1993, Capparelli \& Bernardo, CLU 1332 - Copanello di Staletti (CZ), terrazzi sotto museo Gatti, 27/03/1995, Tassone, CLU 1333 - Strongoli (KR), Pantano, dune marittime, substr. sabbia, esp. E, 0 m, 27/04/1997, Bernardo \& al., CLU 1340 - Marzi (CS), sotto il Ponte Nuovo vicino la confluenza del torrente Ara (Lara) con il fiume Savuto, pendio, $290 \mathrm{~m}$, 18/04/1998, Bartolotta \& al., CLU 1338 - Crotone, lungo la strada per Capo Colonne, a ridosso del Lido Azzurro, 24/01/1999, Anania, CLU 1334 - Sila Greca (CS), torrente Fangaro, sinistra orografica, lungo la strada per Cropalati, 3 km dopo Paludi, 300-400 m, 18/04/2002, Peruzzi \& al., CLU 1341. SICILIA: Ustica, *, 08/05/1853, s.coll., PAL 63760 - Palermo, alla Favorita e nel m.te Pellegrino, 05/05/1895, Baroni, FI - Girgenti, 03/1900, Martelli 56, FI insula Pantelleria, verso Ghirlanda, 20/03/1906, Sommier, FI - Egadi, Marettimo, verso Capo Troia, 27/04/1935, Francini \& Messeri, FI - Motya, 05/05/1956, s.coll., PAL 63764 - Pachino, Contrada Casanova, 09/04/1967, s.coll., CAT 35817 - Ispica (RG), Cozzo Pilato, 06/03/1969, Brullo, CAT 35792 - foce del fiume Cassibile, 16/04/1970, s.coll., CAT 35795 - isola di Lampedusa, gariga fra Cala Galera e Cala Croce, 28/04/1970, Lumini \& Ricceri, FI - Lipari, cava di pomice, 13/05/1972, Brullo, CAT 35804 - Alicudi, 31/05/1980, Brullo, CAT 35801 Filicudi, 28/04/1982, Brullo, CAT 35783 - Panarea, caletta sabbiosa, 20/04/1982, Brullo, CAT 35782 - Salina, Rinella, 30/04/1982, Brullo, CAT 35784 - sughereta di Niscemi, 17/04/1983, Brullo, CAT 35800 - Madonie, Bozzolino, 37 $51^{\prime} \mathrm{N}, 14^{\circ} 06^{\prime} \mathrm{E}, 1000-1200 \mathrm{~m}$, brown soil on quartzarenite, 06/06/1990, Raimondo \& al., OPTIMA It. Med. III 1447, PAL 68780 - Licata (AG), Lido degli Angeli, incolti, 16/02/1992, Pira, PAL 89400 - dintorni di Aci Trezza (CT), 10 m, 26/02/1994, Girelli, Bianchini 20605, VER - Cinisi (PA), Contrada Piano Margi, 650 m, exp. NO, 38 $06^{\prime} 51^{\prime \prime} N, 13^{\circ} 08^{\prime} 09^{\prime \prime} E$, environnement de maquis méditerranéen, 09/04/2000, Certa \& Carratello, PAL 63763 - Termini Imerese (PA), m.te San Calogero, 400 m, incolti su substrato calcareo, 13/12/2000, Marcellino \& D'Agostino, PAL 63761, 63762 - m.ti Climiti (SR), 04/02/2007, Zimmitti, CAT 30930. SARDEGNA: nei prati a Zinigas presso Siliqua, 09/05/1879, Biondi, FI - Capo Spartivento, golfo di Malfatano, 18/04/1894, Martelli, FI Orune, 05/1899, Martelli, FI - Sarrabus, gruppo Sette Fratelli, m.te Casteddu sopra Flumini, 14/04/1925, Negri, FI - Iglesiente, Domusnovas, Grotta di San Giovanni, versante S, m 200 ca., 17/05/1963, Bavazzano \& Ricceri, Fl-Sardegna - Laconi, Lattinazzu, m 800 ca., 24/05/1967, Alias, Fl-Sardegna - Bosa, lungo la costa a N di Torre Argentina, 06/05/1969, Arrigoni \& Ricceri, FI - Dorgali, regione costiera di Plamasera a S di Calagonone, 03/04/1970, Arrigoni \& Ricceri, FI - Siniscola, m.te Albo, rocce e ghiaioni calcarei sotto punta Guturgius, esp. SE, m 200-500, 27/04/1974, Arrigoni \& al., FI-Sardegna - Orani, m.te Gonare, calcari paleozoici della zona culminale, 29/04/1974, Arrigoni \& al., FI-Sardegna - dintorni di Nulvi (SS), 500 m, 09/05/1984, Bianchini \& Di Carlo, Bianchini 7482, VER - isola dell'Asinara, Campo Perdu, lungo la vallecola umida che sale a NO del laghetto, $41^{\circ} 4^{\prime} \mathrm{N}, 8^{\circ} 16^{\prime} \mathrm{E}, 20-40 \mathrm{~m}$, 15/04/2015, Biaggini \& al., Fl. 
Borago pygmaea TOSCANA: isola di Capraia (LI), Vado del Fondo, su stillicidio, 25/06/1997, Pancioli, FI. SARDEGNA: ad rivalum Pizzinuru supra fedinam Ingurtosu, prov. Iglesias, 10/06/1863, Ascherson, FI - Gennamari, 05/1865, Gennari, FI - Lanusei, 1882, Nicotra, FI - luoghi umidi ombrosi a Tempio, 17/07/1887, Fiori, FI - isola Spargi presso la Maddalena, 04/1893, A.Vaccari, FI - circondario di Aritzo, 1935, Porru 9, FI - Bortigiadas (Sassari), Petra, fraz. Figa Ruia, 400 m, roccia madre granito, luoghi freschi, 26/05/1962, Vannelli, FI-Sardegna - Bosa, lungo la costa a nord di Torre Argentina, 06/05/1969, Arrigoni \& Ricceri, FISardegna - Seui, margini della Foresta Montarbu sopra la strada Arqueri - Lago del Flumendosa, 28/09/1977, Arrigoni \& Di Tommaso, Fl-Sardegna - luoghi umidi della valle del Tacquisara, $800 \mathrm{~m}$ ca., 27/05/1979, Biondi, FI - capo Testa, Santa Teresa di Gallura, 0 $\mathrm{m}, 07 / 05 / 1982$, Lucato, VER.

Brunnera TOSCANA: Badia Prataglia (AR), bordi di vie, orti e giardini in paese e a Vetriceta, $840 \mathrm{~m}$, macrophylla 08/06/2006, Selvi, FI-HB 06.22, FI. LAZIO: Tivoli, Villa d'Este, prati e zone incolte, 25/04/2012, Iamonico, Herb. Iamonico [fide D. Iamonico].

Cynoglottis LIGURIA: nei Apennini a Ronco, s.d., Rainer, VER. PIEMONTE: a Rocavion ad Robilant barrelieri subsp. secus viam, et in valle Sturae secus viam demontis, s.d., Bellardi, Herb. Bellardi, TO barrelieri [lectotipo] - Argentera (CN), 25/07/1919, Biadego, VER - Mango d'Alba (CN), margini dei campi, 29/06/1926, Vignolo-Lutati, Gavioli 10486, FI - Roccagrimalda, 10/05/1934, Zercaro, GE - Ovada tra i vigneti, 10/06/1934, Zercaro, GE - Alpi Marittime, val di Gesso, lungo il Gesso presso Madonna Bruna di Aradolo, 670 m, 04/07/1962, Bono, FI - Valle S. Giacomo, Entracqe (CN), 1310 m, 03/07/2011, Lasen, Herb. Lasen. EMILIA ROMAGNA: Appennino reggiano, Santa Maria sul Ventasso, 03/07/1885, Fiori, FI - m.te Alfeo nell'Appennino, s.d., Ricasoli, FI. TOSCANA: Orrido di Botri (LU), 26/05/1935, Maraini, FI La Verna, crinale del m.te Penna da N a S, esp. E, 26/05/1962, Cortini, FI - Preappennino pistoiese-lucchese, Penna di Lucchio, parete rocciosa di SO, 950-1000 m ca., 04/07/1969, Nardi, FI - Garfagnana, Villa Collemandina, Pania di Corfino, roccette esp. SO, 1400-1500 $\mathrm{m}, 03 / 06 / 1987$, Foggi \& al., FI - Appennino lunigianese, Comano (MS), erboso ai margini del sentiero per il cimitero, su calcare, esp. S, 950 m, 17/07/1993, Ferrarini, FI - m.te Cetona (SI) sulla vetta, pascoli e radure rocciose calcare, ca. $1000 \mathrm{~m}, 18 / 06 / 1998$, Selvi, FI-HB 98.07 - sopra Capanne di Careggine verso il m.te Sumbra sui prati presso i ruderi del vecchio paese, 02/07/2005, Gestri, PI. MARCHE: dintorni di Montemonaco, luoghi erbosi e fruticeti radi presso Arato, $775-800 \mathrm{~m}$, suolo arenaceo, humus scarso, 15/05/1987, Brilli-Cattarini \& Gubellini, PESA - gruppo del m.te Tolagna, fruticeti radi e luoghi erbosi nel versante $E$ del m.te Tolagna lungo la bassa valle Canale, 1080-1100 m, suolo calcareo, humus da scarso a più o meno abbondante, 20/05/1987, Brilli-Cattarini \& Gubellini, PESA - gruppo del m.te Catria, macereti e luoghi erboso-sassosi nel basso versante SE del m.te Acuto alle Cave di Pietra, 1000-1025 m, suolo calcareo, humus da subnullo a più o meno abbondante, 01/06/1988, Brilli-Cattarini, PESA - m.ti della Laga, macereti cespugliati nel basso versante NO del Pizzo di Sevo alle Sette Fonti, ca. $1550 \mathrm{~m}$, suolo arenaceo, humus scarso o subnullo, 22/06/1990, Brilli-Cattarini \& Gubellini, PESA gruppo del m.te Pennino, luoghi erboso-sassosi nel basso versante SE del m.te Pennino alle Scurose, 1025-1050 m, suolo calcareo, humus da subnullo a più o meno abbondante, 14/06/1991, Brilli-Cattarini \& al., PESA - gruppo della Serra Santa a S. Maria d'Appennino, luoghi erbosi asciutti, suolo calcareo, humus da nullo o subnullo a più o meno abbondante, 490 m, 06/05/1992, Brilli-Cattarini \& al., PESA - m.ti Sibillini in destra della valle del Fiastrone, tra Monastero e Rio Fessa, pendici sassose, suolo calcareo, humus scarso o subnullo, 650-675 m, 11/05/1992, Brilli-Cattarini \& al., PESA. UMBRIA: salendo da Foligno, Colfiorito, 30/06/1836, Parlatore, FI - Sigillo, versante SE di m.te Cucco, pascoli sassosi cacuminali su calcare, 04/04/1977, Ricceri \& Steinberg, FI - Norcia (PG), Piano Grande di Castelluccio di Norcia, 1250-1300 m, karst plain, 13/06/1990, Millozza, 
SEEM 15401, FI, RO-HG. LAZIO: in montosis Campaniae, Cassino sul m.te Cairo, 06/1872, Terracciano, FI - Vallepietra, alla SS. Trinità sulla rupe, 29/05/1904, Vaccari, FI Cammerata Nuova, fosso Fioio, 1000-1200 m, 30/05/1904, Vaccari, FI - m.te Terminillo (RI), Rio Fuggio, ambiente di forra, 1200 m, 21/06/1993, Scoppola, UTV 1641. ABRUZZO: Tagliacozzo, grotta di Petrella, 01/06/1905, Vaccari, FI - Majella, Campo di Giove, salita al Vado di Cocci, 1000-1200 m, 30/06/1905, Vaccari, FI - Velino, valle di Maselame, sopra Massa d'Albe, 1000-1300 m, 30/06/1906, Vaccari, FI - Ovindoli, rupi esposte a mezzogiorno, 03/06/1929, Chiarugi, FI - Roccaraso alla stazione ferroviaria, $1236 \mathrm{~m}$, 08/06/1951, Chiarugi, FI - L'Aquila, prati lungo la strada fra Passo Capannelle e Assergi, $1400 \mathrm{~m}$ ca., 30/05/1969, Fabbri \& al., FI - m.te Morrone, sentiero per la valle delle Vacche, 13/05/1992, Bigazzi, FI-HB 92.02, FI - Parco Nazionale d'Abruzzo, nei pressi di Barrea (AQ), $1000 \mathrm{~m}, 28 / 04 / 1998$, Di Carlo 36, VER - pressi della cava di calcare a Vigliano e in via Colonnelle, sempre a Vigliano di Scoppito (AQ), 950 m, 10/08/1999, Di Carlo 346, VER - Intermesoli, Pietracamela (TE), 800 m, 26/06/2010, Lasen, Herb. Lasen. MOLISE: San Massimo Matese, 600 m, 11/06/1966, Bianchini 8338, VER. CAMPANIA: Serra delle Puglie, tra Tegiano e Corleto Monforte (SA), sul detrito calcareo, $1100 \mathrm{~m}$ ca., 10/07/1921, Lacaita 198/21, Gavioli 3774, FI - m.te Alburno, da S. Angelo a C. Farina, dal Campo a C. Farina, 15/06/1942, De Philippis, FI - m.ti Picentini, m.te Cervialto, faggete, esp. N, 1600 m, 06/07/1981, Moraldo, FI - Santuario di m.te Vergine, Comunità Montana Partenio (AV), prato, 25/05/1989, Scoppola, UTV 1695. BASILICATA: falde di m.te Serino verso Lagonegro, reg. submontana, 04/06/1899, Fiori, FI - Appennino calabro-lucano, gruppo del m.te Pollino, pascoli di piano di Ruggio, 1500-1550 m ca., 24/07/1972, Nardi \& al., FI. CALABRIA: m.te Pollino, versante S (CS), 1600 m, 03/06/1988, Bianchini \& Di Carlo, Bianchini 13687, 13688, VER.

Hormuzakia SICILIA: Terranova, in arenosis, haud procul a mare, 22/05/1873, Sommier, FI - Licata, aggregata 1886, Reina, PAL 64006 - Girgenti, in arenosis maritimis, 08/1898, Ross H. Sic. 170, FI Siculiana (AG), dune di Siculiana - Torre Salsa, sabbie scarsamente evolute, $10 \mathrm{~m}$, 25/04/1999, Federico, FI - SE Manfria, dune e retrodune, 06/05/1997, Bigazzi \& Selvi, FIAF-HB 97.26.

Lycopsis arvensis LIGURIA: Dolcedo, negli uliveti, 06/1840, Berti, FI - sull'argine del Letimbro presso Savona, 15/05/1900, Mezzana, FI - valle del Trebbia, rocce scistose e diabasiche nei dintroni di Pietranera, 15/07/1977, Arrigoni \& al., FI. PIEMONTE: presso Giaglione, lungo la strada al Cenisio, regione del castagno, 11/08/1877, Aiuti, FI - Terme di Valdieri, orli dell'Orto presso il vecchio stabilimento, $1345 \mathrm{~m}, 23 / 08 / 1899$, Boggiani, FI - Torino, riva sinistra del Po, 07/1929, Zola, Gavioli 6218, FI - Perosa, nei dintorni, 04/07/1937, Negri, FI - Perrero, nei campi salendo a Traverse, 22/07/1937, *, FI - Vercelli (VC), erbosi lungo la riva sinistra della Sesia, 130 m, 09/05/1979, Soldano, Herb. Soldano 648/d - Zimone (BI), muri erbosi presso il cimitero, 07/09/1986, Soldano, Herb. Soldano 5715 - margini stradali erbosi alla Pieve di Santo Stefano Roero, $350 \mathrm{~m}$, sabbie, 20/06/1995, Selvi, FI-HB 95.19, FI - Val di Susa, alla Sacra di S. Michele (TO), 960 m, 17/07/2003, Martini, MFUMartini 9543. VALLE D’AOSTA: presso Aosta a $700 \mathrm{~m}, 18 / 03 / 1897$, Vaccari, FI - Cogne, lidi incolti sopra Cretaz, 14/08/1903, Camperio, FI - valle di Champorcher, a Folliasse (Hône), 630 m ca., 15/04/1988, Bovio 841, Herb. Bovio - lungo la mulattiera Châtillon e Grand Bruson (AO), tra Champillon e Rovarey, 775 m, 17/04/2007, Bovio \& Broglio, FI valle di Gressoney, Gressoney - Saint Jean, a S di Hoaltò, 1407 m, 23/07/2011, Cerutti \& Motta, AO-N.SFV 2678. LOMBARDIA: tra la Lombardia e il Piemonte presso i confini andando da Milano a Torino, 26/07/1841, Parlatore, FI - presso S. Salvatore di Pavia, 27/06/1856, Rampoldi, FI - Alta valtellina, in saxosis prope baln. Bormio, 05/07/1870, Levier, $\mathrm{FI}$ - lungo la strada da Pontagna a Ponte di Legno, regione del larice, $1300 \mathrm{~m}$, 17/07/1870, Parlatore, FI - val Malenco près Sondrio, 30/06/1871, Levier, FI - nel 
Mantovano, s.d., *, FI - Vigevano (PV), siepi lungo il greto della riva destra del Ticino, 25/08/1978, Soldano, Herb. Soldano 648/c. TRENTINO ALTO ADIGE: San Candido, Dobbiaco, strada per i boschi sulla destra della Drava e strada maestra sulla sinistra, 29/07/1922, Chiarugi, FI - val di Vizze, vicino alla presa dell'acquedotto di Sasso, pantano, micascisti, 1560 m, 15/08/1952, Zenari, FI - Lutago, valle Aurina, S. Martino, 15/08/1954, Zenari, FI - Prealpi trentine, val di Gresta, Rovereto (TN), Manzano, 685 m, 05/04/1997, Martini, MFU-Martini 4602 - Pieve di Bono (TN), Agrone, 600 m, 29/06/1997, Scortegagna, MNAV - alta val di Funes presso S.Pietro, incolti presso il maso "Oberpiskojhof", 25/07/2004, Selvi, FI, FI-HB 04.54 - campo di frumento presso Moos di Brunico, 920 m, 27/06/2009, Lasen, Herb. Lasen. VENETO: m.te Baldo, in cultis alle Giare di Valbrutta, 08/1890, Goiran, VER - S. Giorgio di Angarano nella casa di Balestra, 14/06/1896, Saccardo, FI. FRIULI VENEZIA GIULIA: Roveredo in Piano (PN), coltivi in loc. La Viola, 90 m, 17/05/1998, Costalonga \& Tonussi, Costalonga 647, MFU-Forojuliense 10790. EMILIA ROMAGNA: spalti presso Cervarezza nell'Appennino reggiano, 08/07/1882, Pirotta, FI - alto Appennino reggiano, Ligonchio, 10/07/1882, Fiori, FI. TOSCANA: Pian della Vite, tra Linari e la badia di m.te Scalari, presso ad una casa colonica, 24/06/1895, Sommier, FI - Vallombrosa al Saltino, 09/1900, Fiori, FI. UMBRIA: Norcia al Pian Grande di Castelluccio (PG), E 354427 e N 4742520, siti erbosi, 1306 m, 01/07/2014, Conti, APP. ABRUZzO: Palombaro (CH), Piano Laroma, $460 \mathrm{~m}$, incolti, 09/04/1999, Conti \& Manzi, APP 13151. SARDEGNA: Villanova Strisaili, incolto, $818 \mathrm{~m}$, 23/06/2012, Bacchetta, CAG. - L'esemplare di origine campana conservato in FI (Napoli, in horto botanico, in herbosis, 03/1873, Sommier) rappresentava certamente una presenza effimera, frutto di precedente coltivazione.

Lycopsis orientalis PIEMONTE: Monticello d'Alba, tra il selciato di una via del centro abitato, 18/10/1972,

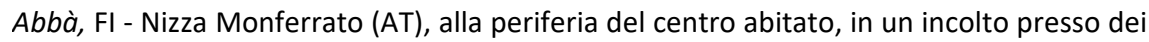
mucchi di macerie, 18/11/1972, Abbà, Fl. - Potrebbero appartenere a questa specie, ma restano da confermare, due popolazioni di recente ritrovamento, una in Veneto (Colli Euganei $(P D), 500 \mathrm{~m}, 04 / 2009$, Cassanego, www.actaplantarum.org), I'altra in Emilia Romagna (S. Demetrio Cesena (FC), $200 \mathrm{~m}$, in terreno sabbioso, 02/2013, Faggi, www.actaplantarum.org).

Melanortocarya LAZIO: in arvis di Tor d'Angelo prope Romam, 19/03/1876, De Notaris, VER - nelle obtusifolia spallette della strada fra la Storta e l'isola Farnese, 05/03/1897, Doria, FI.

Nonea echioides PUGLIA: Barletta, 1860, Bruni, G-DC. - Capitanata, S. Severo in agris, 28/05/1874, Porta \& Rigo, FI. - Indicata da PIGNATTI (1982) anche per la Puglia meridionale, presso Taranto e Otranto.

Nonea lutea PIEMONTE: S. Martino Alfieri (AT), ripa lungo la strada e in una vigna, 16/04/1969, Abbà, TO-HP. VENETO: Verona, presso la città nell'argine del torrente, s.d., Passerini, FI. FRIULI VENEZIA GIULIA: Trieste (TS), avventizia in via Paisiello, 50 m, 10/04/1996, Martini, MFUMartini 4637. EMILIA ROMAGNA: luoghi erbosi in Campo di Marte a Parma, 10/06/1907, Villani, FI - Modena, in arvis, 18/04/1929, Lunardi, Gavioli 14510, FI - Viano (RE), 03/2007, P.Ferrari, www.actaplantarum.org - colline di Traversetolo (PR), 300 m, 03/2011, Ghillani, www.actaplantarum.org. TOSCANA: nata spontaneamente nell'orto botanico di Vallombrosa, 16/07/1902, Fiori - Firenze, nei viottoli dell'orto botanico, 12/04/1916, Savelli, FI. UMBRIA: Perugia, presso la nuova stazione ferroviaria di S. Anna, 13/05/1917, Barsali, FI.

Nonea pulla PIEMONTE: val di Susa, Clavières, pendio erboso sopra Villa Luzzi, 07/1930, Santi, TO - val di Susa, Bruzolo, nei campi a grano lungo l'acquedotto sul fianco sinistro del torrente Dora Riparia, 410 m ca., 22/06/1933, Fontana \& al., FI, TO. VALLE D'AOSTA: valle di 
Cogne, sopra Champlong, pendii a E della cappella di Saint Pierre, 1620-1640 m, 25/06/2005, Bovio \& Poggio 2529, Herb. Bovio. - Indicata da FIORI \& PAOLETTI (1902) anche per la stazione di Draga, sul Carso triestino.

Nonea vesicaria SICILIA: Panormi al fiume Oreto presso la Guadagna, 03/1835, Parlatore, FI - Scoglitti, in arenosis maritimis, 15/05/1873, Sommier, FI - in sylvaticis in arenosis humentibus, Balestrate, 05/1883, Lojacono 611, FI - cimitero di Vittoria (RG), lungo la stradina per "Castelluccio" che entra nella Riserva Naturale "Pino d'Aleppo", in una valletta coltivata a orzo, suolo sabbioso "fossile", 07/05/1997, Bigazzi \& Selvi, FI-HB 97.38, FI.

Pentaglottis LOMBARDIA: Mozzate (VA), via Piccinelli, scarpata sul lato E presso il cavalcavia sulla sempervirens ferrovia, esp. SE, scarpata stradale ombrosa, ca. $270 \mathrm{~m}, 11 / 05 / 2006$, Galasso, SFI E1270, FI. VENETO: Colli Euganei, vicino di Catajo, 1825, Majer, BOLO-Bertoloni. - Esiste una dubbia segnalazione per la Liguria (CONTI \& al. 2005), della quale tuttavia non ci è stato possibile avere alcun riscontro.

Pulmonaria PIEMONTE: Westalpen, Col di Finestre zwischen Susa und Finestrelle, s.d., Kress, HBM angustifolia (orto botanico di Monaco) 385, 386 - N-Seite des Col di Tenda, s.d., Sauer $14866^{\circ}$, HBM (orto botanico di Monaco) 349. LOMBARDIA: Brescianer Alpen, S-Abfall des Pizòccolo, subalpine Grasfluren, ca. 1570 m, 31/05/1973, Sauer 16685, HBM (orto botanico di Monaco) 448. TRENTINO ALTO ADIGE: val di Ledro, in pascuis alpinis m.tis Vies, solo calcareo, 1300-1400 m, 06/1905, Porta, Fl. - Sembrano conformi alla tipica P. angustifolia, ma da confermare mediante indagini cariologiche sulle popolazioni d'origine, anche altri campioni dal Piemonte (Alpe di Varzo, Ossola, 06/1893, Rossi, FIAF 25202 - San Ambrogio, Sacra di San Michele, 09/05/1908, Zola, Gavioli 3767/1, FI - S. Michele di Mondovi, Bicocca di San Giacomo, 18/04/1909, Zola, Gavioli 3767/2, FI), e dal Trentino Alto Adige (m.te Bondone, 02/07/1994, Galesi, CAT 108587); certamente di origine orticola è invece uno siciliano (Aetnae, in arenosis, 1830, s.coll., CAT-Tornabene 7951).

Pulmonaria LIGURIA: Appennino piemontese, Vara Superiore (SV), al margine di un boschetto di australis faggio al passo del Faiallo, 24/05/1979, Abbà, TO-HP. VALLE D'AOSTA: val di Chavannes, 2000-2200 m, 10/08/1900, Vaccari, FI - Aosta, Pont S. Martin, Valeille (Ivery), $800 \mathrm{~m}$ ca., 22/04/1982, Kaplan, AO-N.SFV 2870 - valle di Gressoney, lungo la mulattiera Gressoney La Trinité - Alpenzù Piccolo, 1800 m, 13/06/1993, Bovio \& Cerutti, AO-N.SFV 984 - m.ti di Issogne (AO), nei prati di Cresta, 980 m, 30/04/2006, Bovio, FI. PIEMONTE: Valgrande, strada Corte Bué - Ompio, sopra Alpe Baker, 1000 m, 14/04/1914, Boggiani 303, FI - valle Formazza, sentiero delle Pecore tra Zum Stock e Valscerbiela, 2200-1940 m, 26/07/1919, Boggiani 642 - Ceresole d'Alba (CN), radura nel bosco, quasi al confine con Pralormo (TO), sradicata e coltivata a Chieri (Villa Brea), 25/04/1989, Abbà, TO-HP - Pino Torinese (TO), verso Superga, quasi all'altezza della Cascina dell'Abate, alla sinistra della strada panoramica, versante della valle Gola, 16/05/1989, Abbà, TO-HP - Mont Cenis, s.d., Huguemin, VER. LOMBARDIA: Prealpi berbamasche, da Zambla (BG) a Baita Zuccone (Pizzo Arera), 1253-1800 m, 06/07/1956, Chiarugi \& al., FI - passo Croce Domini (BS), prati ghiaiosi e rocce sopra la Malga Cadino di Branca, 1850-1950 m, 05/08/1969, Steinberg \& Ricceri, FI - Rovagnate (LC), via Spiazzo, $361 \mathrm{~m}$, radura boschiva termofila, 15/03/2012, Villa, MSNM 43424 - Serle (BS), s.d., Pa*, Bianchini 3319, VER. TRENTINO ALTO ADIGE: Tirolia australis. In collibus et montibus circa "Trient." $450 \mathrm{~m}$. s. m., 04/1901, Murr, B 100365437 [materiale originale] - Predazzo, cima del m.te Viezena, 07/1930, Corti, FI - praterie inondate torbo-lacustri della conca del m.te Bondone, 18/06/1962, Moggi \& Ricceri, FI - val di Fassa, dalla stazione della funivia di Col Rodella lungo il sentiero del sasso Lungo, su terreni vulcanici acidi, 08/07/1974, Ricceri \& Steinberg, FI - valle di Trafoi, strada dello Stelvio, nei pressi dell'albergo sotto Stelvio, 2150 m ca., 16/07/1974, Ricceri \& Steinberg, FI - Baselga di Pinè (TN), bosco di abete rosso e larice sul Dosso di Miola, lungo il sentiero che vi sale dalle sponde del lago di Serraia, 1000 m, 18/09/2013, Cecchi \& al., FI-HB 13.71, FI. VENETO: Euganeis, m.te della Madonna supra pagum Rovolone, 02/04/1877, C.Massalongo, VER - m.te Baldo, prati e 
pascoli al passo del Prada, 15/05/1971, Steinberg \& Ricceri, FI - Calcola, Alano, $450 \mathrm{~m}$, castagneto, 13/05/1991, Lasen, Herb. Lasen - Arcugnano (VI), Perarolo, $250 \mathrm{~m}$, prato, 13/03/1992, Scortegagna, MNAV - m.te Belloca, Tregnago (VR), 600 m, 16/04/1996, Ziviani, Bianchini 21686, VER - zona Mavenza-Valpiana, Limana (BL), $800 \mathrm{~m}$, margine bosco, radura, 04/05/1996, Argenti \& Argenti, Herb. Argenti - S. Ambrogio di Valpolicella (VR), verso la Grola, 260 m, 31/03/1999, Di Carlo 178, VER - altopiano di Asiago (VI), Porta Manazzo, $1800 \mathrm{~m}$, margine di mugheta, 01/07/2004, Scortegagna, MNAV - crinale Col Zesta, Vette, $2050 \mathrm{~m}, 11 / 06 / 2007$, Lasen, Herb. Lasen. FRIULI VENEZIA GIULIA: Basovizza (TS), bosco, 350 m, 15/04/1991, Barbo, MFU-Forojuliense 10738 - m.te Joànaz (UD), 09/04/2007, Buccheri, MFU-Forojuliense 10906 - Piancavallo (PN), Casera del medico, 1220 m, 26/04/2008, Buccheri, MFU-Forojuliense 13101 - m.te Porgeit (PN), 15/05/2008, Buccheri, MFU-Forojuliense 14289.

Pulmonaria hirta LIGURIA: Appennino ligure, dintorni del Passo di Giovi, 472 m, 27/04/1975, Peccenini, Herb. Peccenini - Varese ligure, Taglieto, ca. 500 m, 21/06/1990, Ballelli, BOLO. EMILIA ROMAGNA: Piandelagotti, sotto il colle, 23/03/1920, Lunardi, FI. TOSCANA: solco degli Streghi a Campognano in val di Serchio, 08/04/1861, Beccari, FI - Signa, San Romolo, 12/07/1933, Corti \& Corradi, FI - m.te Cetona, 05/07/1935, Corti, FI - Consuma (FI), 13/07/1942, Corradi, FI - Cerbaie, nel bosco di valle Lupitana, 06/05/1951, Contardo, FI [neotipo di P. picta] - fra Larderello e Serrazzano (PI), nel cerreto, $500 \mathrm{~m}$ ca., 29/06/1953, Chiarugi \& al., FI - m.te Senario (FI), versante N, 500 m ca., 27/04/1958, Lanza, FI - Alpi Apuane, dalla Montanina al canalone sotto il ponte per il m.te Procinto, 03/04/1959, Martinoli, FI - S. Casciano, bosco di quercia e prunastro, ca. 200 m, 27/03/1963, Casini, FI - Preappennino pistoiese-lucchese, Penna di Lucchio, pascoli e rupi del crinale a $\mathrm{N}$ della vetta principale, 800-1176 ca., 20/07/1970, Nardi, FI - Dicomano, Pruneta, lungo il torrente, 04/05/1980, Rizzotto, FI - Caprigliola (MS), bosco, 300 m, 03/05/1991, G.Bedini, PIAGR - m.te Labro (GR), 900-950 m, 15/03/1992, Selvi 412, FI - prov. Arezzo: montane Abies-Fagus woods close around the walls of Eremo di Camaldoli, $1100 \mathrm{~m}, 26 / 03 / 2002$, Selvi FI 2356, BM; ibidem, 31/05/2002, Cristofolini, FI 2366, BM [epitipi] - m.te Pisano, sopra Pozzuolo, sentiero CAI verso Gattaiola, 203 m, 05/04/2008, Pierini \& Marchetti, PI - Chianni (PI), m.te Vitalba, strada verso la cima, calcari a calpionelle, argille a palombini, 520-650 m, 21/05/2009, s.coll., PI. UMBRIA: boschi presso Baccano, S. Vito in Monte, 01/04/1886, Cicioni, Herb. Cicioni, PERU 1181911 - M. Malbe nelle selve del versante settentrionale, 04/1889, Cicioni, Herb. Cicioni, PERU 1182011 - Armenzano, alle falde orientali del M. Subasio, 15/05/1909, Chelli, Herb. Cicioni, PERU 1181711 - Collemancio (PG), orno-ostrieto, 03/03/1974, Ubaldi, BOLO 508144. LAZIO: distretto vulcanico laziale, m.ti Cimini, m.te Cimino (VT), faggeta, 900 m, 07/04/2007, Scortegagna, MNAV.

Pulmonaria TRENTINO ALTO ADIGE: Marzola (TN), sentiero bivio II, sella Marzola, $1640 \mathrm{~m}$, officinalis subsp. 19/06/2013, Pupillo, FI 7164 [olotipo]. - Riportato anche per le vicine Val di Sole e la Val marzolae di Chiese (ASTUTI \& al. 2014). Nonostante i dubbi avanzati a tal proposito da AstUTI \& al. (2014), non escludiamo che possano riferirsi a popolazioni di questa entità anche le località citate nel protologo di P. tridentina: Tirolia australis: Mori, Monte Brugino, in dumetis; Tridentum in faucibus torrentis Salé; Goccia d'Oro", 25.04, 25.05, 15/12/1893, Evers 8648, GZU 272989, 272986, 272987, 272988 - In angustiis torrentis Salé; Goccia d'Oro - Val di Ledro, Biacesa, in dumetis Malgae Dromä̈", 03/1891, 05.1895, Evers, GZU 272990, 272991.

Pulmonaria PIEMONTE: Vogogna, lungo la rotabile di Berna, $250 \mathrm{~m}, 03 / 04 / 1914$, Boggiani, FI officinalis subsp. Rovegro, ponte Carletto lungo il canale idroelettrico, 300-400 m, 13/04/1914, Boggiani, officinalis $\mathrm{FI}$ - Leyni, 03/03/1919, Vatova, FI - Borgomanero, 25/05/1919, Vatova \& Negri, FI Augusta Taurinorum, val Salice, prope viam del nebiola, 18/03/1927, Zola, Gavioli 11910, $\mathrm{FI}$ - an der Strasse von Netro nach Graglia bei Biella, ca. 2,4 km nach Netro, von der grossen Kurre, $700 \mathrm{~m}$, 19/08/1976, Bolliger, FI - Moncalvo (AT), margine boschi fra il paese e la stazione di Ponzano, 20/04/1979, Soldano, Herb. Soldano 2544 - Roasio (VC), umidi alla sinistra della strada per Rovasenda al quadrivio di Curavecchia, 12/05/1985, 
Soldano, Herb. Soldano 5534 - laghi di Ivrea (TO), bosco di latifoglie su suolo umificato, 02/04/1995, Bovio 1711, Herb. Bovio. VALLE D'AOSTA: Aosta, in un orto a Chevrod, 14/04/1899, Vaccari, FI. LOMBARDIA: Milano, bosco di Redecesio, 12/03/1876, E.Corti \& Mazzucchelli, FI - Bormio, lungo il *, 15/08/1896, s.coll., FI - Prealpi insubriche, strada Laveno - Caldè (VA), loc. Sasso Galletto, boscaglie e rupi, 27/04/1952, Negri \& Bavazzano, FI - Tignale (BS), m.te Sernifa, 800 m, 16/05/1970, Chiapella, FI - salendo dal lago d'Idro verso Capovalle, 13/05/1971, Steinberg \& Ricceri, FI - Mantova, bosco Fontana, lungo il viale da piazza Carbone all'ingresso, 30/07/1978, Barini 248, FI - Oltrepò pavese, Voghera (PV), fra S. Albano e Mendasco, $600 \mathrm{~m}, 03 / 05 / 1986$, Martini, MFUMartini 4672 - Alpi tra Valnegra e S. Giovanni Bianco, s.d., Rodigher, Gavioli 9041, FI m.te Misma, s.d., Rodigher, Gavioli 9040, FI. TRENTINO ALTO ADIGE: San Martino di Castrozza, sui margini del primo affluente di sinistra del Cismon, a valle di San Martino, 16/07/1892, Bargagli, FI - Cimirlo-Stelar, 690 m, 09/2012, fotografia (Astuti \& al. 2014: 149). VENETO: alveo del Piave, ponte di Cridola, 20/05/1909, Minio, FI - prima di Schievenin, 09/04/1916, Minio, FI - altopiano di Asiago, Granezza, pascolo e ceduo, calcari, 1405 m, 30/05/1951, Zenari, FI - alta pianura veneta (TV), bosco di Vizze, 15 m, 17/04/1983, Martini, MFU-Martini 4674 - Fistere (BL), 380 m, siepe, 30/06/1983, Argenti \& Argenti, Herb. Argenti - m.te Cornetto, faggeta, 1450 m, 18/08/1984, Curti, MNAV Montecchio Precalcino, loc. Bastia, boscaglia di Robinia, castagneto, 18/03/1986, Bettanin, MNAV - Crespano (TV), loc. Valderoa, 21/04/1993, Sburlino, UVV - Valrovina, boscaglia di latifoglie, $300 \mathrm{~m}, 11 / 03 / 1995$, Turcato, MNAV - Salcedo (VI), valle di Sotto, ostrieto, 280 m, 01/05/1995, Scortegagna, MNAV - Sarego (VI), Meledo Alto, 150 m, 14/04/1996, Scortegagna, MNAV - Vajo Borago di Avesa (VR), 250 m, 31/03/1999, Di Carlo \& Tosi, Di Carlo 786, VER - m.ti di Ornella, Livinallongo (BL), 1800 m, margine bosco, 27/06/2010, Argenti \& Argenti, Herb. Argenti. FRIULI VENEZIA GIULIA: Pasiano di Pordenone, 03/03/1906, Pagani, MFU-Forojuliense 10731 - Premariacco, Rocca Bernarda, 01/04/1947, Fornaciari, MFU-Forojuliense 10724 - via al Volaja, $1400 \mathrm{~m}$, 29/06/1951, Fornaciari, MFU-Forojuliense 10722 - m.te S. Simeone, 1000 m, 28/05/1966, Morandini \& al., MFU-Forojuliense 10717 - Prealpi Giulie (UD), m.te Postoncicco, vers. N, 1060 m, 01/07/1984, Martini, MFU-Martini 4673 - Muzzana (UD), Bosco Bando, 5 m, 08/04/1997, Mazzolini, MFU-Martini 4671 - Cividale (UD), Castelmonte, $500 \mathrm{~m}$, 25/04/2001, Dermit, MFU-Martini 4669 - Pielungo (PN), 650 m, 27/03/2002, Dorigo, MFU-Martini 4670 - Gorizia (GO), 23/03/2003, Ussai, MFU-Forojuliense 10715. EMILIA ROMAGNA: Parma, bosco Carrega, 27/04/1919, Minio, FI - Montefiorino, $800 \mathrm{~m}$, 05/1934, Mori, FI - Piandelagotti, boschi, 1200 m, 03/05/1955, Lunardi, FI. TOSCANA: culta in vaso a Orsigna (PT), da pianta raccolta nel bosco lungo il torrente Bagnone sopra lera in Lunigiana, 15/05/2008, Selvi, Fl-HB 08.44, Fl. - La presenza della specie è stata recentemente confermata anche per le Marche ("Montefortino (Fermo), Parco Nazionale dei Monti Sibillini, Gole dell'Infernaccio, (WGS84 $42.914381^{\circ} \mathrm{N} ; 13.260342^{\circ} \mathrm{E}$ ), woods along the pathway going across the gorges of Fiume Tenna, $1000 \mathrm{~m}^{\prime \prime}, 24 / 05 / 2016$, Peruzzi, Fl, Pl; L. Peruzzi \& G. Astuti in BARTOLuCCI \& al. 2016) e per l'Abruzzo ("valle del Venacquaro in loc. Fonte Novello (Fano Adriano), 77.07-77.06 [datum ED50 33T] 10871330 m", 20/04-18/06/2010, Bartolucci \& al., APP; "Colle Abetone-Prati di Incodara-Colle Andreole (Fano Adriano), 76.07-75.06-76.06 [datum ED50 33T] 1775-1615 m", 26/0519/06/2010, Bartolucci \& al., APP; BARTOLUCCI \& al. 2012).

Pulmonaria stiriaca FRIULI VENEZIA GIULIA: valle del torrente Natisone (UD), versante orografico destro (O) fra Antro e Spignon, ca. 500 m, boschi castanili su fleysch, 9846.2/3, 22/04/2007, Poldini, Herb. Poldini.

Pulmonaria LIGURIA: Varazze, in valle Moreana, 29/04/1919, Gresino, Gavioli 12154, FI - tra Memola vallarsae subsp. e la SS Aurelia, in valle di Vara (SP), $100 \mathrm{~m}$ ca., 30/04/1994, Marchetti, MFU-Martini apennina 4676. PIEMONTE: Cuneo, Certosa, 04/03/1946, Berluti, FI [paratipo] - Roccavione (CN), 
s.d., Biadego, VER [paratipo] - Comune di Oncino (CN), frazione Ruera, sul fianco destro della strada che sale verso Ruata, 1070 m, 15/05/2010, Soldano, Herb. Soldano 15025. EMILIA ROMAGNA: Parma, base umida delle sponde del Baganza, 06/04/1920, Minio, FI [paratipo] - Appennino tosco-emiliano, passo del Lupo, 1500 m, 25/05/1955, Chiarugi, FI [paratipo] - Appennino tosco-emiliano, dintorni di Piandelagotti (MO), s.d., Lunardi, FI valle dell'Inferno (PC), m.te Lesima, $1280 \mathrm{~m}$, bosco, 08/06/1986, Crippa-Gliozzi, PI Modigliana (FC), 1988, s.coll., BOLO [paratipo] - Parco Talon, Casalecchio di Reno, 26/04/1996, Puppi, FI 7200 [olotipo], BOLO [isotipo]. TOSCANA: Montopoli, 07/04/1863, Beccari, Fl 7204 [paratipo] - Versilia, tra Stazzema e la foce di Petrosciana, 30/03/1923, Chiarugi, FI 7201 [paratipo] - Preappennino pistoiese-lucchese, ontaneto sul torrente Pescia di Pescia presso il ponte di Castelvecchio, 03/04/1965, Nardi, FI - Firenzuola, bacino idrografico del torrente Diaterna, castagneto con carpino e faggio sul m.te Coloreta, versante N, 13/06/1973, Hoffmann, FI - Alpe della Luna (AR), da case Val di Canale, $682 \mathrm{~m}$, salendo a m.te Maggiore, $1400 \mathrm{~m}$, 05/06/1987, Raffaelli \& al., FI Comano (MS), La Villa del Montale, ai margini della Prada, 300 m, terrazzo fluvio-glaciale, sotto carpini bianchi e cerri, 02/04/1995, Ferrarini, FI. MARCHE: dintorni di Urbisaglia, fruticeti e luoghi boschivi nella selva dell'Abbadia, suolo prevalentemente argillososabbioso ed argilloso-ghiaioso-sabbioso, humus più o meno scarso, 10/05/1969, Brilli Cattarini \& al., PESA - gruppo del m.te Carpegna, luoghi boschivi tra la cantoniera di Carpegna e il Ranco del Cerro, 1000-1025 m, suolo prevalentemente argilloso o argillosocalcareo, humus più o meno abbondante, 18/06/1975, Brilli Cattarini, PESA - gruppo del m.te Nerone, boschi degradati (faggeta) nel versante $S$ del m.te Nerone, tra il Casalino della Fontanella e Pian di Rosato, 1300-1325 m, suolo prevalentemente calcareo, humus più o meno abbondante, 26/05/1977, Brilli Cattarini, PESA - dintorni di Camerino, luoghi boschivi tra la Villa Napoleoni e il Torrone, 700-725 m, suolo argilloso-sabbioso o sabbioso-molassico, humus più o meno abbondante, 05/05/1977, Brilli Cattarini, PESA dintorni di Cupramontana, luoghi boschivi freschi o umidi nella valle della Romita sotto l'Eremo delle Grotte, $225-250 \mathrm{~m}$, suolo prevalentemente sabbioso, humus più o meno abbondante, 25/04/1979, Brilli Cattarini \& Gubellini, PESA - dintorni di Pergola, fruticeti e luoghi boschivi attorno a Montaiate, 550-600 m, suolo prevalentemente arenaceo o arenaceo-argilloso, humus più o meno scarso, 06/05/1967, Brilli Cattarini \& al., PESA dintorni di Fano, luoghi boschivi nella valle del torrente Arzilla presso il Ponte Nuovo, 25$50 \mathrm{~m}$, suolo prevalentemente argilloso-sabbioso, humus più o meno abbondante, 11/04/1974, Brilli Cattarini, PESA - dintorni di Comunanza, luoghi boschivi (carpineto misto, cerreto misto) nella valle del torrente Cinante presso Gesso, $425-450 \mathrm{~m}$, suolo arenaceo, humus più o meno abbondante, 31/05/1982, Brilli Cattarini \& Gubellini, PESA. UMBRIA: Scheggia, presso Gubbio, 680 m, 27/10/1990, Tibiletti, BOLO 508133 [paratipo]. LAZIO: Cammerata Nuova, fosso Fiojo, 1000-1200 m, 30/05/1904, Vaccari, FI - Riano (RM), 1980, Caprolati, RO-HR [paratipo] - m.te Terminillo, 06/1983, Anzalone, RO-HR [paratipo] - m.ti Simbruini, zona Trevi - Valle Pietra, 24/05/1985, Anzalone, RO-HR [paratipo] - Castelli Romani, Rocca Priora (RM), II Cerquone, $550 \mathrm{~m}$, in a Quercus cerris and $Q$. robur wood, 25/03/1992, Iberite \& al., SEEM 16374, FI. ABRUZZO: Sirente, tra Ajelli e i prati di Santa Maria, 900-1500 m, 31/05/1903, Vaccari, FI - Porcinaro, valle di S. Giov., fra Aquila e Teramo, pascoli ad O di Casa Cappelli, 1200-1400 m, 12/06/1906, Vaccari, FI - Ovindoli, Piano di Pezza, 02/06/1929, Chiarugi, FI - Parco Nazionale d'Abruzzo, val Camosciara, 01/08/1953, Chiarugi \& al., FI - Majella, Palena, 1000 m, 07/1987, Cristofolini \& al., BOLO [paratipo]. MOLISE: S. Egidio Matese, boscaglia, 1200 m, 1979, Bianchini, FI. CAMPANIA: m.ti Picentini, m.te Terminio, faggeta, 1400-1750 m, 03/07/1976, Moraldo, FI - Cilento (SA), m.te Cervati, versante O, faggeta di Vallivona, versante destro della valle, $1300 \mathrm{~m}$ ca., 18/04/1986, Moggi \& al. 7839, FI-Cilento - Piano di Pecore, faggeta, $1200 \mathrm{~m}, 05 / 04 / 2003$, Stinca, Herb. Stinca - valle di Amalfi (SA), in silvis pinguibus, $800 \mathrm{~m}$ ca., s.d., Lacaita, Gavioli 3768, FI. BASILICATA: Potenza, in silva Aria Silvana, 900-1200 m, 06/05/1940, Gavioli, FI - strada per Grumentum scavi, 22/04/1995, Galesi, CAT-generale 107317. PUGLIA: Gargano, cerreto-carpineto a metà strada fra S. Giovanni Rotondo e Cagnano Varano, 600-650 m, 27/05/1968, Moggi, FI. CALABRIA: m.ti da Giffone a Serra S. Bruno, 1907, Pasquale \& Lacaita, NAP [paratipo] - Aspromonte (RC), 
torrente Vasì presso S. Eufemia, bosco ripariale a ontano nero, 03/03/2002, Cameriere \& al., FI - Sila Piccola, Villaggio Mancuso (CS), pineta a Pinus nigra nei pressi del centro visite in località Monaco, ca. 1300 m s. I. m., 23/05/2007, Cecchi \& al. 1096, Herb. Cecchi.

Pulmonaria TRENTINO ALTO ADIGE: Tirolia australis, Vallarsa, ca. 1870, Kerner, WU 69919 vallarsae subsp. [lectotipo], WU 71728, 71729, 71730, 71731, 71732, 71733, 71734, 71735, 71736, vallarsae $71737,71738,71739,71740,71741,71742,71743,71744,71745,71746,71747,71748$, 71749, 71750, 71751, 71752, 71753, 71754, 71755 [isolectotipi]. VENETO: m.te Serva, Croda del Sal (BL), 1170 m, margine bosco, 27/05/1983, Argenti \& Argenti, Herb. Argenti - Nogarè (BL), 340 m, bosco alveale, 21/06/1983, s.coll., Herb. Argenti - Lamon, verso Casere Miotti da Le Prese, 690 m, 04/05/1997, Lasen, Herb. Lasen - Fregona (TV), strada per Cansiglio dopo Osigo, 500 m, 14/03/1999, Pavan, MFU-Forojuliense 10736 - Maser (VI), lungo la salita della Forcella Mostaccin sul monte Collalto, 06/06/2006, Cecchi \& Coppi 664, Herb. Cecchi.

Symphytum PIEMONTE: in una vigna a Crea, 14/06/1905, Ferrari, TO-HP - Busca, siepe lungo la strada asperum per l'Eremo, 21/06/1913, Ferrari \& Gola, TO-HP - Alpi Cozie, valle Grana, erbosi secchi a Campomolino, $1150 \mathrm{~m}, 12 / 07 / 1956$, Mondino, TO-HP. TRENTINO ALTO ADIGE: Alto Adige, Vipiteno, imbocco di via Ridanna, prati acquitrinosi verso Casateja, alluvioni miste, 950 m, 15/08/1950, Zenari, FI. FRIULI VENEZIA GIULIA: Monteaperta di Taipana (UD), dietro il bar, 08/1994, Verona, MFU-Forojuliense 10761, 10762. TOSCANA: Mugello, fra Razzolo e la Colla di Casaglia, presso la locanda, 23/06/1907, Sommier, FI - Di questa specie ci è noto anche un secondo campione toscano ("presso Firenze, Cascine, inselvatichita a Scienza dell'Orticoltura, 42 m", 17/05/1929, Fiori, FIAF 25094), ma si trattava di pianta cresciuta in ambiente inidoneo alla sua diffusione, e dunque di un popolamento inevitabilmente effimero; lo stesso vale probabilmente anche per la popolazione segnalata da Fiori (1926) per "Ca' Tron nel Trevigiano", della quale tuttavia non ci è stato possibile avere riscontro tra i reperti esaminati.

Symphytum LIGURIA: Bordighera, ad fossam, 16/04/1908, Gresino, Gavioli 13944, FI - Caravonica bulbosum (IM), presso il paese, 1930, Corradi, PI-Passerini - Sestri Levante (GE), 17/03/1974, Peccenini, Herb. Peccenini - fondo delle doline di Pian di Balè, 500 m, 10/04/1984, O.Cecchi, PI - Caprione (SP), Montemarcello, strada La Ferrara, 01/04/2002, Baldini \& Maccini, PI. PIEMONTE: Novi Ligure (AL), Parco Castello, esp. E, 200 m, scarpata, 27/04/1989, Carrega, TO-HP - Torino collina, Parco Leopardi, 290 m, 15/04/2000, Pandolfo \& Rosenkrantz, TO-HP. LOMBARDIA: in saepibus prope Portisa ad Benacum (BR), calc., 100-200 m, 03/05/1886, Rigo, NAP-Rigo - Milano, zona Baggio, parco delle cave, tra la cava Cabassi e la cava Aurora, 120 m, margine, 13/04/1991, Galasso, MSNM 25929, 25930, 25931 - bassa Brianza, Vimercate, destra idrografica del torrente Molgora, all'altezza dell'asilo di via Galbussera, $191 \mathrm{~m}$, boscaglia di robinie, 30/03/1994, Rovelli, MSNM 34897 - Lentate sul Seveso (MI), P. Groane, scarpatella al margine del bosco intorno allo stagno di Lentate, 15/04/2007, Gariboldi, MSNM 41225, 41226. TRENTINO ALTO ADIGE: in arvis a Selva, 04/1865, Saccardo, FI - Terlan (BZ), Terlan, Weg Nr. 9 Margarethenweg vor Maultasch, Flaumeichen, Hopfenbuchen, Mannaeschen, 275-375 m, $11^{\circ} 14^{\prime}$ 56,8407" E, 46 31' 46,2806" N, 26/04/1999, Stockner, BOZ PVASC 410. VENETO: Trissino (VI), prato, $100 \mathrm{~m}, 08 / 05 / 1886$, M.Savi, MNAV - Minerbe, Legnago, 08/04/1896, Bolzon, FI - Venezia, * Montagnola, giardini, 12/04/1949, Minio, FI Molvena, sponde del torrente Lavarda, 01/05/1986, Bettanin, MNAV - Favorita Valdagno (VI), parco, 200 m, 21/04/1987, M.Savi 479, MNAV - Padova, 19/04/1993, Sburlino, UVV Longare (VI), a margine di un campo di granturco, 06/04/1997, Baron, MNAV - Fontane Bianche di Falzé (TV), 100 m, prato, incolto, 06/05/2003, Argenti \& Argenti, Herb. Argenti - Ca' Chimelli, Euganei, 60 m, 14/04/2004, Lasen, Herb. Lasen - Prai di Castello di Godego (TV), 55 m, riva fossato, 13/04/2009, Argenti \& Argenti, Herb. Argenti. FRIULI VENEZIA GIULIA: Grado, isola del cimitero, 19/04/1964, Fornaciari, MFU-Forojuliense 10747 - 
Latisana (UD), 19/04/1996, Quarin, MFU-Martini 4681 - Udine (UD), viale Vat, $110 \mathrm{~m}$, 25/04/1999, Buccheri, MFU-Martini 9897 - Trieste (TS), S. Giovanni all'interno dell'ex OPP, 65 m, 07/04/2001, Pichierri, MFU-Martini 4679 - Sacile (PN), scarpata canale, 10/04/2001, Argenti \& Argenti, Herb. Argenti. EMILIA ROMAGNA: contorni di Faenza, 04/1864, Caldesi, FI - lungo i fossati nei dintorni di Carpi (MO), 26/04/1883, Pirotta, FI parco fluviale di Casola Valsenio (RA), boscaglie igrofile dei terreni fluviali, 25/04/2000, Buoso, BOLO 506826 - Parco dei Cedri (BO), lungo la golena del fiume Savena, all'ombra di robinie e tigli, zona umida, 45 m, 27/04/2002, Nucciotti, BOLO 40634 - Parma, s.d., Passerini, FI. TOSCANA: Senae in fossis prope il Riluogo, 03/1857, Campani, $\mathrm{FI}$ Pratovecchio, 21/04/1868, Siemoni, FI - Casentino, Giogana, 10/05/1871, Siemoni, FI Sarteano, nell'orto presso il canneto, 29/04/1891, Bargagli, FI - insula Igilium, all'Arinella, 01/03/1897, Sommier, FI - insula Elba, presso Rio Marina, luoghi erbosi lungo il fosso, 30/04/1904, Sommier, FI - Arezzo, lungo i muri fra l'erba sul prato della palestra, 19/04/1911, Savelli, FI - Buggiano, 23/03/1933, Pichi Sermolli 1321, FI - alta valle tiberina, Gelmi pr. Cignano, 04/1937, M.Pichi Sermolli, Pichi Sermolli 7162, FI - isola di Montecristo, vallone di Cala Maestra, nel fosso presso la villa, 01/04/1965, Fabbri \& al., FI - Massa, nel rione della Rocca, 25/03/1979, Marchetti, FI, PI - m.te Argentario (GR), Porto S. Stefano, ex giardino Jacovacci, 05/03/1994, Baldini, FI - Vernio, sulla SS 324, lungo un torrente secco, 04/04/1999, Peruzzi, PI - strada panoramica Molina di Quosa (PI), 200 m, 20/04/2005, Pierini, PI - Firenze (FI), parco delle Cascine, sottobosco dell'area occidentale, tra I'Indiano e piazza J. F. Kennedy, 08/03/2006, Cecchi 447, Herb. Cecchi agro di Montieri presso Travale, siepi e campi attorno al cimitero, ca. 500 m, 21/03/2009, Selvi PI. Etr. Merid. 3234, FI. MARCHE: dintorni di Loreto, luoghi erbosi e campi coltivati lungo il Rio della Lavanderia presso l'azienda Tabacchi, ca. $20 \mathrm{~m}$, suolo argilloso-sabbiosomolassico, 15/04/1964, Brilli-Cattarini, PESA - dintorni di S. Elpidio a Mare, luoghi erbosi nella valle dell'Ete Morto presso Casette d'Ete, $35 \mathrm{~m}$, suolo prevalentemente sabbiosomolassico, 16/04/1964, Brilli-Cattarini, PESA - dintorni di Pergola, luoghi erbosi freschi e campi coltivati sul m.te di S. Croce presso Monterolo, 450-500 m, suolo prevalentemente argilloso od argilloso-calcareo, 20/04/1967, Brilli-Cattarini, PESA - m.ti della Cesana, campi coltivati e luoghi erbosi incolti nel basso versante NE dei m.ti della Cesana lungo il fosso della Cerbaria (valle del Rio Puto), 125-200 m, suolo argilloso, 29/04/1969, BrilliCattarini, PESA - dintorni di Pesaro, luoghi erbosi freschi e campi coltivati lungo il fosso della Biscia presso Borgo S. Maria, 40-50 m, suolo argilloso, humus nullo, 11/04/1971, Brilli-Cattarini \& Sialm, PESA - dintorni di San Severino Marche, campi coltivati e luoghi erbosi incolti freschi lungo il Fosso Grande tra Césolo e Marciano, ca. $275 \mathrm{~m}$, suolo arenaceo o arenaceo-argilloso, humus nullo, 06/05/1971, Brilli-Cattarini \& Sialm, PESA dintorni di Montottone, fruticeti freschi o subumidi lungo il Rio dei Piani presso l'affluenza nell'Ete Vivo, 100-125 m, suolo argilloso-sabbioso, humus nullo o scarso, 30/04/1973, Brilli-Cattarini \& al., PESA - dintorni di Arcevia, fruticeti radi e luoghi erbosi incolti lungo le sponde del torrente Fenella presso Palazzo, $250 \mathrm{~m}$, suolo prevalentemente argilloso-calcareo, humus nullo o scarso, 25/04/1975, Brilli-Cattarini, PESA - dintorni di Ancona, siepi e luoghi erbosi incolti tra il m.te del Trave e il Montirozzo, 150-200 m, suolo prevalentemente argilloso, humus subnullo, 09/04/1981, BrilliCattarini \& Gubellini, PESA - dintorni di Sarnano, siepi e campi incolti nel settore N della Macchia da Foco, 550-575 m, suolo prevalentemente arenaceo, humus nullo o scarso, 13/04/1981, Brilli-Cattarini \& Gubellini, PESA - gruppo del m.te Catria, fruticeti freschi e luoghi boschivi (bosco misto ceduato) nel versante $\mathrm{N}$ del m.te dei Prati di Nocria, 525-550 m, suolo calcareo o più o meno decalcificato, humus da scarso a più o meno abbondante, 26/04/1983, Brilli-Cattarini \& Gubellini, PESA - m.ti della Laga, luoghi erbosi freschi e fruticeti radi a Matera d'Acquasanta, ca. $750 \mathrm{~m}$, suolo arenaceo, humus da scarso a più o meno abbondante, 11/05/1984, Brilli-Cattarini \& Gubellini, PESA. UMBRIA: dintorni di 
Terni, lungo i margini dei fossi, 18/04/1889, Mari, FI - Perugia, lungo un fosso fuori Porta S.ta Croce, 04/1898, Palomba, FI - m.te Subasio, strada delle Carceri, 25/04/1887, Frizzi, CAT-Frizzi 1426. LAZIO: dintorni di Viterbo, alle Patrelle, luoghi umidi, 19/04/1894, Mari, FI - Tivoli, prati e siepi tra I'Ospitale e l'Aniene, 02/04/1905, Vaccari, FI - Monterotondo (RM), 15/03/1951, Steinberg, FI. ABRUZzO: Martinsicuro, luoghi erbosi incolti freschi sull'argine destro del Tronto presso la foce, ca. $5 \mathrm{~m}$, suolo prevalentemente sabbioso o argilloso-sabbioso, humus nullo o subnullo, 09/04/1984, Brilli-Cattarini \& Gubellini, PESA - Parco Nazionale della Majella, bosco di Ateleta (AQ), 14/04/1998, Conti, APP 35981 Vasto $(\mathrm{CH})$, presso il Lebba, $60 \mathrm{~m}$, boschetto di cerro e roverella, 30/03/2002, Conti, APP 37093 - Riserva Naturale di Atri (TE), 150-350 m, 16/04/2009, Conti, APP 37758. CAMPANIA: valle di S. Rocco presso Napoli, 03/1846, Bruni, FI - Caserta, in argillosis humentibus, in sylvis et ad sepes, 90 ped. paris., 05/1872, Terracciano, FI - Hirpinia, Abellinum, locis humidis, arenosis, secus fossas ed ad sepes, $500 \mathrm{~m}$, solo siliceo, 25/04/1907, Trotter, FIE 947bis, FI, GE m.ti Picentini, m.te Raia, bosco misto, 500-700 m, 01/03/1975, Moraldo, $\mathrm{FI}$ - m.te Bulgheria al paese di Acquavena, ca. 700-800 m, 12/05/1994, Bigazzi, FI-HB 94.08 - Penisola Sorrentina, boschi misti e lungo i fossi nei pressi di Agerola, 11/05/1995, Bigazzi \& Selvi, FI-HB 95.21. BASILICATA: Potenza, Montereale ad sepes, 05/1907, Gavioli 3793, FI. PUGLIA: inter segetes prope Gallipoli, 05/1881, Groves, FI - paludi di Otranto, 05/1883, Profeta, FI. CALABRIA: in herbitis olivetorum prope Pizzo, 05/05/1877, Biondi, FI - dintorni di Catanzaro, 15/04/1883, Fiori, FI - dintorni di Cosenza, 24/05/1898, Preda, FI - Papasidero (CS), Massa, 250 m, 09/05/2009, Di Marco, PI - Tarsia (CS), residui lembi di querceto tra i campi di cereali sui colli a ovest del paese, 14/05/2015, Biaggini \& al., FI. SICILIA: Syracusae ad flumen Anapo, 08/04/1873, Sommier, FI - Palermo, ad flumen Oreto in herbosis, 03/1891, Di Giovanni PI. Sic. 415, FI, GE - prope Augusta (CT) secundum rivum Molinello dictum, 04/1905, Cavara, FIE 947, FI, GE - Avola, in arundinetis, in humidiusculis, 3.s.d., Bianca Fl. Dint. Avola 158, FI - Leonforte, 04/1845, Insenga, PAL 63784 - territorio di Piana degli Albanesi, C.da Stretto, negli incolti, 28/03/1994, Di Martino \& Certa, PAL 63797 - Tarsia (CS), residui lembi di querceto tra i campi di cereali sui colli a nord-ovest del paese, 14/05/2015, Biaggini \& al., FI. SARDEGNA: presso Macomer, 29/03/1904, Bicknell \& Pollini, GE.

Symphytum SICILIA: Madonie, castagneto di Castelbuono, 04/1877, Lojacono, FI - Cefalu, al convento gussonei di Gibilmanna, 01/04/1900, Martelli, FI - Nebrodi, faggeta nei pressi del Passo Portella della Femmina Morta, 1100 m, 11/05/1986, Bianchini \& Di Carlo, Bianchini 11533, VER Messina al Campo a Curcuraci, s.d., Sequenza 65, Fl.

Symphytum LIGURIA: boschi di Dolcedo, 05/1886, Berti, FI. PIEMONTE: presso Susa in luoghi umidi, officinale regione del castagno, 09/08/1877, Aiuti, FI - Robilante, 22/08/1888, Bicknell, GE - Alpi Cozie, circondario di Pinerolo, Pomaretto, 17/07/1904, Gestro, FI - Torino, ad marginem viae Rivalto, 25/04/1926, Zola, Gavioli 10496, FI - Biella, vallone inferiore del torrente Oropa, 18/06/1949, Negri, FI - Domodossola, 6.s.d., Rossi, FI. VAL D’AOSTA: Gressoney St. Jean (AO), nei giardini, 24/06/1859, Carestia, FI - Chanversoud, 13/04/1897, Vaccari, FI - laghetti di Lillaz, St. Marcel, luogo umido, fra le canne, $530 \mathrm{~m}$, 24/05/1986, Bovio 517, Herb. Bovio - Grand-Vert, 04/09/1987, Desfayes 87-0181, AO-DESF 65 - St. Vincent, prato umido a S di Toux, 425 m, 24/06/1991, Bovio \& al., AO-N.SFV 728. LOMBARDIA: lungo i fossi di Grumone, 24/08/1871, Parlatore, FI - Milano, lungo i fossi, 05/1895, Camperio, FI - Legnano, 06/05/1906, Tomasi, FI - La Brianza, Erba, lago di Pusiano, 258 m, 04/07/1956, Chiarugi \& al., FI - Mantova, bosco Fontana, sorgente e torrentello alla fontana di San Giuseppe, 06/07/1977, Barini 238, FI. TRENTINO ALTO ADIGE: val di Ledro ad fossas et in umidis, solo calcareo, 400-700 m, 06/1887, Porta, FI - Alpi trentine, val Cadino ai Zocchi, suolo porfirico, 1250 m, 27/06/1922, Fiori, FI - S. Lorenzo di Pusteria, prati, 05/1923, 
Biadego, VER - Merano (TN), boschi sopra Forst, 550 m, 12/09/1953, Piazzoli, MSNM 8536 - val di Fassa (TN), Soraga - Moena e adiacenze, 1200 m, 08/1976, Anzalone, FI lago di Caldaro (BZ), 250 m, 28/06/1980, Curti, Bianchini 18794, VER. VENETO: Bassano a S. Giacomo di Romano, 15/09/1897, Vaccari, FI - Osigo, Colle Carantan, 21/09/1958, Fornaciari, MFU-Forojuliense 10750 - m.te Crocitta (VI), prato, 50-200 m, 15/05/1969, Curti, MNAV - Arsiè, 300 m, 10/05/1976, Lasen, Herb. Lasen - Cinto Caomaggiore (VE), via Reghena, loc. Sega, 7 m, 16/06/1985, Martini, MFU-Martini 4685 - Schio (VI), 200 m, prato, 02/07/1986, Scortegagna, MNAV - Montecchio Precalcino, fosso, 26/06/1987, Bettanin, MNAV - Verona al Pestrino, 60 m, 23/08/1989, Bianchini \& Di Carlo, Bianchini 13555, 13556, VER - Padova, 25/04/1993, Sburlino, UVV - Asiago (VI), val d'Assa, 1400 m, megaforbieto, 26/07/1996, Scortegagna, MNAV - palude del Busatello, argine, S. Pietro in Valle, Gazzo Veronese (VR), 16 m, 01/09/1999, Settin, Di Carlo 1371, VER - Oliosi, frazione di Castelnuovo del Garda (VR), su prato umido a fianco del fiume Tione, $100 \mathrm{~m}$, 29/05/2001, Di Carlo 1812, VER - presso Cencenighe (BL), 20/07/2004, Sburlino, UVV Legnago in loc. Terranegra, riva destra del fiume Adige (VR), 15 m, 22/06/2010, Di Carlo 4006, VER. FRIULI VENEZIA GIULIA: fosso lungo la strada Varmo - Rivignano, presso Cornazai, 20/05/1966, Fornaciari, MFU-Forojuliense 10746 - Pagnacco (UD), 168 m, 04/07/1996, Ortolan, MFU-Martini 4684 - valli del Natisone (UD), Savogna, 05/04/1997, Rizzardini, MFU-Forojuliense 10742 - Tarcento (UD), Cergneu, 300 m, 30/05/1998, Sant, MFU-Martini 4683 - Ronchi dei Legionari (GO), 5 m, 31/05/1998, Sergo, MFUForojuliense 10741 - Pordenone (PD), Borgo Meduna, 24 m, 18/04/1999, Casciano, MFUMartini 4678 - bosco di Carlino, Muzzana (UD), 09/05/2001, Sburlino, UVV. EMILIA ROMAGNA: fossi di Classe presso Ravenna, 06/1864, Caldesi, FI - Finale Emilia, 20/05/1885, Chelussi, FI - in una siepe presso Reggiolo (RE), 19/05/1891, Fiori, FI circondario di Concordia (MO), ai margini dei fossi, 31/05/1895, Mari, FI - Parma, greto Bormioli, 07/06/1919, Minio, FI - Montefiorino, in arvis et secundum fossas, 06/1922, Meori, Gavioli 17949, FI - Modena, villa Ada, 13/05/1929, Lunardi, Gavioli 14489, FI bosco Mesola, 04/07/1964, Stampi, FI. TOSCANA: padule di Bientina, in un prato tra Rio Visona di Compito e la Baracca di Nanni, 13/04/1976, Pistoleni, PI - Massa, erbosi nel rione di Poggioletto, 03/05/1979, Marchetti, PI - Lucca, nei fossati attorno alle mura, 30/08/1999, Peruzzi, PI - Marina di Vecchiano, 11/04/2000, Bottega, PI - riserva del Campolino (Abetone, PT), margine torrente lungo una pista forestale al margine della foresta Fagus-Picea-Abies, arenaria, ca. 1350 m, rara, 07/10/2000, Selvi, FI-HB 00.33 Campi Bisenzio (FI), A.N.P.I.L. Stagni di Focognano, 06/10/2007, Cecchi 1213, Herb. Cecchi. MARCHE: m.te Vettore, 07/1842, Marzialetti, FI - dintorni di Senigallia, scarpate erbose sull'autostrada Bologna-Canosa, all'imbocco $\mathrm{N}$ della galleria del Cavallo, ca. $35 \mathrm{~m}$, suolo argilloso, humus nullo, 13/06/1978, Brilli-Cattarini, PESA - dintorni di Cupramontana, luoghi erbosi umidi sotto I'Eremo delle Grotte, 275-300 m, suolo prevalentemente argilloso-sabbioso, humus scarso, 25/04/1979, Brilli-Cattarini \& Gubellini, PESA - dintorni di Camerano, siepi fresche e luoghi erbosi subumidi lungo la SS Adriatica tra Aspio Terme e Stazione d'Osimo, ca. $30 \mathrm{~m}$, suolo prevalentemente argilloso, humus scarso o subnullo, 11/05/1987, Brilli-Cattarini \& Gubellini, PESA. LAZIO: in humentibus, Fondi lungo il fiume Acquachiara, 08/1870, Terracciano, FI - Roma, rupi della Caffarella, 20/06/1882, Tanfani, FI - in promontorio Circeo, prope Terracina, sotto San Felice, 27/05/1893, Sommier, FI - Velletri, salita da Ninfa a Norma, 04/1910, Vaccari, FI. ABRUZzO: Avezzano (AQ), Vicenna di Castelnuovo, $900 \mathrm{~m}, \mathrm{~N} 42^{\circ} 06.104^{\prime}, \mathrm{E} 13^{\circ} 27.071$ ', 13/05/2006, Conti \& al., APP 36567. CAMPANIA: Salerno, 03/06/1821, Gussone, Herb. Gussone, NAP - ripe del fiume Sarno presso Napoli, s.d., Gussone, Herb. Gussone, NAP. BASILICATA: m.te Vulture, 1840, Terracciano, Herb. Gussone, NAP. CALABRIA [fide BotTeGA \& GARBARI 2003]: Catanzaro, strada della Portella, 15/05/1896, Micheletti, TO - S. Demetrio Corone, s.d., Giuliani, Herb. Tenore, NAP. SICILIA: Collesano, ad *, s.d., 
Gussone, Herb. Gussone, NAP. - L'unico campione accertato per la Sardegna sembrerebbe un campione dell'erbario sardo di Moris, a Torino, privo di dati di raccolta e forse coltivato; la presenza della specie allo stato spontaneo sull'isola resta da confermare.

Symphytum EMILIA ROMAGNA: orto botanico di Bologna, spontaneo sotto i grandi alberi, orientale 26/04/1936, Chiovenda, BOLO 25799. TOSCANA: prope montem Senarium, 05/1843, Bechi, FI - orto botanico di Pisa, 16/05/1910, Fiori, FI - Casal Guidi, Pistoja, presso della Villa, 01/05/1884, Costa Reghini, PI - Firenze, le Cascine, 06/04/1976, Giugnolini, FI Firenze verso Pian del Mugnone, 02/05/1991, Bigazzi, FI-HB 91.03. MARCHE: Cerquete di Cerreto d'Esi (AN), margine stradale, 16/04/2001, Baldoni, SFI 1090, FI. UMBRIA: Perugia, spontaneo nell'orto botanico, 04/1886, Batelli, TO-HG. LAZIO: Roma, palazzo Salviati, via della Lungara 81, vicino al muro di recinzione e sui prati, 13/04/2004, Morgutti, SFI 1307, Fl.

Symphytum TOSCANA: Pisa a Castagnolo, 28/05/1891, Fantozzi, Pichi Sermolli 5755, FI - lago di tanaicense Massaciuccoli, loc. La Piaggetta, 30/08/1999, Peruzzi, PI. - A questa specie sono stati riferiti (Bottega \& Garbari 2003) anche alcuni campioni provenienti dal Friuli Venezia Giulia (es: Burida (PD), torbiera Baregàn, prato superiore magro asciutto, terreno grigio, 06/06/1945, Zenari, PAD), dal Lazio (es: paludi pontine, 04/1879, Cuboni, BOLO 41614) e dalla Campania (Sarno sul fiume, 16/05/1837, Gussone, Herb. Gussone, NAP), cui potremmo aggiungerne altri di incerta identità del Veneto, provenienti dal Bellunese (Polpet, Ponte n/A (BL), 400 m, margine strada, 27/05/1988, Argenti \& Argenti, Herb. Argenti - Pragrande Agordo (BL), 600 m, ruderale, incolto, 31/05/2008, Argenti \& Argenti, Herb. Argenti), ma dall'esame morfologico tali reperti appaiono a stento distinguibili dai comuni esemplari di S. officinale a fiori purpureo-violetti; sono pertanto necessari approfondimenti, specie sul piano cariologico, per meglio definire l'attuale distribuzione di questa entità sul nostro territorio.

Symphytum LIGURIA: valle di Polcevera, 05/1841, Carrega, FI - Appennino ligure-piemontese, valle tuberosum subsp. angustifolium

del rio Lischeo, 25/05/1972, Prossen, GE - colle del Melogno (SV), 24/05/1973, Peccenini,

Herb. Peccenini - La Spezia, Rio Finale tra Riomaggiore e Manarola, 20/03/1979, Nardi \& al., FI - Alpi marittime, m.te Ceppo (IM), 1600 m, 20/06/1993, Bianchini \& Di Carlo, Bianchini 20124, VER - m.te Galero (SV), 1000 m, 27/04/2001, Pareto, TO - Genova, fossatello Angeli, 4.s.d., Gentili, FI - fiumara di Dolcedo, 5.s.d., Berti, FI. PIEMONTE: m.te Orfano, conca di Mergozzo, 250 m, 11/04/1912, Boggiani, FI - Aosta, ad Andrate l. erbosi, 02/05/1913, Bolzon, FI - Roccavione, fonte del Luco, 09/05/1922, Biadego, VER - collina torinese, S. Margherita, 28/04/1929, Tortonese 573, VER - boschi misti freschi presso Varallo Sesia, 500 m, 07/1963, Bono, FI - Alpi Marittime, val Gesso, faggeta di valle di Roaschia, 1200 m, 10/07/1968, Bono, FI - Gignesse (NO), 700 m,bord de la route d'Orta, 15/04/1976, Renard, FI - Oldenico, boschi, 4.s.d., Malinverni, FI. LOMBARDIA: luoghi boschivi della regione subalpina di m.te Ario in val Trompia, 1700-1800 m, 16/07/1864, Parlatore, FI - Milano, prati lungo I'Olona, fuori Porta Magenta, presso S. Giovanni la Vipera, 08/04/1876, E.Corti \& Mazzucchelli, FI - secus vias prope pagum Morbegno, 07/1892, Longa, FI - val Vestino, in valleculis humosis sylvaticis, solo calcareo, 400-500 m, 05/1902, Porta, Gavioli 11504, FI - Prov. di Pavia, locis arenosis aridis subombrosis prope Carbonara non longe a Ticino flumine, solo siliceo, 04/1905, Traverso, FIE 945, FI 7408, FI 7409, GE, MOD [neotipo e isoneotipi di S. tuberosum f. miniatum] - S. Elia, Viggiù, 25/04/1915, Biadego, VER - Montello, 13/04/1926, Burlini, VER - Prealpi insubriche, Varese, m.te Sangiano, bosco, 25/04/1952, Negri \& Bavazzano, FI - gruppo delle Grigne, Piani Resinelli (Lecco), m.te Coltiglione, 03/06/1956, Chiarugi \& al., FI - tra Capovalle e Treviso Bresciano, lungo la strada, 13/05/1971, Steinberg \& Ricceri, FI. TRENTINO ALTO ADIGE: Valsugana, nelle siepi sopra S. Vito, suolo calcareo, s.d., Ambrosi, FI - Tramin a.d. 
Weinstraße (BZ), Tramin, Kellereigenossenschaft, $230 \mathrm{~m}, 1^{\circ} 14^{\prime} 22,979^{\prime \prime} \mathrm{E}$, 46²0'29,0784" N, 21/04/2001, Tratter, BOZ PVASC 6171. VENETO: Lido di Venezia, 1844, Kellnes, VER - Caiada (BL), bosco contrada Caneva, 12/07/1916, Minio, FI - Creazzo, Rivella, sottobosco, 50-200 m, 12/04/1970, Curti, MNAV - altopiano di Asiago, Melette, valle di Campomulo, prato, 1500 m, 09/07/1988, Zamberlan, MNAV - Treponti (PD), 21/03/1993, Sburlino, UVV - Possagno (TV), 400 m, 27/03/1994, Fornasier, MNAV - Schio (VI), Soglio della Guardia, 300 m, *, 09/04/1995, Scortegagna, MNAV - Salcedo (VI), Valle di Sotto, 270 m, vallecole, 01/05/1995, Scortegagna, MNAV - Bassano, m.te Caina, 1000 m, prato - pascolo, 05/05/1996, Franzan, MNAV - m.te Pastello, Lessini, in loc. Molane Fumane (VR), 900 m, 17/05/2001, Di Carlo 1656, VER. FRIULI VENEZIA GIULIA: Verzegnis, torrente Ambiesta, 26/04/1955, Fornaciari, MFU-Forojuliense 10767 - Carso triestino, sentiero da Rupingrande a m.te Lanaro, dolina, 480 m, 23/05/1980, Martini, MFUMartini 4690 - Cormons (GO), 56 m, 28/03/1999, Cociancich, MFU-Martini 4688 - Grado (UD), Fiumicello, 08/04/1999, Pellizzari, MFU-Martini 4687 - Monfalcone (GO), piana di Lisert, 13/04/1999, Buttignon, MFU-Martini 4686 - Braida di Comeglians (UD), 15/05/2004, Sburlino, UVV - Pala Barzana, sopra la Caséra, 1150 m, 07/05/2007, Lasen, Herb. Lasen. EMILIA ROMAGNA: Castiglion de' Pepoli (BO), in sylva castaneorum, 780 m, 29/04/1911, Sommier, FI - Piandelagotti, luoghi ombrosi, 1210 m, 17/05/1954, Lunardi, FI - Forlì, m.te Fumaiolo, prati presso il laghetto ad E del m.te Castelvecchio, $1172 \mathrm{~m}$, 27/05/1967, Raffaelli, FI - Frassinoro, presso le nuove ville, margini faggeta, 1000-1100 m ca., 19/05/1977, Mori, FI - Bagno di Romagna (FC), m.te Penna, 1250 m, esp. O, suolo arenaceo, nella faggeta, 17/05/1983, Padula, FI. TOSCANA: Montopoli, 07/04/1864, Beccari, FI - castagneti di Santa Fiora, 06/1874, Bargagli, FI - Montepiano, nell'abetina, 09/06/1889, Sommier, FI - Marsiliana in Maremma, sotto il castello, 28/04/1905, Sommier, FI - Sargiano (AR), nei boschi, 12/04/1911, Savelli, FI - Appennino aretino, Giogana, suolo siliceo, 1350 m, 30/06/1923, Fiori, FI - Pracchia (PT), luoghi ombrosi nei boschi, 650 m, 04/1930, Tani, FI - m.te Cetona, 29/04/1943, Negri \& Corradi, FI - Cerbaie, lungo il ruscello della valle maggiore, 06/05/1951, Contardo \& Di Moisè, FI Antiappennino toscano, poggio di Montieri, faggeta con Ostrya, vers. N, $1000 \mathrm{~m}$, 15/05/1964, De Dominicis, FI - Gambassi, boschi misti presso il bivio per Montaione, 350400 m, 17/04/1966, Morandi, FI - Firenzuola, bacino idrografico del torrente Diaterna, bosco di faggio tra m.te Freddi e m.te Oggioli, 27/06/1973, Hoffmann, FI - sfagneta di S. Lorenzo (LU), vicino al campo I, lato prospiciente la pineta, 22/04/1976, Mariotti, PI m.te Pisano, passo di Dante, 300 m, 21/05/1977, Baldini \& al., PI - Massa, nel rione di Volpigliano, 12/04/1979, Marchetti, PI - Chianti, passo dei Pecorai, sponda di ruscello, 25/04/1979, Casini, FI - Dicomano, Pruneta, lungo il torrente, 04/05/1980, Rizzotto, FI Pania di Corfino, prati di Corfino, 26/04/1981, Donini, PI - Pratomagno (AR), pascolo cespuglioso nei pressi di Poggio del Lupo, crinale, 1500 m ca., 13/06/1991, Viciani, FI Piombino (LI), valle di Riotorto, 23/03/1993, Cenerini, PI - Riparbella (LI), vicino ad un torrente, 29/03/1999, Peruzzi, PI - strada sterrata da Civitella Marittima (GR), all'entrata della tenuta di Bagnolo, anello dall'entrata della tenuta di Bagnolo verso il poggio L'Aia dei Brogi, viale parafuoco, fosso Lanzo e ritorno dalla strada privata, 405-250 m, 18/05/2003, Sforzi \& Senesi, FI. MARCHE: dintorni di Filottrano, luoghi boschivi nella Selva di S. Paolina (valle del Musone), 75-100 m, suolo argilloso-sabbioso-molassico, 24/04/1964, Brilli-Cattarini, PESA - dintorni di Fano, luoghi boschivi nella valle del torrente Arzilla presso il ponte Nuovo, 50-75 m, suolo prevalentemente argillososabbioso, humus da scarso a più o meno abbondante, 11/04/1974, Brilli-Cattarini, PESA dintorni di Montegaudio, luoghi boschivi nel versante NE del m.te Abollo in loc. Le Cacce Vecchie, 450-475 m, suolo argilloso-calcareo, humus da scarso a più o meno abbondante, 29/05/1974, Brilli-Cattarini, PESA - gruppo del m.te Carpegna, luoghi boschivi tra la cantoniera di Carpegna e m.te Simoncello, 1075-1100 m, suolo prevalentemente 
argilloso-calcareo, humus abbondante, 11/05/1975, Brilli-Cattarini, PESA - dintorni di Rotella, luoghi boschivi nel versante NO del m.te dell'Ascensione in loc. Capitania, 725$825 \mathrm{~m}$, suolo prevalentemente arenaceo, humus da scarso o nullo a più o meno abbondante, 09/06/1976, Brilli-Cattarini, PESA - dintorni di Castelfidardo, luoghi boschivi nel settore occidentale della Selva di Castelfidardo, 25-50 m, suolo argilloso-sabbioso, humus da scarso a più o meno abbondante, 14/04/1977, Brilli-Cattarini, PESA - dintorni di Cupramontana, luoghi boschivi freschi nella valle della Romita sotto l'eremo delle Grotte, 250-275 m, suolo prevalentemente sabbioso, humus più o meno abbondante, 25/04/1979, Brilli-Cattarini \& Gubellini, PESA - dintorni d'Acquapartita, boschi mesofili (faggeta) nel versante $\mathrm{N}$ del m.te Soma presso Lucaraia, ca. $875 \mathrm{~m}$, suolo prevalentemente arenaceo, humus più o meno abbondante, 28/05/1979, Brilli-Cattarini \& Gubellini, PESA - gruppo della Serra Santa, boschi mesofili (faggeta) nel versante NE del m.te Nero, 1000-1100 m, suolo calcareo o più o meno decalcificato, humus da scarso a più o meno abbondante, 01/06/1979, Brilli-Cattarini \& Gubellini, PESA - gruppo del m.te Rotondo, luoghi boschivi (bosco misto mesofilo, carpino-corileto) nel basso versante $\mathrm{N}$ del Poggio delle Battute, $400-425$ m, suolo calcareo o più o meno decalcificato, humus da scarso a più o meno abbondante, 26/04/1982, Brilli-Cattarini \& Gubellini, PESA - dintorni di Fossombrone, luoghi boschivi (carpino-corileto) nel versante NO del m.te Raggio sopra la Ciccoccia, 400-425 m, suolo calcareo, humus più o meno abbondante, 13/05/1982, Brilli-Cattarini \& Gubellini, PESA - m.ti Sibillini, luoghi boschivi (faggeta) nel basso versante NE del m.te Vettore, lungo l'alto fosso del Coleluce, $1400-1425 \mathrm{~m}$, suolo calcareo, humus più o meno abbondante, 18/07/1984, Brilli-Cattarini \& Gubellini, PESA dintorni di S. Angelo in Pontano, luoghi boschivi (querceto misto, castagneto misto) alle Fornacette, 350-375 m, suolo arenaceo, humus da scarso a più o meno abbondante, 23/04/1986, Brilli-Cattarini \& Gubellini, PESA - m.ti della Laga, luoghi boschivi (castagneto) alla Panzanella presso Trisungo, 650-675 m, suolo arenaceo, humus più o meno abbondante, 28/04/1986, Brilli-Cattarini \& Gubellini, PESA - dintorni di Montemonaco, luoghi boschivi (querceto misto più o meno mesofilo) attorno a Cerqueto presso S. Giorgio all'Isola, $750-800 \mathrm{~m}$, suolo arenaceo, humus da scarso a più o meno abbondante, 04/05/1990, Brilli-Cattarini \& al., PESA - dintorni di Pievebovigliana nel versante $\mathrm{E}$ del m.te San Savino presso Colle S. Benedetto, luoghi boschivi (castagneto, querceto misto mesoxerofilo), radure erbose, suolo arenaceo, humus da subnullo (localmente nullo) a più o meno abbondante, 775-800 m, 11/05/1992, Brilli-Cattarini \& al., PESA. UMBRIA: Bettona S Perugia, 500 m, Quercus pubescens - Wald, 13/05/2000, Frey, FI-HB 00.40. LAZIO: m.ti Albani, bosco presso Rocca di Papa, 600-700 m, 27/05/1906, Vaccari, FI - m.ti Aurunci, prati e rocce di m.te Altino, esp. S, ca. 850 m, 21/04/1968, Nardi \& Ricceri, FI - Marano Equo (RM), 10/05/2000, Bottega, PI - distretto vulcanico laziale, m.ti Cimini, m.te Cimino, Soriano del Cimino (VT), 900 m, faggeta, 07/04/2007, Scortegagna, MNAV. ABRUZZO: in herbidis secus vias prope Palena, 24/05/1877, Arcangeli, FI. CAMPANIA: m.te Terminio, V. Sabato, 600-900 m, boschi misti, 30/06/1973, Moraldo, FI - m.ti Picentini, V. Cerreto, bosco misto, 600-700 m, 31/03/1975, Moraldo, FI - Penisola Sorrentina, castagneti presso Agerola, 11/05/1995, Bigazzi \& Selvi, FI-HB 95.20 - in sylvis montium hyrpinorum, Montevergine, s.d., Tenore 113, FI. BASILICATA: Pignola, in querceti loco dicto La Cornalita, solo siliceo, $900 \mathrm{~m}$ ca., 30/04/1922, Gavioli, FI. CALABRIA: Sila, Camigliatello, pendii sottostanti verso la piana, 1290 m, 13/05/1950, Sarfatti \& Corradi, FI - Monte Cocuzza, nei boschi, 13/05/1994, Bigazzi, FI-HB 94.10 - Sila Piccola, Villaggio Mancuso (CS), pineta a Pinus nigra nei pressi del centro visite in località Monaco, ca. 1300 m s. I. m., 23/05/2007, Cecchi \& Coppi 1099, Herb. Cecchi - in pratis montanis e maritimis, Rosarno, Mu*nola, s.d., Pasquale, FI. - La specie è riportata anche per il promontorio del Gargano, in Puglia, da LICHT (2008) 
Symphytum x LIGURIA: Piani di Vallecrosia, sotto gli ulivi, 17/04/1895, Bicknell, Herb. Bicknell, Istituto bicknellii Internazionale di Studi Liguri, Bordighera [lectotipo] - Val Borghetto, presso Bordighera, 12/03/1889, Bicknell, Herb. Bicknell, Istituto Internazionale di Studi Liguri, Bordighera [materiale originale].

Symphytum x EMILIA ROMAGNA: cresciuto nell'Orto Botanico di Ferrara", 01/05/1913, Massalongo, FI ferrariense 7136 [paratipo]/

Symphytum x PIEMONTE: lato strada tra Sesto Calende ed il bivio Arona-Novara, 08/01986, Soldano, uplandicum Herb/ Soldano 5773/ EMILIA ROMAGNA: Nicelli Farini (PC), 1060 m, 5/02009, Romani ${ }^{1}$ / ${ }^{1}$ http://www/actaplantarum/org/acta/galleria1/php?aid=5869/ 


\section{BIBLIOGRAFIA}

ALLIONI 1785 Allioni C., 1785. Flora Pedemontana. Johannes Michael Briolus, Torino.

AltamURA \& al. Altamura L., Colasante M., D’Amato G., 1984. Numeri cromosomici per la flora Italiana: 1984 1022-1036. Informatore botanico italiano 16: 261-270.

AnZALone \& al. 2010 Anzalone B., Iberite M., Lattanzi E., 2010. La Flora vascolare del Lazio. Informatore botanico italiano 42(1): 187-317.

APG 2009 The Angiosperm Phylogeny Group (Bremer B., Bremer K., Chase M. W., Fay M. F., Reveal J. L., Soltis D. E., Soltis P. S., Stevens P. F., Anderberg A. A., Moore M. J., Olmstead R. G., Rudall P. J., Sytsma K. J., Tank D. C., Wurdack K., Xiang J. Q.-Y., Zmarzty S.), 2009. An update of the Angiosperm Phylogeny Group classification for the orders and families of flowering plants: APG III. Botanical Journal of the Linenan Society 161: 105-121.

APG 2016 The Angiosperm Phylogeny Group (Byng J. W., Chase M. W., Christenhusz M. J. M., Fay M. F., Judd W. S., Mabberley D. J., Sennikov A. N., Soltis D. E., Soltis P. S., Stevens P. F. [equally responsible and listed here in alphabetical order only], [with contributions from] Briggs B., Brockington S., Chautems A., Clark J. C., Conran J., Haston E., Möller M., Moore M., Olmstead R., Perret M., Skog L., Smith J., Tank D., Vorontsova M., Weber A., 2016. An update of the Angiosperm Phylogeny Group classification for the orders and families of flowering plants: APG IV. Botanical Journal of the Linenan Society 181: 1-20.

ARCANGELI 1882 Arcangeli G., 1882. Compendio della Flora Italiana. Loescher, Torino.

ARRIGONI 2013 Arrigoni P. V., 2013. Flora dell'Isola di Sardegna 4. Carlo Delfino, Sassari.

Astutı \& al. 2014 Astuti G., Cristofolini G., Peruzzi L., Pupillo P., 2014. A new subspecies of Pulmonaria officinalis (Boraginaceae) from the southern Alps. Phytotaxa 186(3): 148-157.

BACCHetTA \& al. Bacchetta G., Coppi A., Pontecorvo C., Selvi F., 2008. Systematics, phylogenetic 2008 relationships and conservation of the taxa of Anchusa (Boraginaceae) endemic to Sardinia (Italy). Systematics and Biodiversity 6(2): 161-174.

BARToluccl \& al. Bartolucci F., Ranalli N., Bouvet D., Cancellieri L., Fortini P., Gestri G., Di Pietro R., 2012 Lattanzi E., Lavezzo P., Longo D., Marsili S., Peccenini S., Peruzzi L., Salerno G., Soldano A., Tilia A., Turcato C., Viciani D., Wagensommer R. P., Conti F., 2012. Contributo alla conoscenza floristica del settore settentrionale del Gran Sasso d'Italia (Parco Nazionale del Gran Sasso e Monti della Laga) (Abruzzo): resoconto dell'escursione del Gruppo di Floristica (S.B.I.) nel 2010. Informatore Botanico Italiano 44(2): 355-385.

BARToluccl \& al. Bartolucci F., Domina G., Adorni M., Alessandrini A., Angiulli F., M.G. Ardenghi N. M. G., 2016 Banfi E., Barberis G., Bedini G., Bonari G., Calbi M., Fenaroli F., Galasso G., Gestri G., Ghillani L., Gottschlich G., Iberite M., Latini M., Lazzeri V., Nicolella G., Olivieri N., Perrino E. V., Peruzzi L., Pisani G., Roma-Marzio F., Russo G., Scutellà F., Silletti G. N., Stinca A., Wagensommer R. P., Nepi C., 2016. Notulae to the Italian native vascular flora: 1. Italian Botanist 1: 5-15.

BÉGUINOT 1900 Béguinot A., 1900. Piante nuove o rare della Flora romana. Bullettino della Società Botanica Italiana 1900: 121-130.

BERNHARDI 1800 Bernhardi J. J., 1800. Systematisches Verzeichnis der Pflanzen, welche in der Gegend um Erfurt gefunden werden. Hover und Rudolphi, Erfurt.

BERTOLONI 1835- Bertoloni A., 1835-1836. Flora Italica 2(3). Richardus Masi, Bononia. 1836

BigazzI \& RiccerI Bigazzi M., Ricceri C., 1992. Borago morisiana Bigazzi et Ricceri (Boraginaceae), a new 1992 species from Sardinia. Webbia 46(2): 191-202. 
Bıgazzı \& Selvi 1998 Bigazzi M., Selvi F., 1998. Pollen morphology in the Boragineae (Boraginaceae) in relation to the taxonomy of the tribe. Plant Systematics and Evolution 213(1-2): 121151.

BigAzZI \& Selvi 2001 Bigazzi M., Selvi F., 2001. Karyotype morphology and cytogeography in Brunnera and Cynoglottis (Boraginaceae). Botanical Journal of the Linnean Society 136(4): 365-378.

BıgAzzI \& al. 1997 Bigazzi M., Nardi E., Selvi F., 1997. Anchusella, a new genus of Boraginaceae from the Central-Eastern Mediterranean. Plant Systematics and Evolution 205: 241-264.

BıgAzzı \& al. 1999 Bigazzi M., Selvi F., Fiorini G., 1997. A reappraisalof the generic status of Gastrocotyle, Hormuzakia and Phyllocara (Boraginaceae) in the light of micromorphological and karyological evidence. Edinburgh Journal of Botany 56(2): 229-251.

BLAISE \& CARTIER Blaise S., Cartier D., 1982. Notes caryologiques à propos de quelques espèces récoltées 1982 dans I'Apennin central. Informatore Botanico Italiano 14(2-3): 221-225.

BOLLIGER 1978 Bolliger M., 1978. Die Pulmonaria-officinalis-Gruppe in der Schweiz. Bericht der Schweizerischen Botanischen Gesellschaft 88: 30-62.

BOLliger 1982 Bolliger M., 1982. Die Gatting Pulmonaria in Westeuropa. Phanerogamarum Monographiae 8. Cramer, Vaduz.

Bottega \& GarbarI Bottega S., Garbari F., 2003. II genere Symphytum L. (Boraginaceae) in Italia. Revisione 2003 biosistematica. Webbia 58(2): 243-280.

BotTega \& al. 2001 Bottega S., Garbari F., Peruzzi L., 2001. Mediterranean chromosome number reports 11. Flora Mediterranea 11: 436-439.

BRITTON 1951 Britton D. M., 1951. Cytogenetic studies on Boraginaceae. Brittonia 7(4): 233-260.

BRITTON \& Brown Britton N. L., Brown A., 1913. An illustrated flora of the northern United States, Canada 1913 and the British possessions, ed. 2, 3. Scribner's son, New York.

BuCKNALL 1913 Bucknall C., 1913. A revision of the genus Symphytum, Tourn. Journal of the Linnean Society. Botany 41(284): 491-556.

BuXBAUM 1740 Buxbaum J. C., 1740. Plantarum minus cognitarum centuria V. Typographia Academiae, Petropoli.

CAFFERTY \& JaRvis Cafferty S., Jarvis C. E. (ed.), 2004. Typification of linnaean plant names in 2004 Boraginaceae. Taxon 53(3): 799-805.

CAmpo \& Romano Campo G., Romano S., 1995. Numeri cromosomici per la Flora Italiana. Informatore 1995 Botanico Italiano 27(1): 26-34.

CANDolle 1846 Candolle A.L.P.P. de, 1846. Prodromus Systematis Universalis Regni Vegetabilis 10. Masson, Parisiis.

CANDolle \& Lamarck Candolle A.L.P.P. de, Lamarck J. B. de, 1846. Flore française, ed. 3, 3. Agasse, Parisiis. 1805

CAPINERI \& al. 1978 Capineri R., D'Amato G., Marchi P., 1978. Numeri cromosomici per la Flora Italiana. 534-583. Informatore Botanico Italiano 10: 421-465.

CAPINERI 1986 Capineri R., 1986. Numeri cromosomici per la Flora Italiana: 1094-1097. Informatore Botanico Italiano 18: 176-179.

CECCHI \& SeLVI 2009 Cecchi L., Selvi F., 2009. Phylogenetic relationships of the monotypic genera Halacsya and Paramoltkia and the origins of serpentine adaptation in circummediterranean Lithospermeae (Boraginaceae): insights from ITS and matK DNA sequences. Taxon 58(3): 700-714.

CECCHI \& Selvi 2014 Cecchi L., Selvi F., 2014. A synopsis of Boraginaceae subfam. Hydrophylloideae and Heliotropioideae in Italy. Plant Biosystems 148(1): 2-12. Materiale supplementare: 1-29. 
CECCHI \& Selvi Cecchi L., Selvi F., 2015. Synopsis of Boraginaceae subfam. Boraginoideae tribe 2015b Boragineae in Italy. Plant Biosystems 149(4): 630-677.

CECCHI \& al. 2014 Cecchi L., Greuter W., Selvi F., 2014. (859) Proposal to conserve the name Lycopsis pulla (Boraginaceae) with a conserved type. Taxon 65(3): 1132-1133.

CHACón \& al. 2016 Chacón J., Luebert F., Hilger H. H., Ovchinnikova S., Selvi F., Cecchi L., Guilliams C. M., Hasenstab-Lehman K., Sutorý K., Simpson M. G., Weigend M., 2016. The borage family (Boraginaceae s.s.): a revised infrafamilial classification based on new phylogenetic evidence, with emphasis on the placement of some enigmatic genera. Taxon 65(3): 523-546.

CIRILLO 1788 Cirillo D. M. L., 1788. Plantarum Rariorum Regni Neapolitani 1. Neapoli.

Colla 1835 Colla L., 1835. Herbarium Pedemontanum juxta methodum naturalem dispositum 4. Ex typis regiis, Augustae Taurinorum [Torino].

CONTANDRIOPOULOS Contandriopoulos J., 1962. Recherches sur la flore endémique de la Corse et sur sès 1962 origins. Annales de la Faculté des Sciences de Marseille 32: 1-351.

Contı \& al. 1997 Conti F., Manzi A., Pedrotti F., 1997. Liste rosse regionali delle piante d'Italia. WWF Italia, Società Botanica Italiana, Camerino.

CoppI \& al. 2006 Coppi A., Selvi F., Bigazzi M., 2006. Chromosome studies in Mediterranean Boraginaceae. Flora Mediterranea 16: 253-274.

CoppI \& al. 2007 Coppi A., Selvi F., Bigazzi M., 2007. Cromosomi e filogenesi in Borago L. (Boraginaceae). Informatore Botanico italiano 39, Supplemento 1 [La Biosistematica vegetale oggi. Dagli studi tradizionali al DNA]: 127-130.

CoppI \& al. 2008 Coppi A., Mengoni A., Selvi F., 2008. AFLP fingerprinting of Anchusa (Boraginaceae) in the Corso-Sardinian system: genetic diversity, population differentiation and conservation priorities in an insular endemic group threatened with extinction. Biological Conservation 141: 2000-2011.

DANIHELKA \& al. Danihelka J., Chrtek J., Kaplan Z., 2012. Checklist of vascular plants of the Czech 2012 Republic. Preslia 84: 647-811.

DIANA-CoRRIAS 1980 Diana-Corrias S., 1980. Numeri cromosomici per la Flora Italiana: 701-707. Informatore Botanico Italiano 12: 125-129.

D'Amato 1989 D'Amato G., 1989. C-band patterns and caryotype morphology in some Anchusa species. Caryologia 42(3-4): 267-274.

D'AMATO \& MARCHI D'Amato G., Marchi P., 1983. Heterochromatin in Borago officinalis L. (Boraginaceae): 1983 comparison between Feulgen and Giemsa stained heterochromatic segments. Annali di Botanica (Roma) 41: 164-168.

D'Amato \& TrojanI D'Amato G., Trojani Z., 1985. Giemsa banding and karyotype in three species of 1985 Anchusa (Boraginaceae). Caryologia 38: 13-21.

DATTA 1933 Datta S., 1933. Embryological and cytological studies in Nolana atriplicifolia and Nolana prostrata. Journal of the Indian Botanical Society 12: 131-152.

DAVLIANIDZE 1985 Davlianidze M.T., 1985. Chromosome numbers in the representatives of the flora from Georgia. Botaniceskjij Žurnal SSSR 70(5): 698-700 [in Russo].

DAVIS 1979 Davis P. H. (ed.), 1979. Flora of Turkey and the East Aegean islands 6. Edinburgh University Press, Edinburgh.

DMITRIEVA 1986 Dmitrieva S. A., 1986. Chisla khromosom nekotorych vidov rastenij Berezinskogo Biosfernogo Zapovednika. Zapovedniki Belorussii Issledovaniia 10: 24-28.

DMITRIEVA \& Dmitrieva S. A., Parfenov V. I., 1985. Khromosomnye chisla nekotorykh vidov flory Belorussii.

PARFENOv 1983 Izvestiya Akademii Nauk Belorusskoi SSR. Seriya Biologicheskikh Nauk 2: 7-12. 
DMITRIEVA \& Dmitrieva S. A., Parfenov V. I., 1985. Kariologicheskaja kharakteristika nekotorykh vidov PARFENOv 1985 poleznykh rastenij flory Belorussii. Izvestiya Akademii Nauk Belorusskoi SSR. Seriya Biologicheskikh Nauk 6: 3-8.

DOBEŠ \& VITEK 2000 Dobeš C., Vitek E., 2000. Documented chromosome number checklist of Austrian vascular plants. Verlag des Naturhistorischen Museums Wien, Vienna.

Dobeš \& al. 1997 Dobeš C., Hahn B., Morawetz W., 1997. Chromosomenzahlen zur Gefässpflanzen-Flora Österreichs. Linzer Biologische Beiträge 29: 5-43.

Don 1832 Don D., 1832. On the characters and affinities of certain genera chiefly belonging to the flora peruviana. The Edinburgh new philosophical journal 13: 233-244.

EDMONDSON 1977 Edmondson J. R., 1977. The correct name for the Prophet Flower: Arnebia pulchra (Boraginaceae). Willdenowia 8(1): 23-36.

ElenA-Rosselló \& al. Elena-Rosselló J. A., Zapatero M. A. G., Andrés F. N., 1987. Estudios cariológicos en 1987 vegetales nemorales del CW Español, II. Studia Botanica [Salamanca] 6: 159-162.

FERNÁNDES \& LEITÃo Fernándes A., Leitão M. T., 1972. Contribution à la connaissance cytotaxinomique des 1972 Spermatophyta du Portugal. V. Boraginaceae. Boletim da Sociedade Broteriana, série 2, 46: 389-405.

FERNANDES \& Fernandes A., Queirós M., 1971. Sur la caryologie de quelques plantes récoltées

QuEIRós 1971 pendant la Illème Réunion de Botanique Péninsulaire. Memorias da Sociedade Broteriana 31: 343-385.

FIORI 1926 Fiori A., 1926. Nuova Flora Analitica d'Italia 2(2). Ricci, Firenze.

FIORI \& PAOlETTI Fiori A., Paoletti G., 1902. Flora Analitica d'Italia 2(3). Tipografia del Seminario, Padova. 1902

FISCHER \& al. 1937 Fischer F. E. L., Meyer C. A., Trautvetter E. R., 1837. Index tertius seminum, quae Hortus Botanicus Imperialis Petropolitanus pro mutua commutatione offert. Accedunt animadversiones botanicae nonnullae. C. Hintze, San Pietroburgo.

FISCHER \& al. 2008 Fischer M. A., Oswald K., Adler W., 2008. Exkursionsflora für Österreich, Liechtenstein und Südtirol, ed. 3. Land Oberösterreich \& Biologiezentrum der OÖ Landesmuseen, Linz.

FrIGNANI \& al. 2006 Frignani F., Landi M., Zoccola A., Selvi F., 2006. Sulla presenza di Brunnera macrophylla (Boraginaceae) in Toscana, specie esotica nuova per la flora italiana. Informatore Botanico Italiano 38(2): 563-567.

GadelLa 1972 Gadella T.W.J., 1972. Cytological and hybridization studies in the genus Symphytum. Symposia Biologica Hungarica 12: 189-199.

GADELLA 1983 Gadella T.W.J., 1983. Enkele floristische en cytotaxonomische waaremingen op Schiermonnikoog. Gorteria 10: 227-229.

GadelLa 1984 Gadella T.W.J., 1984. Notes on Symphytum (Boraginaceae) in North America. Annals of the Missouri Botanical Garden 71(4): 1061-1067.

Gadella \& Kliphuis Gadella T.W.J., Kliphuis E., 1963. Chromosome numbers of flowering plants in 1963 Netherlands. Acta Botanica Neerlandica 12: 195-230.

GADELLA \& KLIPHUIS Gadella T.W.J., Kliphuis E., 1967. Cytotaxonomic studies in the genus Symphytum. I. 1967 Symphytum officinale L. in the Netherlands. Proceedings of the Koninklijke Nederlandse Akademie van Wetenschappen C 70(3): 378-391.

Gadella \& KliphUIS Gadella T.W.J., Kliphuis E., 1969. Cytotaxonomic studies in the genus Symphytum II. 1969 Crossing experiments between Symphytum officinale L. and Symphytum asperum Lepech. Acta Botanica Neerlandica 18(4): 544-549. 
Gadella \& KliphuIS Gadella T.W.J., Kliphuis E., 1970. Cytotaxonomic investigations in some Angiosperms 1970 collected in the valley of Aosta and in the National Park "Gran Paradiso". Caryologia 23: 363-379.

Gadella \& KliphuIS Gadella T.W.J., Kliphuis E., 1971. Cytotaxonomic studies in the genus Symphytum III. 1971 Some Symphytum hybrids in Belgium and the Netherlands. Biologisch Jaarboek 39: 97107.

GADELLA \& KLIPHUIS Gadella T.W.J., Kliphuis E., 1972. Cytotaxonomic studies in the genus Symphytum IV. 1972 Cytogeographic investigations in Symphytum officinale L. Acta Botanica Neerlandica 21: 169-173.

Gadella \& KLIPHUIS Gadella T.W.J., Kliphuis E., 1973. Cytotaxonomic studies in the genus Symphytum V. 1973 Some notes on W European plants with the chromosome number $2 n=40$. Botanische Jahrbücher für Systematik, Pflanzengeschichte und Pflanzengeographie 93(4): 530-538.

Gadella \& KLIPHUIS Gadella T.W.J., Kliphuis E., 1974. Cytotaxonomic studies in the genus Symphytum VI. 1974 Some notes on Symphytum in Britain. Acta Botanica Neerlandica 23(4): 433-437.

GAdella \& KliPhUIS Gadella T. W. J., Kliphuis E., 1975. Cytotaxonomic studies in the genus Symphytum VII. 1975 Some hybrids between S. asperum Lepech. and S. officinale L. in Denmark. Proceedings of the Koninklijke Nederlandse Akademie van Wetenschappen C 78: 182-188.

Gadella \& KLIPHUIS Gadella T. W. J., Kliphuis E., 1978. Cytotaxonomic studies in the genus Symphytum VIII. 1978 Chromosome number and classification of ten European species. Proceedings of the Koninklijke Nederlandse Akademie van Wetenschappen C 81: 162-172.

GadelLA \& KLIPHUIS Gadella T. W. J., Kliphuis E., 1984. Population variability, hybridization and introgression 1984 in Symphytum officinale L. in the Netherlands. Botanische Jahrbücher für Systematik, Pflanzengeschichte und Pflanzengeographie 104: 519-536.

Gadella \& KliPhuIS Gadella T. W. J., Kliphuis E., 1985. Diploide populaties van de Smeerwortel in Nederland 1985 bedreigd. Gorteria 12: 156-158.

GAdelLA \& al. 1983 Gadella T. W. J., Kliphuis E., Huizing H. J., 1983. Cyto- and chemotaxonomical studies in the sections Officinalia and Coerulea of the genus Symphytum. Botanica Helvetica 93: 169-192.

GaGnIDZE \& al. 1985 Gagnidze R. I., Gviniaschvili T. N., Pataraia M. G., Dzindzolia L. D., 1985. Chromosome numbers in some high elevation species from the Big Caucasus. Botaniceskjij Žurnal SSSR 70(12): 1698-1699 [in Russo].

GeRVAIS 1981 Gervais C., 1981. Liste annotée de nombres chromosomiques de la flore vasculaire du nord-est de l'Amérique. II. Le Naturaliste Canadien 108: 143-152.

GHAFFARI 1996 Ghaffari S. M., 1996. Chromosome studies in some species of Boraginaceae from Iran. Iranian Journal of Botany 7(1): 81-93.

Grau 1968b Grau J., 1968. Cytologische untersuchungen an Boraginaceen I. Mitteilungen der Botanischen Staatssammlung München 7(2): 277-294.

GraU 1971 Grau J., 1971. Cytologische untersuchungen an Boraginaceen II. Mitteilungen der Botanischen Staatssammlung München 9: 177-194.

GREUTER 1965 Greuter W., 1965. Beiträge zur Flora der Südägäis 1-7. Candollea 20: 167-218.

GreUter \& al. 1984 Greuter W., Burdet H. M., Long G., 1984. Med-Checklist 1: Pteridophyta (ed. 2), Gymnospermae, Dicotyledones (Acanthaceae-Cneoraceae). Conservatoire et Jardin Botanique, Genéve.

Gussone 1842 Gussone G., 1842. Florae siculae synopsis 1. Tramater, Napoli.

GuşulEAC 1923 Guşuleac M., 1923. Beiträgen zur Systematische der Anchuseae. Publicaţiunile Societăţii Naturalisţilor din România 6: 78-92. 
GuşulEAC 1927 Guşuleac M., 1927. Die europäischen Arten der Gattung Anchusa Linneo. Buletinul Facultatii de Stiinte din Cernauti 1: 73-123.

GuşulEAC 1929 Guşuleac M., 1929. Species Anchusae generis Linn. hucusque cognitae. Repertorium specierum novarum regni vegetabilis 26: 286-322.

HACIOĞLU \& ERIK Hacioğlu B. T., Erik S., 2011. Phylogeny of Symphytum L. (Boraginaceae) with special 2011 emphasis on Turkish species. African Journal of Biotechnology 10(69): 15483-15493.

HEGI 1927 Hegi G., 1927. Illustrierte Flora von Mittel-Europa 5(3). Lehmanns, München.

HEYWOOD 1972 Heywood V. H. 1972. Flora Europaea: notulae systematicae ad Floram Europaeam spectantes: no. 12. Botanical Journal of the Linnean Society 65(2): 223-269.

HILGER \& al. 2004 Hilger H. H., Selvi F., Papini A., Bigazzi M., 2004. Molecular systematics of Boraginaceae tribe Boragineae based on ITS1 and trnL sequences, with special reference to Anchusa s. I. Annals of Botany 94: 201-212.

Hindáková \& al. Hindáková M., Feráková V., Schwarzová T., 1985. Karyologisches Studium der 2005 slowakischen Flora VII. Acta Facultatis Rerum Naturalium Universitatis Comenianae. Botanica 32: 71-75.

Hitchcock \& Green Hitchcock A. S., Green M. L., 1929. IV. Proposal. International Congress of Botany. 1929 Cambridge (England), 1930. Nomenclature. Proposals by British botanists: 110-199. Wyman, London.

HUIZIG \& al. 1982 Huizing H. J., Gadella T. W. J., Kliphuis E., 1982. Chemotaxonomical investigations of the Symphytum officinale polyploid complex and S. asperum (Boraginaceae): the pyrrolizidine alkaloids. Plant Systematics and Evolution 140: 279-292.

IAMONICO \& al. 2014 Iamonico D., Iberite M., Nicolella G., 2014. Aggiornamento alla flora esotica del lazio (Italia centrale). II. Informatore Botanico Italiano 46(2): 215-220.

ILlaRIo 1935 Illario T., 1935. Osservazioni sull'Anchusa crispa Viviani e su entità affini della Flora sardo-corsa. Archivi di Botanica (Forli): 254-263.

JAARSMA \& al. 1989 Jaarsma T. A., Lohmanns E., Gadella T. W. J., Malingré T. M., 1989. Chemotaxonomy of the Symphytum officinale agg. (Boraginaceae). Plant Systematics and Evolution 167: 113-127.

JAARSMA \& al. 1990 Jaarsma T. A., Lohmanns E., Hendriks H., Gadella T. W. J., Malingré T. M., 1990. Chemoand Karyotaxonomic studies on some rhizomatous species of the genus Symphytum (Boraginaceae). Plant Systematics and Evolution 169: 31-39.

JAFRI \& EL-GADI Jafri S. M. H., El-Gadi A. (ed.), 1979. Flora of Libya 68. Al Faateh University, Tripoli. 1979

JÄGER 2009 Jäger E. J. (ed.), 2009. Exkursionsflora von Deutschland. Gefäßpflanzen: Atlasband. 3. Spektrum Akademischer Verlag, Heidelberg.

JanChen 1953 Janchen E., 1953. Proposal to conserve the name 7096 Nonnea (Boraginaceae). Taxon 2: 210.

JARVIS \& al. 1993 Jarvis C. E., Barrie F. R., Allan D. M., Reveal J. L. (ed.), 1993. A list of linnaean generic names and their types. Regnum Vegetabile 127: 1-100.

Johnson \& Johnson M. A. T., Brandham P. E., 1997. New chromosome numbers in petaloid BRANDHAM 1997 monocotyledons and in other miscellaneous angiosperms. Kew Bulletin 52(1): 121-138.

KAMARI \& al. 1995 Kamari G., Felber, F., Garbari F., 1995. Mediterranean chromosome number reports - 5. Flora Mediterranea 5: 261-373.

KAMARI \& al. 1996 Kamari G., Felber, F., Garbari F., 1996. Mediterranean chromosome number reports - 6. Flora Mediterranea 6: 223-337. 
KAMARI \& al. 2000 Kamari G., Felber, F., Garbari F., 2000. Mediterranean chromosome number reports 10. Flora Mediterranea 10: 381-430.

KazMI 1971 Kazmi S. M. A., 1971. A revision of Boraginaceae of west Pakistan and Kashmir. Journal of the Arnold Arboretum 52: 110-136, 334-363, 486-522, 666-690.

KeRner 1878 Kerner A., 1878. Monographia Pulmonariarum. Libraria Academica Wagneriana, Oeniponte.

KIRÁLY \& al. 2011 Király G., Virók V., Molnár V. A., 2011. Új magyar füvészkönyv. Magyarország hajtásos növényei. Határozókulcsok [New Hungarian herbal. The vascular plants of Hungary. Identification key]. Aggteleki Nemzeti Park Igazgatóság, Jósvafő.

KIRCHNER 2004 Kirchner D. E., 2004. Molekulare Phylogenie und Biogeographie der gattung Pulmonaria L. (Boraginaceae). PhD Thesis. Mainz, Aachen.

KIRSCHNER \& al. Kirschner J., Kirschnerová L., Štěpánek J., 2007. Generally accepted plant names based 2007 on material from the Czech Republic and published in 1753-1820. Preslia 79: 323-365.

KoBrLová \& al. Kobrlová L., Hroneš M., Koutecký P., Štech M., Trávníček B., 2016. Symphytum 2016 tuberosum complex in central Europe: cytogeography, morphology, ecology and taxonomy. Preslia 88: 77-112.

Krahulcová 1988 Krahulcová A. 1988. Selected chromosome counts of the Czechoslovak flora I. Folia Geobotanica et Phytotaxonomica 23: 375-381.

KUBITZKI \& al. 2016 Kubitzki K., Kadereit J. W., Bittrich V. (ed.), 2016. Families and genera of vascular plants. Flowering Plants. Eudicots. Aquifoliales, Boraginales, Bruniales, Dipsacales, Escalloniales, Garryales, Paracryphiales, Solanales (except Convolvulaceae), Icacinaceae, Metteniusaceae, Vahliaceae. Springer Verlag, Berlin.

KURTTO 1981 Kurtto A., 1981. Taxonomical status of Symphytum floribundum and S. $\times$ ferrariense (Boraginaceae). Annales Botanici Fennici 18(1): 13-21.

KURTTO 1982 Kurtto, A. 1982: Taxonomy of the Symphytum asperum aggregate (Boraginaceae), especially in Turkey. Annales Botanici Fennici 19(3): 177-192.

KURTTO 1985 Kurtto A., 1985. Taxonomy of Symphytum ottomanum, S. pseudobulbosum and S. orientale (Boraginaceae). Annales Botanici Fennici 22(4): 319-331.

LACAITA 1927 Lacaita C., 1927. Rettifiche alla "Monografia Pulmonariarum" di Kerner. Nuovo Giornale Botanico Italiano 34: 1163-1165.

LÅNGSTRÖM \& CHASE Långström E., Chase M. W., 2002. Tribes of Boraginoideae (Boraginaceae) and 2002 placement of Antiphytum, Echiochilon, Ogastemma and Sericostoma: a phylogenetic analysis based on atpB plastid DNA sequence data. Plant Systematic and Evolution 234: 137-153.

LEHMANN 1821 Lehmann J. G. C., 1821. Icones rariorum plantarum e familia asperifoliarum 1. Perthes \& Besser, Hamburg.

LEPECHIN 1798 Lepechin I. I., 1798. Symphyti asperi nova species. Nova Acta Academiae Scientiarum Imperialis Petropolitanae 14: 442-444.

LEVITSKY 1940 Levitzki G. A., 1940. The karyotypes of some pairs of related forms of plants. Botaniceskii Zhurnal 25: 292-296.

LIEBLEIN 1784 Lieblein F. K., 1784. Flora Fuldensis oder Verzeichniss der im dem Fürstenthume Fuld wildwachsenden Bäume, Sträuche und Pflanzen zum Gebrauch der hiesigen academischen Vorlesungen entworfen. Andreaischen Buchhandlung, Frankfurt am Main.

LINNEO 1753 Linneo C., 1753. Species plantarum. Salvius, Holmia.

LIPPERT 2006 Lippert W., 2006. Chromosomenzahlen von Pflanzen aus Bayern und anderen Gebieten. Berichte der Bayerischen Botanischen Gesellschaft 76: 85-110. 
LITARDIÈre 1955 Litardière R. de, 1955. Prodrome de la Flore Corse 3(2). Lechevalier, Paris.

LÖFLING 1758 Löfling P., 1758. Iter hispanicum. Lars Salvii, Stockholm.

Loon 1987 Loon J. C. van (ed.), 1987. A cytotaxonomical atlas of the Balkan flora. Cramer, Berlin \& Stuttgart.

LövE 1971 Löve A. (ed.), 1971. IOPB chromosome number reports XXXII. Taxon 20(2-3): 349-356.

Löve 1977 Löve A. (ed.), 1977. IOPB Chromosome Number Reports LVII. Taxon 26(4): 443-452.

LövE 1980b Löve A. (ed.), 1980. IOPB chromosome number reports LXVIII. Taxon 29(4): 533-547.

LÖVE 1980c Löve A. (ed.), 1980. IOPB chromosome number reports LXIX. Taxon 29(5-6): 703-730.

LöVE 1981 Löve A. (ed.), 1981. IOPB chromosome number reports LXXV. Taxon 30(4): 829-861.

LöVE 1982a Löve A. (ed.), 1982. IOPB chromosome number reports LXXV. Taxon 31(2): 342-368.

LövE 1982b Löve A. (ed.), 1982. IOPB chromosome number reports LXXVI. Taxon 31(3): 574-598.

LÖVE 1983 Löve A. (ed.), 1983. IOPB chromosome number reports LXXVIII. Taxon 32(1): 138-141.

LÖVE 1984 Löve A. (ed.), 1984. IOPB chromosome number reports LXXXII. Taxon 33(1): 126-134.

LÖVE \& LöVE 1956 Löve A., Löve D., 1956. Cytotaxonomical conspectus of the Icelandic Flora. Acta Horti Gothoburgensis 20: 65-291.

LÖVE \& KJelQUIST Löve A., Kjelquist E., 1974. Cytotaxonomy of Spanish plants IV. Di cotyledons. 1974 Caesalpiniaceae-Asteraceae. Lagascalia 4: 153-211.

LÖVKVIST \& HULTGÅRD Lövkvist B., Hultgård U. M, 1999. Chromosome numbers in south Swedish vascular 1999 plants. Opera Botanica 137: 1-42.

LUEBERT \& al. 2016 Luebert F., Cecchi L., Frohlich M. W., Gottschling M., Guilliam C. M., Hasenstab-Lehman K. E., Hilger H. H., Miller J. S., Mittelbach M., Nazaire M., Nepi M., Nocentini D., Ober D., Olmstead R. G., Selvi F., Simpson M. G., Sutorý K., Valdés B., Walden G. K., Weigend M., 2016. Familial classification of the Boraginales. Taxon 65(3): 502-522.

LUQUE 1980 Luque T., 1980. Numeros cromosomicos de algunas Boraginaceas de Portugal. Boletim da Sociedade Broteriana, série 2, 2(53): 663-670.

LUQUE 1983 Luque T., 1983. Estudio cariológico de Boragináceas españolas, I. Anchusa. Lagascalia 12: 81-97.

LUQUE 1989 Luque T., 1989. Estudio cariológico de Boragináceas españolas, IV. Boletim da Sociedade Broteriana, série 2, 62: 211-220.

LUQUE 1995 Luque T., 1995. Karyology of Nonea Medicus (Boraginaceae) in Spain; relationships between genera of Boragineae Barbier \& Mathez (Anchuseae DC.). Botanical Journal of the Linnean Society 117(4): 321-331.

MÁsovský 1974 Májovský J. (ed.), 1974. Index of chromosome numbers of Slovakian flora. Part 4. Acta Facultatis Rerum Naturalium Universitatis Comenianae. Botanica 23: 1-23.

MÁJovský 1976 Májovský J. (ed.), 1976. Index of chromosome numbers of Slovakian flora. Part 5. Acta Facultatis Rerum Naturalium Universitatis Comenianae. Botanica 25: 1-18.

Májovský 1978 Májovský J. (ed.), 1978. Index of chromosome numbers of Slovakian flora. Part 6. Acta Facultatis Rerum Naturalium Universitatis Comenianae. Botanica 26: 1-42.

MÁJovskÝ \& MuRIN Májovský J., Murin A., 1980. Cytotaxonomy of the genus Pulmonaria L. in Slovakia. 1980 Boletim da Sociedade Broteriana, sér. 2 2(53): 725-739.

MÁJovskÝ \& Májovský J., Uhríková A., 1985. Karyotaxonomisches Studium einiger Arten der UHRíkovÁ 1985 slowakischen Flora V. Acta Facultatis Rerum Naturalium Universitatis Comenianae. Botanica 32: 59-65. 
MaLAKHOVA 1990 Malakhova L. A., 1990. Kariologocheskij analiz prirodnykh populjacij redkich i ischezajushchikh rastenij na juge Tomskoj Oblasti. Byulleten' Glavnogo Botaniceskogo Sada. Moscow \& Leningrad [San Pietroburgo] 155: 60-66.

MAnsion \& al. 2009 Mansion G., Selvi F., Guggisberg A., Conti E., 2009. Origin of mediterranean insular endemics in the Boraginales: integrative evidence from molecular dating and ancestral area reconstruction. Journal of Biogeography 36: 1282-1296.

Markova \& IVANova Markova M., Ivanova P., 1970. Kariologische untersuchungen der vertreter der fam.

1970 Boraginaceae, Labiatae und Scrophulariaceae in Bulgarien. Izvestiya na Botanicheskiya Institut 20: 93-98.

Markova \& IVANOVA Markova M., Ivanova P., 1971. Kariologische untersuchungen der vertreter der fam.

1971 Boraginaceae, Labiatae und Scrophulariaceae in Bulgarien II. Izvestiya na Botanicheskiya Institut 21: 123-131.

MARTIUS 1817 Martius C. F. P., 1817. Plantae nonnullae horti academici Monacensis descriptae atque illustratae. Denkschriften der Koniglich Akademie der Wissenschaften zu München 5e: 175-192.

McNeILl \& al. 2012 McNeill J., Barrie F. R., Buck W.R., Demoulin V., Greuter W., Hawksworth D. L., Herendeen P. S., Knapp S., Marhold K., Prado J., Prud'homme van Reine W. F., Smith J. H., Wiersema J. H. Turland N. J. (ed.), 2012. International Code of Nomenclature for algae, fungi, and plants (Melbourne Code) adopted by the Eighteenth International Botanical Congress Melbourne, Australia, July 2011. Koeltz Scientific Books. Koenigstein.

MeKkI \& al. 1987 Mekki L., Hart H., El-Alfy N. Z., Dewedar A., Gadella T. W. J., 1987. The Giemsa c-banded karyotype of diploid Symphytum officinale (Boraginaceae). Acta Botanica Neerlandica 36(1): 33-37.

Merxmüller \& Grau Merxmüller H., Grau J., 1969. Dysploidie bei Pulmonaria. Revue Roumaine de Biologie 1969 14(1): 57-63.

MĚsíčEK \& Měsíček J., Javurková-Jarolímová V., 1992. List of chromosome numbers of the Czech JAVURKOVÁ- vascular plants. Academia, Praha.

JAROLÍMOVÁ 1992

MIREK \& al. 2002 Mirek Z., Piękosz-Mirkowa H., Zając A., Zając M., 2002. Flowering plants and pteridophytes of Poland. A checklist. W. Szafer Institute of Botany, Polish Academy of Sciences, Kraków.

MizIANTI \& al. 1981 Mizianti M., Frey L., Mirek Z., 1981. Contribution to the knowledge of the chromosome numbers of Polish vascular plants. Fragmenta Floristica et Geobotanica 27: 19-29.

MoRIS 1827 Moris G. G., 1827. Stirpium sardoarum elenchus 1. Ex typis regiis, Cagliari.

MoRIS 1829 Moris G. G., 1829. Stirpium sardoarum elenchus 3. Torino.

MoRIS 1845 Moris G. G., 1845. Enumeratio Seminum regii horti botanici taurinensis anno 1845. Torino.

MoRIs 1858-1859 Moris G. G., 1858-1859. Flora sardoa 3. Ex regio typographeo, Torino.

MülLER 1777 Müller O. F., 1777. Flora Danica 4(12). Hauniae [Copenhagen].

MülLeR \& KUHLMANN Müller A., Kuhlmann M., 2003. Narrow flower specialization in two European bee 2003 species of the genus Colletes (Hymenoptera: Apoidea: Colletidae). European Journal of Entomology 100(4): 631-635.

Murín \& MÁJovskı Murín A., Májovski J., 1982. Die Bedeutung der Polyploidie in der Entwicklung der in 1982 der Slowakei wachsenden Arten der Gattung Symphytum L. Acta Facultatis Rerum Naturalium Universitatis Comenianae Botanica 29: 1-25. 
Murín \& MÁJovskı Murín A., Májovski J., 1987. Karyological study of the Slovak flora XIX. Acta Facultatis 1987 Rerum Naturalium Universitatis Comenianae Botanica 34: 3-20.

MURR 1899 Murr J., 1899. Beiträge zur Flora von Tirol und Vorarlberg. XI. Deutsche Botanische Monatsschrift 17(4-5): 49-52.

NePI \& al. 2010 Nepi C., Peccenini S., Peruzzi L. (ed.), 2010. Notulae alla Checklist della flora italiana: 9. Informatore Botanico Italiano 42(1): 369-386.

Nocca 1800 Nocca D., 1800. Ticinensis horti academici plantae selectae. Galeazzi, Pavia.

PARfenov \& Parfenov V. I., Dmitrieva S. A., 1988. Kariologicheskaja kharakteristika predstavitelej

DIMITRIEVA 1988 flory sosudistykh rastenij Berezinskogo biosfernogo zapovednika. Zapovedniki Belorussii Issledovaniia 12: 3-8.

Parlatore \& Caruel Parlatore F., Caruel T., 1886. Flora Italiana 6(3). Le Monnier, Firenze. 1886

PASSERINI \& al. 1928 Passerini N., Pampanini R., Negri G., Chiarugi A., 1928. Adunanza del giorno 14 aprile 1928 [della Società Botanica Italiana]. Nuovo Giornale Botanico Italiano, nuova serie, 35: 301-304.

PASTA \& Lo CAScio Pasta S., Lo Cascio, P., 2002. Contributi alla conoscenza botanica delle isole minori 2002 circumsiciliane. II. Note tassonomiche e geobotaniche sulla flora delle Isole Eolie. Naturalista siciliano 26(3-4): 131-145.

PaWlowski 1962 Pawlowski B., 1962. Annotationes de Pulmonariis. Acta Societatis Botanicorum Poloniae 31: 229-238.

PAWLOWSKI 1971 Pawlowski P., 1971. Symphyta mediterranea nova vel minus cognita. Fragmenta Floristica et Geobotanica 17(1): 17-37.

Perring 1975 Perring F. H., 1975. BSBI Symphytum survey. Watsonia 10: 296-297.

Peruzzi 2003 Peruzzi L., 2003. Numeri cromosomici per la Flora Italiana 1415-1420. Informatore Botanico Italiano 35(1): 81-84.

PeruzzI 2010 Peruzzi L., 2010. Notulae 1736-1737. Informatore Botanico Italiano 42(2): 528-532.

Peruzzi \& Cesca Peruzzi L., Cesca G., 2003. Numeri cromosomici per la Flora Italiana 1421-1433. 2003 Informatore Botanico Italiano 35(1): 85-91.

Peruzzı \& al. 2000 Bottega S., Garbari F., Peruzzi L., 2010. Aspetti biosistematici di popolazioni toscane afferenti a Symphytum gr. officinale (Boraginaceae). $95^{\circ}$ Congresso della Società Botanica Italiana. Abstract: 71.

PeruzzI \& al. 2001 Peruzzi L., Garbari F., Bottega S., 2001. Symphytum tanaicense (Boraginaceae) new for the Italian Flora. Willdenowia 31: 33-41.

PIGNATTI 1982 Pignatti S., 1982. Flora d'Italia 2. Edagricole, Bologna.

PIROTTA 1886 Pirotta R., 1886. Di una pianta nuova per la flora italiana. Atti della Società dei Naturalisti di Modena, ser. 3, 2: 118-120.

Pogan \& al. 1989 Pogan E., Jankun A., Sawicka Z., 1990. Further studies in chromosome numbers of Polish angiosperms, part 22. Acta Biologica Cracoviensia, Series Botanica 31: 1-17.

Probatova \& al. Probatova N. S., Rudyka E. G., Shatalova S. A., 2001. Chromosome numbers in some 2001 plant species from the environs of Vladivostok city (Primorsky Region). Botaničeskij Žhurnal (Moscow \& Leningrad) 86(1): 168-172.

PUPPI \& CRISTOFOLINI Puppi G., Cristofolini G., 1991. Sul significato del binomio Pulmonaria saccharata Miller. 1991 Webbia 45(2): 221-234. 
PUPPI \& CRISTOFOLINI Puppi G., Cristofolini G., 1996. Systematics of the complex Pulmonaria saccharata - $P$.

1996 vallarsae and related species (Boraginaceae). Webbia 51(1): 1-20.

QUILICHINI \& Quilichini A., Debussche M., 2000. Seed dispersal and germination patterns in a rare DebussCHE 2000 Mediterranean island endemic (Anchusa crispa Viv., Boraginaceae). Acta Oecologica 21: 303-313.

REICHENBACH 1831 Reichenbach H. G. L., 1831. Flora germanica excursoria ex affinitate regni vegetabilis naturali disposita, sive principia synopseos plantarum in Germania terrisque in Europa media adjacentibus sponte nascentium cultarumque frequentius 1. Carolum Cnobloch, Lipsia.

RIVInUs 1690 Rivinus A. Q., 1690. Ordo Plantarum, quae sunt flore irregulare monopetalo. Fleischer, Leipzig.

Rossı \& al. 2013 Rossi G., Montagnani C., Gargano D., Peruzzi L., Abeli T., Ravera S., Cogoni A., Fenu G., Magrini S., Gennai M., Foggi B., Wagensommer R. P., Venturella G., Blasi C., Raimondo F. M., Orsenigo S. (eds.), 2013. Lista Rossa della Flora Italiana 1. Policy Species e altre specie minacciate. Comitato Italiano IUCN e Ministero dell'Ambiente e della Tutela del Territorio e del Mare, Roma.

SANDBRINK \& al. Sandbrink J. M., Van Brederode J., Gadella T. W. J., 1990. Phylogenetic relationships in 1990 the genus Symphytum L. (Boraginaceae). Proceedings, Koninklijke Nederlandse Akademie van Wetenschappen 93: 295-334.

SAUER 1973 Sauer W., 1973. Beitrag zur Kenntnis von Pulmonaria stiriaca und Verwandten (Boraginaceae). Mitteilungen der Botanischen Staatssammlung München 11: 1-32.

SAUER 1974 Sauer W., 1974. Beitrag zur Kenntnis von Pulmonaria montana Lejeune and P. mollis Wulfen (recte: Wolff) ex Hornemann in Mitteleuropa. Phyton 16(1-4): 239-264.

SAUER 1975 Sauer W., 1975. Karyo-systematische Untersuchungen an der Gattung Pulmonaria (Boraginaceae): Chromosomen-Hahlen, Karyotyp-Analysen und Allgemeine Hinweise auf die Entwicklungsgeschichte Bibliotheca Botanica 131: 1-85.

SAUER 1987 Sauer W., 1987. The Pulmonaria dacica group: its affinities with central and south-east European allies and with the genus Paraskevia (Boraginaceae). Plant Systematics and Evolution 155(1-4): 257-276.

SAUER \& Gruber Sauer W., Gruber G., 1979. Beitrag zur Kenntnis der Gattung Pulmonaria in Bayern: 1979 kritische Sippen, Verbreitung und allgemeine Hinweise auf die Arealbildung. Berichte der Bayerischen Botanischen Gesellschaft 50: 127-160.

SEKOVSKI \& Sekovski Z., Jovanovska M., 1983. Chromosome atlas of some Macedonian JoVANOVSKA 1983 angiosperms. IV. Godisen zbornik. Bioloski fakultet na Univerzitetot "Kiril i Metodij". Skopje 36: 73-86.

Selvi 1998 Selvi F., 1998. Floral biometrics in the Anchusa undulata L. group (Boraginaceae) from the central-eastern Mediterranean. Botanical Journal of the Linnean Society 128: 251270.

Selvi \& Bigazzı 1998 Selvi F., Bigazzi M., 1998. Anchusa L. and allied genera (Boraginaceae) in Italy. Plant Biosystems 132(2): 113-142.

Selvi \& Bigazzı 2001 Selvi F., Bigazzi M., 2001. The Nonea pulla group (Boraginaceae) in Turkey. Plant Systematics and Evolution 227: 1-26.

Selvi \& BigazzI 2002 Selvi F., Bigazzi M., 2002. Chromosome studies in Turkish species of Nonea (Boraginaceae): the role of polyploidy and descending dysploidy in the evolution of the genus. Edinburgh Journal of Botany 59(3): 405-420.

Selvi \& Bıgazzı 2003 Selvi F., Bigazzi M., 1998. Revision of genus Anchusa (Boraginaceae-Boragineae) in Greece. Botanical Journal of the Linnean Society 142: 431-454. 
Selvi \& al. 1996 Selvi F., Nardi E., Bigazzi M., 1996. The ultimate types of Anchusa L. and Lycopsis L. (Boraginaceae). Taxon 45(2): 305-307.

SElvi \& al. 1997 Selvi F., Bigazzi M., Bacchetta G., 1997. Anchusa formosa (Boraginaceae), a new species from southern Sardinia (Italy). Plant Biosystems 131(2): 103-111.

Selvi \& al. 2002 Selvi F., Papini A., Bigazzi M., 2002. Systematics of Nonea (Boraginaceae-Boragineae): new insights from phenetic and cladistic analyses. Taxon 51(4): 719-739.

Selvi \& al. 2004 Selvi F., Papini A., Hilger H. H., Bigazzi M., Nardi E., 2004. The phylogenetic relationships of Cynoglottis (Boraginaceae-Boragineae) inferred from ITS, $5.8 \mathrm{~S}$ and trnL sequence variation. Plant Systematics and Evolution 246(3-4): 195-209.

Selvi \& al. 2006a Selvi F., Coppi A., Bigazzi M., 2006. Karyotype variation, evolution and phylogeny in Borago (Boraginaceae), with emphasis on subgenus Buglossites in the Corso-Sardinian system. Annals of Botany 98: 857-868.

Selvi \& al. 2006b Selvi F., Bigazzi M., Hilger H. H., Papini A. 2006. Molecular phylogeny, morphology and taxonomic recircumscription of the generic complex Nonea/Elizaldia/Pulmonaria/Paraskevia (Boraginaceae-Boragineae). Taxon 55(4): 907918.

Semerenko 1990 Semerenko L. V., 1990. Chromosome numbers of some flowering plants from the Berezinsky Biosphere Reservation (the Byelorussian Soviet Socialist Republic). Botaničeskij Žhurnal (Moscow \& Leningrad) 75: 279-282.

SHIRATO \& al. 1985 Shirato K., Shintani T., Nakanishi G., Kamizy A., 1985. Chromosome numbers in genus Symphytum. Chromosome Information Service 38: 21-23.

SIBTHORP \& SMITH Sibthorp J., Smith J. E., 1806. Flora Graeca 1(1). Taylor \& Co., London. 1806

SIBTHORP \& SMITH Sibthorp J., Smith J. E., 1813. Flora Graeca 2. Taylor \& Co., London. 1813

SIDHU 1979 Sidhu M. K., 1979. Distributional and cytological studies of the weed flora of cultivable fields of Patiala district (Panjab). PhD Thesis, Patiala.

SIMONKAI 1904 Simonkai L., 1904. A Magyar-Királyság területén honos Pulmonaria-k fagai, fajtái és kiválobb életjelenségei. Novenytani Kozlemenyek 3: 100-115.

SKALINSKA 1974 Skalinska M., 1974. Further studies in chromosome numbers of Polish angiosperms. X. Acta Biologica Cracoviensia, Series Botanica 17: 133-164.

SMITH 1932 Smith S. G., 1932. Cytology of Anchusa and its relation to the taxonomy of the genus. Botanical Gazette 94: 394-403.

SteARn 1986 Stearn W. T., 1986. The Greek species of Symphytum (Boraginaceae). Annales Musei Goulandris 7: 175-220.

STACE 1995 Stace C. A. (ed.), 1995. IOPB chromosome data 9. International Organization of Plant Biosystematists: Newsletter (Zürich) 24: 10-23.

Stepanov 1994 Stepanov N. V., 1994. Chromosome numbers in some nemoral species of the West Sayan (Krasnoyarsk region). Botaničeskij Žhurnal (Moscow \& Leningrad) 79(3): 125-128.

StePANov \& Stepanov N. V., Muratova E. N., 1995. Chromosome numbers of some taxa of higher plants MURATOVA 1995 of Krasnoyarsk territory. Botaničeskij Žhurnal (Moscow \& Leningrad) 80(6): 114-116.

SteARn 1985 Stearn W. T., 1985. The Greek species of Symphytum (Boraginaceae). Annales Musei Goulandris 7: 175-220.

SteVEns 2016 Stevens P. F., 2016. Angiosperm Phylogeny Website. http://www.mobot.org/MOBOT/research/APweb/

[ultimo accesso 11/03/2016] 
StREY 1931 Strey M., 1931. Karyologische studien an Boraginoideae. Planta 14: 682-730.

SUzUKA 1950 Suzuka O., 1950. Chromosome numbers in pharmaceutical plants. I. Seiken Jiho 4: 5758.

SVENSSON 1925 Svensson H. G., 1925. Zur Embryologie der Hydrophyllaceen, Borraginaceen und Heliotropiaceen: mit besonderer Rücksicht auf die Endospermbildung. PhD Thesis. Almqvist \& Wiksell, Uppsala.

Talavera \& al. 2012 Talavera S., Andrés C., Arista M., Fernández Piedra M. P., Gallego M. J., Ortiz P. L., Romero Zarco C., Salgueiro F. J., Silvestre S., Quintanar A. (ed.), 2012. Flora Iberica 11, Gentianaceae-Boraginaceae. Real Jardín Botánico, CSIC, Madrid.

TARNAVSCHI 1935 Tarnavschi I. T., 1935. Studii caryo-systematice la genul 'Pulmonaria' L., cu accentuarea morfologiei chromosomilor şi meiosei. Buletinul Facultății de științe din Cernăuți 9(1-2): 45-122.

TARNAVSCHI 1948 Tarnavschi I. T., 1948. Die Chromosomenzahlen der Anthophyten-Flora von Rumänien mit einem Ausblick auf das Polyploidie-Problem. Buletinul Grădinii Botanice şi al Muzeului Botanic de la Universitatea din Cluj 28, Supliment: 1-130.

TAUSCH 1829 Tausch I. F., 1829. Botanische Beobachtungen. Flora 12(2): 641-656.

TENORE 1812 Tenore M., 1812. Flora Napolitana 1 [folii 11-15]: 41-60.

TeNORE 1831 Tenore M., 1831. Sylloge Plantarum Vascularium Florae Neapolitanae Hucusque Detectarum. Fibreni, Neapoli.

Thomas \& al. 2008 Thomas D. C., Weigend M., Hilger H. H., 2008. Phylogeny and systematics of Lithodora (Boraginaceae - Lithospermeae) and Its affinities to the monotypic genera Mairetis, Halacsya and Paramoltkia based on ITS1 and trnL sequence data and morphology. Taxon 57(1): 79-97.

TISCHLER 1935 Tischler G., 1935. Die Bedeutung der Polyploidie für die Verbreitung der Angiospérmen. Botanische Jahrbücher für Systematik, Pflanzengeschichte und Pflanzengeographie 67: 1-36.

TUtin \& al. 1972 Tutin T. G., Heywood V. H., Burges N. A., Moore D. M., Valentine D. H., Walters S. M., Webb D. A. (ed.), 1972. Flora Europaea 3. Cambridge University Press, Cambridge.

VALDÉs 1981b Valdés B., 1981. Anchusa undulata L. en Andalucía Occidental. Lagascalia 10: 104-110.

VALDÉs \& al. 1978 Valdes B., Pastor P., Ubera J., 1978. [Numeros cromosomicos para la flora Española. 144] Numeros 1-14. Lagascalia 7(2): 192-199.

VALSECCHI 1976 Valsecchi F., 1976. II genere Anchusa in Sardegna. Webbia 30: 43-68.

VALSECCHI 1988 Valsecchi F., 1988. Le piante endemiche della Sardegna. Bollettino della Società sarda di Scienze Naturali 26: 311-319.

VASUDEVAN 1975 Vasudevan K. N., 1975. Contribution to the cytotaxonomy and cytogeography of the flora of the western Himalayas (with an attempt to compare it with the flora of the Alps). Part II. Bericht der Schweizerischen Botanischen Gesellschaft 85: 210-252.

VEST 1821 Vest L. C. von, 1821. Aufzählung einiger neuen pflanzenarten. Flora 4(10): 146-160.

VIVIANI 1802 Viviani D., 1802. Elenchus plantarum horti botanici J. Car. Dinegro. De Planis, Genua.

Vosa \& PISTolesi Vosa C. G., Pistolesi G., 2004. Chromosome numbers and distribution of the genus 2004 Pulmonaria (Boraginaceae) in Tuscany and neighbouring areas. Caryologia 57(1): 121-126.

VURAL \& TAN 1983 Vural M., Tan K., 1983. New taxa and records from Turkey. Notes from the Royal Botanic Garden, Edinburgh. 41: 65-75.

WcIsŁo 1972 Wcisło H., 1972. Karyological studies in Symphytum L. Acta Biologica Cracoviensia 15: 153-163. 
WEIGEND \& al. 2009 Weigend M., Gottschling M., Selvi F., Hilger H. H., 2009. Marbleseeds are gromwells Systematics and evolution of Lithospermum and allies (Boraginaceae tribe Lithospermeae) based on molecular and morphological data. Molecular phylogenetics and Evolution 52: 755-768.

WeIGEND \& al. Weigend M., Gottschling M., Selvi F., Hilger H. H., 2010. Fossil and extant western 2010a hemisphere Boragineae, and the polyphyly of "Trigonotideae" Riedl (Boraginaceae: Boraginoideae). Systematic Botany 35(2): 409-419.

Weigend \& al. 2013 Weigend M., Luebert F., Selvi F., Brokamp G., Hilger H. H., 2013. Multiple origins for Hound's tongues (Cynoglossum L.) and Navel seeds (Omphalodes Mill.) - the phylogeny of the borage family (Boraginaceae s.str.). Molecular Phylogenetics and Evolution 68(3): 604-618.

WEIGEND \& al. 2014 Weigend M., Luebert F., Gottschling M., Couvreur T. L. P., Hilger H. H., Miller J. S., 2014. From capsules to nutlets - phylogenetic relationships in the Boraginales. Cladistics 30(5): 508-518.

ZANONI 1742 Zanoni G., 1742. Rariorum stirpium historia. Laelii a Vulpe, Bononiae. 
Figura 1

Relazioni filogenetiche tra le

11 famiglie dell'ordine Boraginales (albero semplificato da LUEBERT \& al. 2016). In grassetto i nomi dei taxa presenti in Italia.

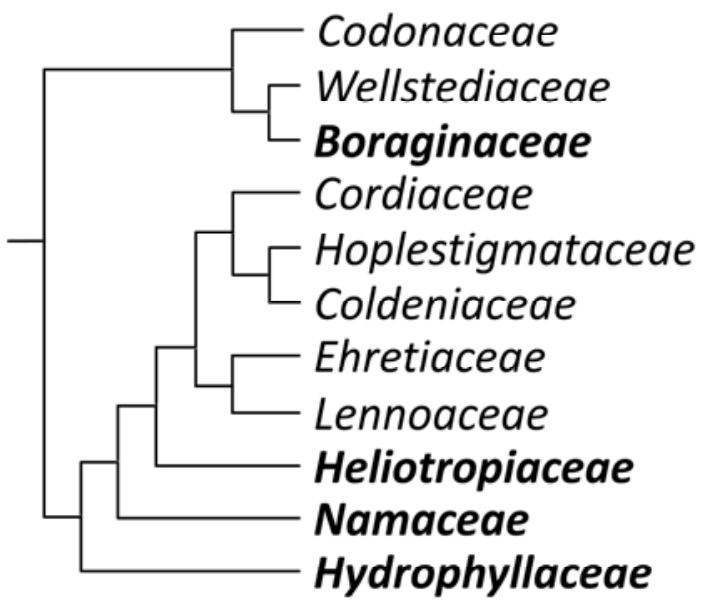


Figura 2

Relazioni filogenetiche tra le

11 tribù della famiglia Boraginaceae

(albero semplificato da

CHACON \& al. 2016). In grassetto i nomi dei taxa presenti in Italia.

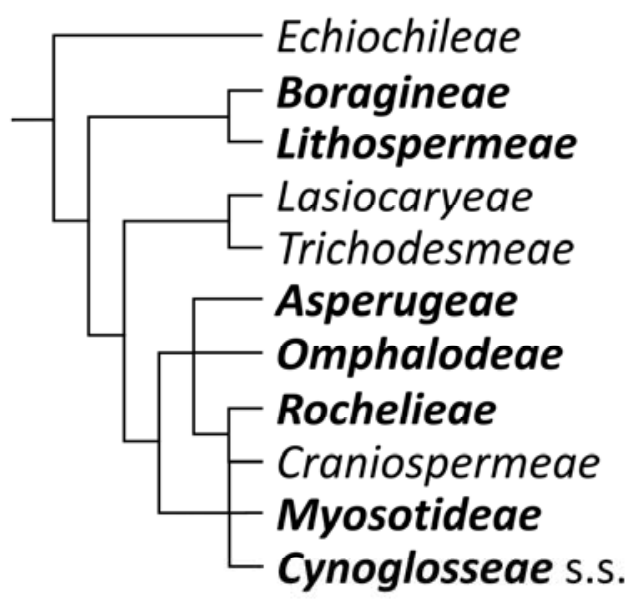


Figura 3

Relazioni filogenetiche tra i

generi della tribù

Boragineae

(albero semplificato da

HILGER \& al. 2004

WEIGEND \& al. 2010). In grassetto i nomi dei taxa presenti in Italia.

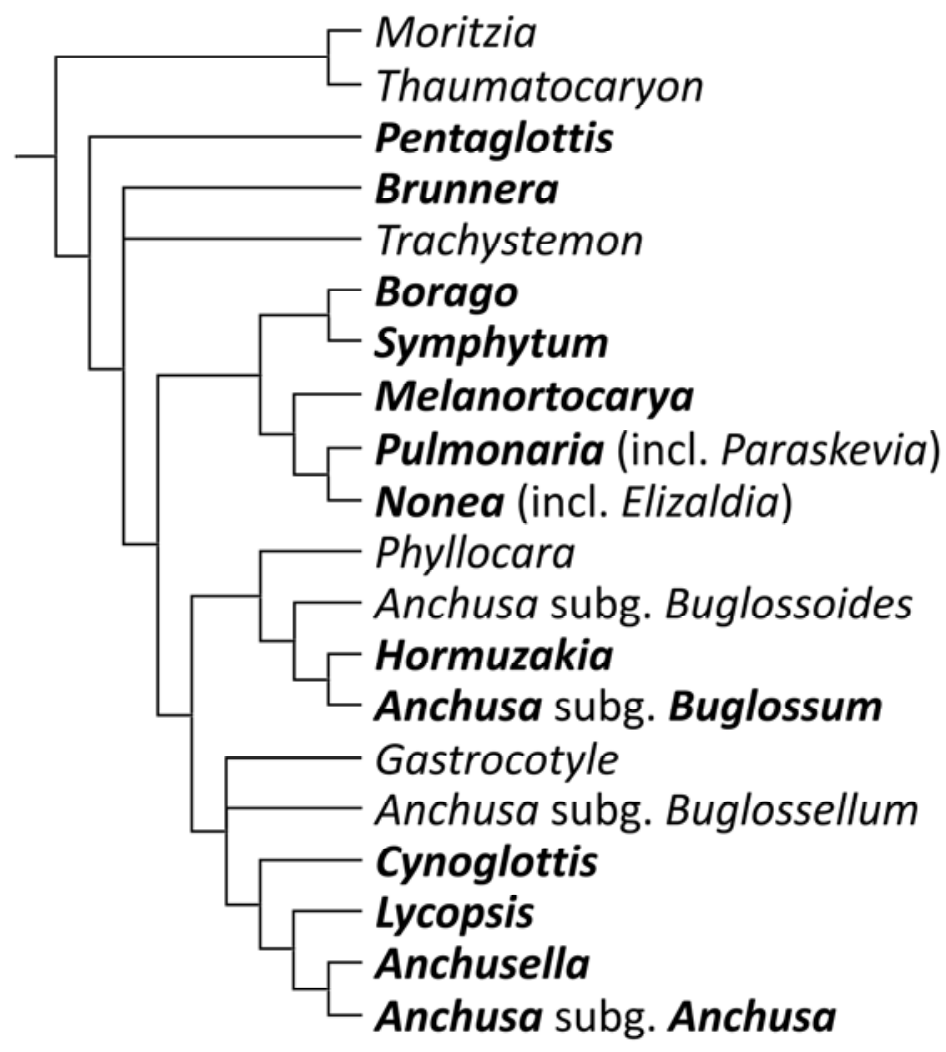


Figura 4

Pentaglottis sempervirens:

(A) habitus; (B) lembo corollino; (C, D) mericarpo in visione laterale e frontale.

Barra di scala: $A=3 \mathrm{~cm}$;

$B-D=3 \mathrm{~mm}$.

Disegno di L. Cecchi (Mozzate (VA), 2006, Galasso, SFI E1270, FI).

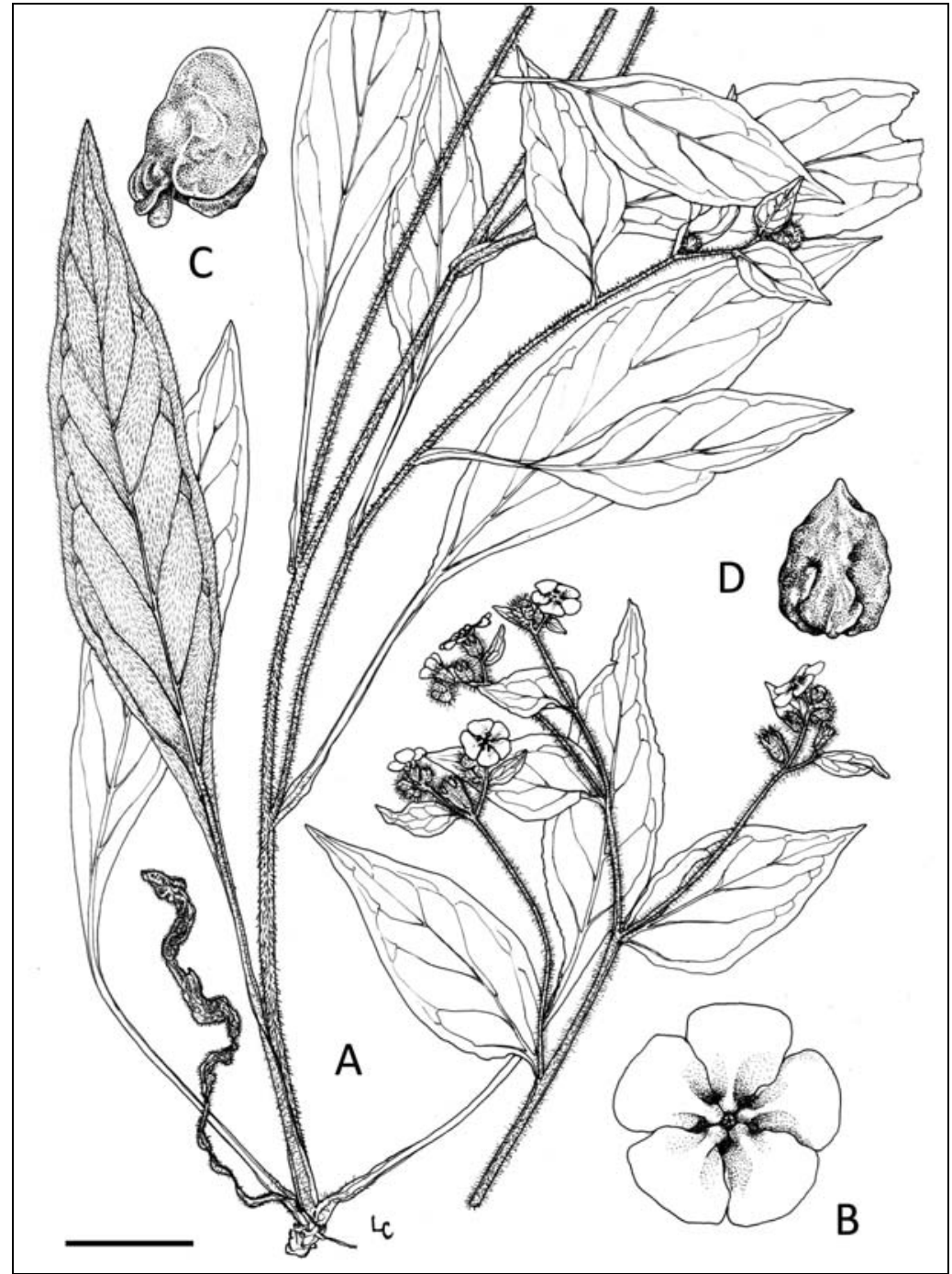


Figura 5

Distribuzione italiana di Pentaglottis sempervirens.

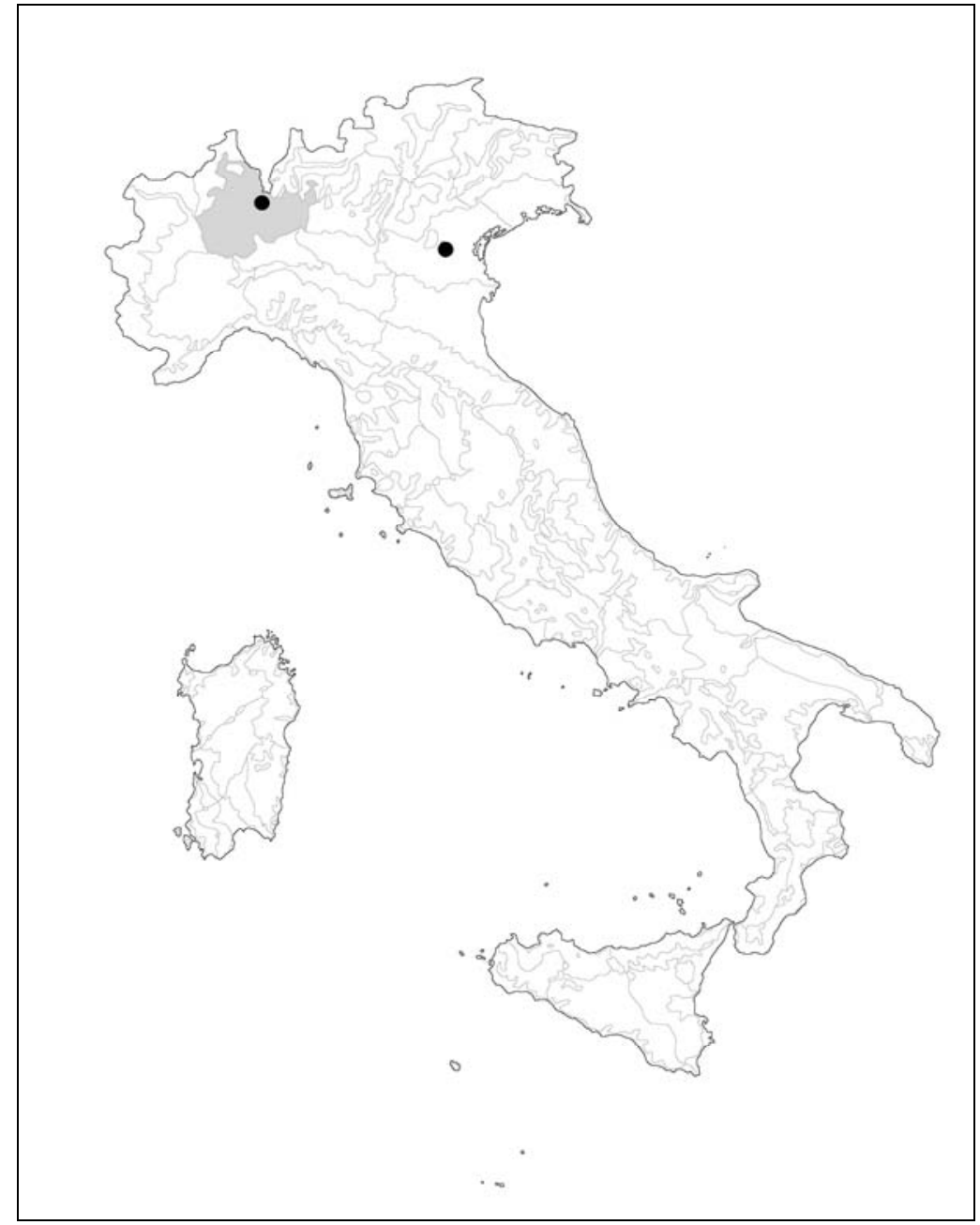




\section{Figura 6}

Brunnera macrophylla:

(A) habitus; (B) foglia

basale; (C) cima fiorale; (D) mericarpo in visione

laterale.

Barra di scala:

$\mathrm{A}=16 \mathrm{~cm} ; \mathrm{B}=4 \mathrm{~cm}$;

$C=8 \mathrm{~mm} ; \mathrm{D}=4 \mathrm{~mm}$.

Disegno di L. Cecchi

(A-C: Badia Prataglia (AR), 2006, Selvi, FIAF HB

06.22 .

B: ibidem, 2005, Frignani \& al., FIAF HB 05.01).

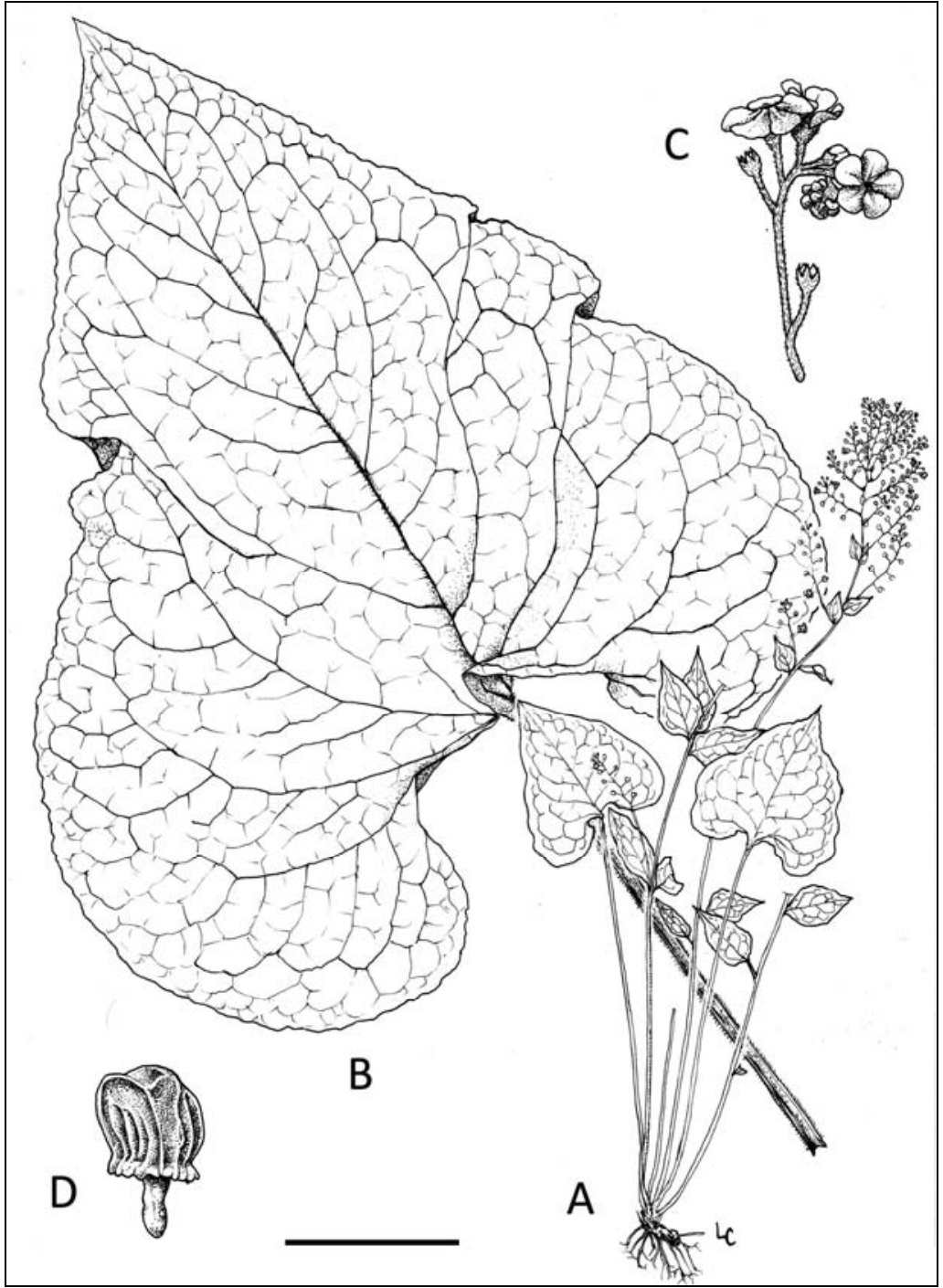


Figura 7

Distribuzione italiana di Brunnera macrophylla.

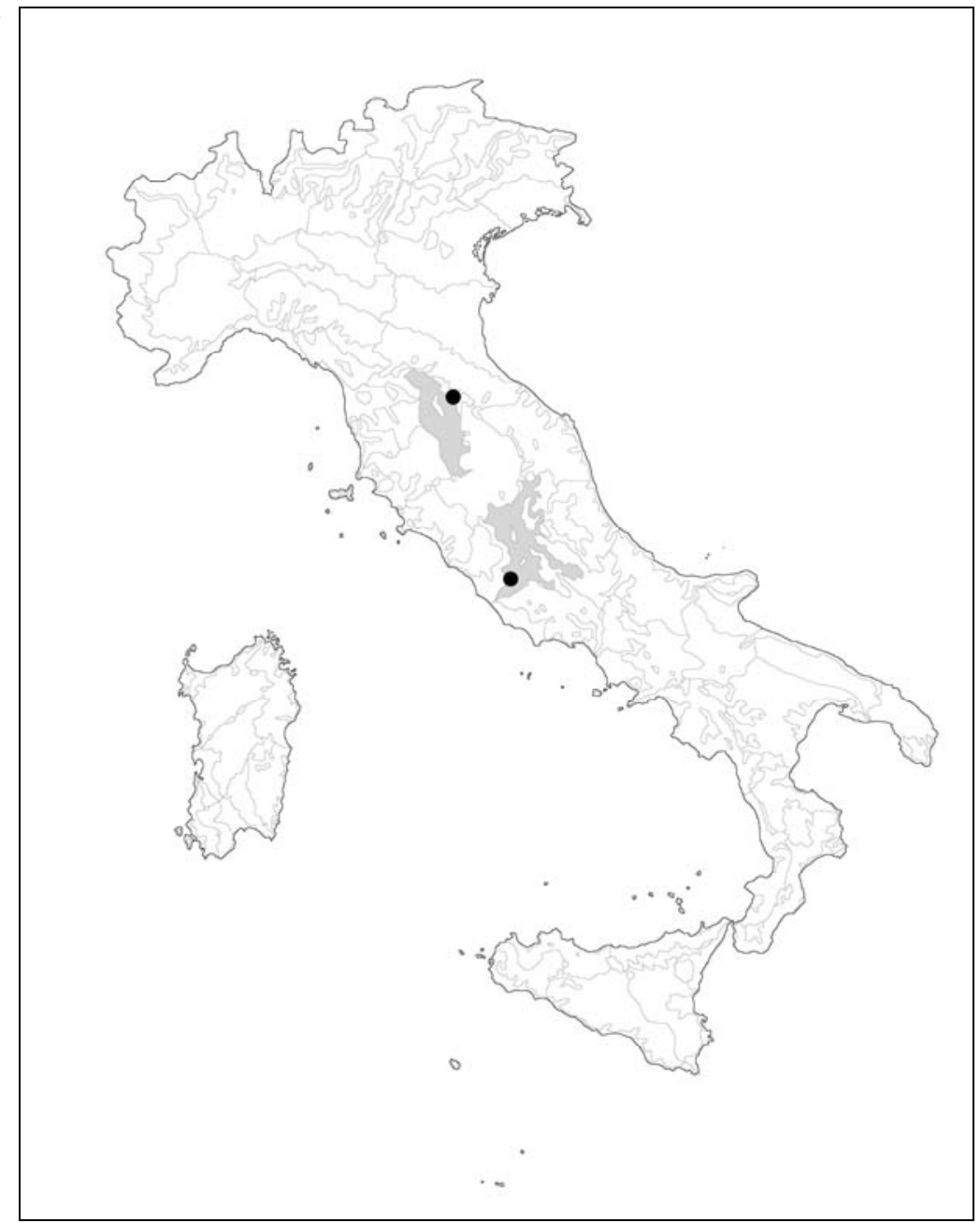


Figura 8

Borago morisiana: (A, B) habitus; (C) fiore; (D) corolla aperta; (E) stame in visione dorsale; (F) mericarpo in visione laterale.

Barra di scala: $A, B=2 \mathrm{~cm}$;

$C-E=4 \mathrm{~mm} ; \mathrm{F}=2 \mathrm{~mm}$.

Disegno di A. Maury (da BIGAZZI \& RICCERI 1992: 192).

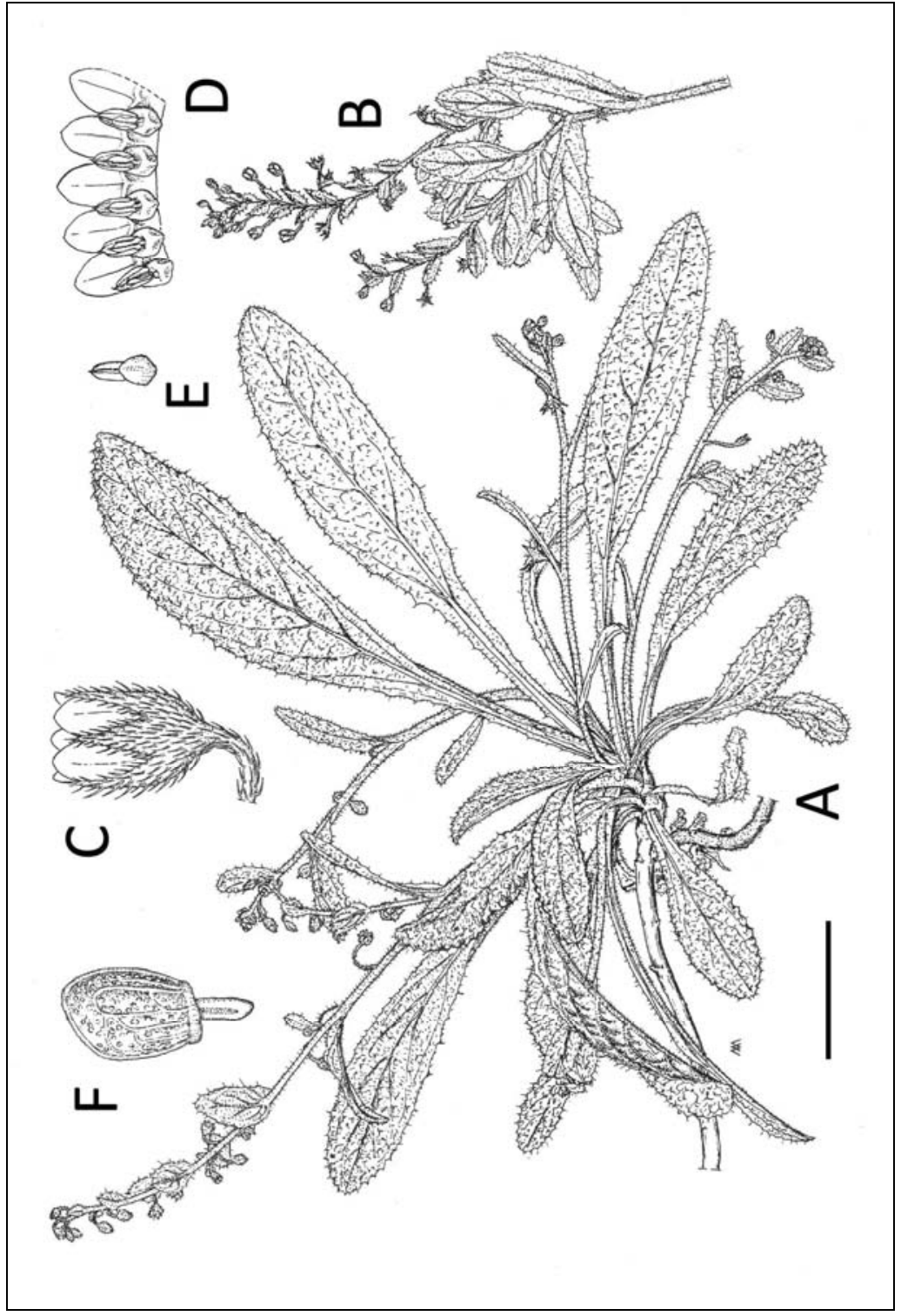


Figura 9

Distribuzione italiana di Borago morisiana (la stella indica il locus classicus).

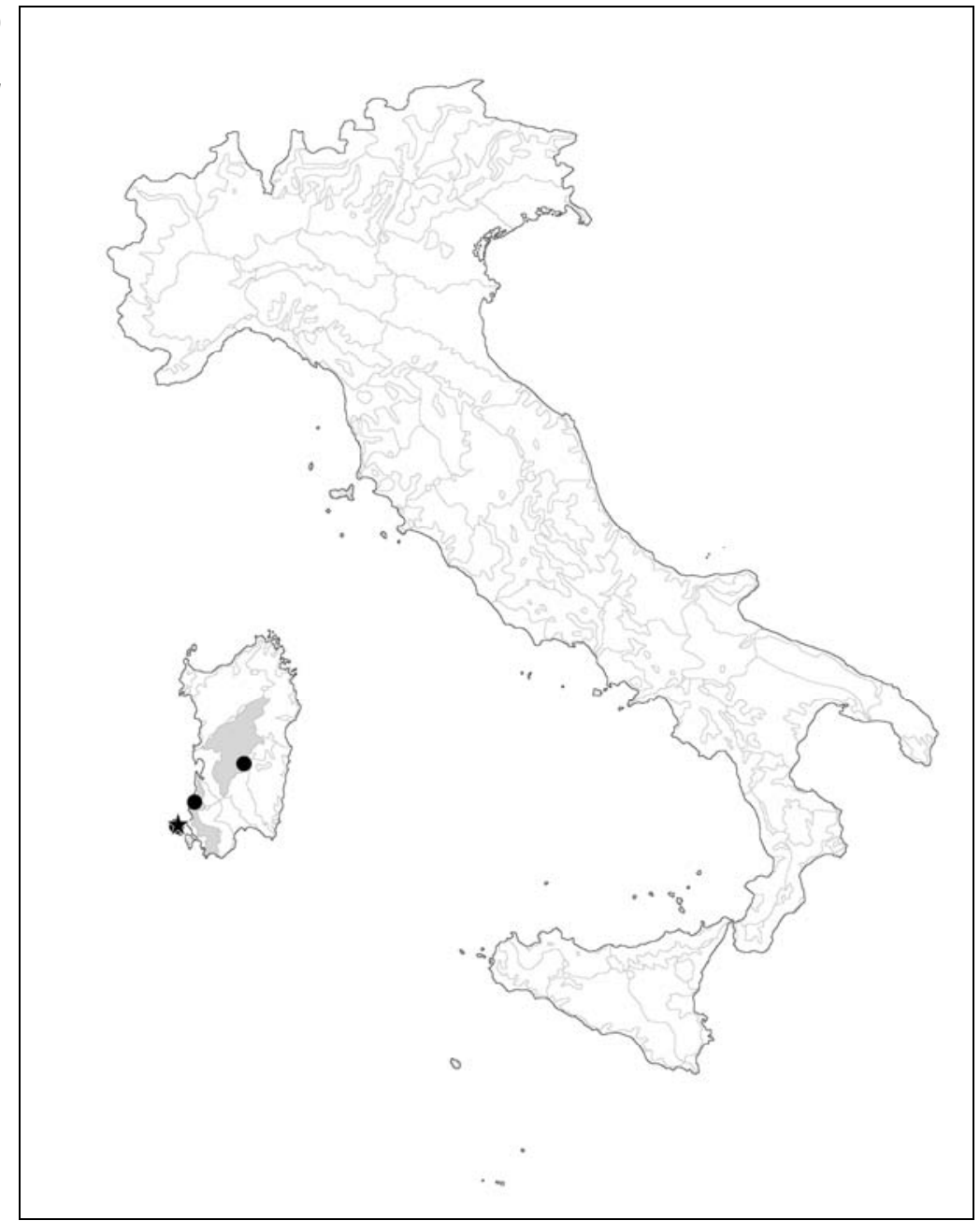




\section{Figura 10}

Borago officinalis: (A) habitus; (B) dettaglio dell'indumento fogliare; (C) fiore dal lato abassiale; (D) corolla chiusa in visione laterale, dettaglio dell'interno e gineceo; $(E)$ mericarpo in visione laterale.

Barra di scala: $A=5 \mathrm{~cm} ; C, D$ $=1 \mathrm{~cm} ; \mathrm{B}, \mathrm{E}=5 \mathrm{~mm}$. Disegno di A. Maury (Sardegna; da ARRIGONI 2012: 224, per gentile concessione di P. V. Arrigoni).

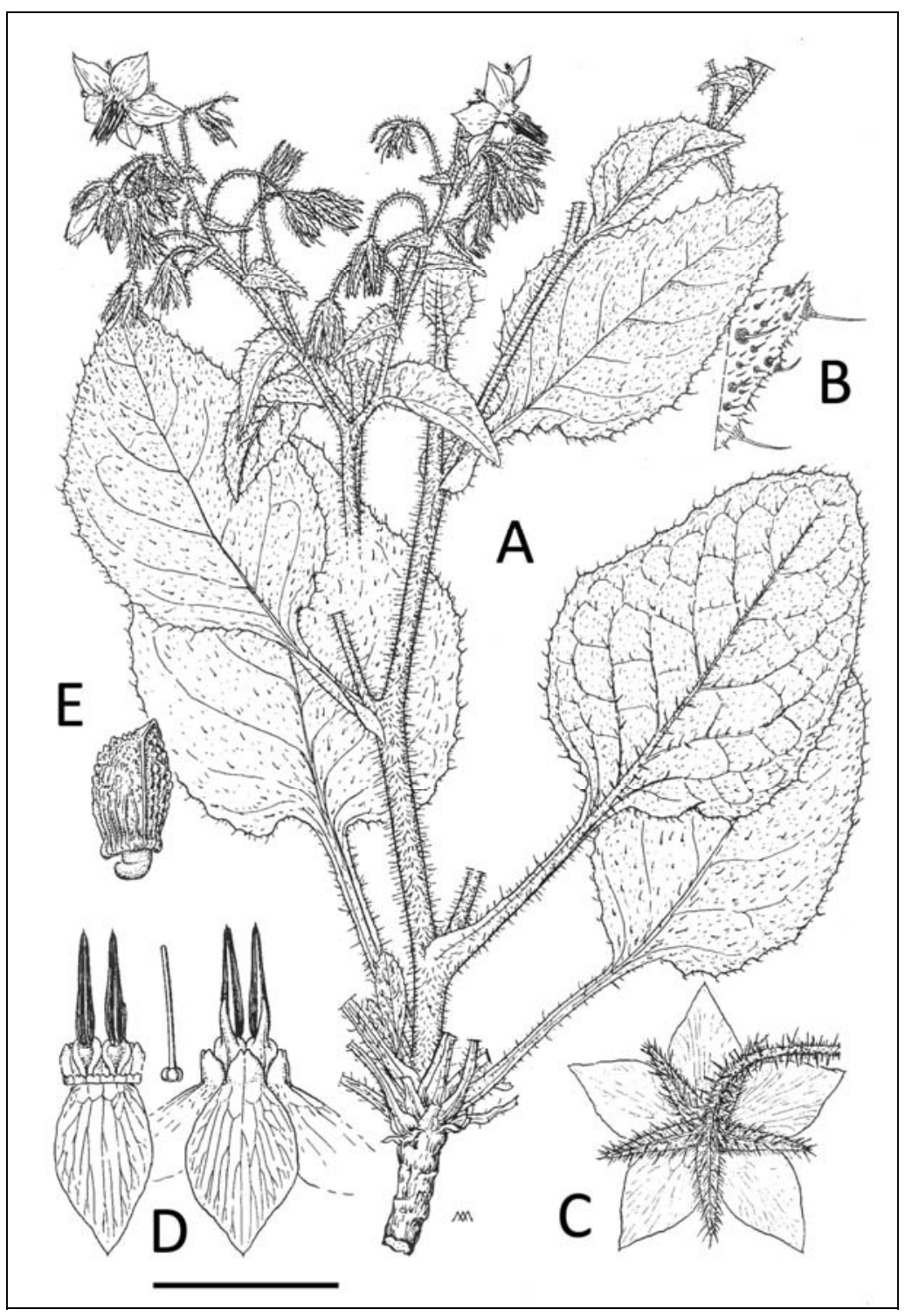


Figura 11

Distribuzione italiana di Borago officinalis.

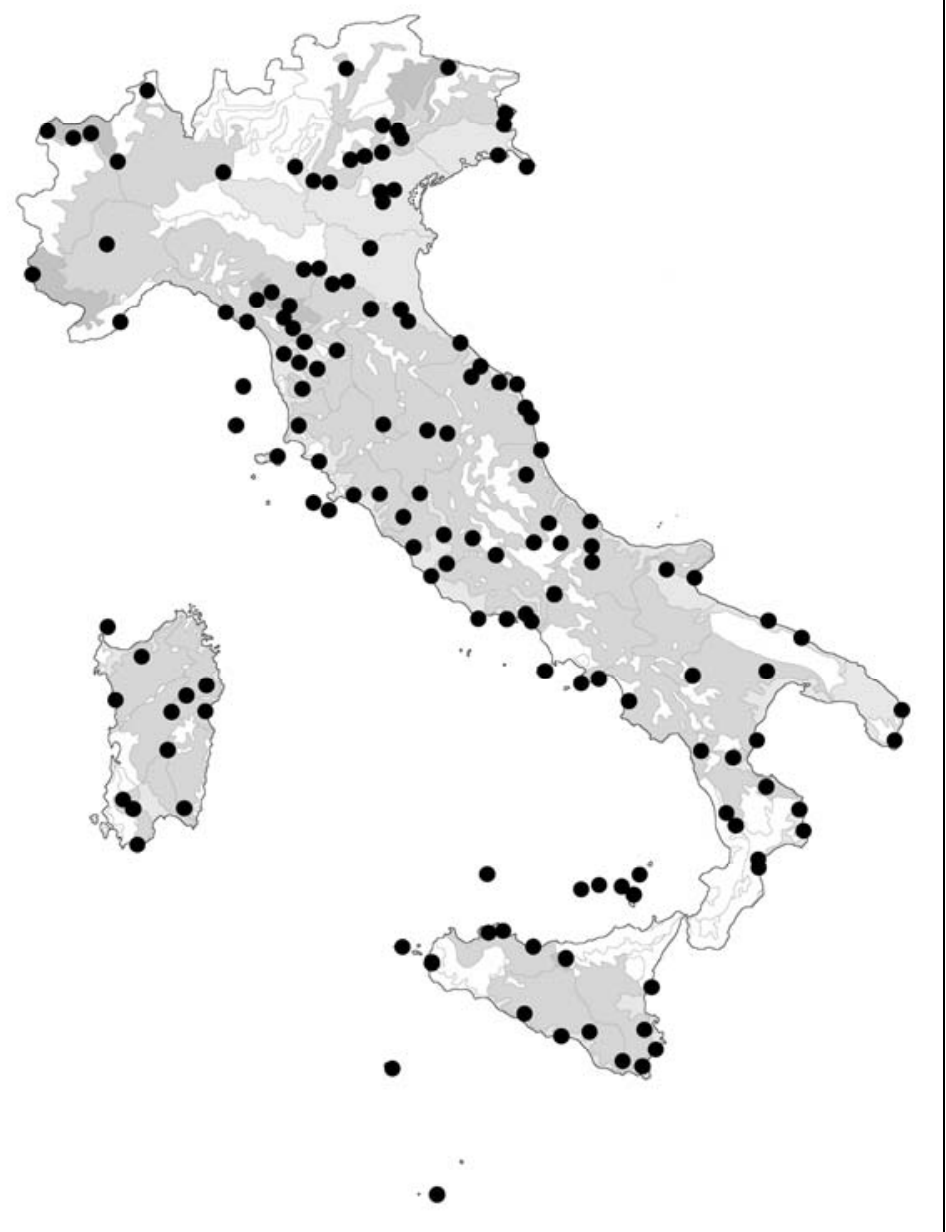




\section{Figura 12 \\ Borago pygmaea: \\ (A) habitus; (B)}

infiorescenza; (C) dettaglio

di una cima fiorifera; (D)

mericarpo in visione

laterale.

Barra di scala: $A, B=10 \mathrm{~cm}$;

$C=2 \mathrm{~cm} ; D=1 \mathrm{~cm}$.

Disegno di L. Cecchi

(A-C : coltivata a Firenze da pianta originaria dell'isola di Capraia (LI), da foto in vivo;

$\mathrm{D}$ : Ingurtosu, Iglesias (Cl), 1863, Ascherson, FI).

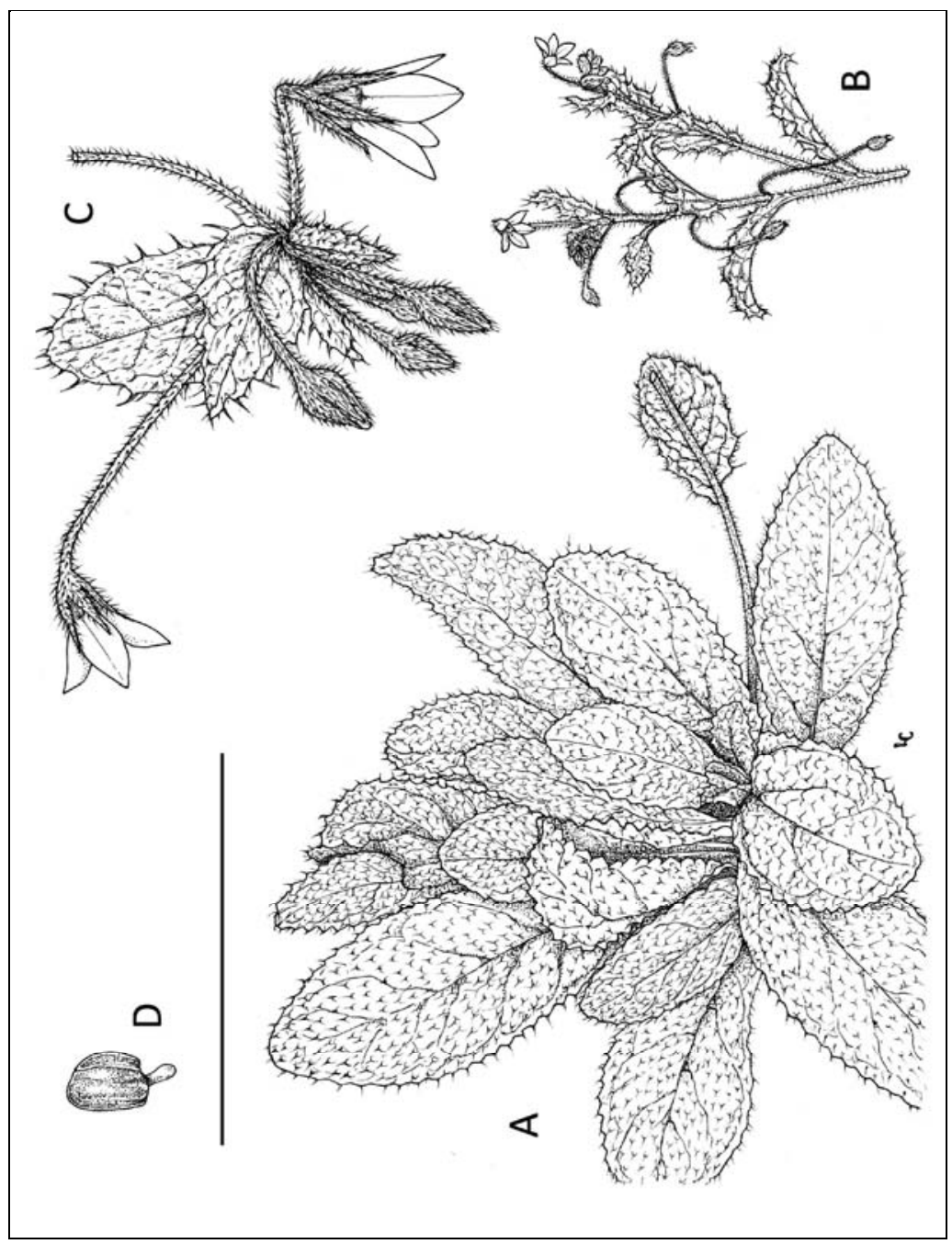


Figura 13

Distribuzione italiana di Borago pygmaea.

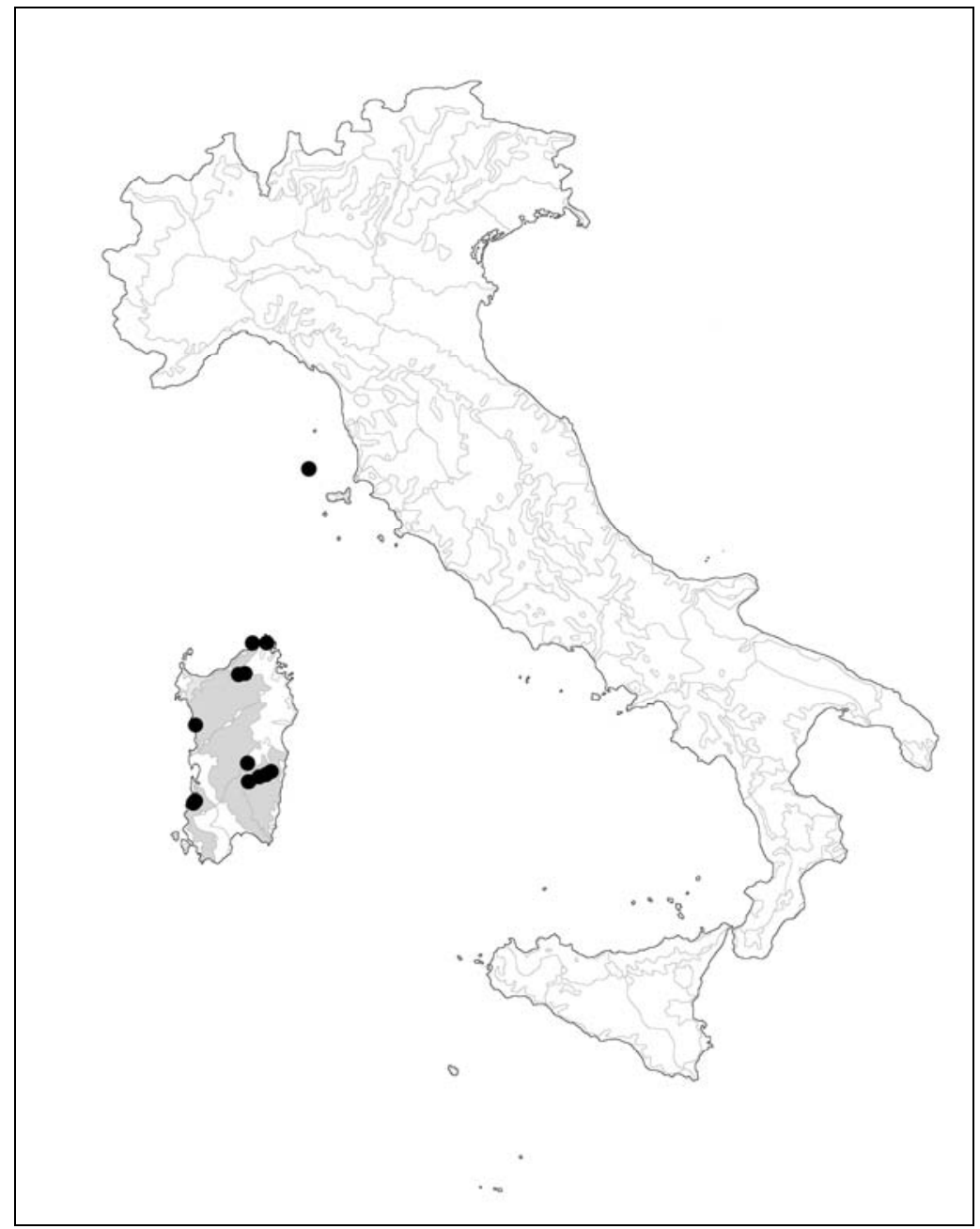


Figura 14

Symphytum asperum:

(A) habitus; (B) mericarpo (senza elaiosoma) in visione laterale.

Barra di scala: $A=10 \mathrm{~cm} ; B=1 \mathrm{~cm}$. Disegno di L. Cecchi (Val Ridanna (BZ), 1950, Zenari, FI)

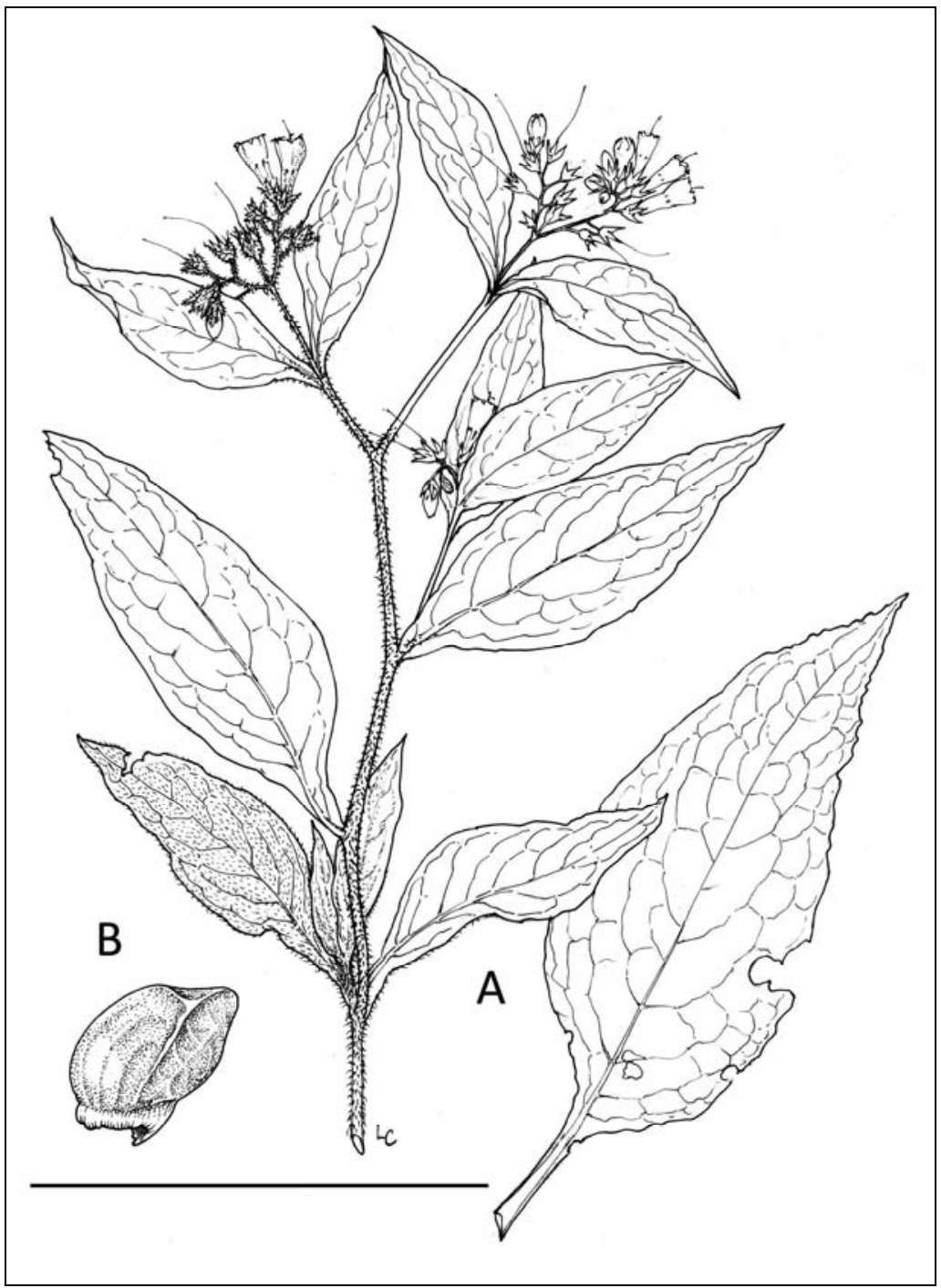


Figura 15

Distribuzione italiana di Symphytum asperum.

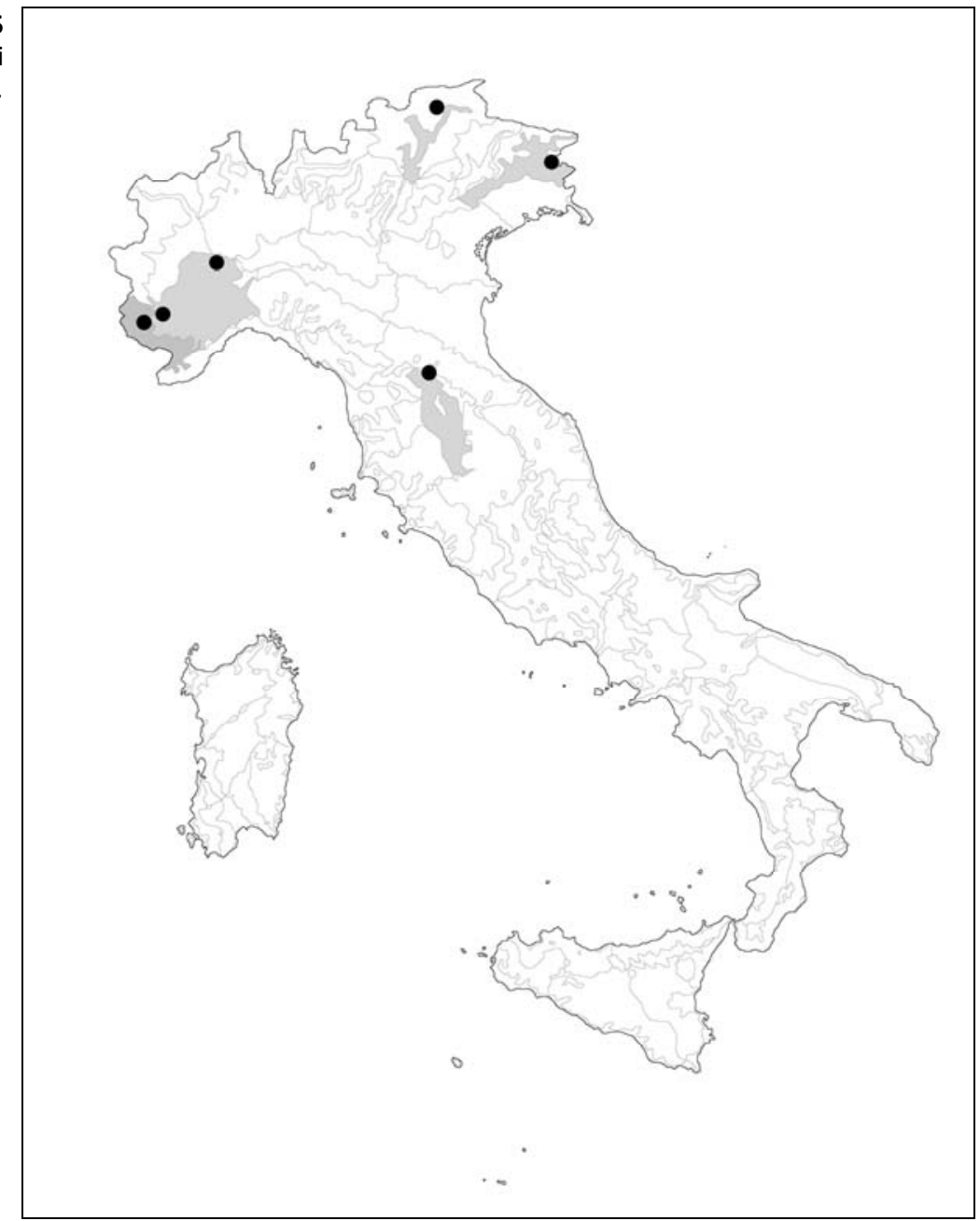


Figura 16

Symphytum bulbosum:

(A) habitus; (B) mericarpo in visione laterale. Barra di scala: $A=10 \mathrm{~cm} ; B=1 \mathrm{~cm}$. Disegno di L. Cecchi (A: Monte Argentario (GR), 1993, Baldini, Fl.

$\mathrm{B}$ : Firenze $(\mathrm{FI})$, sine die, Sommier, FI).

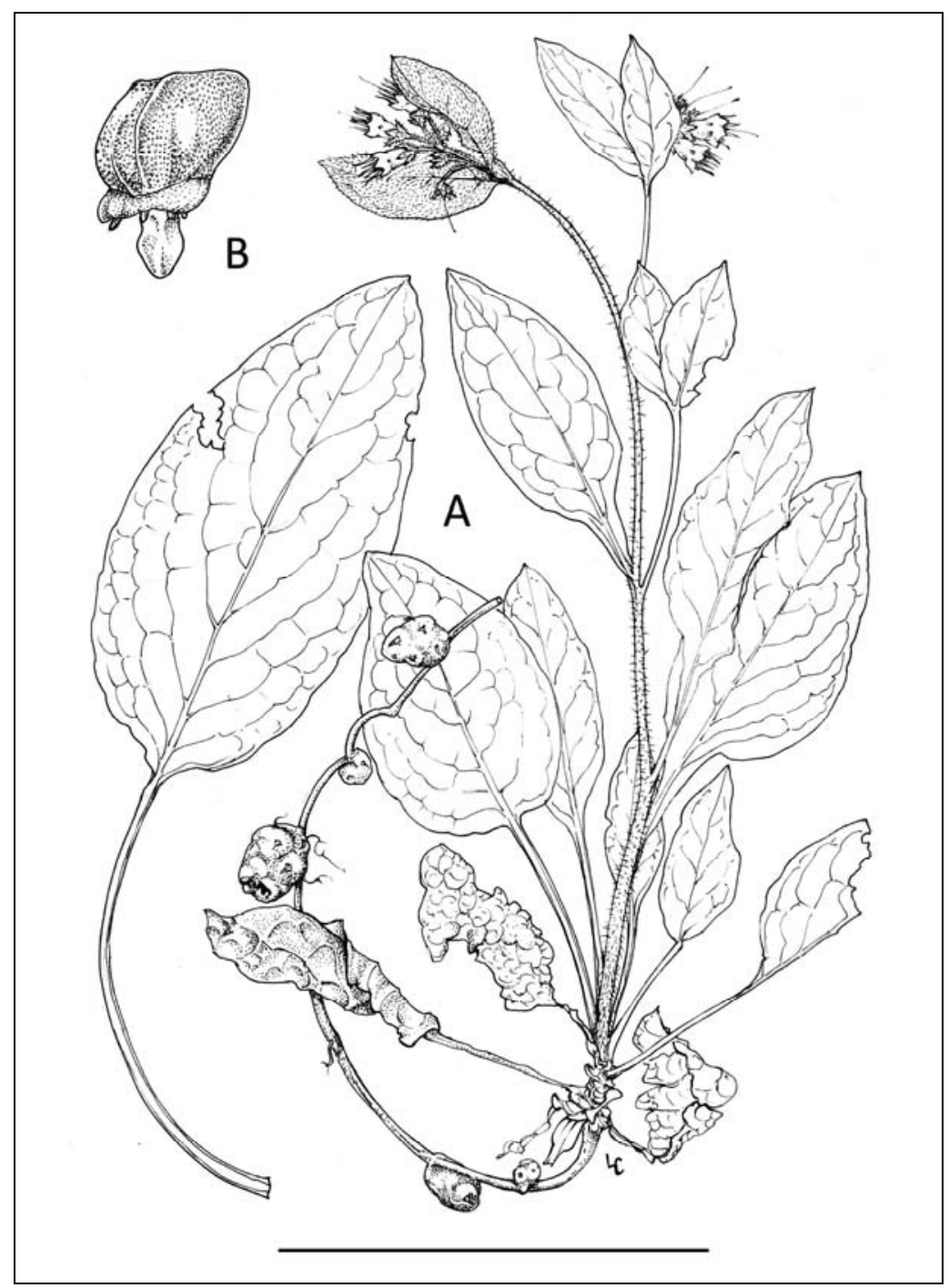


Figura 17

Distribuzione italiana di Symphytum bulbosum.

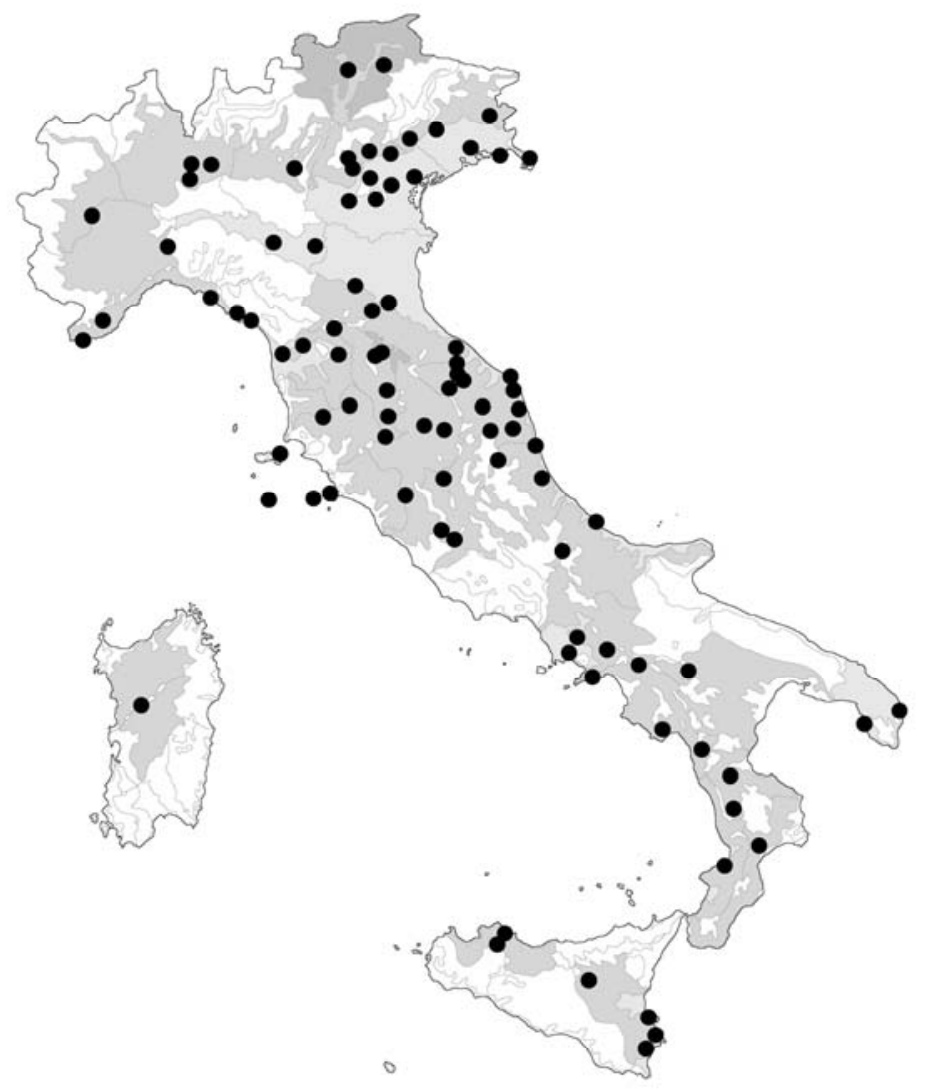


Figura 18

Symphytum gussonei:

(A) habitus; (B) fiore; (C) corolla aperta. Barra di scala: $A=3 \mathrm{~cm}$; $B, C$ $=7,5 \mathrm{~mm}$. Disegno di L. Cecchi (Gibilmanna (PA), 1887, Lojacono, FI; Messina, sine die, Nicotra, FI).

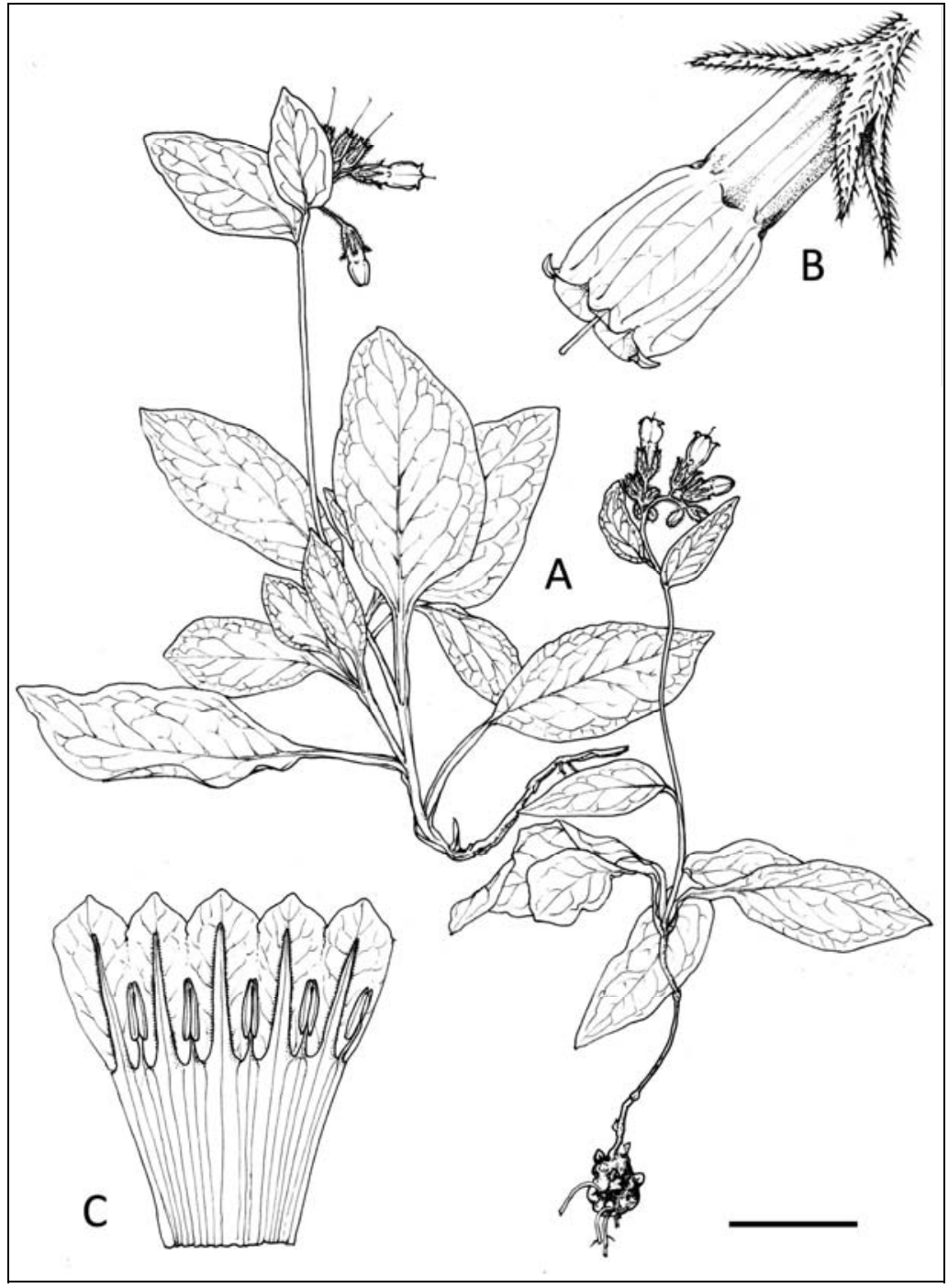


Figura 19

Distribuzione italiana di Symphytum gussonei.

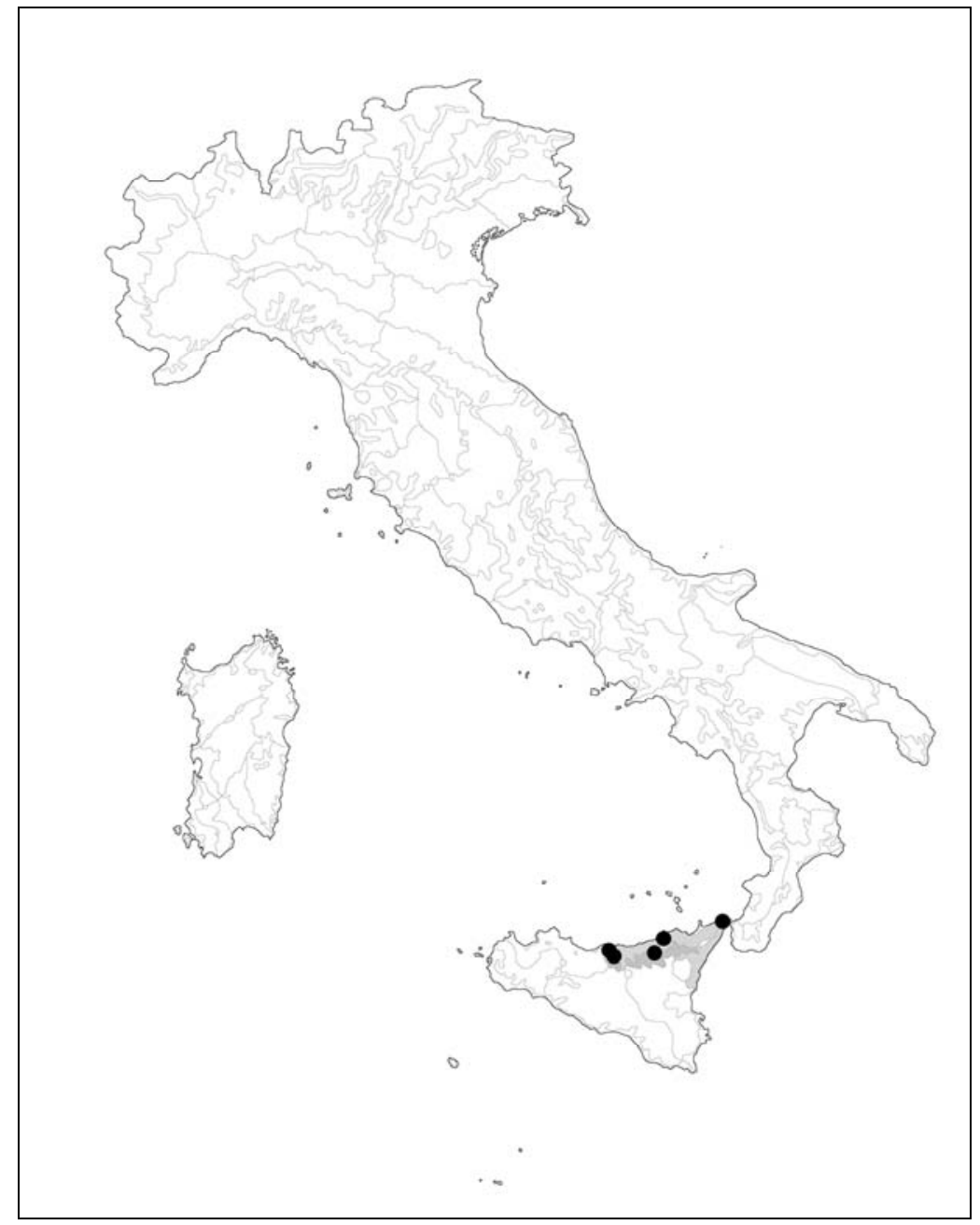


Figura 20

Symphytum officinale:

(A) fronda con infiorescenze; $(B, C)$ radici e foglie basali. Barra di scala: $A, B=10 \mathrm{~cm}$; $\mathrm{C}=1 \mathrm{~m}$. Disegno di L. Cecchi (A: Aosta (AO), 1899, Vaccari, $\mathrm{Fl}$. B: Campi Bisenzio (FI), 2005, Bartolini \& Cecchi, Fl).

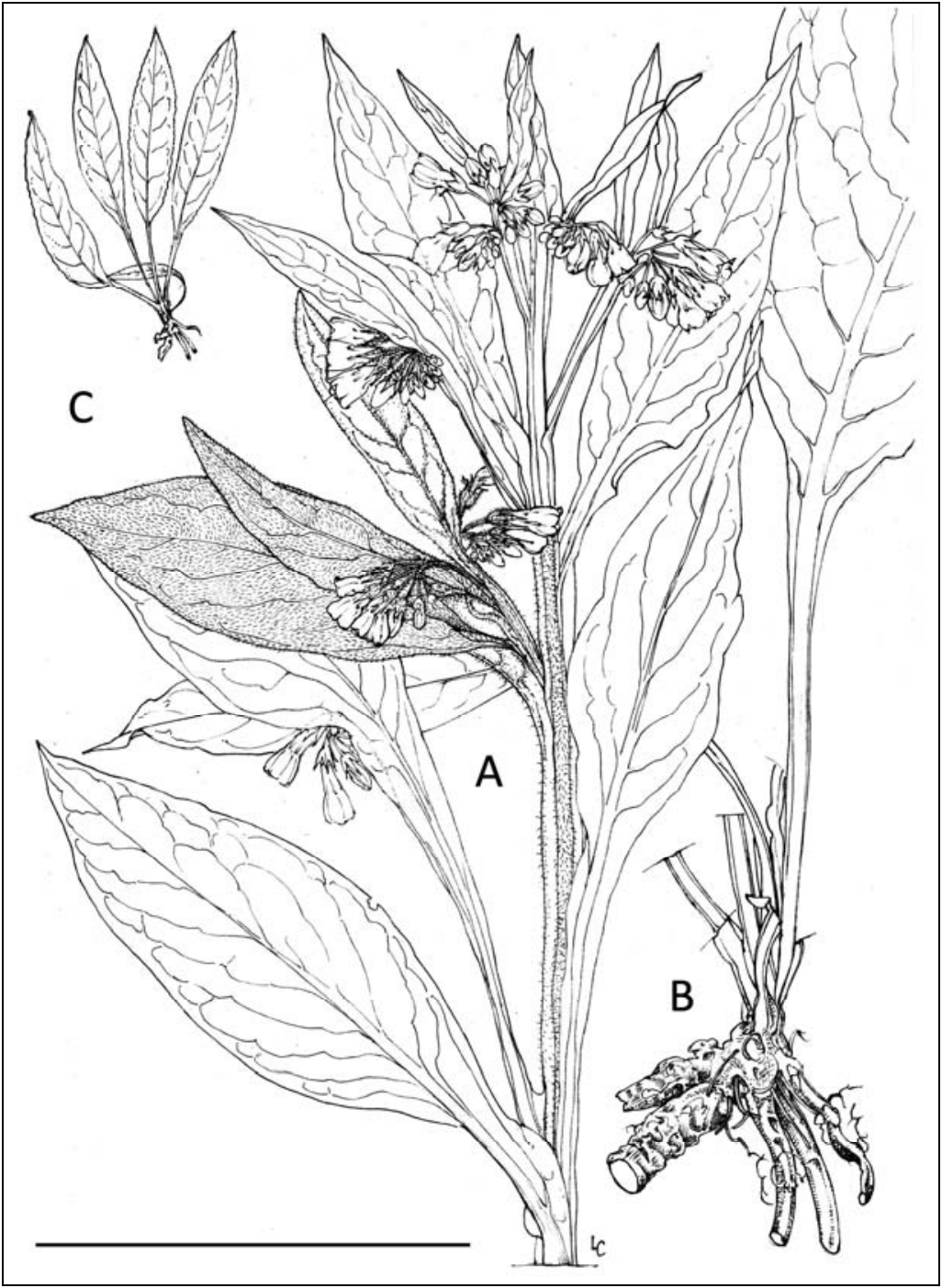


Figura 21

Distribuzione italiana di Symphytum officinale.

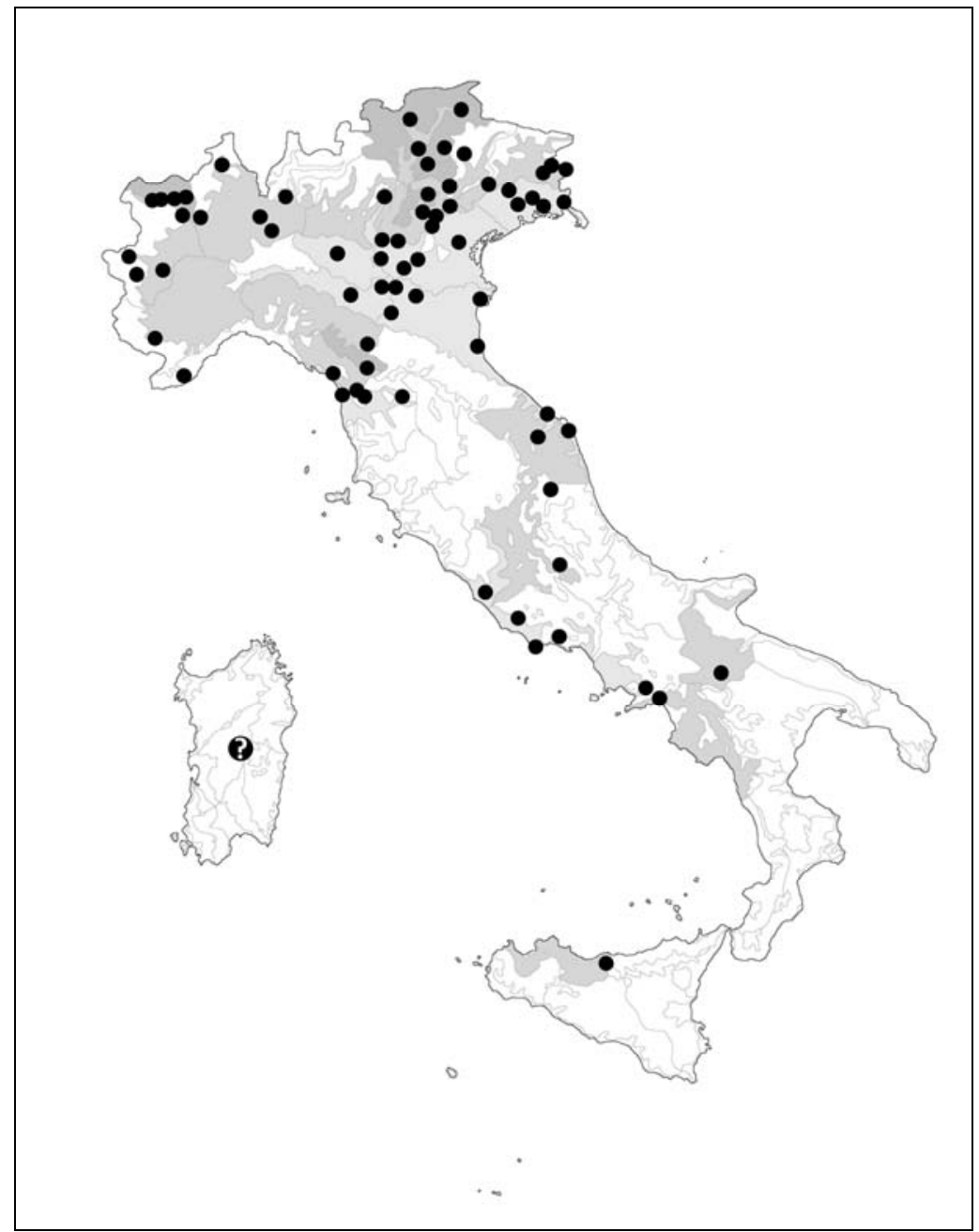


Figura 22

Symphytum orientale: (A) infiorescenza; (B) foglia basale; (C) fiore; (D) corolla aperta; $(E)$ calice fruttifero in sezione. Barra di scala: $A, B=3 \mathrm{~cm}$; $\mathrm{C}-\mathrm{E}=3 \mathrm{~mm}$. Disegno di L. Cecchi (campagna di Peretola (FI), dal vivo).

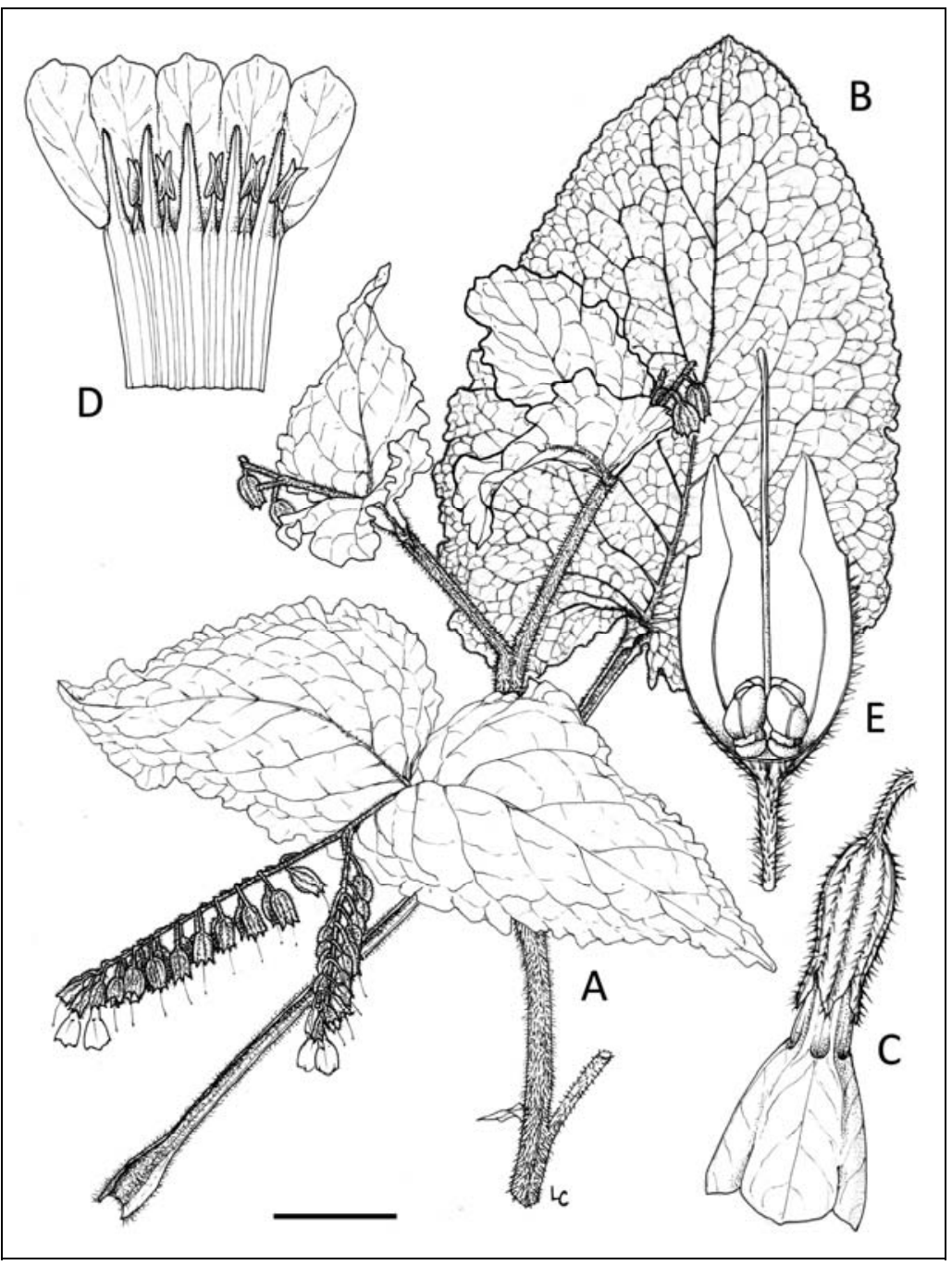


Figura 23

Distribuzione italiana di Symphytum orientale.

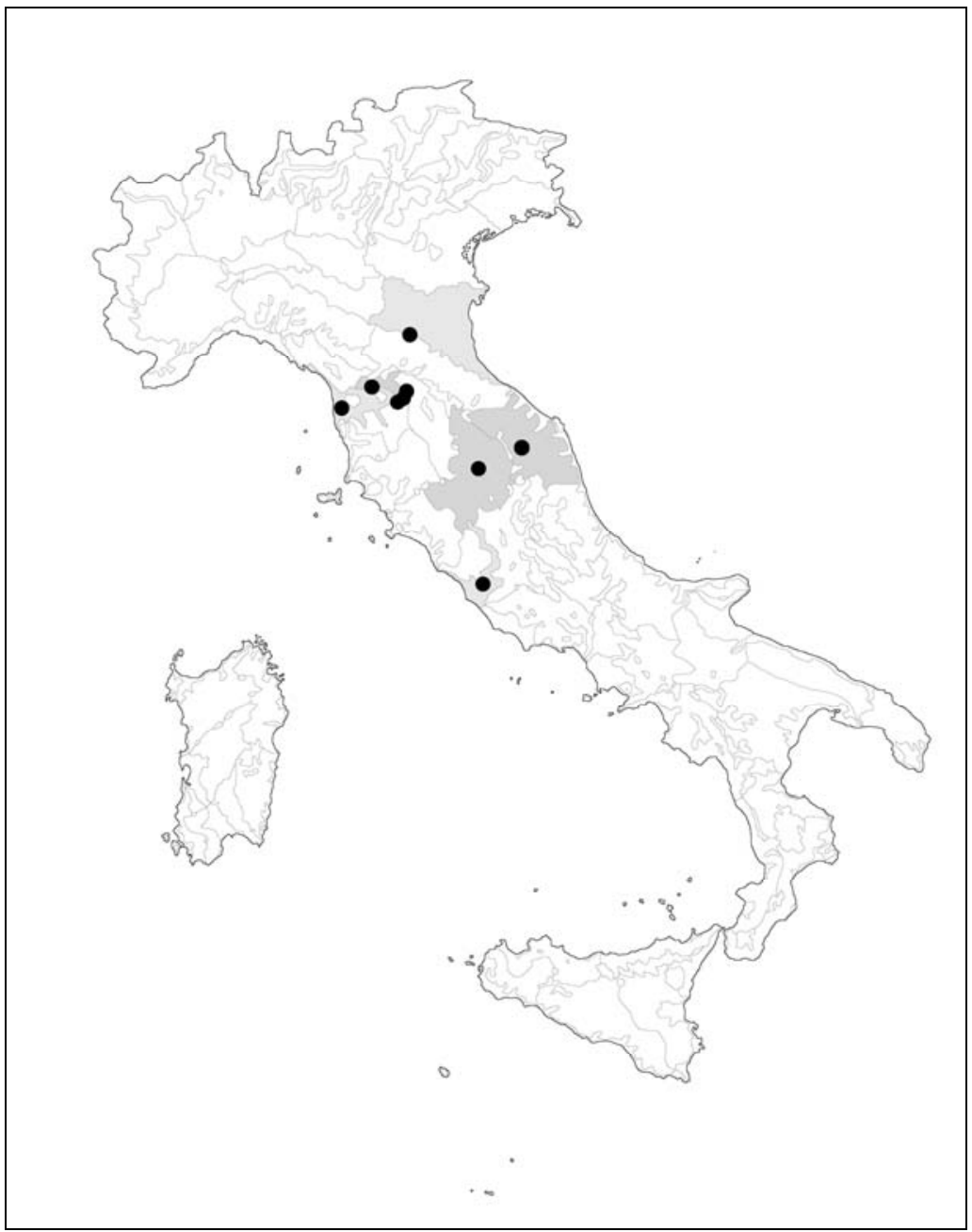


Figura 24

Symphytum tanaicense:

(A) habitus; (B) fiore; (C) corolla aperta; (D) mericarpo in visione laterale.

Barra di scala: $A=10 \mathrm{~cm}$; $B$ $C=1 \mathrm{~cm} ; D=5 \mathrm{~mm}$.

Disegno di A. Maury (in vivo da pianta coltivata nell'orto botanico di Pisa, origine: lago di Massaciuccoli (LU), 1999, Peuzzi \& Bottega 388/1999; da PeruzzI \& al. 2001: 35 , per gentile concessione dell'editore di Willdenowia).

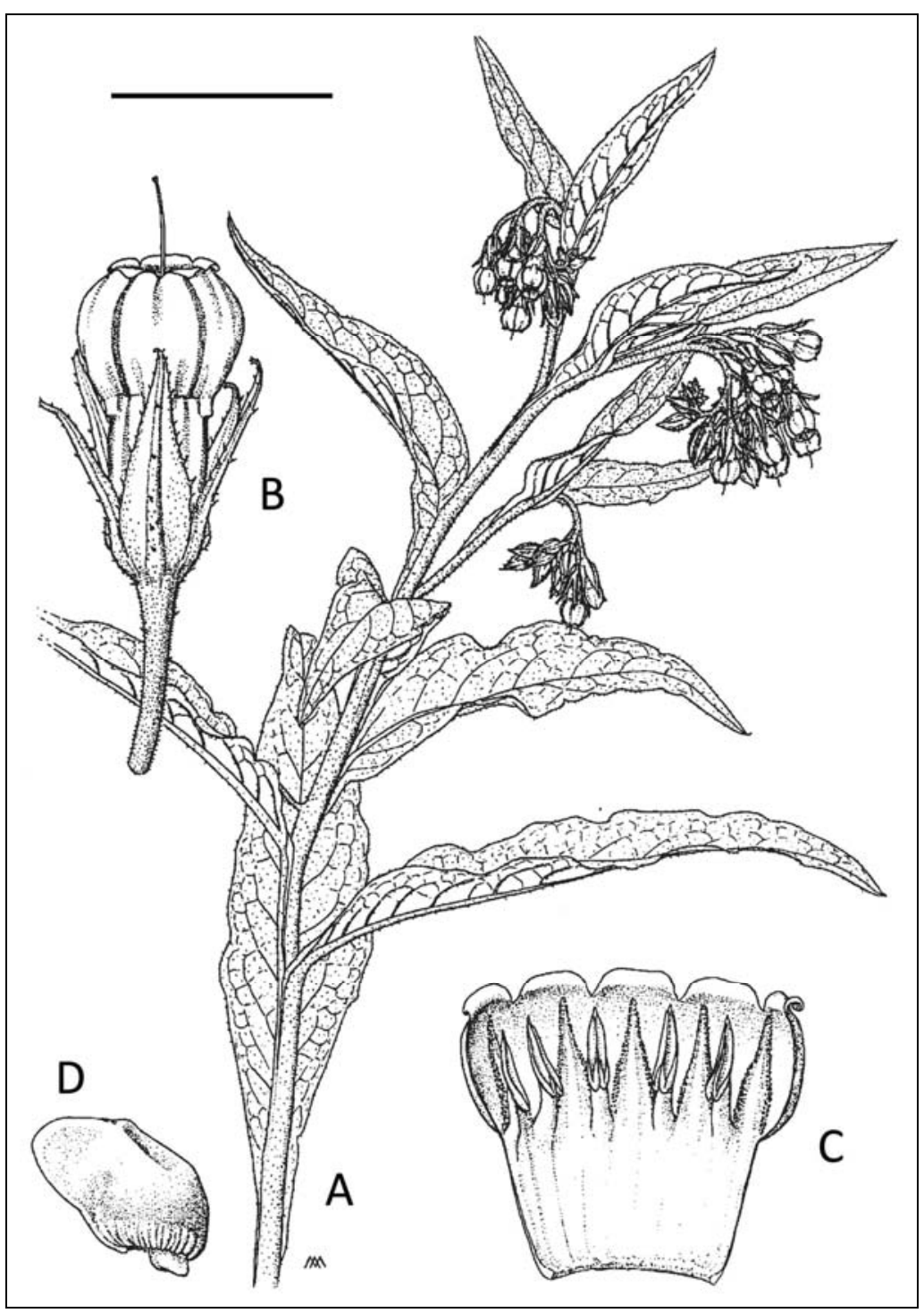


Figura 25

Distribuzione italiana di Symphytum tanaicense.

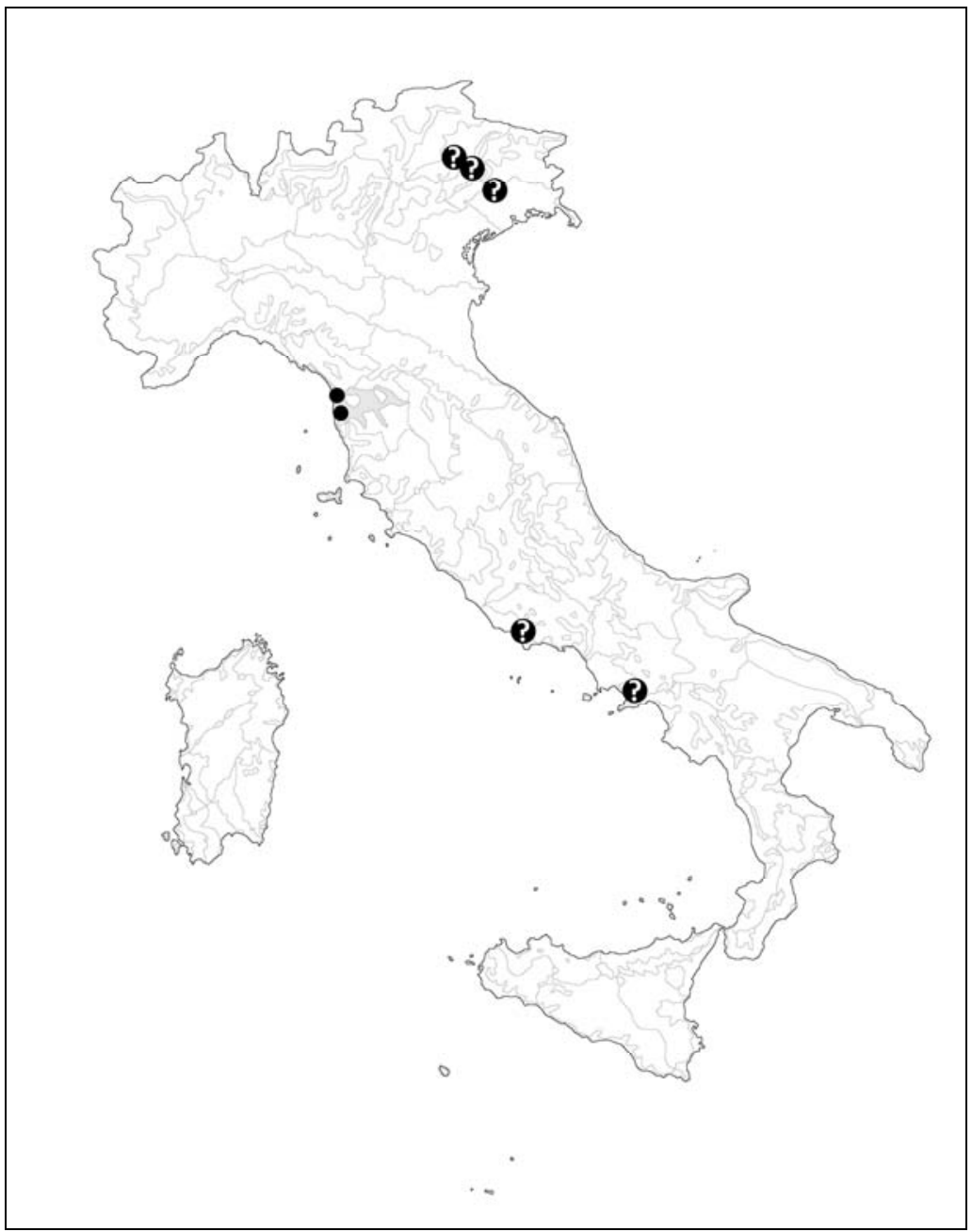


Figura 26

Symphytum tuberosum subsp. angustifolium:

(A) fronda con infiorescenze; $(B, C)$ radici e foglie basali. Barra di scala: $A, B=10 \mathrm{~cm}$; $\mathrm{C}=1 \mathrm{~m}$.

Disegno di L. Cecchi

(A: monte Spaccato (TS), 1873, Solla, FI.

B: Vittorio Veneto (TV), 1894, Pampanini, FI. C: Varallo Sesia (VC), 1963, Bono, FI).

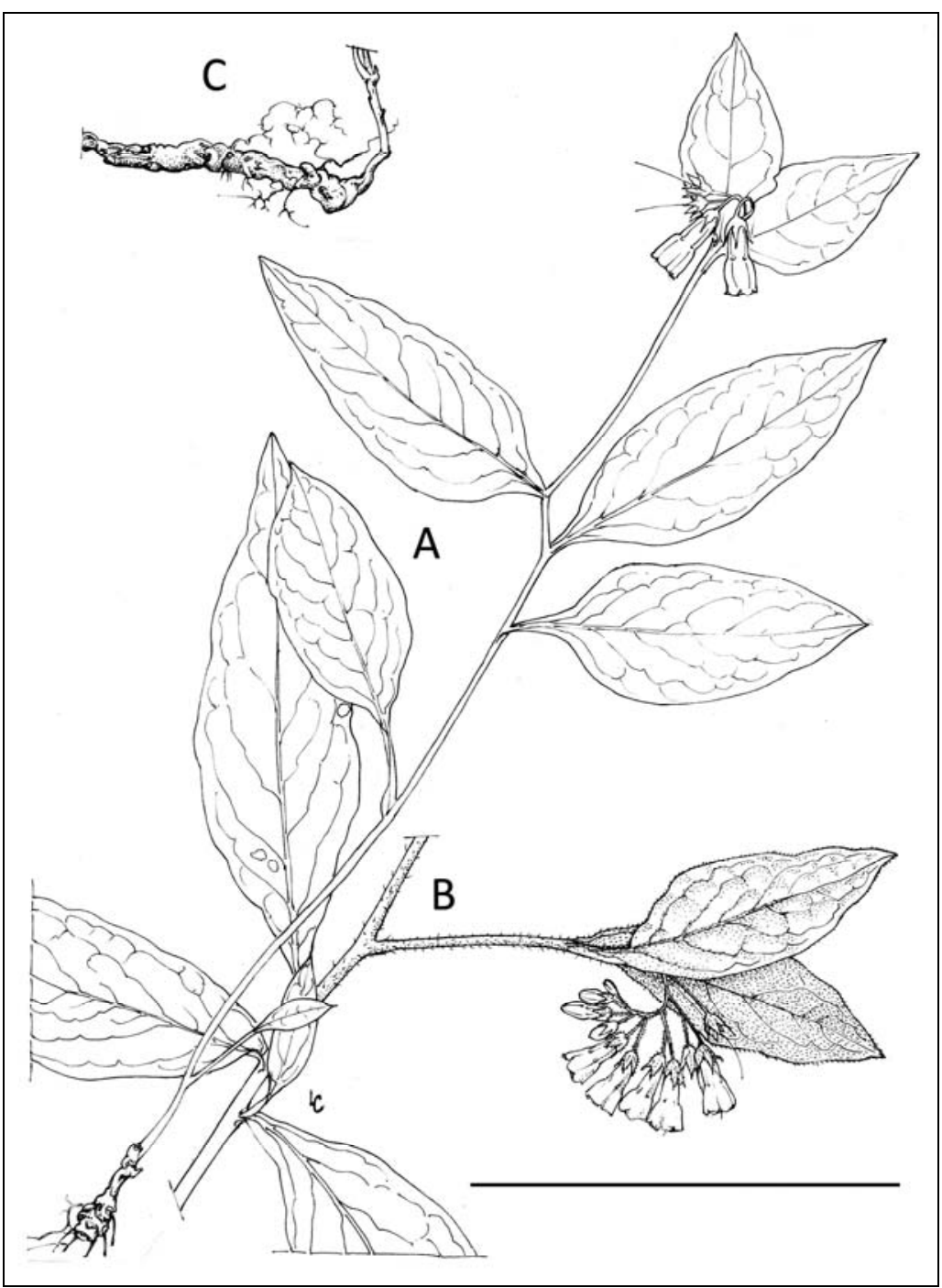


Figura 27

Distribuzione italiana di Symphytum tuberosum.

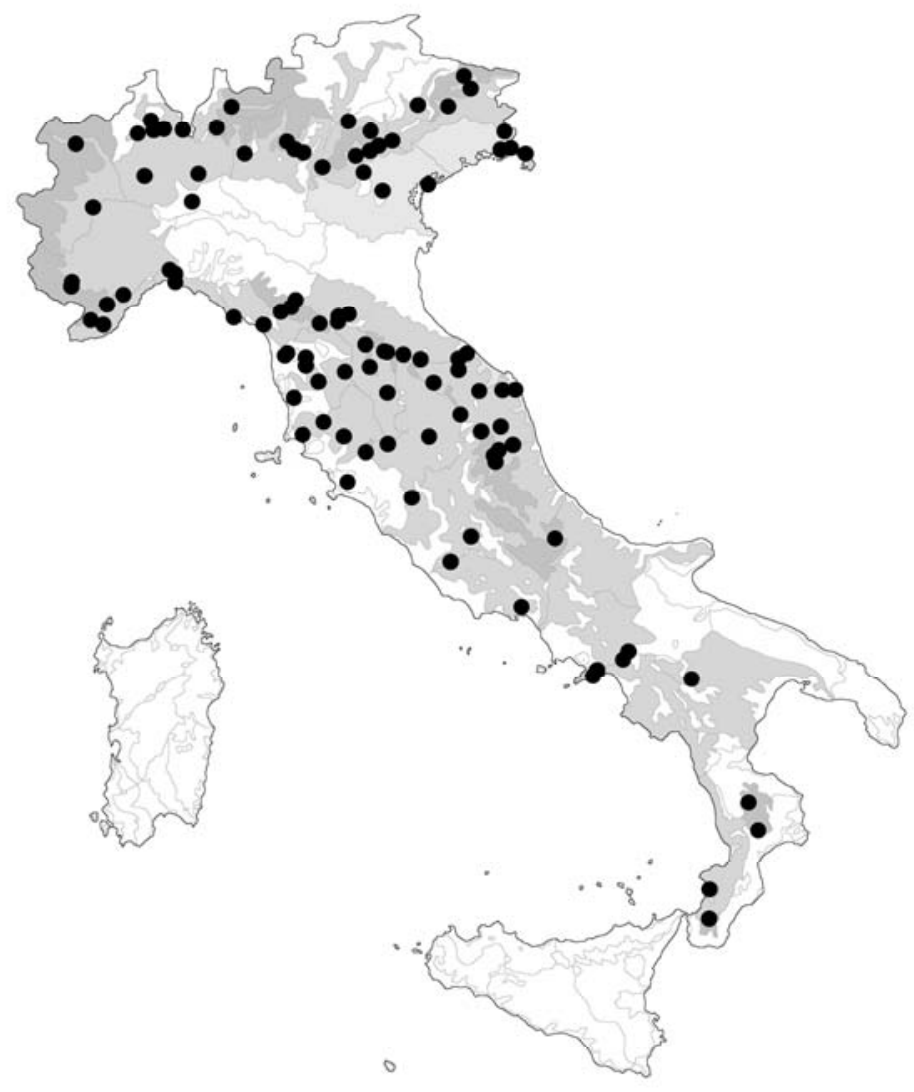




\section{Figura 28}

Melanortocarya obtusifolia: (A, B) habitus; (C) dettaglio dell'infiorescenza; (D) calice fruttifero; $(E)$ mericarpo in visione laterale. Barra di scala: $A, B=10 \mathrm{~cm}$; $C, D=2 \mathrm{~cm} ; E=1 \mathrm{~cm}$. Disegno di L. Cecchi (A, C- E: Israele, Neve Ilan, 1996, Bigazzi \& Selvi, FI-HB 96.12.

B: Tor D'Angelo (RM), 1876. De Notaris, Herb. Goiran, VER).
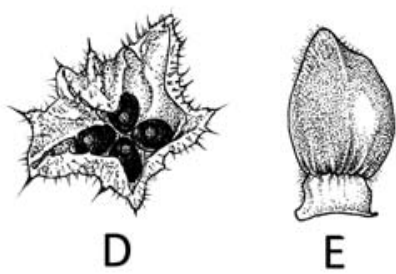
E
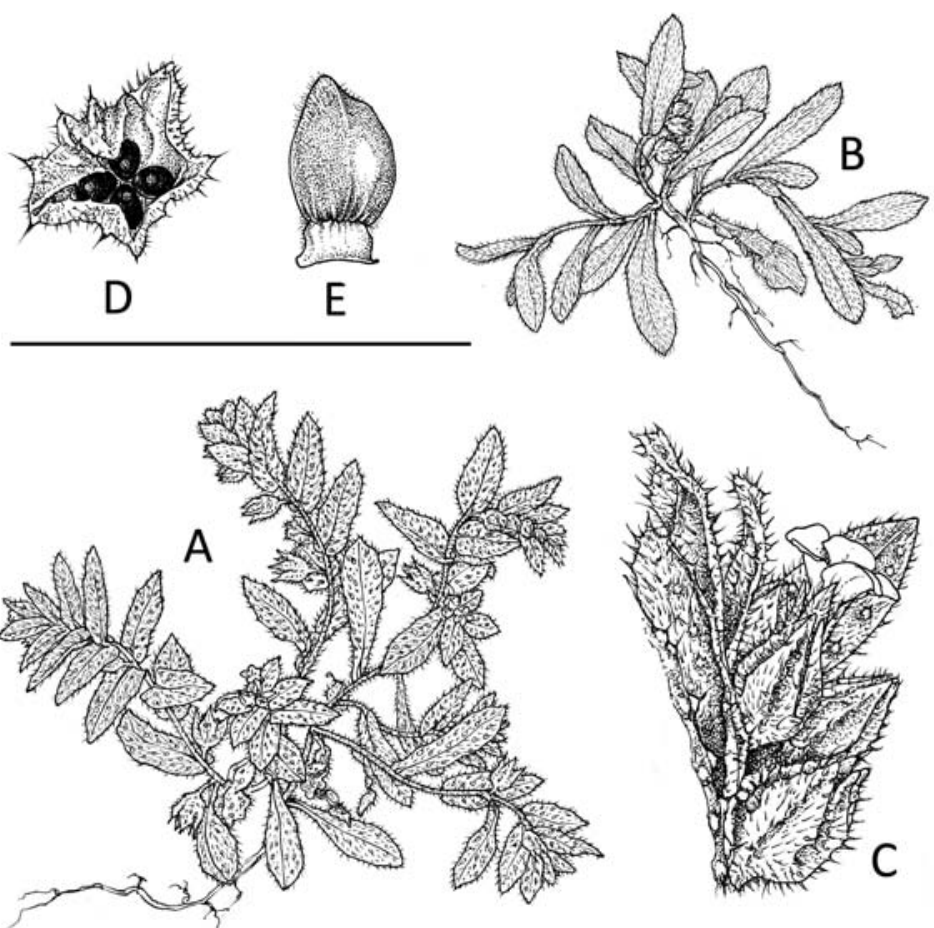
Figura 29

Distribuzione italiana di Melanortocarya obtusifolia.

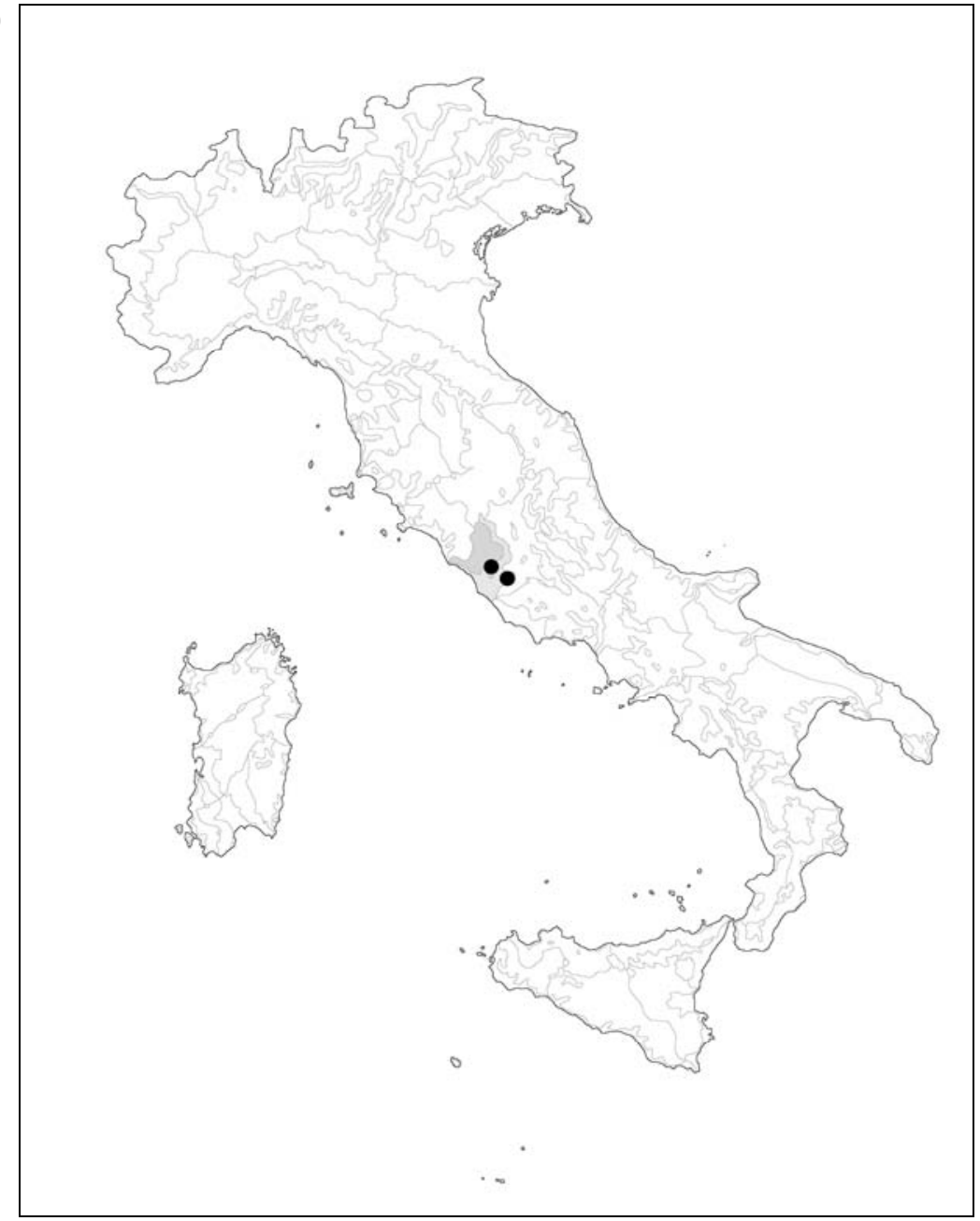


Figura 30

Pulmonaria angustifolia: (A) habitus; (B) dettaglio dell'indumento sulla pagina superiore delle foglie basali adulte; (C) corolla chiusa;

(D) corolla aperta.

Barra di scala: $A=2 \mathrm{~cm}$; $B-D=4 \mathrm{~mm}$.

Disegno di L. Cecchi (val di Ledro (TN), 1905, Porta, FI).

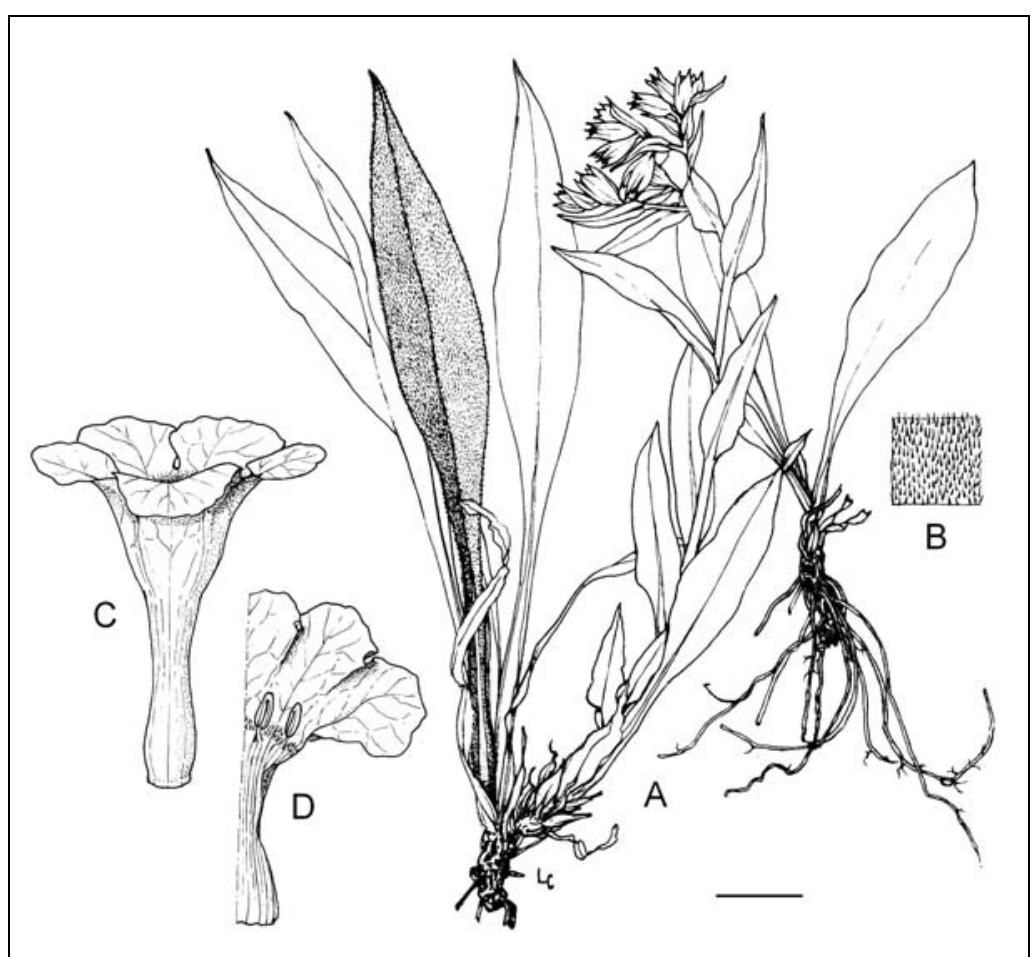


Figura 31

Pulmonaria australis:

(A) habitus; (B) dettaglio dell'indumento sulla pagina superiore delle foglie basali adulte.

Barra di scala: $A=2 \mathrm{~cm}$; $B=4 \mathrm{~mm}$. Disegno di L. Cecchi (bacino medio del Natisone (UD), Minio, FI).

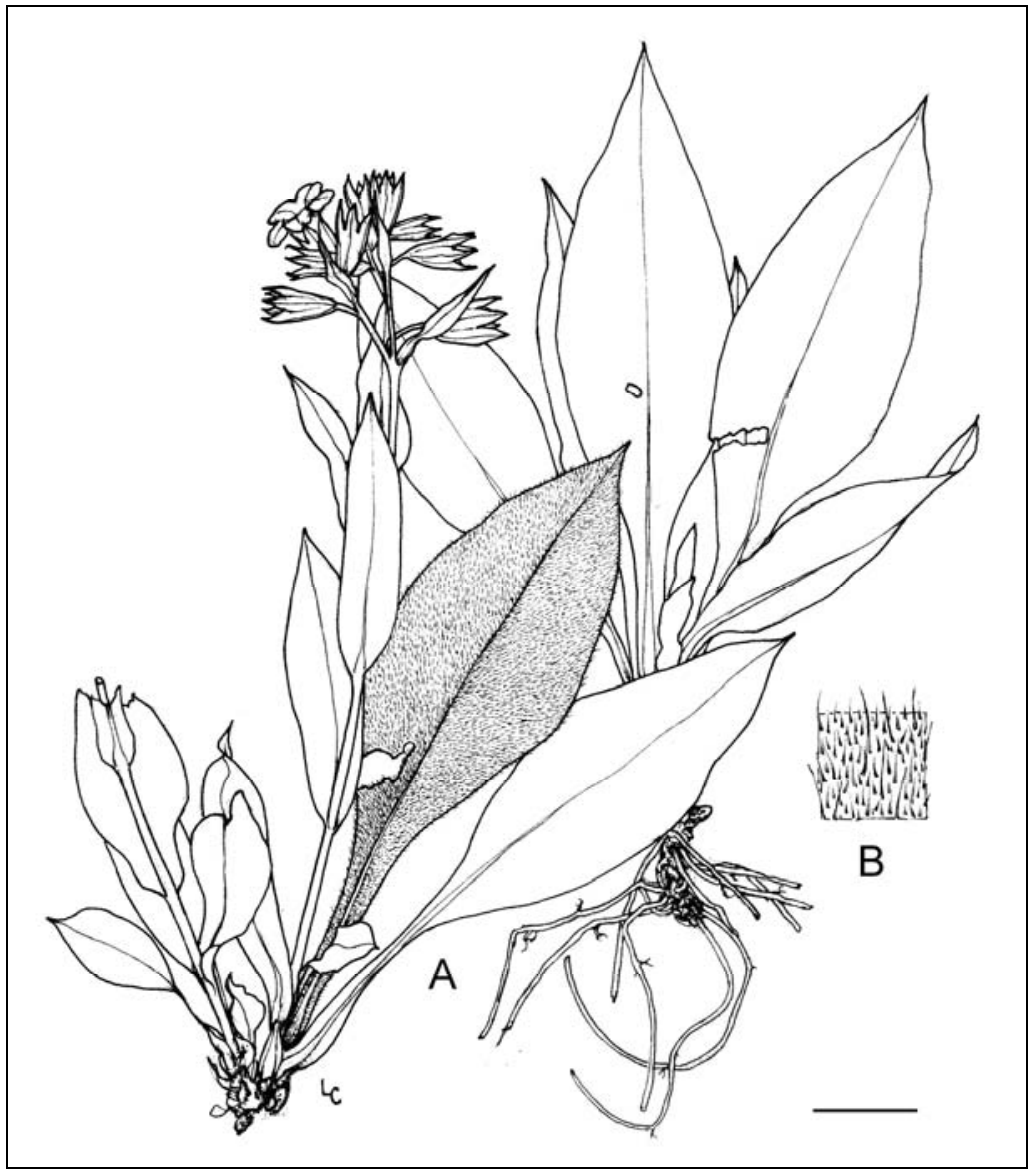


Figura 32

Distribuzione italiana delle specie del complesso di Pulmonaria angustifolia s.l.: $P$. angustifolia s.s. (tondi bianchi) e $P$. australis (tondi neri; la stella indica il locus classicus).

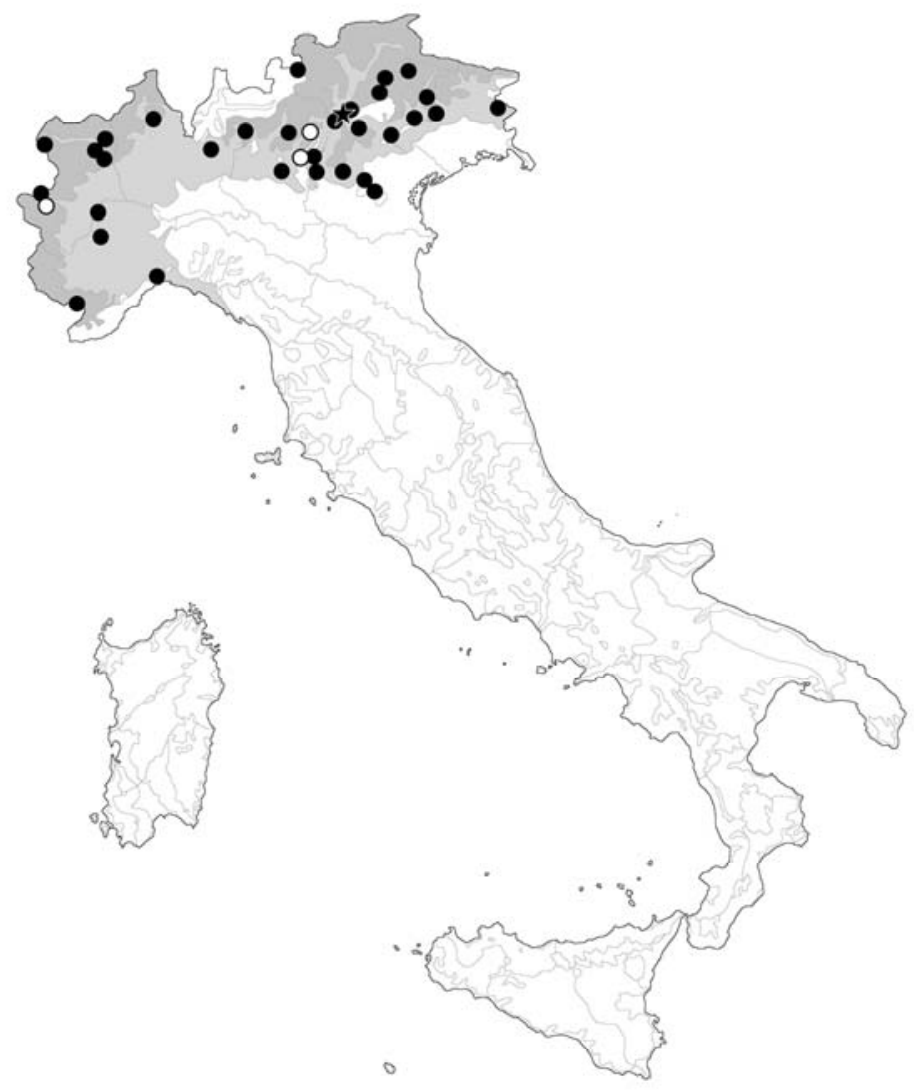


Figura 33

Pulmonaria hirta:

(A) habitus primaverile; (B)

foglia basale adulta; (C)

rosetta autunnale; (D)

dettaglio dell'indumento sulla pagina superiore delle

foglie basali adulte.

Barra di scala:

$\mathrm{C}=20 \mathrm{~cm}$;

$A, B=10 \mathrm{~cm} ; D=2 \mathrm{~cm}$.

Disegno di L. Cecchi

(A: Camaldoli (AR), 2002,

epitipo, Selvi, FI002356.

C: ibidem, 2013, da foto in

vivo. $\mathrm{B}, \mathrm{D}$ : La Consuma (AR), 1942, Corradi, FI).

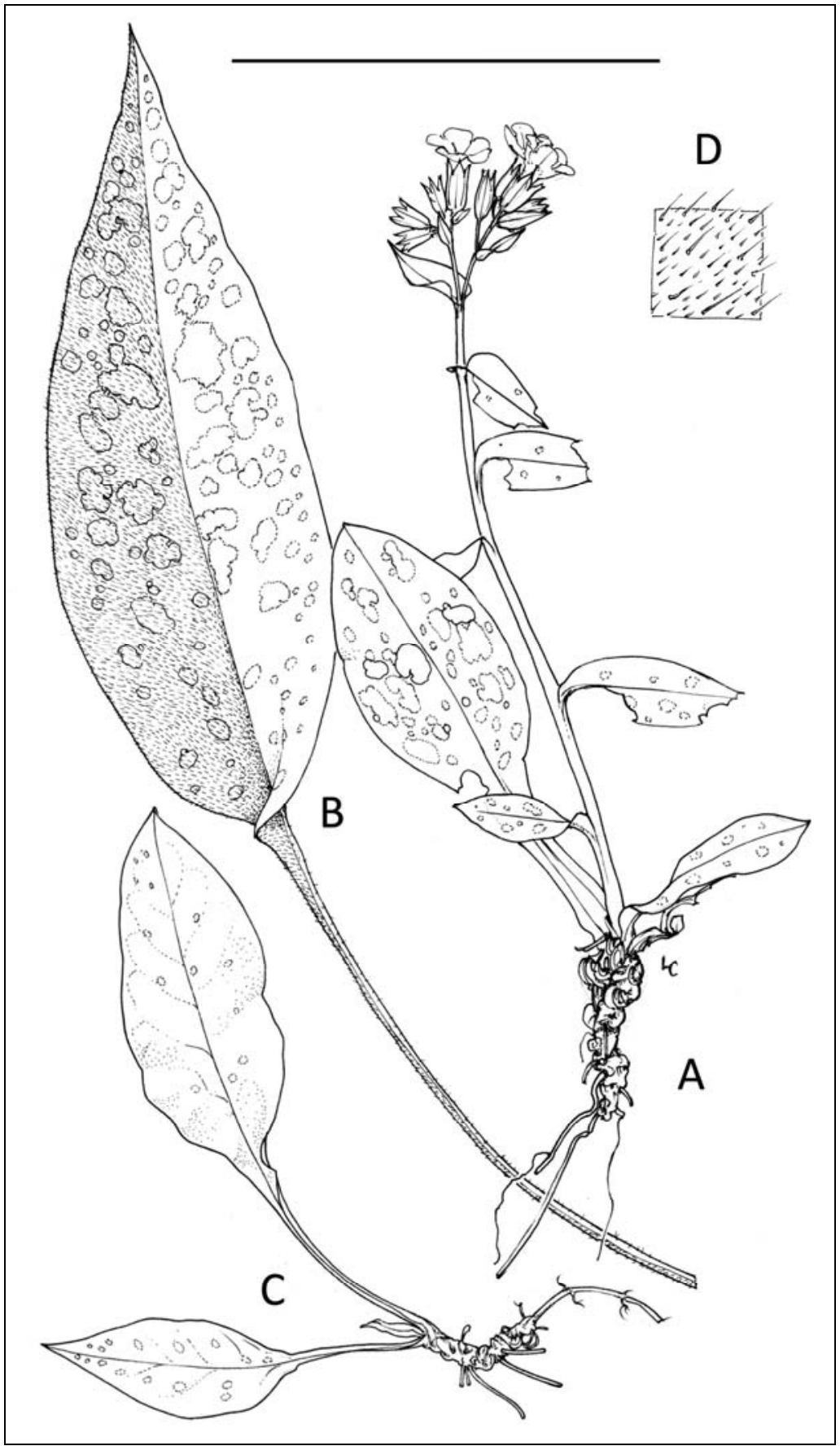


Figura 34

Distribuzione italiana di Pulmonaria hirta (la stella indica il locus classicus).

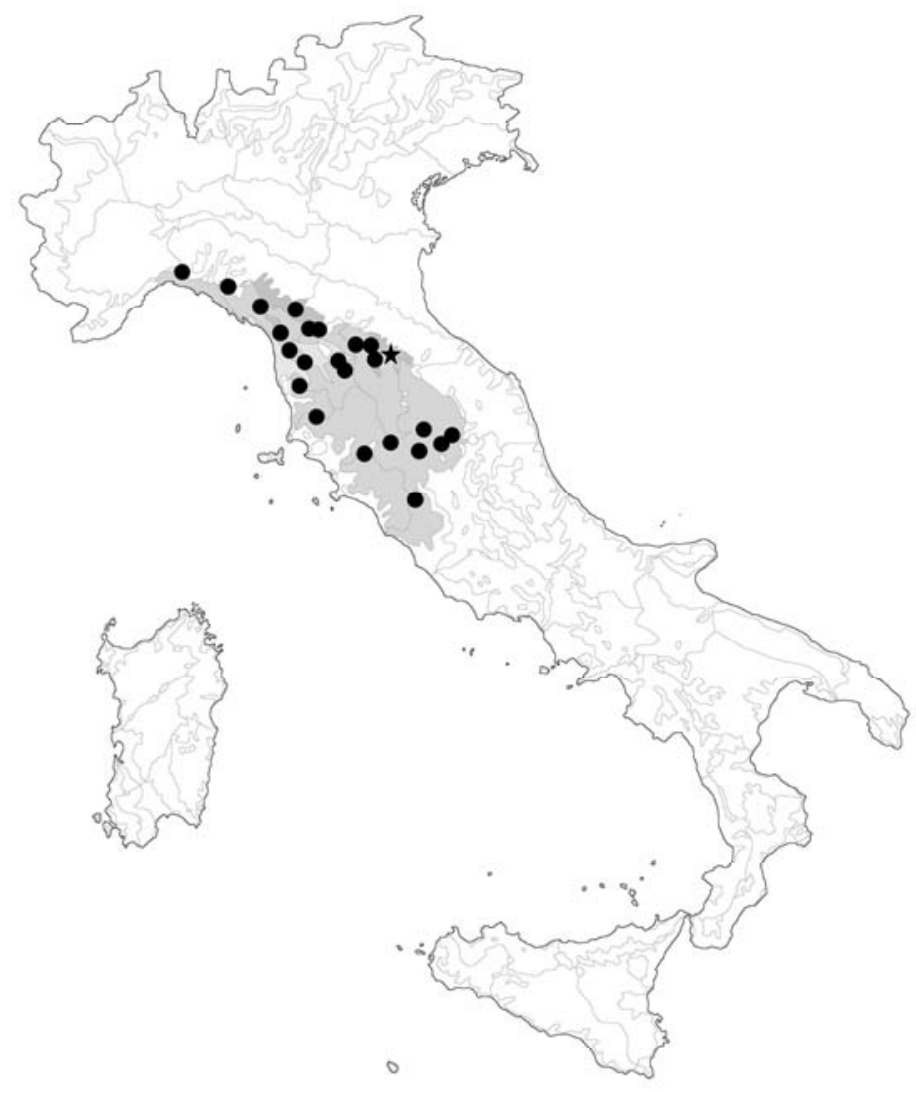




\section{Figura 35 \\ Pulmonaria officinalis} subsp. officinalis: (A) habitus; (B) foglia basale adulta; (C) dettaglio dell'indumento sulla pagina superiore delle foglie basali adulte.

Pulmonaria officinalis subsp. marzolae:

(D) habitus; (E) dettaglio dell'indumento sulla pagina superiore delle foglie basali adulte.

Barra di scala: $A, B, D=10 \mathrm{~cm} ; C, E=2 \mathrm{~cm}$. Disegno di L. Cecchi (A: pianta spontaneizzata a Charvensod (AO), 1899, Vaccari, $\mathrm{Fl}$.

B, C: Mantova (MN), 1977, Barini, FI.

D, E: olotipo, Sella Marzola (TN), 2013, Pupillo, FI 7164).

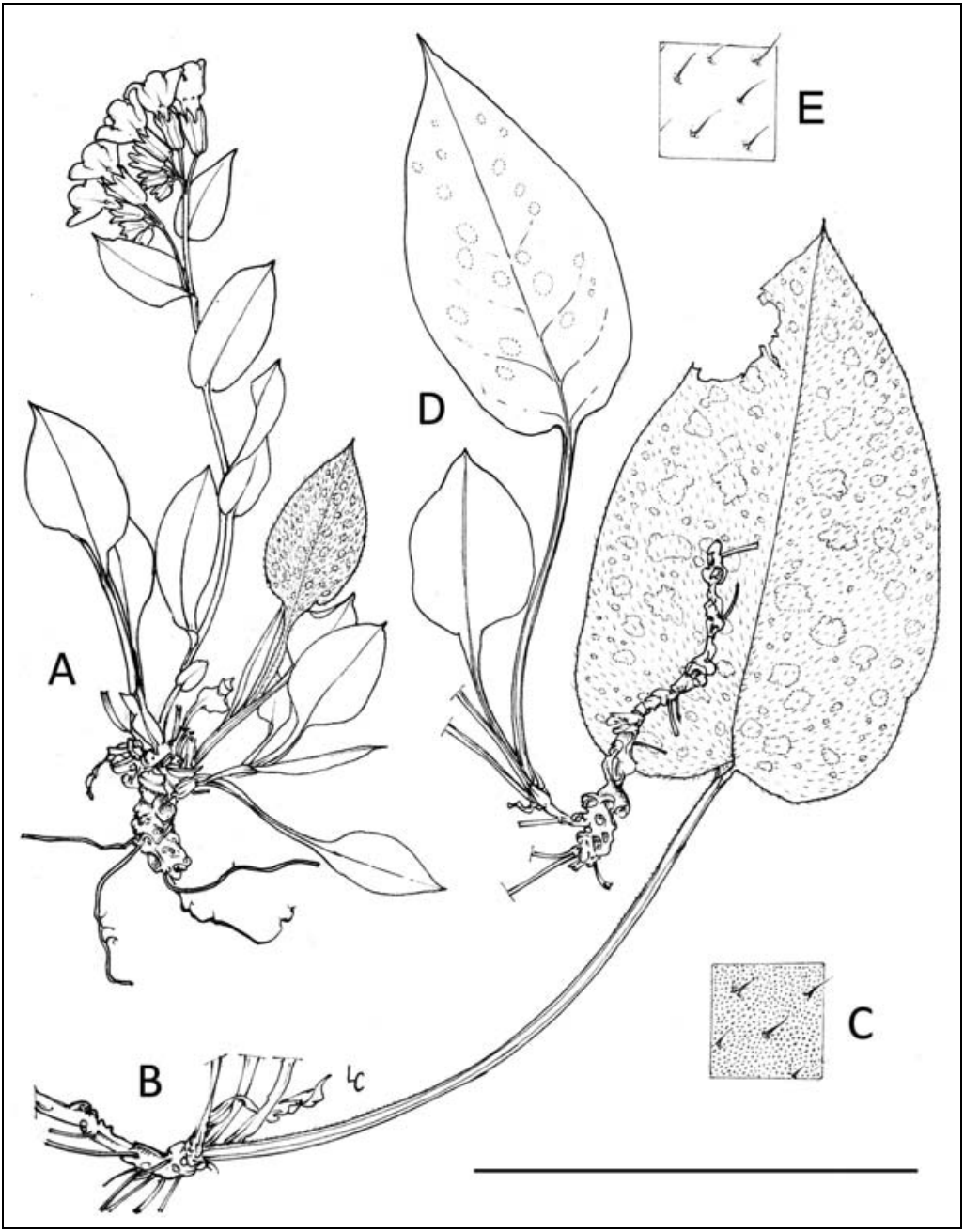


Figura 36

Distribuzione italiana di Pulmonaria officinalis subsp. officinalis (tondi neri) e subsp. marzolae (stella bianca, che indica il locus classicus).

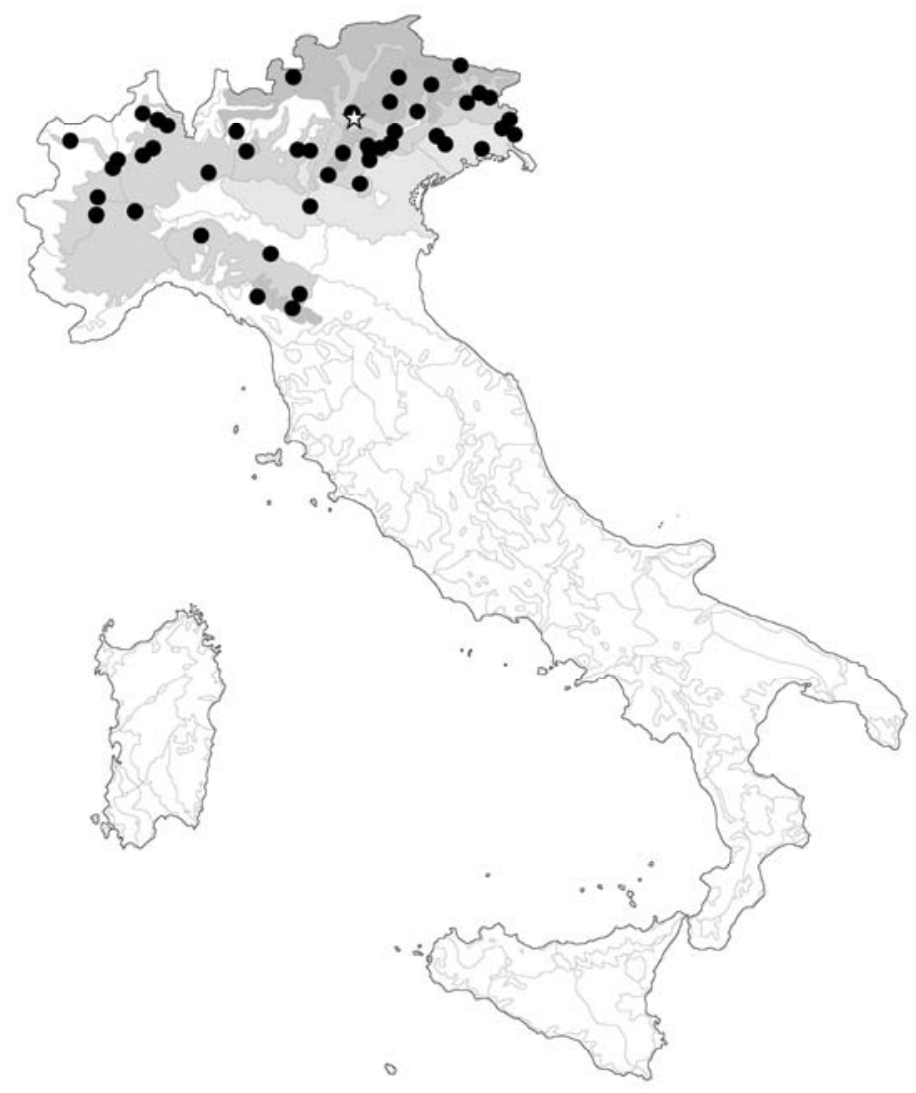


Figura 37

Pulmonaria stiriaca: (A, B) habitus; (C) dettaglio dell'indumento sulla pagina superiore delle foglie basali adulte.

Barra di scala: $A=10 \mathrm{~cm}$; $B \approx 20 \mathrm{~cm} ; D=2 \mathrm{~cm}$. Disegno di L. Cecchi (A: valle del Natisone (UD), 2007, Poldini 9846.2/3, Herb. Poldini (Trieste).

B: Slovenia, Ljubljana ("prope Labacum"), 1885, Deschmann \& Paulin, $\mathrm{Fl}$. Exs. Austro-Hung. $n^{\circ} 928, \mathrm{P}$ 508147).

C: Austria, Graz, 1949, Höpflinger, FI).

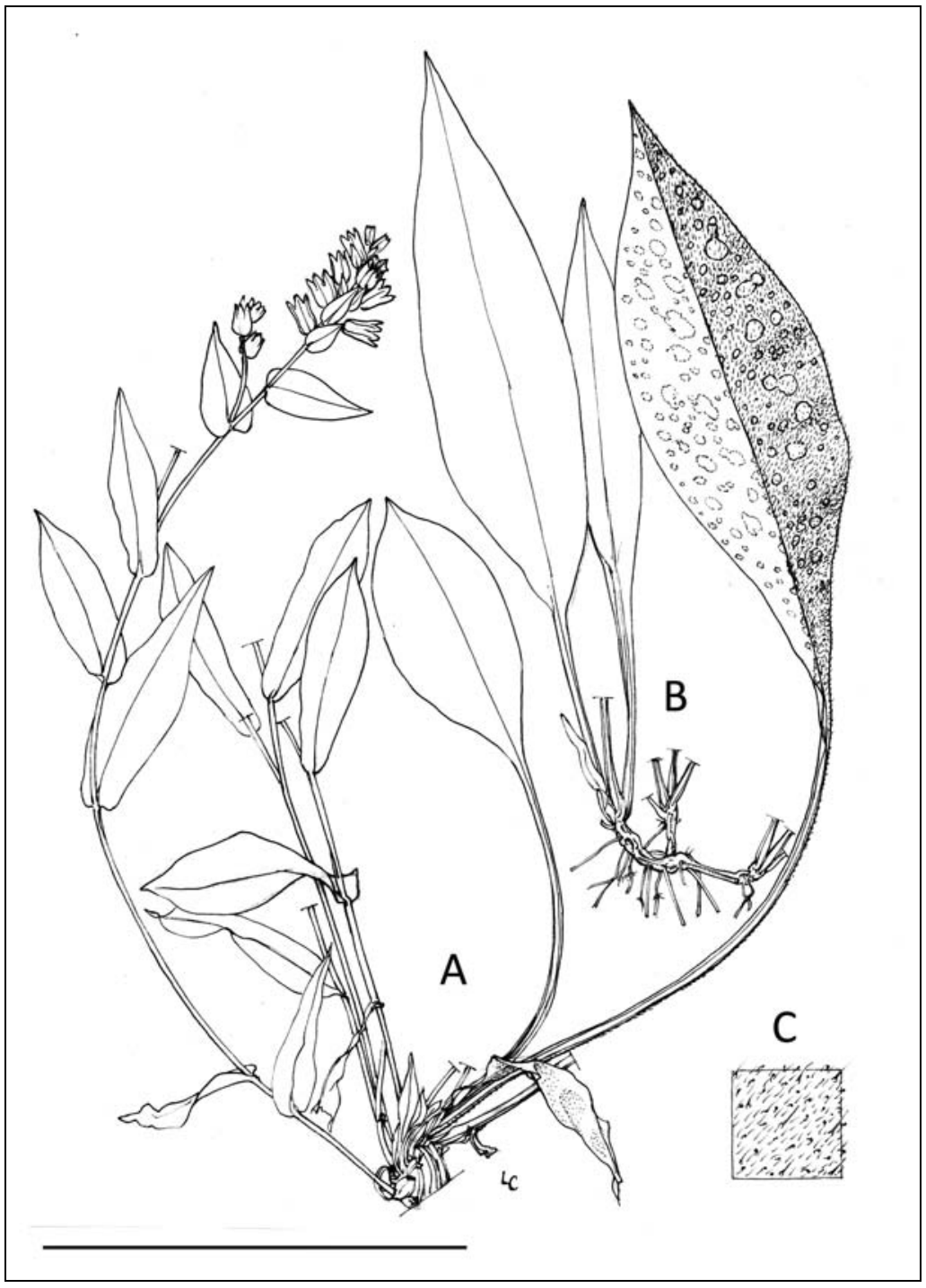


Figura 38

Distribuzione italiana di Pulmonaria stiriaca.

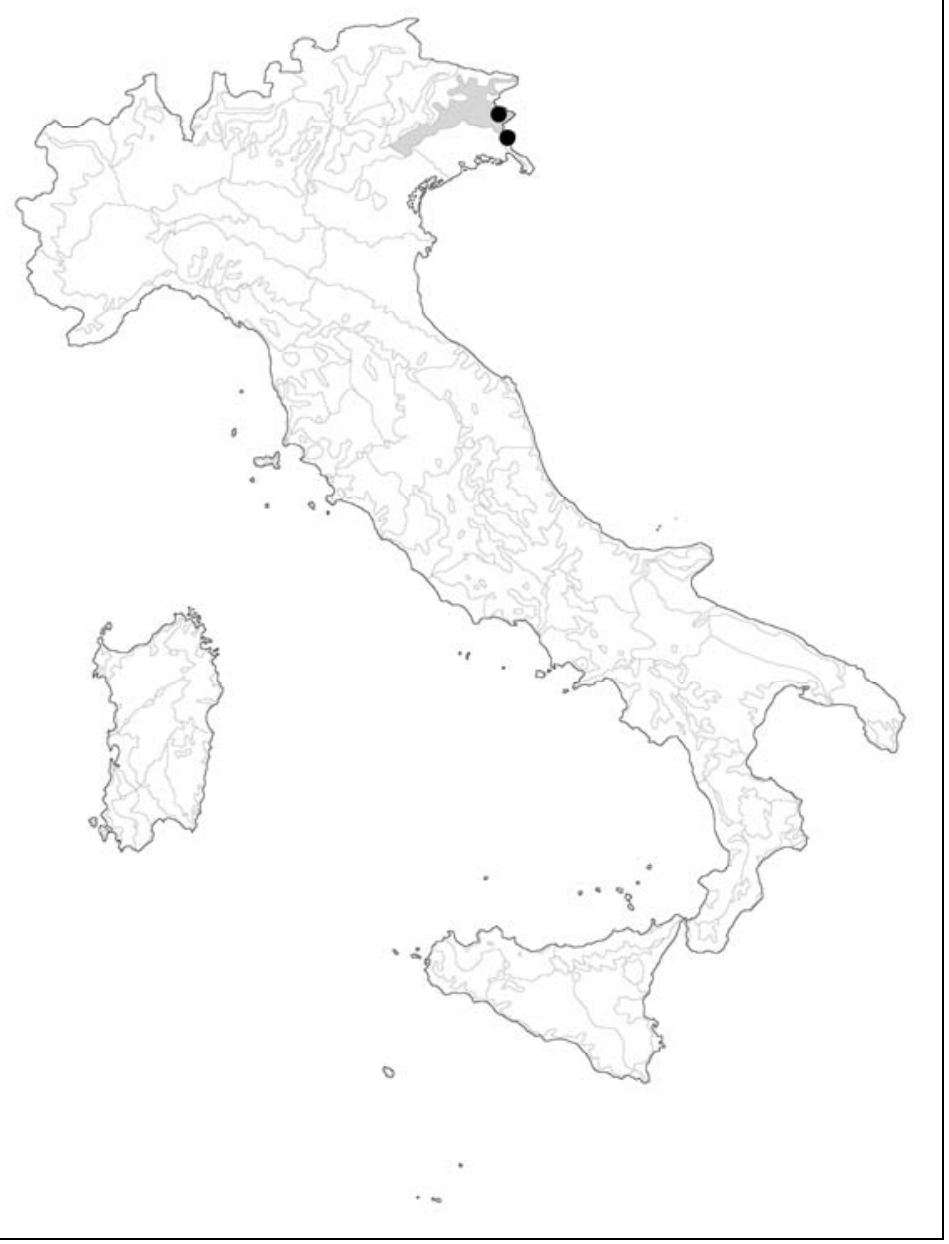




\section{Figura 39 \\ Pulmonaria vallarsae} subsp. vallarsae:

(A) foglia basale adulta; (B) infiorescenza; (E) dettaglio dell'indumento sulla pagina superiore delle foglie basali adulte.

Pulmonaria vallarsae subsp. apennina:

(C) habitus; (D) rosetta primaverile. Barra di scala: A, C, D = $20 \mathrm{~cm}$; $B=10 \mathrm{~cm} ; E=2 \mathrm{~cm}$. Disegno di L. Cecchi (A, E: Pian delle Fugazze (TN), 2013, Cecchi \& al. HB 13.74, FI. B: Bellunese (BL), sine die, Minio, FI. C, D: Casalecchio di Reno (BO), 2013, Cecchi \& Selvi HB 13.77, FIAF).

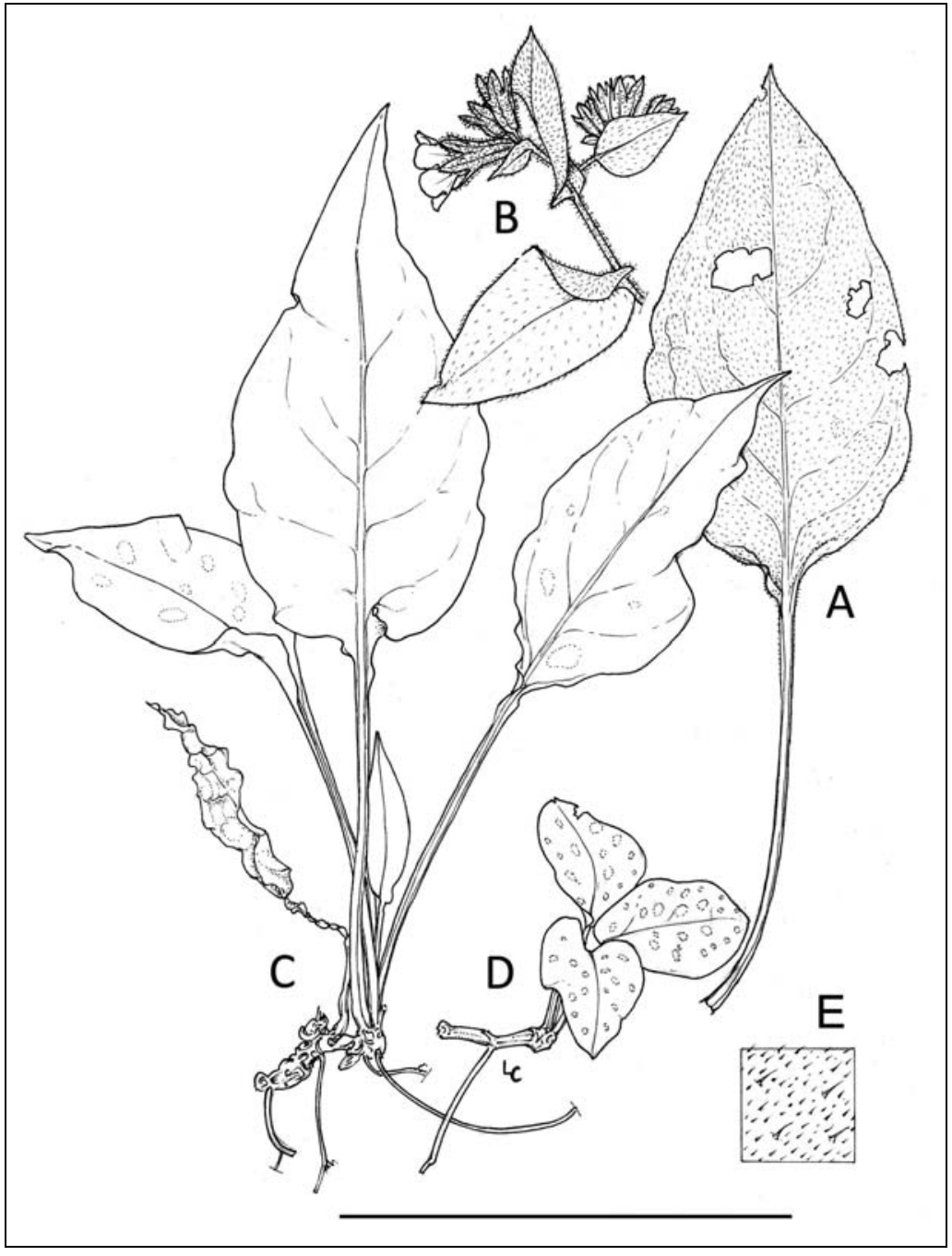


Figura $\mathbf{4 0}$

Distribuzione italiana di Pulmonaria vallarsae subsp. vallarsae (tondi bianchi) e subsp. apennina (tondi neri). Le stelle indicano $\mathrm{i}$ loci classici delle due sottospecie.

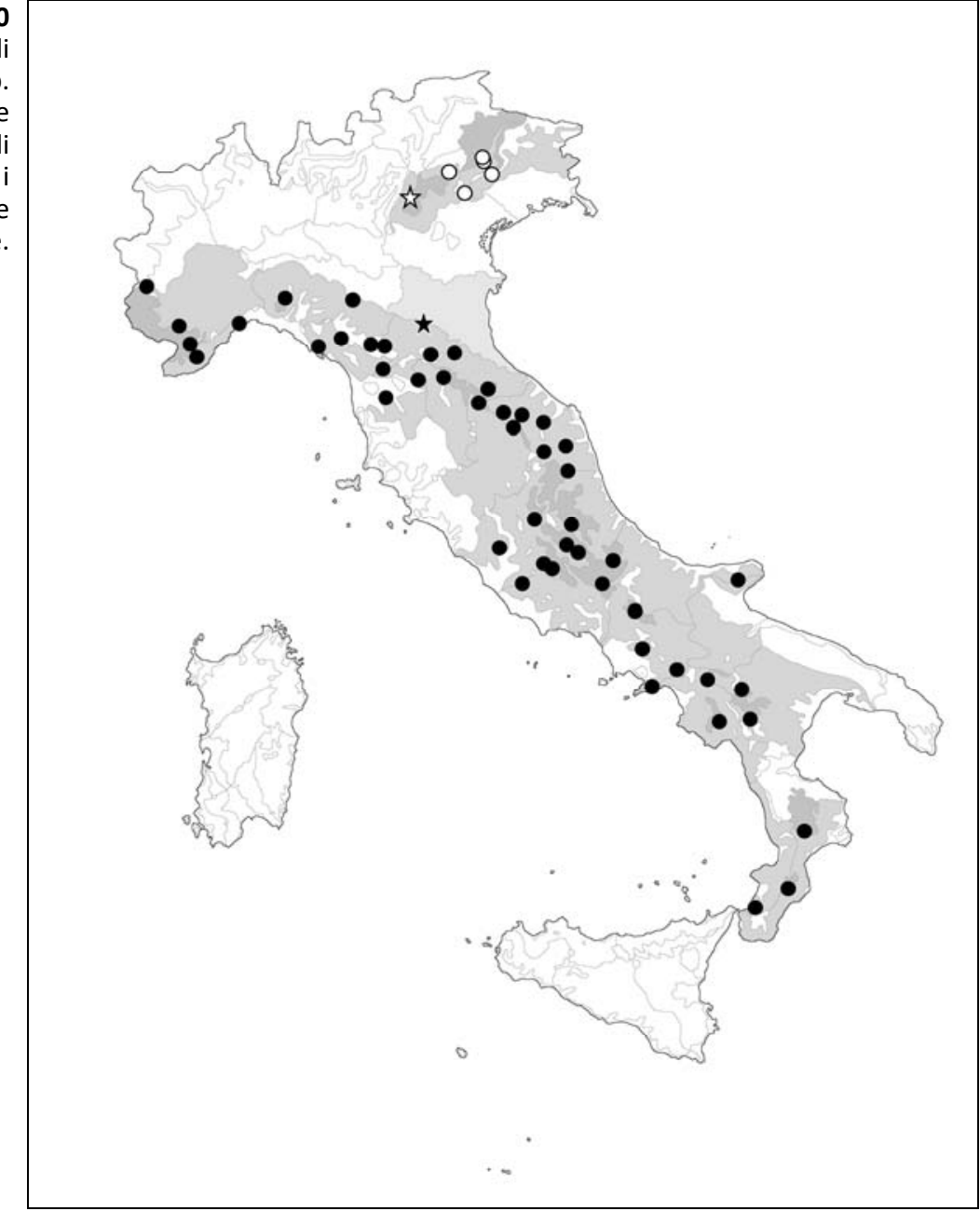


Figura 41

Nonea echioides:

(A) habitus; (B) cima con fiori e calici fruttiferi in accrescimento; (C) fiore; (D) mericarpo in visione laterale.

Barra di scala: $A=10 \mathrm{~cm}$; $\mathrm{B}$ $\approx 4 \mathrm{~cm} ; C=2 \mathrm{~cm} ; D=1 \mathrm{~cm}$. Disegno di L. Cecchi (A: San Severo (FG), Porta \& Rigo, 1874, Fl;

B, C: Spagna, Andalusia, Sierra de Maria, 2011, da foto in vivo [cfr. Cecchi \& al. HB 11.19], FI.

D: Turchia, Mersin, 1997, Bigazzi \& Selvi HB 97.31, FI).

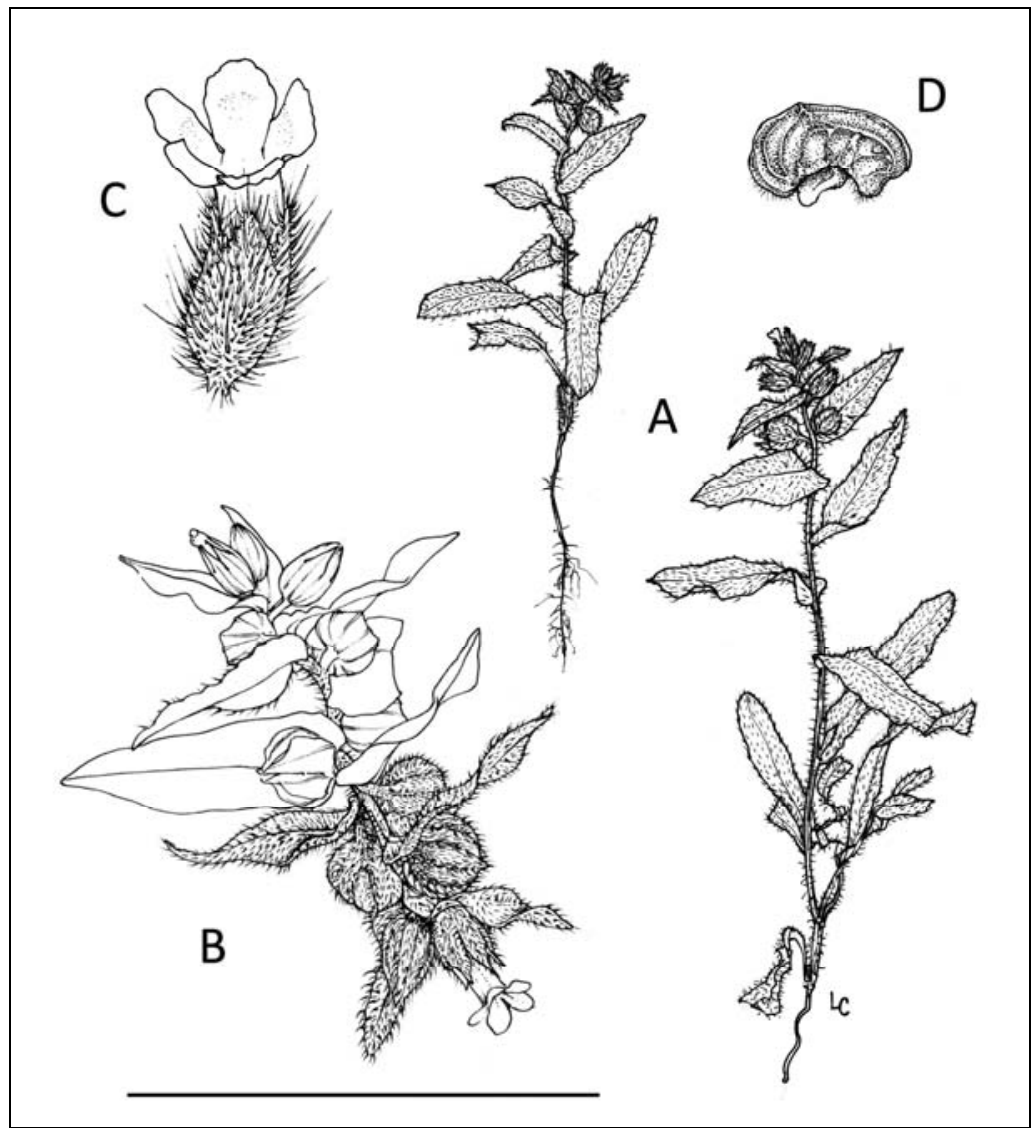


Figura 42

Distribuzione italiana di Nonea echioides.

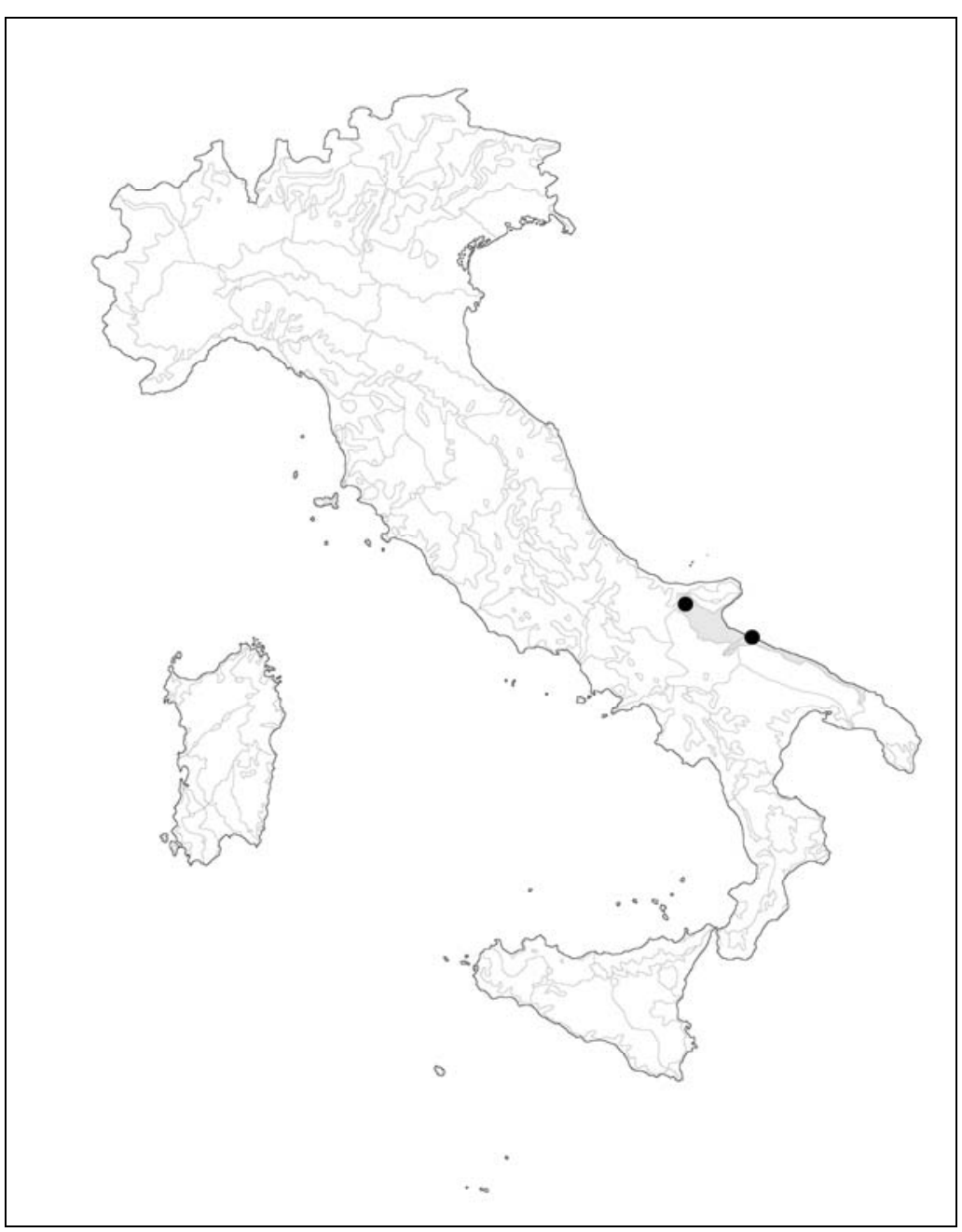




\section{Figura 43}

Nonea lutea:

(A) habitus; (B) mericarpo in visione laterale.

Barra di scala: $A=3 \mathrm{~cm}$; B: 3 $\mathrm{mm}$. Disegno di L. Cecchi (A: spontaneizzata nell'orto botanico di Firenze $(\mathrm{FI})$, 2010, Selvi HB 10.20, FIAF.

B: Perugia (PG), 1917, Barsali, FI).

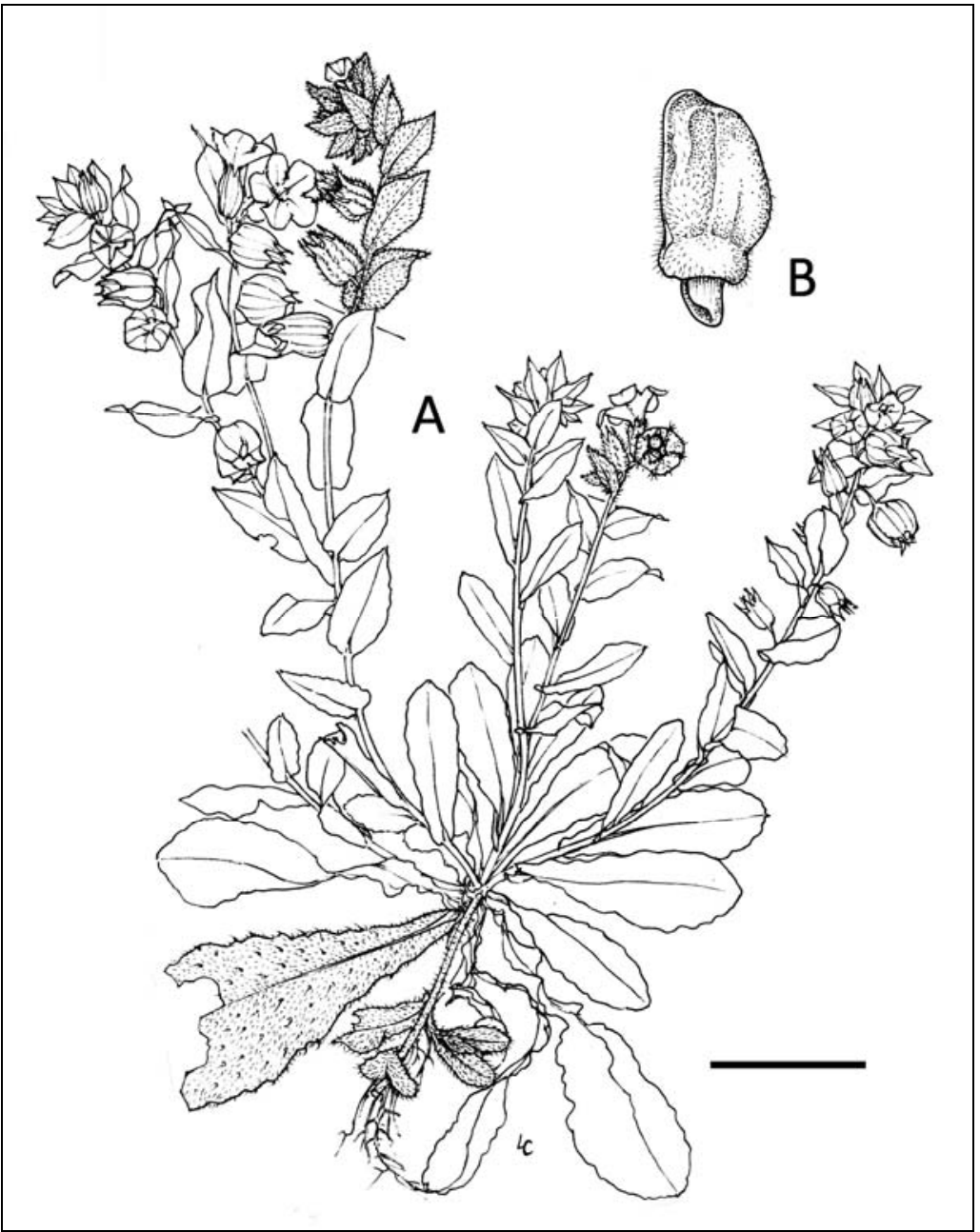


Figura 44

Distribuzione italiana di Nonea lutea.

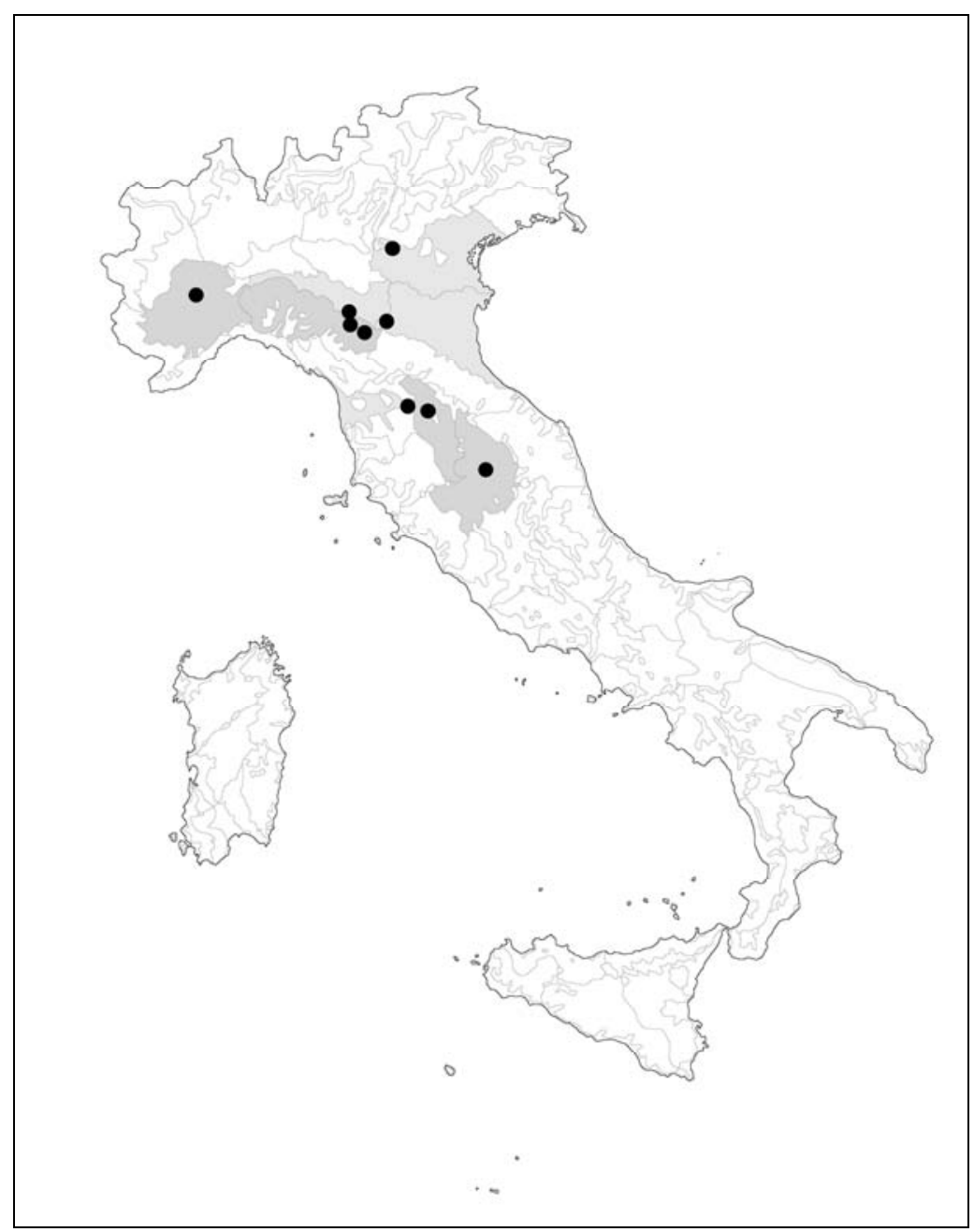




\section{Figura 45 \\ Nonea pulla \\ (A) habitus; (B) parte} dell'infiorescenza con calici fruttiferi; (C) nucula in visione laterale.

Barra di scala: $A=3 \mathrm{~cm}$; $B, C=3 \mathrm{~mm}$.

Disegno di L. Cecchi (Val di Susa (TO), Bruzolo,

Fontana \& al., 1933, FI).

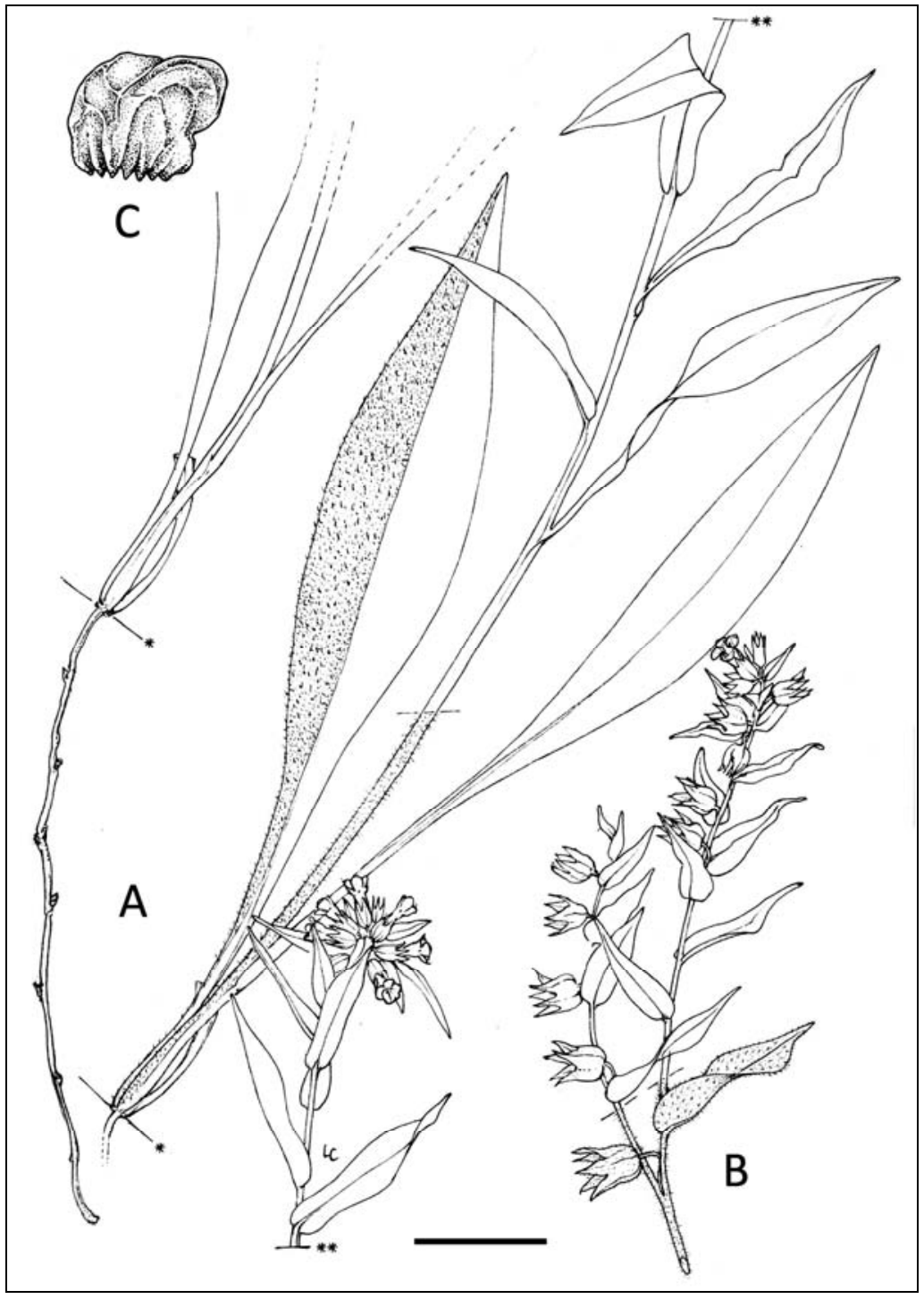


Figura 46

Distribuzione italiana di

Nonea pulla.

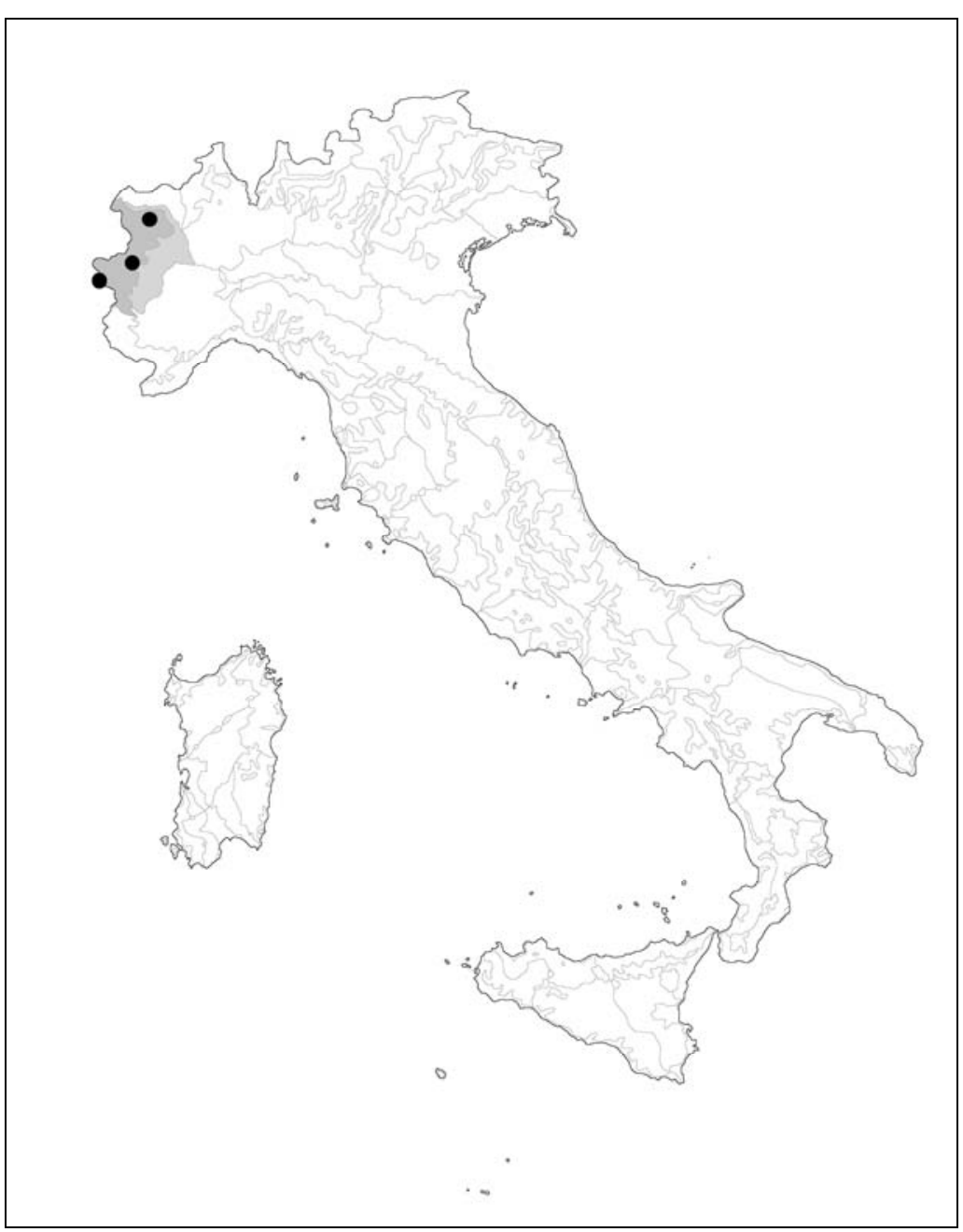


Figura 47

Nonea vesicaria:

(A) habitus; (B) fiore; (C)

calice fruttifero.

Barra di scala: $A=3 \mathrm{~cm}$;

$B, C=6 \mathrm{~mm}$.

Disegno di L. Cecchi

(A: Vittoria (RA), Riserva

Naturale "Pino d'Aleppo",

1997, Bigazzi \& Selvi, HB

97.38, FI;

B: ibidem, da foto in vivo di

G. Di Gregorio,

http://www.actaplantarum.

org).

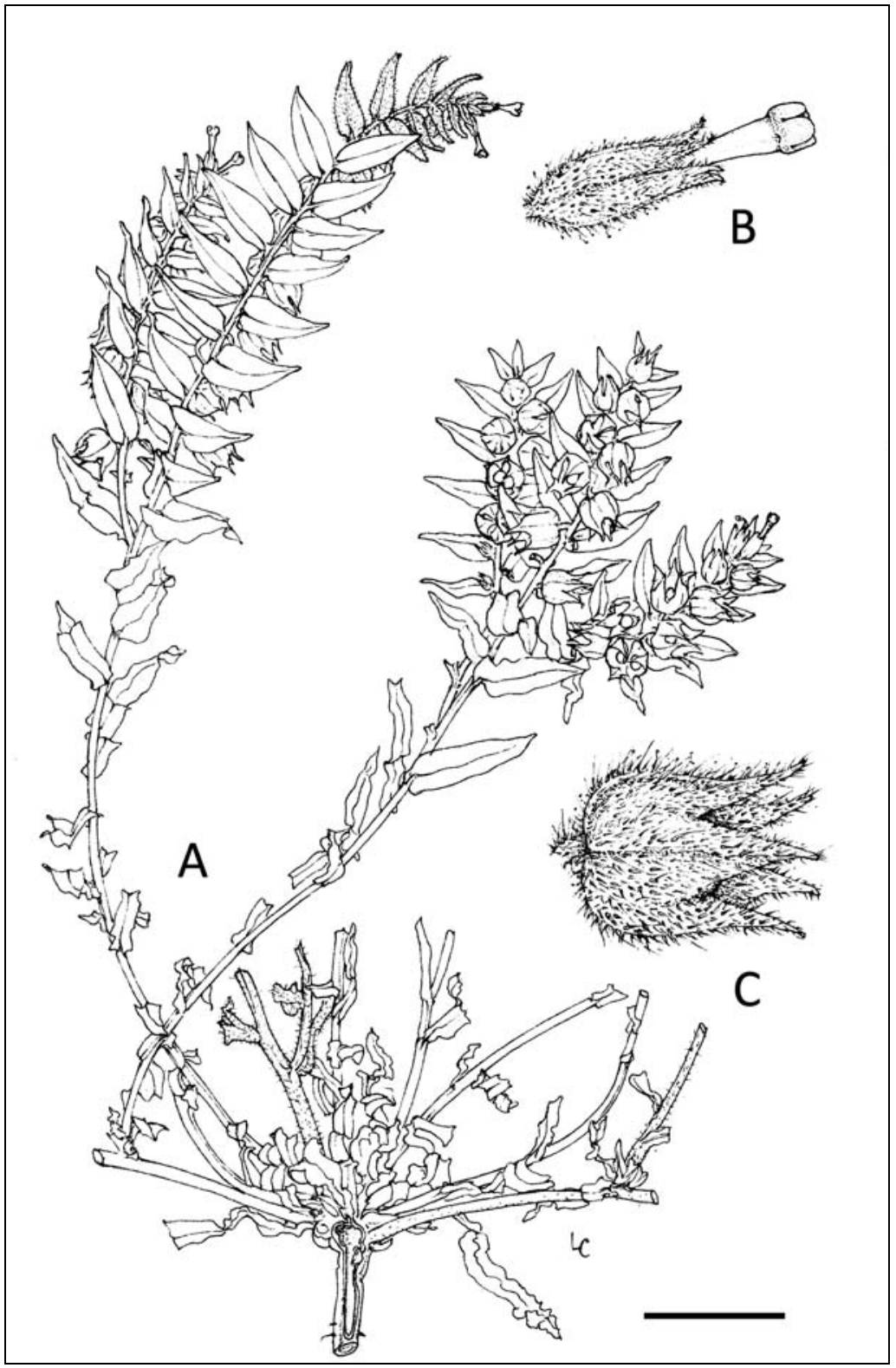


Figura 48

Distribuzione italiana di Nonea vesicaria.

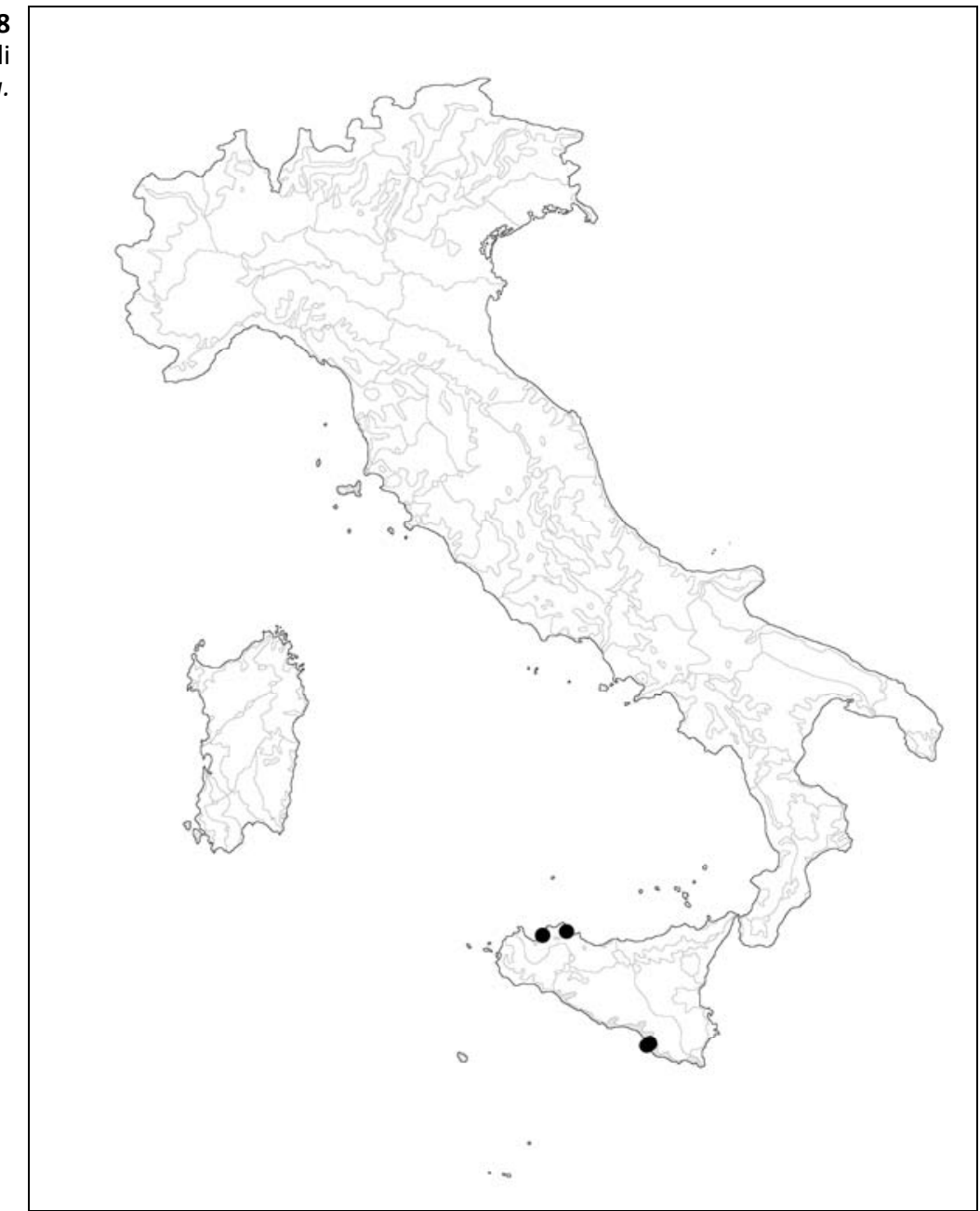

0 
Figura 49

Hormuzakia aggregata:

(A) habitus; (B) fiore con brattea; (C) corolla aperta; (D) gineceo; (E) mericarpo in visione laterale. Barra di scala: $A=5 \mathrm{~cm} ; B, C$ $=1 \mathrm{~mm} ; \mathrm{D}, \mathrm{E}=5 \mathrm{~mm}$. Disegno di A. Maury. (Italia, Sicilia, Manfria, 1997, Bigazzi \& Selvi, FIAF-HB 97.26).

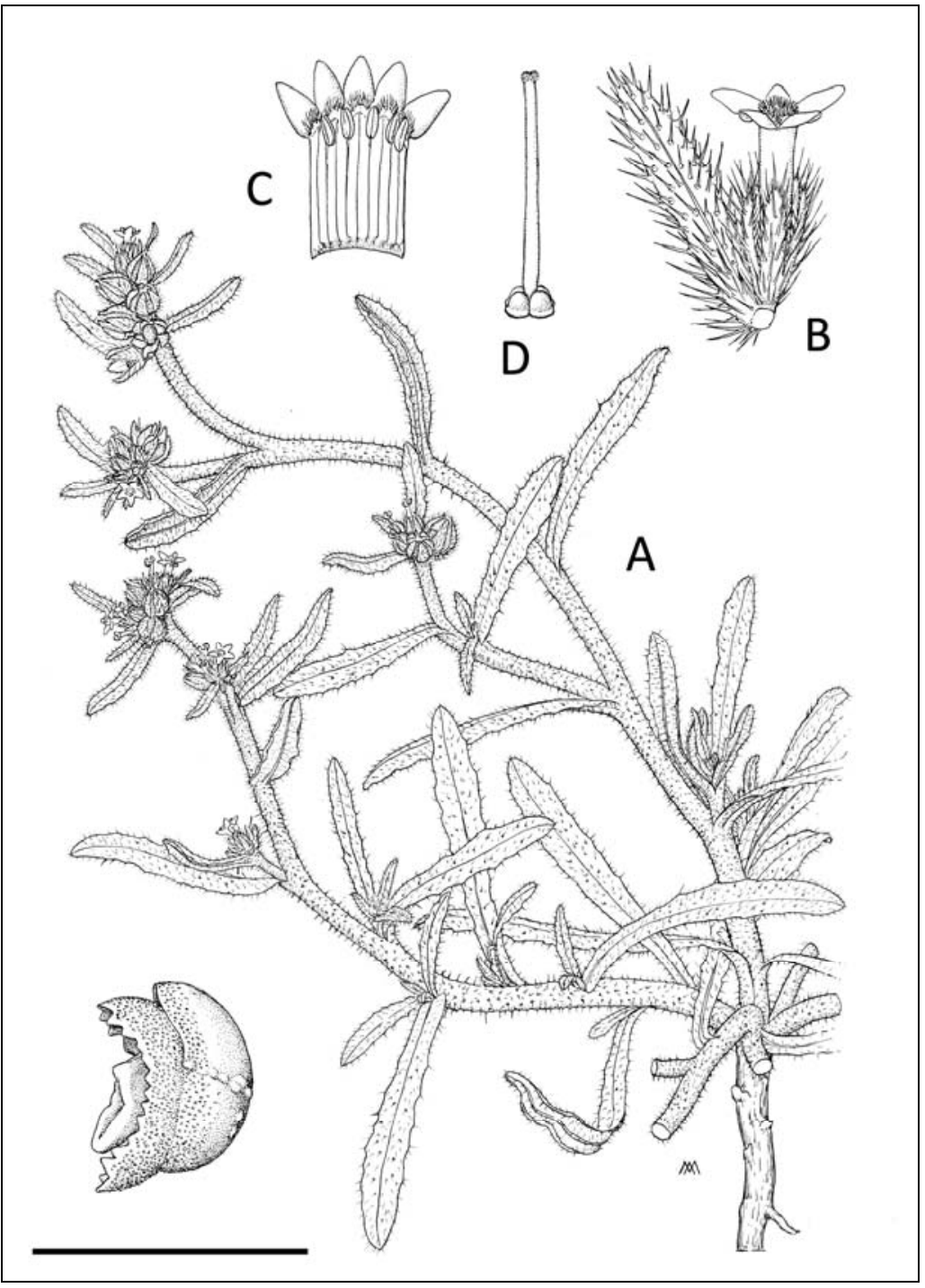


Figura 50

Distribuzione italiana di Hormuzakia aggregata.

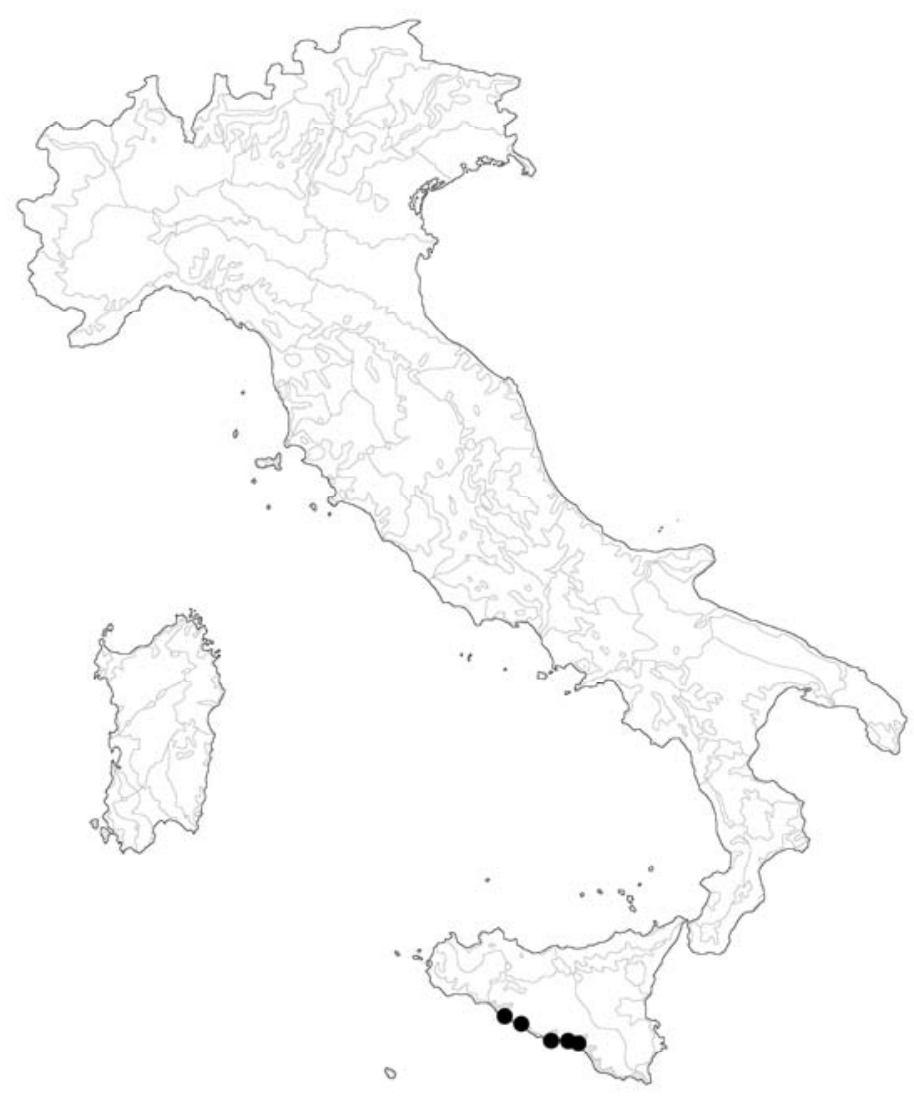

0 
Figura 51

Cynoglottis barrelieri subsp. barrelieri: (A, B) habitus; (C) fiore con brattea; (D) corolla aperta; (E) gineceo; $(F)$ mericarpo in visione laterale. Barra di scala: $A, B=5 \mathrm{~cm}$; $C, D=1 \mathrm{~cm} ; E, F=5 \mathrm{~mm}$. Disegno di A. Maury (da Selvi \& BIGAZZI 1998: 126).

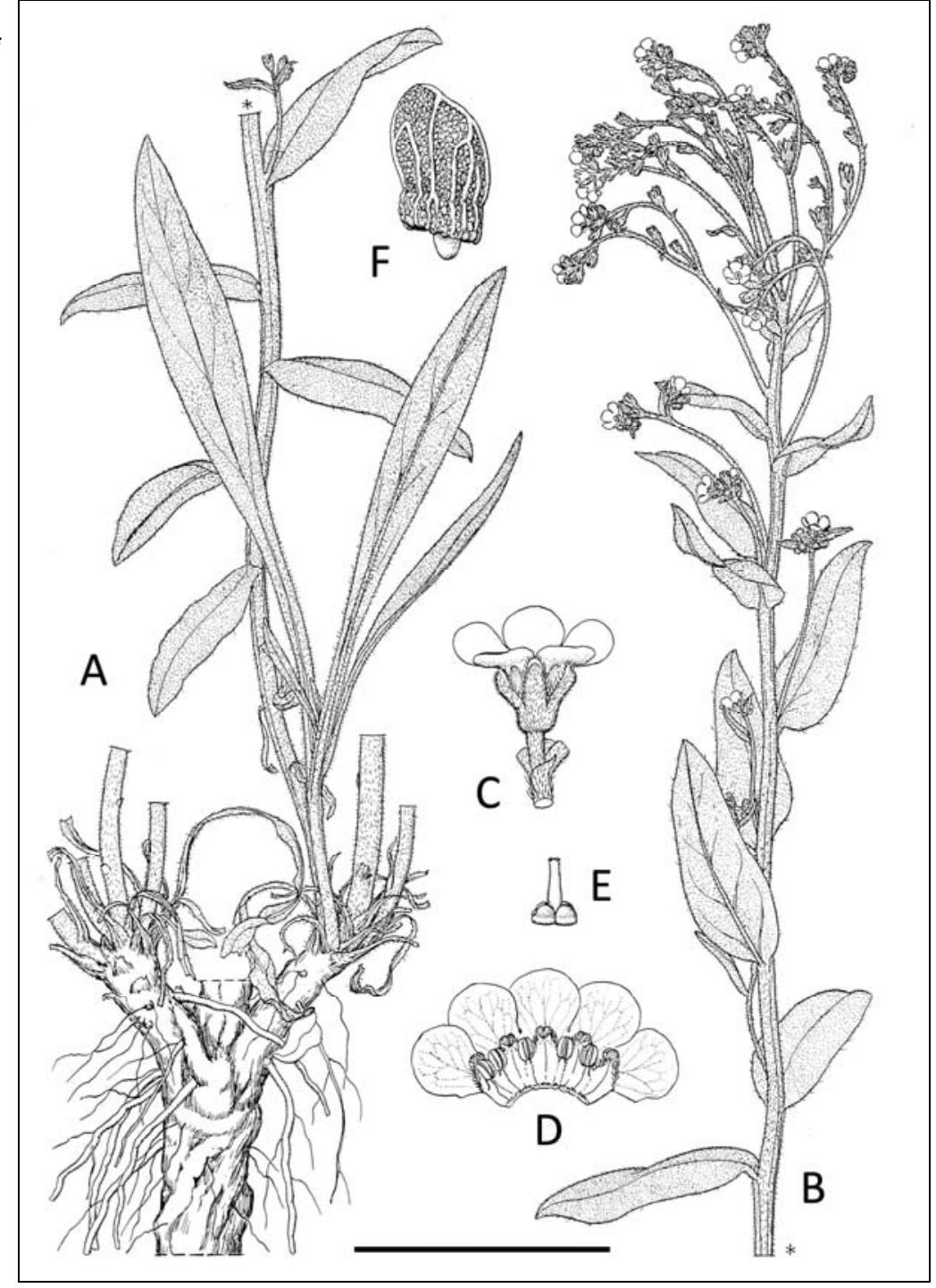


Figura 52

Distribuzione italiana di Cynoglottis barrelieri subsp. barrelieri (la stella indica il locus classicus).

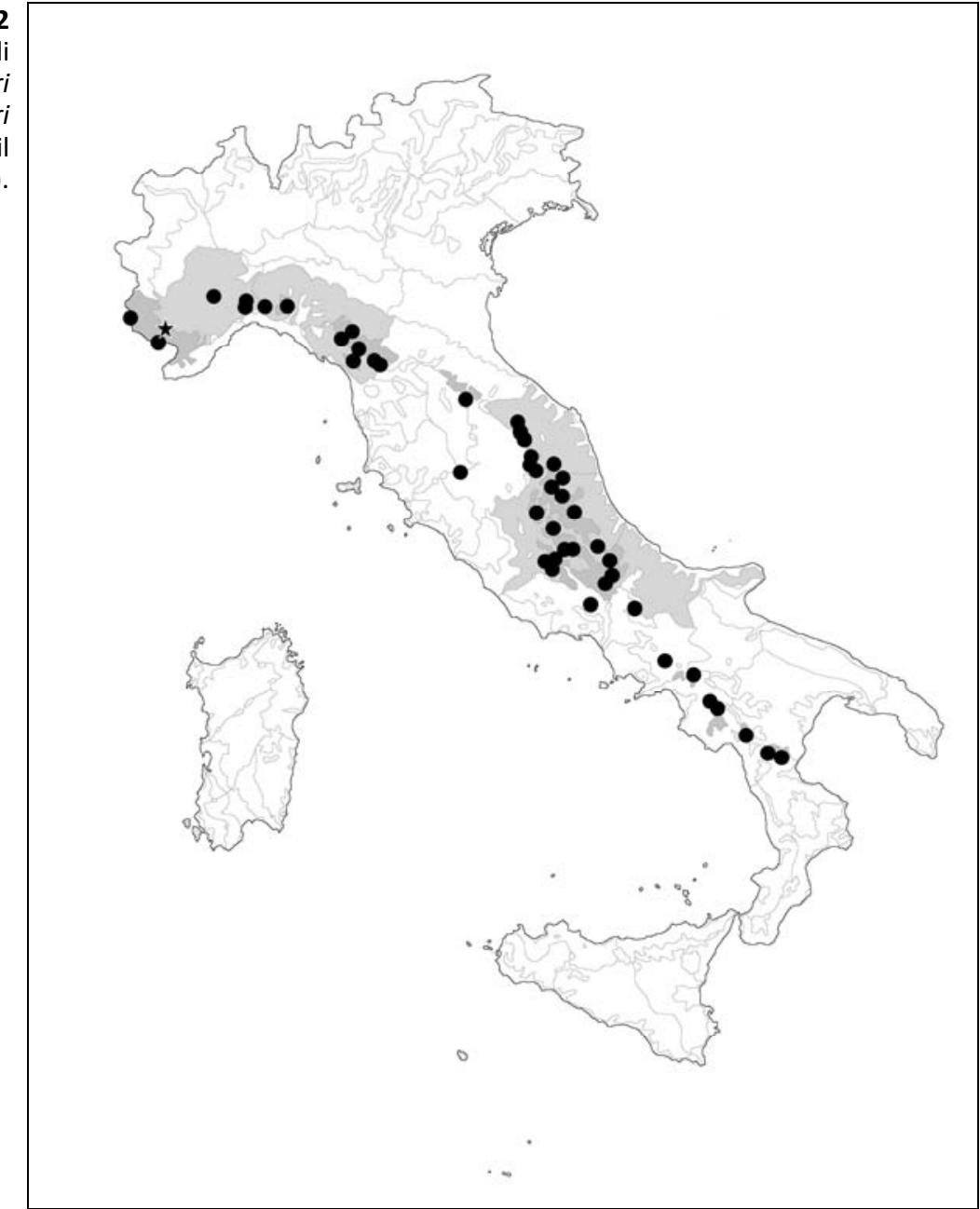

0 


\section{Figura 53 \\ Lycopsis arvensis:}

(A) habitus; (B) fiore in sezione longitudinale; (C, D) corolla chiusa e aperta; (E) gineceo; $(F, G)$ mericarpo in visione ventro-laterale e dorsale.

Barra di scala: $A=3 \mathrm{~cm}$; $B-E$ $=6 \mathrm{~mm} ; \mathrm{F}-\mathrm{G}=3 \mathrm{~mm}$. Disegno di A. Maury (da SelvI \& BIgAZZI 1998: 132).

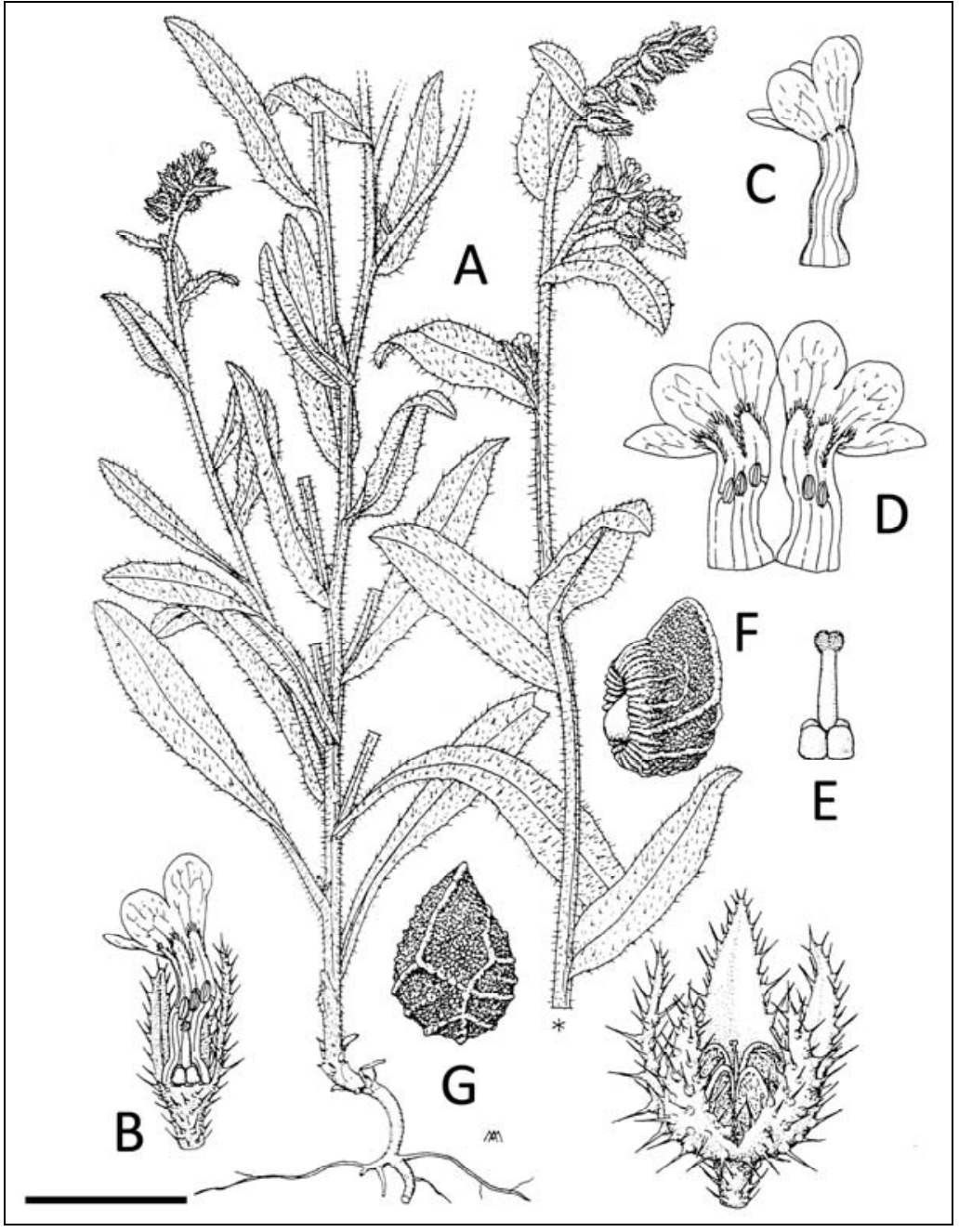


Figura 54

Distribuzione italiana di Lycopsis arvensis.

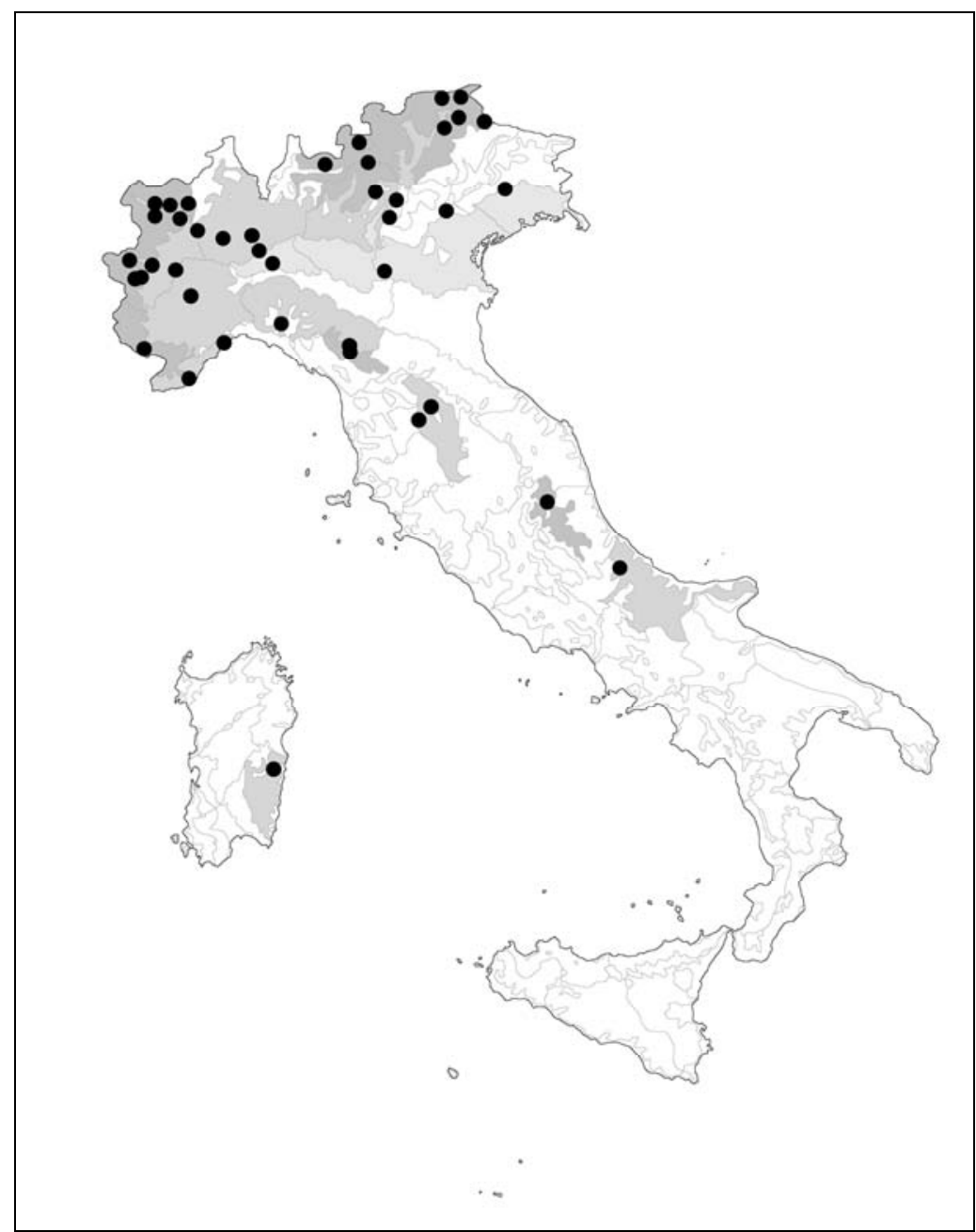

o 
Figura 55

Lycopsis orientalis:

(A) habitus; (B) fiori con brattee; (C) calice fruttifero;

(D) mericarpo in visione laterale. Barra di scala: $A=10 \mathrm{~cm} ; B$, $C=2 \mathrm{~cm} ; D=1 \mathrm{~cm}$. Disegno di L. Cecchi

(A: Turkia, Demirkent, Bigazzi \& Selvi HB 98.12, FI; B-D: Italia, Veneto, Colli Euganei, 2009, Cassanego, da foto in vivo di Actaplantarum).

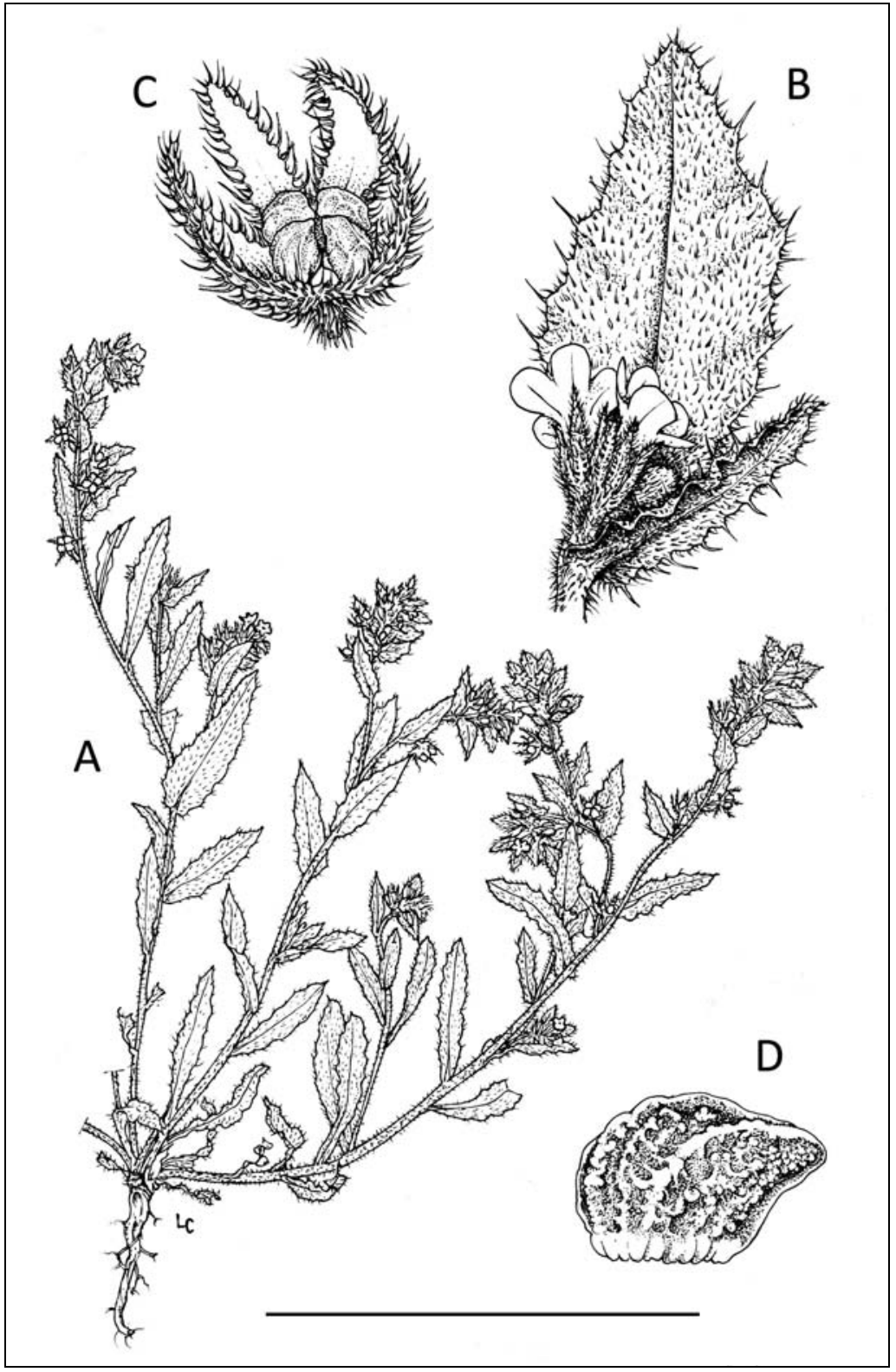


Figura 56

Distribuzione italiana di Lycopsis orientalis.

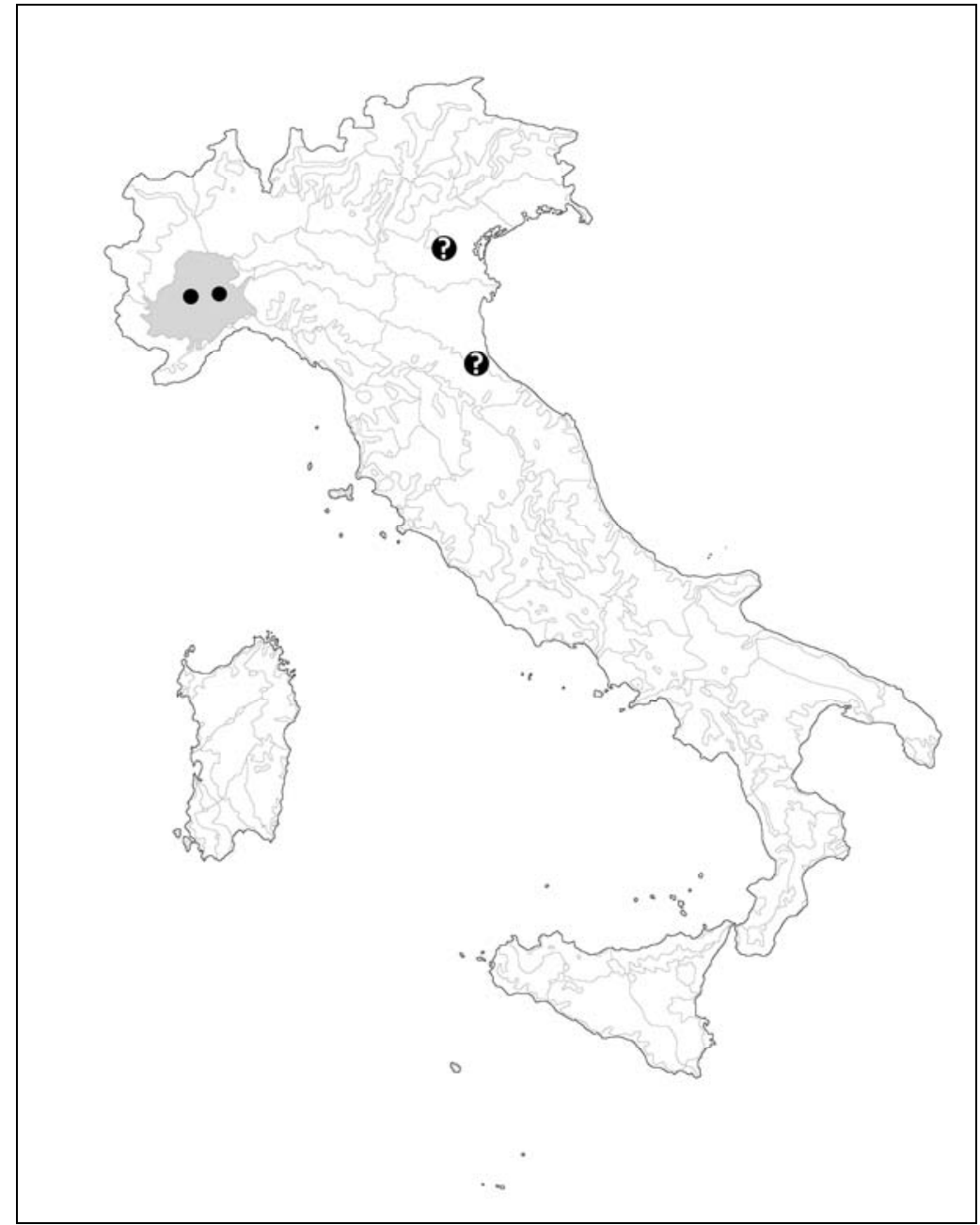


Figura 57

Anchusella cretica: (A) habitus; (B) fiore con brattea; (C) corolla chiusa;

(D) corolla aperta; (D) gineceo; $(E, F)$ gineceo in visione laterale e frontale:

(G); calice fruttifero; $(\mathrm{H}, \mathrm{I})$ mericarpo in visione dorsale e ventro-laterale. Barra di scala: $A=5 \mathrm{~cm}$; $B-$ $D, G=1 \mathrm{~cm} ; E, F, H, I=5$ $\mathrm{mm}$. Disegno di A. Maury (da BıgAZZI \& al. 1997: 258).

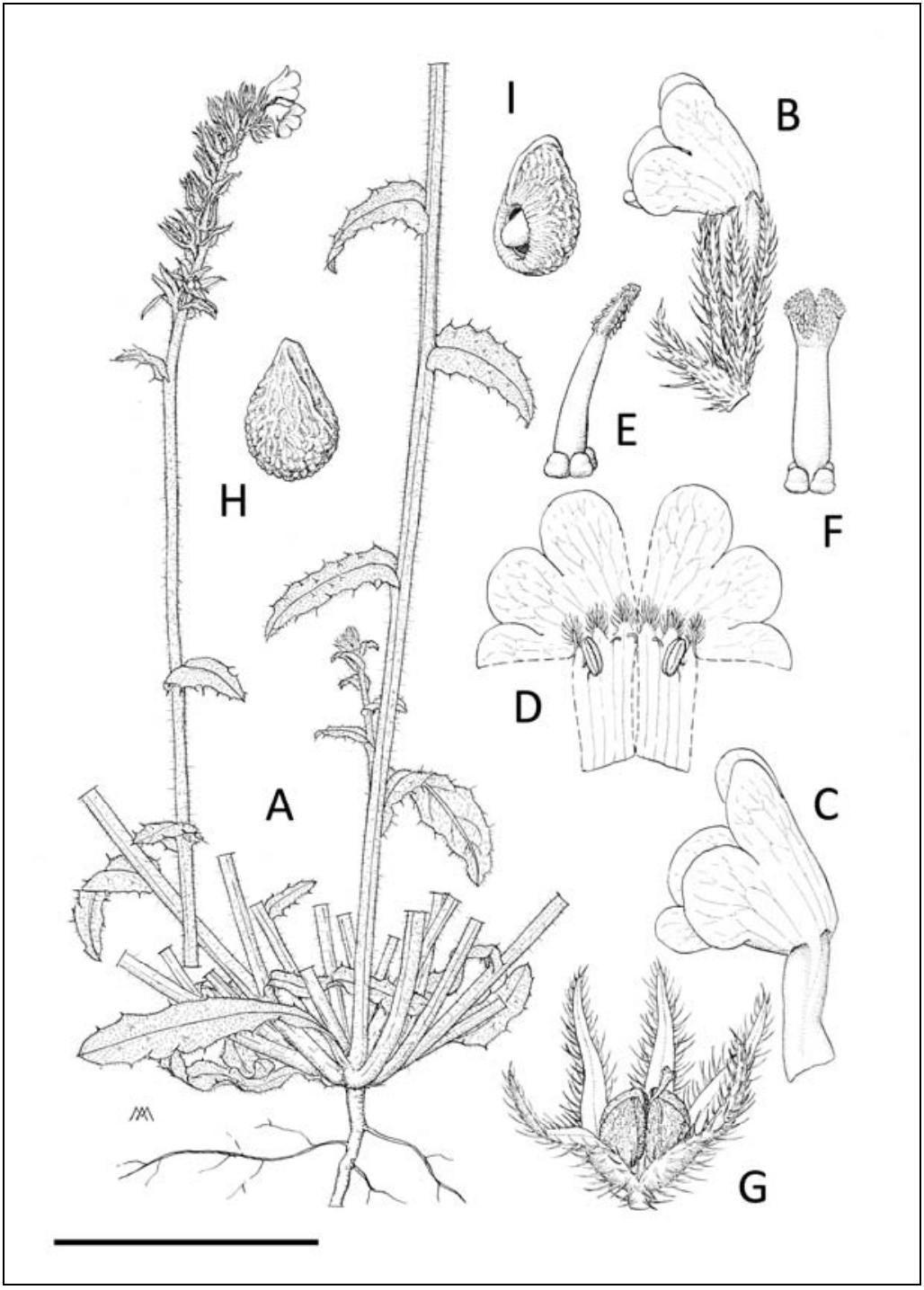


Figura 58

Distribuzione italiana di Anchusella cretica.

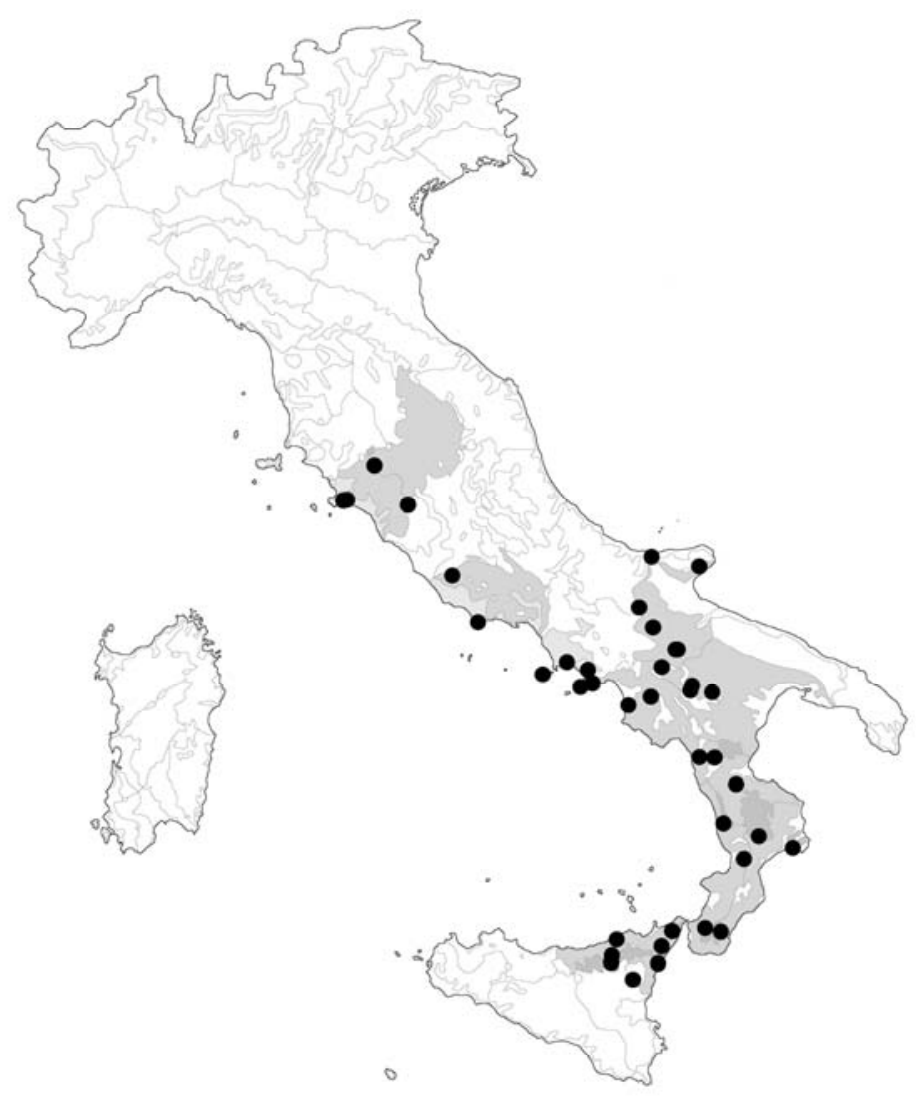

0 
Figura 59

Anchusa azurea:

(A) habitus; (B) fiore con

brattea; (C) corolla aperta;

(D) gineceo; (E) mericarpo in visione ventro-laterale. Barra di scala: $A=5 \mathrm{~cm}$; ,

$C, E=1 \mathrm{~cm} ; D=5 \mathrm{~mm}$.

Disegno di A. Maury (da Selvi \& BIgAZZI 1998: 131).

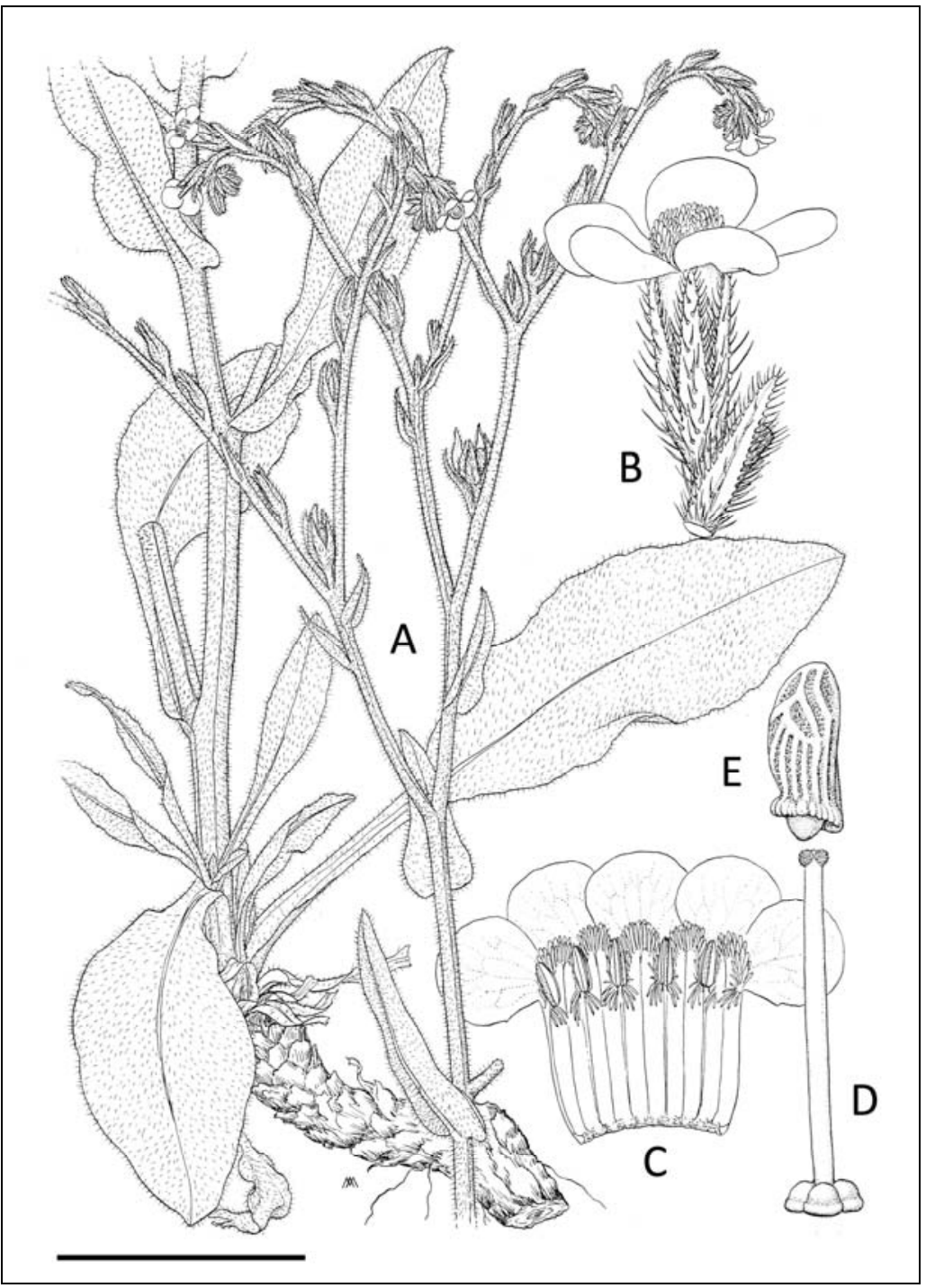


Figura 60

Distribuzione italiana di Anchusa azurea (la stella indica il locus classicus).

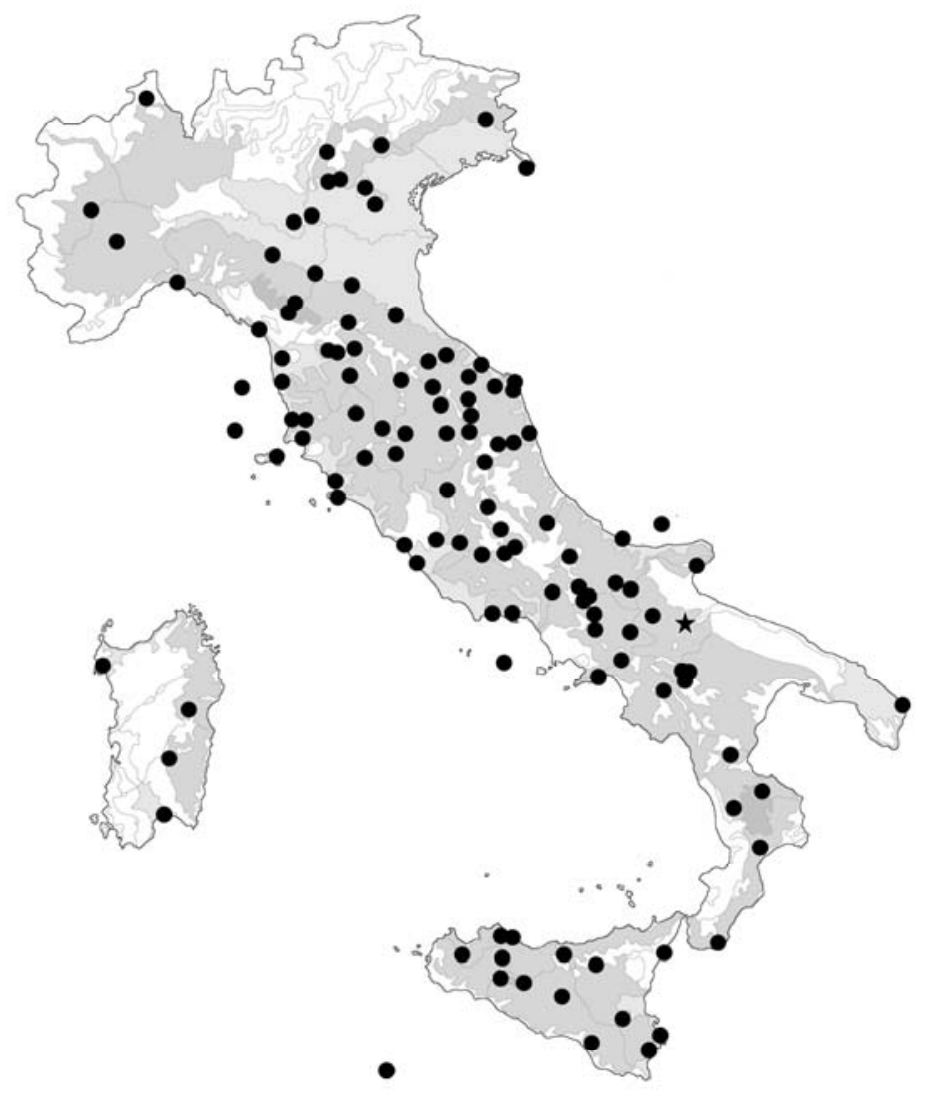

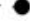


Figura 61

Anchusa capellii:

(A) habitus; (B) fiore con brattea; (C) corolla aperta; (D) gineceo; (E) mericarpo in visione ventro-laterale. Barra di scala: $A=5 \mathrm{~cm}$; $B, C$ $=1 \mathrm{~cm} ; \mathrm{D}, \mathrm{E}=5 \mathrm{~mm}$.

Disegno di A. Maury da pianta del locus classicus (da SelVI 1998: 265).

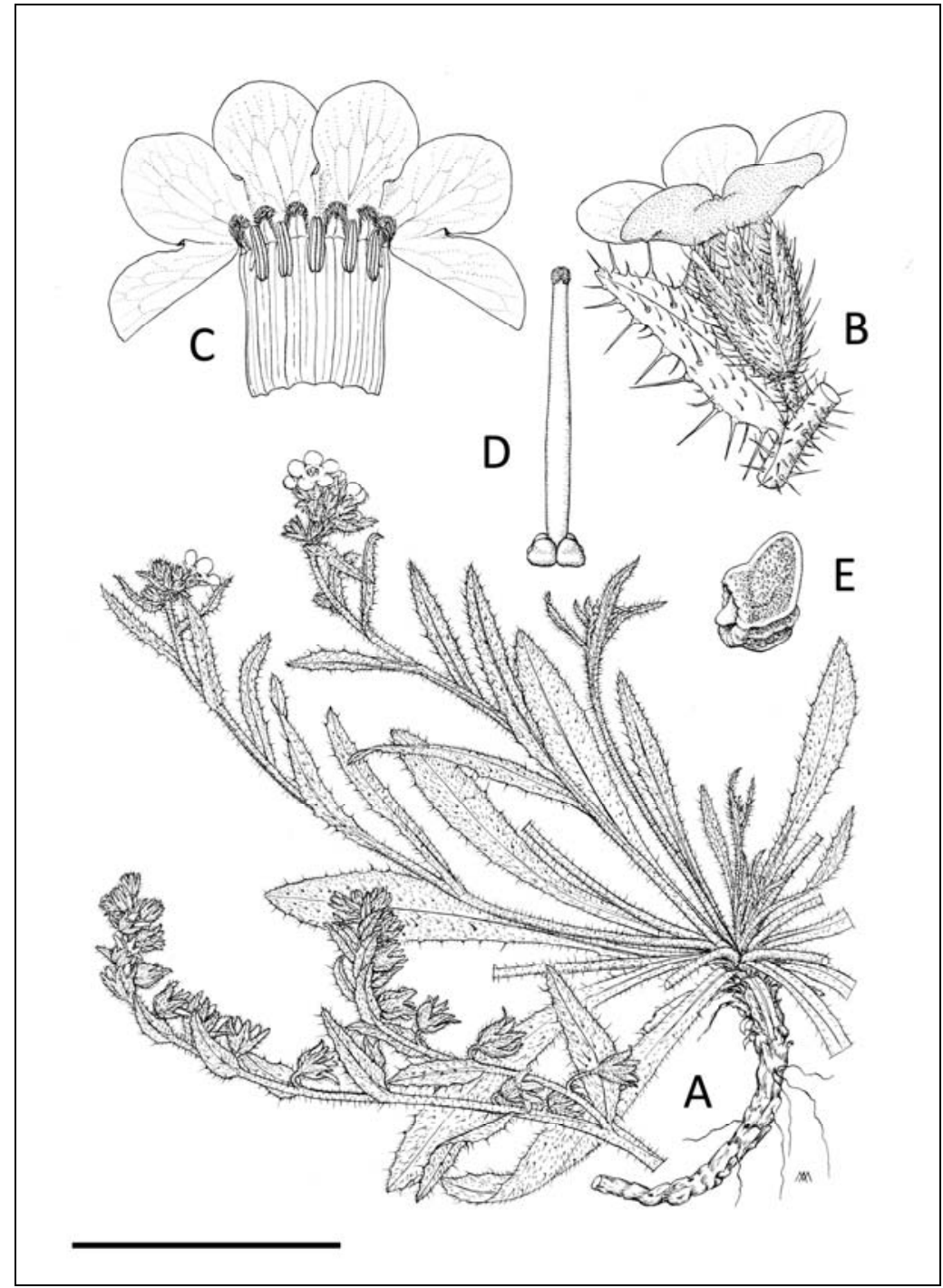


Figura 62

Distribuzione italiana di

Anchusa capellii

(la stella indica il

locus classicus).

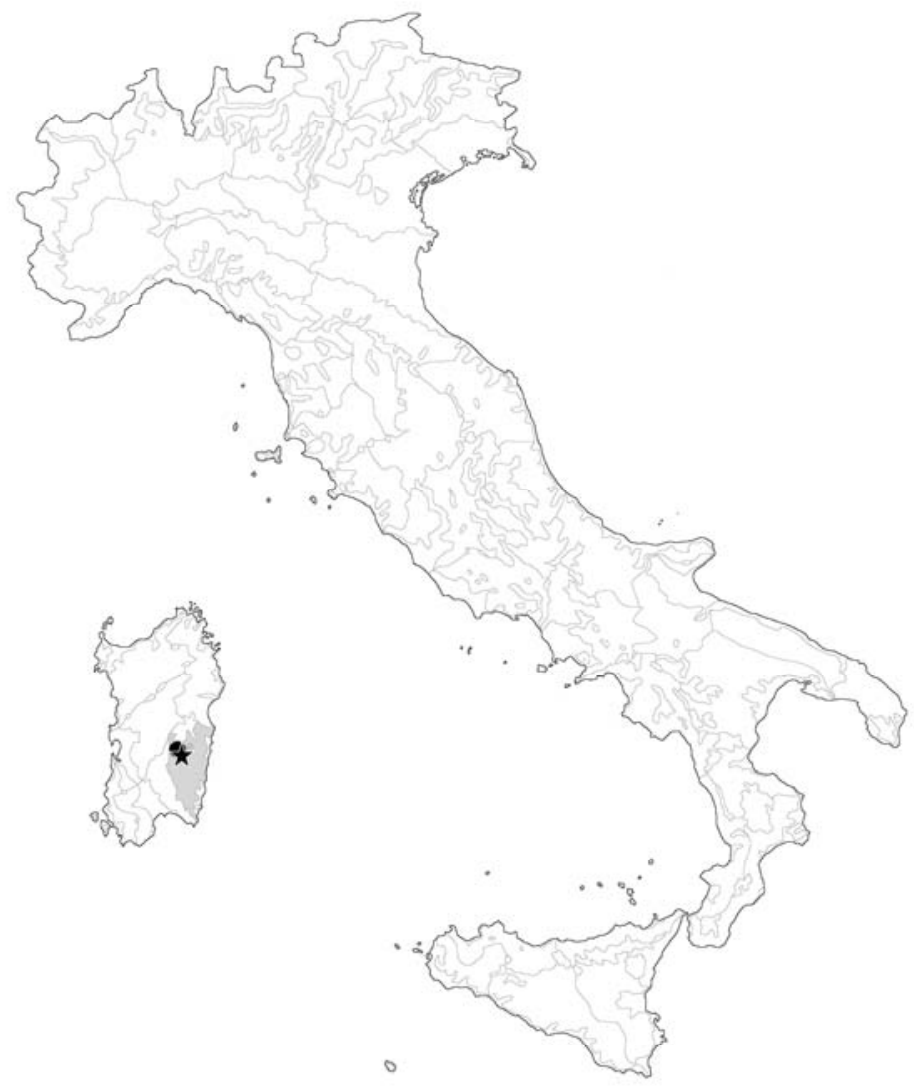




\section{Figura 63 \\ Anchusa crispa subsp. crispa:}

(A) habitus; (B) fiore con brattea; (C) corolla aperta; (D) gineceo; $(E)$ mericarpo in visione laterale. Anchusa crispa subsp. maritima:

(F) fiore con brattea. Barra di scala: $A=5 \mathrm{~cm} ; B$, $C, F=1 \mathrm{~cm} ; \mathrm{D}, \mathrm{E}=5 \mathrm{~mm}$.

Disegno di A. Maury

(A, B, D-F modificato da Selvi \& BigAZZI 1998: 135; C, inedito).

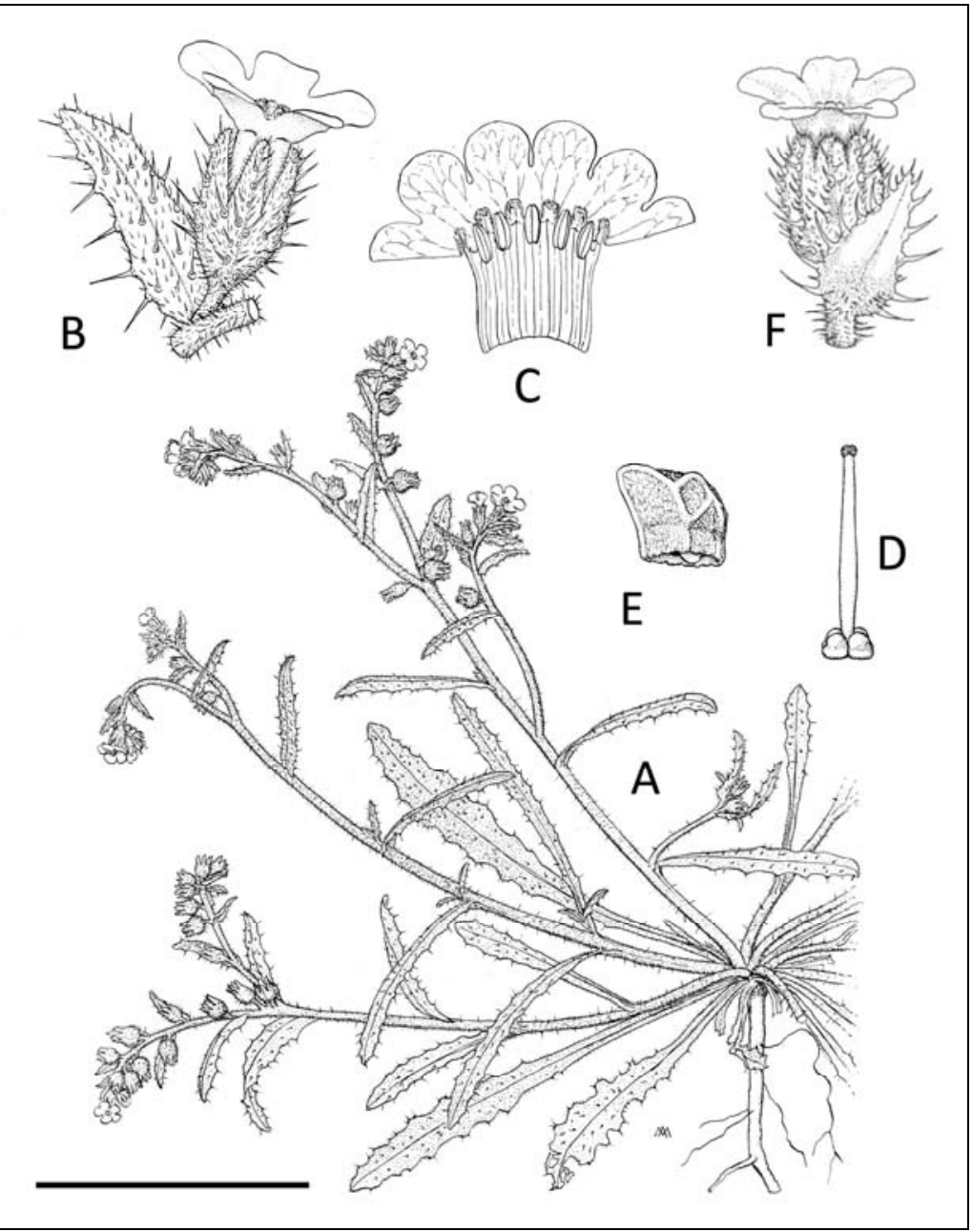


Figura 64

Distribuzione italiana di

Anchusa crispa subsp. crispa (tondi neri) e subsp. maritima (tondi bianchi; la stella indica il locus classicus).

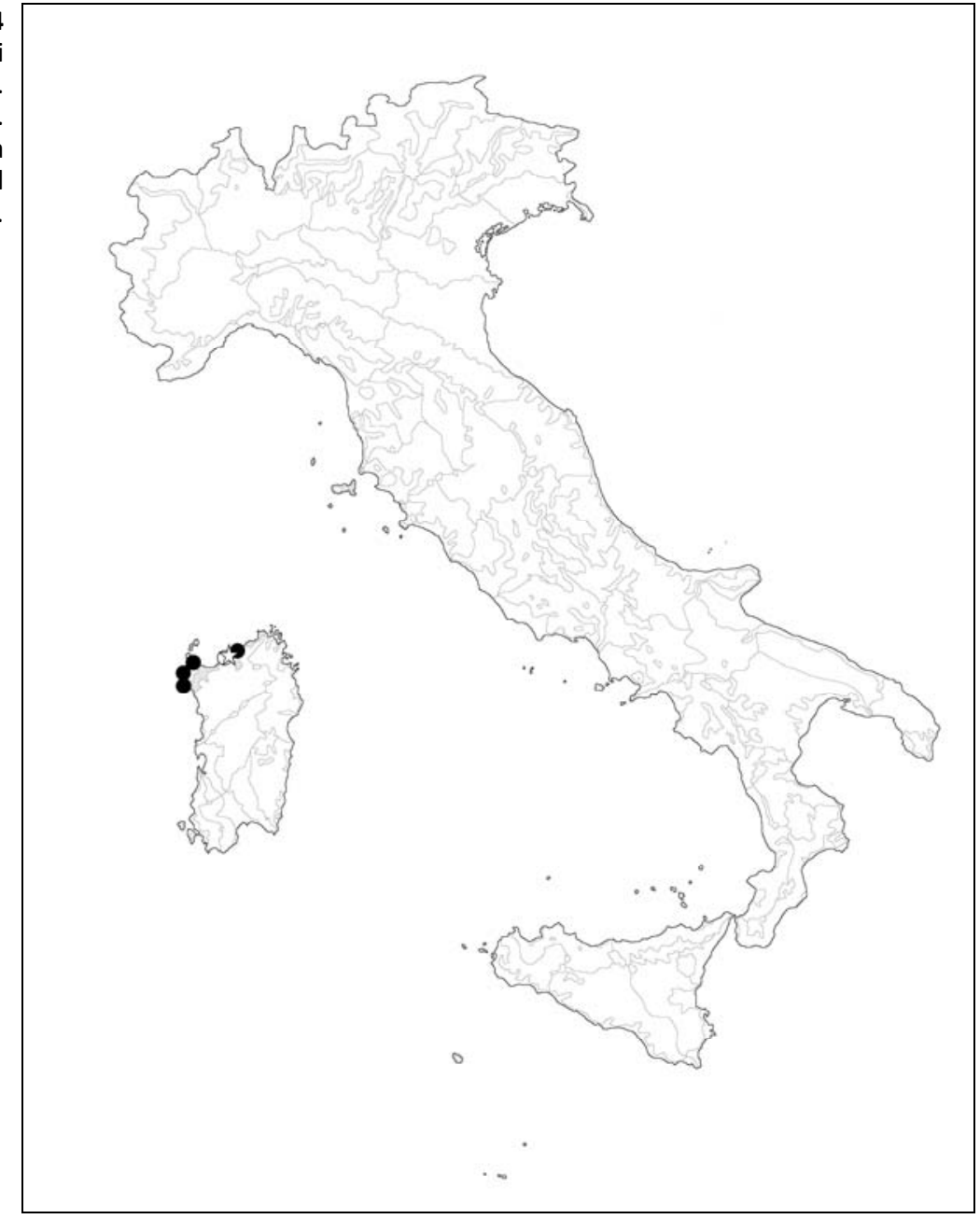


Figura 65

Anchusa formosa:

(A) habitus; (B) fiore con brattea; (C) corolla aperta;

(D) gineceo; $(E, F)$ mericarpo in visione laterale e latero-dorsale. Barra di scala: $A=5 \mathrm{~cm}$;,$C$ $=1 \mathrm{~cm} ; \mathrm{D}-\mathrm{F}=5 \mathrm{~mm}$.

Disegno di A. Maury (da Selvi \& al. 1997: 104).

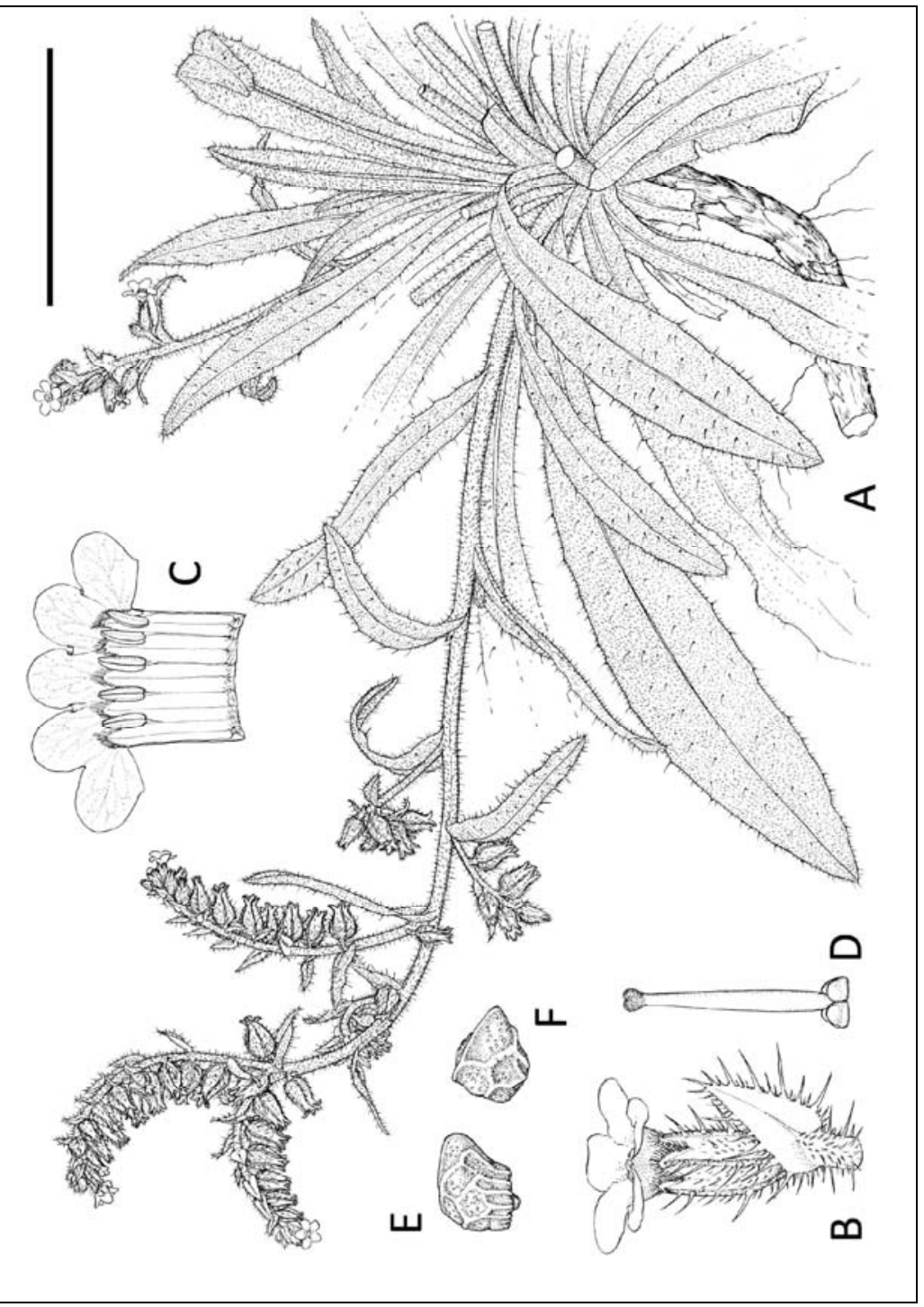


Figura 66

Distribuzione italiana di Anchusa formosa (la stella indica il locus classicus).

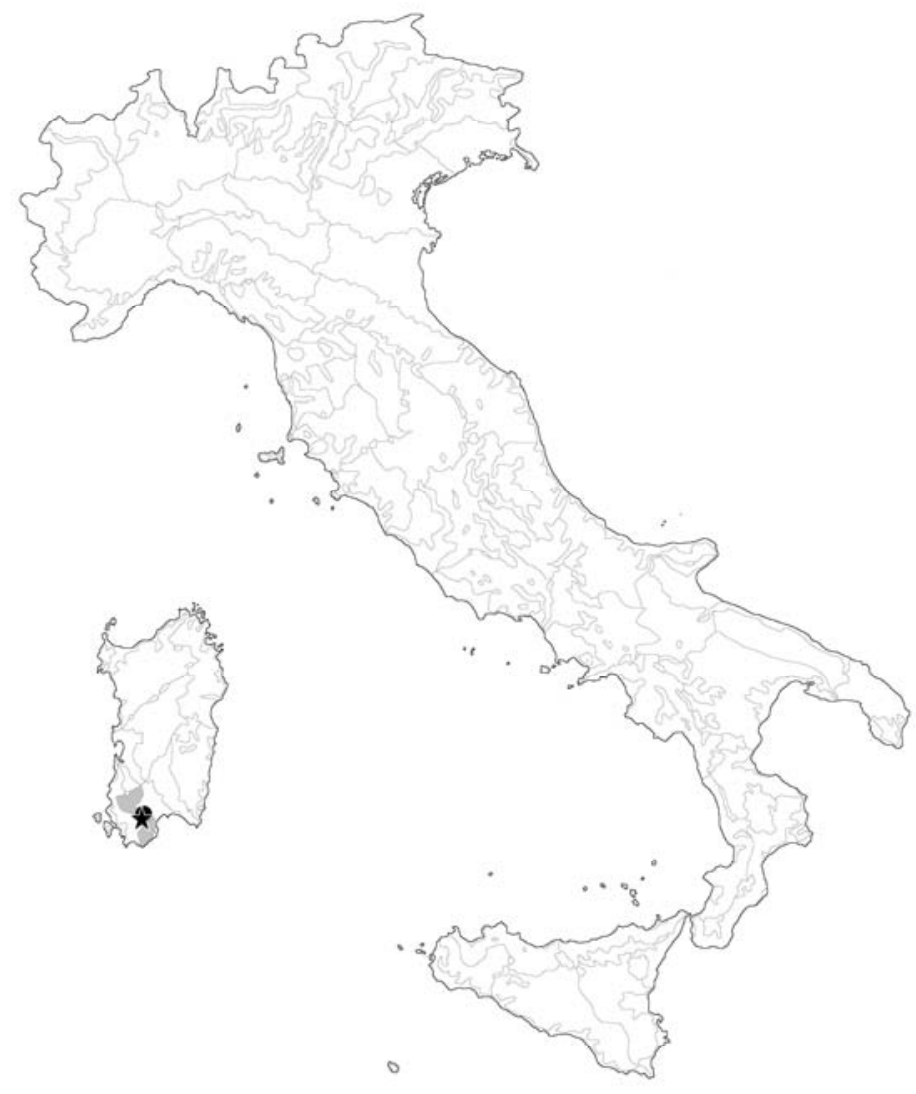




\section{Figura 67 \\ Anchusa litorea:}

(A, B, C) habitus; (D)

dettaglio dell'infiorescenza.

Barra di scala: $10 \mathrm{~cm}$; D: $x 7$.

Disegno di L. Cecchi

(A: spiaggia di Arborea,

1981, Mossa, CAG;

B, C: Is Arenas di Arbus, da

foto in vivo;

D: esemplare coltivato in orto botanico a Firenze, da foto in vivo).

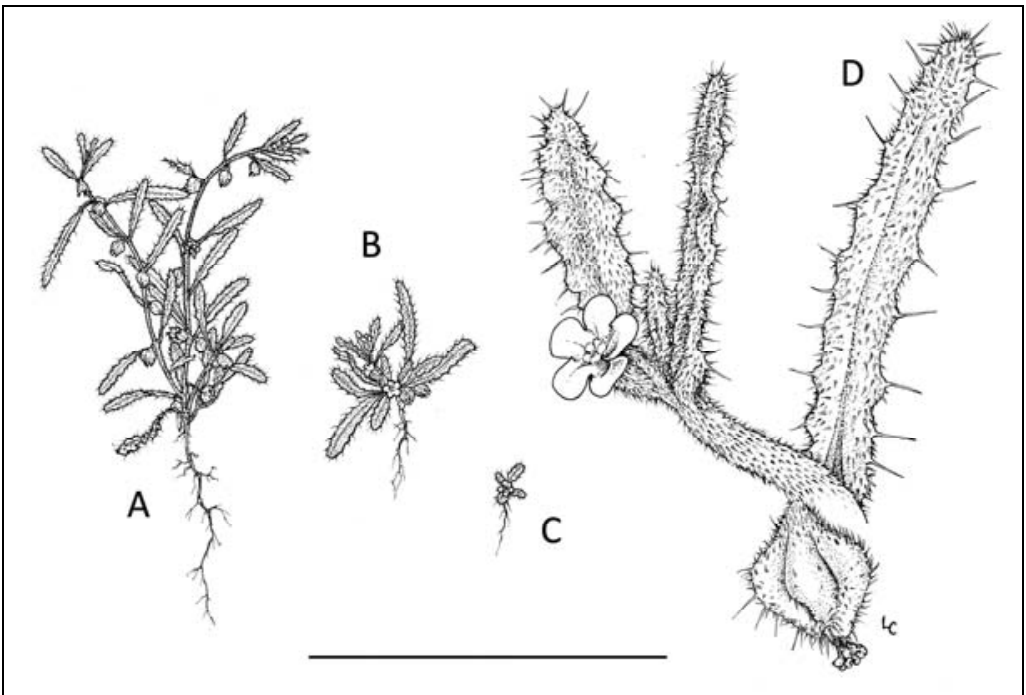


Figura 68

Distribuzione italiana di Anchusa litorea (la stella indica il locus classicus).

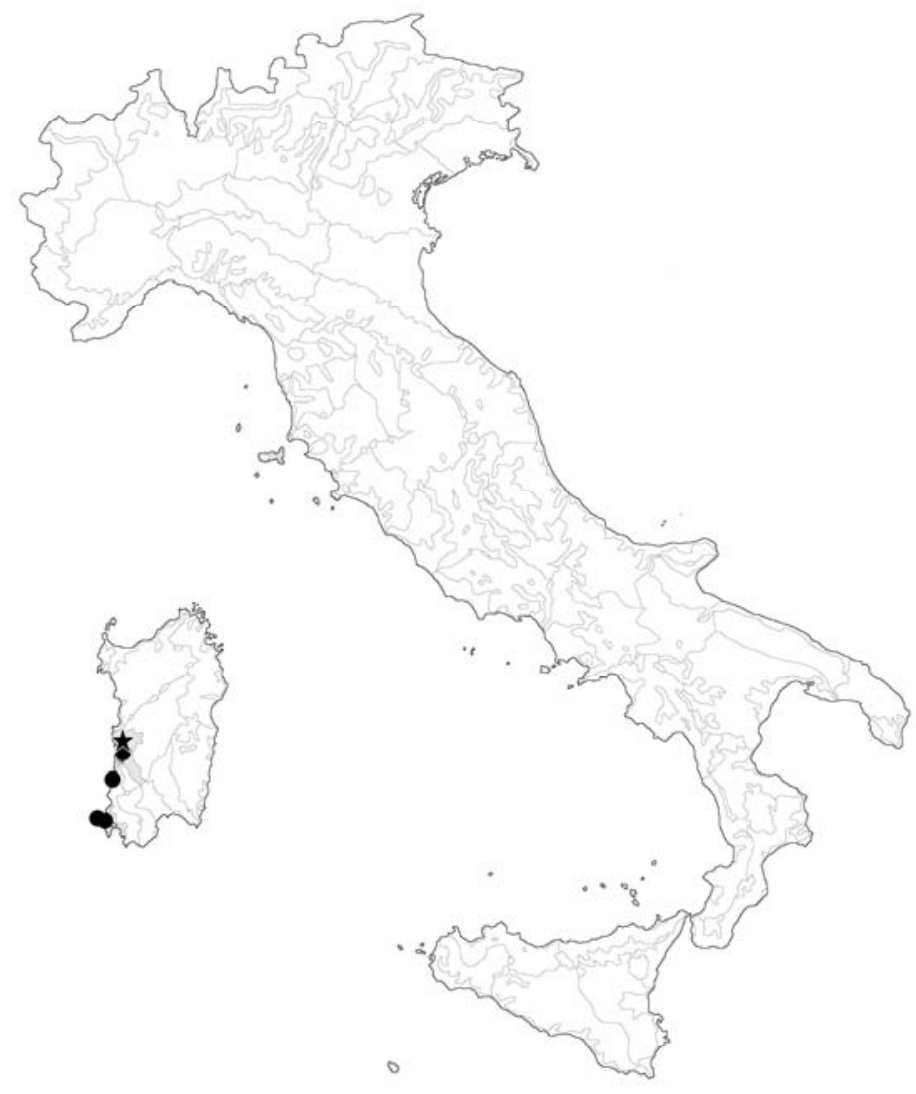


Figura 69

Anchusa montelinasana: (A) habitus; (B) fiore con brattea; (C) mericarpo in visione laterale. Barra di scala: $A=5 \mathrm{~cm}$; $B=$ $1 \mathrm{~cm} ; \mathrm{C}=5 \mathrm{~mm}$. Disegno di A. Maury (da BACCHETTA \& al. 2008: 167).

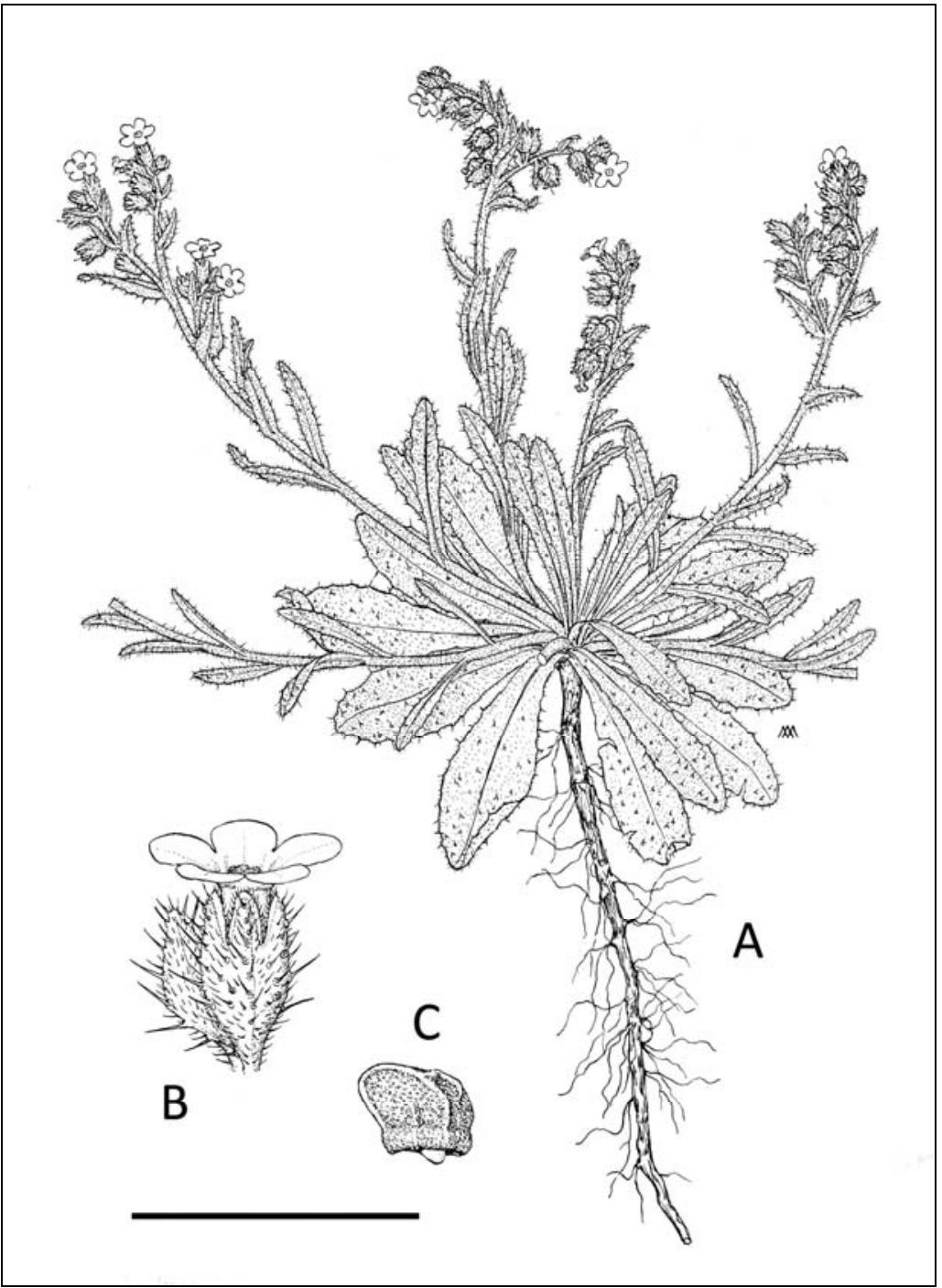


Figura 70

Distribuzione italiana di Anchusa montelinasana (locus classicus).

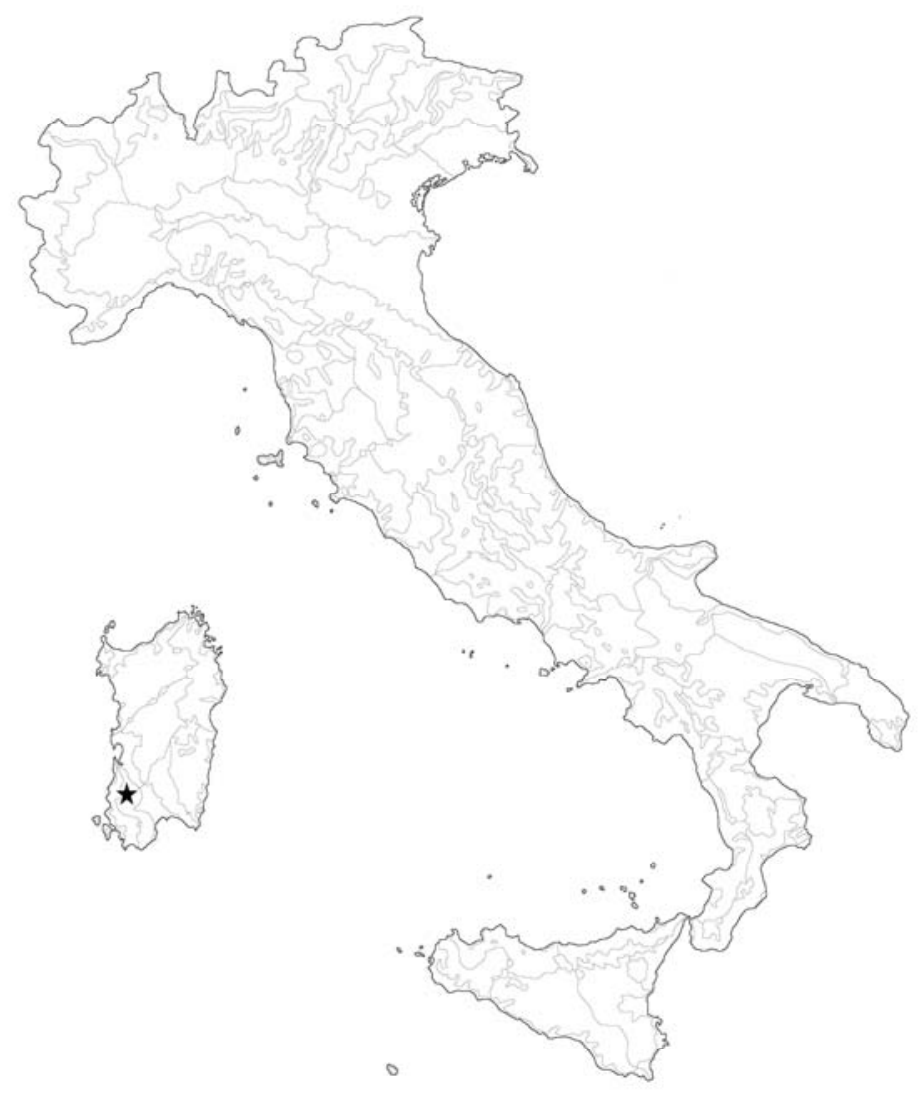


Figura 71

Anchusa ochroleuca:

(A) habitus; (B) cima fruttifera.

Barra di scala: $10 \mathrm{~cm}$.

Disegno di L. Cecchi.

(A: Italia, Friuli Venezia

Giulia, Stazione di Draga

(TS), 1894, Marchesetti, FI.

B: Italia, Toscana, valle del

Frigido (MS), 1890, Pellegrini, $\mathrm{FI})$.

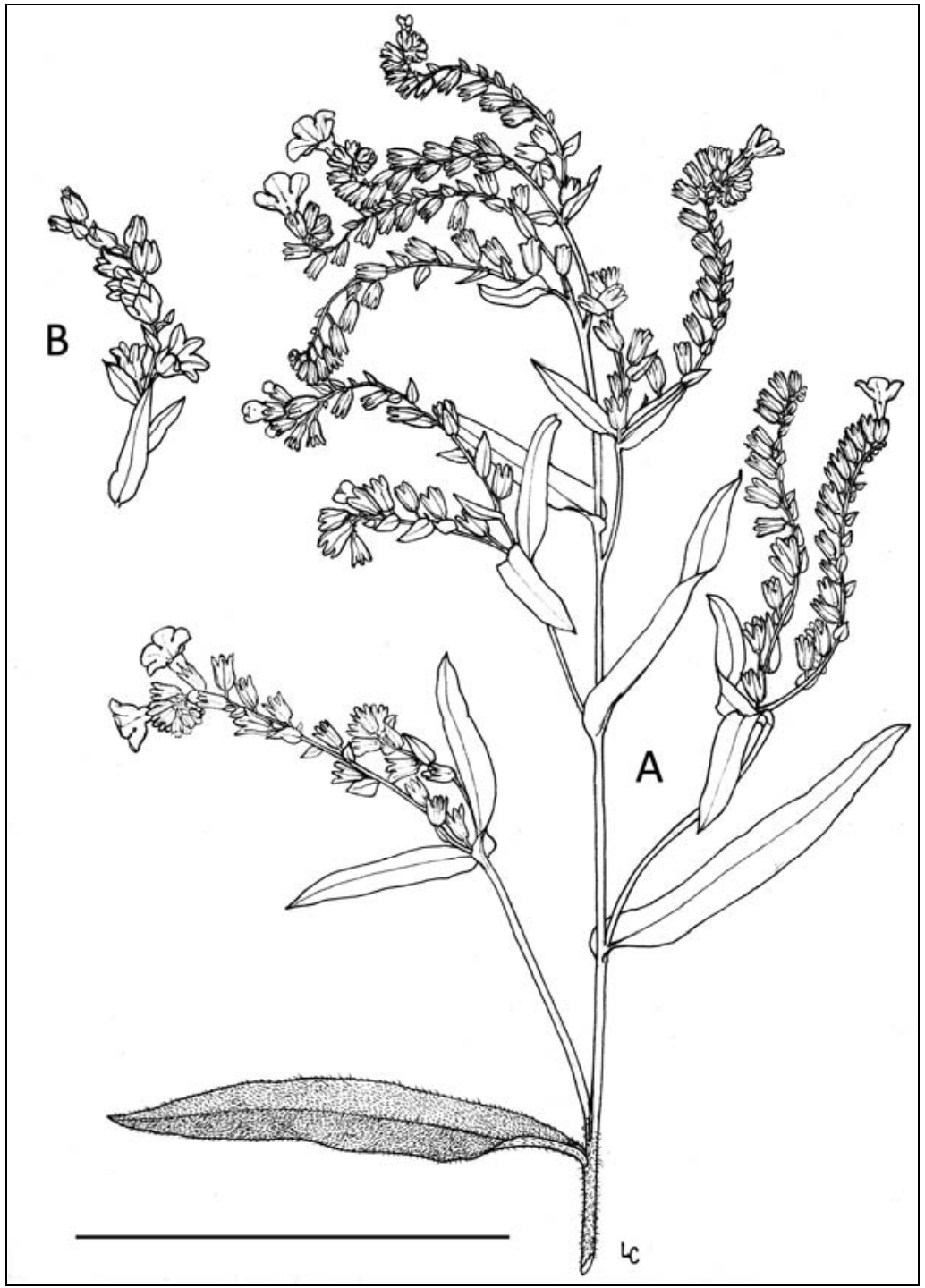


Figura 72

Distribuzione italiana di

Anchusa ochroleuca.

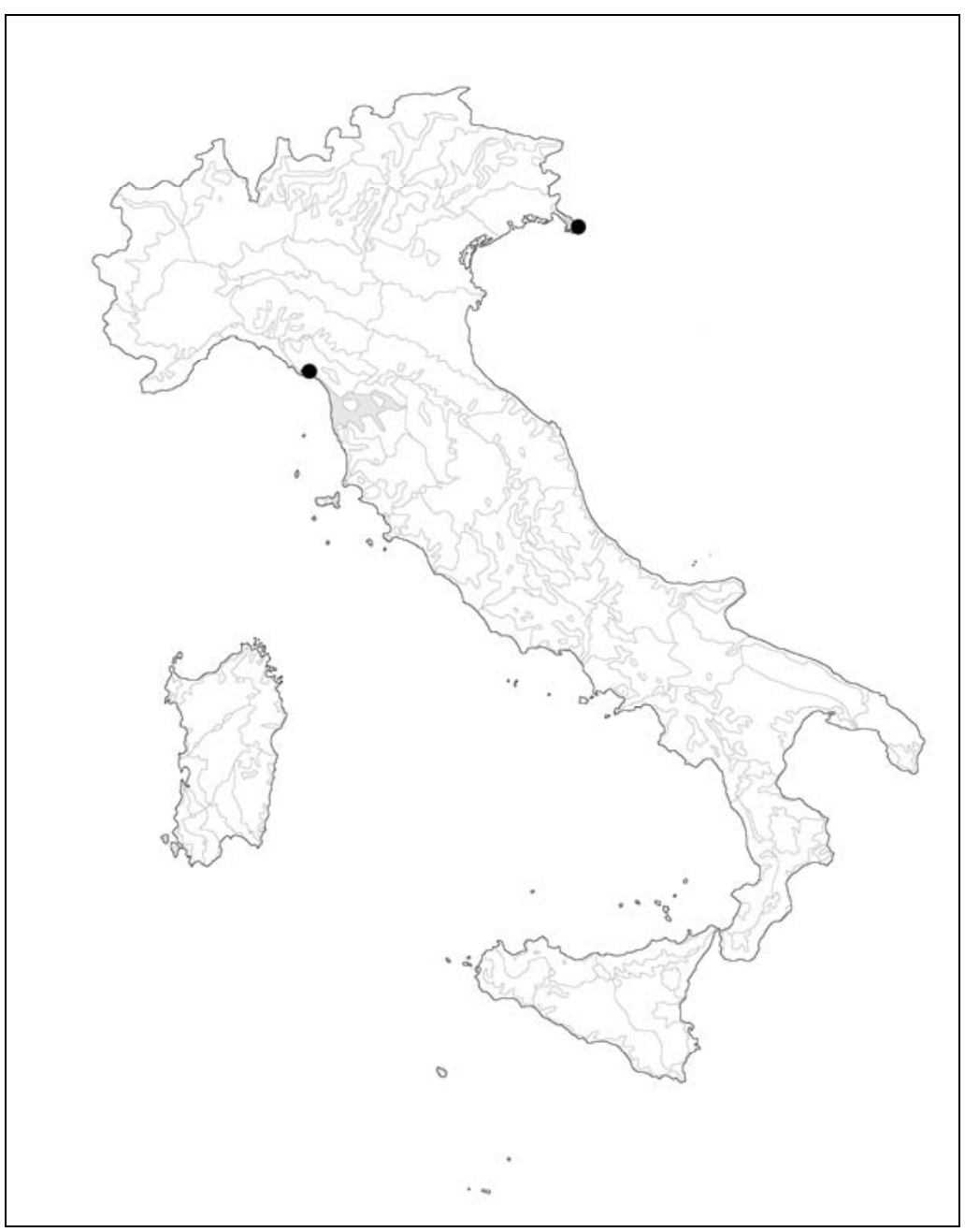


Anchusa officinalis subsp.

officinalis:

(A) habitus; (B) calice

fruttifero; (C, D) corolla chiusa e aperta; (E) gineceo;

$(F, G)$ mericarpo in visione

ventro-laterale e dorsale.

Barra di scala: $A=3 \mathrm{~cm}$; $B-E$

$=6 \mathrm{~mm} ; \mathrm{F}-\mathrm{G}=3 \mathrm{~mm}$.

Disegno di A. Maury

(modificato da Selvi \&

BIGAZZI 1998: 132).

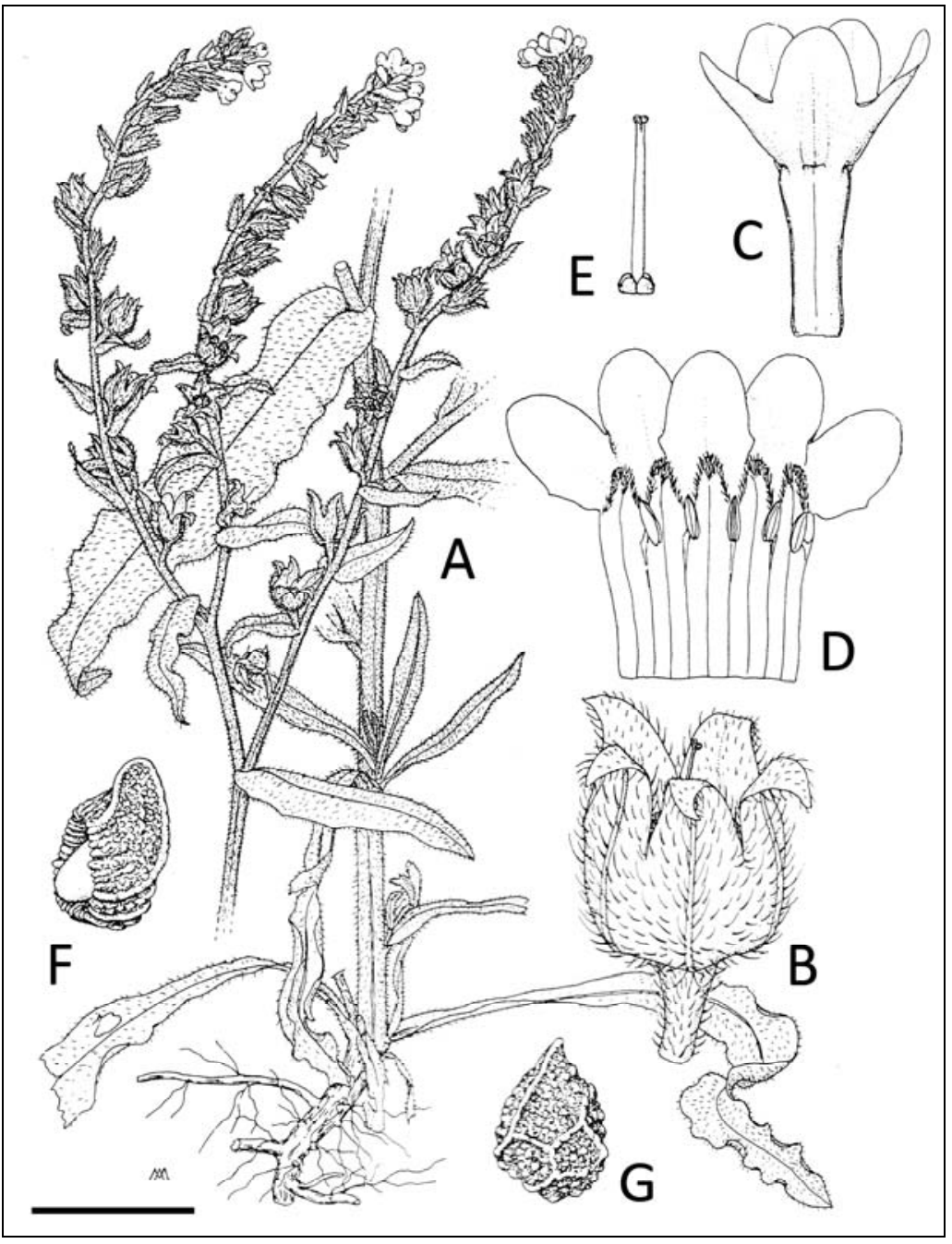


Figura 74

Distribuzione italiana di Anchusa officinalis subsp. officinalis

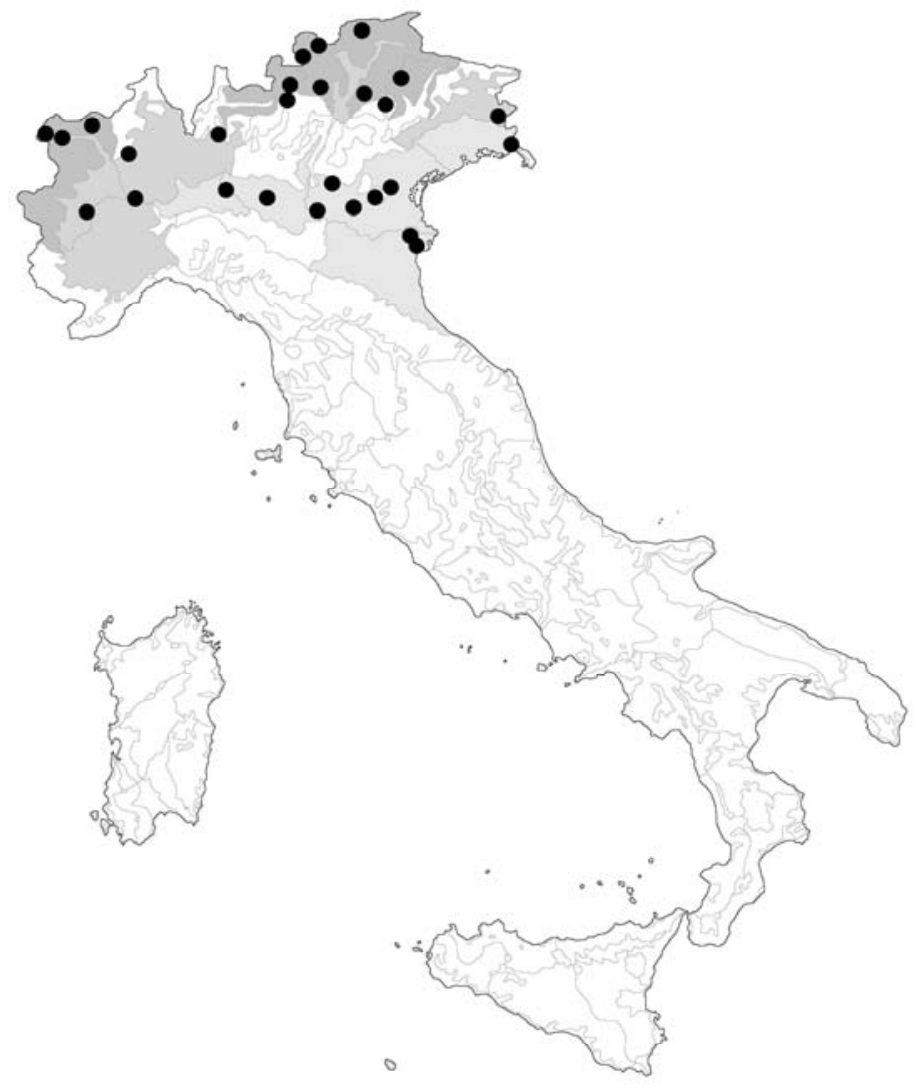




\section{Figura 75}

Anchusa sardoa:

(A) habitus; (B) fiore con

brattea; (C) superficie interna del sepalo; (D) corolla aperta; (E) gineceo;

(F) mericarpo in visione laterale.

Barra di scala: $A=5 \mathrm{~cm}$; B-D

$=1 \mathrm{~cm} ; \mathrm{E}, \mathrm{F}=5 \mathrm{~mm}$.

Disegno di A. Maury

(da Selvi \& BigazZI 1998:

137).

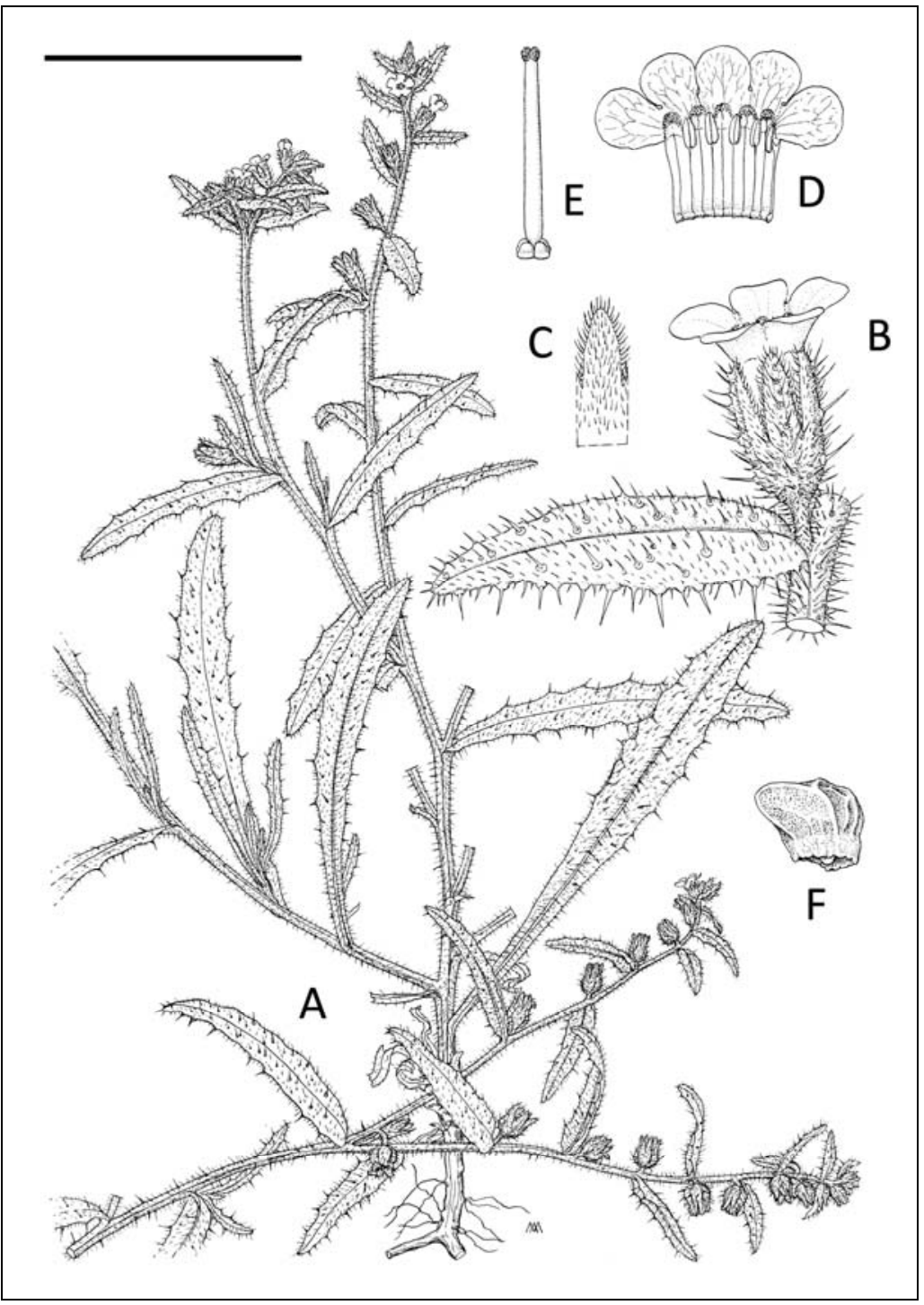


Figura 76

Distribuzione italiana di Anchusa sardoa (locus classicus).

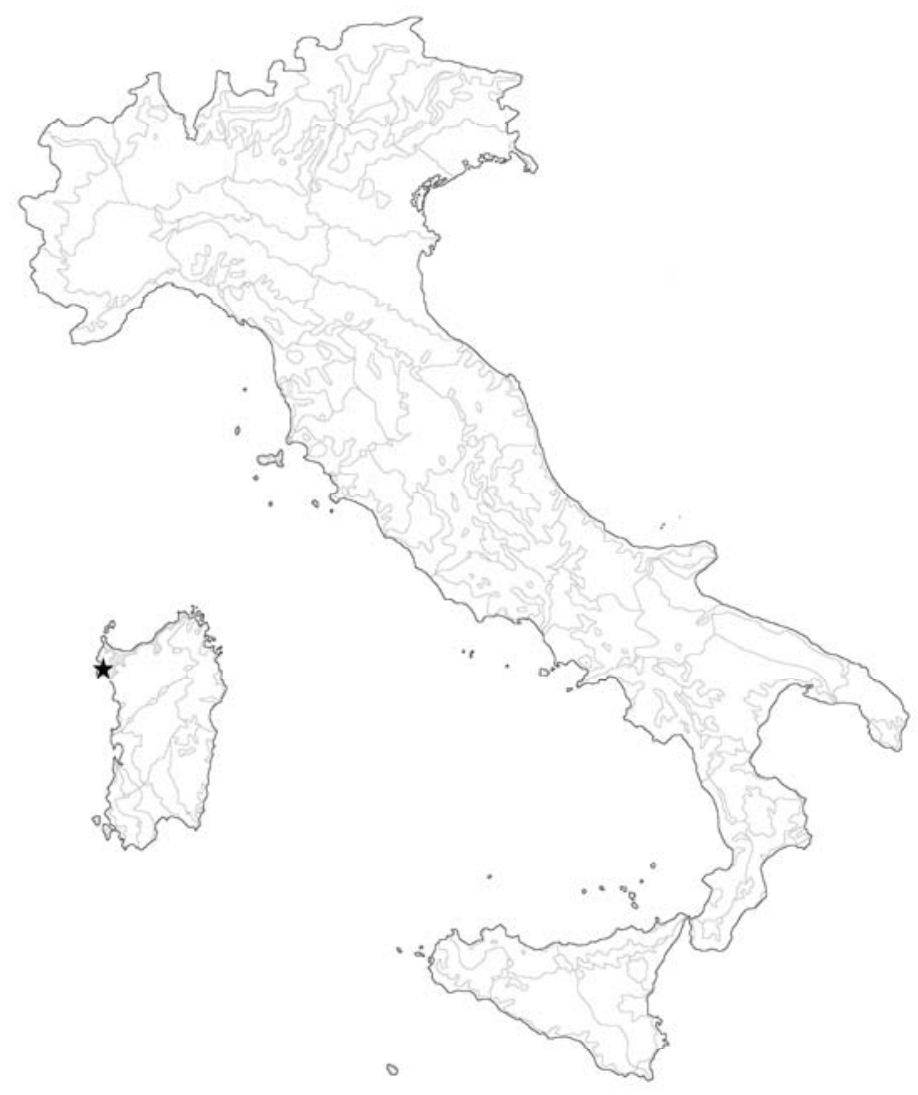




\section{Figura 77 \\ Anchusa undulata subsp. hybrida}

(da pianta dell'Italia centrale):

(A) habitus; (B) fiore con brattea; (C) corolla aperta; $(E)$ gineceo; $(F)$ mericarpo in visione laterale. Anchusa undulata subsp. undulata (da pianta della Grecia nord-occidentale): (D) corolla aperta. Barra di scala: $A=5 \mathrm{~cm} ; B$, $C, D, F=1 \mathrm{~cm} ; E=5 \mathrm{~mm}$. Disegno di A. Maury (da SelvI 1998: 263).
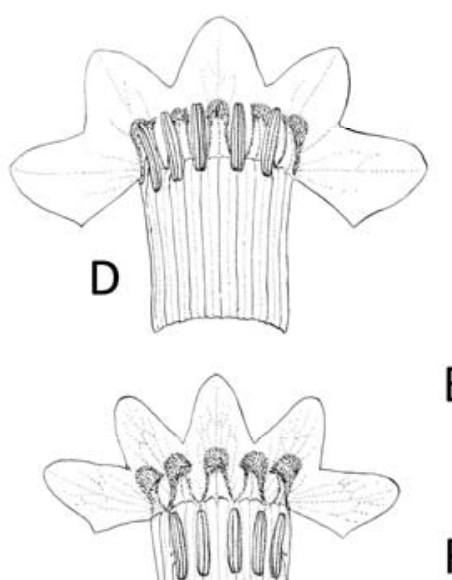

E

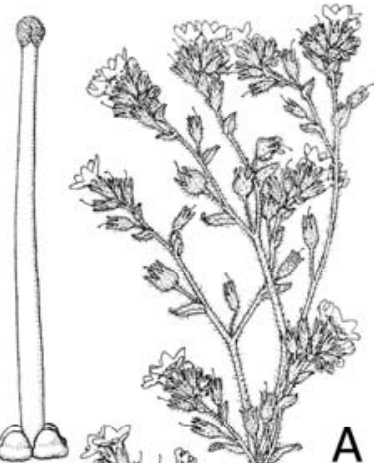

C

B

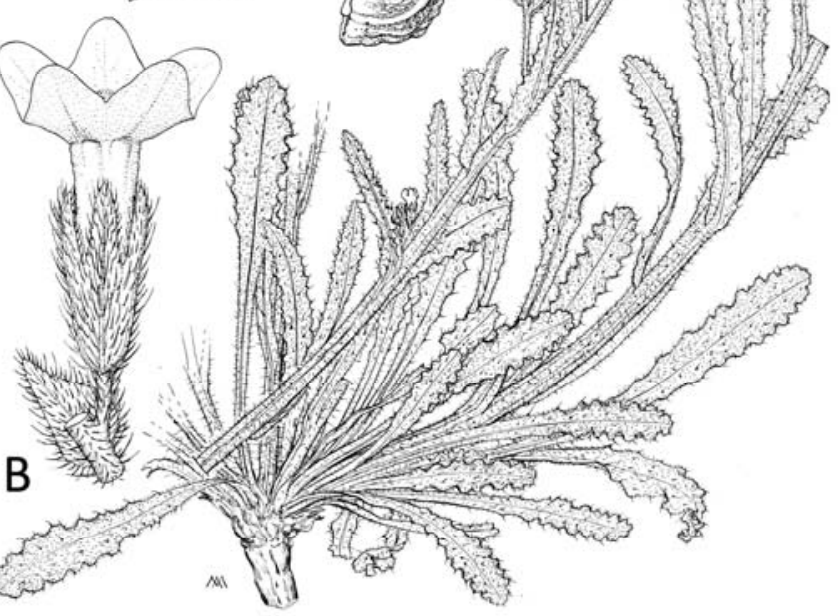


Figura 78

Distribuzione italiana di Anchusa undulata subsp. hybrida (la stella indica il locus classicus).

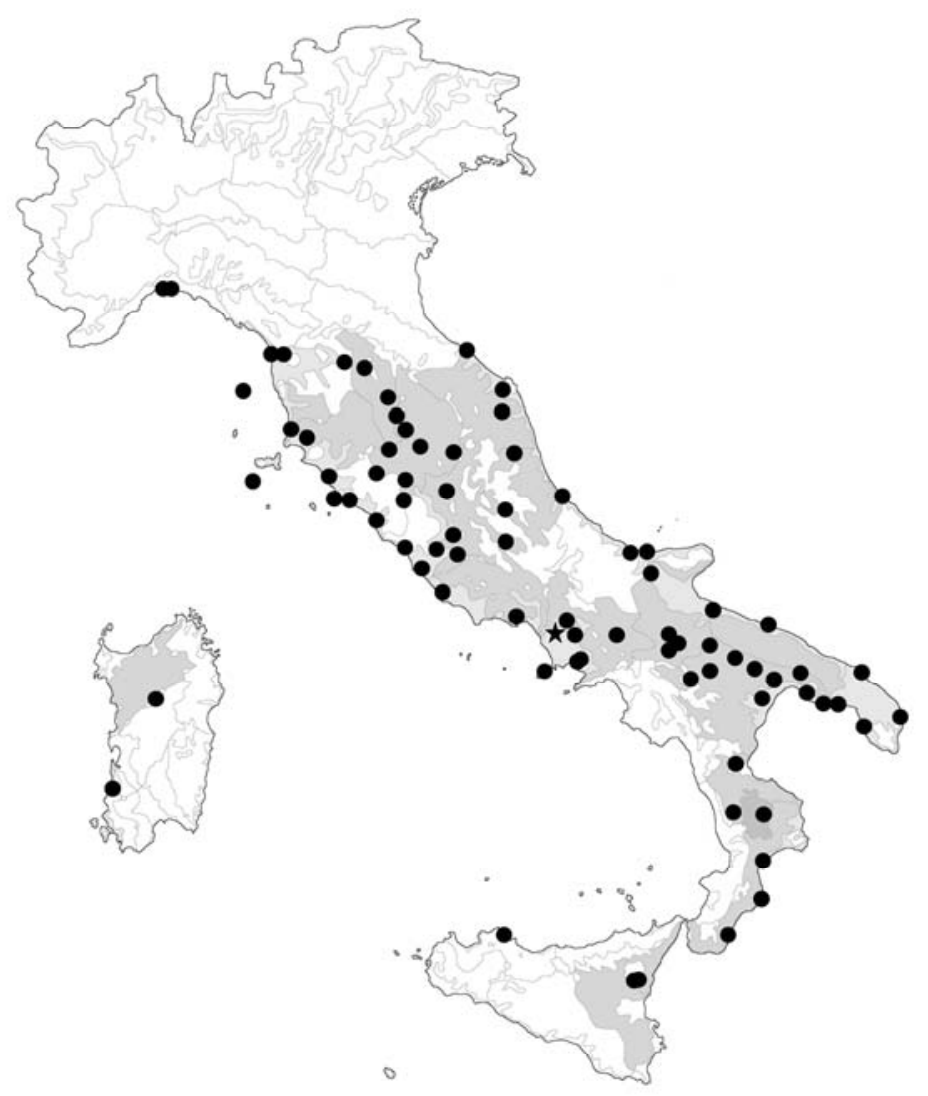

